\title{
Searches for New Physics at MiniBooNE: Sterile Neutrinos and Mixing Freedom
}

\author{
by \\ Georgia S. Karagiorgi \\ Submitted to the Department of Physics \\ in Partial Fulfillment of the Requirements for the Degree of \\ Doctor of Philosophy in Physics \\ at the

\section{MASSACHUSETTS INSTITUTE OF TECHNOLOGY}

September 2010

(c) 2010 Georgia S. Karagiorgi. All Rights Reserved.

The author hereby grants to MIT permission to reproduce and distribute publicly paper and electronic copies of this thesis document in whole or in part.

Signature of Author:

Department of Physics July 6,2010

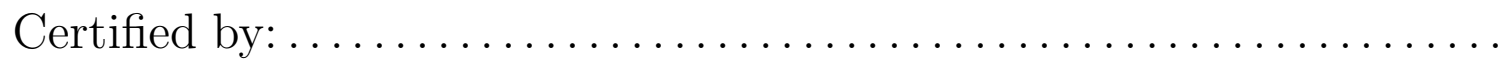

Janet M. Conrad Professor of Physics Thesis Supervisor

Accepted by: .

Krishna Rajagopal Chairman, Departmental Committee on Graduate Students 


\title{
Searches for New Physics at MiniBooNE: Sterile Neutrinos and Mixing Freedom
}

by

\author{
Georgia S. Karagiorgi
}

\author{
Submitted to the Department of Physics \\ on July 6, 2010, in partial fulfillment of the \\ requirements for the Degree of \\ Doctor of Philosophy in Physics
}

\begin{abstract}
The MiniBooNE experiment was designed to perform a search for $\nu_{\mu} \rightarrow \nu_{e}$ oscillations in a region of $\Delta m^{2}$ and $\sin ^{2} 2 \theta$ very different from that allowed by standard, threeneutrino oscillations, as determined by solar and atmospheric neutrino experiments. This search was motivated by the LSND experimental observation of an excess of $\bar{\nu}_{e}$ events in a $\bar{\nu}_{\mu}$ beam which was found compatible with two-neutrino oscillations at $\Delta m^{2} \sim 1 \mathrm{eV}^{2}$ and $\sin ^{2} 2 \theta<1 \%$. If confirmed, such oscillation signature could be attributed to the existence of a light, mostly-sterile neutrino, containing small admixtures of weak neutrino eigenstates. In addition to a search for $\nu_{\mu} \rightarrow \nu_{e}$ oscillations, MiniBooNE has also performed a search for $\bar{\nu}_{\mu} \rightarrow \bar{\nu}_{e}$ oscillations, which provides a test of the LSND two-neutrino oscillation interpretation that is independent of $C P$ or CPT violation assumptions. This dissertation presents the MiniBooNE $\nu_{\mu} \rightarrow \nu_{e}$ and $\bar{\nu}_{\mu} \rightarrow \bar{\nu}_{e}$ analyses and results, with emphasis on the latter. While the neutrino search excludes the two-neutrino oscillation interpretation of LSND at 98\% C.L., the antineutrino search shows an excess of events which is in agreement with the twoneutrino $\bar{\nu}_{\mu} \rightarrow \bar{\nu}_{e}$ oscillation interpretation of LSND, and excludes the no oscillations hypothesis at $96 \%$ C.L. Even though the neutrino and antineutrino oscillation results from MiniBooNE disagree under the single sterile neutrino oscillation hypothesis, a simple extension to the model to include additional sterile neutrino states and the possibility of $C P$ violation allows for differences between neutrino and antineutrino oscillation signatures. In view of that, the viability of oscillation models with one or two sterile neutrinos is investigated in global fits to MiniBooNE and LSND data, with and without constraints from other oscillation experiments with similar sensitivities to those models. A general search for new physics scenarios which would lead to effective non-unitarity of the standard $3 \times 3$ neutrino mixing matrix, or mixing freedom, is also performed using neutrino and antineutrino data available from MiniBooNE.
\end{abstract}

Thesis Supervisor: Janet M. Conrad

Title: Professor of Physics 


\section{Acknowledgments}

I owe this work to my advisor, Janet Conrad. Janet, you have been not only an unending source of inspiration for me these past five years, but also the reason I joined neutrino physics in the first place. Our very first meeting during the Columbia graduate student open house in 2005 was what introduced me to the excitement of neutrinos, while our interaction since then has made me appreciate the richness of this field. Thank you for the opportunity of becoming your advisee, and for your guidance and dedication over these past five years.

I can't express how grateful I am to all the people I've worked most closely with during these past five years: I must begin with Michel Sorel, who has been like an older brother to me since the summer of 2005, introducing me to his work on sterile neutrinos and supporting me as I followed in his footsteps; Mike Shaevitz, for looking over my shoulder on practically every analysis I have performed as a graduate student-Mike, I'm looking forward to continuing working with you for many years to come; Alexis Aguilar-Arevalo and Dave Schmitz, thank you for helping me tame this monster called CombinedFit, and for so patiently guiding me through the intricate details of the MiniBooNE appearance analysis.

There are many others with whom I have frequently interacted these past three years and learned so much from, be it physics or lessons in life: Sam Zeller, Bill Louis, Richard Van de Water, Chris Polly, Steve Brice, Byron Roe, Bonnie Fleming, Teppei Katori, Peter Fisher, Zelimir Djurcic, Van McGary, Geoff Mills, Josh Spitz, Zarko Pavlovic, Kendall Mahn, Niki Saoulidou, thank you, I've learned so much from you.

Thank you MiniBooNE collaboration, for your support, guidance, and constructive criticism over the past three years, which helped me see this analysis through. Of course, I could have never done this without each and every one of you. And thank you MicroBooNE collaboration, for doing your part in keeping the future of neutrino physics exciting.

I must humbly express my thank you to Boris Kayser. Boris, thank you for your valuable contributions to the field of neutrino physics. Thank you for our long and 
enlightening discussions on mixing freedom, and thank you for continually making me realize how much we assume and how little we know for fact when it comes to neutrinos.

Thank you parents, sisters, brothers and friends for your love and support over the past five years. Thank you for believing in me, encouraging me when times got difficult, and for your understanding all those times I forgot to call, email, or wish you happy birthday on your actual birthday. I am looking forward to being a more responsible daughter, sister and friend in my post-doctoral life!

And last but not least, thank you Stephen, for keeping me sane through all of this. I love you, and I can't wait to marry you! 


\section{Contents}

1 Neutrinos in the Standard Model $\quad 17$

1.1 The Standard Model of Particle Physics _ . . . . . . . . . . . . 17

1.1.1 In Search of a Final Theory of Particle Physics . . . . . . . . 17

1.1.2 Standard Model Particles and Their Interactions . . . . . . . 18

1.1.3 Massless Neutrinos in the Standard Model . . . . . . . . . . 20

1.2 The First Clues to Neutrino Mass . . . . . . . . . . . . . . . . . . . 23

1.3 Extending the Standard Model to Accommodate Neutrino Mass and Leptonic Mixing . . . . . . . . . . . . . . . . . . . . 27

1.3.1 Dirac Neutrino Mass . . . . . . . . . . . . . . . . . 27

1.3.2 On the Lightness of Neutrino Mass: The See-Saw Model . . . 28

1.3.3 Incorporating Neutrino Mixing . . . . . . . . . . . . 31

1.3.4 Neutrino Oscillations . . . . . . . . . . . . . . . . . 32

1.3.5 An Instructive Example: Two-Neutrino Oscillations . . . . . . 35

1.4 Results of the Last Two Decades . . . . . . . . . . . . . . . 38

1.4.1 Carving Out the Three-Neutrino Oscillation Parameters . . . 38

1.4.2 Direct Limits on Neutrino Mass . . . . . . . . . . . . . . 46

1.4.3 Constraints from Cosmology . . . . . . . . . . . . . 47

2 Neutrinos as Windows to New Physics 49

2.1 Mixing Freedom . . . . . . . . . . . . . . . . . 50

2.1 .1 Underlying Scenarios . . . . . . . . . . . . . . . . 51

2.1.2 How Mixing Freedom Can Arise: Light Sterile Neutrinos . . . 52

2.1.3 Physical Effects of Mixing Freedom . . . . . . . . . . . 55 
2.1.4 Experimental Constraints . . . . . . . . . . . . 57

2.2 A Close Look at Light Sterile Neutrinos _ . . . . . . . . . . 63

2.2.1 Light Sterile Neutrino Oscillation Formalism . . . . . . . . . 63

2.2 .2 The LSND Signal . . . . . . . . . . . . . . . . . . . . . 69

2.2.3 Constraints to Light Sterile Neutrino Models . . . . . . . . . 74

2.3 Synopsis of Thesis . . . . . . . . . . . . . . . 75

3 The MiniBooNE Experiment $\quad 77$

3.1 Overview . . . . . . . . . . . . . . . . . . . . . . . . . 77

3.2 The Booster Neutrino Beam . . . . . . . . . . . . . . 79

3.2 .1 Primary Proton Beam . . . . . . . . . . . . . 79

3.2 .2 Proton Target . . . . . . . . . . . . . . . . . . 80

3.2 .3 Focusing of Secondary Mesons . . . . . . . . . . . . . . 81

3.2.4 Secondary Meson Decay Pipe . . . . . . . . . . . . . . 82

3.3 The MiniBooNE Detector $\ldots \ldots \ldots \ldots$

3.3.1 Detector Description . . . . . . . . . . . . . . 86

3.3.2 Active Detector Components and Electronics . . . . . . . . . 89

3.3.3 Detector Calibration Systems _ . . . . . . . . . . . . 92

3.4 Predicting the Neutrino Event Rates at MiniBooNE . . . . . . . . . 92

3.4.1 Neutrino Flux Prediction . . . . . . . . . . . . . . . . . . 92

3.4 .2 Neutrino Interaction Simulations _ . . . . . . . . . . 97

3.4.3 Neutrino Cross-Sections at $\sim 1 \mathrm{GeV} \ldots \ldots \ldots$. . . . . 98

3.4.4 Neutrino Event Rate Predictions . . . . . . . . . . . . 107

3.5 Neutrino Events in the MiniBooNE Detector . . . . . . . . . . . . 109

3.5.1 Event Signatures . . . . . . . . . . . . . . . . . . . 109

3.5.2 Isolating Neutrino Events _ . . . . . . . . . . . . . . 112

3.5.3 Event Reconstruction . . . . . . . . . . . . . . . . . . 114

4 The MiniBooNE $\nu_{e}$ and $\bar{\nu}_{e}$ Appearance Searches 118

4.1 Overview . . . . . . . . . . . . . . . . . . . . . . . . . . . . . . 119

4.2 CCQE Event Selection . . . . . . . . . . . . . . . . . . 122 
4.2 .1 Preliminary Event Selection _. . . . . . . . . . . . . . 122

4.2.2 $\nu_{\mu}$ CCQE Event Selection Requirements . . . . . . . . . 123

$4.2 .3 \nu_{e}$ CCQE Event Selection Requirements . . . . . . . . . . 124

4.3 Central Values . . . . . . . . . . . . . . . . . . . . . . . . . . . . . . . . . 129

4.3.1 $\nu_{\mu}$ CCQE Data and Predicted Samples: Neutrino Mode . . . . 130

4.3.2 $\nu_{\mu}$ CCQE Data and Predicted Samples: Antineutrino Mode . 131

4.3.3 $\nu_{e}$ CCQE Predicted Samples: Neutrino Mode . . . . . . . . . 134

4.3.4 $\nu_{e}$ CCQE Predicted Samples: Antineutrino Mode . . . . . . . 136

4.4 Oscillation Fit Method . . . . . . . . . . . . . . . . . . 137

$4.4 .1 \chi^{2}$ Definition . . . . . . . . . . . . . . 137

4.4.2 The $\nu_{\mu}$ CCQE Constraint in the $\nu_{e}$ and $\bar{\nu}_{e}$ Appearance Fits . . 140

4.4.3 Scaling the Covariance Matrix . . . . . . . . . . . . . . . . 142

4.4.4 Finding the Best-fit Oscillation Parameters . . . . . . . . . . 144

4.4.5 On the Drawing of Confidence Level Intervals . . . . . . . . . 144

4.4.6 Quantifying Goodness-of-fit . . . . . . . . . . . . . . 147

4.5 Constraining the $\nu_{e}$ CCQE Background Predictions $\ldots \ldots \ldots \ldots$

4.5.1 Intrinsic $\nu_{e}$ and $\nu_{\mu}$ CCQE Mis-identified Backgrounds . . . 148

4.5.2 Mis-identified NC $\pi^{0}$ Backgrounds . . . . . . . . . . . . . 150

4.5.3 Mis-identified $\Delta \rightarrow N \gamma$ Backgrounds $\ldots \ldots \ldots \ldots$

4.5.4 Dirt Backgrounds . . . . . . . . . . . . . . . . . . . 154

4.6 Accounting for Systematic and Statistical Uncertainties . . . . . . 155

4.6.1 Flux Systematic Uncertainties . . . . . . . . . . . . . . 159

4.6.2 Cross-Section Systematic Uncertainties . . . . . . . . . . . 163

4.6.3 Detector Systematic Uncertainties . . . . . . . . . . . . . . 164

4.6.4 Statistical Uncertainties _. . . . . . . . . . . . . . 166

4.7 Sensitivity to $\nu_{e}$ and $\bar{\nu}_{e}$ Appearance $\ldots \ldots \ldots \ldots \ldots \ldots$

5 MiniBooNE Appearance Results 171

$5.1 \quad \nu_{e}$ Appearance Results . . . . . . . . . . . . . . . . . . . 171

5.1 .1 Oscillation Search . . . . . . . . . . . . . . . . 171 
5.1 .2 The Low Energy Excess . . . . . . . . . . . . . . . . 174

$5.2 \bar{\nu}_{e}$ Appearance Results . . . . . . . . . . . . . . . . . . . . . 182

5.2 .1 Oscillation Search . . . . . . . . . . . . . . . . . . 182

5.2.2 A Closer Look at the Antineutrino Mode Excess . . . . . . . . 189

5.2.3 On the Possibility of a Low Energy Excess in Antineutrinos . 200

5.3 Sensitivity to $\bar{\nu}_{e}$ Appearance for $10.0 \times 10^{20}$ POT . . . . . . . . . 201

6 Interpretation of MiniBooNE and LSND Appearance Results in Global Light Sterile Neutrino Oscillation Fits 205

7 A Search for Mixing Freedom at MiniBooNE 226

7.1 General Assumptions for a Search for Instantaneous Transitions . . . 227

7.2 Predicting MiniBooNE Event Rates When Mixing Is Free . . . . . . 229

7.3 Fit Method . . . . . . . . . . . . . . . . . 231

7.3.1 Fit I: $\nu_{\mu} \rightarrow \nu_{e}$ Transitions $\ldots \ldots \ldots \ldots \ldots \ldots$

7.3.2 Fit II: $\nu_{\mu} \rightarrow \nu_{e}, \nu_{\mu} \rightarrow \nu_{\mu}$ and $\nu_{e} \rightarrow \nu_{\theta}$ Transitions $\ldots \ldots .232$

7.4 Fit Results . . . . . . . . . . . . . . . . . . . . . 237

7.4.1 Fit I: $\nu_{\mu} \rightarrow \nu_{e}$ Transitions $\ldots \ldots \ldots \ldots \ldots 237$

7.4.2 Fit II: $\nu_{\mu} \rightarrow \nu_{e}, \nu_{\mu} \rightarrow \nu_{\mu}$ and $\nu_{e} \rightarrow \nu_{q}$ Transitions $\ldots \ldots .238$

7.5 Final Remarks . . . . . . . . . . . . . . . . . . . . 241

8 Conclusions 245

A MiniBooNE Analysis Tools and Cross Checks 248

A.1 Reweighting . . . . . . . . . . . . . . . . . . . 248

A.2 The $\nu_{\mu}-\nu_{e}$ Combined Fit Constraint . . . . . . . . . . 249

A.3 Obtaining a Shape-only Error Matrix . . . . . . . . . . . . . 252

A.4 Absorber Studies in Antineutrino Mode . . . . . . . . . . . . . 253

B Future Searches: MicroBooNE 259

B.1 Introduction . . . . . . . . . . . . . . . . . . . . . . . . 259

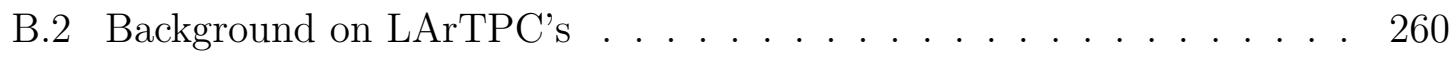


B.3 The MicroBooNE Detector . . . . . . . . . . . . . . . . . . . . 261

B.4 Neutrino Interactions in MicroBooNE . . . . . . . . . . . 267

B.5 MicroBooNE Physics Potential _. . . . . . . . . . . . . . . 268

B.5.1 Investigation of MiniBooNE Low Energy Excess . . . . . . . 270

B.5.2 Neutrino Oscillations . . . . . . . . . . . . . . . . . . 272

B.5.3 Antineutrino Oscillations . . . . . . . . . . . . . . . . . . . . 274

B.6 R\&D Studies for LAr Scintillation Light Detection . . . . . . . . . 276

B.6.1 TPB As a VUV to Visible Wavelength Shifting Material . . . 278

B.6.2 Light Collection Efficiency of Two Geometry Configurations 280

B.6.3 Coating Options and Plate Materials _ . . . . . . . . . . 282

B.6.4 Light Transmittance and Fluorescence Studies . . . . . . . . 287

B.6.5 Summary of R\&D Studies . . . . . . . . . . . . . . . . . 291 


\section{List of Figures}

1-1 Particle spectrum of the Standard Model . . . . . . . . . . . . . 19

1-2 Summary of interactions between particles in the Standard Model . . 20

1-3 Solar neutrino flux measurements from SNO . . . . . . . . . 26

1-4 Disappearance and appearance oscillation effects . . . . . . . . . . 37

1-5 Three-neutrino mass spectrum . . . . . . . . . . . . . . . . 39

1-6 Summary of Super-K, K2K, and MINOS oscillation results . . . . . 41

1-7 Summary of KamLAND oscillation results . . . . . . . . . . 43

1-8 Oscillation parameters from solar and reactor neutrino experiments . 44

1-9 Current experimental limits on $\sin ^{2} \theta_{13}$ from $\mathrm{CHOOZ} \ldots \ldots . . . .45$

$2-1 \quad(3+1)$ neutrino mass spectrum $\ldots \ldots \ldots \ldots \ldots \ldots \ldots$

$2-2 \quad(3+2)$ neutrino mass spectrum $\ldots \ldots \ldots \ldots \ldots \ldots \ldots$

$2-3 \quad$ LSND experimental layout . . . . . . . . . . . . 70

2-4 Neutrino flux spectra at the LSND detector . . . . . . . . 70

2-5 LSND observed excess of $\bar{\nu}_{e}$ events $\ldots \ldots \ldots \ldots \ldots$

2-6 LSND-allowed oscillation parameters . . . . . . . . . . . 74

3-1 Schematic drawing of the MiniBooNE experiment at Fermilab . . . 78

3-2 The booster neutrino beamline at Fermilab . . . . . . . . . 80

3-3 Proton beam timing structure . . . . . . . . . . . . . . . 81

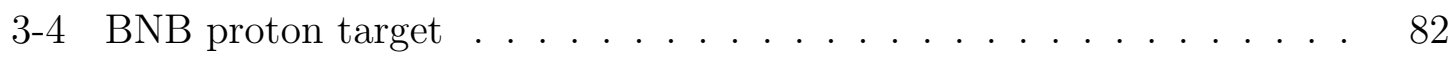

3-5 The MiniBooNE magnetic horn and its effect on the neutrino flux . . 83

3-6 Protons delivered to the BNB target over time . . . . . . . . . 84

3-7 Absorber configuration in the BNB decay pipe . . . . . . . . . 85 
3-8 Effect of absorber running on the antineutrino event rates . . . . . 86

3-9 The MiniBooNE detector . . . . . . . . . . . . . . . . . . 87

3-10 The MiniBooNE veto and tank PMT's . . . . . . . . . . . . . . . . 89

3-11 PMT signal digitization . . . . . . . . . . . . . . . . 90

3-12 The MiniBooNE cosmic ray muon calibration system . . . . . . . . 91

3-13 Stability of reconstructed Michel energy over time . . . . . . . . . . . 91

3-14 Neutrino flux spectra at the MiniBooNE detector . . . . . . . . . 95

3-15 Neutrino charged-current cross-sections at $\sim 1 \mathrm{GeV}$. . . . . . . . . . 99

3-16 The main neutrino CC interaction processes in MiniBooNE . . . . . . 100

3-17 The main neutrino NC interaction processes in MiniBooNE . . . . . . 100

3-18 MiniBooNE $\nu_{\mu}$ CCQE data and MonteCarlo prediction . . . . . . . . 104

3-19 The effect of pion absorption in single pion production . . . . . . . 105

3-20 PMT hit topologies for electrons, muons, or photons . . . . . . . . . . 111

3-21 Time distribution of PMT hits for one- and two-subevent events . . . 112

3-22 Subevent distribution within the beam trigger window after pre-cuts . 113

3-23 Neutrino interactions per POT as a function of time . . . . . . . . . . 114

3-24 Track parameters used in event reconstruction . . . . . . . . . . 116

4-1 External interactions contributing as background . . . . . . . . 126

4-2 Effect of $\nu_{e}$ CCQE PID cuts . . . . . . . . . . . . . . . 128

4-3 Neutrino mode $\nu_{\mu}$ CCQE central values . . . . . . . . . . . . . . 130

4-4 Neutrino mode $\nu_{\mu}$ CCQE prediction breakup . . . . . . . . . . . . . . 132

4-5 Antineutrino mode $\nu_{\mu}$ CCQE prediction breakup . . . . . . . . 133

4-6 Antineutrino mode $\nu_{\mu}$ CCQE central values . . . . . . . . . . . 134

4-7 $\nu_{e}$ CCQE background prediction in neutrino mode . . . . . . . . 135

$4-8 \quad \nu_{e}$ CCQE signal prediction in neutrino mode . . . . . . . . . . 136

4-9 $\quad \nu_{e}$ CCQE background prediction in antineutrino mode . . . . . . . . 139

4-10 $\nu_{e}$ CCQE signal prediction in antineutrino mode . . . . . . . . . . 140

4-11 Ancestor phase-space overlap for $\nu_{e}$ and $\nu_{\mu}$ flux $\ldots \ldots$. . . . . . 141

4-12 Effect of $\nu_{\mu}$ CCQE constraint on $\bar{\nu}_{e}$ appearance sensitivity $\ldots \ldots$. . 142 
4-13 Covariance matrix collapse . . . . . . . . . . . . . . . . . . 143

4-14 Fake data $\Delta \chi_{i}^{2}$ distribution at the null point $\ldots \ldots \ldots \ldots . . .146$

4-15 Effect of $\mathrm{NC} \pi^{0}$ rate correction in neutrino mode . . . . . . . . . 154

4-16 Effect of $\mathrm{NC} \pi^{0}$ rate correction in antineutrino mode . . . . . . 155

4-17 Effect of systematic uncertainties on $\bar{\nu}_{e}$ appearance sensitivity $\ldots . .160$

4-18 Effect of $E_{\nu}^{Q E}>475 \mathrm{MeV}$ on appearance sensitivity . . . . . 168

4-19 MiniBooNE's sensitivity to $\nu_{\mu} \rightarrow \nu_{e}$ oscillations in neutrino mode . . 169

4-20 MiniBooNE's sensitivity to $\bar{\nu}_{\mu} \rightarrow \bar{\nu}_{e}$ oscillations in antineutrino mode 170

5-1 Neutrino mode $\nu_{e}$ CCQE data and background distributions $\ldots \ldots .172$

5-2 MiniBooNE limit to $\nu_{\mu} \rightarrow \nu_{e}$ oscillations in neutrino mode . . . . . 174

5-3 Limit to $\nu_{\mu} \rightarrow \nu_{e}$ oscillations in neutrino mode for $E_{\nu}^{Q E}>200 \mathrm{MeV}$. 175

5-4 $\quad E_{v i s}$ and $E_{\nu}^{Q E}$ event excess distributions in neutrino mode . . . . 176

5-5 $Q^{2}, \cos \theta_{z}$, and $E_{v i s}$ event distributions in neutrino mode . . . . 178

5-6 Excess to single-photon background shape comparisons in $\nu$ mode . . 179

5-7 Excess to single-electron background shape comparisons in $\nu$ mode . . 180

5-8 Antineutrino mode $\nu_{e}$ CCQE data and background distributions . . . 183

5-9 Antineutrino mode $\nu_{e}$ CCQE data and best-fit distributions $\ldots \ldots .184$

5-10 $E_{\nu}^{Q E}$ event excess distribution in antineutrino mode $\ldots \ldots \ldots . . .186$

5-11 MiniBooNE allowed regions for $\bar{\nu}_{\mu} \rightarrow \bar{\nu}_{e}$ oscillations: $E_{\nu}^{Q E}>475 \mathrm{MeV} 188$

5-12 MiniBooNE allowed regions for $\bar{\nu}_{\mu} \rightarrow \bar{\nu}_{e}$ oscillations: $E_{\nu}^{Q E}>200 \mathrm{MeV} 188$

5-13 $Q^{2}, \cos \theta_{z}$, and $E_{v i s}$ event distributions in antineutrino mode . . . 190

5-14 Excess to $\bar{\nu}_{\mu} \rightarrow \bar{\nu}_{e}$ oscillation signal comparisons in $\bar{\nu}$ mode . . . . . 191

5-15 Excess to single-photon background shape comparisons in $\bar{\nu}$ mode . . 192

5-16 Excess to single-electron background shape comparison in $\bar{\nu}$ mode . . 193

5-17 Projected $\bar{\nu}_{\mu} \rightarrow \bar{\nu}_{e}$ sensitivity for $10.0 \times 10^{20}$ POT . . . . . . . . 201

5-18 Projected $\bar{\nu}_{\mu} \rightarrow \bar{\nu}_{e}$ oscillation fit results for $10.0 \times 10^{20}$ POT $\ldots \ldots .202$

5-19 Projected exclusion of no oscillation hypothesis vs. POT . . . . . . 203

5-20 Projected $\bar{\nu}_{\mu} \rightarrow \bar{\nu}_{e}$ oscillation signal significance vs. POT . . . . . 204

7-1 Constraints to $\left|\left(U U^{\dagger}\right)_{e \mu}\right|,\left|\left(U U^{\dagger}\right)_{e e}\right|$, and $\left|\left(U U^{\dagger}\right)_{\mu \mu}\right|$ from $\nu$ mode $\ldots .240$ 
7-2 Constraints to $\left|\left(U U^{\dagger}\right)_{\mu e}\right|,\left|\left(U U^{\dagger}\right)_{e e}\right|$, and $\left|\left(U U^{\dagger}\right)_{\mu \mu}\right|$ from $\bar{\nu}$ mode . . . 240

7-3 Neutrino mode data vs. mixing freedom best-fit prediction . . . . . . 242

7-4 Antineutrino mode data vs. mixing freedom best-fit prediction . . . . 243

8-1 MiniBooNE observed excess distributions as a function of $L / E$. . . . 246

A-1 Observed $\nu_{e}$ CCQE events per POT during antineutrino running . . 256

A-2 Observed $\nu_{e}$ CCQE event rate over time in antineutrino mode . . . 256

A-3 $\nu_{e}$ CCQE $E_{\nu}^{Q E}$ distributions for different absorber running periods . . 257

A-4 Ratio distributions for different absorber running periods . . . . . . . 258

B-1 Schematic of the MicroBooNE detector . . . . . . . . . . . . . . . 262

B-2 PMT arrangement in the detector . . . . . . . . . . . . . 263

B-3 PMT supporting frame . . . . . . . . . . . . . . . 264

B-4 PMT and custom-made PMT base . . . . . . . . . . . . . 264

B-5 MicroBooNE $d E / d x$ differentiation capability $\ldots \ldots$. . . . . . . . . 269

B-6 Expected energy spectrum for electron-like events . . . . . . . . . . . 271

B-7 Expected energy spectrum for photon-like events . . . . . . . . . . . . 272

B-8 Sensitivity to $\nu_{\mu} \rightarrow \nu_{e}$ oscillations . . . . . . . . . . 273

B-9 $\nu_{\mu} \rightarrow \nu_{e}$ signal expectations in neutrino mode . . . . . . . . . 274

B-10 Sensitivity to $\bar{\nu}_{\mu} \rightarrow \bar{\nu}_{e}$ oscillations in antineutrino mode $\ldots . . . .275$

B-11 $\bar{\nu}_{\mu} \rightarrow \bar{\nu}_{e}$ signal expectations in antineutrino mode . . . . . . . . 276

B-12 Estimates for improved $\bar{\nu}_{\mu} \rightarrow \bar{\nu}_{e}$ oscillation sensitivity $\ldots \ldots \ldots$. . 278

B-13 TPB absorption and emission spectra for LAr scintillation light . . . 279

B-14 Spectral efficiency of MicroBooNE PMT's . . . . . . . . . . . . . . . 280

B-15 Potential light collection system configurations in MicroBooNE . . . . 281

B-16 Angular efficiency of 8-inch Hamamatsu R5912 PMT's . . . . . . . . 282

B-17 Relative light collection efficiency for two configurations . . . . . . . 283

B-18 Evaporatively coated TPB plate . . . . . . . . . . . . . 285

B-19 Testing of TPB-PS drip-coated acrylic plates in liquid nitrogen . . . . 286

B-20 Vacuum test setup for TPB-coated plates . . . . . . . . . . . . . 286 
B-21 Plate transmittance measurement setup . . . . . . . . . . . . 287

B-22 Transmittance measurements for various TPB-coated plates . . . . 288

B-23 Transmittance measurements for TPB-PS drip-coated plates . . . . . 289

B-24 TPB fluorescence measurement setup . . . . . . . . . . . . . 289

B-25 Fluorescence spectra for various TPB coatings on acrylic plates . . . 290

B-26 Summary of fluorescence and transmittance measurements . . . . . 293 


\section{List of Tables}

2.1 Underlying physics scenarios which lead to mixing freedom . . . . . . 51

2.2 Experimental sources of constraints to mixing freedom . . . . . . . 57

2.3 Neutrino and antineutrino oscillation probabilities under $C P, C P T$. 68

2.4 Experimental constraints from short-baseline neutrino experiments . . 75

3.1 MiniBooNE mineral oil properties . . . . . . . . . . . . . . . 88

3.2 Processes taken into account in the MiniBooNE BNB simulation . . . 94

3.3 Neutrino flux contributions in neutrino mode . . . . . . . . . . . 96

3.4 Neutrino flux contributions in antineutrino mode . . . . . . . . . . 97

3.5 NUANCE cross-section parameters . . . . . . . . . . . . . . 98

3.6 MiniBooNE neutrino and antineutrino mode event rates . . . . . . 108

4.1 PID cut coefficients used in $\nu_{\mu}$ CCQE event selection . . . . . . . . 124

4.2 Summary of $\nu_{\mu}$ CCQE selection cuts . . . . . . . . . . . . 125

4.3 PID cut coefficients used in $\nu_{e}$ CCQE event selection . . . . . . . . 127

4.4 Summary of $\nu_{e}$ CCQE selection cuts . . . . . . . . . . . . . . 129

$4.5 \nu_{e}$ CCQE selection efficiency . . . . . . . . . . . . . 129

4.6 Expected number of $\nu_{e}$ CCQE events: $\nu_{e}$ appearance analysis . . . . . 137

4.7 Expected number of $\nu_{e}$ CCQE events: $\bar{\nu}_{e}$ appearance analysis . . . . . 138

$4.8 \Delta \chi^{2}$ cuts for various confidence level intervals . . . . . . . . . . . . . 146

$4.9 \pi^{0}$ and $\Delta \rightarrow N \gamma$ corrections and systematic uncertainties . . . . . 152

4.10 Summary of contributing systematic uncertainties . . . . . . . . . 157

4.11 Low energy systematic uncertainties . . . . . . . . . . . . 158

4.12 High energy systematic uncertainties . . . . . . . . . . . . 159 
4.13 Systematic uncertainties in the MiniBooNE flux prediction . . . . . . 161

4.14 Cross-section uncertainties in the MiniBooNE event rate prediction . 164

4.15 Generated MonteCarlo event statistics . . . . . . . . . . . . . 166

5.1 Excess to background shape comparisons in neutrino mode . . . . . . 181

5.2 Best-fit $\bar{\nu}_{\mu} \rightarrow \bar{\nu}_{e}$ oscillation parameters . . . . . . . . . . . . . 185

5.3 Event summary for the $\nu_{e}$ and $\bar{\nu}_{e}$ appearance analyses . . . . . . . . . 187

$5.4 \chi^{2}$ summary for antineutrino mode oscillation fits . . . . . . . . . 187

5.5 Background investigations in $\bar{\nu}$ mode: $E_{v i s} \ldots \ldots$. . . . . . . . 195

5.6 Background investigations in $\bar{\nu}$ mode: $\cos \theta_{z} \ldots \ldots$. . . . . . . . 196

5.7 Background investigations in $\bar{\nu}$ mode: $Q^{2} \ldots \ldots$. . . . . . . . . . 197

5.8 Intrinsic $\nu_{e}$ background investigations . . . . . . . . . . . . . . . . . . 198

7.1 MiniBooNE limits on $\nu_{\mu} \rightarrow \nu_{e}$ instantaneous transitions: $\nu$ mode . . . 237

7.2 MiniBooNE limits on $\bar{\nu}_{\mu} \rightarrow \bar{\nu}_{e}$ instantaneous transitions: $\bar{\nu}$ mode . . . 237

7.3 MiniBooNE limits on $\nu_{\mu} \rightarrow \nu_{e}, \nu_{\mu} \rightarrow \nu_{\mu}$ and $\nu_{e} \rightarrow \nu_{\theta}$ instantaneous transitions: $\nu$ mode . . . . . . . . . . . . . . . . . . . . . . . 238

7.4 MiniBooNE limits on $\bar{\nu}_{\mu} \rightarrow \bar{\nu}_{e}, \bar{\nu}_{\mu} \rightarrow \bar{\nu}_{\mu}$ and $\bar{\nu}_{e} \rightarrow \bar{\nu}_{\phi}$ instantaneous transitions: $\bar{\nu}$ mode . . . . . . . . . . . . . . . . . . . . . . . 239

7.5 Summary of constraints to mixing freedom from MiniBooNE . . . . . 243

B.1 Highlights in LArTPC development prior to MicroBooNE . . . . . . . 261

B.2 MicroBooNE BNB neutrino event rates . . . . . . . . . . . . . 266

B.3 Differences in MicroBooNE and MiniBooNE event reconstruction . . 267

B.4 Estimates for MicroBooNE $\bar{\nu}_{\mu} \rightarrow \bar{\nu}_{e}$ oscillation excess significance . . 277 


\section{Chapter 1}

\section{Neutrinos in the Standard Model}

Following the detection of the neutrino in the late 1950s by Fred Reines and Clyde Cowan, particle physics went through a spectacular flowering which culminated in the formulation of the Standard Model. We begin this chapter by briefly introducing the Standard Model and its building blocks. We then describe neutrino properties within the Standard Model circa 1970s, and examine how the field of neutrino physics has evolved over time into its current form, driven by experimental discoveries in the last couple of decades.

\subsection{The Standard Model of Particle Physics}

\subsubsection{In Search of a Final Theory of Particle Physics}

The Standard Model of particle physics was conceived in the 1970s, based on a compilation of laws, theories, and empirical observations made as early as the midnineteenth century. Since its conception, it has been scrutinized by hundreds of particle physics experiments, the overwhelming majority of which have been able to verify its accuracy and predictive power to remarkable precision. It is truly inspiring that a single set of underlying forces, symmetries and elementary building blocks seem sufficient to describe the underlying physics of phenomena ranging from thermonuclear reactions and nuclear burning in the center of the Sun, to the hot, dense, 
early universe following the Big Bang, to phenomena at distance scales so small, that they are only accessible at particle accelerators. More recent discoveries such as that of the top quark in $1995[1,2]$, or the tau neutrino in 2000 [3], both of which were predicted based on fundamental concepts in the theory, have further reinforced our current picture of particle physics theory.

Nevertheless, despite the many successes, new particle physics observations and several experimental mysteries which have appeared over the last few decades have rendered our current picture of the Standard Model incomplete. Aside from the lack of a consistent theory of gravity, and the fact that there are far too many arbitrary parameters and unfounded relationships to justify it being the final theory, there have been several discoveries that indicate that our understanding of particle physics is a work in progress. One of those is the discovery of neutrino mass, which is discussed in the following sections.

\subsubsection{Standard Model Particles and Their Interactions}

The building blocks of the Standard Model (SM) are shown in Fig. 1-1. Standard Model particles are divided into fermions (spin- $\frac{1}{2}$ particles) and bosons (integer-spin particles). Fermions are the fundamental constituents that make up all visible matter in the universe, and they are further divided into two families, quarks and leptons. Each fermion family consists of three generations, identified primarily through their mass.

The gluons ( 8 in total), photon, $Z$ and $W^{ \pm}$are the SM bosons. Those are the mediators of the three fundamental forces by which SM particles interact: the strong, electromagnetic, and weak force, respectively. The higgs boson is the only hypothesized SM particle, predicted by electroweak theory $[5,6,7]$, and it is responsible for giving massive particles, including the higgs itself, their masses.

The three fundamental interactions in the SM are summarized in Fig. 1-2. Each interaction is characterized by an intrinsic strength. The weak interaction has a relative strength of $\mathcal{O}\left(10^{-13}\right)$, and it is experienced by all SM particles that carry weak charge (all fermions, and the $W^{ \pm}$and $Z$ bosons). Next follows the electromagnetic 


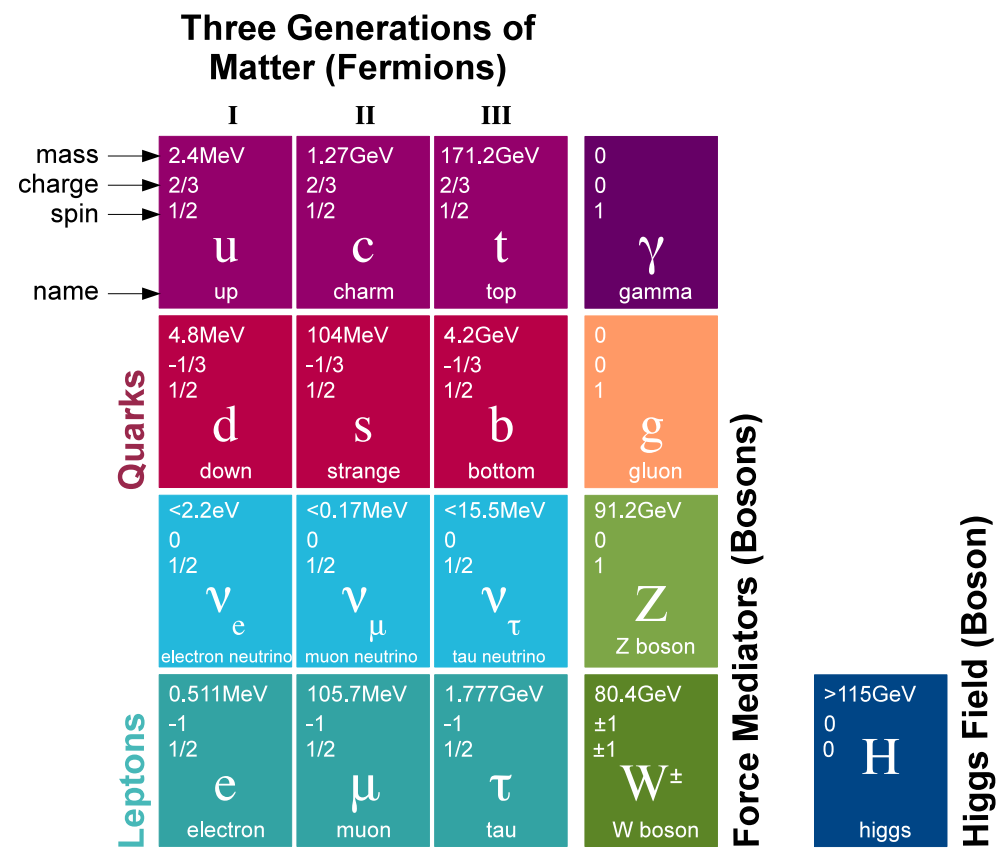

Figure 1-1: The particle spectrum of the Standard Model. The figure is from [4], with modifications.

interaction, with a relative strength of $\mathcal{O}\left(10^{-2}\right)$, which is experienced by all particles that have non-zero electric charge. Finally, the strong interaction has a relative strength of $\mathcal{O}(1)$, and it is experienced only by quarks and their composite objects, which carry color charge.

One of the limitations of the SM is that it cannot explain why the weak and electromagnetic interaction strengths differ from that of the strong interaction by many orders of magnitude. There are theories beyond the SM which predict that the electromagnetic, weak, and strong forces become equal, or "unified", at some very high energy scale (generically between $10^{14}-10^{19} \mathrm{GeV}$ ), which is beyond our current experimental reach (for a brief review, see [9] and references within). In that case, the observed discrepancy in the SM intrinsic strengths can be attributed to some symmetry which is effectively broken at a lower energy scale. Those theories are referred to as Grand Unified Theories (GUTs) and form the basis for many extensions to the SM. 


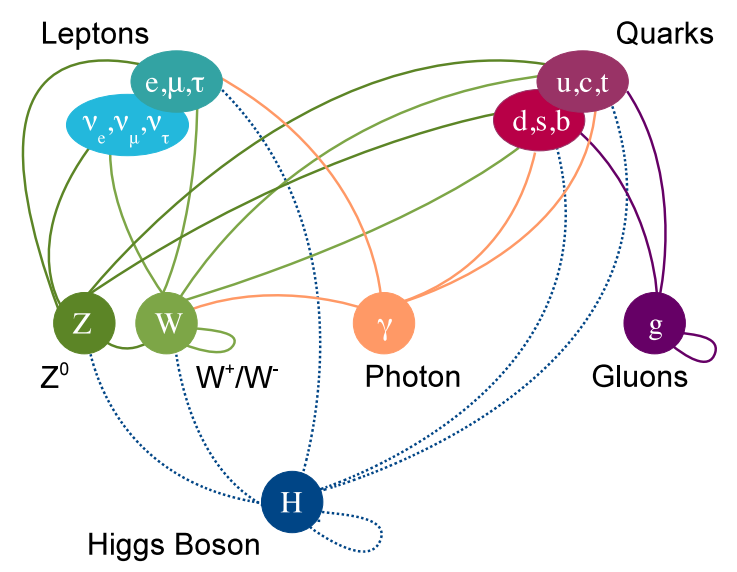

Figure 1-2: Summary of interactions between particles described in the Standard Model. Green, orange, and purple lines signify weak, electromagnetic, and strong interactions, respectively. Closed loops signify self-interaction. All massive particles are coupled to the higgs field (a hypothetical particle) through their mass, as indicated by the dotted blue lines. The figure is from [8], with modifications.

Nevertheless, the SM itself does provide an explanation for the difference between the electromagnetic and weak interaction strengths, through electroweak theory $[5$, $6,7]$ and the higgs mechanism for electroweak symmetry breaking $[10,11,12,13,14]$. In fact, the discovery of the $W$ and $Z$ gauge bosons in $1983[15,16]$ has been one of the triumphs of the SM, and in particular electroweak theory, as both particles had been predicted as massive bosons within the theory and as the source of asymmetry between the weak and electromagnetic interaction strengths.

\subsubsection{Massless Neutrinos in the Standard Model}

Neutrinos, and in particular what we now identify as electron neutrinos, were first postulated by Wolfgang Pauli in 1930. Pauli's motivation in introducing this particle was to explain the non-discrete energy spectrum of electrons emitted in the $\beta$-decay of radioactive nuclei. In a "desperate" attempt to rescue the sacred law of conservation of energy, Pauli proposed the existence of this additional, nearly-massless and electrically 
neutral particle. ${ }^{1}$ This particle had to be emitted along with the other $\beta$-decay products, therefore randomly sharing available energy in the reaction and naturally smearing the energy of the outgoing electron. Thus, the neutrino (meaning "little neutral one") was born.

Because they are neutral leptons, neutrinos only interact with matter through the weak force, with a typical cross-section of the order of $10^{-38} \mathrm{~cm}^{2},{ }^{2}$ and are therefore extremely hard to detect. Neutrino interactions with other fermions in the SM occur either through the exchange of a $W^{ \pm}$boson (charged-current, or CC interaction), or through the exchange of a $Z$ boson (neutral-current, or NC interaction). Furthermore, the coupling of leptons to the $W^{ \pm}$takes place strictly within a particular generation,

$$
\left(\begin{array}{c}
\nu_{e} \\
e
\end{array}\right),\left(\begin{array}{c}
\nu_{\mu} \\
\mu
\end{array}\right), \text { and }\left(\begin{array}{c}
\nu_{\tau} \\
\tau
\end{array}\right) \text {. }
$$

That is, the coupling allows for $W^{-} \rightarrow e^{-} \bar{\nu}_{e}, W^{-} \rightarrow \mu^{-} \bar{\nu}_{\mu}, W^{-} \rightarrow \tau^{-} \bar{\nu}_{\tau}$, but there is no cross-generational coupling of the form $W^{-} \rightarrow e^{-} \bar{\nu}_{\mu}$, for example. The same is true for $\mathrm{NC}$ interactions, i.e. $Z \rightarrow \nu_{e} \bar{\nu}_{e}$, but not $Z \rightarrow \nu_{e} \bar{\nu}_{\mu}$. In typical neutrino experiments, neutrinos are produced through CC interactions always ${ }^{3}$ in association with a charged lepton of the same generation (or flavor), e.g.,

$$
\begin{gathered}
n \rightarrow p e^{-} \bar{\nu}_{e}, \\
\pi^{+} \rightarrow \mu^{+} \nu_{\mu}
\end{gathered}
$$

and are detected in an analogous way.

Due to its weakly-interacting nature, the discovery of the neutrino came 26 years after it was first theoretically proposed, when Clyde Cowan and Fred Reines made use of inverse $\beta$-decay $\left(\bar{\nu}_{e} p \rightarrow n e^{+}\right)$to detect electron antineutrinos from a nuclear

\footnotetext{
${ }^{1}$ In Pauli's own words, "I have done a terrible thing. I have postulated a particle that cannot be detected."

${ }^{2}$ The cross-section varies with neutrino energy, and is further dependent on the type of interaction.

${ }^{3}$ This is true within the SM. In Chapter 2 we will consider beyond-SM models where this is not true.
} 
reactor [17]. The low event rates detected by Cowan and Reines also confirmed the weak nature of neutrinos. The discovery of the muon neutrino followed in 1962, by Leon Lederman, Melvin Schwartz, and Jack Steinberger, using a higher energy neutrino beam than what had been previously available [18], which was required in order for the muon neutrino to be able to kinematically produce a muon. The tau neutrino was finally detected four decades later by the DONuT experiment [3], prompted by the discovery of the tau lepton [19] in 1975.

For a long time, neutrinos were believed to be massless particles. In fact, the concept of massless neutrinos was considered so fundamental that it was interwoven into the theory of weak interactions in the 1950s and 1960s. By that, we refer to the parity-violating nature of weak interactions, which we discuss next. Of course, we now know-and shall see later on - that is no longer the case: neutrinos have mass, and neutrino mass and the parity-violating nature of weak interactions are two independent properties.

Around the same time as Cowan and Reines' discovery, several realizations took place concerning the nature of weak interactions. In particular, the discovery of parity violation in weak decays by T. D. Lee and C. N. Yang in 1956 [20] and by C. S. Wu and collaborators in 1957 [21] established the unique nature of weak interactions: unlike strong and electromagnetic processes, weak processes are not invariant under a mirror reflection of their coordinate system (parity operation, or $P$ ). What $\mathrm{Wu}$ and collaborators had found in their experiment was that the spin of the electron emitted in $\beta$-decays of ${ }^{60} \mathrm{Co}$ was always aligned in the opposite direction of its momentum. This was a revolutionary observation, since both parallel and anti-parallel orientations were expected if parity was a conserved symmetry. Following those discoveries, the helicity of the neutrino was determined to be left-handed in 1958, by Goldhaber et al. $[22]$.

The discovery of parity violation led to the formulation of a "two-component theory" of massless, spin- $\frac{1}{2}$ neutrinos [23], which was founded on the basis that neutrinos were pure left-helicity states (i.e. chirally left-handed), whereas antineutrinos were pure right-helicity states (i.e. chirally right-handed). Soon after that, the "V-A" the- 
ory of weak interactions was developed, according to which the $W^{ \pm}$bosons coupled exclusively to left-handed particles and right-handed antiparticles [24]. As this theory was to form the basis of electroweak theory on which the SM was later based on, the left-handed nature of neutrinos and right-handed nature of antineutrinos was thus "permanently" embedded into the SM theory of weak interactions.

The absence of a right-handed neutrino (or left-handed antineutrino) is precisely what prohibited one from constructing a neutrino Dirac mass term in the SM Lagrangian, which must follow the form

$$
-\mathcal{L}_{\text {mass }}=m\left(\bar{\psi}_{L} \psi_{R}+\psi_{L} \bar{\psi}_{R}\right)
$$

expressed in terms of the right- and left-handed projections, $\psi_{R, L}=\frac{1}{2}\left(1 \pm \gamma_{5}\right) \psi$, of any given fermion field $\psi$. Since neutrinos were left-handed states, such term could not be constructed. Thus, neutrinos in the SM as it was originally conceived in the 1970s are, by construction, massless.

\subsection{The First Clues to Neutrino Mass}

The first evidence for non-zero neutrino mass, although not recognized as such until decades later, came in 1968. At the time, Ray Davis and collaborators were trying to detect solar neutrinos in an effort to test theoretical models of nuclear fusion in the sun, developed by theoretical astrophysicist John Bahcall. Davis' experiment made use of the CC interaction of $\nu_{e}$ on chlorine atoms,

$$
\nu_{e}+C l \rightarrow e^{-}+A r,
$$

in order to detect what was thought to be $\nu_{e}$ arriving at the earth's surface after having been produced in nuclear reactions in the center of the sun. To their surprise, the $\nu_{e}$ event rate Davis and collaborators measured was only a third of that expected according to Bachall's calculations [25].

For decades later, other radiochemical and water Cherenkov experiments tried 
to measure the ratio of observed $\nu_{e}$ flux to that predicted by Bachall et al. All of those experiments observed ratios ranging between 0.3-0.6. The range in observed ratios, as well as the fact that each experiment was sensitive to different solar neutrino energies, complicated the picture even further. No combination of adjustments in the solar model could reproduce the energy-dependent effects observed by all experiments. Thus, a controversy began, known as the "solar neutrino problem".

By the time of Davis' experiment, it was already known that two distinct flavors of neutrinos, namely the muon neutrino and the electron neutrino, exist [18]. The speculation that neutrinos may transform from one flavor to another surfaced as early as the same year, by V. N. Gribov and B. Pontecorvo [26]. The concept of neutrino oscillations was first proposed by Pontecorvo in 1957, although only within the context of $\nu-\bar{\nu}$ oscillations, analogous to $K^{0}-\bar{K}^{0}$ mixing in the quark sector. Maki, Nakagawa and Sakata later suggested that transitions between different flavors of neutrinos may also occur, if neutrinos had mass. ${ }^{4}$ Of course, this (radical at the time) interpretation was not immediately accepted.

Meanwhile, a new experimental neutrino anomaly had surfaced. Almost two decades after Davis' experiment, large water Cherenkov detectors started being used in searches for proton decay, a rare process predicted by GUTs. Due to their low background design, those experiments were also capable of detecting neutrinos produced by cosmic ray interactions in the earth's atmosphere. In fact, atmospheric neutrino interactions in the detectors of such experiments contributed as a significant background to the search for proton decay events.

Cosmic ray showers produce primarily muon neutrinos and antineutrinos from $\pi^{+} \rightarrow \mu^{+} \nu_{\mu}$ and $\pi^{-} \rightarrow \mu^{-} \bar{\nu}_{\mu}$ decays. The atmospheric neutrino flux spectra are fairly well-known, from studies of the atmospheric muon flux produced hand-in-hand. Therefore, when the Kamiokande experiment in Japan observed a deficit of atmospheric muon neutrinos in 1988 [27], in an attempt to study backgrounds to a search for proton decay, the effect was too striking to ignore.

In addition to Kamiokande, other experiments at the time, such as the IMB ex-

\footnotetext{
${ }^{4}$ We will see why in the following section.
} 
periment in the U.S. [28] detected similar deficits; however, other experiments did not $[29,30]$. A second mystery was immediately born, reminiscent of the solar neutrino deficit. What was different with respect to the solar neutrino measurements was that the missing neutrinos were of muon rather than electron flavor, and the neutrino energy scale for solar vs. atmospheric neutrinos differed by approximately three orders of magnitude.

The solar neutrino deficit was finally resolved in 2001, from solar neutrino measurements provided by the Sudbury Neutrino Observatory (SNO) experiment [31]. A viable interpretation of the solar deficit results was based on neutrino flavor transitions, and relied on the fact that past radiochemical experiments were only sensitive to $\nu_{e}$ interactions. This was a consequence of both the low energy of solar neutrinos, and the actual experimental designs. Using deuterium in heavy water, SNO was sensitive to $\mathrm{CC}$ interactions of electron neutrinos, as well as $\mathrm{NC}$ interactions of all three neutrino flavors, which, unlike CC interactions, have no low energy threshold. The SNO results are summarized in Fig. 1-3. The ratio of CC to NC interactions measured by SNO determined that, even though nuclear reactions in the sun are not energetic enough to produce muon or tau neutrinos, neutrinos from the sun clearly arrive at the earth in more than just $\nu_{e}$ flavor. Furthermore, the total flux measured by SNO through NC interactions was consistent with theoretical predictions of solar neutrino flux by Bachall et al..

The SNO measurement was a victory for both solar theory and neutrino experiments, but presented a conflict with the SM as originally conceived. Electroweak theory tells us that in a CC interaction, which is the interaction experiments exploit in order to tag the generation (or flavor) of an incoming neutrino, a $\nu_{e}$ always produces an $e$, and never a $\mu$ or $\tau$, and so forth; i.e., weak interactions conserve lepton generational number. With that in mind, the experimental results discussed above suggested that what must be produced as a neutrino of flavor $\alpha$, interacts in the detector as what must be a neutrino of flavor $\beta$. In other words, whatever is responsible for this observed flavor transition, must have to do with neutrino propagation, and cannot be a production or detection effect. This is when the theory of neutrino 


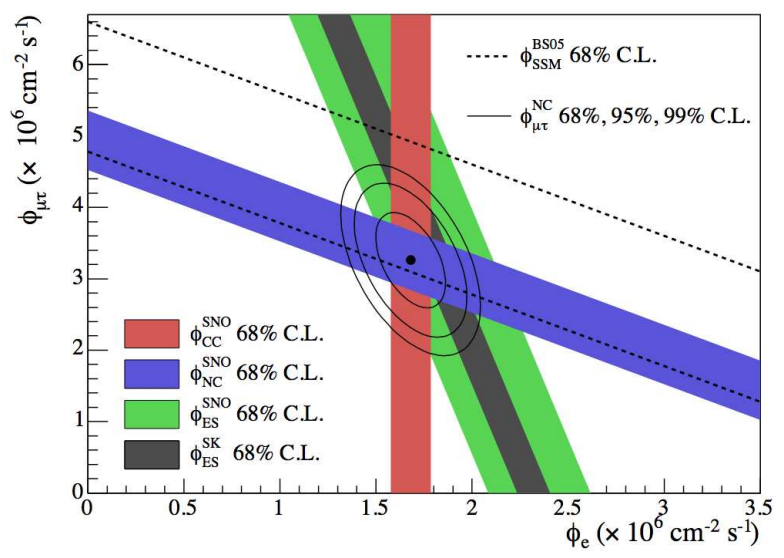

Figure 1-3: Solar neutrino flux measurements from SNO. The figure shows the flux of muon and tau neutrinos versus the flux of electron neutrinos measured by SNO. The colored bands correspond to the observed fluxes for CC (red), NC (blue), and elastic scattering (green) interactions. The solar model expectation for the NC flux is shown by the dashed bands. The gray band corresponds to a separate elastic scattering measurement of solar neutrinos made at Super-Kamiokande, which is in good agreement with SNO data. The figure is from [32].

flavor-changing oscillations by Maki, Nakagawa, and Sakata, mentioned earlier, was embraced.

Neutrino oscillation is a process by which a neutrino of flavor $\alpha=e, \mu$, or $\tau$ produced in association with a charged lepton of flavor $\alpha$, after propagating a distance $L \neq 0$, is detected as a neutrino of flavor $\beta \neq \alpha$ through production of a charged lepton of flavor $\beta$ in its interaction. This process comes about naturally if one assumes that neutrinos have mass and that leptons mix. Both assumptions are theoretically appealing. Firstly, even though the SM postulates so, there is no reason why neutrinos should be massless. In fact, this postulate would make neutrinos unique, as the only massless fermions in the SM. Secondly, mixing is a phenomenon that has already been established in the quark sector, and it is only natural to allow for such possibility in the lepton sector.

The following section describes how the SM can be extended to account for that possibility. 


\subsection{Extending the Standard Model to Accommo- date Neutrino Mass and Leptonic Mixing}

\subsubsection{Dirac Neutrino Mass}

Perhaps the simplest way to incorporate neutrino mass in the SM is to assume neutrinos are Dirac particles, and that right-handed neutrinos exist in nature. ${ }^{5}$ Then, the SM Lagrangian acquires an additional Dirac mass term, of the form

$$
-\mathcal{L}_{m_{\nu}}^{D}=m_{\nu} \bar{\nu} \nu=m_{\nu}\left(\bar{\nu}_{L} \nu_{R}+\bar{\nu}_{R} \nu_{L}\right)
$$

for each neutrino generation.

Of course, right-helicity neutrinos are not observed in nature for two reasons: 1) Because of the nature of the weak interaction, which acts on and creates only chirally left-handed states, right-helicity neutrinos can only participate in weak processes at the order of $m_{\nu} / E$; and 2) given current neutrino mass limits (see Sec. 1.4.2) and neutrino energies we currently have sensitivity to, effects due to these amplitudes are too small to observe experimentally.

In this scenario, neutrinos acquire their mass through their coupling with the higgs field, like all other massive SM fermions. The neutrino mass, $m_{\nu}$, is then given by $h^{\nu} v / \sqrt{2}$, in terms of a Yukawa coupling constant, $h^{\nu}$, and the higgs vacuum expectation value, $v$. Note that, while this scenario can easily accommodate nonzero neutrino masses, it begs the question of why neutrino masses are so small in comparison to other SM fermions, or $h^{\nu} \ll h^{\ell, q}$.

\footnotetext{
${ }^{5}$ This corresponds to the most minimal extension to the SM which can accommodate non-zero neutrino masses.
} 


\subsubsection{On the Lightness of Neutrino Mass: The See-Saw Model}

An alternative scenario, and one of the most popular explanations of the lightness of neutrinos relative to quarks and charged leptons, is the See-Saw model. ${ }^{6}$ This model was proposed in 1979 by Murray Gell-Mann, Pierre Ramond and Richard Slansky, and independently by Tsutomu Yanagida [42].

In a See-Saw model, the addition of a Majorana mass term to the neutrino mass Lagrangian leads to two (or, typically, more than three) physical neutrino mass states which are Majorana particles, i.e. they satisfy $\nu=\bar{\nu}$. One of these Majorana neutrinos, $\nu$, is very light, and is identified as the familiar neutrino. The other one, $N$, is very heavy, and possibly non-weakly interacting. ${ }^{7}$ In this scheme, the masses of $\nu$ and $N$ are related to the typical quark or charged lepton mass scale, $m_{q, l}$, by

$$
m_{\nu} m_{N}=m_{q, l}^{2}
$$

implying that as the mass of the heavy neutrino increases, the mass of the light neutrino decreases. Hence, the term "See-Saw". For typical neutrino and quark or lepton masses, $m_{N}$ acquires a value reminiscent of the symmetry-breaking scale in GUTs. That makes the See-Saw model particularly promising from a theoretical stand point.

In the following paragraphs, we consider the See-Saw theoretical framework in more detail, as we will be referring to this framework when we discuss further extensions to the SM in Chapter 2.

In the most minimal, neutrino mass accommodating extension of the SM, we saw

\footnotetext{
${ }^{6}$ There are alternative models for introducing small neutrino masses, generally subdivided within loop models, or models with extra dimensions. It should also be noted that there are many types of See-Saw models, namely Type I and III See-Saw, based on fermionic exchange [33, 34, 35, 36], Type II, based on scalar exchange [37, 38], Low-Scale See-Saw, etc. Useful reviews can be found in $[39,40,41]$. We limit this discussion to the Type-I See-Saw model.

${ }^{7}$ It is usually assumed that the $N$ states are so heavy that they have decayed early in the time evolution of the universe.
} 
that a Dirac neutrino mass term is added to the SM Lagrangian, $\mathcal{L}_{m_{\nu}}$, of the form

$$
-\mathcal{L}_{m_{\nu}}^{D}=m_{D} \overline{\nu_{R}^{0}} \nu_{L}^{0}+\text { h.c. }
$$

where h.c. stands for hermitian conjugate, and the superscript 0 denotes weak eigenstates, so that $\nu_{L}^{0}$ is the three-dimensional left-handed vector of neutrino weak eigenstates

$$
\nu_{L}^{0} \equiv\left(\begin{array}{c}
\nu_{L e}^{0} \\
\nu_{L \mu}^{0} \\
\nu_{L \tau}^{0}
\end{array}\right)
$$

and similarly for $\nu_{R}^{0}$. In the See-Saw model, the same applies, but one also has the freedom to add a Majorana mass term to $-\mathcal{L}_{m_{\nu}}^{D}$ of Eq. 1.7. A Majorana mass term is constructed out of $\nu_{L}^{0}$ or $\nu_{R}^{0}$ alone, such as

$$
-\mathcal{L}_{m_{\nu}}^{M}=\frac{m_{R}}{2} \overline{\left(\nu_{R}^{0}\right)^{c}} \nu_{R}^{0}+\text { h.c. }
$$

where the superscript $c$ denotes charge conjugation, making use of the fact that, for neutral particles, $\nu^{c}=C(\bar{\nu})^{T}$. The effect of this operation is to convert the fundamental neutrino fields into their own antiparticles.

By adding in a Majorana mass term, the neutrino mass Lagrangian becomes $[43,33]$

$$
-\mathcal{L}_{m_{\nu}}=-\mathcal{L}_{m_{\nu}}^{M}-\mathcal{L}_{m_{\nu}}^{D}=\frac{m_{R}}{2} \overline{\left(\nu_{R}^{0}\right)^{c}} \nu_{R}^{0}+m_{D} \overline{\nu_{R}^{0}} \nu_{L}^{0}+\text { h.c. },
$$

given in terms of neutrino weak eigenstates. The term can then be diagonalized to obtain the observable mass eigenstates of this scenario, $\nu$ and $N$, in terms of the above weak eigenstates and masses.

The resulting mass-diagonalized Lagrangian is

$$
\begin{aligned}
-\mathcal{L}_{m_{\nu}} & =\frac{1}{2} \bar{n} D_{\nu} n \\
& =\frac{1}{2}\left(\begin{array}{ll}
\bar{\nu} & \bar{N}
\end{array}\right)\left(\begin{array}{cc}
m_{D}^{2} / m_{R} & 0 \\
0 & m_{R}
\end{array}\right)\left(\begin{array}{l}
\nu \\
N
\end{array}\right)
\end{aligned}
$$




$$
=\frac{1}{2} \frac{m_{D}^{2}}{m_{R}} \bar{\nu} \nu+\frac{1}{2} \frac{m_{D}^{2}}{m_{R}} \bar{N} N
$$

The six components of $n$ are the six observable neutrino mass eigenstates: $\nu$ (three states) and $N$ (three states), with masses $m_{\nu}=m_{D}^{2} / m_{R}$ and $m_{N}=m_{R}$, respectively. Evidently, the See-Saw relation $m_{\nu} m_{N}=\left(m_{D}^{2} / m_{R}\right) m_{R}=m_{D}^{2}$ holds. In this model, it is then assumed that $m_{D} \sim m_{q, l}$, which elegantly avoids the issue of the smallness of neutrino mass mentioned in Sec. 1.3.1, and that $m_{R} \gg m_{D}$, so that, depending on the value of $m_{R}$, one can explain the observable light neutrino masses we know of.

The physical interpretation of this scheme is that there are three very heavy neutrinos $\left(m_{R}\right.$ comes from physics at some high mass scale), and three very light ones, their lightness being driven by how large $m_{R}$ is. In fact, a characteristic prediction of the See-Saw, using what we already know about $m_{q, l}$ and $m_{\nu}$, is that $m_{N}=$ $m_{R}=m_{q, l}^{2} / m_{\nu} \sim m_{\text {top }}^{2} / 0.05 \mathrm{eV}^{2} \sim 10^{15} \mathrm{GeV}$, which reminiscent of the GUT scale $\left(\sim 2 \times 10^{16} \mathrm{GeV}\right)$. Therefore, the theoretical prejudice is that neutrino masses are a window towards GUTs, or some other physics beyond the SM energy scale.

Furthermore, both $\nu$ states and $N$ states have left- and right-handed (helicity) components, i.e.,

$$
\nu=\nu_{L}+\nu_{L}^{c}, \text { and } N=N_{L}+N_{L}^{c}
$$

In the case of the light neutrinos, $\nu$, the fact that right-handed neutrinos have never been experimentally observed is attributed to the left-handed nature of the weak interaction, as well as the lightness of the neutrino mass, as the energies required to reverse the helicities of neutrinos produced in meson decays are far beyond what any existing or planned accelerators can provide. The argument is the same as for Dirac right-handed neutrinos.

Finally, using the expressions in Eq. 1.12, it can be shown that $\nu=\bar{\nu}$ and $N=\bar{N}$, that is, $\nu$ and $N$ are Majorana particles. 


\subsubsection{Incorporating Neutrino Mixing}

Under the assumption that neutrinos have mass, we assume that there are three neutrino mass eigenstates, $\nu_{1}, \nu_{2}$, and $\nu_{3}$, which are the analogs of the charged lepton mass eigenstates, $e, \mu$, and $\tau$. The key realization which will lead to neutrino flavor transitions is that these mass states do not necessarily identify with the observable weak neutrino eigenstates, $\nu_{e}, \nu_{\mu}$, and $\nu_{\tau}$; they can be mixed. This mixing is due to the nature of the weak sector.

The physics of neutrino oscillation can be obtained by taking a closer look at the weak interaction coupling the $W$ boson to a charged lepton and a neutrino. Let's consider, for example, the leptonic $W^{+}$decay $W^{+} \rightarrow \ell_{\alpha}^{+}+\nu_{\alpha}$, where $\alpha=e, \mu$ or $\tau$. The Hamiltonian density for the $\mathrm{CC}$ weak interaction is given in terms of the above weak eigenstates by

$$
H_{C C}=g W^{+} \sum_{\alpha=e, \mu, \tau} i \ell_{\alpha}^{+} \gamma_{\mu}\left(1+\gamma_{5}\right) \nu_{\alpha}+\text { h.c. },
$$

where $g$ is the semi-weak coupling constant of the SM, $g=e / \sin \theta_{W}$, with $e$ being the electric charge of the proton, and $\theta_{W}$ the Weinberg angle.

The oscillation physics arises by making the connection that the neutrino weak eigenstate $\nu_{\alpha}$, which is what we define to be produced in $W^{+}$decay in association with a charged lepton $\ell_{\alpha}$, is a linear superposition of neutrino mass eigenstates $\nu_{i}$,

$$
\left|\nu_{\alpha}\right\rangle=\sum_{i=1-3} U_{\alpha i}^{*}\left|\nu_{i}\right\rangle
$$

This is referred to as lepton mixing, and it implies that the weak interaction coupling a $W$ boson with a charged lepton and a neutrino can couple a charged lepton mass eigenstate, $\ell_{\alpha}$, with any neutrino mass eigenstate $\nu_{i}$. In that case, Eq. 1.13 becomes

$$
H_{C C}=g W^{+} \sum_{\alpha=e, \mu, \tau} i l_{\alpha}^{+} \gamma_{\mu}\left(1+\gamma_{5}\right) \sum_{i=1-3} U_{\alpha i}^{*} \nu_{i}+\text { h.c. }
$$


The mixing matrix

$$
U=\left(\begin{array}{ccc}
U_{e 1} & U_{e 2} & U_{e 3} \\
U_{\mu 1} & U_{\mu 2} & U_{\mu 3} \\
U_{\tau 1} & U_{\tau 2} & U_{\tau 3}
\end{array}\right)
$$

also called the PMNS matrix in honor of Pontecorvo, Maki, Nakagawa and Sakata, is analogous to the CKM matrix which is responsible for quark mixing. $U$ is responsible for lepton mixing, and, in the most minimal extension of the SM to include neutrino masses and mixing, it is expected to be a $3 \times 3$ unitary matrix. Furthermore, it can have both real and imaginary parts, and can therefore lead to $C P$ violation in leptonic mixing, as will be discussed later.

Note that as there are only three charged lepton mass eigenstates, as well as three generations of quarks, it is only natural to assume that there are only three neutrino mass eigenstates $(i=1-3$ in Eq. 1.14). It is important, however, to remember is that the three-neutrino scenario is a minimal scheme, inspired byand following theoretical prejudice for - a model which resembles the three family structure of quarks and charged leptons. We now know that neutrinos are different from the quark and charged lepton sectors and that there is no fundamental symmetry in nature forcing a definite number of neutrino mass states to equal three. In more complex SM extensions, it is possible that more than three neutrino mass eigenstates exist, and that the $U$ matrix is greater than $3 \times 3$. We will consider such extensions in Chapter 2.

\subsubsection{Neutrino Oscillations}

Neutrino oscillation is a consequence of neutrino masses and leptonic mixing, and it manifests when one considers how neutrino states evolve in time. The wavefunction 
for a neutrino born as $\nu_{\alpha}$, as a function of its proper time,$^{8} \tau$, is given by

$$
\left|\nu_{\alpha}(\tau)\right\rangle=\sum_{i} U_{\alpha i}^{*} e^{-i m_{i} \tau_{i}}\left|\nu_{i}(0)\right\rangle
$$

and can be rewritten as a function of laboratory-frame parameters as

$$
\left|\nu_{\alpha}(t)\right\rangle=\sum_{i} U_{\alpha i}^{*} e^{-i\left(E_{i} t-p_{i} x\right)}\left|\nu_{i}(0)\right\rangle
$$

where $\left|\nu_{i}(0)\right\rangle$ are identified as the $\left|\nu_{i}\right\rangle$ in Eq. 1.14.

To calculate the probability for a neutrino with flavor $\alpha$ to oscillate into a neutrino with flavor $\beta$ after propagating some distance, we consider the amplitude for the following process: a neutrino born with an $\ell_{\alpha}^{+}$, propagating some distance $L$ in laboratory frame, and being detected in association with an $\ell_{\beta}^{-}$. Making use of Eq. 1.18, the amplitude for the process, squared, yields the following oscillation probability:

$$
P\left(\nu_{\alpha} \rightarrow \nu_{\beta}\right)=\left|\left\langle\nu_{\beta} \mid \nu_{\alpha}(t)\right\rangle\right|^{2}=\left|\sum_{i} U_{\alpha i}^{*} e^{-i\left(E_{i} t-p_{i} L\right)} U_{\beta i}\right|^{2}
$$

where $i$ runs over all neutrino mass eigenstates which can be kinematically produced in the decay which produced $\nu_{\alpha}$, and is assumed to run over all three SM neutrino mass eigenstates, $i=1-3$, since we know all three neutrinos are extremely light.

It is important to point out the exponential propagator term in Eq. 1.17. We will see next that this propagator, which is $m_{i}$-dependent, introduces a relative phase between any two distinct mass eigenstate components produced in the decay. The resulting interference between different neutrino mass eigenstates is what causes neutrino flavor oscillations.

By carrying out the product in Eq. 1.19, and exploiting the unitarity of the mixing matrix $U$, we obtain

$$
P\left(\nu_{\alpha} \rightarrow \nu_{\beta}\right)=\delta_{\alpha \beta}
$$

\footnotetext{
${ }^{8}$ In the following derivation we use the convention $\hbar=c=1$.
} 


$$
\begin{aligned}
& -4 \sum_{i>j} \operatorname{Re}\left\{U_{\alpha i}^{*} U_{\alpha j} U_{\beta i} U_{\beta j}^{*}\right\} \sin ^{2}\left(\frac{1}{2}\left(\left(p_{i}-p_{j}\right) L-\left(E_{i}-E_{j}\right) t\right)\right) \\
& +\sum_{i>j} \operatorname{Im}\left\{U_{\alpha i}^{*} U_{\alpha j} U_{\beta i} U_{\beta j}^{*}\right\} \sin \left(\left(p_{i}-p_{j}\right) L-\left(E_{i}-E_{j}\right) t\right)
\end{aligned}
$$

Next, we note that, for relativistic neutrinos, $p_{i} \sim p_{j} \sim E$, and $t \sim L / \bar{v}$, where $\bar{v}=\left(p_{i}+p_{j}\right) /\left(E_{i}+E_{j}\right)$ is an approximation of the average velocities for the $\nu_{i}$ components of the beam. Therefore,

$$
\left(p_{i}-p_{j}\right) L-\left(E_{i}-E_{j}\right) t=\frac{p_{i}^{2}-p_{j}^{2}}{p_{i}+p_{j}} L-\frac{E_{i}^{2}-E_{j}^{2}}{p_{i}+p_{j}} L \simeq\left(m_{j}^{2}-m_{i}^{2}\right) \frac{L}{2 E}
$$

and Eq. 1.20 becomes

$$
\begin{aligned}
P\left(\nu_{\alpha} \rightarrow \nu_{\beta}\right)= & \delta_{\alpha \beta} \\
& -4 \sum_{i>j} \operatorname{Re}\left\{U_{\alpha i}^{*} U_{\alpha j} U_{\beta i} U_{\beta j}^{*}\right\} \sin ^{2}\left(\frac{\left(m_{j}^{2}-m_{i}^{2}\right) L}{4 E}\right) \\
& +\sum_{i>j} \operatorname{Im}\left\{U_{\alpha i}^{*} U_{\alpha j} U_{\beta i} U_{\beta j}^{*}\right\} \sin \left(\frac{\left(m_{j}^{2}-m_{i}^{2}\right) L}{2 E}\right) .
\end{aligned}
$$

The resulting probability in Eq. 1.22 indeed oscillates, with an oscillation amplitude which is dependent on the level of mixing between the mass and weak eigenstates, and an oscillation frequency which is dependent on neutrino masses and the experimental parameters $L$ and $E$.

Note that three conditions must be met in order to have neutrino oscillations:

1. At least two of the neutrino masses must be non-zero $\left(m_{i} \neq 0\right)$.

2. Neutrino masses must be distinct $\left(\Delta m_{i j}^{2} \neq 0\right)$.

3. There must be leptonic mixing, i.e. $U \neq I$, where $I$ is the identity matrix.

If any one of the above conditions is not met, then Eq. 1.22 reduces to $\delta_{\alpha \beta}$, and there are no neutrino oscillations.

Equation 1.22 applies for neutrino oscillations. To obtain the oscillation probability in the case of antineutrinos, one replaces $U$ in Eq. 1.22 with its complex conjugate. 
The effect is a sign flip in front of the imaginary component term in the oscillation probability. If $U$ is complex, the sign flip implies different oscillation probabilities for neutrinos versus antineutrinos. Since $P\left(\nu_{\alpha} \rightarrow \nu_{\beta}\right)$ and $P\left(\bar{\nu}_{\alpha} \rightarrow \bar{\nu}_{\beta}\right)$ are related by the $C P$ operation, $P\left(\nu_{\alpha} \rightarrow \nu_{\beta}\right) \neq P\left(\bar{\nu}_{\alpha} \rightarrow \bar{\nu}_{\beta}\right)$ implies $C P$ violation, and vice versa.

The importance of the possibility of $C P$ violation in the neutrino sector deserves special mention. This was a long-recognized possibility, originally motivated by the discovery of $C P$ violation by J. W. Cronin and V. Fitch in the neutral kaon system [44] and later by the realization that three generations of neutrinos existed. To this date, $C P$ violation in the three neutrino sector has never been observed. The implications of such possibility may prove important in our understanding of the evident matterantimatter asymmetry in the universe [45].

\subsubsection{An Instructive Example: Two-Neutrino Oscillations}

Equation 1.22 gives the neutrino oscillation probability for any number of neutrino generations. However, it is cumbersome to interpret in a scenario with more than two neutrino states. Fortunately, experimental neutrino oscillation signatures so far, as we shall see in the following section, are consistent with cases in which only a single $\Delta m^{2}$ driven frequency dominates and only two neutrinos participate in oscillation with a significant amplitude. ${ }^{9}$ Therefore, it is sufficient and more instructive to consider a two-neutrino oscillation scenario before discussing experimental results.

In a two-neutrino oscillation approximation, mixing is described by a $2 \times 2$ unitary mixing matrix of the form

$$
U=\left(\begin{array}{cc}
\cos \theta & \sin \theta \\
-\sin \theta & \cos \theta
\end{array}\right)
$$

so that

$$
\left(\begin{array}{c}
\nu_{\alpha} \\
\nu_{\beta}
\end{array}\right)=\left(\begin{array}{cc}
\cos \theta & \sin \theta \\
-\sin \theta & \cos \theta
\end{array}\right)\left(\begin{array}{c}
\nu_{1} \\
\nu_{2}
\end{array}\right) .
$$

\footnotetext{
${ }^{9}$ This is also referred to as "one mass scale dominance" [46].
} 
Note that when $\theta=0$, there is no mixing: $\nu_{\alpha}=\nu_{1}$, and $\nu_{\beta}=\nu_{2}$.

From Eq. 1.22, the probability of a neutrino of flavor $\alpha$ oscillating into a neutrino of flavor $\beta$ when only two mass eigenstates exist, is given by

$$
P\left(\nu_{\alpha} \rightarrow \nu_{\beta}\right)=\delta_{\alpha \beta}-4\left|U_{\alpha 2}^{*} U_{\beta 2} U_{\alpha 1} U_{\beta 1}^{*}\right| \sin ^{2}\left(1.267 \frac{\Delta m^{2} L}{E}\right)
$$

and substituting in the mixing matrix elements from Eq. 1.24 yields

$$
P\left(\nu_{\alpha} \rightarrow \nu_{\beta}\right)=\delta_{\alpha \beta}-\sin ^{2} 2 \theta \sin ^{2}\left(1.267 \frac{\Delta m^{2} L}{E}\right)
$$

where we have included the factors of $\hbar$ and $c$ omitted in Eq. 1.22, assuming $E$ is the neutrino energy in $\mathrm{GeV}, L$ is the distance traveled by the neutrino in $\mathrm{km}$, and $\Delta m^{2}=m_{2}^{2}-m_{1}^{2}$ is the squared-mass difference of the two neutrino mass eigenstates, $\nu_{1}$ and $\nu_{2}$, in units of $\mathrm{eV}^{2}$. From Eq. 1.26, we see that neutrino oscillations can manifest either as $\nu_{\alpha}$ "disappearance" $(\beta=\alpha)$, or $\nu_{\beta}$ "appearance" $(\beta \neq \alpha)$. In the case of disappearance, one starts with a known flux of $\nu_{\alpha}$, and at $L \neq 0$ observes a flux of $\nu_{\alpha}$ weighted by the survival probability

$$
P\left(\nu_{\alpha} \rightarrow \nu_{\alpha}\right)=1-\sin ^{2} 2 \theta \sin ^{2}\left(1.267 \frac{\Delta m^{2} L}{E}\right)
$$

which is always $\leq 1$. In the case of appearance, one instead observes the appearance of neutrinos of flavor $\beta$ in a beam of $\nu_{\alpha}$, with a probability given by

$$
P\left(\nu_{\alpha} \rightarrow \nu_{\beta \neq \alpha}\right)=\sin ^{2} 2 \theta \sin ^{2}\left(1.267 \frac{\Delta m^{2} L}{E}\right)
$$

which is always $\geq 0$ and $\leq 1$. The two effects are illustrated in Fig. 1-4, for an experiment which uses a $\nu_{\alpha}$ beam, with a small intrinsic content of $\nu_{\beta}$.

Note that Eq. 1.26 will lead to a significant oscillation signal for any experiment with $L / E$ such that $\Delta m^{2} L / E \sim \mathcal{O}(1)$.

As a final note, in the two-neutrino oscillation scenario we have considered, $U$ is real by definition (see Eq. 1.23). Similarly, in a two-neutrino approximation, $U$ is also 


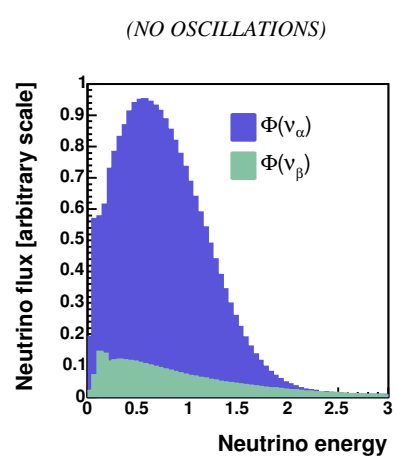

(NO OSCILLATIONS)

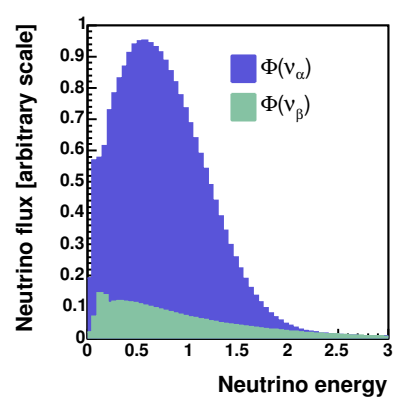

(NO OSCILLATIONS)

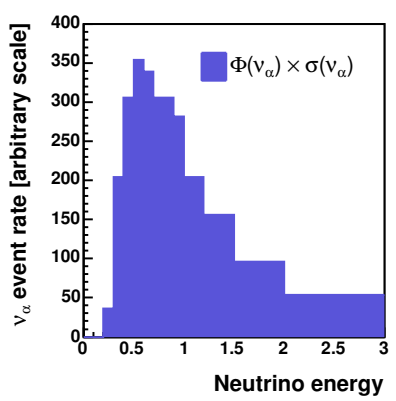

(NO OSCILLATIONS)

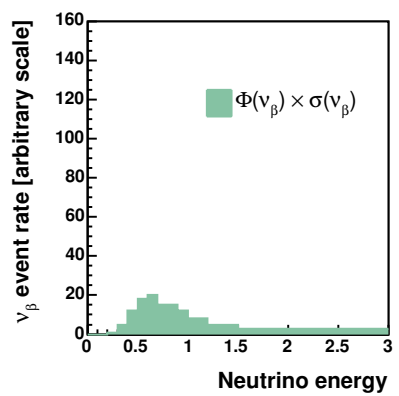

(OSCILLATIONS)

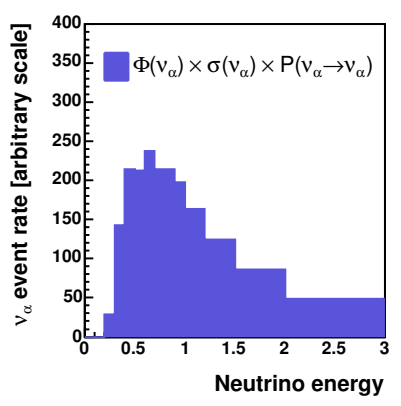

(OSCILLATIONS)

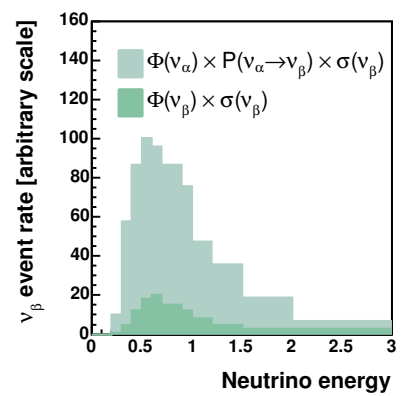

Figure 1-4: Cartoon illustration of disappearance (top) and appearance (bottom) oscillation effects, for an experiment illuminated with a neutrino flux as shown in the left panel. At $L=0$, or assuming no neutrino oscillations, the experiment would observe the neutrino event spectra as shown in the middle panels. At $L \neq 0$, and assuming neutrino oscillations, the experiment would the neutrino event spectra as shown in the right panels.

real to the level at which the approximation holds. Therefore, in experiments where oscillations are dominated by a single $\Delta m^{2}$, in which case the two-neutrino oscillation approximation is sufficient to describe the data, $C P$ violation, if any, is only expected to be a second-order effect; i.e., any difference in oscillation probabilities for neutrinos versus antineutrinos is expected to be negligible. 


\subsection{Results of the Last Two Decades}

\subsubsection{Carving Out the Three-Neutrino Oscillation Parame- ters}

Today, most neutrino oscillation experimental data can be fit into a three-neutrino oscillation picture, where there are two independent $\Delta m_{i j}^{2} \equiv m_{j}^{2}-m_{i}^{2}$, and three independent mixing angles, $\theta_{12}, \theta_{23}$, and $\theta_{13}$, which can be used to parametrize the neutrino mixing matrix in Eq. 1.16 as follows:

$$
\begin{aligned}
U & =\left(\begin{array}{lll}
U_{e 1} & U_{e 2} & U_{e 3} \\
U_{\mu 1} & U_{\mu 2} & U_{\mu 3} \\
U_{\tau 1} & U_{\tau 2} & U_{\tau 3}
\end{array}\right) \\
& =\left(\begin{array}{lll}
1 & 0 & 0 \\
0 & c_{23} & s_{23} \\
0 & -s_{23} & c_{23}
\end{array}\right)\left(\begin{array}{ccc}
c_{13} & 0 & s_{13} e^{-i \delta} \\
0 & 1 & 0 \\
-s_{13} e^{i \delta} & 0 & c_{13}
\end{array}\right)\left(\begin{array}{ccc}
c_{12} & s_{12} & 0 \\
-s_{12} & c_{12} & 0 \\
0 & 0 & 1
\end{array}\right) \\
& =\left(\begin{array}{llll}
c_{12} c_{13} & s_{12} c_{13} & \\
-s_{12} c_{23}-c_{12} s_{23} s_{13} e^{i \delta} & c_{12} c_{23}-s_{12} s_{23} s_{13} e^{i \delta} & s_{23} c_{13} \\
s_{12} s_{23}-c_{12} c_{23} s_{13} e^{i \delta} & -c_{12} s_{23}-s_{12} c_{23} s_{13} e^{i \delta} & c_{23} c_{13}
\end{array}\right),
\end{aligned}
$$

where $c_{i j}=\cos \theta_{i j}$ and $s_{i j}=\sin \theta_{i j}$, and $\delta$ is a Dirac $C P$-violating phase which is zero only when $U$ is real. ${ }^{10}$

The two independent $\Delta m^{2}$ values, $\Delta m_{12}^{2}$ and $\Delta m_{23}^{2}$, control the solar and atmospheric neutrino oscillations, respectively. The mixing angles $\theta_{12}$ and $\theta_{23}$ also control the solar and atmospheric neutrino oscillations, while the third independent angle in the three neutrino mixing matrix, $\theta_{13}$, is constrained to be zero within uncertainty by data from short-baseline reactor-based neutrino experiments, which we will discuss

\footnotetext{
${ }^{10}$ To be precise, there is an additional degree of freedom which may come from neutrinos being Majorana particles. In that case, the expression for $U$ in Eq. 1.29 should be multiplied by $\times \operatorname{diag}\left(e^{i \alpha_{1} / 2}, e^{i \alpha_{2} / 2}, e^{i \alpha_{3} / 2}\right)$, where the phases $\alpha_{i}$, known as Majorana phases, have physical consequences only if neutrinos are Majorana particles, and influence neutrinoless double-beta decay and other processes $[47,48,49]$. Note, however, that those phases do not affect neutrino oscillation, regardless of whether neutrinos are Majorana or not.
} 


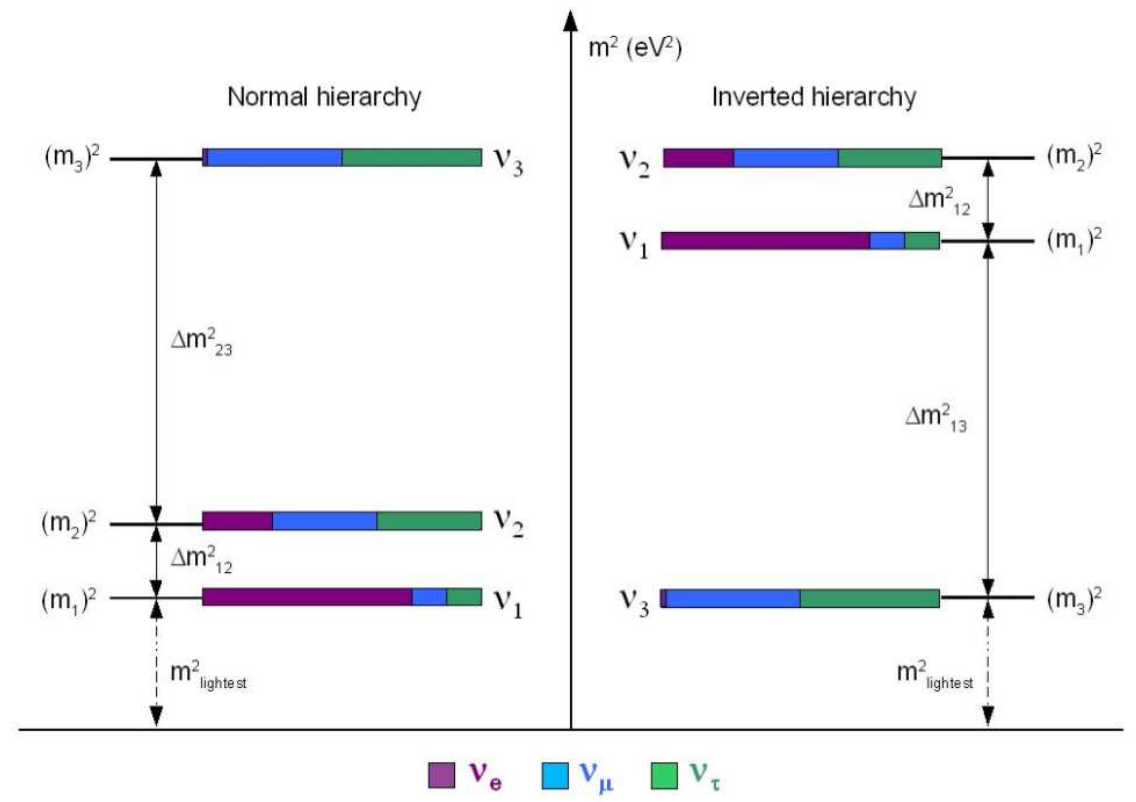

Figure 1-5: Three-neutrino mass spectrum in the most minimal, neutrino mass accommodating extension of the Standard Model. Left: normal hierarchy. Right: inverted hierarchy. The electron, muon, and tau content of each mass eigenstate, denoted by colored bands, represents the level of mixing of each mass eigenstate with each weak eigenstate, $\left|U_{\alpha i}\right|^{2}$. The electron neutrino content of the third mass eigenstate, $\left|U_{e 3}\right|^{2}=\sin ^{2} \theta_{13}$, has yet to be measured, and is constrained to be zero within uncertainties by reactor experiments. The figure is from [50].

next.

Despite the fact that we do not know the actual value of $\theta_{13}$, the two distinct oscillation signatures coming from the solar and atmospheric sectors suggest a neutrino mass spectrum as that in Fig. 1-5. It should be noted that while oscillation experiments so far have been able to determine the size of the $\Delta m_{23}^{2}$ to remarkable precision, they are insensitive to its sign. That is because current neutrino oscillation searches are only sensitive enough to employ the two-neutrino approximation in Sec. 1.3.5, where the oscillation probability is sensitive to $\sin ^{2}\left(1.267 \Delta m^{2} L / E\right)$. Therefore, the neutrino mass spectrum can either have the form shown on the left of Fig. 1-5, which resembles the bottom-near-degeneracy seen in the mass spectra of charged leptons and quarks, referred to as "normal hierarchy", or the form shown on the right of 
Fig. 1-5, referred to as "inverted hierarchy". ${ }^{11}$

Analysis of all present neutrino oscillation data in three-neutrino oscillation fits [52], which accounts for possible sub-dominant effects, tells us that, at the $3 \sigma$ confidence level,

$$
\begin{array}{r}
\theta_{12}=34.4_{-3.7}^{+4.8} \text { degrees } \\
\theta_{23}=45_{-9.3}^{+9.9} \text { degrees } \\
\theta_{13} \leq 12.9 \text { degrees } \\
\Delta m_{12}^{2}=7.6_{-0.5}^{+0.7} \times 10^{-5} \mathrm{eV}^{2} \\
\left|\Delta m_{23}^{2}\right|=2.4_{-0.4}^{+0.4} \times 10^{-3} \mathrm{eV}^{2},
\end{array}
$$

and there are no constraints on $\delta$. The above numbers are consistent with results from individual experiments, which use the simple two-neutrino approximation to extract oscillation parameters from their data.

The following subsections briefly review results from each sector separately.

\section{The $\left(\Delta m_{23}^{2}, \sin ^{2} 2 \theta_{23}\right)$ sector}

Results from atmospheric and accelerator neutrino experiments are consistent with oscillations at $\Delta m_{23}^{2} \sim 2.5 \times 10^{-3} \mathrm{eV}^{2}$, and $\sin ^{2} 2 \theta_{23} \sim 1$.

The first concrete evidence of oscillations at the level suggested by $\Delta m_{23}^{2}$ and $\sin ^{2} 2 \theta_{23}$ came from Super-Kamiokande in 1998 [53]. This experiment was a successor to the Kamiokande experiment, mentioned in Sec. 1.2, which was the first experiment to observe an atmospheric neutrino deficit. Compared to Kamiokande, Super-Kamiokande (or Super-K) had additional sensitivity to the direction of incoming muon neutrinos, and not only confirmed the deficit but also conclusively proved

\footnotetext{
${ }^{11}$ With future long-baseline neutrino oscillation experiments, it may be possible to determine the sign of $\Delta m^{2}$ by comparing neutrino and antineutrino oscillation probabilities for neutrinos passing through dense matter, based on the fact that the sign of the matter potential experienced in a neutrino beam is opposite for neutrinos and antineutrinos [51]. Those matter effects affect neutrino propagation and lead to differences in neutrino and antineutrino oscillation probabilities which are dependent on the mass hierarchy. Note that this effect also mimics the effect of true $C P$ violation, which can make the two difficult to disentangle.
} 


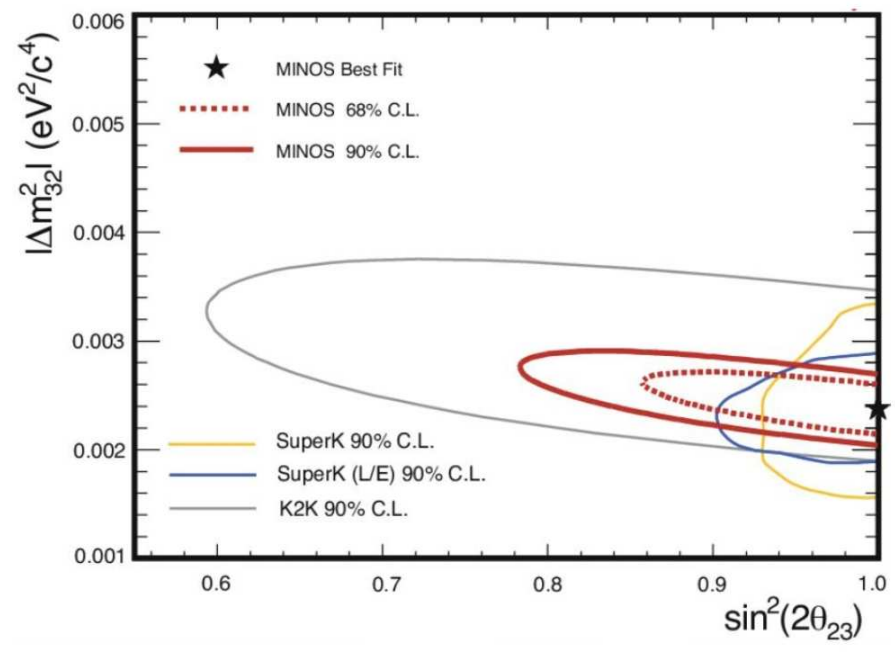

Figure 1-6: Summary of Super-K [54, 55], K2K [57], and MINOS [56] oscillation results. The plot is taken from [58].

that the flux of atmospheric muon neutrinos coming through the earth was lower than the flux of those coming from above the detector [54]. Based on symmetry arguments, this result suggested that some mechanism must exist by which the $\nu_{\mu}$ flux changes as a function of distance traveled by the neutrinos. Furthermore, while the experiment lacked sensitivity to tau neutrinos, it had sufficient sensitivity to atmospheric electron neutrinos. Because the measured electron neutrino event rate was found consistent with theoretical flux predictions, the effect was interpreted as $\nu_{\mu} \rightarrow \nu_{\tau}$ transitions with an oscillation probability amplitude $>90 \%$. A latter analysis of Super-K data [55], using the reconstructed direction and energy of the incoming neutrinos in the range of 1-10 GeV, was able to reconstruct an $L / E$-dependent event distribution and measure the mass splitting $\Delta m_{\text {atm }}^{2} \sim 2-3 \times 10^{-3} \mathrm{eV}^{2}$ with better precision.

Still, Super-K results suffered from uncertainties in the modeling of atmospheric neutrino fluxes, and therefore confirmation and more precise determination of the parameters followed using man-made, accelerator-based beams of neutrinos at a slightly lower energy than atmospheric neutrinos. The MINOS [56] and K2K [57] experiments are both accelerator-based muon neutrino disappearance experiments. Both experiments look for $\nu_{\mu}$ disappearance using a two-detector approach. A near detector located $\sim 1 \mathrm{~km}$ from the neutrino source is used to normalize the expected event 
rate in the absence of oscillations at a far detector located several hundred kilometers away. A deficit and energy dependent distortion of the $\nu_{\mu}$ event rate at $1-10 \mathrm{GeV}$ in the distant detector is therefore evidence for the same $\nu_{\mu} \rightarrow \nu_{\tau}$ oscillations as seen in atmospheric neutrino experiments. K2K was able to determine an energy-dependent discrepancy in their observed spectrum of $\nu_{\mu}$ events which was consistent with an $L / E$-dependent oscillation hypothesis, while MINOS in a similar manner was able to provide a precise measurement of $\Delta m_{23}^{2} \cdot{ }^{12}$

The oscillation parameters determined by atmospheric and accelerator-based neutrino experiments are summarized in Fig. 1-6.

\section{The $\left(\Delta m_{12}^{2}, \sin ^{2} 2 \theta_{12}\right)$ sector}

As discussed in Sec. 1.2, the solar neutrino problem that began with Davis' experiment was eventually resolved in 2001, when results from the SNO experiment proved definitively that both experiment and theory were correct [31]. The SNO measurement was consistent with results from solar radiochemical experiments under the Mikheyev-Smirnov-Wolfenstein large mixing angle (LMA) solution of the solar neutrino problem [59]. In this model, neutrinos produced as $\nu_{e}$ in the center of the sun, evolve into $\nu_{2}$ mass eigenstates adiabatically, due to a matter effect potential they experience as they propagate outwards. ${ }^{13}$ Therefore, what SNO and other solar neutrino experiments detect are in fact $\nu_{2}$ mass eigenstates, which are mixtures of $\nu_{e}, \nu_{\mu}$, and $\nu_{\tau}$ weak eigenstates, as shown in Fig. 1-5. The ratio of fluxes measured through the CC to NC observed rates by SNO therefore corresponds to $\left|U_{e 2}\right|^{2} / \sum_{\alpha}\left|U_{\alpha 2}\right|^{2}=\left|U_{e 2}\right|^{2}$. A global analysis of solar neutrino data determines $\theta_{12} \sim 34^{\circ}[60]$.

The first experiment to successfully demonstrate neutrino oscillations as a function of $L / E$ was the KamLAND reactor-based experiment in Japan [61]. In doing so, KamLAND helped solidify the LMA solution of the solar neutrino problem and determined $\Delta m_{12}^{2}$. The experiment detected electron antineutrinos from several power reactors in Japan, with an average distance of $180 \mathrm{~km}$, and it was able to recon-

\footnotetext{
${ }^{12}$ In fact, MINOS provides the most precise measurement of $\Delta m_{23}^{2}$ to date.

${ }^{13}$ For a useful review of matter effects, see, e.g. [45] and references within.
} 

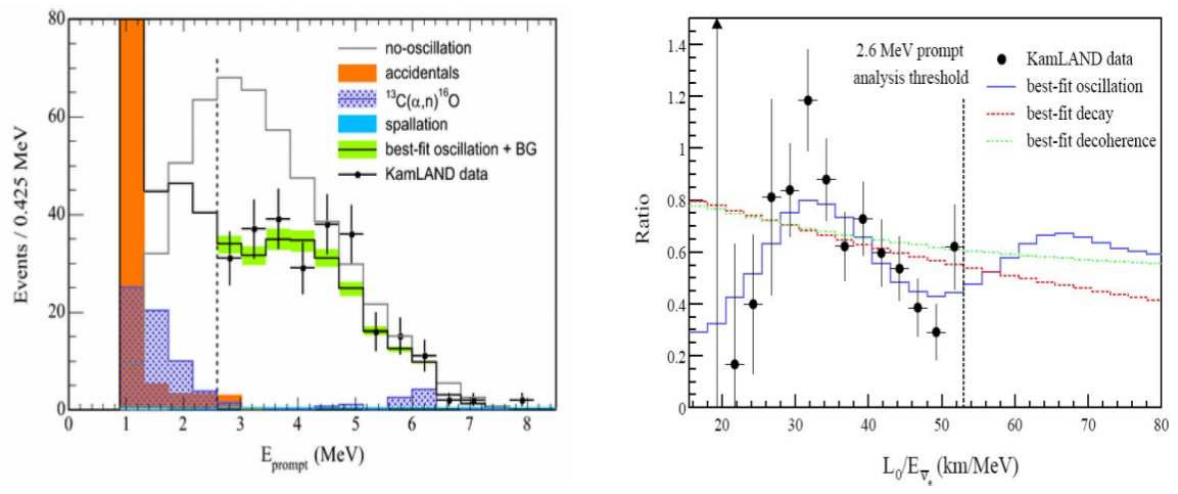

Figure 1-7: Summary of KamLAND oscillation results. The left panel shows the energy distribution of events for the no oscillation hypothesis compared to data. The right panel shows the ratio of data to a no oscillation prediction, as a function of $L / E$. The blue histogram shows the best fit oscillation hypothesis. The figure is from $[61]$.

struct the energy of each neutrino by measuring the energy of the outgoing electron in inverse $\beta$ decay neutrino interactions. The neutrino energy ranged between 0 and $10 \mathrm{MeV}$. The result, summarized in Fig. 1-7 showed that only $60 \%$ of the expected electron antineutrinos from a no oscillation prediction made it to the KamLAND detector, consistent with $\theta_{12} \sim 30^{\circ}$. The location of the minimum ratio of observed to predicted events determined the size of $\Delta m_{12}^{2}$ to be consistent with $\sim 7 \times 10^{-5} \mathrm{eV}^{2}$. To this date, the KamLAND results yield the most precise measurement of $\Delta m_{12}^{2}$.

The $\left(\Delta m_{12}^{2}, \tan ^{2} \theta_{12}\right)$ regions allowed by solar neutrino experiments and KamLAND are shown in Fig. 1-8. A joint analysis of KamLAND and solar neutrino data assuming CPT invariance yields [62]

$$
\begin{array}{r}
\tan ^{2} \theta_{12}=0.47_{-0.05}^{+0.06} \\
\Delta m_{12}^{2}=(7.59 \pm 0.21) \times 10^{-5} \mathrm{eV}^{2} .
\end{array}
$$

Note that both $\theta_{23}$ and $\theta_{12}$ are large, in striking contrast to all quark mixing angles $[45]$. 


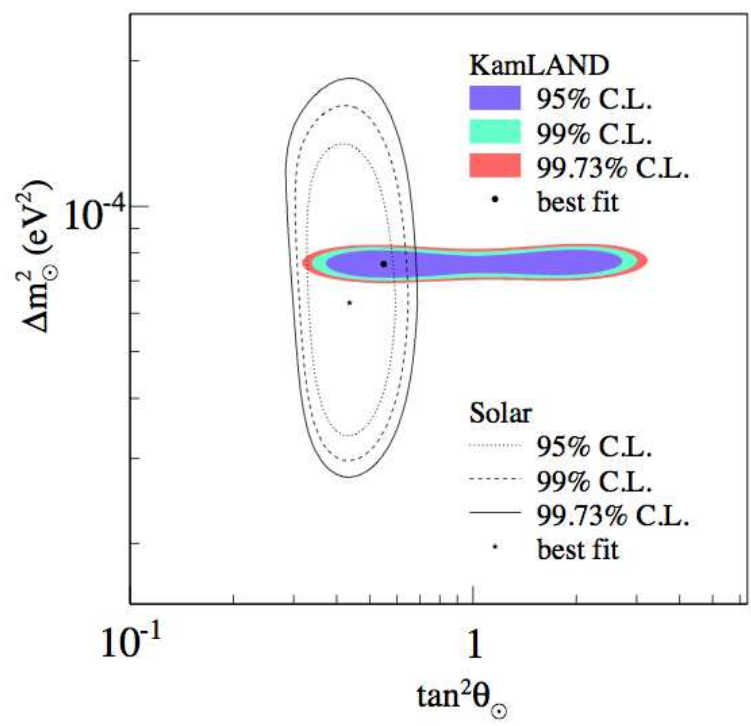

Figure 1-8: Allowed regions for $\left(\Delta m_{12}^{2}, \sin ^{2} 2 \theta_{12}\right)$ from solar [31] and reactor [61] neutrino experiments. The figure is taken from [61].

The $\left(\Delta m_{13}^{2}, \sin ^{2} 2 \theta_{13}\right)$ sector

So far we have seen that $\theta_{12}, \theta_{23}, \Delta m_{12}^{2}$, and $\Delta m_{23}^{2}$ are constrained by solar/reactor neutrino experiments and atmospheric/accelerator neutrino experiments. The remaining oscillation parameters, $\theta_{13}$ and $\Delta m_{13}^{2}$ are constrained by reactor-based neutrino oscillation experiments looking for $\bar{\nu}_{e}$ disappearance at experimental baselines much smaller than KamLAND's. This shorter baseline is used to allow for maximal sensitivity to (the much larger) $\Delta m_{13}^{2}$, expected to have a value of $\left|\Delta m_{23}^{2} \pm \Delta m_{12}^{2}\right|,{ }^{14}$ which enters the $\left(1.27 \Delta m^{2} L / E\right)$ phase in the oscillation probability.

The amplitude of such oscillations is expected to be small, based on atmospheric neutrino oscillation results, which show no significant excess of $\nu_{e}$ events compared to background prediction, and therefore attribute the missing $\nu_{\mu}$ flux to maximal $\nu_{\mu} \rightarrow \nu_{\tau}$ oscillations. Therefore, it is particularly attractive to look for $\Delta m^{2}$-driven oscillations at reactors, which produce a relatively abundant (high-statistics) and well-understood (low-systematics) flux of electron antineutrinos.

\footnotetext{
${ }^{14}$ The sign depends on whether the three-neutrino spectrum is normal, or inverted, as discussed earlier.
} 


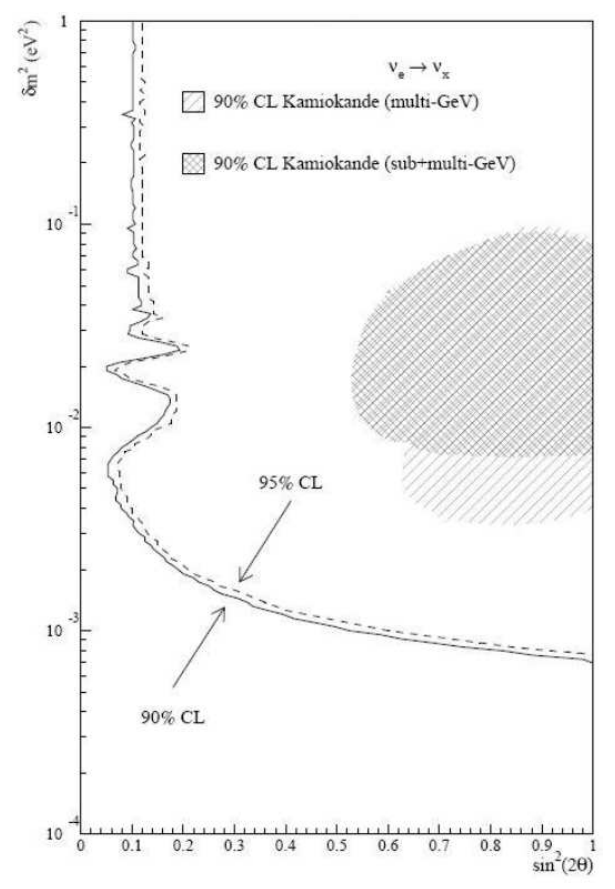

Figure 1-9: Current limits on $\sin ^{2} \theta_{13}$ from the CHOOZ experiment [63]. The region to the right of the solid and dashed curves are excluded at $90 \%$ and $99 \%$ C.L., respectively.

So far, reactor-based short-baseline experiments have not been able to observe $\nu_{e} \rightarrow \nu_{\ell}$ oscillations driven by $\Delta m_{13}^{2}$. The strongest constraints come from the CHOOZ experiment [63] which places a limit to $\sin ^{2} \theta_{13}$ as shown in Fig. 1-9. From short-baseline reactor experiments and other data [64, 65], $\sin ^{2} \theta_{13}$ is constrained to less than 0.032 , at $2 \sigma$ [66]. However, a recent three-neutrino analysis [67] using all available neutrino oscillation data, and invoking unitarity of the neutrino mixing matrix, suggests that $\sin ^{2} \theta_{13}$ is non-zero at the level of $1 \sigma$. Near-future reactor-based short-baseline experiments such as Double-Chooz [68], RENO [69], and Daya Bay [70], will have $\theta_{13}$ sensitivities beyond current experimental limits, and are expected to address this possibility in the near future.

As alluded to earlier, accelerator-based long-baseline experiments are also sensitive to $\theta_{13}$. The caveat of such search is that oscillations due to $\theta_{13}$ are small relative to the dominant $\theta_{23}$ channel. Currently, K2K and MINOS are limited by low $\nu_{e}$ signal statistics [71]. The intrinsic $\nu_{e}$ flux is also low, which makes it difficult to constrain $\nu_{e}$ 
flux and cross-section systematics. However, the upcoming T2K experiment in Japan [72] and $\mathrm{NO} \nu \mathrm{A}$ experiment in the U.S. [51] are each designed with a high-intensity beam, specifically intended for such a search, and will have increased sensitivity to $\theta_{13}$.

It should be noted that precise determination of $\theta_{13}$, and specifically a measurement $\theta_{13} \neq 0$ is crucial from the perspective of searches for $C P$ violation in the neutrino sector. As seen from Eq. 1.29, the $C P$-violating phase $\delta$, which is the only phase in the $3 \times 3 U$ matrix that can produce $C P$ violation in neutrino oscillations, enters $U$ only in combination with $\sin \theta_{13}$. Therefore, the size of $C P$ violation effects in neutrino oscillations depends on the size of this parameter. If $\theta_{13}$ is zero, then there can be no observable $C P$ violation in neutrino oscillations.

\subsubsection{Direct Limits on Neutrino Mass}

While neutrino oscillation experiments have demonstrated that neutrinos have nonzero and distinct masses, and have been able to precisely measure mass-squared differences, they are insensitive to absolute neutrino masses. That is, they cannot tell us how far from zero the mass spectra in Fig. 1-5 are. They can, however, provide a lower limit for the mass of the heaviest neutrino mass eigenstate, which can obviously not be any less than $\sqrt{\Delta m_{\text {atm }}^{2}}$. Direct limits on absolute neutrino masses are instead obtained $^{15}$ by studying the kinematics of various weak decays in laboratory-based experiments.

Specifically, experiments such as Katrin [73], MARE [74] and Mainz [75] aim to measure the effective mass of the electron neutrino by studying the endpoint of the energy spectrum of electrons in tritium beta decay. Depending on the energy resolution of the experiment, the different mass eigenstates which make up the electron neutrino produced in the decay introduce kinks to the electron energy spectrum with sizes and positions dependent on the overall mixing and mass of each state. Assum-

\footnotetext{
${ }^{15}$ Here, we limit our discussion of constraints to those applicable to Dirac neutrinos. In the case of Majorana neutrinos, neutrinoless double-beta decay experiments can also provide constraints on neutrino masses. Those will be discussed in Chapter 2, as they are applicable in new physics scenarios considered within that chapter.
} 
ing that the energy resolution of the experiment is much larger than $m_{i}$, which is the current experimental situation, the effective mass of the electron neutrino emitted in the decay is defined as

$$
m_{\nu_{e}}^{2}=\sum_{i}\left|U_{e i}\right|^{2} m_{i}^{2} .
$$

This is precisely the parameter measured by those experiments. The larger the mass

of the $\nu_{i}$ with the most appreciable coupling to an electron $\left(\left|U_{e i}\right|^{2}\right)$, the higher $m_{\nu_{e}}^{2}$ will be. This result therefore is different for normal versus inverted hierarchies.

Currently, the best direct limits on the effective electron neutrino mass come from the Mainz experiment [75], and correspond to $m_{\nu_{e}}<2.3 \mathrm{eV}$ at $95 \%$ C.L.

Similarly, limits on the effective muon neutrino mass come from studying $\pi^{+} \rightarrow$ $\mu^{+} \nu_{\mu}$ decay at rest, which constrains

$$
m_{\nu_{\mu}}^{2}=\sum_{i}\left|U_{\mu i}\right|^{2} m_{i}^{2} .
$$

Current experimental limits are consistent with $m_{\nu_{\mu}}<0.19 \mathrm{MeV}$ at $90 \%$ C.L. [45].

Limits on the effective tau neutrino mass can be obtained from studies of kinematics of $\tau^{-} \rightarrow 2 \pi^{-} \pi^{+} \nu_{\tau}$ decays, or $\tau^{-} \rightarrow 3 \pi^{-} 2 \pi^{+}\left(\pi^{0}\right) \nu_{\tau}$ decays. Current limits from [76] correspond to

$$
m_{\nu_{\tau}}^{2}=\sum_{i}\left|U_{\tau i}\right|^{2} m_{i}^{2}<18.2 \mathrm{MeV}
$$

at $95 \%$ C.L.

\subsubsection{Constraints from Cosmology}

Observational cosmology provides another way to address the question of the absolute scale of neutrino mass, since non-zero neutrino masses contribute to the energy density of the universe, and therefore play a fundamental role in its evolution. For example, Big Bang Nucleosynthesis (BBN) data constrain the primordial abundances of Helium and other elements, which are sensitive to the neutrino energy density (or effective number of neutrino types) during the BBN era. Moreover, non-zero neutrino masses, and in particular the amount of relativistic versus non-relativistic neutrino species in 
the early universe, will have implications on large scale structure formation in the universe and cosmic microwave background anisotropies observed today [77].

Current cosmological data constrain the sum of the masses of all light neutrino mass eigenstates $\nu_{i}$ that may exist and were in thermal equilibrium in the early universe to

$$
\sum m_{i}<(0.17-2.0) \mathrm{eV}
$$

depending on underlying cosmological assumptions [78, 79].

It should be emphasized that the limits obtained from cosmology are modeldependent and require assumptions beyond just the existence of three neutrino mass eigenstates as suggested in Fig. 1-5 (see, e.g., [80]). They are, in that sense, indirect. 


\section{Chapter 2}

\section{Neutrinos as Windows to New Physics}

The discovery of neutrino mass begs the question:

Is neutrino mass a reflection of new physics which is deeper than a simple extension to the Standard Model?

In addressing this, one may also consider the one oscillation experiment which does not fit the three-neutrino picture described in Chapter 1: The Liquid Scintillator Neutrino Detector, or LSND. Consideration of the surprising results of this experiment has led the author and her collaborators in $[81,82]$ to further explore a phenomenological model which is described in this chapter, that of light sterile neutrinos. This model can be couched within a larger context, called "mixing freedom", which is being explored by the author and her collaborators, J. Conrad and B. Kayser, separately, in a paper now in draft.

The chapter begins with a discussion on neutrino phenomenology with mixing freedom, which is often a consequence of possible extensions to the Standard Model beyond three-neutrino mixing. We discuss an example of how mixing freedom can arise in Sec. 2.1.2, and present some general experimental signatures of mixing freedom in Sec. 2.1.3. Relevant experimental constraints are discussed in Sec. 2.1.4. Particular emphasis is placed on the light sterile neutrino scenario, which is discussed in Sec. 2.2 as a possible interpretation of the LSND experimental anomaly, the latter 
being presented in Sec. 2.2.2. The narrow phenomenological picture considered in view of the LSND results introduces multiple light sterile neutrinos, where the work by the author explored the possibility of $C P$ violation within the model, as a way to overcome current experimental constraints from other neutrino oscillation experiments. At the end of this chapter, we provide a map for how the phenomenology presented in this chapter will be applied to analyses reported in the rest of this thesis.

\subsection{Mixing Freedom}

Mixing freedom refers precisely to mixing via a matrix whose experimentally accessible part is non-unitary, and is often a consequence of extending the neutrino spectrum from 3 to $N$ neutrinos. A simple way to do so is by extending the $\nu_{L}^{0}$ in Eq. 1.8 to beyond three neutrino generations, such that

$$
\nu_{L}^{0} \equiv\left(\begin{array}{c}
\nu_{L e}^{0} \\
\nu_{L \mu}^{0} \\
\nu_{L \tau}^{0} \\
\nu_{L s_{1}}^{0} \\
\nu_{L s_{2}}^{0} \\
\cdots
\end{array}\right)
$$

where $s_{i}$ are new "flavor" eigenstates, which do not couple to the $W$ and $Z$ bosons. We will see the reason for this requirement in a Sec. 2.1.4. The corresponding neutrino states are called "sterile" neutrinos.

In the context of Eq. 2.1, the neutrino mixing matrix, $U$, becomes $N \times N$, and while it may be unitary in its entirety, the part of $U$ which may be accessible experimentally is generally expected to be non-unitary.

We refer to two specific examples of such extensions in the following section, and discuss one of them in more detail in Sec. 2.1.2. Experimental consequences and constraints within the context of neutrino experiments are discussed in Secs. 2.1.3 and 2.1.4, respectively. 


\begin{tabular}{l|l}
\hline \hline Scenario 1: & $\begin{array}{l}\text { See-Saw with light sterile neutrinos } \\
\text { Such sterile neutrinos have small enough masses }(<10 \mathrm{eV}) \text { to be } \\
\text { components of conventional neutrino beams. }\end{array}$ \\
\hline Scenario 2: & $\begin{array}{l}\text { See-Saw with sterile neutrinos at two distinct mass scales } \\
\text { This is an extension of scenario 1, where both very light }(<10 \mathrm{eV}) \\
\text { and heavier }(>10 \mathrm{GeV}) \text { sterile neutrinos exist. The latter cannot } \\
\text { be emitted in meson decays, and therefore cannot be part of a conventional } \\
\text { neutrino beam. }\end{array}$ \\
\hline \hline
\end{tabular}

Table 2.1: Underlying physics scenarios which lead to mixing freedom. These models are discussed in more detail in [88].

\subsubsection{Underlying Scenarios}

There are several underlying physics scenarios that can lead to mixing freedom, or extension of the $3 \times 3$ neutrino mixing matrix to $N \times N$. Many such scenarios have been already heavily explored within the literature, including heavy or light sterile neutrinos or new lepton generations. Those scenarios usually lead to exotic oscillation signatures or non-standard neutrino interactions beyond those expected in the threegeneration scheme of Sec. 1.3 (see, e.g., [83, 84, 85, 86, 87]).

Table 2.1 summarizes two particular models that we will refer to in this chapter, chosen as examples from Ref. [88]. The first scenario, See-Saw with light sterile neutrinos, will be discussed in more detail in the following subsection.

Depending on the underlying scenario, mixing freedom can manifest itself in neutrino experiments differently. We discuss a particular effect of mixing freedom, relevant to scenario 2, in Sec. 2.1.3. However, in general, the ways in which mixing freedom manifests will depend on how the new leptons in each scenario modify the accessible part of the mixing matrix. The "accessible part" of the mixing matrix refers to the sub-matrix of the leptonic mixing matrix $U$, in each scenario, which couples $\ell_{\alpha}=e, \mu, \tau$ to the neutrino mass eigenstates which are light enough to be produced in typical neutrino experiments (i.e., neutrino masses of order $10 \mathrm{eV}$ or less).

One defines the leptonic mixing matrix $U$ in terms of the couplings of the leptons 
to the $W$ boson in the electroweak CC Lagrangian, as previously,

$$
-\mathcal{L}_{W}=\frac{g}{\sqrt{2}} \sum_{\alpha, i} \overline{\ell_{L \alpha}} \gamma^{\lambda} U_{\alpha i} \nu_{L i} W_{\lambda}^{-}+h . c .
$$

except, in the case of mixing freedom, $\alpha$ runs over the charged lepton flavors, $e, \mu, \tau$, as well as any additional ones which may exist in the scenario under consideration, while $i$ runs over the neutrino mass eigenstates, whose number is $N \geq 3$.

Scenario 1 is an underlying model which is very instructive to consider within the context of mixing freedom. The next section considers this model in detail, and provides an example of how mixing freedom arises. ${ }^{1}$

\subsubsection{How Mixing Freedom Can Arise: Light Sterile Neutri- nos}

To see the degree to which the accessible part of the mixing matrix, $A$, retains aspects of unitarity in this particular scenario, one may repeat the exercise of Sec. 1.3.2, starting with Eq. 1.10, except now one replaces $\nu_{L}^{0}$ (and $\nu_{R}^{0}$ ) with an $N$-dimensional vector, as defined in Eq. 2.1, and the sub-matrices $0, m_{D}$, and $m_{R}$ in the mass matrix

$$
\mathcal{M}_{\nu} \equiv\left(\begin{array}{cc}
0 & m_{D}^{T} \\
m_{D} & m_{R}
\end{array}\right)
$$

which appears in $\mathcal{L}_{m_{\nu}}$, are all $N \times N$ rather than $3 \times 3$.

The mass matrix $\mathcal{M}_{\nu}$ may be diagonalized using a $(2 N) \times(2 N)$ matrix, $Z$, which unitary and of the from

$$
Z=\left(\begin{array}{ll}
V & Y \\
X & W
\end{array}\right)
$$

where the sub-matrices $V, W, X$, and $Y$ are all $N \times N$. Note that, given that

\footnotetext{
${ }^{1}$ It should be noted that models with sterile neutrinos sometimes involve new heavy gauge bosons, $W^{\prime}$ and/or $Z^{\prime}$, which can couple to sterile neutrinos. Such models can be addressed by the Tevatron experiments at Fermi U.S. National Accelerator Laboratory, or the upcoming Large Hadron Collider experiments at CERN. Here, we limit ourselves to discussions within the context of neutrino experiments.
} 
$m_{D} \ll m_{R}, X$ and $Y$ are much smaller than $V$ and $W$, so that $V$ and $W$ are each unitary to an excellent approximation.

The left-handed component $\nu_{L}$ of the $2 N$-dimensional vector of neutrino mass eigenstates is then related to the weak eigenstate vectors $\nu_{L}^{0}$ and $\nu_{R}^{0}$ by

$$
\left(\begin{array}{c}
\nu_{L}^{0} \\
\left(\nu_{R}^{0}\right)^{c}
\end{array}\right)=Z \nu_{L} .
$$

Thus, since $Y \ll V$,

$$
\nu_{L \beta}^{0} \simeq \sum_{i=1, \ldots, N} V_{\beta i} \nu_{L i}^{\text {light }}
$$

where the sum runs over the $N$ light neutrino mass eigenstates $\nu_{L i}^{\text {Light }}$, and not over their GUT-scale heavy See-Saw partners.

Now let us return back to the SM leptonic CC interaction Lagrangian,

$$
-\mathcal{L}_{W}=\frac{g}{\sqrt{2}} \sum_{\beta=e, \mu, \tau} \overline{\ell_{L \beta}^{0}} \gamma^{\lambda} \nu_{L \beta}^{0} W_{\lambda}^{-}+\text {h.c. }
$$

where $\ell_{L \beta}^{0}$ is the left-handed charged lepton weak eigenstate of flavor $\beta$. In the case of charged leptons, $\ell_{L \beta}^{0}$ is related to the left-handed components of the charged lepton mass eigenstates, $\ell_{L \alpha}$, by

$$
\ell_{L \beta}^{0}=\sum_{\alpha=e, \mu, \tau} B_{\beta \alpha} \ell_{L \alpha}
$$

where $B$ is a $3 \times 3$ unitary matrix. ${ }^{2}$ Using this relation and Eq. 2.6, Eq. 2.7 becomes

$$
\begin{aligned}
-\mathcal{L}_{W}= & \frac{g}{\sqrt{2}} \sum_{\alpha=e, \mu, \tau} \sum_{i=1, \ldots, N} \overline{\ell_{L \alpha}} \gamma^{\lambda}\left(\sum_{\beta=e, \mu, \tau} B_{\alpha \beta}^{\dagger} V_{\beta i}\right) \nu_{L i}^{l i g h t} W_{\lambda}^{-} \\
& + \text {h.c. }
\end{aligned}
$$

\footnotetext{
${ }^{2}$ Note that, in the case of charged leptons, $i$ and $\alpha$ indices are interchangeable.
} 
Comparing the above equation with Eq. 2.2, which defines $U$, we identify

$$
U_{\alpha i}=\sum_{\beta=e, \mu, \tau} B_{\alpha \beta}^{\dagger} V_{\beta i} ; \quad \alpha=e, \mu, \tau, \text { and } i=1, \ldots, N .
$$

One may "complete" the $3 \times N U$ matrix of Eq. 2.10 by adding rows in such a way that $U$ becomes an $N \times N$ square unitary matrix. We may define an $N \times N$ unitary matrix $\mathcal{B}$ by

$$
\mathcal{B}^{\dagger}=\left(\begin{array}{cc}
B^{\dagger} & 0^{T} \\
0 & I_{N-3}
\end{array}\right),
$$

where $B$ is the $3 \times 3$ unitary matrix introduced previously, $I_{N-3}$ is the $(N-3)$-dimensional identity matrix and 0 is an $(N-3) \times 3$ block of zeroes. Then, to the level at which $V$ is unitary, which is an excellent approximation,

$$
U_{\text {complete }} \equiv \mathcal{B}^{\dagger} V
$$

is an $N \times N$ square unitary matrix whose $e, \mu$, and $\tau$ rows are those of the mixing matrix $U$ given by Eq. 2.10. $U_{\text {complete }}$ is identified to the complete unitary mixing matrix. Only its $e, \mu$, and $\tau$ rows will affect neutrino oscillations.

Because we assume that all light neutrinos in this scenario have masses $m_{i}<10 \mathrm{eV}$, and can therefore be emitted by conventional neutrino sources, the entire $e, \mu$, and $\tau$ rows of $U_{\text {complete }}$ are experimentally accessible, and are identified with $A$, a $3 \times \mathrm{N}$ matrix consisting of these three rows.

Taking a closer look at $A$, we find that

$$
\sum_{i} A_{\alpha i} A_{\beta i}^{*}=\delta_{\alpha \beta} ; \quad \alpha, \beta=e, \mu, \tau
$$


with $i$ running over mass eigenstates with masses $m_{i}<10 \mathrm{eV}$. However,

$$
\sum_{\alpha=e, \mu, \tau} A_{\alpha i}^{*} A_{\alpha j}=\sum_{\alpha=e, \mu, \tau} V_{\alpha i}^{*} V_{\alpha j} ; \quad i, j \text { running over mass eigenstates with } m_{i}<10 \mathrm{eV}
$$

Since $V$ is an $N \times N$ unitary matrix with $N>3$, but the right-hand side of Eq. 2.14 sums only over its first three rows, in general this sum does not yield $\delta_{i j}$. Thus, in the present scenario the accessible part of the neutrino mixing matrix does not satisfy the unitarity condition

$$
\sum_{\alpha=e, \mu, \tau} A_{\alpha i}^{*} A_{\alpha j}=\delta_{i j} ; \quad i, j \text { running over mass eigenstates with } m_{i}<10 \mathrm{eV} .
$$

\subsubsection{Physical Effects of Mixing Freedom}

In this section, we consider some physical effects of mixing freedom within the context of neutrino experiments.

Depending on the nature of the new leptons, the consequences for neutrino experiments may include instantaneous flavor change of neutrinos emitted in weak decays (a long-recognized possibility [89]), anomalous neutrino oscillation patterns, oscillation of the $e-\mu-\tau$ flavor-summed neutrino oscillation probability, oscillation of the $\mathrm{NC}$ event rate in a neutrino beam, and enriched patterns of $C P$ violation [88]. Here, we focus on the effect of instantaneous transitions.

We begin by deriving the probability for neutrino oscillation which one would measure experimentally in the presence of mixing freedom. We follow a similar approach as in Sec. 1.3.4, except this time we do not invoke unitarity.

Using the Kayser-Stodolsky [90] approach to deriving neutrino oscillation amplitudes, whether $A$ is unitary or not, the amplitude squared for a neutrino of energy $E$ to be born with an $\ell_{\alpha}$ and then to make an $\ell_{\beta}$, after propagating some distance $L$, is given by

$$
R_{\alpha \beta}=\left|\sum_{i} A_{\alpha i}^{*} A_{\beta i} e^{-i m_{i}^{2} \frac{L}{2 E}}\right|^{2}
$$




$$
\begin{aligned}
= & \left|\left(A A^{\dagger}\right)_{\alpha \beta}\right|^{2} \\
& -4 \sum_{i>j} \operatorname{Re}\left\{A_{\alpha i}^{*} A_{\beta i} A_{\alpha j} A_{\beta j}^{*}\right\} \sin ^{2}\left(\Delta m_{i j}^{2} \frac{L}{4 E}\right) \\
& +2 \sum_{i>j} \operatorname{Im}\left\{A_{\alpha i}^{*} A_{\beta i} A_{\alpha j} A_{\beta j}^{*}\right\} \sin \left(\Delta m_{i j}^{2} \frac{L}{2 E}\right),
\end{aligned}
$$

where $i$ and $j$ run over masses less than $10 \mathrm{eV}$. The above expression applies to neutrinos both produced and detected through CC interactions.

From Eq. 2.16, it is clear that when $A$ is unitary, or more specifically when its rows are orthonormal vectors, ${ }^{3}\left|\left(A A^{\dagger}\right)_{\alpha \beta}\right|=\delta_{\alpha \beta}$, and therefore Eq. 2.16 reduces to that of Eq. 1.22, as in standard $\nu_{\alpha} \rightarrow \nu_{\beta}$ oscillations. The difference, however, is that now sums are allowed among all $N>3$ light neutrino mass eigenstates which are components of the neutrino beam.

In the event, however, that the rows of $A$ are not orthonormal vectors (e.g., in the case of scenario 2, where heavier sterile neutrino mass eigenstates would not contribute to the summation of Eq. 2.13), $\left|\left(A A^{\dagger}\right)_{\alpha \beta}\right| \neq \delta_{\alpha \beta}$. The effect of this term is to introduce $L$-independent transitions. Specifically, when $L=0$, one would still expect to observe $\nu_{\alpha} \rightarrow \nu_{\beta}$ transitions, where $\alpha \neq \beta$.

This consequence becomes more intuitive from the perspective of disappearance, $\nu_{\alpha} \rightarrow \nu_{\not \alpha}$, where $\left|\left(A A^{\dagger}\right)_{\alpha \alpha}\right| \neq 1$. This introduces a normalization change to the expected event rate at $L=0$, or instantaneous disappearance. For example, in the case of scenario 2, this disappearance of $\alpha$ content in the beam is due to the fact that the beam is physically missing the neutrino mass eigenstates with masses greater than $10 \mathrm{eV}$, and their corresponding $\alpha$ content. Those mass eigenstates are missing simply because they are too heavy to be emitted at neutrino production.

It should be noted that instantaneous disappearance would only be detectable in the case where an experiment compares rates from a first-principles prediction to the observed rates in the detector. It cannot be observed in near-far neutrino detector experiments because the transition occurs at the neutrino production vertex - well before the near detector. In most cases, first principles predictions have poor precision;

\footnotetext{
${ }^{3}$ This holds in scenario 1.
} 


\begin{tabular}{l|cc}
\hline \hline Source of constraints & Scenario 1 & Scenario 2 \\
\hline \hline Cosmology & model-dep. & model-dep. \\
Tritium beta decay & weak & weak \\
$0 \nu \beta \beta$ & model-dep. & model-dep. \\
Electroweak decays & & weak \\
$\mu \rightarrow e \gamma$ experiments & & strong \\
$\Gamma_{\text {invis }}(Z)$ & & weak \\
Neutrino experiments & strong & weak \\
\hline \hline
\end{tabular}

Table 2.2: Summary of experimental sources of constraints to mixing freedom scenarios in Tab. 2.1 [88]. These are discussed in more detail in Sec. 2.1.4.

however, in Sec. 2.1.4 we consider examples where this is not the case.

As a final note, when calculating oscillation probabilities, Eq. 2.16 must be modified by a normalization factor of $1 /\left|\left(A A^{\dagger}\right)_{\alpha \alpha}\left(A A^{\dagger}\right)_{\beta \beta}\right|$. This comes from considering the fact that the neutrino states $\nu_{\alpha}$ and $\nu_{\beta}$ in the quantum-mechanical treatment approach of Eq. 2.16 must be unit-normalized [91, 89]. Those factors are important to include when calculating expected event rates in searches for mixing freedom.

\subsubsection{Experimental Constraints}

\section{Constraints from cosmology}

Both scenarios in Tab. 2.1 introduce new degrees of freedom. In the case where the new degrees of freedom are light (i.e., $m_{i} \sim 1 \mathrm{MeV}$ or less), relevant constraints can be provided by several cosmological observables.

Specifically, data from Cosmic Microwave Background (CMB) and Large Scale Structure (LSS) surveys are sensitive to the number of relativistic neutrino species in the early universe, and can be used to constrain the number and total mass of light neutrino species with weak couplings. A recent analysis from WMAP [92], provides currently the strongest constraints on the effective number of neutrino species in the early universe, $N_{\nu}=4.34_{-0.88}^{+0.86}$ (68\% C.L.), and the total neutrino mass, $\sum m_{\nu}<0.58$ eV (95\% C.L.). These limits are rather model-dependent and can easily be evaded. For example, most models assume that sterile neutrinos in the early universe are present with the same thermal abundance as the standard, "active "neutrino flavors. 
Thermal abundances can easily be suppressed (see, e.g. [93, 94]), resulting in weaker limits. While cosmological constraints are difficult to interpret as limits on the level of non-unitarity of $A$, they can offer information on the viability of various underlying mixing freedom scenarios.

\section{Constraints from direct limits on neutrino mass}

In the case of scenarios 1 and 2 , constraints to the mass and mixing of light sterile neutrinos can also be provided by tritium beta decay. In this case, the constraints come entirely from kinematic considerations, and are therefore less model-dependent than cosmological constraints. Beta decay experiments limit the effective electron neutrino mass, $m_{\nu_{e}}^{2}=\sum_{i}\left|U_{e i}\right|^{2} m_{i}^{2}{ }^{4}$ Light sterile neutrino states with mass $m_{i}$ on the order of $\sim 1 \mathrm{eV}$ and mixing $U_{e i}$ will also contribute to $m_{\nu_{e}}^{2}$ according to $m_{\nu_{e}}=\sum_{i}\left|U_{e i}\right|^{2} m_{i}$ $[95,96]$. Constraints are very weak, but present. For mass and mixing parameters of light sterile neutrinos allowed by global analyses of short-baseline neutrino oscillation experiments (see, e.g., [81], or Chapter 6 for current status), limits from tritium beta decay are relatively weak, since best-fit parameters from these models contribute to $m_{\nu_{e}}$ by less than $0.1 \mathrm{eV}$. Stronger constraints on $m_{\nu_{e}}$ are expected from the forthcoming KATRIN [73] experiment.

Neutrinoless double-beta decay $(0 \nu \beta \beta)$ experiments such as CUORE [97] can also provide constraints to the masses and mixings of extra neutrino states, assuming they are Majorana particles. Those experiments try to measure the amplitude for a process where two neighboring nucleons exchange a $\nu_{e}$ and $\beta$-decay simultaneously. The amplitude of this process is expected to be non-zero for Majorana neutrinos, but highly suppressed by a factor of $\left(m_{\nu} / E_{\nu}\right)$. In the case of light Majorana neutrinos, with $m_{i}^{2} \ll Q^{2}$, where $Q^{2}$ is the momentum transfer in the interaction, sterile neutrino states contribute to the effective neutrino mass $m_{\beta \beta}$ measured in $0 \nu \beta \beta$ as $\sum_{i} U_{e i}^{2} m_{i}$, whereas heavier sterile neutrinos $\left(m_{i} \gg Q^{2}\right)$ contribute as $\sum_{i} U_{e i}^{2} / m_{i}[98]$. However, these constraints are also model-dependent. For example, Ref. [98] considers the

\footnotetext{
${ }^{4}$ Currently, the best limits come from the Mainz measurement [75] of $m_{\nu_{e}}^{2}=\left(-0.6 \pm 2.2_{\text {stat }} \pm\right.$ $\left.2.1_{\text {sys }}\right) \mathrm{eV}^{2}$, which yields $m_{\nu_{e}}<2.3 \mathrm{eV}$ at $95 \%$ C.L.
} 
effective neutrino mass $m_{\beta \beta}$ in a scenario with five light and a single heavy sterile neutrino of mass $m_{6}$; they illustrate the dependence of $m_{\beta \beta}$ on $m_{6}$ and point out some of the caveats of a Majorana model, as the new phases which are introduced can naturally lead to interference and cancellation effects.

\section{Constraints from electroweak measurements}

A constraint that is discussed often within the context of non-unitarity [89] is the LEP measurement of the invisible width of the $Z$. $Z$ decays into $e^{+} e^{-}, \mu^{+} \mu^{-}, \tau^{+} \tau^{-}$, $q \bar{q}(q=u, d, s, c, b)$ and $\nu \bar{\nu}$, with the partial width to $\nu \bar{\nu}$ decays given by

$$
\Gamma_{i n v}=\frac{1}{12 \pi} \frac{G_{F}}{\sqrt{2}} M_{Z}^{3} N_{\nu} \simeq 0.181 N_{\nu} \mathrm{GeV}
$$

where $N_{\nu}$ is the number of neutrino generations (weak eigenstates) that couple to the $Z$ boson through the neutral current weak interaction. The partial width measured by the LEP experiments yields [99]

$$
N_{\nu}=2.984 \pm 0.008
$$

Here, we point out that this constraint is only relevant in specific cases. To see this, recall that LEP measures $\Gamma[Z \rightarrow \nu \bar{\nu}]$, that is,

$$
\Gamma_{\nu \bar{\nu}}=\sum_{i, j} \Gamma\left[Z \rightarrow \nu_{i} \bar{\nu}_{j}\right]
$$

summed over all kinematically allowed mass eigenstate combinations. In the SM, as well as scenarios with sterile neutrinos where all neutrino mass eigenstates are less than $M_{Z} / 2$, and assuming the $\mathrm{SM} Z$-coupling to neutrinos,

$$
A m p\left[Z \rightarrow \nu_{\alpha}^{0} \bar{\nu}_{\beta}^{0}\right]=\delta_{\alpha \beta}
$$

where $\alpha, \beta$ are the three active flavors. Then, considering all $N$ neutrino mass eigen- 
states, where $N>3$,

$$
\begin{aligned}
A m p\left[Z \rightarrow \nu_{i} \bar{\nu}_{j}\right] & =\sum_{\alpha, \beta}\left\langle\nu_{i} \bar{\nu}_{j} \mid \nu_{\alpha}^{0} \bar{\nu}_{\beta}^{0}\right\rangle\left\langle\nu_{\alpha}^{0} \bar{\nu}_{\beta}^{0} \mid Z\right\rangle \\
& =\sum_{\alpha} U_{\alpha i}^{*} U_{\alpha j},
\end{aligned}
$$

and,

$$
\begin{aligned}
\sum_{i, j}^{N} \Gamma\left[Z \rightarrow \nu_{i} \bar{\nu}_{j}\right] & =\sum_{i, j}^{N} \sum_{\alpha} U_{\alpha i}^{*} U_{\alpha j} \sum_{\beta} U_{\beta i} U_{\beta j}^{*} \\
& =\sum_{\alpha, \beta} \delta_{\alpha \beta} \delta_{\alpha \beta}=\sum_{\alpha=1}^{3} 1=3
\end{aligned}
$$

just as with a unitary $3 \times 3 U$. Hence, this constraint is not applicable, as long as all $N m_{i}$ satisfy $m_{i}<M_{Z} / 2$.

As pointed out in Ref. [89], the tightest constraints on mixing freedom arise from precision rare decay experiments and electroweak decays, as there exist scenarios which would manifest as modified $W$ couplings. Such models affect $W$ decays, charged meson decays, and charged lepton decays in a way analogous to non-universality. A standard way to test non-universality comes from examining the ratio of $\mu \bar{\nu}_{\mu}$ to $e \bar{\nu}_{e}$ in pion decay, which is sensitive to $\left|\left(A A^{\dagger}\right)_{\mu \mu}\right| /\left|\left(A A_{e e}^{\dagger}\right)\right|$. This ratio method is attractive as a way to search for non-standard physics in meson decays, as it is effectively independent of the pion form factor. Stronger limits can be extracted from $\mu$ decay to $e \gamma$, which is sensitive to $\left|\left(A A^{\dagger}\right)_{\mu e}\right|^{2} /\left|\left(A A^{\dagger}\right)_{\mu \mu}\left(A A^{\dagger}\right)_{e e}\right|$. However, extraction of limits from muon decay takes as input the Fermi constant, $G_{F}$, which is extracted assuming unitarity.

Although the $Z$ width, $m_{W}$, and electroweak measurements can provide strong constraints on several mixing freedom scenarios, it is also important to consider mixing freedom within the strict context of neutrino experiments. This provides a general test for new physics, in a model-independent way [88]. We review the most straightforward methods in which searches for mixing freedom can be performed in neutrino oscillation experiments in the following paragraphs. 


\section{Constraints from neutrino oscillation experiments}

Instantaneous flavor transitions (see Sec. 2.1.3) are possibly the least model-dependent way to search for mixing freedom with neutrino experiments, in the limit of heavy degrees of freedom, or in the limit of large $\Delta m^{2}$ relative to the sensitivity of the experiment. Instantaneous disappearance results in a normalization difference between the observed neutrino event rate and that predicted from first physics principles. Instantaneous appearance results in a normalization and, often, shape change (due to flux and cross-section shape differences for neutrinos of different flavors) between observed and predicted spectra.

The accuracy to which those normalization effects can be measured is currently limited by the accuracy to which the absolute flux and cross-section normalizations in neutrino experiments are known. Such searches are challenging, due to large uncertainties on neutrino production and interaction cross-sections from first principles, especially for accelerator-based neutrino beams. In those cases, typical flux and crosssection theoretical uncertainties are on the order of $20 \%$. Near-far detector comparisons, which are usually employed to cancel flux and cross-section systematic uncertainties in neutrino oscillation searches, are insensitive to instantaneous transition effects, or in the high- $\Delta m^{2}$ limit, since both are $L$-independent effects. Atmospheric and solar neutrino detectors are also limited by the level at which atmospheric and solar neutrino fluxes are understood.

Searches for instantaneous $\nu_{e}$ disappearance at reactor-based experiments such as Texono [100], or other very short baseline reactor-based experiments which are designed to measure $\nu-e$ scattering with high precision could be sensitive to instantaneous $\nu_{e}$ disappearance. However uncertainties in flux prediction for those experiments is on the order of $30 \%$.

Appearance-style $\nu_{\mu} \rightarrow \nu_{e}$ instantaneous transition searches are far more straightforward as a method for searching for mixing freedom, although caveats may exist in the way that various experiments treat their systematic uncertainties or other flux and cross-section systematic effects in their data. We consider such issues in Chapter 7, 
where we attempt to use the publicly available MiniBooNE neutrino and antineutrino data $[101,102]$ to search for $\nu_{\mu} \rightarrow \nu_{e}$ instantaneous transitions.

The authors of [89] have derived constraints on the non-unitarity of the $3 \times 3$ assumed accessible neutrino mixing matrix using information from neutrino experiments alone. In their combined analysis, K2K, CHOOZ, KamLAND, and SNO oscillation data provide constraints to the $e$-row of $A$, whereas $\mathrm{K} 2 \mathrm{~K}$ and Super-K oscillation data provide constraints to the $\mu$-row of $A$. Imposing additional constraints from near detectors at KARMEN [103], MINOS, NOMAD [104] and Bugey [64] allows disentangling degeneracies in $\left|A_{\mu 1}\right|$ and $\left|A_{\mu 2}\right|$ elements, so that the constraints on the $3 \times 3$ (not necessarily unitary) mixing matrix can be translated, at 90\% C.L., to

$$
|A|=\left(\begin{array}{ccc}
0.75-0.89 & 0.45-0.66 & <0.27 \\
0.00-0.69 & 0.22-0.81 & 0.57-0.85 \\
? & ? & ?
\end{array}\right)
$$

Notice that there are no constraints on the $\tau$-row from neutrino experiments if one does not impose unitarity.

In the minimal unitarity violation analysis scheme considered in [89], the above neutrino oscillation data constrain deviations from unitarity (i.e., deviations from $\left.\left|A A^{\dagger}\right|-\mathbb{I}=0\right)$ to be smaller than 0.10 at $90 \%$ C.L. in all elements of $\left|A A^{\dagger}\right|$, except for $\left|A A^{\dagger}\right|_{\tau \tau}$, which is unconstrained. Combining the constraints from neutrino experiments with the more stringent constraints from weak decays (which are of the order $10^{-5}-10^{-2}$ ) results in limits on the deviation from unitarity in the leptonic sector of less than 0.05 in terms of $\left|A^{\dagger} A\right|$ at $90 \%$ C.L. [89].

The most challenging channel for mixing freedom searches within neutrino experiments is the $\nu_{e} \rightarrow \nu_{\tau}$ instantaneous appearance channel. Since there are no available high-energy $\nu_{e}$ beams (accelerator-based neutrino beams are $\nu_{\mu}$-dominated beams, and beta-beam neutrino fluxes are limited to below a few $\mathrm{GeV}$ in energy), there are no existing direct limits on this process, and at the very least render this search currently extremely difficult. Searching for non-zero instantaneous $\nu_{e} \rightarrow \nu_{\tau}$ transitions relies on assumptions of $C P$ and $C P T$ conservation (i.e., $U U_{\tau e}^{\dagger}=U U_{e \tau}^{\dagger}$ ), through a 
search for $\nu_{\tau} \rightarrow \nu_{e}$ instantaneous transitions. The OPERA experiment is currently the only experiment using a $\nu_{\tau}$ beam, with only $10 \%$ of the neutrino flux actually being $\nu_{\tau}$, and is therefore significantly background-dominated, which also makes it particularly hard to search in this channel with high enough precision.

\subsection{A Close Look at Light Sterile Neutrinos}

In this section, we focus on the light sterile neutrino scenario, in particular within the context of oscillation experiments. We begin by discussing the light sterile neutrino oscillation formalism, and then discuss an experimental signature which was found consistent with this hypothesis.

\subsubsection{Light Sterile Neutrino Oscillation Formalism}

Generally, in a world with sterile neutrinos, there are $N>3$ neutrino mass eigenstates, and the neutrino mixing matrix is greater than $3 \times 3$, but unitary in its entirety.

As in standard three-neutrino oscillations, under the assumptions of CPT invariance and unitarity of (the full, bigger than $3 \times 3$ ) $U$, the probability for a neutrino produced with flavor $\alpha$ and energy $E$ to be detected as a neutrino of flavor $\beta$, after traveling a distance $L$, is given by Eq. 1.22,

$$
\begin{gathered}
P\left(\nu_{\alpha} \rightarrow \nu_{\beta}\right)=\delta_{\alpha \beta}-4 \sum_{i>j} \operatorname{Re}\left\{U_{\alpha i}^{*} U_{\beta i} U_{\alpha j} U_{\beta j}^{*}\right\} \sin ^{2} x_{i j}+ \\
2 \sum_{i>j} \operatorname{Im}\left\{U_{\alpha i}^{*} U_{\beta i} U_{\alpha j} U_{\beta j}^{*}\right\} \sin 2 x_{i j}
\end{gathered}
$$

where Re and Im indicate the real and imaginary parts of the product of mixing matrix elements, respectively; $\alpha, \beta \equiv e, \mu, \tau$, or $s ; i, j=1, \ldots, N$; and $x_{i j} \equiv 1.27 \Delta m_{i j}^{2} L / E$.

As in standard three-neutrino oscillations, in the case of antineutrinos, the oscillation probability is obtained from Eq. (2.24) by replacing the mixing matrix $U$ with its complex-conjugate matrix. Therefore, if the mixing matrix is not real, neutrino and antineutrino oscillation probabilities can differ.

For $N$ neutrino species, there are, in general, $(N-1)$ independent mass splittings, 
$N(N-1) / 2$ independent moduli of parameters in the unitary mixing matrix, which is assumed to be $N \times N$, and $(N-1)(N-2) / 2$ Dirac $C P$-violating phases that may be observed in oscillations.

For sterile neutrinos with masses of order $1 \mathrm{eV}$, the first oscillation maximum for typical neutrino beam energies of a few $\mathrm{MeV}$ to a few $\mathrm{GeV}$ corresponds to a few meters to a few $\mathrm{km}$ from the neutrino source. These baselines are relatively short compared to those of solar or atmospheric oscillations, and therefore experiments that have sensitivity to sterile neutrino oscillations are referred to as short-baseline oscillation experiments.

For short-baseline experiments that are sensitive only to $\nu_{\mu} \rightarrow \nu_{\mu}, \nu_{e} \rightarrow \nu_{\phi}$, and $\nu_{\mu} \rightarrow \nu_{e}$ transitions, which is the current experimental situation, the set of observable parameters is reduced considerably. Firstly, oscillations due to atmospheric and solar mass splittings, $\Delta m_{21}^{2}$ and $\Delta m_{32}^{2}$, can be neglected. ${ }^{5}$ Secondly, $U_{\tau i}$ elements of the mixing matrix do not enter in oscillation probabilities measured by these experiments. In this case, the number of observable parameters is reduced to $N-3$ independent mass splittings, $2(N-3)$ moduli of mixing matrix parameters, and $N-4 C P$-violating phases.

The simplest light sterile neutrino oscillation scenario is one where one mostlysterile neutrino mass eigenstate exists, in addition the three already-known standard neutrinos $(N=4)$. The scenario is depicted schematically in Fig. 2-1.

In the scenario of Fig. 2-1, referred to as a $(3+1)$ model, for three active plus one sterile neutrino, the size of the electron and muon flavor content of the extra state, $\left|U_{e 4}\right|^{2}$ and $\left|U_{\mu 4}^{2}\right|$, respectively, is limited by unitarity arguments of the $4 \times 4$ neutrino mixing matrix, $U$, and from considerations of solar and atmospheric neutrino data. Specifically, the equations

$$
\sum_{i=1-4}\left|U_{e i}\right|^{2}=1
$$

and

$$
\sum_{i=1-4}\left|U_{\mu i}\right|^{2}=1
$$

\footnotetext{
${ }^{5}$ One equivalently can set $m_{1}=m_{2}=m_{3}=0$.
} 


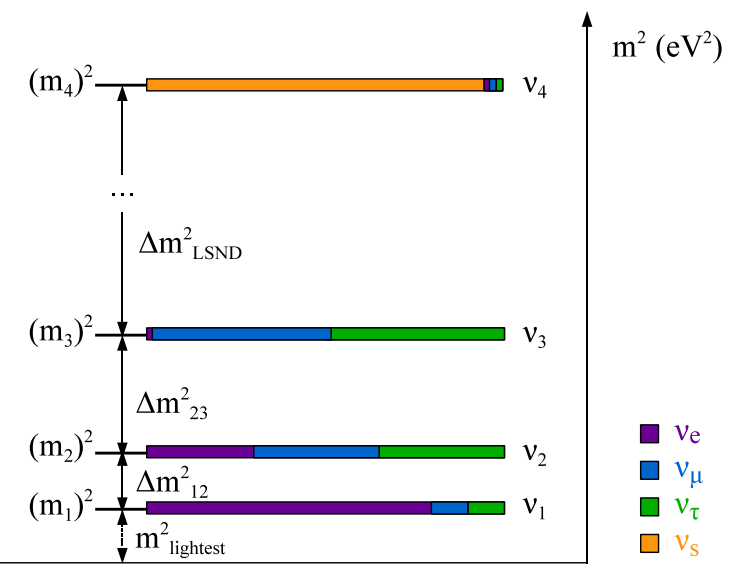

Figure 2-1: The neutrino mass spectrum in the simplest light sterile neutrino oscillation scenario, referred to as " $(3+1)$ ".

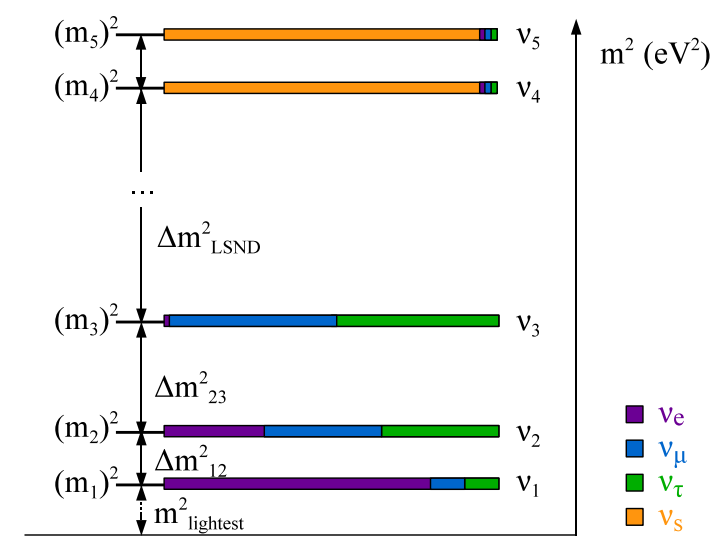

Figure 2-2: The neutrino mass spectrum in the $(3+2)$ light sterile neutrino oscillation scenario.

must hold, where the values of $\left|U_{e 1}\right|,\left|U_{e 2}\right|$, and $\left|U_{e 3}\right|$ are constrained by KamLAND, solar, and CHOOZ data, and the values of $\left|U_{\mu 1}\right|,\left|U_{\mu 2}\right|$, and $\left|U_{\mu 3}\right|$ are constrained by atmospheric, K2K, and MINOS data.

The remaining flavor content of this fourth mass eigenstate is sterile, and is nonweakly interacting. This requirement is imposed by measurements of the invisible width of the $Z$ boson, $\Gamma_{i n v}$, in studies of $Z$ production in $e^{+} e^{-}$collisions at LEP, discussed in the previous section. The invisible width corresponds to decays of $Z$ to neutrinos, $Z \rightarrow \nu \bar{\nu}$, and is sensitive to the number of light $\left(m_{i}<M_{Z} / 2\right)$ neutrino species which are weakly charged, $N_{\nu_{a}}$. A combined result from the four LEP exper- 
iments at center-of-mass energies near the $Z$ mass, which is where the cross-section of this process becomes large, constraints the number of weakly interacting neutrinos to $N_{\nu_{a}}=2.984 \pm 0.008$ [99]. In terms of $N$ light neutrinos, the result is interpreted as

$$
\begin{aligned}
N_{\nu_{a}} & =\sum_{i, j}^{N} \Gamma\left(Z \rightarrow \nu_{i} \bar{\nu}_{j}\right)=\sum_{i, j}^{N}\left|A m p\left[Z \rightarrow \nu_{i} \bar{\nu}_{i}\right]\right|^{2} \\
& =\sum_{i, j}^{N}\left(\sum_{\alpha=1}^{N_{\text {active }}} U_{\alpha i}^{*} U_{\alpha j} \sum_{\beta=1}^{N_{\text {active }}} U_{\beta i} U_{\beta j}^{*}\right) \\
& =\sum_{\alpha, \beta=1}^{N_{\text {active }}} \delta_{\alpha \beta} \delta_{\alpha \beta}=\sum_{\alpha=1}^{N_{\text {active }}} 1=N_{\text {active }}
\end{aligned}
$$

which suggests that there can only be three active flavors. If the sterile flavor was weakly interacting, then LEP would measure a larger $N_{\nu_{a}}$.

In terms of the flavor content of the fourth, mostly sterile neutrino mass eigenstate, the appearance probability in the $(3+1)$ oscillation hypothesis under the above approximations, reduced from Eq. 2.24, is

$$
P\left(\nu_{\mu} \rightarrow \nu_{e}\right)=4\left|U_{\mu 4}\right|^{2}\left|U_{e 4}\right|^{2} \sin ^{2}\left(1.27\left(m_{4}^{2}-m_{1}^{2}\right) L / E\right)
$$

which can be rewritten in terms of $\Delta m_{41}^{2}=m_{4}^{2}-m_{1}^{2}$ and $\sin ^{2} 2 \theta_{\mu e}=4\left|U_{\mu 4}\right|^{2}\left|U_{e 4}\right|^{2}$ to resemble the two-neutrino-approximation appearance probability of Eq. 1.28:

$$
P\left(\nu_{\mu} \rightarrow \nu_{e}\right)=\sin ^{2} 2 \theta_{\mu e} \sin ^{2}\left(1.27 \Delta m_{41}^{2} L / E\right)
$$

Similarly, the disappearance probability is given by

$$
\begin{aligned}
P\left(\nu_{\alpha} \rightarrow \nu_{\alpha}\right) & =1-4\left|U_{\alpha 4}\right|^{2}\left(1-\left|U_{\alpha 4}\right|^{2}\right) \sin ^{2}\left(1.27 \Delta m_{41}^{2} L / E\right) \\
& \equiv 1-\sin ^{2} 2 \theta_{\alpha \alpha} \sin ^{2}\left(1.27 \Delta m_{41}^{2} L / E\right)
\end{aligned}
$$

where $\alpha=e$, or $\alpha=\mu$.

In the next-to-minimal extension, two mostly-sterile neutrino mass eigenstates are assumed, in which case the neutrino mass spectrum looks as in Fig. 2-2. In 
this scenario, the two mass splittings, $\Delta m_{41}^{2}$ and $\Delta m_{51}^{2}$, are both on the order of 0.1-100 $\mathrm{eV}^{2}$. Thus, oscillations occur simultaneously at two slightly different $L / E$, yielding, experimentally, a more complicated oscillation signature as a function of energy. Specifically, the disappearance probability is given by

$$
\begin{array}{r}
P\left(\nu_{\alpha} \rightarrow \nu_{\alpha}\right)=1-4\left[\left(1-\left|U_{\alpha 4}\right|^{2}-\left|U_{\alpha 5}\right|^{2}\right)\right. \\
\left(\left|U_{\alpha 4}\right|^{2} \sin ^{2} x_{41}+\left|U_{\alpha 5}\right|^{2} \sin ^{2} x_{51}\right)+ \\
\left.\left|U_{\alpha 4}\right|^{2}\left|U_{\alpha 5}\right|^{2} \sin ^{2} x_{54}\right],
\end{array}
$$

for $\alpha=e, \mu$, and

$$
\begin{array}{r}
P\left(\nu_{\alpha} \rightarrow \nu_{\beta \neq \alpha}\right)=4\left|U_{\alpha 4}\right|^{2}\left|U_{\beta 4}\right|^{2} \sin ^{2} x_{41}+ \\
4\left|U_{\alpha 5}\right|^{2}\left|U_{\beta 5}\right|^{2} \sin ^{2} x_{51}+ \\
8\left|U_{\alpha 5}\right|\left|U_{\beta 5}\right|\left|U_{\alpha 4}\right|\left|U_{\beta 4}\right| \sin x_{41} \sin x_{51} \cos \left(x_{54}-\phi_{45}\right)
\end{array}
$$

in the case of appearance, for $\alpha=\mu$ or $\beta=e$

One important consequence of this scenario is an interference oscillation term in each of Eqs. 2.31 and 2.32, which is dominant at an $L / E$ determined by $\Delta m_{54}^{2}=$ $\Delta m_{51}^{2}-\Delta m_{41}^{2}$. In the case of appearance, this term is also dependent on a Dirac $C P$-violating phase, $\phi_{45}$, given by,

$$
\phi_{45}=\arg \left(U_{\mu 5}^{*} U_{e 5} U_{\mu 4} U_{e 4}^{*}\right)
$$

which differs for neutrinos and antineutrinos by an overall "-" sign. ${ }^{6}$ A non-zero value for this $C P$-violating phase therefore leads to differences in appearance probabilities of neutrinos and antineutrinos for searches performed at the same $L / E$.

It should be noted that there are no $C P$-violating terms in the disappearance probability. In fact, in any $C P T$-conserving neutrino oscillation scenario, there can

\footnotetext{
${ }^{6}$ The appearance formula for antineutrino oscillations are obtained by substituting $\phi_{45} \rightarrow-\phi_{45}$ in Eq. 2.32.
} 


\begin{tabular}{l|c|c}
\hline \hline Conserved symmetry & $\begin{array}{c}\text { Single- } \Delta m^{2} \text { approximation } \\
\mathbf{( 3 + 1 )}\end{array}$ & $\begin{array}{c}\text { Two- } \Delta m^{2} \text { approximation } \\
\mathbf{( 3 + 2 )}\end{array}$ \\
\hline \hline$C P$ and $C P T$ & $P\left(\nu_{\alpha} \rightarrow \nu_{\beta}\right)=P\left(\bar{\nu}_{\alpha} \rightarrow \bar{\nu}_{\beta}\right)$ & $P\left(\nu_{\alpha} \rightarrow \nu_{\beta}\right)=P\left(\bar{\nu}_{\alpha} \rightarrow \bar{\nu}_{\beta}\right)$ \\
& $P\left(\nu_{\alpha} \rightarrow \nu_{\alpha}\right)=P\left(\bar{\nu}_{\alpha} \rightarrow \bar{\nu}_{\alpha}\right)$ & $P\left(\nu_{\alpha} \rightarrow \nu_{\alpha}\right)=P\left(\bar{\nu}_{\alpha} \rightarrow \bar{\nu}_{\alpha}\right)$ \\
\hline$C P T$ & $P\left(\nu_{\alpha} \rightarrow \nu_{\beta}\right)=P\left(\bar{\nu}_{\alpha} \rightarrow \bar{\nu}_{\beta}\right)$ & $P\left(\nu_{\alpha} \rightarrow \nu_{\beta}\right) \neq P\left(\bar{\nu}_{\alpha} \rightarrow \bar{\nu}_{\beta}\right)$ \\
& $P\left(\nu_{\alpha} \rightarrow \nu_{\alpha}\right)=P\left(\bar{\nu}_{\alpha} \rightarrow \bar{\nu}_{\alpha}\right)$ & $P\left(\nu_{\alpha} \rightarrow \nu_{\alpha}\right)=P\left(\bar{\nu}_{\alpha} \rightarrow \bar{\nu}_{\alpha}\right)$ \\
\hline & $P\left(\nu_{\alpha} \rightarrow \nu_{\beta}\right) \neq P\left(\bar{\nu}_{\alpha} \rightarrow \bar{\nu}_{\beta}\right)$ & $P\left(\nu_{\alpha} \rightarrow \nu_{\beta}\right) \neq P\left(\bar{\nu}_{\alpha} \rightarrow \bar{\nu}_{\beta}\right)$ \\
& $P\left(\nu_{\alpha} \rightarrow \nu_{\alpha}\right) \neq P\left(\bar{\nu}_{\alpha} \rightarrow \bar{\nu}_{\alpha}\right)$ & $P\left(\nu_{\alpha} \rightarrow \nu_{\alpha}\right) \neq P\left(\bar{\nu}_{\alpha} \rightarrow \bar{\nu}_{\alpha}\right)$ \\
\hline
\end{tabular}

Table 2.3: Relationship between neutrino and antineutrino oscillation probabilities under various $C P$ and $C P T$ assumptions.

be no difference between neutrino and antineutrino disappearance probabilities. To see this, consider the following reasoning. By charge conjugation symmetry $(C)$,

$$
P\left(\nu_{\alpha, L} \rightarrow \nu_{\beta, L}\right) \equiv P\left(\bar{\nu}_{\alpha, L} \rightarrow \bar{\nu}_{\beta, L}\right)
$$

Then, by parity conservation $(P)$,

$$
P\left(\bar{\nu}_{\alpha, L} \rightarrow \bar{\nu}_{\beta, L}\right) \equiv P\left(\bar{\nu}_{\alpha, R} \rightarrow \bar{\nu}_{\beta, R}\right)
$$

And, finally, by time reversal symmetry $(T)$,

$$
P\left(\bar{\nu}_{\alpha, R} \rightarrow \bar{\nu}_{\beta, R}\right) \equiv P\left(\bar{\nu}_{\beta, R} \rightarrow \bar{\nu}_{\alpha, R}\right)
$$

Therefore, for $\alpha=\beta, C P T$ conservation implies that $P\left(\nu_{\alpha, L} \rightarrow \nu_{\alpha, L}\right) \equiv P\left(\bar{\nu}_{\alpha, R} \rightarrow\right.$ $\left.\bar{\nu}_{\alpha, R}\right)$; i.e., the disappearance probability is the same for neutrinos and antineutrinos. Note that Eqs. 2.34 and 2.35 also imply that the appearance probability for neutrinos and antineutrinos must be the same if $C P$ is conserved.

Table 2.3 summarizes neutrino and antineutrino oscillation probability equalities under assumptions of different symmetries between neutrinos and antineutrinos. 


\subsubsection{The LSND Signal}

The Liquid Scintillator Neutrino Detector (LSND) experiment [105] was designed with high sensitivity to $\bar{\nu}_{\mu} \rightarrow \bar{\nu}_{e}$ flavor transitions, and observed an excess of electron antineutrino events above expected background with a $3.8 \sigma$ significance. When interpreted as two-neutrino oscillations, this excess was indicative of oscillations with a small mixing amplitude, $\sin ^{2}(2 \theta)$, of the order of less than $1 \%$, and a large $\Delta m^{2} \sim 1 \mathrm{eV}^{2}$. LSND's sensitivity to neutrino oscillations being maximal for $\Delta m^{2} \sim$ $1 \mathrm{eV}^{2}$ was determined by its $L[\mathrm{~m}] / E[\mathrm{MeV}]$ experimental parameters.

\section{LSND experimental setup}

The layout of the LSND experiment is illustrated in Fig. 2-3. The experiment ran at Los Alamos U.S. National Laboratory from 1993 to 1998, and studied antineutrinos produced primarily in decays of $\mu^{+}$at rest. The $\pi^{+}$beam was generated by a high-intensity, $798 \mathrm{MeV}$ proton beam from the LANSCE accelerator facility at Los Alamos National Laboratory, incident on water and other high-Z targets. Protontarget interactions produced $\pi^{+}$(and $\pi^{-}$, at a $\frac{1}{8} \times$ smaller production cross-section, most of which were absorbed in the beam stop and surrounding shielding materials, along with any $\mu^{-}$from $\pi^{-}$which decayed in flight). Approximately $5 \%$ of the $\pi^{+}$ produced at the proton target decayed in flight to produce primarily $\mu^{+}$and $\nu_{\mu}$, while the remaining $\pi^{+}$and subsequent $\mu^{+}$decayed at rest within a copper beam stop, located downstream but extremely close to the proton target, producing $\nu_{\mu}$ and $\bar{\nu}_{\mu}$, respectively.

The beam stop configuration resulted in two significantly different neutrino flux spectra at the detector, shown in Fig. 2-4: one at high energy (60-200 MeV), consisting

primarily of $\nu_{\mu}$ from $\pi^{+}$decay in flight (DIF), and one at low energy (20-60 MeV), consisting primarily of $\bar{\nu}_{\mu}$ from $\mu^{+}$decay at rest (DAR). The data sample used in the LSND search for oscillations was dominated by the DAR sample.

The center of the LSND detector was located $30 \mathrm{~m}$ downstream the beam stop. The detector was a cylindrical vessel, filled with 167 metric tons of liquid scintillator. 


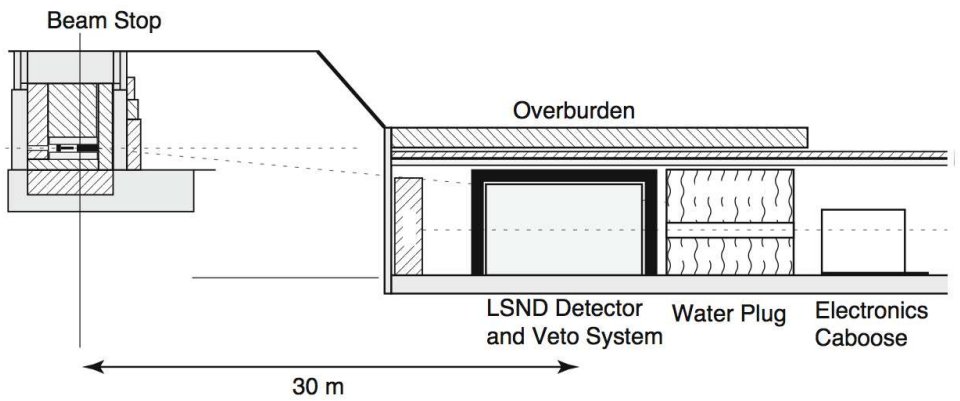

Figure 2-3: The LSND experimental layout.
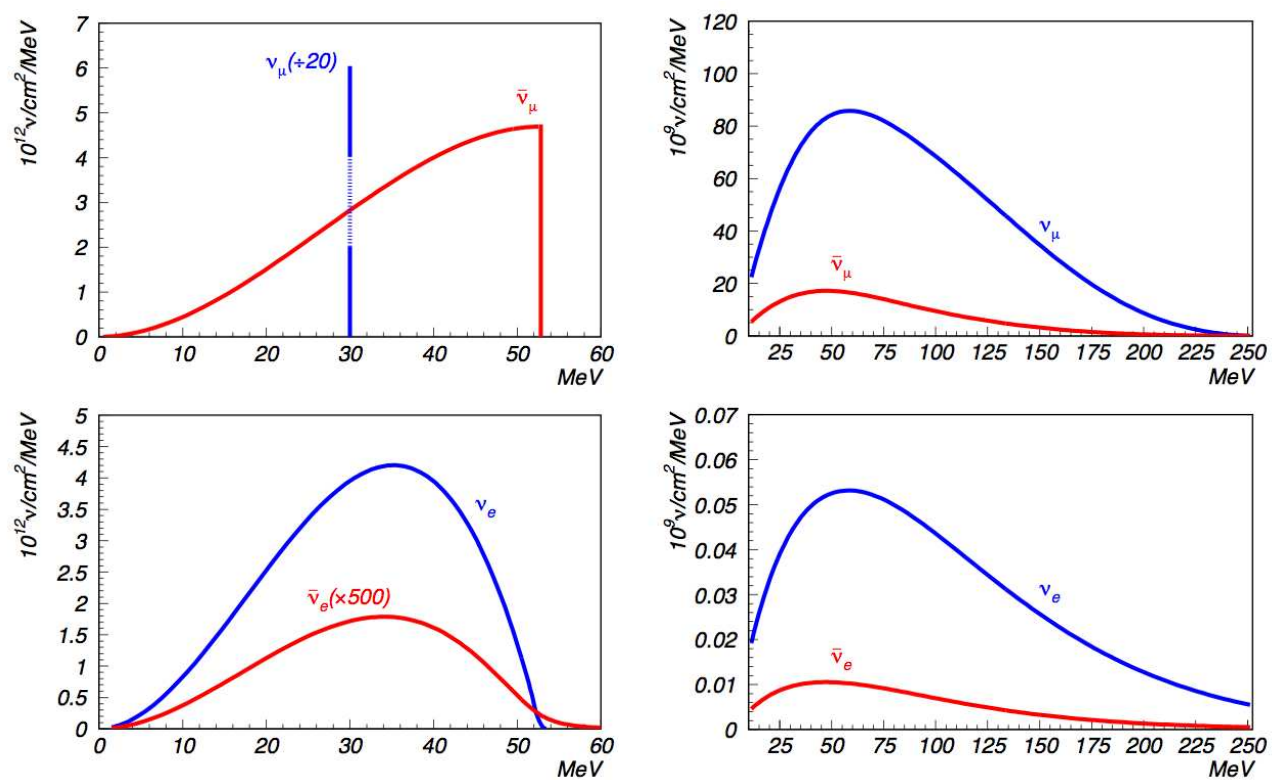

Figure 2-4: The decay at rest (left panels) and decay in flight (right panels) neutrino flux spectra at the LSND detector. 
The vessel's inner surfaces were lined with uniformly spaced photomultiplier tubes. Due to a relatively low scintillator concentration, the detector was sensitive to both scintillation and Cherenkov light produced by products of neutrino interactions within the mineral oil.

The detector was surrounded on all sides except the bottom by a veto liquidscintillator vessel, also lined with photomultiplier tubes, which tagged through-going cosmic ray muons for background rejection purposes. The veto region also included an inner shell filled with lead shot, which acted as extra shielding to neutron and low energy photon environmental backgrounds.

\section{LSND's search for $\bar{\nu}_{\mu} \rightarrow \bar{\nu}_{e}$ oscillations}

In LSND's DAR data set, the $\bar{\nu}_{e}$ flux was calculated to be only $\sim 8 \times 10^{-4}$ as large as the $\bar{\nu}_{\mu}$ flux. Therefore, the flux from $\mu^{+}$DAR provided high sensitivity to $\bar{\nu}_{\mu} \rightarrow \bar{\nu}_{e}$ flavor transitions, and was used to perform a search for $\bar{\nu}_{\mu} \rightarrow \bar{\nu}_{e}$ oscillations in 1996, and 2001 [106, 107], the latter being summarized here.

The $\bar{\nu}_{e}$ detection signature was inverse beta decay $\left(\bar{\nu}_{e} p \rightarrow e^{+} n\right)$, followed by neutron capture on a free proton $(n p \rightarrow d \gamma)$. The first part of this two-fold signature, $\bar{\nu}_{e} p \rightarrow e^{+} n$, produced a pattern of scintillation and Cherenkov light in the detector characteristic of an $e^{+}$with an endpoint energy of $52 \mathrm{MeV}$, which could be recognized through timing and pulse-height information recorded on the array of photomultiplier tubes in the detector. The second part, neutron capture, resulted in a characteristic 2.2 MeV photon from the $n p \rightarrow d \gamma$ reaction. Because LSND was insensitive to the charge of the outgoing lepton, this two-fold signature was necessary to differentiate against events from $\nu_{e}$ which were produced in $\pi^{+}$and $\mu^{+}$decays and interacted in the detector through $\nu_{e} C \rightarrow e^{-} B$. If there were any $\bar{\nu}_{\mu} \rightarrow \bar{\nu}_{e}$ oscillations, LSND expected to see a relative excess of such correlated event signatures above background prediction.

The $\bar{\nu}_{e}$ background rate in the DAR data sample was calculated to be almost four orders of magnitude smaller relative to the expected $\bar{\nu}_{\mu}$ rate. Backgrounds included mostly interactions of intrinsic $\bar{\nu}_{e}$ in the beam: $\bar{\nu}_{e}$ from $\pi^{-}$decaying in flight (sup- 
pressed, due to the small branching fraction of $\pi^{-} \rightarrow e^{-} \bar{\nu}_{e}$ relative to $\left.\pi^{-} \rightarrow \mu^{-} \bar{\nu}_{\mu}\right)$ and $\mu^{-}$decaying at rest, following $\pi^{-} \rightarrow \mu^{-} \bar{\nu}_{\mu}$ decays in flight (also suppressed, since most $\mu^{-}$were captured in the beam stop before decaying, and also due to the low cross-section for $\pi^{-}$production at the proton target relative to $\pi^{+}$). Backgrounds also included mis-identified $\bar{\nu}_{\mu} C \rightarrow \mu^{+} n X$ interactions of $\bar{\nu}_{\mu}$ from $\pi^{+}$decaying in flight. As the dominant DAR flux component, $\bar{\nu}_{\mu}$ could mimic $\bar{\nu}_{e} C \rightarrow e^{+} n X$ through $\bar{\nu}_{\mu} C \rightarrow \mu^{+} n X$, if the energy of the outgoing $\mu^{+}$was sufficiently low, or if the $\mu^{+}$decayed too quickly, leading to a correlated $e^{+} / \gamma$ event. Such backgrounds were taken into account in the analysis. Other backgrounds which resulted from accidental $e^{+} / \gamma$ coincidences were also taken into account, for example neutrons in the beam stop creeping through the veto and capturing in the detector in coincidence with a beam $\nu_{e}$ interacting in the detector.

The $e^{+}$energy in the analysis ranged between 20-60 MeV. This lower limit was placed to reject mostly accidental backgrounds (such as backgrounds from ${ }^{12} B$ or other radioactive decays) below $20 \mathrm{MeV}$, while the upper limit was chosen as the upper kinematic limit of the $\bar{\nu}_{\mu}$ flux from muon decay at rest.

Further selection and classification of $\bar{\nu}_{e}$ events as $e^{+} / \gamma$-correlated events versus accidental backgrounds was made by considering a likelihood parameter, $R_{\gamma}$, defined as the likelihood that the reconstructed $\gamma$ is correlated divided by the likelihood that the $\gamma$ is accidental. This parameter depended on the distance between the reconstructed positron and neutron capture events, the time difference between them, and the reconstructed energy of the photon from neutron capture.

\section{A possible hint for New Physics?}

The LSND search for $\bar{\nu}_{e}$ events [107] revealed a total beam event excess of $87.9 \pm$ $22.4 \pm 6.0$ events, over a background of $30.0 \pm 6.0$ events. This excess is shown as a function of the positron energy reconstructed in each event in Fig. 2-5 (left), for events with $R_{\gamma}>10$, which correspond to a clean sample of any expected $\bar{\nu}_{e}$ signal. The excess is consistent with $\bar{\nu}_{e} p \rightarrow e^{+} n$ scattering, shown in blue, above the expected background prediction, shown in red and green. 

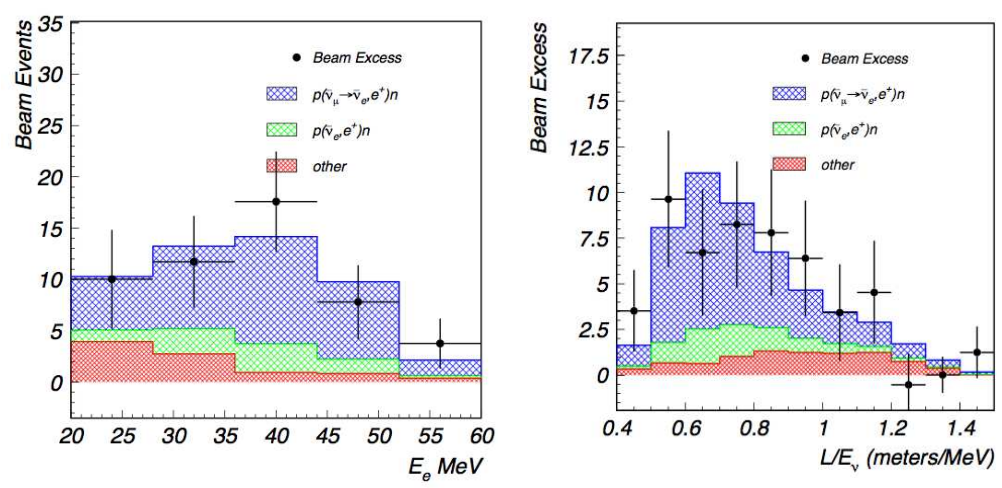

Figure 2-5: The LSND observed excess of $\bar{\nu}_{e}$ events. The left panel shows the observed data (black points) and expected background and best-fit signal from $\bar{\nu}_{\mu} \rightarrow \bar{\nu}_{e}$ oscillations (shaded stacked histograms) as a function of the measured energy of the outgoing positron. The right panel shows the same information as a function of $L / E_{\nu}$, where $L$ is the distance traveled by the neutrino in the lab frame, corresponding to the distance from the beam stop to the reconstructed positron vertex, and $E_{\nu}$ is the energy of the neutrino calculated given the reconstructed positron energy, $E_{e}$, and reconstructed positron angle, assuming two-body kinematics. The figure is taken from [107].

The simplest, two-neutrino oscillation interpretation of this excess, illustrated in Fig. 2-5 (right), requires $\Delta m^{2} \sim 1 \mathrm{eV}^{2}$ and $\sin ^{2} 2 \theta_{\mu e} \sim 0.003$. Of course, this cannot be accommodated within three-neutrino mixing in the Standard Model, which only allows for two independent $\Delta m^{2}$ scales, already determined to be orders of magnitude smaller than $1 \mathrm{eV}^{2}$. However, a phenomenological picture has been adopted over the last decade as a possible explanation, which assumes the existence of a neutrino mass eigenstate on the order of 1-10 eV, containing a small admixture of electron and muon flavor, such that $4\left|U_{e 4}\right|^{2}\left|U_{\mu 4}\right|^{2}<1 \%$. This interpretation corresponds to the $(3+1)$ oscillation hypothesis considered in Sec. 2.2.1, depicted schematically in Fig. 2-1, and results in an appearance oscillation probability given by Eq. 2.28 in terms of the above parameters. The remaining of this fourth mass eigenstate must be mostly of sterile flavor, in agreement with results from $\Gamma_{i n v}$, as discussed earlier.

In addition to a $\bar{\nu}_{\mu} \rightarrow \bar{\nu}_{e}$ search, LSND was also able to perform a (less sensitive) $\nu_{\mu} \rightarrow \nu_{e}$ search using the $\nu_{\mu}$-dominated DIF data set. In that case, LSND observed an excess of $18.1 \pm 6.6 \pm 4.0$ events. The DIF data was also included in the above search 

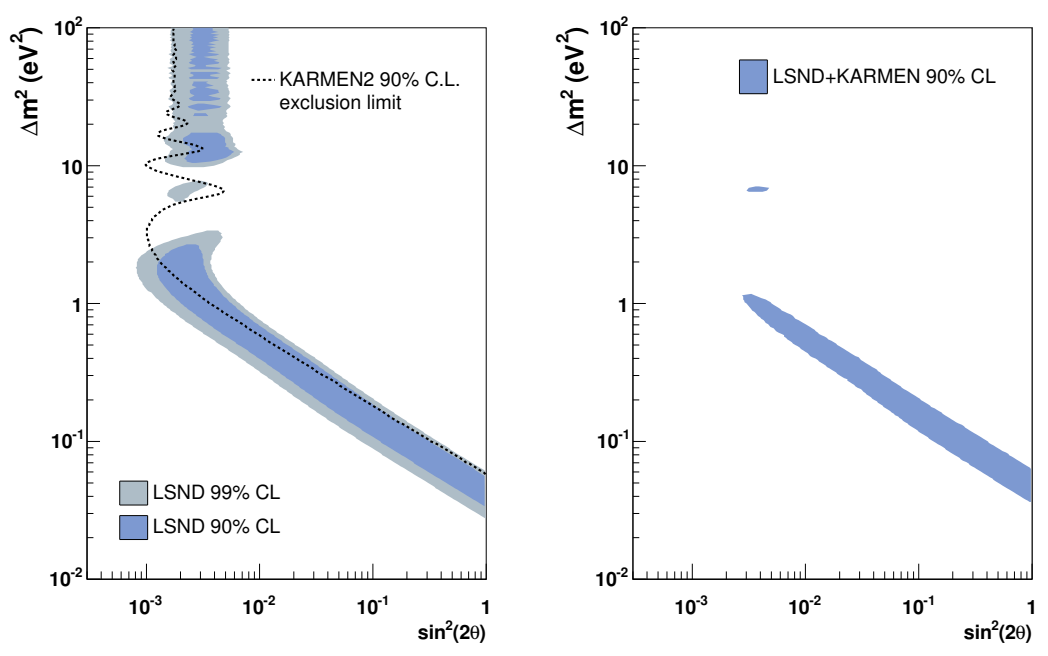

Figure 2-6: The oscillation parameter space allowed by LSND DAR and DIF data under a two-neutrino oscillation approximation. The left panel shows the allowed (color filled) regions obtained from the analysis of LSND data [107]. The overlapped limit is from KARMEN [108], and experiment which ran before LSND, and excluded values in parameter space to the right of the dotted line. A combined analysis of the two results [109] yields the allowed region shown on the right panel, at 90\% C.L.

for oscillations [107]. The result, however, was mainly driven by the DAR excess.

When the DAR and DIF LSND data are fit to a $(3+1)$ hypothesis and under a twoneutrino oscillation approximation (which is valid since the $\Delta m^{2}$ determined by LSND is much larger than $\Delta m_{\text {solar }}^{2}$ and $\Delta m_{\text {atm }}^{2}$ ), they allow for sets of parameters shown within the $90 \%$ and $99 \%$ C.L. allowed regions of Fig. 2-6 (left). The best fit parameters correspond to $\left(\Delta m^{2}, \sin ^{2} 2 \theta\right)=\left(1.2 \mathrm{eV}^{2}, 0.003\right)$, and an oscillation probability of $0.26 \%$.

\subsubsection{Constraints to Light Sterile Neutrino Models}

Aside from LSND, as of 2007, three other experiments had searched for $\bar{\nu}_{\mu} \rightarrow \bar{\nu}_{e}$ oscillations with similar sensitivity to $\Delta m^{2} \sim 0.01-100 \mathrm{eV}^{2}$. Those are the KARMEN [108], E776 [110] and NOMAD [104] experiments, all of which have seen no evidence for such oscillations.

The strongest limits come from KARMEN, which excludes a portion, but not all of the parameter region favored by LSND, as shown in Fig. 2-6. The search performed by KARMEN is also a direct test of the LSND signal, being that KARMEN was also 


\begin{tabular}{lccccc}
\hline \hline Data set & Channel & $\begin{array}{c}L / E \\
{[\mathrm{~km} / \mathrm{GeV}]}\end{array}$ & $\begin{array}{c}\text { Optimal } \Delta m^{2} \\
{\left[\mathrm{eV}^{2}\right]}\end{array}$ & $\begin{array}{c}\sin ^{2} 2 \theta \\
\text { at optimal } \Delta m^{2}\end{array}$ & Ref. \\
\hline \hline Appearance: & & & & & \\
LSND & $\bar{\nu}_{\mu} \rightarrow \bar{\nu}_{e}$ & $0.5-1.4$ & 2.0 & {$[1.2-3.2] \times 10^{-3}$} & {$[105,107]$} \\
KARMEN2 & & $0.3-1.1$ & 3.0 & $<1.0 \times 10^{-3}$ & {$[108]$} \\
NOMAD & $\nu_{\mu} \rightarrow \nu_{e}$ & $0.002-0.3$ & 30 & $<1.0 \times 10^{-3}$ & {$[104]$} \\
\hline Disappearance: & & & & & \\
Bugey & $\bar{\nu}_{e} \rightarrow \bar{\nu}_{q}$ & $2-50$ & 0.6 & $<1.3 \times 10^{-2}$ & {$[64]$} \\
CHOOZ & & $100-400$ & 0.006 & $<5.0 \times 10^{-2}$ & {$[63]$} \\
CCFR84 & $\nu_{\mu} \rightarrow \nu_{\mu}$ & $0.004-0.03$ & 900 & $<2.0 \times 10^{-1}$ & {$[111]$} \\
CDHS & & $0.02-1.5$ & 0.3 & $<5.3 \times 10^{-1}$ & {$[112]$} \\
\hline \hline
\end{tabular}

Table 2.4: Summary of short-baseline data sets which provide constraints to the LSND-allowed sterile neutrino oscillation parameters. The table indicates the oscillation channel that each experiment constrains, the $L / E[\mathrm{~km} / \mathrm{GeV}]$ parameters and corresponding optimal $\Delta m^{2}$, as well as $\sin ^{2} 2 \theta$ constraints at $90 \%$ C.L., in a twoneutrino oscillation approximation [113].

a $\mu^{+}$decay at rest experiment. Nevertheless, a joint analysis [109] of the results from both experiments finds that a mass splitting of $0.2-1 \mathrm{eV}^{2}$ and a mixing amplitude of 0.003-0.03, for example, could adequately explain both data sets. The analysis yields a $64 \%$ compatibility of the two experimental outcomes.

Constraints to the $(3+1)$ allowed parameters also come from several short-baseline $\nu_{e}$ and $\nu_{\mu}$ disappearance experiments, which independently constrain $\left|U_{e 4}\right|$ and $\left|U_{\mu 4}\right|$, respectively. Those are summarized in Tab. 2.4. Specifically, short-baseline $\nu_{\mu}$ disappearance experiments constrain $\sin ^{2} 2 \theta=4\left|U_{\mu 4}\right|^{2}\left(1-\left|U_{\mu 4}\right|^{2}\right)$, while short-baseline $\nu_{e}$ disappearance experiments constrain $\sin ^{2} 2 \theta=4\left|U_{e 4}\right|^{2}\left(1-\left|U_{e 4}\right|^{2}\right)$.

\subsection{Synopsis of Thesis}

At this point, one of the fundamental and actively pursued questions in neutrino physics is the question of sterile neutrinos [45]. The MiniBooNE experiment [114] at Fermi National Accelerator Laboratory was launched with the goal of conclusively confirming or refuting the LSND oscillation signal. The following chapters present the MiniBooNE experiment and analysis followed to search for $\nu_{\mu} \rightarrow \nu_{e}$ and $\bar{\nu}_{\mu} \rightarrow$ $\bar{\nu}_{e}$ oscillations at the level suggested by LSND. The results presented in Chapter 5 correspond to work by the author. 
Chapter 6 will consider the MiniBooNE neutrino and MiniBooNE and LSND antineutrino results in global fits to sterile neutrino oscillation models, with explicit constraints from the null short-baseline experiments listed in Tab. 2.4, and additional constraints from atmospheric oscillation experiments. The fit results presented in that chapter are new, building upon and differing from previous work by the co-authors of Ref. [82].

Given MiniBooNE's $L \sim 0$ baseline and high sensitivity to small amplitude $\nu_{\mu} \rightarrow$ $\nu_{e}$ transitions, the MiniBooNE neutrino and antineutrino appearance data sets may be used to perform a more general search for mixing freedom due to additional leptons which may exist in a wide range of underlying physics scenarios, leading to effective non-unitarity of the $3 \times 3$ neutrino mixing matrix. The fit method and results from such search are presented in Chapter 7 and correspond to work by the author.

Chapter 8 provides a brief summary of results from the above three searches. Future prospects on addressing both LSND and MiniBooNE results are discussed within the context of a new experiment, MicroBooNE [115], in Appendix B. The sensitivity studies presented are primarily work by the author, based on experimental parameter specifications from [115]. Appendix B also discusses R\&D studies for the MicroBooNE experiment, performed in collaboration with the MicroBooNE Active Detectors Working Group. 


\section{Chapter 3}

\section{The MiniBooNE Experiment}

The MiniBooNE experiment is located at Fermi National Accelerator Laboratory. Motivated by the LSND results discussed in Chapter 2, the experiment makes use of a dominantly muon-flavor neutrino beam to look for $\nu_{\mu} \rightarrow \nu_{e}$ or $\bar{\nu}_{\mu} \rightarrow \bar{\nu}_{e}$ oscillations driven by a $\Delta m^{2} \gg \Delta m_{\text {solar }}^{2}$ or $\Delta m_{\text {atm }}^{2}$.

While two-neutrino oscillations (driven by a single $\Delta m^{2}$ ) imply identical oscillation probabilities for neutrinos and antineutrinos, ${ }^{1}$ MiniBooNE has performed oscillation searches both using a neutrino beam, looking for $\nu_{\mu} \rightarrow \nu_{e}$ oscillations, and using an antineutrino beam, looking for $\bar{\nu}_{\mu} \rightarrow \bar{\nu}_{e}$ oscillations. The former is a much higher statistics and, therefore, more powerful search, while the latter serves as a direct test of the LSND oscillation interpretation, which was driven by antineutrino oscillations.

\subsection{Overview}

MiniBooNE's goal [116] was to confirm or refute the LSND oscillation interpretation using a similar neutrino baseline to energy ratio $(L[\mathrm{~m}] / E[\mathrm{MeV}])$ to that of LSND, thus maintaining sensitivity to neutrino oscillations at the same $\Delta m^{2}\left[\mathrm{eV}^{2}\right]$. A key requirement in doing so was to change both $L$ and $E$, while keeping their ratio the same, in order to change the detection method and associated experimental systematics.

\footnotetext{
${ }^{1}$ This assumes $C P T$ conservation.
} 


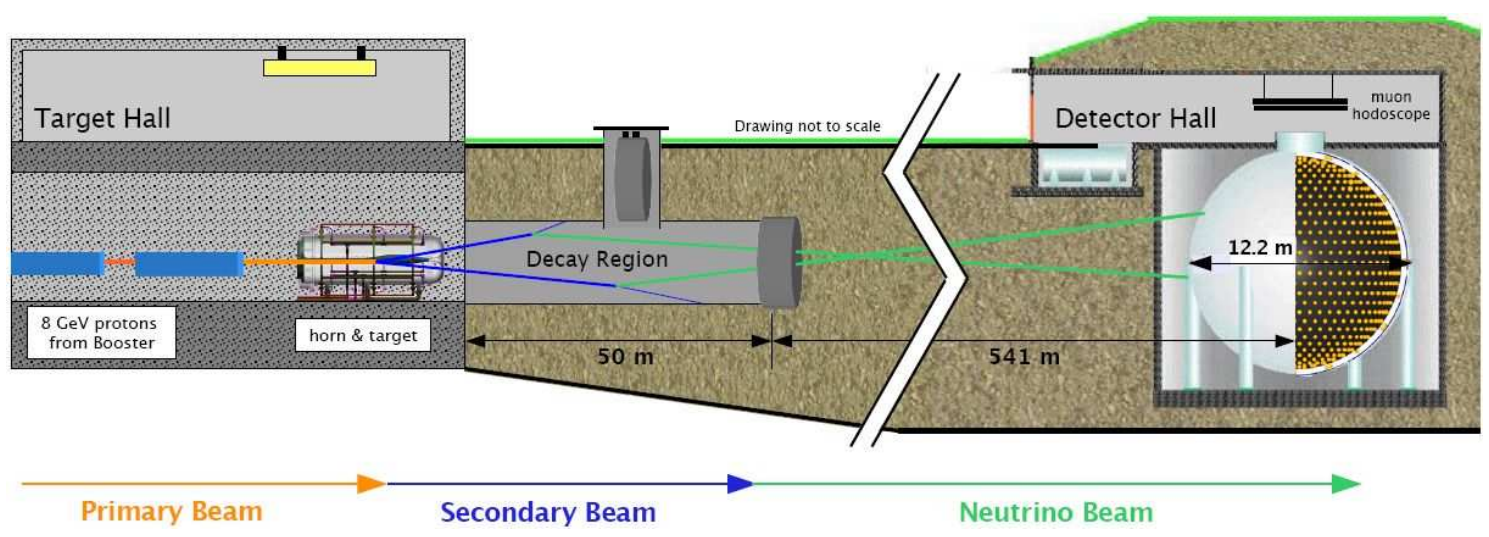

Figure 3-1: Schematic drawing of the MiniBooNE experiment at Fermi National Accelerator Laboratory. The neutrino beam is produced by decaying mesons produced in proton-beryllium interactions. After traveling approximately 500 meters, neutrinos with a mean energy of approximately $800 \mathrm{MeV}$ interact in the MiniBooNE detector.

A schematic of the MiniBooNE experiment is shown in Fig. 3-1. It is a shortbaseline $(L=541 \mathrm{~m})$ oscillation experiment making use of the $8 \mathrm{GeV}$ proton beam from the Fermilab booster, which impinges on a beryllium target in order to generate a neutrino or an antineutrino beam $>99 \%$ pure in muon flavor. The mean energy of the neutrino flux viewed by the MiniBooNE detector is a few hundred MeV. Those two parameters make the experiment most sensitive to two-neutrino oscillations at a mass splitting $>0.01 \mathrm{eV}^{2}$.

A key factor that dictates the experimental parameters of MiniBooNE is the very small $(<0.5 \%)$ oscillation probability suggested by the LSND results. Typical flux uncertainties for accelerator neutrino beams such as the one used by MiniBooNE are more than an order of magnitude larger than any expected oscillation effect. This makes a $\nu_{\mu}$ disappearance search at that level particularly challenging for a single detector. $^{2}$ In the case of the appearance search, however, using a neutrino beam where the intrinsic $\nu_{e}$ contamination is less than $1 \%$, together with a measurement of the high-statistics $\nu_{\mu}$ component of the beam, used to constrain flux and crosssection systematics, sufficient sensitivity to small-amplitude $\nu_{\mu} \rightarrow \nu_{e}$ oscillations can

\footnotetext{
${ }^{2} \nu_{\mu}$ and $\bar{\nu}_{\mu}$ disappearance searches have in fact been performed at MiniBooNE using shape-only, energy-dependent information [117], and are discussed in detail in [118].
} 
be achieved. With that said, a high-intensity neutrino beam is required, in order to generate high enough $\nu_{\mu} \rightarrow \nu_{e}$ event statistics, as well as a detector with the ability to efficiently detect $\nu_{e}$ events while rejecting more than $99 \%$ of $\nu_{\mu}$ events.

\subsection{The Booster Neutrino Beam}

The booster neutrino beamline (BNB) at Fermilab is divided in three beam stages, as outlined in Fig. 3-1:

1. A primary beam of protons extracted from the Fermilab booster synchrotron, incident on a beryllium target.

2. A secondary beam of mesons produced in inelastic collisions of the proton beam with the beryllium nuclei.

3. A tertiary beam of neutrinos and other particles (mostly muons) produced by decays of secondary mesons.

\subsubsection{Primary Proton Beam}

The schematic layout of the BNB is shown in Fig. 3-2, and is described in detail in [119]. $8 \mathrm{GeV}(8.9 \mathrm{GeV} / c$ momentum) protons from the Fermilab booster are directed to the BNB and aimed toward a thick beryllium target, located underground of the MI-12 Target Service Building in Fig. 3-2. The proton beam timing structure as it arrives at the beryllium target is composed of a series of "pulse trains", separated by 2-3 seconds, as illustrated in Fig. 3-3. The typical proton beam contains $4 \times 10^{12}$ protons delivered in a spill approximately $1.6 \mu$ s in duration.

A monitoring system measures both the time and intensity of the beam spills. The absolute number of protons on target (POT) delivered is measured by two toroids upstream of the target. The error on the delivered POT prior to March 2003 was 3\%, and $1.7 \%$ since then. A second system of beam position monitors (BPM) and a multiwire chamber determine the beam width, position, and direction. The beam position 


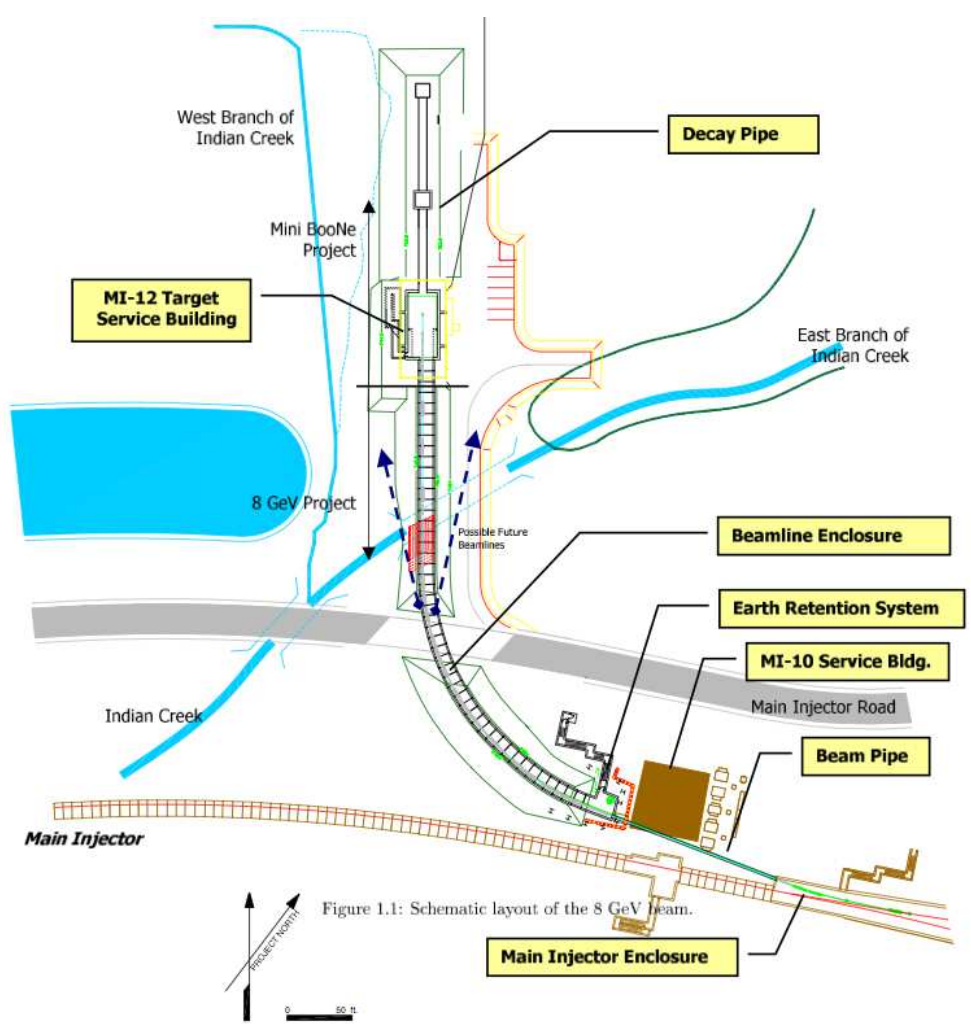

Figure 3-2: The booster neutrino beamline at Fermilab. The figure is from [119].

is centered about the beryllium target axis to within $\sigma_{x}=1.51 \mathrm{~mm}$ and $\sigma_{y}=0.75$ $\mathrm{mm}$ at the upstream face of the beryllium target, in horizontal $(x)$ and vertical $(y)$ directions, respectively.

\subsubsection{Proton Target}

The beryllium target, shown in the top panel of Fig. 3-4, measures $71.1 \mathrm{~cm}$ long $(1.7$ proton interaction lengths) and $0.96 \mathrm{~cm}$ in diameter. It is made out of seven "slugs" of beryllium, and is surrounded by a $0.9 \mathrm{~cm}$ thick sleeve also made of beryllium and supported by fins which allow for cooling through air circulation. Beryllium is specifically chosen as the proton target due to its low $\mathrm{Z}$, which minimizes radiative (energy) losses of incident protons, before they have a chance to interact inelastically with the target material and produce secondary mesons: $\pi^{ \pm}, K^{ \pm}, K^{0}$. 


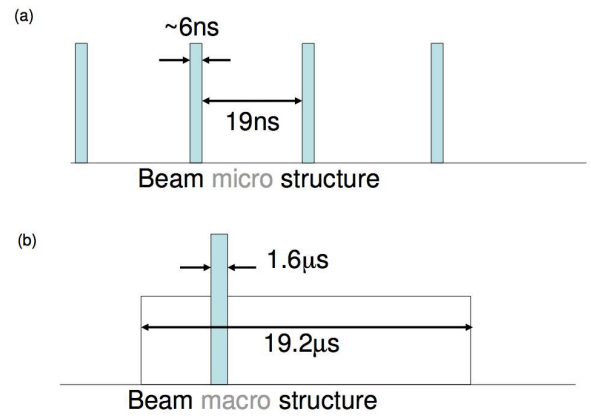

Figure 3-3: The proton beam timing structure. The figure is taken from [120].

\subsubsection{Focusing of Secondary Mesons}

The proton target is located along the axis of a cylindrical, focusing electromagnet ("horn") so that both the target and the horn axes coincide with the primary proton beam axis. The target-horn configuration is shown in the bottom panel of Fig. 3-4. The horn is made of two concentric shells, made of conducting aluminum, connected at the downstream end with a half-donut end cap. The horn geometry is illustrated in more detail on the left in Fig. 3-5.

The horn current is pulsed in coincidence with the proton beam hitting the beryllium target, producing a magnetic field which focuses either positively or negatively charged secondaries (depending on field polarity) in the direction of the detector. The peak electric current in neutrino running mode (producing a neutrino beam) is $174 \pm 1$ $\mathrm{kA}$, and in antineutrino mode (producing an antineutrino beam) is $-174 \pm 1 \mathrm{kA}$. The polarity flip is what selects the sign of the neutrino beam (neutrinos or antineutrinos) in each running mode. The effect of the current on the neutrino flux at MiniBooNE is illustrated on the right in Fig. 3-5. In addition to maximizing the neutrino flux in the energy range most relevant to the oscillation search, magnetic focusing also maximizes the sign-selection purity of the neutrino beam; i.e., it minimizes the amount of antineutrinos in the beam in neutrino mode, and vice versa.

Neutrino running at MiniBooNE took place from September 2002 through December 2006, and October 2007 through April 2008. Antineutrino running took place 

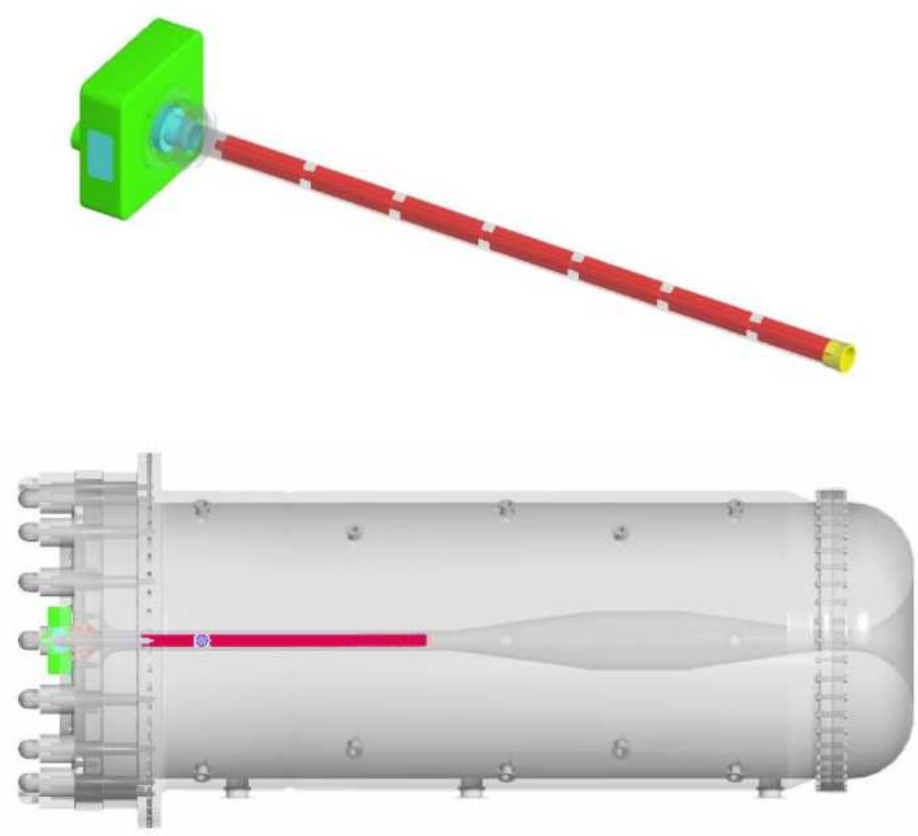

Figure 3-4: The BNB proton target. The top panel illustrates the target geometry. The beryllium components are shown in magenta color. The bottom panel illustrates the location of the target inside the upstream end of the magnetic focusing horn, shown in metallic gray color.

from January 2006 through July 2007, and still continues today since April 2008, with a goal of nearly doubling current antineutrino event statistics by the end of 2011. Fig. 3-6 shows the total number of protons delivered to the BNB target since the commissioning of the beamline in 2002. Aside from scheduled accelerator shutdowns, shown by gaps in the weekly rate distribution, the POT delivery rate has been steady at $>1 \times 10^{18}$ per week. The oscillation analysis results which will be presented in Chapter 5 apply to both neutrino and antineutrino running, using data samples that correspond to $6.46 \times 10^{20}$ POT and $5.66 \times 10^{20}$ POT, respectively.

\subsubsection{Secondary Meson Decay Pipe}

Downstream the target $(259 \mathrm{~cm}$ from its upstream end), a $214 \mathrm{~cm}$ long concrete collimator, with an aperture of $30 \mathrm{~cm}$ (upstream) to $35.5 \mathrm{~cm}$ (downstream) radius, absorbs secondary particles coming out at large angles with respect to the beam 

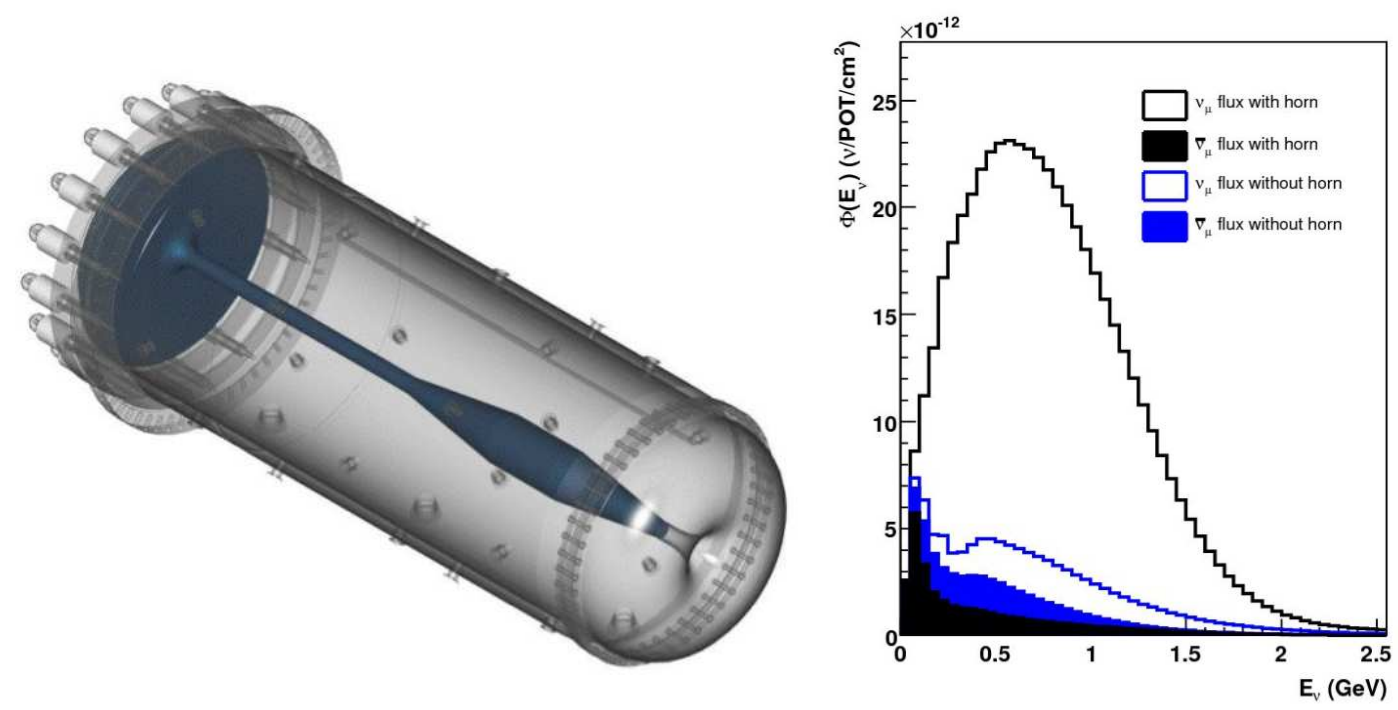

Figure 3-5: The MiniBooNE magnetic horn and its effect on the neutrino flux. The MiniBooNE magnetic horn, made of conductive aluminum, is shown on the left. The conductor geometry generates a $\propto 1 / R$ magnetic field which focuses either positively charged or negatively charged secondaries, depending on polarity, in the forward direction toward the MiniBooNE detector. The effect of magnetic field focusing on the neutrino flux expected in neutrino mode is shown on the right [50].

direction, that would otherwise not contribute to the neutrino beam seen at the detector. Immediately past the collimator, secondary mesons, including $\pi^{ \pm}, K^{ \pm}$, and $K^{0}$, enter a $50 \mathrm{~m}$ long decay pipe filled with air at atmospheric temperature and pressure. The charged mesons are allowed to decay in flight, producing neutrinos and other tertiary charged particles.

A set of ten retractable absorber plates is suspended half-way through $(25 \mathrm{~m})$ and above the decay pipe, as illustrated in Fig. 3-7. The plates were intended for studies of beam systematics. Lowering the absorber plates would result in reducing the relative neutrino rates from long-lived particles, e.g., suppressing electron neutrino rates from pion decays relative to kaon decays, by absorbing the former mesons before they have a chance to decay. Two of the absorber plates were accidentally ${ }^{3}$ deployed and remained in the decay region during part of the antineutrino running at MiniBooNE.

\footnotetext{
${ }^{3}$ The plates fell due to failure of the hardened-steel chains supporting the plates, which corroded from radiation exposure within the beamline environment.
} 

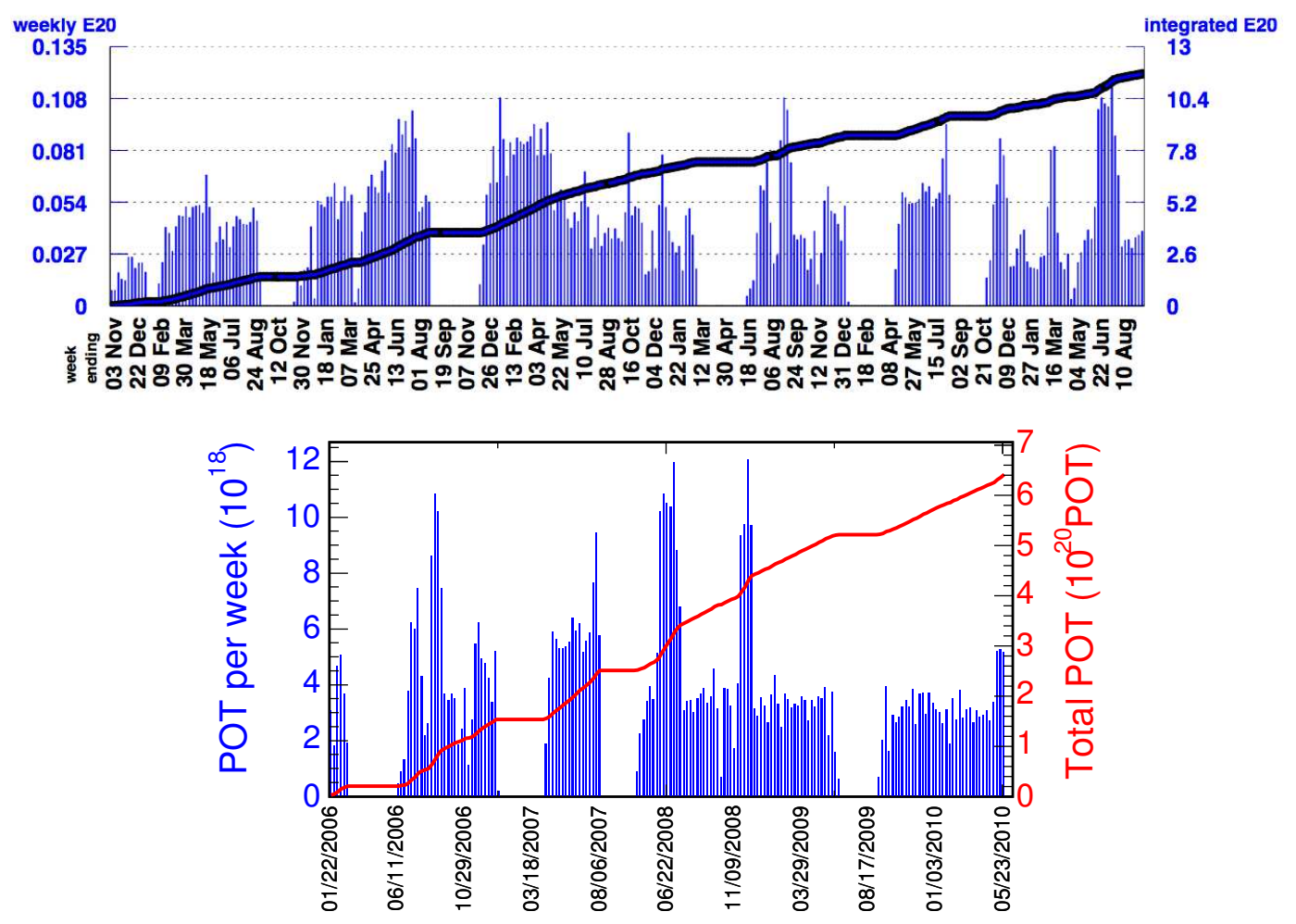

Figure 3-6: Protons delivered to the BNB target per week, since beamline commissioning in September 2002. The blue and red lines track the total POT delivered in neutrino (top figure) and antineutrino (bottom figure) running modes, respectively.

The first plate, identified as the rightmost of the two shaded plates in Fig. 3-7, fell during the 2006 accelerator shutdown, while the second one fell on August 29th, 2009. The plates were removed during a subsequent shutdown. For each plate inserted in the decay volume, the relative reduction in the $\nu_{\mu}$ (and $\bar{\nu}_{\mu}$ ) flux was approximately $10 \%$, as illustrated in Fig. 3-8. Modifications in the expected neutrino fluxes were taken into account in predicting antineutrino mode event rates, as described in App. A.4.

At the end of the decay region (50 $\mathrm{m}$ from the target), a beam dump made of steel and concrete absorbs all remaining particles other than neutrinos. Embedded in the beam dump is an array of gas proportional counters that measure the number of muons penetrating the dump. The $50 \mathrm{~m}$ decay length was chosen to reduce decays of long-lived muons, which would otherwise contribute to the $\nu_{e}$ appearance search background. 


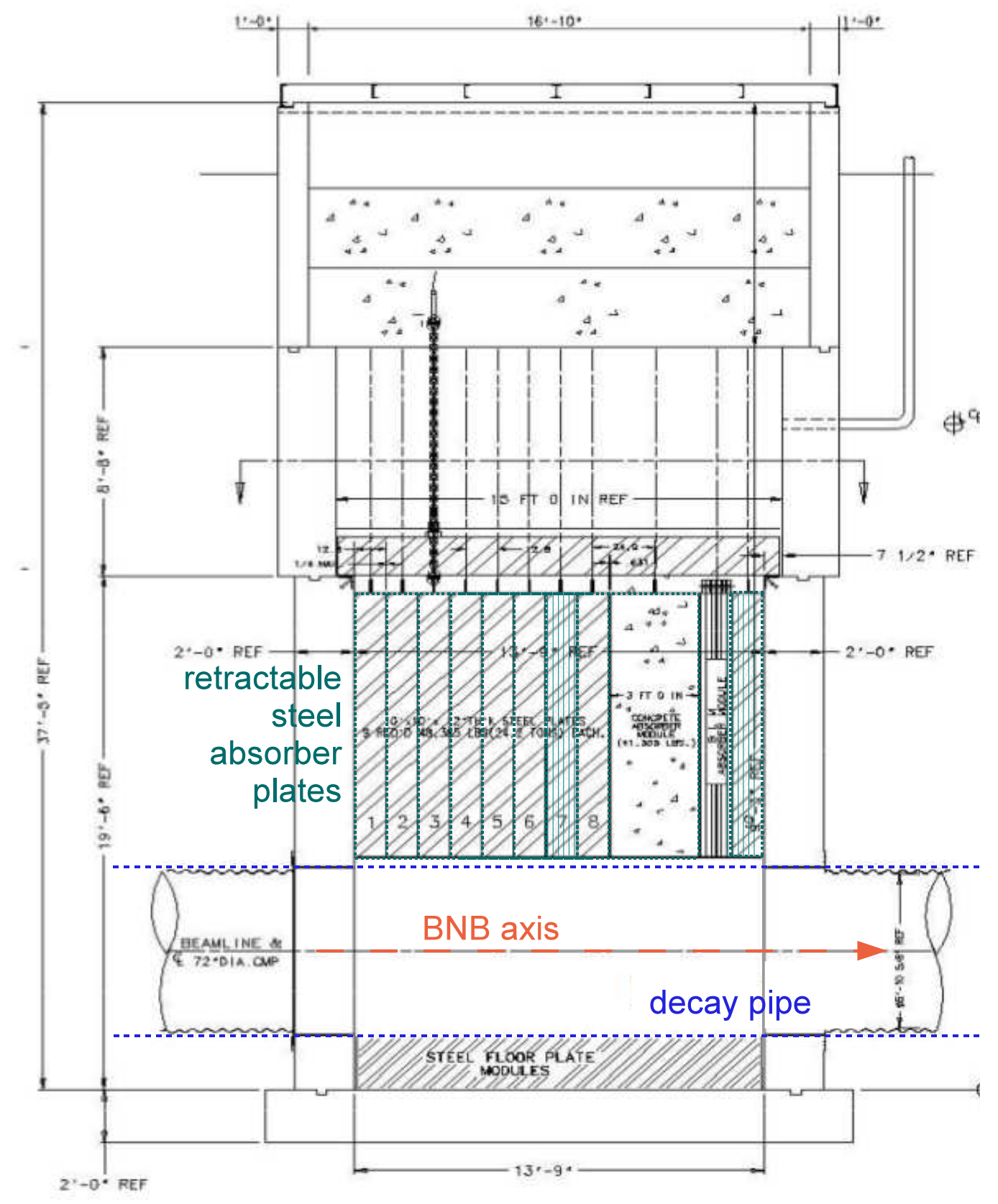

Figure 3-7: Absorber configuration in BNB decay pipe. The ten $10^{\prime} \times 10^{\prime}$ retractable steel (and steel+concrete) absorber plates are identified in green. The two plates identified in shaded green color accidentally fell into the decay pipe during part of the antineutrino running at MiniBooNE. The figure is from [121], with modifications. 


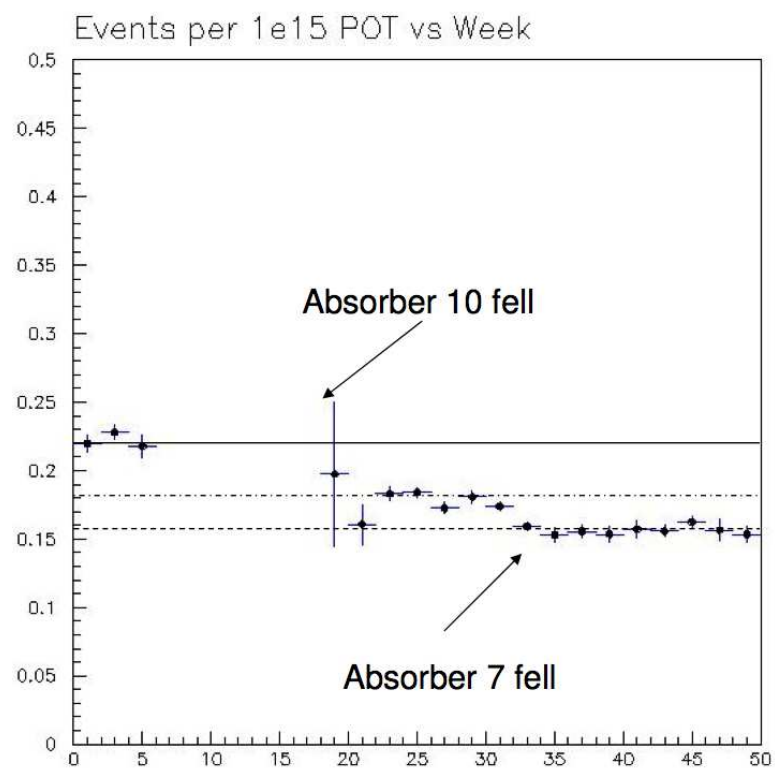

Figure 3-8: Effect of absorber on the antineutrino event rate over time. The first absorber fell during the 2006 accelerator shutdown, followed by the second one on August 29, 2006. The exact time periods can be identified by the reduction in absolute neutrino event rate in the detector. The figure is by R. Van de Water.

\subsection{The MiniBooNE Detector}

\subsubsection{Detector Description}

The MiniBooNE detector, shown in Fig. 3-9, is a spherical steel vessel, $12.2 \mathrm{~m}$ in diameter. It is composed of two optically isolated regions: an inner spherical region of $11.5 \mathrm{~m}$ diameter, and an outer spherical shell of $35 \mathrm{~cm}$ thickness. Both regions are filled with Exxon/Mobil Marcol 7 mineral oil (pure $\mathrm{CH}_{2}$ ), which acts as both the target for neutrino interactions and the propagating medium for the particles produced in those interactions. The target mass of the detector, 818 tons, was chosen so that MiniBooNE would see a statistically significant number of events $(\sim 1000$ events) from LSND-like oscillations for $10.0 \times 10^{20}$ POT [122].

The majority of final state particles in neutrino interactions studied in MiniBooNE are electrons, muons, pions, and protons, most of which are produced above 

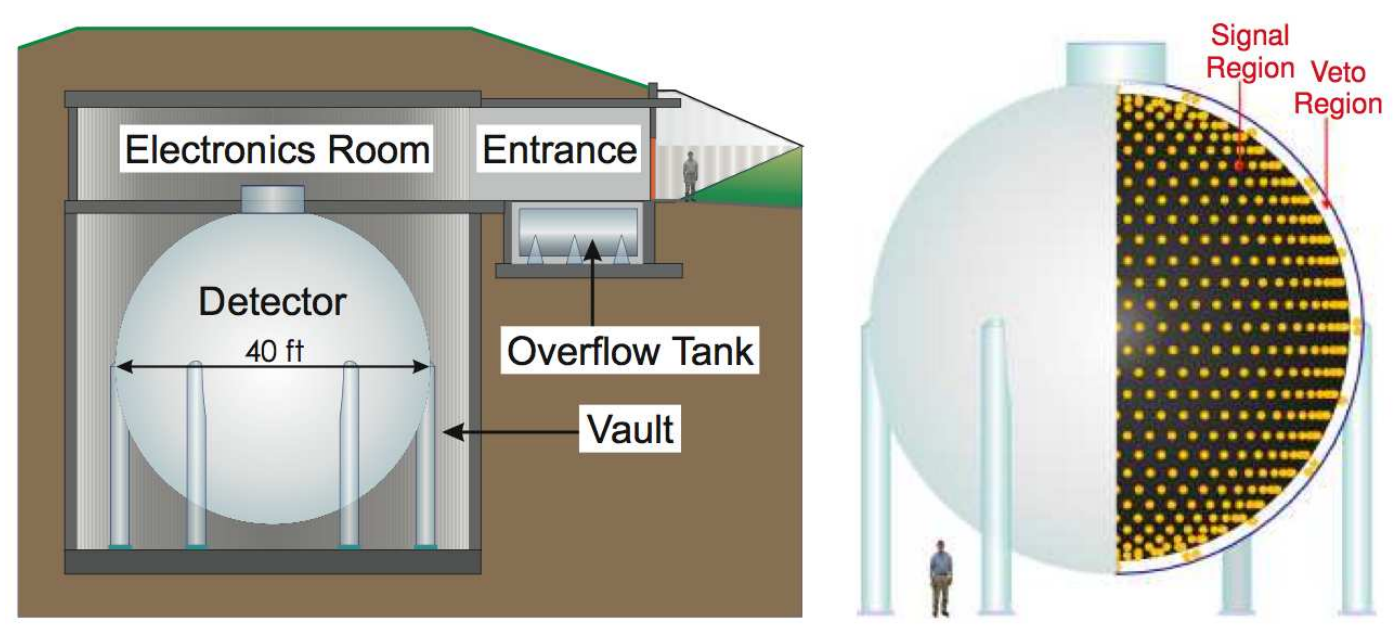

Figure 3-9: Schematic drawing of MiniBooNE detector enclosure (left) and MiniBooNE detector (right). The MiniBooNE detector is a spherical vessel filled with mineral oil and lined with photomultiplier tubes. The photomultiplier tubes detect Cherenkov and scintillation light produced by outgoing particles in neutrino interactions. The detector is located in a $13.7 \mathrm{~m}$ in diameter, underground, cylindrical vault. Detector electronics are housed in a hall above the vault, and under $3 \mathrm{~m}$ of earth overburden, providing shielding against cosmic ray muons, as well as insulation against temperature fluctuations. Also shown is an overflow tank, which allows for thermal expansion and recirculation of the oil in the MiniBooNE tank. The electronics hall is accessed via an opening, shown on the right. The figure is from [122], and is not to scale.

Cherenkov threshold. Cherenkov light produced by outgoing charged particles and detected by photomultiplier tubes lining the inner surfaces of the detector is used as the main signature in identifying and classifying neutrino interactions in MiniBooNE.

Table 3.1 summarizes the properties of the mineral oil used in MiniBooNE, and justifies its selection over water, another commonly used medium in Cherenkov detectors. The detector material was specifically chosen due to its high Cherenkov to scintillation light yield. Weak scintillation results in the production of delayed isotropic light for particles with sub-Cherenkov velocities, and, unlike Cherenkov light, it offers no directional information. Therefore, neutrino detection and event identification relies mainly on the detection of Cherenkov light patterns and, to a much smaller degree, scintillation light.

The inner region of the detector (tank) acts as the neutrino beam target. The 


\begin{tabular}{|c|c|c|}
\hline Property & Mineral oil & $\overline{\text { Water }}$ \\
\hline $\begin{array}{ll}\text { Density } & \\
\text { Index of refraction } & \\
\text { Extinction length }^{5} & \\
\text { Rayleigh scattering length } \\
\text { Muon capture rate } & \\
\text { Cherenkov threshold: } & e \\
& \pi \\
& \mu \\
& p \\
& \end{array}$ & $\begin{array}{c}0.86 \mathrm{~g} / \mathrm{cm}^{3} \\
1.47^{4} \\
>25 \mathrm{~m} \text { at } 460 \mathrm{~nm} \\
52 \mathrm{~m} \text { at } 442 \mathrm{~nm} \\
8 \% \\
0.7 \mathrm{MeV} / c \\
190 \mathrm{MeV} / c \\
144 \mathrm{MeV} / c \\
1280 \mathrm{MeV} / \mathrm{c}\end{array}$ & $\begin{array}{c}20 \% \\
0.8 \mathrm{MeV} / c \\
212 \mathrm{MeV} / c \\
160 \mathrm{MeV} / c \\
1423 \mathrm{MeV} / c\end{array}$ \\
\hline
\end{tabular}

Table 3.1: MiniBooNE mineral oil properties, and comparison to those of water, another commonly used medium in Cherenkov detectors. Mineral oil has a higher index of refraction and lower density, which lead to higher Cherenkov light yield. The higher index of refraction also allows for better event vertex resolution and lower Cherenkov thresholds for charged particles commonly produced in the MiniBooNE detector. The lower density also leads to small dispersion and long attenuation lengths for 320-600 nm light, as well as a relatively low muon capture rate. An additional advantage of mineral oil is that it is non-reactive and non-conducting, so that one can safely immerse electronic components in it.

tank inner surface is instrumented with 1280 8-inch Hamamatsu photomultiplier tubes (PMT's), evenly spaced, and facing towards the center of the tank, with a total photocathode coverage of $11.3 \%$. This is illustrated in Fig. 3-10. The inner tank surface is painted with non-reflective, black paint, to minimize Cherenkov light reflections, which would otherwise complicate event reconstruction and particle identification by mimicking delayed, isotropic scintillation light (see Sec. 3.5.3).

The purpose of the outer (veto) region is to detect charged particles entering or exiting the tank. The cosmic ray muon rate at the detector location is approximately $10 \mathrm{kHz}$. Rejecting events with significant veto activity provides rejection of cosmic ray events, neutrino events with vertices outside the detector, and neutrino events with tracks that escape the main tank volume. Both surfaces of the veto region are lined with a total of 240 8-inch Hamamatsu PMT's, and are painted with white, reflective paint. These PMT's are facing directions that are tangent to the detector radius at each location, as shown in Fig. 3-10. This maximizes the detection efficiency of light from through-going particles. The cosmic ray muon rejection efficiency provided by the veto region was measured to be $99.99 \%$ [122]. 

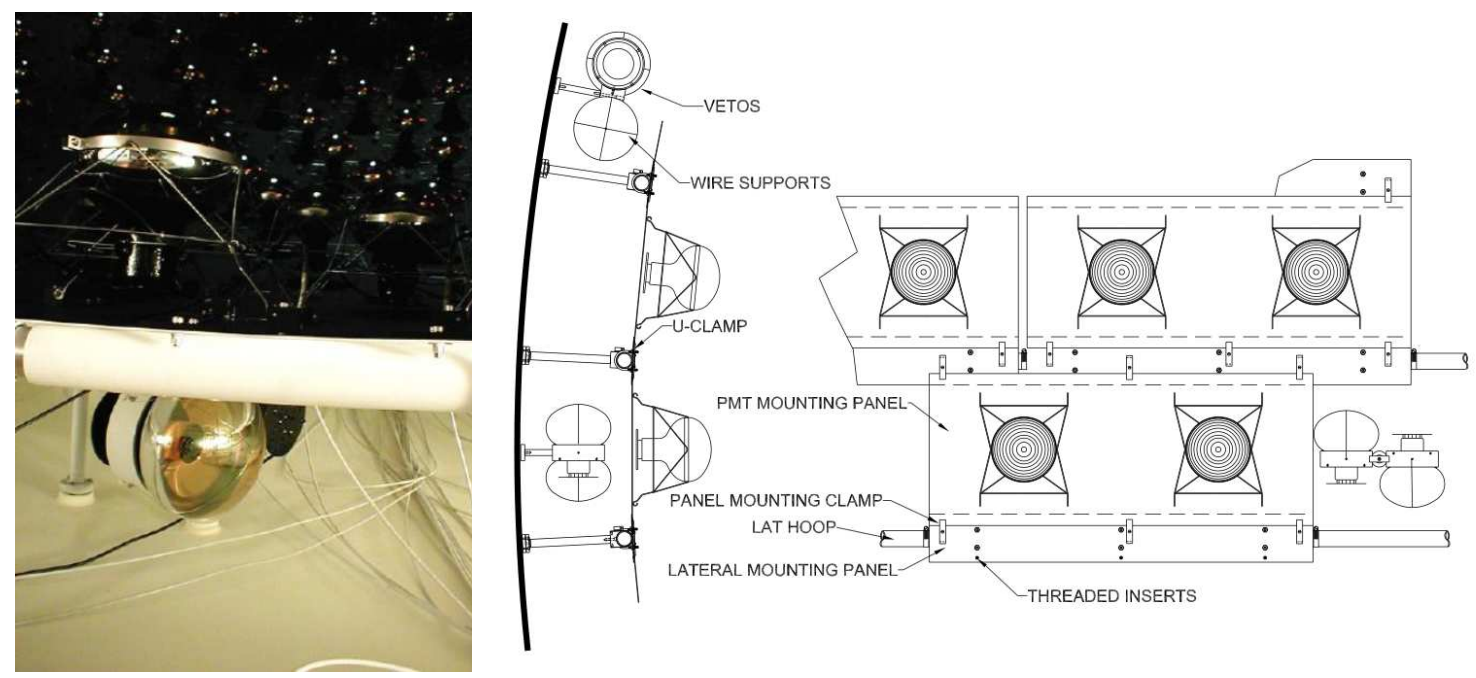

Figure 3-10: The MiniBooNE veto and tank photomultiplier tubes (PMT's). On the left is an actual cross-sectional picture of the MiniBooNE veto region (region painted with white, reflective paint) and the outer edge of the main tank region (region painted with black, non-reflective paint). On the right is an engineering drawing [122] of the MiniBooNE PMT support system for the veto region and main tank.

\subsubsection{Active Detector Components and Electronics}

All installed PMT's make up the active detector component of the MiniBooNE detector. Charge $(q)$ and timing $(t)$ information is read out separately from each PMT when the charge on a PMT is greater than $2 \mathrm{mV},{ }^{6}$ defined as a "hit". PMT information is digitized and stored via an analog-to-digital converter (ADC) unit in intervals of $200 \mu \mathrm{s}$, following any trigger signal.

Before being installed in the MiniBooNE detector, the PMT's were tested for gain, dark noise rate, charge resolution, timing resolution, and double-pulsing rate. The PMT time response and light collection efficiency as a function of light incident angle have also been characterized in external measurements using pulsed LED light $[123,124]$.

Several triggers are used in MiniBooNE, discussed in detail in [122]. Here, we summarize the three main ones: the beam trigger, synchronized with the accelerator clock, signals the ADC $5 \mu$ s before and in coincidence with each beam spill, providing

\footnotetext{
${ }^{6}$ The PMT base resistance and gain are set such that $2 \mathrm{mV}$ corresponds to $\sim 0.1$ p.e..
} 


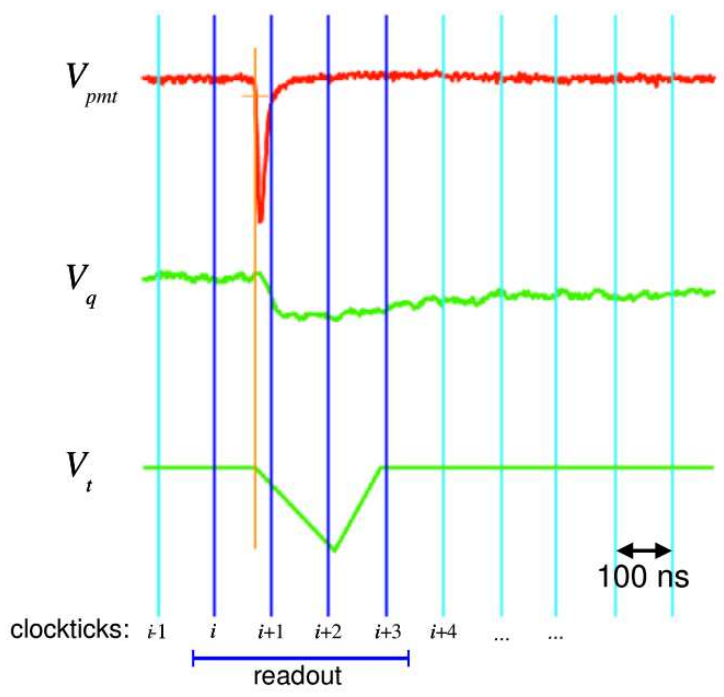

Figure 3-11: PMT signal digitization process. The times signify synchronization with the accelerator/beam trigger clock.

beam-on data used in the MiniBooNE oscillation search; the strobe trigger signals the ADC at $2.01 \mathrm{~Hz}$ (asynchronously with the accelerator clock), providing a sample of beam-off events to be used for cosmic ray muon studies, and studies of related background systematics; and the laser calibration trigger signals the ADC in coincidence with a laser system installed in the detector, at $3.33 \mathrm{~Hz}$, providing in situ calibration of individual PMT gains, as well as data to be used for oil stability checks. After each beam trigger, all other triggers (and their systems) are suspended for the duration of the beam trigger readout window, which is $19.2 \mu \mathrm{s}$ long.

Figure 3-11 illustrates the electronics response to a PMT signal. The fast charge output by the PMT (PMT anode signal), $V_{P M T}$, is integrated and stretched $\left(V_{Q}\right)$ before it can be sampled through a discriminator at $10 \mathrm{MHz}$. Once a threshold value of $>2 \mathrm{mV}$ registers for $V_{P M T}$, the discriminator fires and simultaneously generates a $200 \mathrm{~ns}$ hold-off gate and a time ramp signal, $V_{T}$. The hold off gate ensures that, after being read, a PMT cannot be read out again for $\sim 200$ ns. The ADC unit subsequently digitizes the integrated charge $\left(V_{Q}\right)$ and time $\left(V_{T}\right)$ traces for each PMT on every 100 ns clock tick, and those correspond to the $q$ and $t$ measurements recorded and used in further data analysis and event reconstruction. 

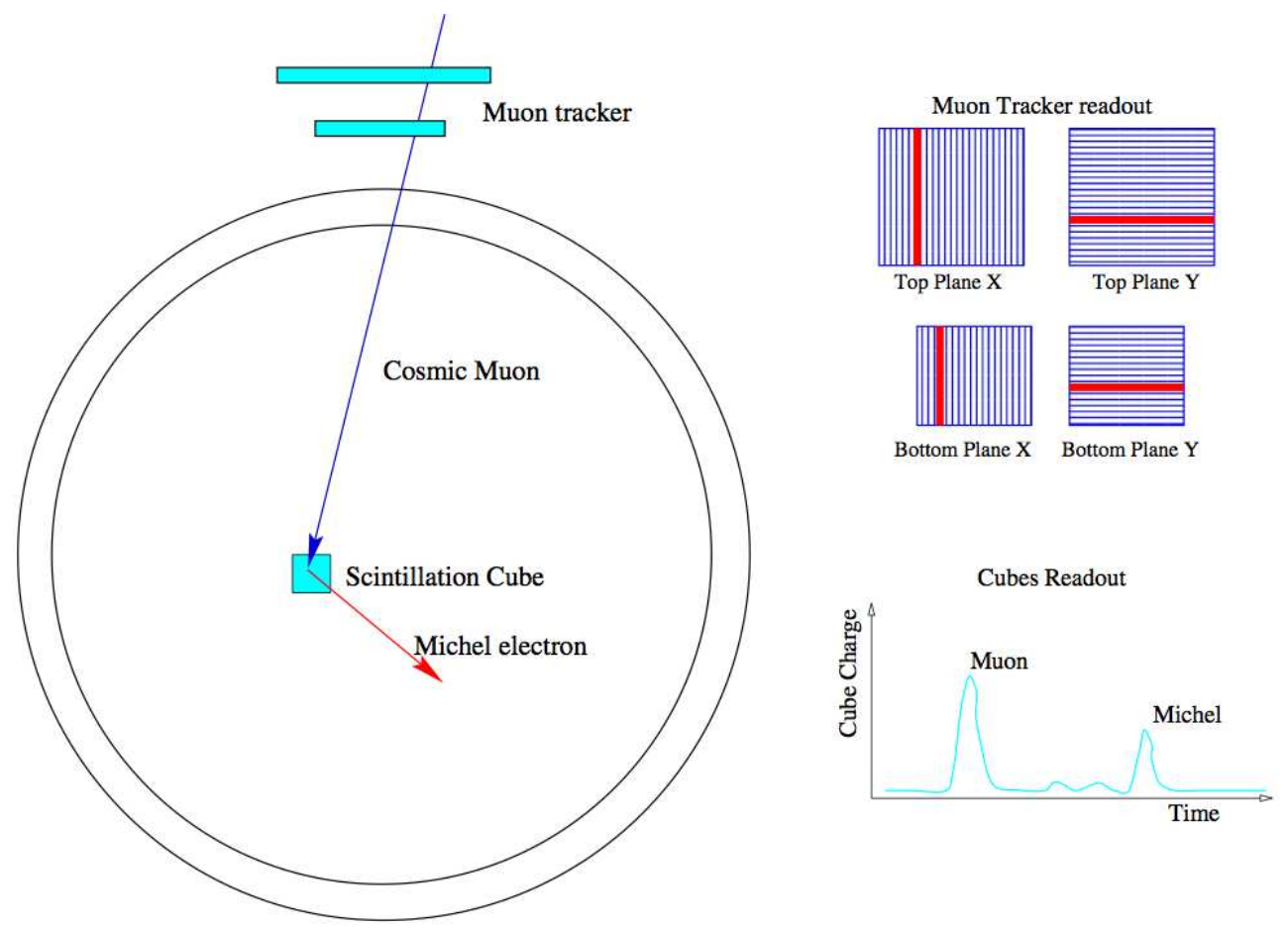

Figure 3-12: The cosmic ray muon calibration system. The muon hodoscope is shown above the MiniBooNE detector. The rate of muons passing through the muon tracker and one cube is $\sim 0.5 \mathrm{~Hz}$, and the rate of muons passing through the tracker and stopping in any of the cubes is approximately 100 per month.

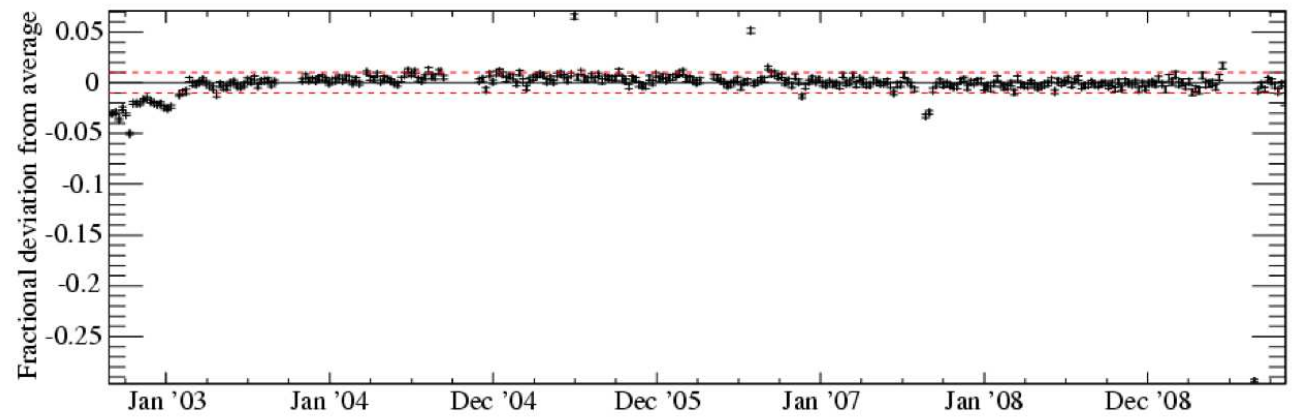

Figure 3-13: Stability of reconstructed Michel energy over time, for antineutrino running. The mean reconstructed energy has been stable within $1 \%$ over the entire antineutrino run. Similar stability was seen in neutrino mode running. The figure is by C. Green and H. Ray. 


\subsubsection{Detector Calibration Systems}

Cosmic ray muons which enter the MiniBooNE tank and decay within six optically sealed scintillator cubes dispersed through the tank volume provide a way to calibrate and determine the energy and angular resolution of the detector. This calibration system is demonstrated in Fig. 3-12. Incoming cosmic ray muons are identified with the use of a muon hodoscope, located above the detector, which can determine throughgoing muons' trajectories with $1.9^{\circ}$ angular resolution. Events with a muon stopping in one of the scintillation cubes, which have a well-defined range, are used for muon energy calibration measurements in the $100-800 \mathrm{MeV}$ range. ${ }^{7}$ Such (stopping) events are identified by the coincident signals of the tracker, scintillator cubes, ${ }^{8}$ and tank PMT's. The electrons from muon decay (Michel electrons) themselves are used for electron energy calibration measurements at $\sim 50 \mathrm{MeV}$ (Michel spectrum endpoint).

In addition to energy calibrations, these samples provide a way of verifying or tuning track reconstruction algorithms for the tank PMT's, since the muon directions are well determined independently of light reconstruction. They also allow for detector stability checks. For example, Fig. 3-13 illustrates the reconstructed Michel energy calibration spectra stability over the first half of the MiniBooNE antineutrino run.

For detailed information on calibration measurements, see Ref. [122].

\subsection{Predicting the Neutrino Event Rates at Mini- BooNE}

\subsubsection{Neutrino Flux Prediction}

The neutrino flux prediction starts with the simulation of the proton beam impinging on the beryllium target, and ends with the simulation of neutrinos produced in secondary and tertiary particle decays in the MiniBooNE beamline, within the angular acceptance of the detector. A detailed discussion can be found in [125]. Here, we pro-

\footnotetext{
${ }^{7}$ Detector through-going muons are also used for calibrations at higher energies $\sim 1 \mathrm{GeV}$.

${ }^{8}$ Each scintillator cube is read out through an optical fiber terminating on a 1-inch PMT.
} 
vide a brief summary with emphasis on information most relevant to the MiniBooNE $\nu_{e}$ and $\bar{\nu}_{e}$ appearance searches.

The BNB simulation uses Geant4-based software [126, 127, 128] for modeling primary and secondary particle production and propagation through the beamline geometry.

The parameters that mostly affect the prediction of the BNB neutrino flux are those related to meson production in primary $p-B e$ interactions. The four most relevant types of secondaries for neutrino flux calculations are $\pi^{+}, \pi^{-}, K^{+}$, and $K_{L}^{0}$, followed by $p$ and $n$, all of which are produced in inelastic interactions of the primary proton beam with the $B e$ target. Because the variation in available Geant4 hadron-production models is of order $50-100 \%$, the flux prediction makes use of a custom physics model for simulating the production of secondary mesons in primary $p-B e$ interactions, both in terms of their relative multiplicities, and in terms of their kinematics. The custom physics model is described in detail in Refs. [125, 50], and summarized as follows.

Primary $p-B e$ interactions at $8.9 \mathrm{GeV} / c$ proton kinetic energy are parametrized in terms of double-differential inelastic cross-sections, $\frac{d^{2} \sigma}{d p d \Omega}(p, \theta)$, for the production of each secondary. The parametrization is made as a function of longitudinal $\left(p_{Z}\right)$ and transverse $\left(p_{T}\right)$ incident proton momentum, ranging from $0-10 \mathrm{GeV}$ and $0-1 \mathrm{GeV}$, respectively. Existing production data from the HARP [129], E910 [130], and eight other kaon production experiments $[131,132,133,134,135,136,137,138]$, as well as data from Abe et al. [139] on neutral kaon production, available in slightly different $\left(p_{Z}, p_{T}\right)$ parameter space than that relevant to MiniBooNE, are used in several parametrization fits in order to extrapolate the double-differential cross-sections in the $\left(p_{Z}, p_{T}\right)$ parameter space relevant to MiniBooNE.

Available $\pi^{ \pm}$and $K^{0}$ production data from HARP [129] and E910 [130] and from E910 ${ }^{9}$ and Abe et al. [139], respectively, are fit according to a Sanford and Wang parametrization [141], while $K^{+}$production data from the above eight separate kaon experiments are fit to a simpler parametrization based on Feynman Scaling [142, 143].

\footnotetext{
${ }^{9}$ Analysis of the E910 [140] data by J. Link.
} 


\begin{tabular}{l}
\hline \hline BNB simulation processes \\
\hline \hline proton beam optics \\
target geometry and materials \\
magnetic horn focusing \\
primary proton-beryllium interactions \\
secondary kinematics \\
secondary hadronic and electromagnetic interactions \\
secondary decays \\
\hline \hline
\end{tabular}

Table 3.2: Processes taken into account in the MiniBooNE BNB simulation. For more details, see Ref. [125].

$K^{-}, p$ and $n$ production does not make use of parameterizations of existing data. Instead, the production cross-section tables are filled using the MARS [144] simulation program predicted cross-sections. Note, however, that $K^{-}$are produced at a rate which is three orders of magnitude suppressed relative to $\pi^{ \pm}$production. A more detailed description of the parametrization fitting procedure can be found in [125], including fit results.

The multiplicity of each secondary species is obtained with respect to the total inelastic production cross-section by integrating the corresponding parametrized double-differential cross-sections over the MiniBooNE proton kinematic phase-space, accounting for other proton elastic and inelastic processes as well.

Each generated secondary in the simulation is tracked through the beamline geometry until it decays (producing the neutrino beam) or gets absorbed. The simulation accounts for the effect of the magnetic horn, particle decays, as well as electromagnetic processes and hadronic interactions (both for primary protons and secondary particles) in the beamline geometry/materials, also defined within the simulation. A list of all processes taken into account is summarized in Tab. 3.2. A detailed discussion of the BNB simulation can be found in Refs. [125, 113].

After being produced in meson and/or subsequent lepton decays, neutrinos travel from their production point to the MiniBooNE detector essentially uninterrupted. ${ }^{10}$. A Fortran-based program [113] is used for propagating neutrinos from their point of production in the beamline to the detector, which properly accounts for neutrino

\footnotetext{
${ }^{10}$ Matter effects (see Sec. 1.4.1) are also negligible, due to the relatively low energy of neutrinos and the short baseline of the experiment.
} 

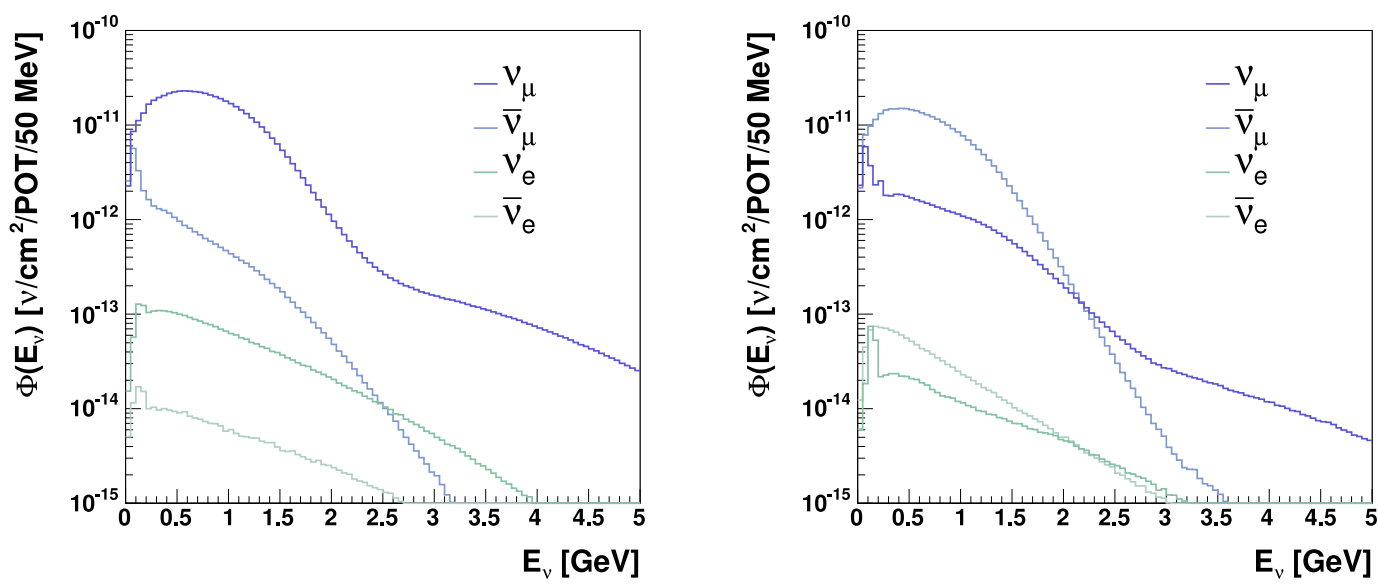

Figure 3-14: Neutrino flux spectra at the MiniBooNE detector in neutrino (left) and antineutrino (right) running configurations.

kinematics. In this step, the MiniBooNE detector is treated as a $610 \mathrm{~cm}$ radius vertical disk with its center located $541 \mathrm{~m}$ from the upstream face of the beryllium target, and $1.89 \mathrm{~m}$ above the neutrino beam direction.

The resulting neutrino flux prediction as viewed by the MiniBooNE detector is shown in Fig. 3-14 for neutrino (left) and antineutrino (right) running configurations. The flux, $\Phi\left(E_{\nu}\right)$, is given as a function of true neutrino energy for each type of neutrino, $\nu_{\mu}, \bar{\nu}_{\mu}, \nu_{e}$, and $\bar{\nu}_{e}$. The integrated contribution for each neutrino species is shown in Tabs. 3.3 and 3.4 for neutrino and antineutrino running modes, respectively, broken down further in terms of neutrino parent. The broken-down contributions per parent can be found as functions of $E_{\nu}$ in [125].

There are several differences between the predicted neutrino and antineutrino mode fluxes at MiniBooNE. The most striking difference is in statistics. Assuming the same number of POT for neutrino and antineutrino running, the total flux seen at the detector in antineutrino running is suppressed relative to that in neutrino running by approximately a factor of two. This is due to momentum and angular distribution differences in the production of the various secondary mesons $\left(\pi^{+}, K^{+}, \pi^{-}, K^{-}\right.$, etc. $)$ that contribute to the neutrino beam, as well as the resulting focusing power of the magnetic horn per secondary species. 


\begin{tabular}{l|rr|rr}
\hline \hline & \multicolumn{2}{|c|}{$\nu_{\mu}$} & $\bar{\nu}_{\mu}$ \\
\hline Fraction of total & & $93.6 \%$ & & $5.86 \%$ \\
\hline Composition & $\pi^{+}:$ & $96.72 \%$ & $\pi^{-}:$ & $89.74 \%$ \\
& $K^{+}:$ & $2.65 \%$ & $\mu^{+}$from $\pi^{+}:$ & $4.54 \%$ \\
& $\pi^{+}$from $K^{+}:$ & $0.26 \%$ & $K^{-}:$ & $0.51 \%$ \\
& $\pi^{+}$from $K^{0}:$ & $0.04 \%$ & $K^{0}:$ & $0.44 \%$ \\
& $K^{0}:$ & $0.03 \%$ & $\pi^{-}$from $K^{0}:$ & $0.24 \%$ \\
& $\mu^{-}$from $\pi^{-}:$ & $0.01 \%$ & $\mu^{+}$from $K^{+}:$ & $0.06 \%$ \\
& Other: & $0.30 \%$ & $\pi^{-}$from $K^{-}:$ & $0.03 \%$ \\
& & & Other: & $4.43 \%$ \\
\hline \hline Fraction of total & \multicolumn{2}{|r|}{$\nu_{e}$} & $\bar{\nu}_{e}$ \\
\hline Composition & $\mu^{+}$from $\pi^{+}:$ & $51.64 \%$ & & $0.05 \%$ \\
\hline & $K^{+}:$ & $37.28 \%$ & $\mu^{-}$from $\pi^{-}:$ & $19.33 \%$ \\
& $K_{L}^{0}:$ & $7.39 \%$ & $K^{-}:$ & $4.07 \%$ \\
& $\pi^{+}:$ & $2.16 \%$ & $\pi^{-}:$ & $1.26 \%$ \\
& $\mu^{+}$from $K^{+}:$ & $0.69 \%$ & $\mu^{-}$from $K^{-}:$ & $0.07 \%$ \\
& Other: & $0.84 \%$ & Other: & $4.62 \%$ \\
\hline \hline
\end{tabular}

Table 3.3: Integrated flux contribution for each neutrino species in neutrino running mode [125].

A second difference in the two running modes is that of wrong-sign flux contributions. In neutrino mode, the integrated antineutrino (wrong-sign) contamination of $\bar{\nu}_{\mu}$ and $\bar{\nu}_{e}$ in the beam is $5.9 \%$, whereas, in antineutrino mode, the wrong-sign contamination is much larger, at $15.9 \%$. Understanding the wrong-sign contamination of the beam and corresponding systematics in antineutrino running is crucial, especially in theoretical frameworks where neutrinos and antineutrinos are subject to different oscillation probabilities. While the MiniBooNE detector is unable to distinguish a single neutrino interaction from a corresponding antineutrino interaction, there are kinematic variables in specific reconstructed event samples that can be used to cross-check the wrong-sign predictions. Those are discussed in Sec. 4.5.1.

In both neutrino and antineutrino running configurations, the intrinsic $\nu_{e}$ and $\bar{\nu}_{e}$ contamination is less than $1 \%$ at the peak of the $\nu_{\mu}$ and $\bar{\nu}_{\mu}$ fluxes, although the contamination rises at higher energy, above $\sim 2 \mathrm{GeV}$, where contribution from neutral kaon decays dominates. Understanding the intrinsic $\nu_{e}$ and $\bar{\nu}_{e}$ content of the MiniBooNE flux is crucial for the appearance analysis, as intrinsic $\nu_{e}$ and $\bar{\nu}_{e}$ in the beam will contribute as significant background to any expected (small-amplitude) oscilla- 


\begin{tabular}{l|rr|rr}
\hline \hline & \multicolumn{2}{|c|}{$\nu_{\mu}$} & $\bar{\nu}_{\mu}$ \\
\hline Fraction of total & & $15.71 \%$ & & $83.73 \%$ \\
\hline Composition & $\pi^{+}:$ & $88.79 \%$ & $\pi^{-}:$ & $98.37 \%$ \\
& $K^{+}:$ & $7.53 \%$ & $K^{-}:$ & $0.18 \%$ \\
& $\mu^{-}$from $\pi^{-}:$ & $1.77 \%$ & $\pi^{-}$from $K^{0}:$ & $0.05 \%$ \\
& $K^{0}:$ & $0.26 \%$ & $K^{0}:$ & $0.05 \%$ \\
& Other: & $2.00 \%$ & $\mu^{+}$from $\pi^{+}:$ & $0.03 \%$ \\
& & & $\pi^{-}$from $K^{-}:$ & $0.02 \%$ \\
& & & Other: & $1.30 \%$ \\
\hline \hline Fraction of total & \multicolumn{2}{|c}{$\nu_{e}$} & $\bar{\nu}_{e}$ \\
\hline Composition & $K^{+}:$ & $51.72 \%$ & $\mu^{-}$from $\pi^{-}:$ & $0.45 .67 \%$ \\
& $K^{0}:$ & $31.56 \%$ & $K^{0}:$ & $16.51 \%$ \\
& $\mu^{+}$from $\pi^{+}:$ & $13.30 \%$ & $K^{-}:$ & $3.08 \%$ \\
& $\pi^{+}:$ & $0.83 \%$ & $\pi^{-}:$ & $2.58 \%$ \\
& $\mu^{+}$from $K^{+}:$ & $0.41 \%$ & $\mu^{-}$from $K^{-}:$ & $0.06 \%$ \\
& Other: & $2.17 \%$ & Other: & $2.10 \%$ \\
\hline \hline
\end{tabular}

Table 3.4: Integrated flux contribution for each neutrino species in antineutrino running mode. The antineutrino mode prediction assumes no absorber plates in the beamline [125].

tions. Nevertheless, there are several ways of indirectly constraining the intrinsic $\nu_{e}$ and $\bar{\nu}_{e}$ background contribution in either running mode, and those are discussed in more detail in Sec. 4.5.

\subsubsection{Neutrino Interaction Simulations}

Neutrino interactions at MiniBooNE are simulated in mineral oil and materials surrounding the detector starting with the v3 NUANCE [145] event generator. This is a simulation program which uses a comprehensive list of built-in neutrino interaction cross-sections over the range of $\sim 100 \mathrm{MeV}$ to $1 \mathrm{TeV}$. Ninety-nine distinct neutrino reactions are modeled separately for each neutrino type in the MiniBooNE flux, and are summed to produce the total neutrino cross-section.

By default, MiniBooNE uses the built-in NUANCE cross-section parameterizations and tunes the underlying cross-section parameters where necessary. For example, as will be discussed in the following sections, the $m_{A}$ parameter appearing in the nucleon axial vector form factor and the Pauli blocking parameter, $\kappa$, used to 


\begin{tabular}{l|cc}
\hline \hline $\begin{array}{l}\text { Cross-section } \\
\text { parameter }\end{array}$ & $\begin{array}{c}\text { Value for } \nu_{e} \text { and } \bar{\nu}_{e} \\
\text { appearance analysis }\end{array}$ & Correlations \\
\hline \hline$m_{A}(\mathrm{QE})$ & $1.23 \pm 0.077 \mathrm{GeV}$ & $\rho\left(m_{A}, \kappa\right)=-0.875$ \\
$m_{A}^{H}(Q E)$ & $1.13 \pm 0.10 \mathrm{GeV}$ & - \\
$\kappa$ & $1.022 \pm 0.021$ & $\rho\left(\kappa, m_{A}\right)=-0.875$ \\
$\Delta s$ & $0.0 \pm 0.1 \mathrm{MeV}$ & - \\
$E_{B}$ & $34.0 \pm 9.0 \mathrm{MeV}$ & - \\
$p_{F}$ & $220.0 \pm 30.0 \mathrm{MeV}$ & - \\
$m_{A}^{1 \pi}$ & $1.10 \pm 0.275$ & $\rho\left(m_{A}^{1 \pi}, m_{A}^{c o h}\right)=1$ \\
$m_{A}^{1 \pi}$ & $1.10 \pm 0.10 \mathrm{GeV}$ & - \\
$m_{A}^{N \pi}$ & $1.30 \pm 0.52 \mathrm{GeV}$ & - \\
$m_{A}^{c o h}$ & $1.030 \pm 0.275 \mathrm{GeV}$ & $\rho\left(m_{A}^{c o h}, m_{A}^{1 \pi}\right)=1$ \\
\hline \hline
\end{tabular}

Table 3.5: NUANCE cross-section parameter values used as inputs to the neutrino and antineutrino event rate predictions for the MiniBooNE $\nu_{e}$ and $\bar{\nu}_{e}$ appearance analyses. The values for $m_{A}$ and $\kappa$ were extracted from fits to MiniBooNE data. Note that the $\kappa$ value used in the $\nu_{e}$ appearance analysis differs from [146], for historical reasons. $m_{A}^{H}(Q E)$ and $m_{A}^{1 \pi, H}$ were used for the antineutrino mode analysis, only.

parametrize neutrino quasi-elastic scattering on carbon and hydrogen, were adjusted by fits to MiniBooNE $\nu_{\mu}$ and $\bar{\nu}_{\mu}$ CCQE data [146]. Table 3.5 summarizes the crosssection parameters used in predicting the neutrino and antineutrino event rates at MiniBooNE, defined within the NUANCE simulation package, and associated uncertainties. We will refer to these settings in Sec. 4.5, and will be discussing assigned cross-section systematic uncertainties in detail in Sec. 4.6.2.

Before we consider event rates and event signatures in the MiniBooNE detector, it is useful to discuss neutrino cross-sections in the energy range covered by the MiniBooNE predicted flux spectra (0-3 GeV). In the following discussion, we focus on the most dominant cross-sections in that range, as well as the ones which are most likely to induce background rates to the MiniBooNE appearance searches.

\subsubsection{Neutrino Cross-Sections at $\sim 1 \mathrm{GeV}$}

Fig. 3-15 summarizes (muon) neutrino and antineutrino charged-current (CC) crosssections in the $0-100 \mathrm{GeV}$ range. The neutrino cross-sections in the $0-3 \mathrm{GeV}$ range, which is the energy range that overlaps with that of the neutrino flux seen at MiniBooNE, are the ones most relevant for predicting event rates at MiniBooNE. The 

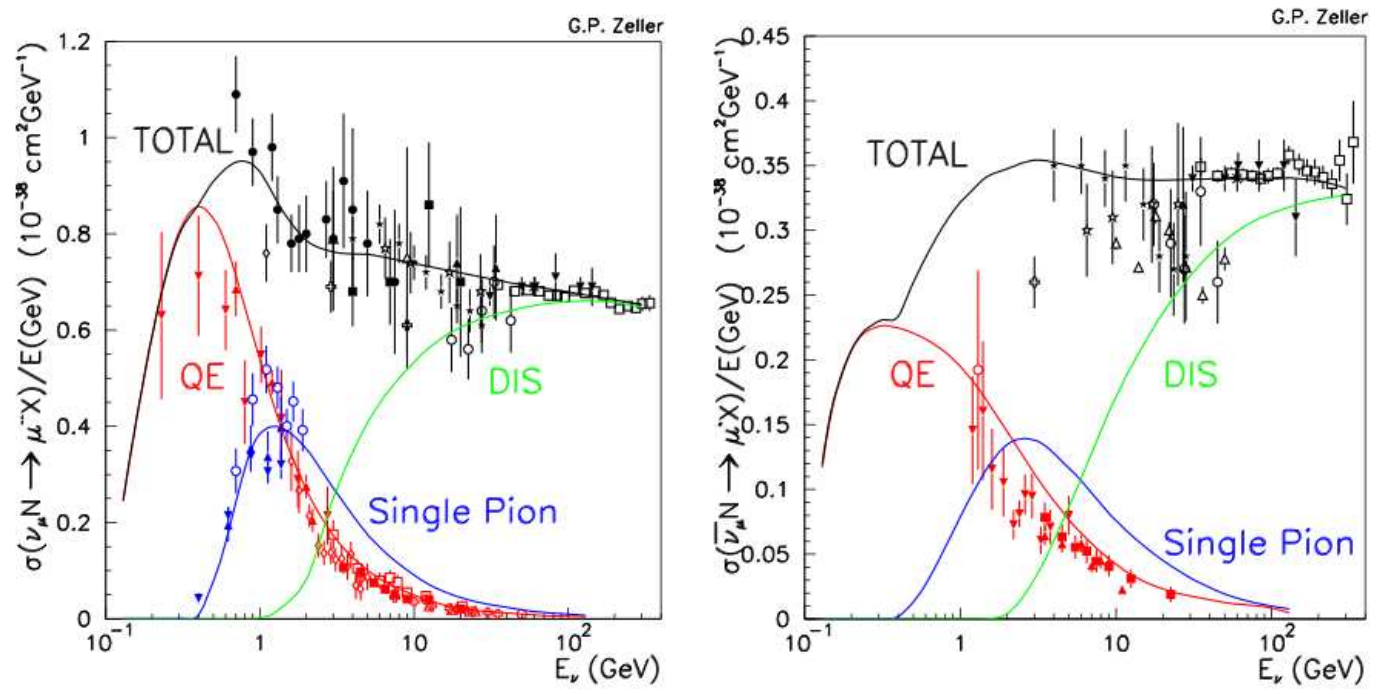

Figure 3-15: Neutrino charged-current cross-sections at $\sim 1 \mathrm{GeV}$. Left: neutrino crosssections. Right: antineutrino cross-sections. The dominant cross-sections for MiniBooNE are those of charged-current quasi-elastic and single pion production processes. The solid lines correspond to the NUANCE neutrino event generator [145] predictions, while the data points are from available measurements prior to MiniBooNE. The figures are by G. Zeller.

dominant cross-section is that of charged-current quasi-elastic (CCQE) scattering, both for the $\nu_{\mu}$ flux, and for the $\nu_{e}$ flux. For a $\nu_{e}$ appearance search, MiniBooNE makes use of primarily two samples: $\nu_{\mu}$ CCQE, which is the most abundant interaction sample, and $\nu_{e}$ CCQE, which is the main interaction channel for any signal from $\nu_{\mu} \rightarrow \nu_{e}$ oscillations. The corresponding interaction diagrams are shown in Fig. 3-16, and have the same signature in MiniBooNE independent of whether they are induced by a neutrino or an antineutrino.

We will see in the next section that neutral-current (NC) interactions, in particular single $\pi^{0}$ and resonant $\Delta$ production, can significantly contribute as a background to the appearance search at MiniBooNE. The most relevant NC $\pi^{0}$ and $\Delta$ interaction diagrams for the $\nu_{e}$ appearance search at MiniBooNE are shown in Fig. 3-17.

In the following subsections, we discuss the main interaction processes in Figs. 3-16 and 3-17 in more detail. 

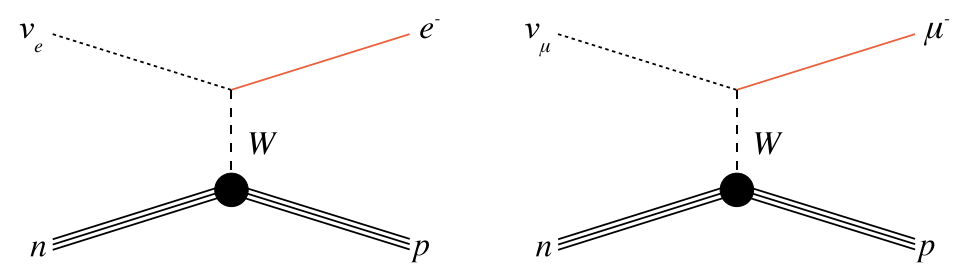

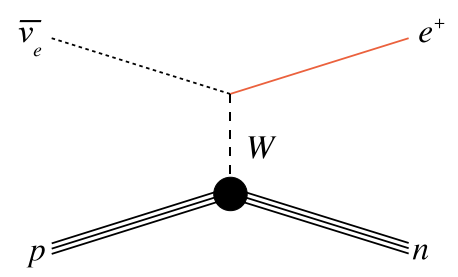

(a)

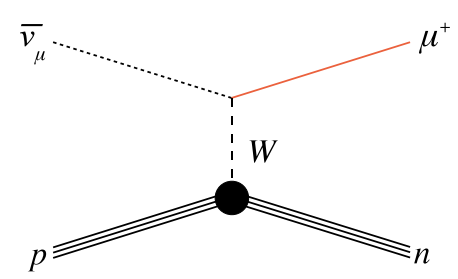

(b)

Figure 3-16: Schematic diagrams of the main neutrino (top) and antineutrino (bottom) CC interaction processes in the MiniBooNE detector. (a) CCQE events contributed from the intrinsic $\nu_{e}$ component of the neutrino beam or from a possible $\nu_{e}$ beam component due to $\nu_{\mu} \rightarrow \nu_{e}$ oscillations. (b) CCQE events contributed from the dominant $\left(\nu_{\mu}\right)$ component of the neutrino beam.

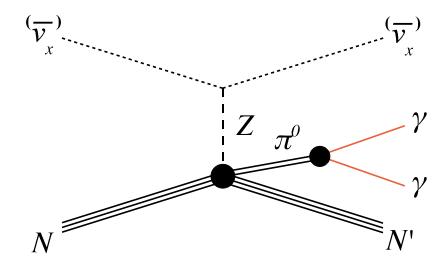

(a)

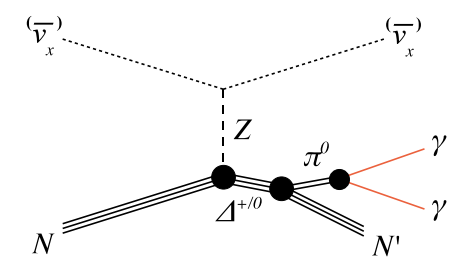

(b)

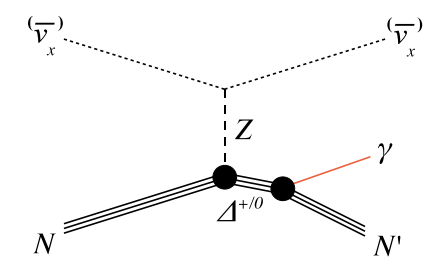

(c)

Figure 3-17: Schematic diagrams of the main neutrino and antineutrino NC interaction processes in MiniBooNE. (a) NC coherent $\pi^{0}$ production. (b) NC resonant $\pi^{0}$ production. (c) NC resonant $\Delta$ production, followed by $\Delta$ radiative decay. The majority of these events come from NC interactions of the $\nu_{\mu}$ and $\bar{\nu}_{\mu}$ components of the neutrino or antineutrino beam. 


\section{Charged-Current Quasi-Elastic Interactions}

At neutrino energies of a few hundred $\mathrm{MeV}$, which is where the MiniBooNE flux peaks, the dominant neutrino interaction is CCQE scattering. In this interaction, a neutrino interacts through $W$-boson exchange with a proton or neutron within a nucleus, producing a charged lepton, as shown in Fig. 3-16.

In a CCQE interaction, lepton flavor is conserved. Therefore, the flavor of the outgoing lepton is indicative of the flavor of the neutrino that induced the interaction. MiniBooNE makes use of this assumption in order to determine the flavor of incoming neutrinos. A relatively small $\nu_{e}$ signal rate can be therefore identified over the dominant $\nu_{\mu}$ rate by the presence of an electron rather than a muon being produced in the interaction.

Because uncertainties on this process are relatively large, MiniBooNE relies on its own measurement of the CCQE cross-section, which uses a high-statistics $(\sim 150,000$ reconstructed events in neutrino mode) $\nu_{\mu}$ CCQE sample [146], in order to predict the $\nu_{e}$ CCQE signal (and background) event rate, as it shares the same cross-section. The $\nu_{e}$ CCQE cross-section uncertainties are effectively reduced by exploiting the cross-section correlations between the two samples, as will be discussed in Sec. 4.5.

The MiniBooNE neutrino and antineutrino CCQE cross-sections are calculated based on the Llewellyn Smith [147] formalism, in which the differential CCQE crosssection for a neutrino of energy $E_{\nu}$, scattering off of a bare nucleon with mass $m_{N}$, and producing a charged lepton $\ell$ with mass $m_{\ell}$, is given by

$$
\frac{d \sigma}{d Q^{2}}=\frac{G_{F}^{2} m_{N}}{8 \pi E_{\nu}^{2}}\left[A \pm \frac{(s-u)}{m_{N}} B+\frac{(s-u)^{2}}{m_{N}^{4}} C\right]
$$

where $+/$ - refers to antineutrino/neutrino scattering. $Q^{2}=-q^{2}$ is the four-momentumsquared transfer in the interaction; $s$ and $u$ are the standard Mandelstam variables so that $(s-u)=4 m_{N} E_{\nu}-Q^{2}-m_{\ell}^{2}$; and $G_{F}$ is the Fermi constant. For $\nu_{e}$ scattering, $m_{\ell}$ corresponds to the mass of the electron, while for $\nu_{\mu}$ scattering, $m_{\ell}$ corresponds to the mass of the muon. As can be seen from the form of the cross-section, the two interactions will be highly correlated for $E_{\nu} \gg m_{\ell}$. 
Because neutrino scattering occurs on composite objects, and not point-like objects, the three parameters, $A, B$ and $C$ are dependent on nuclear form factors with further $Q^{2}, m_{\ell}$, and $m_{N}$ dependence. More explicitly, the form factors on which $A, B$ and $C$ depend on are: the vector form factors $F_{1}$ and $F_{2}$; the axial-vector form factor

$$
F_{A}=\frac{g_{A}}{\left(1+Q^{2} / m_{A}^{2}\right)}
$$

and the pseudo-scalar form factor

$$
F_{P}=\frac{2 m_{N}^{2}}{m_{\pi}^{2}+Q^{2}} F_{A} .
$$

$m_{\pi}, \mu_{p}$ and $\mu_{n}$ are the pion mass and proton and neutron anomalous magnetic moments, and $m_{V}, m_{A}$ and $g_{A}$ are the vector mass, axial mass and axial coupling parameters, respectively.

$F_{1}$ and $F_{2}$ are determined using empirical fits from from [148], and are relatively well known. However, $F_{A}$ and $F_{P}$ are not, and so the uncertainty in the cross-section is dominated by uncertainties in the axial-vector form factor, $F_{A}$. The parameter $g_{A}$ in $F_{A}$ is well determined through $\beta$ decay experiments (at $Q^{2}=0$ ). However, $m_{A}$ is less understood. There have been several measurements of this value, obtained from the $Q^{2}$ distribution of CCQE neutrino-nucleon scattering events. The world's data on neutrino interactions on deuterium give $m_{A}=1.03 \mathrm{GeV}$ [149], and are uncertain at the level of 20-40\%; on the other hand, recent measurements on oxygen [150] from the $\mathrm{K} 2 \mathrm{~K}$ experiment suggest a higher value. ${ }^{11}$

In order to minimize uncertainties, MiniBooNE treats $m_{A}$ as an adjustable parameter in the model, and relies on an in situ measurement of $m_{A}$ (on carbon) by studying reconstructed $\nu_{\mu}$ CCQE events as a function of $Q^{2}$. The $m_{A}$ parameter affects both the shape and the normalization of the distribution (at the level of $\sim 30 \%$ ). Because $\nu_{\mu}$ oscillations due to sterile neutrinos are expected to be small, and their effect on the $Q^{2}$ shape distribution is expected to be less pronounced than that of

\footnotetext{
${ }^{11}$ The higher value is also consistent with recent results from SciBooNE [151] and MiniBooNE [146], which are both on higher-Z neutrino targets.
} 
$m_{A}$, extraction of this parameter at MiniBooNE is not expected to mask any possible disappearance effects [117].

In a CCQE interaction, the kinematic distributions of the outgoing particles are further complicated by the fact that the interacting nucleon is not a free particle at rest, but rather subject to Fermi motion within the carbon nucleus, as well as other effects that have to do with the fact that the nucleons are bound to each other. MiniBooNE uses the Smith-Moniz relativistic Fermi gas model [152] within the NUANCE neutrino event generator to model these effects. This model is dependent on the binding energy and Fermi momentum of the nucleus, $E_{B}=34 \mathrm{MeV}$ and $p_{F}=220$ $\mathrm{MeV}$ for ${ }^{12} \mathrm{C}$, and allows for adjustment of an extra parameter, $\kappa$, which is used to model nuclear effects related to Pauli blocking. These effects suppress the crosssection at low $Q^{2}$. As in the case of $m_{A}, \kappa$ is not well constrained by available $\nu_{\mu}$ CCQE cross-section measurements [146], and its value was therefore also determined by $Q^{2}$-dependent fits to MiniBooNE reconstructed $\nu_{\mu}$ CCQE data.

The values of $m_{A}$ and $\kappa$ used in the appearance analyses presented in this thesis were extracted from fits to neutrino mode data and verified by fits to antineutrino mode data [153], and are $m_{A}=1.23 \mathrm{GeV}$ and $\kappa=1.022$. Figure 3-18 illustrates the effect of $m_{A}$ and $\kappa$ on the MiniBooNE $\nu_{\mu}$ CCQE data to MonteCarlo prediction agreement.

Since the detector medium is $\mathrm{CH}_{2}$, in antineutrino mode, the CCQE interaction also has a non-negligible contribution from scattering on hydrogen that one must account for, in addition to CCQE scattering on carbon. Roughly $25 \%$ of the antineutrino CCQE scatters are on hydrogen rather than carbon. An additional parameter, $M_{A}^{Q E, H}$, was used in the antineutrino analysis to parametrize hydrogen CCQE scattering. ${ }^{12}$

\footnotetext{
${ }^{12}$ In neutrino mode, the target nucleon in a CCQE interaction is always a neutron, by definition. Therefore, there are no CCQE interactions on hydrogen.
} 


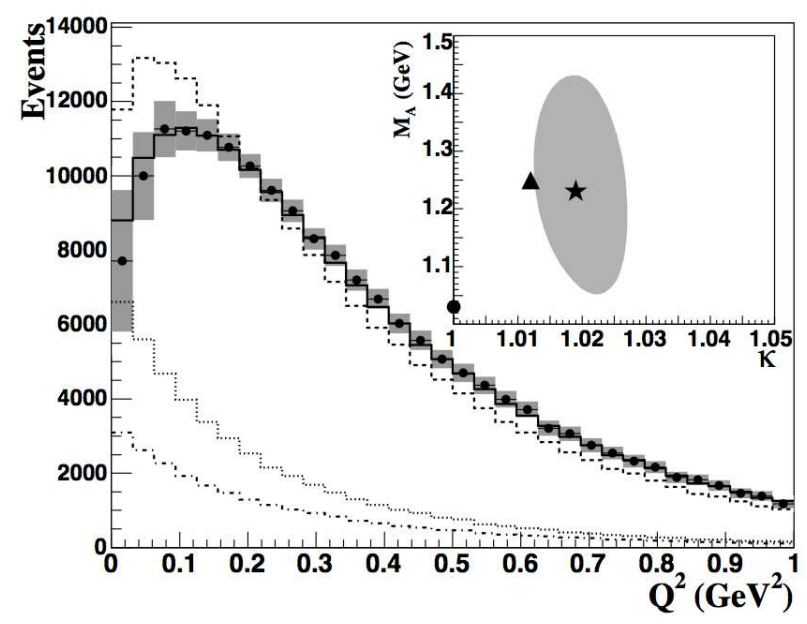

Figure 3-18: MiniBooNE neutrino mode $\nu_{\mu}$ CCQE data and MonteCarlo prediction, after $m_{A}$ and $\kappa$ corrections [146]. The dashed black line corresponds to the NUANCE prediction $\left(m_{A}=1.03 \mathrm{GeV}\right.$ and $\kappa=1.0$, shown by the black circle in the inset in comparison with the MiniBooNE best-fit parameters and associated $1 \sigma$ uncertainty), while the solid black line corresponds to the NUANCE prediction with $m_{A}=1.23 \mathrm{GeV}$ and $\kappa=1.019$ extracted from $Q^{2}$-dependent, relatively-normalized fits to MiniBooNE data. The points correspond to data with statistical (almost negligible) error bars. The gray band indicates systematic uncertainty. The MonteCarlo curves in this figure have been relatively normalized to data.

\section{Single Pion Production}

As shown in Fig. 3-15, the next most abundant process in MiniBooNE is CC single pion $(\mathrm{CC} \pi)$ production. Single pion production in fact can proceed either through $W$ exchange $(\mathrm{CC} \pi)$ or through $Z$ exchange ( $\mathrm{NC} \pi)$ :

$$
\begin{array}{ll}
\nu_{\ell}+N \rightarrow \ell^{-}+N^{\prime}+\pi & (\mathrm{CC} \pi) \\
\nu_{\ell}+N \rightarrow \nu_{\ell}+N^{\prime}+\pi & (\mathrm{NC} \pi) .
\end{array}
$$

Furthermore, both CC $\pi$ and NC $\pi$ production can occur either via $\Delta$ resonance production (primarily), or via coherent scattering. In the case of resonance production, $N\left({ }^{\prime}\right)$ in Eq. 3.4 denotes a nucleon, while in the case of coherent production it denotes a nucleus.

One might expect that such interactions can be easily identified by the presence of the pion produced in the interaction. However, both $\mathrm{CC}$ and $\mathrm{NC} \pi$ production 

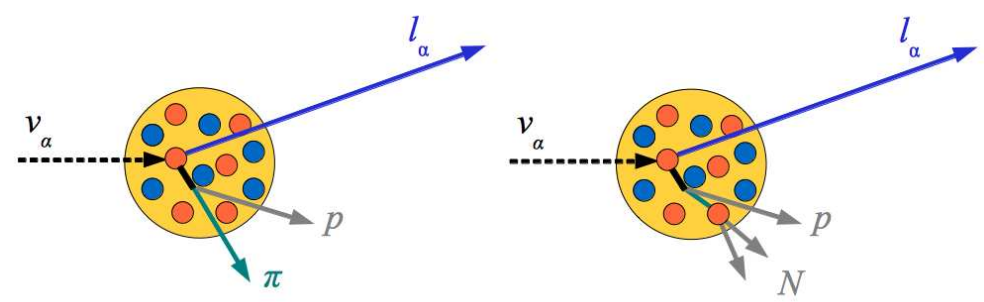

Figure 3-19: The effect of pion absorption in single pion production neutrino interactions in the MiniBooNE detector. When the pion is absorbed within the nucleus, the resulting final states are indistinguishable from those of a CCQE interaction in the MiniBooNE detector.

processes are subject to nuclear effects, which may lead to absorption of the outgoing pion, for example, as demonstrated in Fig. 3-19. In that case, CC $\pi$ production can contribute as irreducible background to the CCQE samples. More specifically, because pion absorption causes missing energy in event reconstruction, it primarily affects CCQE measurements at lower reconstructed neutrino energies.

The CC $\pi$ cross-section is parametrized by its own axial mass parameter, $m_{A}^{1 \pi}$ (on carbon). MiniBooNE relies on the NUANCE default value for this parameter, but has extracted a measurement of $\mathrm{CC} \pi^{+}$to CCQE cross-section ratio in neutrino mode [154], which allows for a more precise estimation of mis-identified rates, once nuclear and other final state effects are taken into account. The measurement also constrains the relative contributions from coherent $(<1 \%)$ versus resonant production.

It should be noted that, as in the case of CCQE, in antineutrino mode, single pion production can also occur through antineutrino scattering on hydrogen. This process is also taken into account in predicting event rates at MiniBooNE and is instead parametrized by $m_{A}^{1 \pi, H}$.

Understanding NC single pion production is particularly crucial in the $\nu_{e}$ appearance search. As already mentioned, MiniBooNE looks for $\nu_{e}$ events by looking for a single electron produced in the detector. We will see later on that events which produce a single photon in the detector can be mis-identified as $\nu_{e}$ CCQE interactions. As shown in the left and middle diagrams of Fig. 3-17, $\mathrm{NC} \pi^{0}$ production results in a $\pi^{0}$ produced in the tank, which promptly $\left(\tau \sim 8.4 \times 10^{-17} \mathrm{~s}\right)$ decays into two photons. 
In the event that one of the two photons is lost in the reconstruction process, that particular interaction will contribute as a mis-identified background to the $\nu_{e}$ CCQE search.

Because the $\mathrm{NC} \pi^{0}$ cross-section is poorly understood, MiniBooNE relies once more on an in situ measurement of the $\mathrm{NC} \pi^{0}$ interaction rate in the detector, in order to be able to accurately predict the rate at which such events can be mis-identified as background [155]. ${ }^{13}$ The MiniBooNE NC $\pi^{0}$ rate measurements are performed in both neutrino and antineutrino mode with high-statistics NC $\pi^{0}$ event samples reconstructed in each case. In doing so, MiniBooNE also constrains the rates of both coherent and resonant $\mathrm{NC} \pi^{0}$ production in each running mode, independently.

\section{Neutral-Current $\Delta$ Resonance Production}

In the case of $\mathrm{NC} \Delta$ resonance production, shown in the middle and right diagrams in Fig. $3-17$, a $\Delta$ is produced via the excitation of a nucleon, $N$, and subsequently decays into a pion plus the original nucleon, $N$, in the overwhelming majority of $\Delta$ resonance production events. However, a $\Delta$ can also decay radiatively, through the emission of a single photon, $\Delta \rightarrow N \gamma$, as shown on the right of Fig. 3-17. This process is extremely rare, and is related to the $\pi^{0}+N$ decay mode through a branching fraction of $0.52-0.60 \%$ [45]. Nevertheless, because it produces a single photon in the detector, it provides an irreducible background to the $\nu_{e}$ appearance search.

Radiative $\Delta$ decay is also constrained by the MiniBooNE NC $\pi^{0}$ rate measurement, as will be discussed in Sec. 4.5.

\section{Final State Interactions}

Pion charge exchange and absorption within the nucleus affect the $\nu_{\mu}$ and $\nu_{e}$ CCQE backgrounds, and are therefore accounted for in the MiniBooNE neutrino event rate predictions, as they can make CC $\pi$ interactions look indistinguishable from CCQE. Other final state effects, affecting mostly the $\nu_{e}$ CCQE background prediction, include

\footnotetext{
${ }^{13}$ It should be noted that the probability that a $\pi^{0}$ is mis-identified as an electron depends directly on the kinematics of the $\pi^{0}$ decay photons.
} 
photonuclear absorption ${ }^{14}$ of final state photons in the detector [157], radiative $\mu^{-}$ capture on carbon and explicit modeling [158] of subsequent final state neutrons and protons, which are absent from Geant3.

Following neutrino interaction simulations, final state electromagnetic and weak interactions in MiniBooNE are modeled within Geant3, expanded to accommodate $\pi^{0} \rightarrow \gamma e^{+} e^{-}$and $\mu \rightarrow e \nu \bar{\nu}$ decays, while hadronic interactions are modeled by GCALOR [159], and cross-checked with predictions by GFLUKA [160], for systematics studies. The latter processes account for photonuclear interactions on carbon, radiative $\pi^{-}$capture in pion production processes, radiative decay of $\Delta$ resonances produced in pion-carbon interactions, and $\pi^{ \pm}-\mathrm{C}$ (strong) elastic scattering. Uncertainties in hadronic processes contribute most dominantly to final state uncertainties, and are taken into account in event rate predictions as discussed in Sec. 4.6.2.

\subsubsection{Neutrino Event Rate Predictions}

Table 3.6 summarizes the absolute rates of all types of interactions expected in the MiniBooNE detector for both neutrino running and antineutrino running, before final state interactions.

\footnotetext{
${ }^{14}$ This process was omitted in the original neutrino mode appearance search [156].
} 


\begin{tabular}{|c|c|c|c|c|c|}
\hline Neutrino Running & & & "Antineutrino Running & & \\
\hline Process & Interaction rate & Fraction & Process & Interaction rate & Fraction \\
\hline$\overline{\text { Total }}$ & $\overline{2,201,699}$ & $100 \%$ & Total & $\overline{537,590}$ & $100 \%$ \\
\hline$\nu_{\mu} n \rightarrow \mu^{-} p$ & 868,377 & $39.4 \%$ & $\bar{\nu}_{\mu} p \rightarrow \mu^{+} n$ & $\overline{157,922}$ & $29.4 \%$ \\
\hline$\nu_{\mu} N \rightarrow \nu_{\mu} N$ & 373,926 & $17.0 \%$ & $\bar{\nu}_{\mu} N \rightarrow \bar{\nu}_{\mu} N$ & 73,313 & $13.6 \%$ \\
\hline$\nu_{\mu} N \rightarrow \mu^{-} N^{\prime} \pi^{+/ 0}(\operatorname{res} \mathrm{CC} \pi)$ & 566,479 & $25.7 \%$ & $\bar{\nu}_{\mu} N \rightarrow \mu^{+} N^{\prime} \pi^{-/ 0}(\operatorname{res}$ CC $\pi)$ & 46,146 & $8.6 \%$ \\
\hline$\nu_{\mu} N \rightarrow \nu_{\mu} N^{\prime} \pi^{ \pm / 0}($ res NC $\pi)$ & 199,103 & $9.0 \%$ & $\bar{\nu}_{\mu} N \rightarrow \bar{\nu}_{\mu} N^{\prime} \pi^{ \pm / 0}($ res NC $\pi)$ & 30,483 & $5.7 \%$ \\
\hline$\nu_{\mu} A \rightarrow \mu^{-} A \pi^{+}(\operatorname{coh} \mathrm{CC} \pi)$ & 34,028 & $1.5 \%$ & $\bar{\nu}_{\mu} A \rightarrow \mu^{+} A \pi^{-}(\operatorname{coh} \mathrm{CC} \pi)$ & 14,633 & $2.7 \%$ \\
\hline$\nu_{\mu} A \rightarrow \nu_{\mu} A \pi^{0}(\operatorname{coh} \mathrm{NC} \pi)$ & 20,027 & $0.9 \%$ & $\bar{\nu}_{\mu} A \rightarrow \bar{\nu}_{\mu} A \pi^{0}(\operatorname{coh} \mathrm{NC} \pi)$ & 8963 & $1.7 \%$ \\
\hline Other $\nu_{\mu}$ & 98,201 & $4.5 \%$ & Other $\bar{\nu}_{\mu}$ & 14548 & $2.7 \%$ \\
\hline Total $\nu_{\mu}$ & $2,160,141$ & $98.1 \%$ & Total $\bar{\nu}_{\mu}$ & 346008 & $64.4 \%$ \\
\hline Total $\bar{\nu}_{\mu}$ & 26,752 & $1.2 \%$ & Total $\nu_{\mu}$ & 186795 & $34.7 \%$ \\
\hline$\nu_{e} n \rightarrow e^{-} p$ & 4,753 & & $\bar{\nu}_{e} p \rightarrow e^{+} n$ & 745 & \\
\hline$\nu_{e} N \rightarrow \nu_{e} N$ & 1,989 & & $\bar{\nu}_{e} N \rightarrow \bar{\nu}_{e} N$ & 321 & \\
\hline$\nu_{e} N \rightarrow e^{-} N^{\prime} \pi^{+/ 0}(\operatorname{res} \mathrm{CC} \pi)$ & 4,062 & & $\bar{\nu}_{e} N \rightarrow e^{+} N^{\prime} \pi^{-/ 0}(\operatorname{res} \mathrm{CC} \pi)$ & 326 & \\
\hline$\nu_{e} N \rightarrow \nu_{e} N^{\prime} \pi^{ \pm / 0}(\operatorname{res} \mathrm{NC} \pi)$ & 1,352 & & $\bar{\nu}_{e} N \rightarrow \bar{\nu}_{e} N^{\prime} \pi^{ \pm / 0}(\operatorname{res} \mathrm{NC} \pi)$ & 174 & \\
\hline$\nu_{e} A \rightarrow e^{-} A \pi^{+}(\operatorname{coh} \mathrm{CC} \pi)$ & 233 & & $\bar{\nu}_{e} A \rightarrow e^{+} A \pi^{-}(\operatorname{coh}$ CC $\pi)$ & 76 & \\
\hline$\nu_{e} A \rightarrow \nu_{e} A \pi^{0}(\operatorname{coh} \mathrm{NC} \pi)$ & 119 & & $\bar{\nu}_{e} A \rightarrow \bar{\nu}_{e} A \pi^{0}(\operatorname{coh} \mathrm{NC} \pi)$ & 39 & \\
\hline Other $\nu_{e}$ & 1,653 & & Other $\bar{\nu}_{e}$ & 159 & \\
\hline Total $\nu_{e}$ & 14,161 & $0.6 \%$ & Total $\bar{\nu}_{e}$ & 1840 & $0.3 \%$ \\
\hline Total $\bar{\nu}_{e}$ & 645 & $<0.1 \%$ & Total $\nu_{e}$ & 2947 & $0.5 \%$ \\
\hline
\end{tabular}

Table 3.6: Relative rates (flux times cross-section) of interactions at MiniBooNE in neutrino and antineutrino running modes (before final state interactions). The rates assume fiducial volume of a $610 \mathrm{~cm}$ radius sphere. The event rates are obtained assuming $10.0 \times 10^{20} \mathrm{POT}$ in each running mode. For definition of resonant and coherent processes, see Sec. 3.4.3. The majority of "other" are NC and CC deep inelastic scattering events. The event rates in antineutrino mode assume no absorber plates in the beamline. 


\subsection{Neutrino Events in the MiniBooNE Detector}

The propagation of final state particles and simulation of light production and propagation in the MiniBooNE detector, up until the point where individual photons are absorbed in a PMT photocathode and have the chance to produce a photoelectron, uses a Geant4-based MonteCarlo, where the detector and surroundings' geometry and materials (and their properties) are specified within the simulation.

Light simulation also takes into account Cherenkov radiation, intrinsic scintillation of the oil and UV fluorescence, as well as wavelength-dependent absorption and reflection of light, and PMT efficiencies. A set of 35 adjustable parameters is used as input to the optical modeling, in order to describe processes and parameters such as extinction length, index of refraction, scintillation yield, fluorescence yield, reflections, PMT angular efficiency, etc. Those parameters have been tuned using external measurements and calibration data $[161,162]$.

A more detailed description of the detector simulation is given elsewhere [163].

The following section begins with a review of the three main event signatures relevant to the MiniBooNE appearance analysis, which correspond to $\nu_{\mu} \mathrm{CCQE}$, $\nu_{e} \mathrm{CCQE}$, and $\mathrm{NC} \pi^{0}$ interactions. Given those signatures, a brief description of the MiniBooNE particle reconstruction and identification method will be given in Sec. 3.5.3.

\subsubsection{Event Signatures}

As stated in Sec. 3.3, the MiniBooNE PMT's are sensitive to both Cherenkov and (to a smaller extent) scintillation light produced by charged particles in the detector. Cherenkov photons are emitted at an angle $\theta_{C}$ relative to a charged particle's trajectory, where $\cos \theta_{C}=1 / n \beta$ ( $n$ being the index of refraction in the MiniBooNE oil, and $\beta=v / c$ of the charged particle). The radiation is azimuthally symmetric about the

particle's track direction, resulting in a ring-like pattern that can be identified on the PMT array. Scintillation photons, on the other hand, are emitted isotropically and are delayed with respect to the Cherenkov light. 
In the case of $\nu_{\mu}$ and $\nu_{e}$ CCQE interactions, the outgoing proton is most often produced below Cherenkov threshold, and observation of scintillation light from the proton is overwhelmed by light produced by the outgoing lepton. In the case of $\bar{\nu}_{\mu}$ and $\bar{\nu}_{e}$ CCQE events, where the recoil nucleon is always a neutron, only light produced by the outgoing lepton will be observed.

At energies above $200 \mathrm{MeV}$, a muon is considered a minimum-ionizing particle. Therefore, a muon produced in a $\nu_{\mu}$ CCQE interaction traverses the MiniBooNE detector with minimal chance of radiative energy loss or deviation from its course due to multiple Coulomb scattering. As a result, a muon produces a Cherenkov cone which is seen by the PMT array as a ring with a well-defined outer edge. As the muon loses energy, the cone angle becomes smaller $\left(\cos \theta_{C} \propto 1 / \beta\right)$, and therefore the Cherenkov ring may become filled. A muon ring pattern also becomes filled as the muon approaches the tank wall. Those light patterns are characteristic of $\nu_{\mu} \mathrm{CCQE}$ interactions.

On the other hand, single electrons produced in the MiniBooNE tank lose energy primarily via Bremsstrahlung. As such, an electron quickly loses its energy as it travels through the detector medium through electromagnetic showers. This produces a significantly different ring pattern from that of a muon: electrons produce diffuse, open rings. Figure 3-20 illustrates the two different signatures. An open, fuzzy ring is the characteristic signature for any $\nu_{e}$ signal from $\nu_{\mu} \rightarrow \nu_{e}$ oscillations which interacts through CCQE scattering.

The third class of events, NC $\pi^{0}$, produce a $\pi^{0}$ which decays into two photons. Those will photoconvert (with a mean conversion length of $67 \mathrm{~cm}$ ) within the oil and induce electromagnetic showers which are indistinguishable from those induced by electrons, as shown in Fig. 3-20. Being able to efficiently identify both photon rings is crucial for the appearance analysis. In cases where the two photons produced in $\pi^{0}$ decay overlap ${ }^{15}$ or the photons are produced back-to-back with one having too low energy to produce a reconstructible ring, those events will contribute as background

\footnotetext{
${ }^{15}$ A typical $\pi^{0}$ momentum in MiniBooNE is $0.3 \mathrm{GeV}$, which results in somewhat boosted $\gamma \gamma$ final state in the lab frame.
} 


\section{particle track Cherenkov ring}

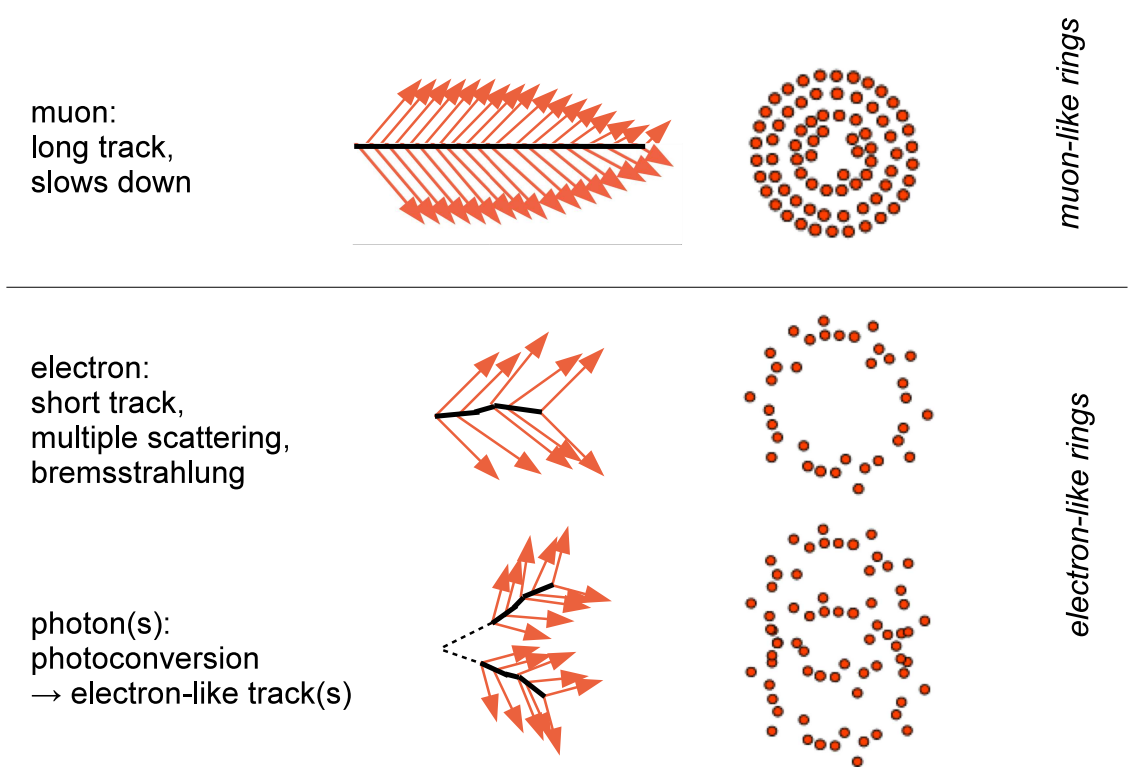

Figure 3-20: PMT hit topologies due to light from electrons, muons, or photons in the MiniBooNE detector. Electrons and photons produce fuzzy, open rings, while muons produce well-defined, and/or filled Cherenkov rings. The quantity, spatial distribution, and arrival times of photons provide information on the location, direction, and energy of a charged particle produced in the MiniBooNE detector. 


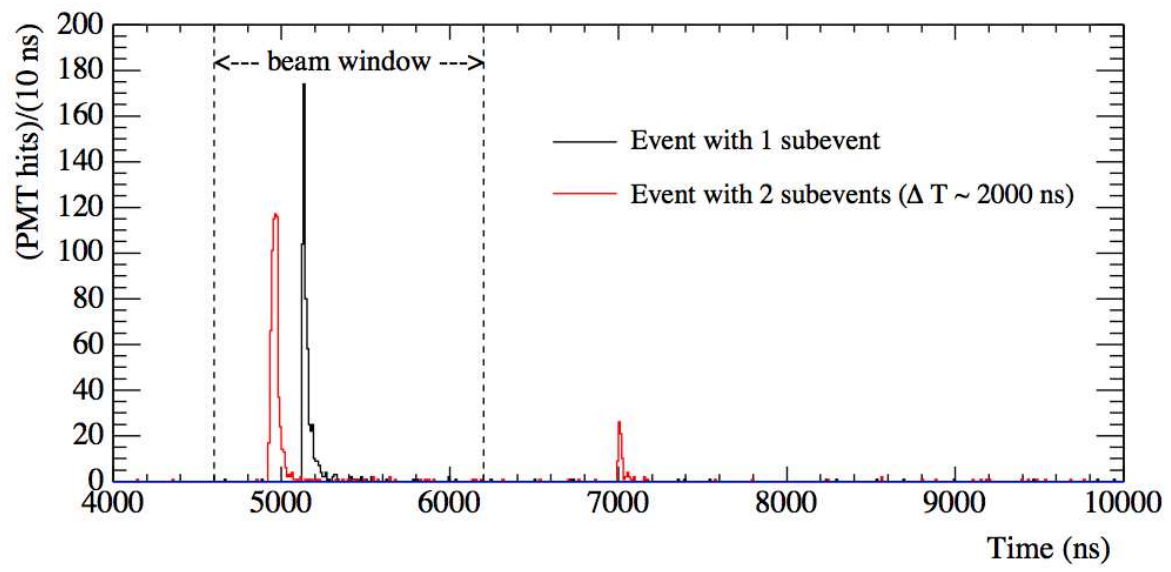

Figure 3-21: Time distribution of PMT hits recorded for an event with one (black) and two (red) subevents, relative to the $1.6 \mu$ s beam spill window, defined by the dashed vertical lines. The two-subevent is characteristic of a muon decaying at rest within the detector, where the second subevent, delayed by $\sim 2.2 \mu \mathrm{s}$, corresponds to a Michel electron.

to the $\nu_{e}$ CCQE appearance search. Irreducible background is also contributed by radiative $\Delta$ decays, which always produce a single photon in the detector.

\subsubsection{Isolating Neutrino Events}

Neutrino events in the MiniBooNE detector are isolated by looking for clusters of recorded PMT hits as a function of time. Depending on the type of neutrino interaction, each event can consist of one or more clusters. For example, $\nu_{\mu}$ or $\bar{\nu}_{\mu} \mathrm{CCQE}$ events will typically generate two clusters, one corresponding to light from the muon produced in the neutrino interaction, and one corresponding to light produced by the Michel electron coming from the decay of the outgoing muon, approximately 2.2 $\mu$ s later, as illustrated in Fig. 3-21. On the other hand, $\nu_{e}$ or $\bar{\nu}_{e}$ CCQE events will generate only one cluster, corresponding to light from the electron produced in the neutrino interaction. If more than one clusters occur within an event, each cluster is identified as a sub-event, defined somewhat more precisely as a cluster of PMT hits where at least 10 hits occur, and any two consecutive hits within the cluster are separated by no more than $10 \mathrm{~ns}$.

A clean sample of beam events in the MiniBooNE detector can be isolated with 

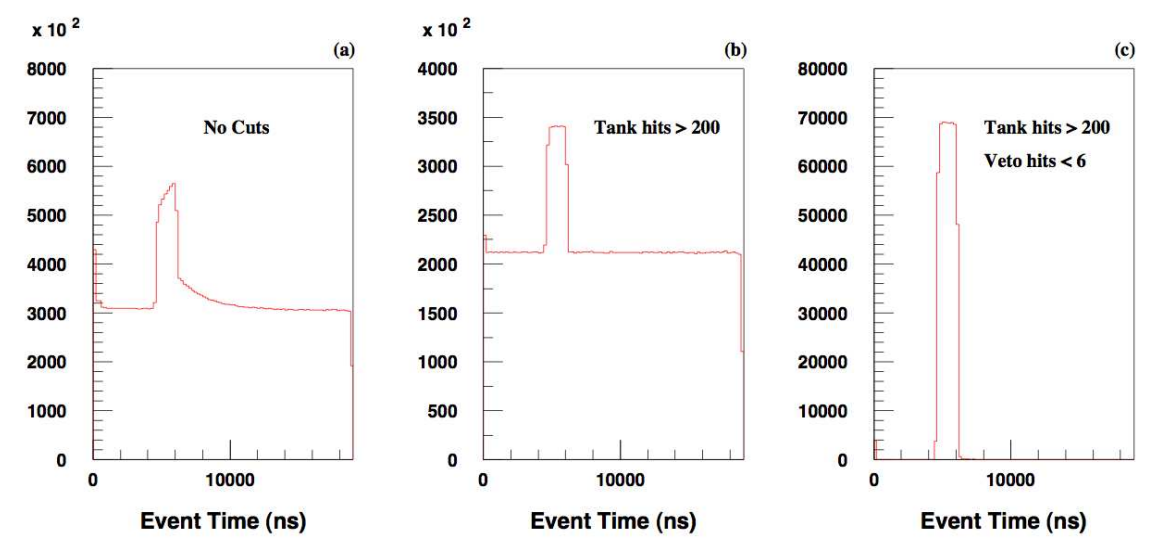

Figure 3-22: Time distribution of subevents within the $19.8 \mu$ s beam trigger window with: no cuts (left), more than 200 tank PMT hits (middle), and more than 200 tank PMT hits and less than 6 veto PMT hits (right). The figure is from [122].

a set of "pre-cuts", designed to look for (sub-)events which occur in coincidence with the beam time window and satisfy minimal veto activity and some requirement for minimum energy deposited in the main tank. This is demonstrated in Fig. 3-22. Starting with all subevents within the $19.8 \mu$ s beam trigger window, shown on the left of Fig. 3-22, the minimum tank hits requirement ensures that low energy events from Michel electrons are removed, with the remaining subevents shown in the middle panel. A sequential maximum veto hits requirement further rejects any remaining, more energetic cosmic ray background, with the remaining sub-event distribution as a function of time shown in the right panel.

With the above set of pre-cuts applied, one can perform simple checks of the stability of the detector and beam by monitoring the number of neutrino interactions detected per POT, as illustrated in Fig. 3-23, looking for possible long term variations that could be expected due to, for example, deterioration of oil purity. From the resulting distributions, a flat line fit can be performed in each running mode, which shows that the event rates in either running mode are consistent within a less than $1 \%$ variation in time. This is also within the assigned POT rate systematic uncertainty, as will be discussed in Sec. 4.6.1. 

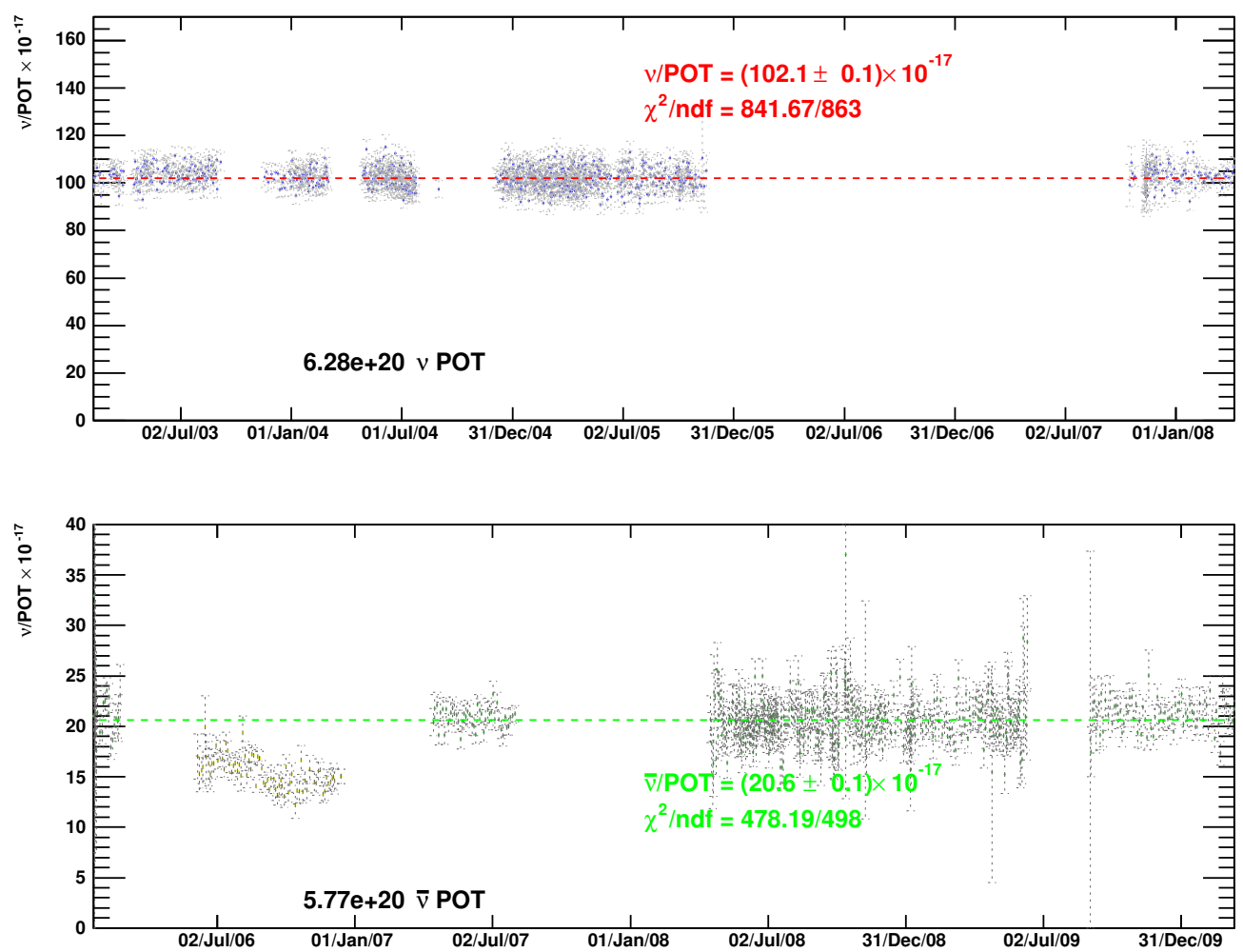

Figure 3-23: Neutrino interactions per POT as a function of time. The top plot corresponds to neutrino running mode. The bottom plot corresponds to antineutrino running mode. The $\chi^{2}$ 's are from a fit to a flat line using only statistical uncertainties. The figure is by A. A. Aguilar-Arevalo.

\subsubsection{Event Reconstruction}

With a clean sample of beam-on events as identified above, the MiniBooNE detector is then able to further identify the charged particle(s) produced in each neutrino interaction by means of the topology of their emitted Cherenkov and/or scintillation light patterns. In obtaining observed event rates for the MiniBooNE $\nu_{e}$ and $\bar{\nu}_{e}$ appearance analyses, the basis of event selection relies in particular on being able to distinguish between a muon and an electron produced in a CCQE interaction, or an electron produced in a CCQE interaction and a single photon produced in a $\mathrm{NC} \pi^{0}$ or $\Delta$ radiative decay interaction.

The MiniBooNE event reconstruction is described in detail in [164, 163]. Event reconstruction relies on a maximum likelihood fitting algorithm used to reconstruct 
the basic properties (position, direction, and energy) of charged particle tracks, given the charges and times measured by the PMT's lining the interior of the MiniBooNE detector. The likelihoods returned from fitting a set of measured charges and times to different event hypotheses are also used to categorize each event as a signal $\nu_{e}$ event or as one of two background-contributing $\nu_{\mu}$ processes: $\nu_{\mu}$ CCQE scattering and NC $\pi^{0}$ production.

We note that since the ring profiles of a $\mu^{-}$and a $\mu^{+}$, and an $e^{-}$and an $e^{+}$do not differ, the detector cannot differentiate (on an event-by-event basis) a $\nu_{\mu}$ from a $\bar{\nu}_{\mu}$ interaction, or a $\nu_{e}$ from a $\bar{\nu}_{e}$ interaction, and therefore the same reconstruction method applies in both neutrino and antineutrino mode searches.

Generally, all events are reconstructed under four hypotheses: a single electron track, a single muon track, two photon tracks, and two photon tracks with an assumed invariant mass $m_{\gamma \gamma}=m_{\pi^{0}}$, used to constrain their reconstructed kinematics. In each hypothesis, a particle track, for example caused by a single electron or muon, is uniquely defined in terms of seven input parameters $\vec{\alpha}=\left(x_{0}, y_{0}, z_{0}, t_{0}, \theta_{0}, \phi_{0}, E_{0}\right)$, as illustrated in Fig. 3-24 (left): the initial track vertex $\left(x_{0}, y_{0}, z_{0}\right)$ with respect to the center of the tank, the initial time $t_{0}$ of the event, and the direction $\left(\theta_{0}, \phi_{0}\right)$ and kinetic energy $E_{0}$ of the particle. Similarly, $\mathrm{NC} \pi^{0}$ candidate events are defined by 14 parameters, as illustrated on the right of Fig. 3-24, including the conversion distance of the two photons produced in $\pi^{0}$ decay, $s_{1}$ and $s_{2}$, where the energy of each photon is measured in terms of the Cherenkov and scintillation light produced in its electromagnetic shower. From those parameters, the energy of each photon, and relative angle between the two photon directions $\left(\theta_{\gamma \gamma}\right)$ can be reconstructed, and used to calculate the invariant mass

$$
m_{\gamma \gamma}^{2}=2 E_{1} E_{2}\left(1-\cos \theta_{\gamma \gamma}\right)
$$

This parameter is constrained to $m_{\gamma \gamma}=m_{\pi^{0}}$ under the fourth hypothesis, by removing 


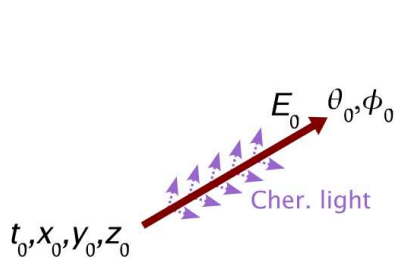

(a) single $\mathrm{e} / \mu$ track

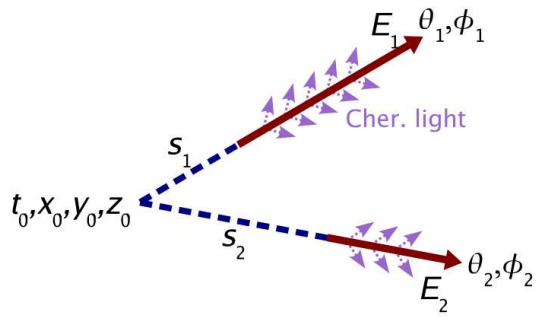

(b) two $y$ tracks

Figure 3-24: Track parameters for (a) a single muon or electron track and (b) two photon tracks. The two track parameters can be constrained such that the invariant mass of the two photons is always $m_{\pi^{0}}$.

$E_{2}$ as a free parameter and setting it to

$$
E_{2}=\frac{m_{\pi^{0}}^{2}}{2 E_{1}\left(1-\cos \theta_{\gamma \gamma}\right)}
$$

The likelihood that a set of reconstructed parameters $\vec{\alpha}=(x, y, z, t, \theta, \phi, E)$ produces an observed set of PMT measurements is expressed as

$$
\mathcal{L}(\vec{\alpha})=\prod_{i=1}^{1280} P\left(q_{i} \mid \vec{\alpha}\right) \cdot P\left(t_{i} \mid \vec{\alpha}\right)
$$

where $P\left(q_{i} \mid \vec{\alpha}\right)$ and $P\left(t_{i} \mid \vec{\alpha}\right)$ correspond to the probability to measure charge $q_{i}$ and at time $t_{i}$ in PMT $i$ for an event with parameters $\vec{\alpha}$, respectively. ${ }^{16}$ Given any set of measured values $q_{i}$ and $t_{i}$, the most likely track parameters are determined by minimizing the negative logarithm of the likelihood function

$$
-\log (\mathcal{L}(\vec{\alpha}))=-\sum_{i=1}^{1280} \log \left(P\left(q_{i} \mid \vec{\alpha}\right)\right)-\sum_{i=1}^{1280} \log \left(P\left(t_{i} \mid \vec{\alpha}\right)\right)
$$

with respect to $\vec{\alpha}$ under each hypothesis: a single electron track (or electron-like ring), a single muon track (or muon-like ring), two photon tracks (or photon-like rings), and two photon tracks with an invariant mass $m_{\gamma \gamma}=m_{\pi^{0}}$.

\footnotetext{
${ }^{16} P\left(q_{i} \mid \vec{\alpha}\right)$ and $P\left(t_{i} \mid \vec{\alpha}\right)$ are dependent on the type and energy of the track, and their values are tabulated in advance to data reconstruction using MonteCarlo simulations for each type of track at various energies of interest.
} 
The maximum likelihoods returned under the first, second, and fourth hypothesis, $\mathcal{L}_{e}, \mathcal{L}_{\mu}$, and $\mathcal{L}_{\pi^{0}}$, can then be used to determine which hypothesis is a most likely description for the event. For example, the quantities

$$
R_{e / \mu} \equiv \log \frac{\mathcal{L}_{e}}{\mathcal{L}_{\mu}}=\log \mathcal{L}_{e}-\log \mathcal{L}_{\mu}
$$

and

$$
R_{e / \pi^{0}} \equiv \log \frac{\mathcal{L}_{e}}{\mathcal{L}_{\pi^{0}}}=\log \mathcal{L}_{e}-\log \mathcal{L}_{\pi^{0}}
$$

are used to determine, for a given event, whether the electron hypothesis is preferred over the muon and $\pi^{0}$ hypotheses, respectively. The convention is such that a more positive number is more electron-like. The reconstructed quantity $m_{\gamma \gamma}$, which corresponds to the parameters which maximize Eq. 3.8 under the third hypothesis, provides an additional handle on differentiation of single-photon events from $\mathrm{NC} \pi^{0}$ interactions with respect to true CCQE events in the appearance search, as will be seen in Sec. 4.2.3.

The reconstructed kinematics of an event, $\vec{\alpha}$, will be determined under the most likely hypothesis.

Assuming CCQE kinematics for all (single-track hypothesis) reconstructed events in the CCQE samples used for the $\nu_{e}$ appearance searches, the neutrino energy can be reconstructed in terms of the scattering angle $\left(\theta_{\ell}\right)$, total energy $\left(E_{\ell}\right)$, and momentum $\left(p_{\ell}\right)$ of the outgoing lepton. Because reconstructing the track of the outgoing nucleon is practically impossible in MiniBooNE, the reconstructed neutrino energy is approximated assuming that the nucleon participating in the interaction is at rest, so that

$$
E_{\nu}^{Q E}\left(\left|\vec{p}_{p}\right|=0\right) \equiv E_{\nu}^{Q E}=\frac{2 m_{n} E_{\ell}+m_{p}^{2}-m_{n}^{2}-m_{\ell}^{2}}{2\left(M_{n}-E_{\ell}+\cos \theta_{\ell} \sqrt{E_{\ell}^{2}-m_{\ell}^{2}}\right)},
$$

in terms of the reconstructed scattering angle $\theta_{\ell}=\vec{p}_{\ell} \cdot \vec{U}_{\nu} /\left|\vec{p}_{\ell}\right|, \vec{U}_{\nu}$ being the direction of the incident neutrino beam. $m_{n, p, \ell}$ are the neutron, proton, and lepton masses. For antineutrino CCQE scattering, the same reconstructed neutrino energy definition is assumed; neutron-proton mass differences are ignored. 


\section{Chapter 4}

\section{The MiniBooNE $\nu_{e}$ and $\bar{\nu}_{e}$ Appearance Searches}

In this chapter, we present the method by which MiniBooNE searches for $\nu_{\mu} \rightarrow \nu_{e}$ and $\bar{\nu}_{\mu} \rightarrow \bar{\nu}_{e}$ appearance in neutrino and antineutrino running mode, respectively. The method followed for the neutrino mode search is that of [101], and the method followed for the antineutrino search is that of [165], with the exception of the $\chi^{2}$ definition used in the oscillation fit method. ${ }^{1}$

Both MiniBooNE appearance analyses are blind, in the sense that the reconstruction method and all selection requirements were defined and finalized prior to looking at $\nu_{e}$ candidate data, using only MonteCarlo generated samples, or using data samples that the MonteCarlo predicted would have minimal overlap with any possible signal prediction.

\footnotetext{
${ }^{1}$ The $\chi^{2}$ definition used throughout this thesis is the same for neutrino and antineutrino mode, corresponding to that of [101], which, in the case of the antineutrino search, presents the only difference with respect to [165]. The fit method will be discussed in Sec. 4.4.
} 


\subsection{Overview}

The MiniBooNE search for an LSND-like signal assumes simple, two-neutrino oscillations, described by the oscillation probability in Eq. 1.28, i.e.,

$$
P\left(\nu_{\mu} \rightarrow \nu_{e}\right)=\sin ^{2} 2 \theta \sin ^{2}\left(1.267 \Delta m^{2} L_{\nu} / E_{\nu}\right),
$$

and using the standard units of $\Delta m^{2}$ in $\mathrm{eV}^{2}, L_{\nu}$ in $\mathrm{m}$, and $E_{\nu}$ in $\mathrm{MeV}$.

In a simple, two-neutrino oscillation scenario, the above oscillation probability does not distinguish among neutrinos and antineutrinos. However, in performing two separate searches, one sensitive to only $\nu_{\mu} \rightarrow \nu_{e}$ oscillations, and one sensitive to $\bar{\nu}_{\mu} \rightarrow \bar{\nu}_{e}$ oscillations, conclusions can be drawn on the possibility that neutrinos and antineutrinos may yield different allowed oscillation probabilities, and the results can then be used in investigations of the possibility of $C P$-violating oscillations [81]. Such investigation will be considered in Chapter 6 .

We note that in neutrino mode, the search is performed by looking for $\nu_{\mu} \rightarrow \nu_{e}$ oscillations, including a small contribution (1.2\%) from $\bar{\nu}_{\mu} \rightarrow \bar{\nu}_{e}$ oscillations due to the wrong-sign $\left(\bar{\nu}_{\mu}\right)$ component of the neutrino beam, as will be seen in Sec. 4.3.3. In antineutrino mode, the search assumes only $\bar{\nu}_{\mu} \rightarrow \bar{\nu}_{e}$ oscillations, and no contribution to the signal prediction from the wrong sign $\left(\nu_{\mu}\right)$ component of the beam oscillating into $\nu_{e}$.

Once again, the reader is reminded that, on an event by event basis, neutrino and antineutrino events are indistinguishable in MiniBooNE. Therefore, aside from the assumption of only $\bar{\nu}_{\mu} \rightarrow \bar{\nu}_{e}$ oscillations in antineutrino mode, the appearance search method described in this chapter applies equivalently to both the $\nu_{e}$ appearance and the $\bar{\nu}_{e}$ appearance search. For the remainder of this chapter, $\nu$ refers to both $\nu$ and $\bar{\nu}$, unless explicitly stated otherwise. For example, when referring to the antineutrino mode appearance search, $\nu_{e}$ CCQE includes all possible $\nu_{e}$ and $\bar{\nu}_{e}$ CCQE events.

The search for a possible signal due to oscillations is performed by comparing, in each running mode, the reconstructed sample of $\nu_{e}$ CCQE observed events $\left(\nu_{e}\right.$ CCQE data) to that predicted by MonteCarlo assuming no oscillations $\left(\nu_{e}\right.$ CCQE background 
prediction). If an excess is found above background prediction, it is tested under various oscillation predictions using a $\chi^{2}$ statistic quantifying the agreement between observed data, $D$, and the background prediction, $B$, plus a possible oscillation signal due to any set of oscillation parameters, $S\left(\Delta m^{2}, \sin ^{2} 2 \theta\right)$.

A signal contribution, $N^{F}$, assuming $100 \%$ oscillation probability, is obtained from the convolution of $\nu_{\mu}$ flux ${ }^{2}$ predicted by the MonteCarlo in each running mode, $\Phi^{\nu_{\mu}}\left(E_{\nu}\right)$, by the $\nu_{e}^{3}$ CCQE cross-section, $\sigma^{\nu_{e}}\left(E_{\nu}\right)$, and $\nu_{e}$ CCQE selection efficiencies, $\epsilon\left(E_{\nu}\right)$,

$$
N^{F}=\sum_{k} F^{k}\left(E_{\nu}\right)=\Phi^{\nu_{\mu}}\left(E_{\nu}\right) \sigma^{\nu_{e}}\left(E_{\nu}\right) \epsilon\left(E_{\nu}\right)
$$

where $N^{F}$ stands for the number of "fully-oscillated" (or "fullosc") events, and $F^{k}$ is the assigned weight of the $k^{\text {th }}$ MonteCarlo-predicted fullosc event. ${ }^{4}$ MonteCarlo truth information available for this sample, specifically $E_{\nu}$ and $L_{\nu}$, corresponding to the true neutrino energy and true neutrino travel distance (in the lab frame, defined as the distance between the neutrino production vertex and the neutrino interaction vertex) for each event in the sample, are used to weigh each fullosc event, $k$, by the oscillation probability of Eq. 4.1,

$$
S\left(\Delta m^{2}, \sin ^{2} 2 \theta\right)=\sum_{k} F^{k} \times \sin ^{2} 2 \theta \sin ^{2}\left(1.267 \Delta m^{2} L_{\nu} / E_{\nu}\right)
$$

The resulting signal prediction is re-evaluated for any set of oscillation parameters in consideration, added to the $\nu_{e}$ CCQE background prediction, and compared to the observed data in terms of $i=1, \ldots, N$ bins of reconstructed neutrino energy, $E_{\nu}^{Q E}$, by way of the $\chi^{2}$ statistic

$$
\chi^{2}\left(\Delta m^{2}, \sin ^{2} 2 \theta\right)=\sum_{i, j=1}^{N}\left(D_{i}-\left(B_{i}+S_{i}\left(\Delta m^{2}, \sin ^{2} 2 \theta\right)\right) \mathcal{M}_{i j}^{-1}\right.
$$

\footnotetext{
${ }^{2} \nu_{\mu}$ and $\bar{\nu}_{\mu}$ flux in neutrino mode, and $\bar{\nu}_{\mu}$ flux in antineutrino mode.

${ }^{3}$ And/or $\bar{\nu}_{e}$, as applicable.

${ }^{4}$ By definition, $F^{k} \equiv 1$ if the MonteCarlo is generated with a number of events corresponding to data POT. MonteCarlo, however, is generated with MonteCarlo POT $\gg$ data POT.
} 


$$
\left(D_{j}-\left(B_{j}+S_{j}\left(\Delta m^{2}, \sin ^{2} 2 \theta\right)\right) .\right.
$$

In the above expression, $\mathcal{M}_{i j}$ is an $N \times N$ covariance matrix which includes, on each of its diagonal elements, $\mathcal{M}_{i i}$, systematic and statistical uncertainties (squared) on the sum of signal and background prediction in each corresponding bin $i$, as well as bin-to-bin systematic correlations, in its off-diagonal elements. The set of oscillation parameters which minimizes the $\chi^{2}$ statistic will correspond to the best fit $\Delta m^{2}$ and $\sin ^{2} 2 \theta$ oscillation parameters.

Of course, following the above approach from a fit to $\nu_{e}$ CCQE data and background prediction alone from first principles would result in a relatively weak sensitivity to oscillations, since many of the $\nu_{e}$ backgrounds as well as any oscillation signal prediction are subject to large flux and cross-section systematic uncertainties (on the order of $\sim 20 \%$ ). Instead, MiniBooNE employs a simultaneous fit of $\nu_{e}$ CCQE and $\nu_{\mu} \mathrm{CCQE}$ distributions to search for oscillations. In this way, the information gained by comparing the observed high statistics $\nu_{\mu} \mathrm{CCQE}$ event sample to that predicted by MonteCarlo can be used to constrain the uncertainties and absolute rate of the $\nu_{e}$ CCQE prediction (both signal and background).

Specifically, the $\nu_{\mu}$ CCQE sample is fit side-by-side with the $\nu_{e}$ CCQE sample, using a covariance matrix which is extended to include all systematic and statistical uncertainties of both samples, and, as we will see, powerful systematic correlations between the two samples. This technique works effectively as a ratio fit, often used in particle physics analyses to cancel systematic uncertainties. ${ }^{5}$ Exploiting the correlations between the $\nu_{e}$ and $\nu_{\mu}$ CCQE event rates is what allows for a (partial) cancellation of flux and cross-section systematic uncertainties. The $\nu_{\mu}-\nu_{e}$ combined fit method is described in more detail in Sec. 4.4.

In the following discussion, we describe how the central value predictions for the $\nu_{e}$ and $\nu_{\mu}$ CCQE samples used in the MiniBooNE $\nu_{e}$ and $\bar{\nu}_{e}$ appearance analyses are obtained. We present the central value predictions in Sec. 4.3.

\footnotetext{
${ }^{5}$ For example, disappearance experiments $[68,56]$ employ a far-to-near ratio fit which leads to cancellation of flux, cross-section, and, if identical detectors are used, detector systematic uncertainties.
} 


\subsection{CCQE Event Selection}

As described in Sec. 3.5.3, after reconstruction, each event can be classified either as $\nu_{e}$ CCQE-like or $\nu_{\mu}$ CCQE-like and described by its corresponding reconstructed kinematic parameters. For the purpose of the appearance analysis, three MonteCarlo predicted event samples are constructed: a $\nu_{\mu}$ CCQE sample, a background $\nu_{e}$ CCQE sample, and a fullosc $\nu_{e}$ CCQE sample. Those are then compared to two observed event samples reconstructed in parallel: $\nu_{\mu}$ CCQE and $\nu_{e}$ CCQE. ${ }^{6}$

The event selection cuts in the MiniBooNE oscillation analysis, described in the following subsections, are applied on an event-by-event basis.

\subsubsection{Preliminary Event Selection}

As described in Sec. 3.5.2, the first step in event selection is the rejection of beamunrelated backgrounds. These are backgrounds originating mostly from cosmic ray events with products that reach the MiniBooNE detector, which can mimic neutrino interaction signatures at MiniBooNE's neutrino beam energies. Unlike beam events, beam-unrelated events occur both within, and outside the beam timing window, and therefore a beam timing cut provides powerful rejection.

Two additional cuts are used, to identify events with sufficient energy deposition in the main tank rather than the veto region, characteristic of beam related neutrino events, or events that are contained within the tank, as described in Sec. 3.5.2. The two cuts require that the first subevent corresponds to more than 200 tank PMT hits, and less than 6 veto PMT hits.

The above three pre-cuts yield a $>99.99 \%$ rejection of beam unrelated backgrounds. An additional cut regarding the number of subevents is then applied to split the remaining events into two main categories, a two-subevent sample, characteristic of $\nu_{\mu}$ CCQE interactions, and a one-subevent sample, characteristic of $\nu_{e}$ CCQE interactions and respective backgrounds (referring to Fig. 3-21.)

\footnotetext{
${ }^{6}$ Auxiliary samples are also considered, and used to constrain backgrounds to the $\nu_{e}$ appearance analysis. Those are discussed in Sec. 4.5.
} 
After pre-cuts, additional kinematic selection cuts are used to further increase the purity of each sample. The following subsections describe each respective set of cuts.

\subsection{2 $\nu_{\mu}$ CCQE Event Selection Requirements}

All events in the $\nu_{\mu}$ CCQE sample are identified by the two-subevent structure, with the first subevent subject to the pre-cuts in Sec. 4.2.1. For the second subevent (Michel electron), a maximum of 200 tank and a maximum of six veto PMT hits are required. A minimum time cut of 1000 ns between the first and second subevents is also placed to ensure PMT stability for proper charge response.

Subsequent selection cuts make use of reconstructed variables obtained under either the muon or the electron hypothesis. The first subevent vertex reconstructed under the muon hypothesis, $\vec{R}_{1, \mu}$, defined with respect to the center of the track, is required to occur within the fiducial volume defined by $\left|\vec{R}_{1, \mu}\right|<500 \mathrm{~cm}$. The fiducial volume requirement ensures proper simulation, and reduces uncertainties due to PMT angular efficiency variations, low-wavelength light (which has short extinction length), or reflections, for example. The same requirement is applied to the track end-point, $\left|\vec{R}_{1, \mu}^{e n d}\right|$, defined under the muon hypothesis as

$$
\left|\vec{R}_{1, \mu}^{e n d}\right|=\left|\vec{R}_{1, \mu}+2 \Delta_{\text {mid }}\left(E_{\mu}\right) \vec{U}_{1, \mu}\right|
$$

where $\Delta_{\text {mid }}\left(E_{\mu}\right)$ is a muon energy-dependent range function that gives the mean distance from the vertex to the track midpoint [163], and $\vec{U}_{1, \mu}$ is the reconstructed three-direction of the muon track.

The neutrino energy reconstructed from the outgoing muon energy and angle, $E_{\nu}^{Q E}$, is required to satisfy $E_{\nu}^{Q E}>150 \mathrm{MeV}$.

A cut on the separation distance between the muon and decay electron vertices, as a function of the reconstructed muon energy, is also applied to provide rejection against backgrounds, mostly from CC $\pi^{ \pm}$interactions [163]. This cut exploits the almost flat $\frac{d E}{d x}$ of muons in the MiniBooNE energy range to reject background events which typically have more energy than is expected given the reconstructed muon 


\begin{tabular}{ccc}
\hline \hline Sample & $a_{0}(\mathbf{c m})$ & $a_{1}(\mathbf{c m} / \mathbf{M e V})$ \\
\hline \hline Data & -32.0 & 0.532 \\
MonteCarlo & -34.8 & 0.539 \\
\hline \hline
\end{tabular}

Table 4.1: The particle identification (PID) cut coefficients $a_{0}$ and $a_{1}$ used in data and MonteCarlo $\nu_{\mu}$ CCQE event selection. The numbers are extracted empirically from separate fits to data and MonteCarlo, as described in Ref. [163].

range. The cut is defined as a function of the reconstructed muon energy, $E_{1, \mu}$, and the longitudinal separation distance

$$
\Delta_{\|}=\left(\vec{R}_{2, e}-\vec{R}_{1, \mu}\right) \cdot \vec{U}_{1, \mu}
$$

where $\vec{R}_{2, e}$ is the electron track vertex reconstructed under the electron hypothesis. In terms of the above two reconstructed parameters, events are required to satisfy

$$
\left|a_{0}+a_{1} E_{1, \mu}-\Delta_{\|}\right|<50 \mathrm{~cm}
$$

where $a_{0}$ and $a_{1}$ are two coefficients determined by fits to $\nu_{\mu}$ CCQE prediction and data separately according to [163]. ${ }^{7}$ The resulting coefficients are summarized in Tab. 4.1.

A summary of all $\nu_{\mu}$ CCQE selection cuts is provided in Tab. 4.2.

\subsection{3 $\nu_{e}$ CCQE Event Selection Requirements}

All $\nu_{e}$ CCQE events are identified by the one-subevent structure, and the pre-cuts described in Sec. 4.2.1. Following the same reasoning as for $\nu_{\mu}$ CCQE events, only events with a reconstructed vertex (under the electron track hypothesis) at a radius less than $500 \mathrm{~cm}$ are considered. ${ }^{8}$

\footnotetext{
${ }^{7}$ In an ideal world, the resulting coefficients extracted separately from data and MonteCarlo should be identical; however, uncertainties in detector optical properties and nuclear final state interactions lead to the MonteCarlo prediction being a different representation from real data. The resulting numbers from fits to MonteCarlo variants with detector optical properties and nuclear final state interactions yield comparable results. (See [163] for a more detailed description.)

${ }^{8}$ This defines the fiducial volume in the MiniBooNE appearance analysis, corresponding to $\sim 450$ tons.
} 


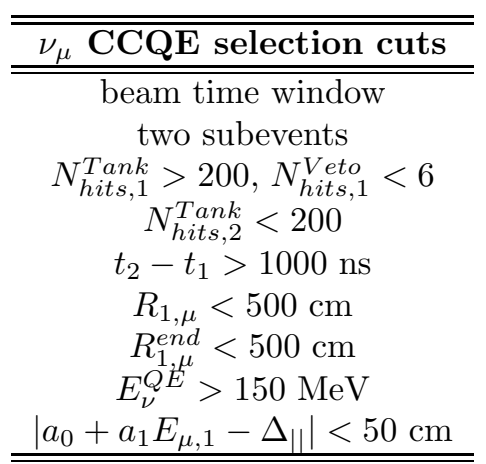

Table 4.2: Summary of $\nu_{\mu}$ CCQE selection cuts. See text for parameter definitions and coefficient values.

Each event is also required to have a track endpoint under the muon hypothesis that is less than $488 \mathrm{~cm}$. This minimizes the number of muon neutrinos mis-identified as electron neutrinos, which would result from muons decaying too close to the fiducial volume boundary.

A minimum visible energy cut, $E_{v i s}=E_{e}-m_{e}>140 \mathrm{MeV}$, is required to reject $\mathrm{NC}$ events, which deposit less visible energy than their corresponding CC interactions due to the (unobserved) escaping neutrino.

An additional kinematics-based cut is used to reject mis-identified events due to interactions that take place outside the detector, or close to the fiducial volume boundary. Events with a vertex outside the fiducial volume boundary are referred to as "dirt" events, and the ones contributing most to the $\nu_{e}$ CCQE background are usually single photons from $\mathrm{NC} \pi^{0}$ interactions, as illustrated in Fig. 4-1 (left), that penetrate the veto unobserved and photoconvert within the fiducial volume. Those events have characteristically low visible energy, and they are usually reconstructed with a vertex at high radius and a track that points toward the center of the detector. Therefore, a large majority of them is rejected by an $E_{v i s}$-dependent cut of a geometrical parameter $R_{\text {back-to-wall }}$, defined in terms of the reconstructed three-vertex $\vec{R}_{e}$ and track direction $\vec{U}_{e}$ under the electron hypothesis, as

$$
R_{\text {back-to-wall }}=\vec{R}_{e} \cdot \vec{U}_{e}+\left(\left(\vec{R}_{e} \cdot \vec{U}_{e}\right)^{2}-\left|\vec{R}_{e}\right|^{2}+R_{0}^{2}\right)^{1 / 2}
$$

The above quantity corresponds to the reconstructed length that a photon would 

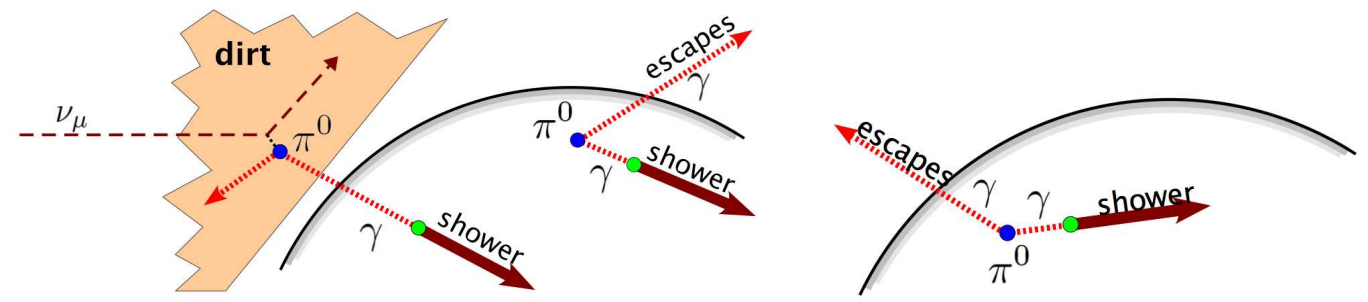

Figure 4-1: External interactions contributing as background to the $\nu_{e}$ appearance signal. The left panel illustrates how a neutrino interaction in the dirt leads to a single photon converting in the analysis fiducial volume, while the right panel illustrates how a $\pi^{0}$ decaying near the wall of the tank can lead to one of the photons escaping the fiducial volume undetected, before converting into an electromagnetic shower.

have to traverse as it enters the active detector before producing an observed track, defined with respect to the detector wall, at radius $R_{0}$. The cut is defined in terms of $E_{v i s}$ and $R_{b a c k-t o-w a l l}$ to reject events with

$$
R_{\text {back-to-wall }}<a_{0 b}-a_{1 b} E_{\text {vis }}, \text { and } R_{\text {forward-to-wall }}>a_{0 f}
$$

where $a_{0 b}=347.3 \mathrm{~cm}, a_{1 b}=0.595 \mathrm{~cm} / \mathrm{MeV}$, and $a_{0 f}=100 \mathrm{~cm}$, and $R_{\text {forward-to-wall }}$ is defined in an analogous fashion to reject events occurring close to the boundary with products escaping the fiducial volume, as in Fig. 4-1 right.

As discussed in Sec. 3.5.1, a large contribution of mis-identified backgrounds comes from $\nu_{\mu}$-induced NC $\pi^{0}$ events. Those are rejected by requiring that the reconstructed $\pi^{0}$ mass obtained under the two-photon-track hypothesis, $m_{\gamma \gamma}$, is safely smaller than the true $\pi^{0}$ mass, as illustrated in the top left and right panels of Fig. 4-2. This requirement is applied using a quadratic function in terms of reconstructed $E_{e}$,

$$
0<m_{\gamma \gamma}^{2}<a_{0}+a_{1} E_{e}+a_{2} E_{e}^{2}
$$

where $a_{0}, a_{1}$, and $a_{2}$ are given in Tab. 4.3.

Two more particle identification cuts are applied, as illustrated in the middle and bottom panels Fig. 4-2, which further enhance the rejection power against misidentified NC $\pi^{0}$ and also reject other $\nu_{\mu}$-induced backgrounds. Those cuts are based 


\begin{tabular}{l|lll}
\hline \hline PID variable & Coefficients & & \\
\hline \hline$m_{\gamma \gamma}^{2}$ & $a_{0}: 32.033 \mathrm{MeV}^{2}$ & $a_{1}: 7.41657 \times 10^{-3} \mathrm{MeV}$ & $a_{2}: 2.73787 \times 10^{-5}$ \\
$\log \left(\mathcal{L}_{e} / \mathcal{L}_{\mu}\right)$ & $b_{0}: 1.335 \times 10^{-2}$ & $b_{1}: 3.467 \times 10^{-2} \mathrm{GeV}^{-1}$ & $b_{2}:-8.259 \times 10^{-3} \mathrm{GeV}^{-2}$ \\
$\log \left(\mathcal{L}_{e} / \mathcal{L}_{\pi}\right)$ & $d_{0}: 2.471 \times 10^{-3}$ & $d_{1}: 4.115 \times 10^{-3} \mathrm{GeV}^{-1}$ & $d_{2}:-2.738 \times 10^{-2} \mathrm{GeV}^{-2}$ \\
\hline \hline
\end{tabular}

Table 4.3: PID cut coefficients used in $\nu_{e}$ CCQE event selection. The $c_{i}$ and $d_{i}$ cut values have been optimized to maximize sensitivity to $\nu_{\mu} \rightarrow \nu_{e}$ oscillations at $\Delta m^{2} \sim$ $1 \mathrm{eV}^{2}[163]$.

on the maximum likelihoods returned by the event reconstruction algorithm, defined in Eqs. 3.9 and 3.10. The first cut is used to reject mostly $\nu_{\mu}$ CCQE events that satisfy the one-subevent requirement. Even though the majority of $\nu_{\mu}$ CCQE interactions can be identified through their two-subevent structure, approximately $10 \%$ of the time the outgoing $\mu$ captures on carbon, or happens to decay too quickly so that the $\mu$ and $e$ subevents overlap. In those cases, the Cherenkov ring profile of each event can be used to identify the neutrino flavor and provide further differentiation. Events that satisfy

$$
\log \left(\mathcal{L}_{e} / \mathcal{L}_{\mu}\right)>c_{0}+c_{1} E_{e}+c_{2} E_{e}^{2},
$$

where $c_{0}, c_{1}$, and $c_{2}$ are given in Tab. 4.3, are accepted as $\nu_{e}$ CCQE events. The second cut is used to reject mostly remaining $\mathrm{NC} \pi^{0}$ events that satisfy the single-track hypothesis, and exploits the difference between the light pattern from a single electron track to that of two overlapping, ${ }^{9}$ or back-to-back photon tracks where one of the photons has too low energy to produce a reconstructible ring. A similar requirement,

$$
\log \left(\mathcal{L}_{e} / \mathcal{L}_{\pi^{0}}\right)>d_{0}+d_{1} E_{e}+d_{2} E_{e}^{2},
$$

where $d_{0}, d_{1}$, and $d_{2}$ are given in Tab. 4.3 , is applied to increase the $\nu_{e}$ CCQE purity.

A summary of all $\nu_{e}$ CCQE selection cuts is provided in Tab. 4.4.

The $\nu_{e}$ CCQE selection efficiency (after pre-cuts) obtained on MonteCarlo predicted samples is shown for both signal and background $\nu_{e}$ CCQE events in Tab. 4.5.

\footnotetext{
${ }^{9} \mathrm{~A}$ typical $\pi^{0}$ momentum in MiniBooNE is $0.3 \mathrm{GeV}$, which results in somewhat boosted $\gamma \gamma$ final state in the lab frame.
} 

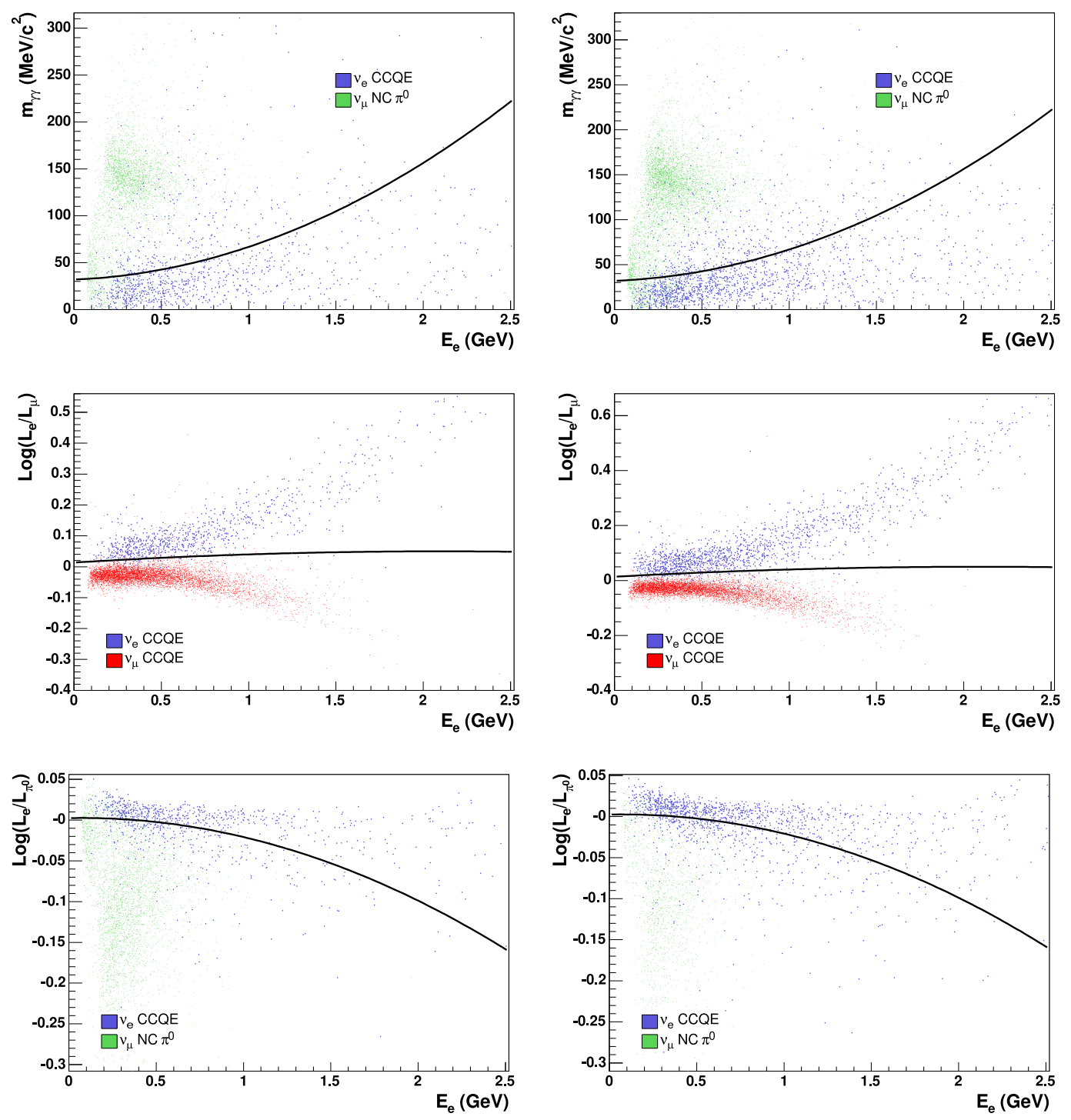

Figure 4-2: Illustrating the effect of the three $\nu_{e}$ CCQE PID cuts used in the MiniBooNE $\nu_{e}$ appearance analysis: $m_{\gamma \gamma}, \mathcal{L}_{e} / \mathcal{L}_{\mu}$, and $\mathcal{L}_{e} / \mathcal{L}_{\pi^{0}}$. Left: neutrino mode. Right: antineutrino mode. 


\begin{tabular}{c}
\hline \hline$\nu_{e}$ CCQE selection cuts \\
\hline \hline beam time window \\
one subevent \\
$N_{\text {hits }}^{\text {Tank }}>200, N_{\text {hits }}^{\text {Veto }}<6$ \\
$R_{e}<500 \mathrm{~cm}$ \\
$R_{\mu}^{\text {end }}<488 \mathrm{~cm}$ \\
$E_{\text {vis }}>140 \mathrm{MeV}$ \\
$0<m_{\gamma \gamma}<a_{0}+a_{1} E_{e}+a_{2} E_{e}^{2}$. \\
$R_{\text {back-to-wall }}^{e}<a_{0 b}-a_{1 b} E_{e}$ \\
$R_{\text {forward-to-wall }}^{e}>a_{0 f}$ \\
$\log \left(\mathcal{L}_{e} / \mathcal{L}_{\mu}\right)>c_{0}+c_{1} E_{e}+c_{2} E_{e}^{2}$ \\
$\log \left(\mathcal{L}_{e} / \mathcal{L}_{\pi^{0}}\right)>d_{0}+d_{1} E_{e}+d_{2} E_{e}^{2}$ \\
\hline \hline
\end{tabular}

Table 4.4: Summary of $\nu_{e}$ CCQE selection cuts. See text for parameter definitions and coefficient values.

\begin{tabular}{l|rr}
\hline \hline Analysis & Background efficiency & Signal efficiency \\
\hline \hline Antineutrino & $1.2 \%$ & $22.9 \%$ \\
Neutrino & $0.82 \%$ & $20.1 \%$ \\
\hline \hline
\end{tabular}

Table 4.5: $\nu_{e}$ CCQE selection efficiency in each running mode, relative to events after pre-cuts, calculated using MonteCarlo predicted samples.

\subsection{Central Values}

After $\nu_{e}$ and $\nu_{\mu}$ CCQE selection requirements, the following central values are obtained:

- $\nu_{e}$ CCQE data

- $\nu_{e}$ CCQE predicted background

- $\nu_{e}$ CCQE fullosc

- $\nu_{\mu}$ CCQE data

- $\nu_{\mu}$ CCQE prediction (background)

each as a function of $E_{\nu}^{Q E}$, in each running mode. Below we present all but the first one, which we save for Chapter 5 .

The neutrino mode central values correspond to $6.46 \times 10^{20}$ POT, and correspond to those in [101], and the antineutrino mode central values correspond to $5.66 \times 10^{20} \mathrm{POT}$, from $[165]$. 


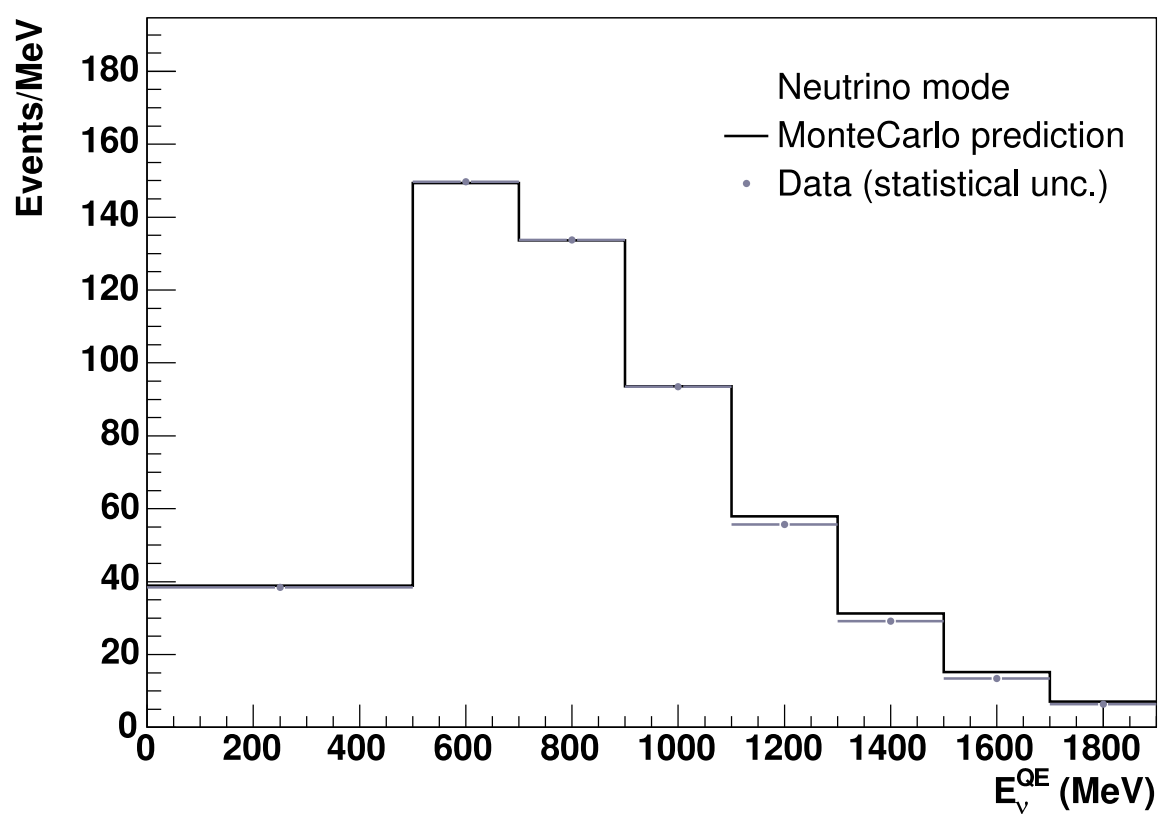

Figure 4-3: The MiniBooNE $\nu_{\mu}$ CCQE central values (data and MonteCarlo) in the MiniBooNE $\nu_{e}$ appearance fit (neutrino mode). The error bars correspond to data statistical uncertainties.

\subsection{1 $\nu_{\mu}$ CCQE Data and Predicted Samples: Neutrino Mode}

The $\nu_{\mu}$ CCQE observed and predicted samples are included in the $\nu_{e}$ appearance fit as a function of eight (8) variable-width bins of reconstructed neutrino energy, $E_{\nu}^{Q E}$, ranging from 0 to $1900 \mathrm{MeV}$, as illustrated in Fig. 4-3.

The data distribution in Fig. 4-3 corresponds to a sample of 117,099 data events satisfying $\nu_{\mu}$ CCQE selection requirements. The data sample is compared to the $\nu_{\mu}$ CCQE MonteCarlo prediction which has been corrected to match the observed $\nu_{\mu}$ CCQE data through a normalization factor of 1.28 , applied to events from $\pi^{+}$ decays in the beam. This normalization correction is covered by flux and crosssection uncertainties, and is accounted for in the neutrino mode oscillation fit by a reduction in the quoted effective degrees of freedom by one unit.

After correction, the $\nu_{\mu}$ CCQE prediction corresponds to 117,024 events, with $74.7 \%$ purity in true CCQE events. CC $\pi^{+}$interactions are the dominant source of 
background (19.0\%). The sample composition is $97.8 \%$ from $\nu_{\mu}$ produced in pion decays and $1.4 \%$ from $\nu_{\mu}$ produced in kaon decays. The wrong-sign content of the reconstructed event sample, included in the numbers quoted above, is $1.2 \%$. The composition of the predicted $\nu_{\mu}$ CCQE sample is given in Fig. 4-4, in terms of neutrino parent, neutrino type ( $\nu$ versus $\bar{\nu})$, and type of neutrino interaction, respectively.

\subsection{2 $\nu_{\mu}$ CCQE Data and Predicted Samples: Antineutrino Mode}

In antineutrino mode, a sample of 24,711 data events satisfy $\nu_{\mu}$ CCQE selection requirements. This sample is compared to a MonteCarlo prediction which has been corrected to match the observed $\nu_{\mu}$ CCQE data through a normalization factor of 1.20 applied to events from $\pi^{-}$decays in the beam, and 0.99 applied to events from $\pi^{+}$decays in the beam. These normalization factors are extracted from a data to MonteCarlo fit to the angular distributions of the outgoing $\mu^{+}$and $\mu^{-}[166]$, using MonteCarlo truth information for a sample of reconstructed $\nu_{\mu}$ CCQE events which has significant overlap with the $\nu_{\mu}$ CCQE sample presented here. The above two factors result in an overall $+13 \%$ normalization correction, which is covered by flux and cross-section uncertainties.

After correction, the sample contains $94.8 \% \bar{\nu}_{\mu}$ and $\nu_{\mu}$ produced in pion decays, and $2.3 \% \bar{\nu}_{\mu}$ and $\nu_{\mu}$ produced in kaon decays. The wrong-sign (neutrino) content of the sample, included in the numbers quoted above, is $22.7 \%$. The majority of events $(71.3 \%)$ are true CCQE interactions, with $\mathrm{CC} \pi^{ \pm}$interactions being the dominant source of background (20.6\%). The composition of the predicted sample is shown in Fig. 4-5 as a function of reconstructed muon neutrino energy, in terms of neutrino parent, neutrino type $(\nu$ versus $\bar{\nu})$, and type of neutrino interaction.

The $\nu_{\mu}$ CCQE observed and predicted samples are included in the $\bar{\nu}_{e}$ appearance fit as a function of the same eight (8) variable-width bins of $E_{\nu}^{Q E}$ as in neutrino mode, ranging from 0 to $1900 \mathrm{MeV}$, as shown in Fig. 4-6. The reduction of a factor of $\sim 5$ on the overall event rate relative to neutrino mode (see Fig. 4-3) for similar POT is 

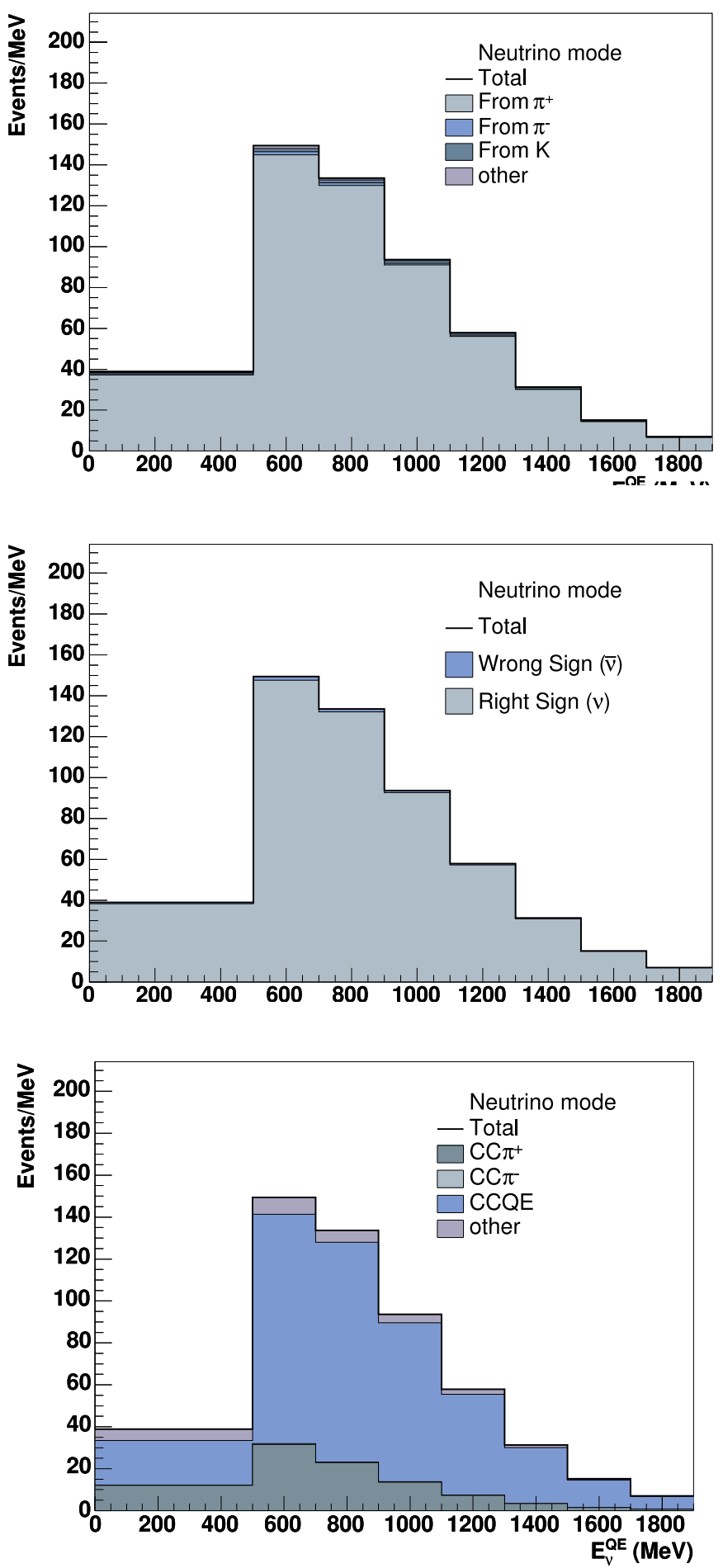

Figure 4-4: Neutrino mode distributions for the MiniBooNE $\nu_{\mu}$ CCQE central value prediction, after $N_{\pi}$ correction. Events are broken down by neutrino parent (top), neutrino type (middle), and type of neutrino interaction (bottom). 

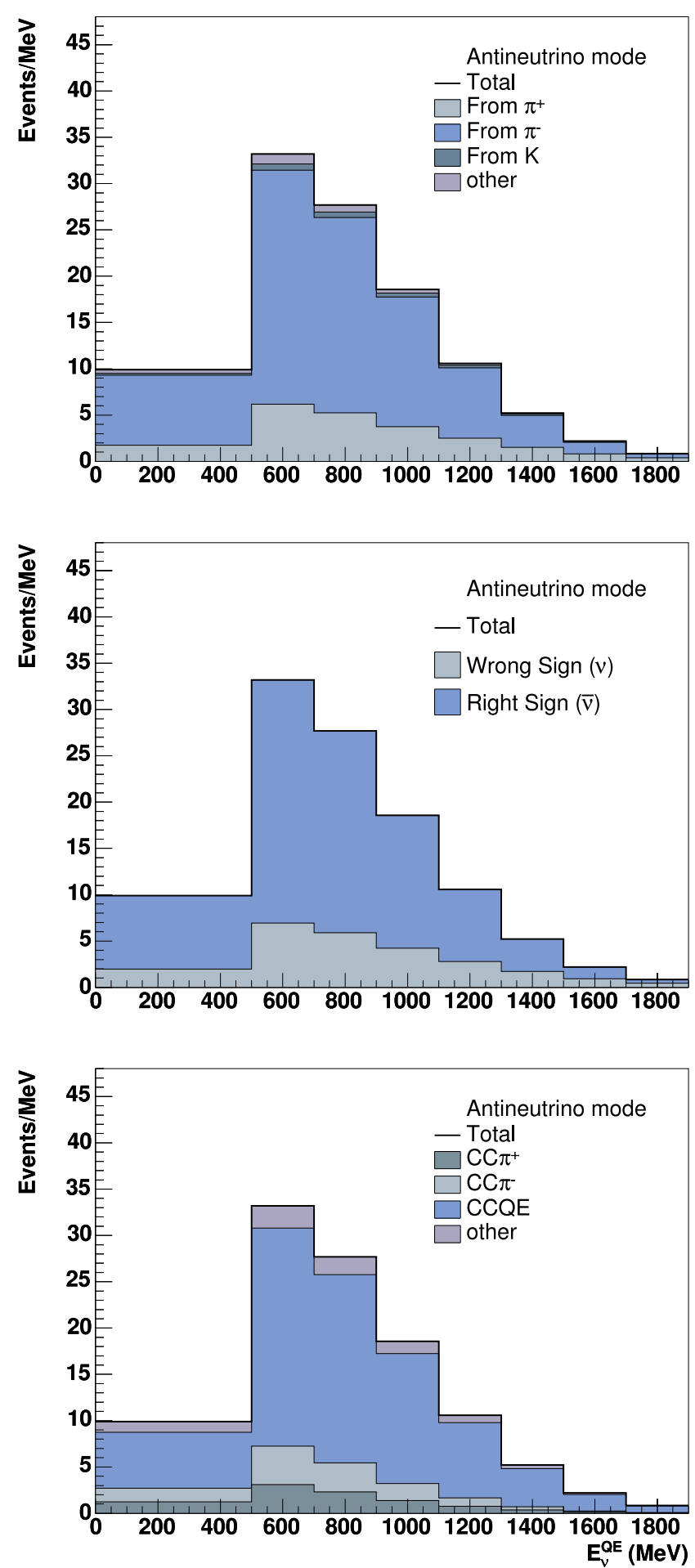

Figure 4-5: Antineutrino mode distributions for the MiniBooNE $\nu_{\mu}$ CCQE central value prediction, after $N_{\pi}$ correction. Events are broken down by neutrino parent (top), neutrino type (middle), and type of neutrino interaction (bottom). 


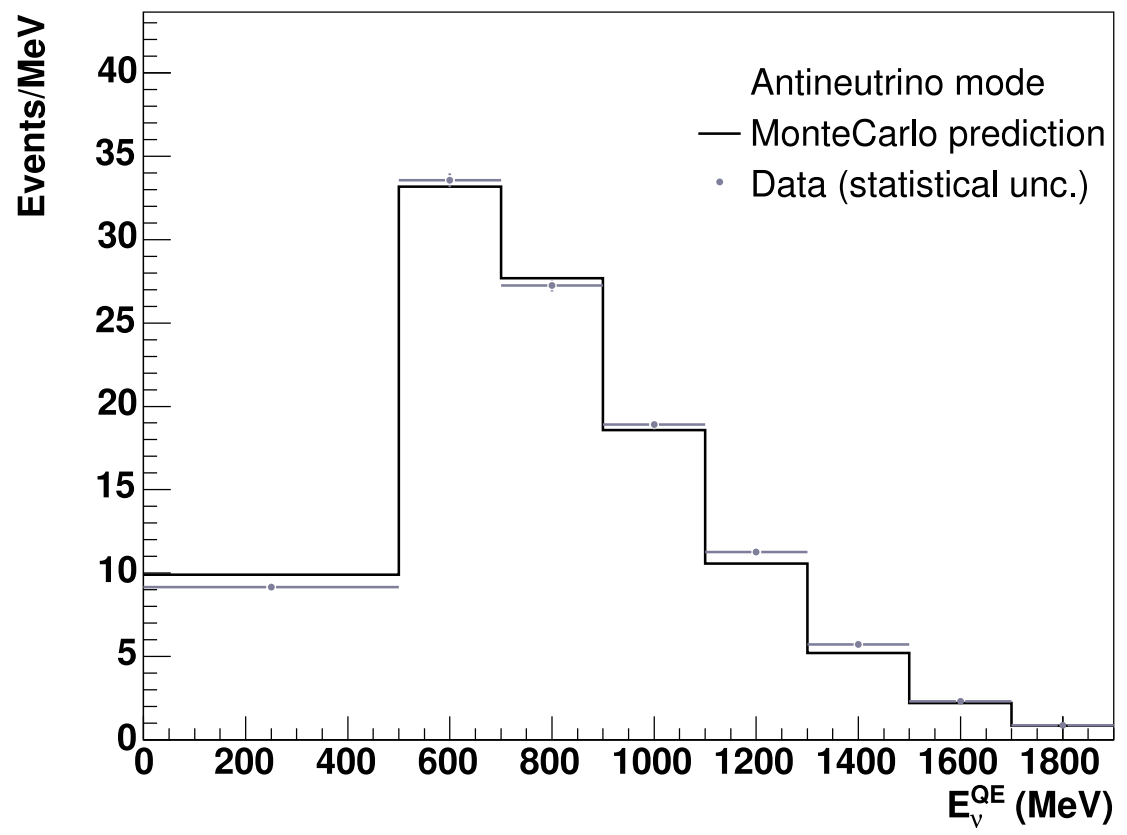

Figure 4-6: The MiniBooNE $\nu_{\mu}$ CCQE central values (data and MonteCarlo) in the MiniBooNE $\bar{\nu}_{e}$ appearance fit (antineutrino mode). The error bars correspond to data statistical uncertainties.

due to convoluted meson production and focusing at the proton target and neutrino vs. antineutrino interaction cross-section differences, mentioned in Sec. 3.4.1.

\subsection{3 $\nu_{e}$ CCQE Predicted Samples: Neutrino Mode}

In neutrino mode, the final $\nu_{e}$ CCQE background prediction corresponds to 921.5 events in the $200<E_{\nu}^{Q E}<3000 \mathrm{MeV}$ range. The backgrounds are contributed at higher energy mostly by beam intrinsic $\nu_{e}$ from $K^{+}$and $K^{0}$ decays, which interact in the detector through either the CCQE or the $\mathrm{CC} \pi^{ \pm}$channel. At low reconstructed neutrino energy (200-475 MeV) the dominant background contribution comes from mis-identified $\nu_{\mu}$ events interacting though $\mathrm{NC}$ channels, and correspond to mostly $\mathrm{NC} \pi^{0}, \Delta$, and dirt events, as discussed in Sec. 4.2.3. A detailed breakup of events for different ranges in $E_{\nu}^{Q E}$ is given in Tab. 4.6.

The sample is included in the $\nu_{e}$ appearance fit to oscillations as a function of 


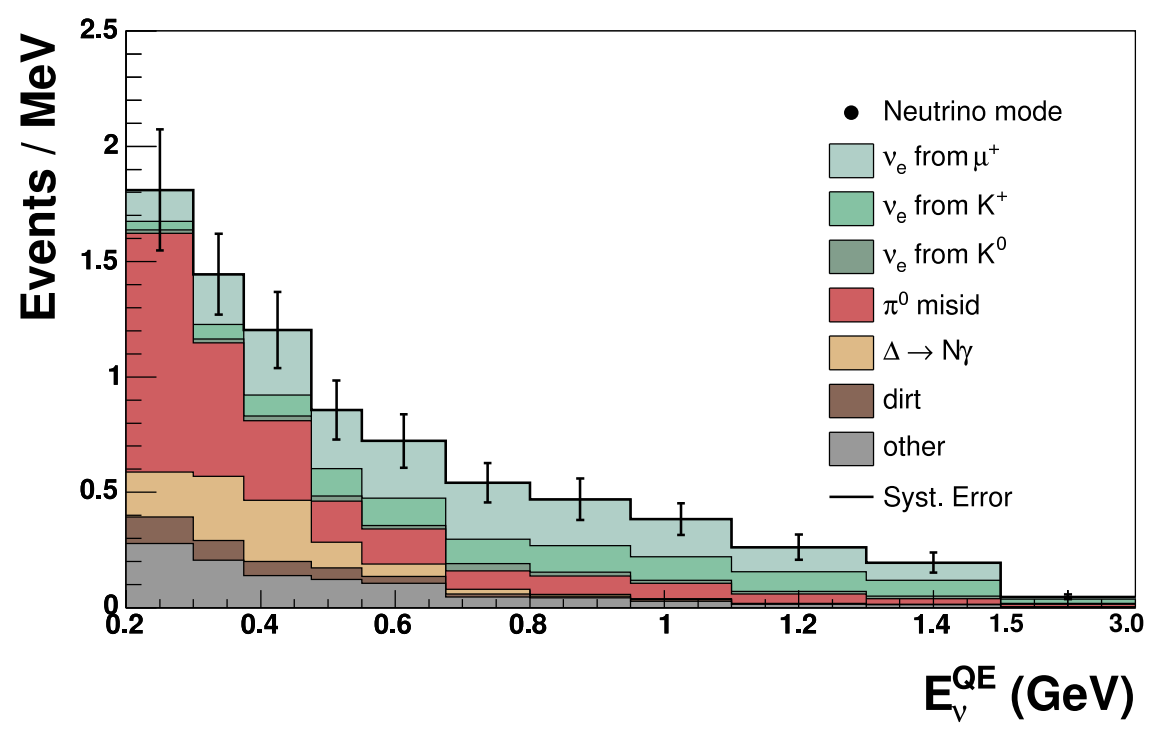

Figure 4-7: The $\nu_{e}$ CCQE background predictions in the MiniBooNE $\nu_{e}$ appearance fit, with systematic-only uncertainties (before $\nu_{\mu} \mathrm{CCQE}$ constraint). For fits to $E>$ $475 \mathrm{MeV}$, the lowest three bins $(200-475 \mathrm{MeV})$ are removed from the fit. The various background contributions have been corrected as will be discussed in Sec. 4.5.

eleven (11) or eight (8) bins of $E_{\nu}^{Q E}$, ranging from 200 to $3000 \mathrm{MeV}$ or 475 to 3000 $\mathrm{MeV}$, respectively, as shown in Fig. 4-7. In the 475-3000 MeV range, a total of 511.7 $\nu_{e}$ CCQE background events are expected.

The expected signal prediction in neutrino mode, assuming a flat $26 \%$ oscillation probability suggested by the LSND result, is shown in Fig. 4-8 as a function of the same $E_{\nu}^{Q E}$ binning as the $\nu_{e}$ background prediction. This sample is obtained simply by scaling the predicted fullosc events by the above flat oscillation probability. As in the case of the $\nu_{\mu}$ CCQE sample, a small wrong-sign contribution of $1.2 \%$ is included in the total signal prediction. The wrong-sign fullosc is allowed to oscillate and is treated exactly like the right-sign fullosc in the neutrino mode fit. If $\nu_{\mu} \rightarrow \nu_{e}$ oscillations at the LSND level indeed occur in nature, MiniBooNE would expect to observe a total of 271.0 events above background prediction (511.73 events) in the $475<E_{\nu}^{Q E}<3000$ $\mathrm{MeV}$ energy range. 


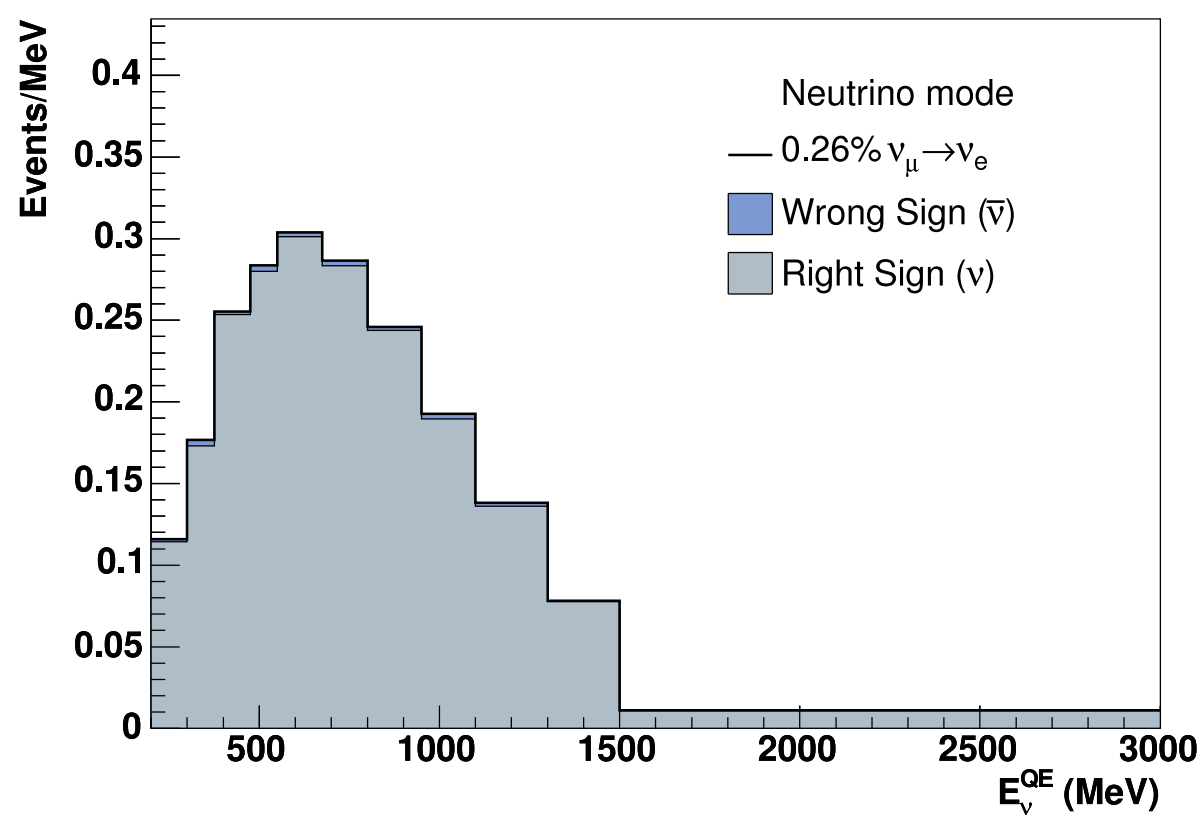

Figure 4-8: The $\nu_{e}$ CCQE signal prediction in the MiniBooNE $\nu_{e}$ appearance fit, assuming all $\nu_{\mu}$ and $\bar{\nu}_{\mu}$ in the beam oscillate with a flat $0.26 \%$ probability. The signal prediction has been corrected as will be discussed in Sec. 4.5.

\subsection{4 $\nu_{e}$ CCQE Predicted Samples: Antineutrino Mode}

Table 4.7 shows the number of predicted $\nu_{e}$ CCQE background events for different ranges of $E_{\nu}^{Q E}$ in antineutrino mode. A total of 231.7 background events are expected over the full 200-3000 MeV range. Again, as in the case of the neutrino mode appearance search, most intrinsic $\nu_{e}$ backgrounds pile up at higher energy, while mis-identified $\mathrm{NC} \pi^{0}, \Delta$, and dirt events are reconstructed at low energy, between 200-475 MeV. The sample is included in the $\bar{\nu}_{e}$ appearance fit to oscillations as a function of eight (8) or eleven (11) bins of $E_{\nu}^{Q E}$, ranging from 475 to $3000 \mathrm{MeV}$ or 200 to $3000 \mathrm{MeV}$, respectively, as shown in Fig. 4-9. In the 475-3000 MeV range, a total of $132.3 \nu_{e}$ CCQE background events are expected.

The antineutrino background distribution shown in Fig. 4-9 has a similar breakup to that in neutrino mode, shown in Fig. 4-7, except that at higher energy the relative contribution of intrinsic $\nu_{e}$ from $K^{0}$ decays in antineutrino mode is larger. This is due 


\begin{tabular}{l|ccc}
\hline \hline Process & 200-300 MeV & 300-475 MeV & 475-1250 MeV \\
\hline \hline$\nu_{e}$ from $K^{ \pm}$ & 4.15 & 14.11 & 82.06 \\
$\nu_{e}$ from $K^{0}$ & 2.24 & 4.06 & 21.85 \\
$\nu_{e}$ from $\pi^{+} \rightarrow \mu^{+}$decays & 13.60 & 44.67 & 153.90 \\
$\nu_{e}$ from $\pi^{-} \rightarrow \mu^{-}$decays & 0.00 & 0.64 & 2.19 \\
Other $\nu_{e}$ & 0.63 & 0.16 & 2.04 \\
\hline$\nu_{\mu}$ CCQE & 8.24 & 17.07 & 11.54 \\
External events & 11.54 & 12.33 & 11.45 \\
NC $\Delta \rightarrow N \gamma$ & 19.55 & 47.20 & 19.44 \\
NC $\pi^{0}$ & 103.07 & 77.80 & 70.30 \\
Other $\nu_{\mu}$ & 18.05 & 10.74 & 16.57 \\
\hline Total & 181.06 & 228.78 & 391.36 \\
\hline LSND best fit & 6.19 & 36.29 & 139.58 \\
$P\left(\nu_{\mu} \rightarrow \nu_{e}\right)=0.26 \%$ & 11.60 & 38.79 & 182.77 \\
\hline \hline
\end{tabular}

Table 4.6: The expected number of events for different $E_{\nu}^{Q E}$ ranges from all of the backgrounds in the $\nu_{e}$ appearance analysis and for the LSND central expectation of $\nu_{\mu} \rightarrow \nu_{e}$ oscillations. Neutrino mode, $6.46 \times 10^{20}$ POT. The events correspond to both neutrino and antineutrino contributions, the latter being $38.8 \%$ of the total background in the $200-3000 \mathrm{MeV}$ range.

to a relative suppression of horn focusing power for negatively charged vs. positively charged mesons, with respect to neutral mesons, in neutrino vs. antineutrino mode. Neutral mesons are not affected by the horn focusing.

Because the antineutrino mode appearance search is designed as a direct test of LSND antineutrino oscillations, only signal contributed by the wrong-sign component of the flux is used to account for any possible oscillations observed in the data. The antineutrino signal prediction as a function of $E_{\nu}^{Q E}$, assuming a flat, $0.26 \%$ oscillation probability, is shown in Fig. 4-10. If $\bar{\nu}_{\mu} \rightarrow \bar{\nu}_{e}$ oscillations at the LSND level indeed occur in nature, MiniBooNE would expect to observe a total of 33.1 events above background prediction (126.0 events) in the $475<E_{\nu}^{Q E}<3000 \mathrm{MeV}$ energy range.

\subsection{Oscillation Fit Method}

\subsection{1 $\chi^{2}$ Definition}

The MiniBooNE fit to oscillations is performed using the reconstructed $\nu_{e}$ and $\nu_{\mu}$ CCQE samples as a function of $E_{\nu}^{Q E}$ and the following $\chi^{2}$ statistic, quantifying data 


\begin{tabular}{l|ccc}
\hline \hline Process & 200-300 MeV & 300-475 MeV & 475-1250 MeV \\
\hline \hline$\nu_{e}$ from $K^{ \pm}$ & 2.06 & 6.09 & 18.61 \\
$\nu_{e}$ from $K^{0}$ & 1.32 & 3.81 & 21.20 \\
$\nu_{e}$ from $\pi^{+} \rightarrow \mu^{+}$decays & 0.55 & 1.54 & 5.02 \\
$\nu_{e}$ from $\pi^{-} \rightarrow \mu^{-}$decays & 3.41 & 7.95 & 26.37 \\
Other $\nu_{e}$ & 0.51 & 0.75 & 2.05 \\
\hline$\nu_{\mu}$ & 1.74 & 2.56 & 2.04 \\
External events & 2.87 & 3.29 & 2.63 \\
NC $\Delta \rightarrow N \gamma$ & 4.17 & 8.22 & 3.37 \\
NC $\pi^{0}$ & 24.11 & 17.47 & 12.57 \\
Other $\nu_{\mu}$ CCQE & 3.65 & 3.38 & 4.22 \\
\hline Total & 44.38 & 55.05 & 98.08 \\
\hline LSND best fit & 1.14 & 6.23 & 21.39 \\
$P\left(\bar{\nu}_{\mu} \rightarrow \bar{\nu}_{e}\right)=0.26 \%$ & 2.36 & 6.72 & 29.13 \\
\hline \hline
\end{tabular}

Table 4.7: The expected number of events for different $E_{\nu}^{Q E}$ ranges from all of the backgrounds in the $\bar{\nu}_{e}$ appearance analysis and for the LSND central expectation ( $0.26 \%$ oscillation probability) of $\bar{\nu}_{\mu} \rightarrow \bar{\nu}_{e}$ oscillations. Antineutrino mode, $5.66 \times 10^{20}$ POT. The events correspond to both antineutrino and neutrino contributions, the latter being $42.8 \%$ of the total background in the $200-3000 \mathrm{MeV}$ range.

to Monte Carlo agreement:

$$
\chi^{2}=\sum_{i, j=1}^{N_{e}+N_{\mu}}\left(D_{i}-P_{i}\right) \mathcal{M}_{i j}^{-1}\left(D_{j}-P_{j}\right),
$$

where:

- $N_{e}$ is the number of $E_{\nu}^{Q E}$ bins for observed $\nu_{e}$ CCQE events.

- $N_{\mu}$ is the number of $E_{\nu}^{Q E}$ bins for observed $\nu_{\mu}$ CCQE events.

- $D_{i}=\left(D_{j}^{\nu_{e}}\left(j=1, \ldots, N_{e}\right), D_{j}^{\nu_{\mu}}\left(j=1, \ldots, N_{\mu}\right)\right)$ is a histogram composed of two side-by-side histograms of 1) observed $\nu_{e}$ CCQE events, $D_{j}^{\nu_{e}}$, and 2) observed $\nu_{\mu}$ CCQE events, $D_{j}^{\nu_{\mu}}$, as functions of $N_{e}$ and $N_{\mu} E_{\nu}^{Q E}$ bins, respectively.

- $P_{i}=\left(\left(B_{j}^{\nu_{e}}+S_{j}\right)\left(j=1, \ldots, N_{e}\right), B_{j}^{\nu_{\mu}}\left(j=1, \ldots, N_{\mu}\right)\right)$ is a histogram composed of two side-by-side histograms of 1) predicted $\nu_{e}$ CCQE background events, $B_{j}^{\nu_{e}}$, plus predicted $\nu_{e}$ CCQE signal events, $S_{j}$, and 2) predicted $\nu_{\mu}$ CCQE events, $B_{j}^{\nu_{\mu}}$. 


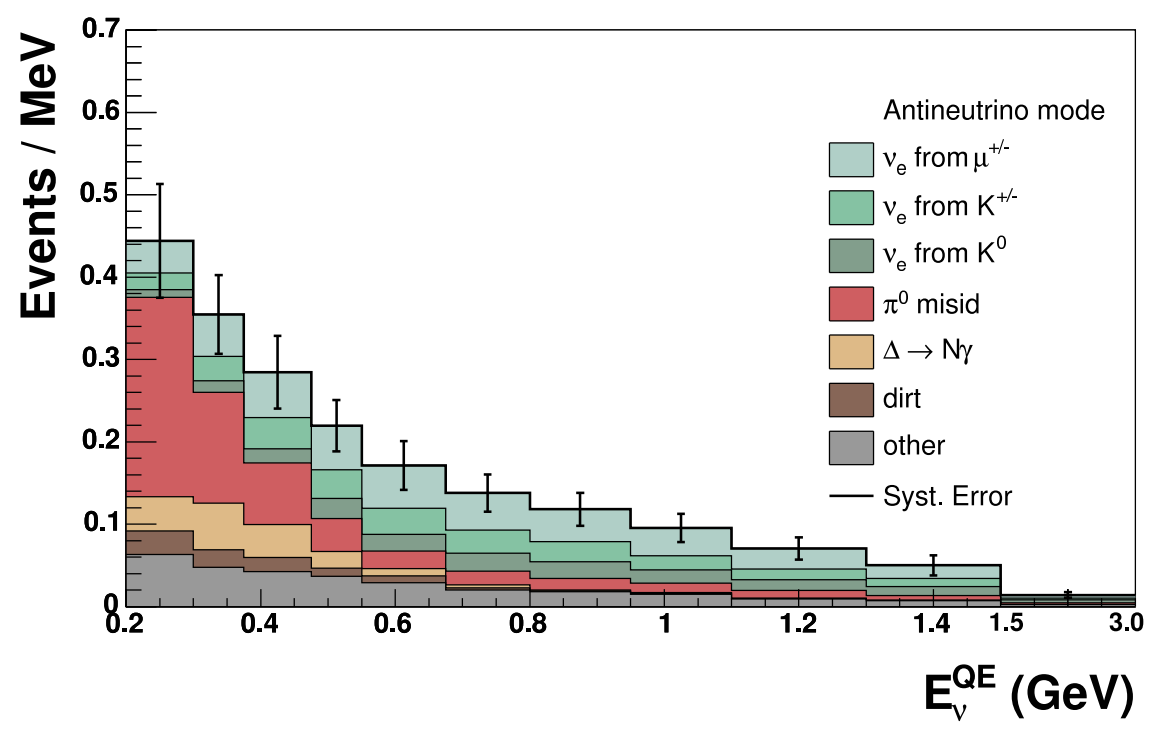

Figure 4-9: The $\nu_{e}$ CCQE background predictions in the MiniBooNE $\bar{\nu}_{e}$ appearance fit. For fits to $E>475 \mathrm{MeV}$, the lowest three bins (200-475 MeV) are removed from the fit. The various background contributions have been corrected as will be discussed in Sec. 4.5. The dashed black line corresponds to the LSND best-fit signal expectation.

- $\mathcal{M}_{i j}^{-1}$ is the inverse of the total $\left(N_{e}+N_{\mu}\right) \times\left(N_{e}+N_{\mu}\right)$ covariance matrix $\mathcal{M}_{i j}$, which includes all systematic and statistical uncertainties for $P_{i}$, and bin-to-bin systematic correlations.

The number of signal events, $S_{i}$, predicted per $E_{\nu}^{Q E}$ bin $i=1, \ldots, N_{e}$ is obtained from the $\nu_{e}$ CCQE fullosc sample as described in Sec. 4.1, except, rather than calculating $S_{j}$ on an event by event basis, an energy-averaged event distribution is obtained for each $\Delta m^{2}$, which is then simply modulated by $\sin ^{2} 2 \theta$ during the fit. That approach is followed in order to reduce computing power requirements in the fits. 


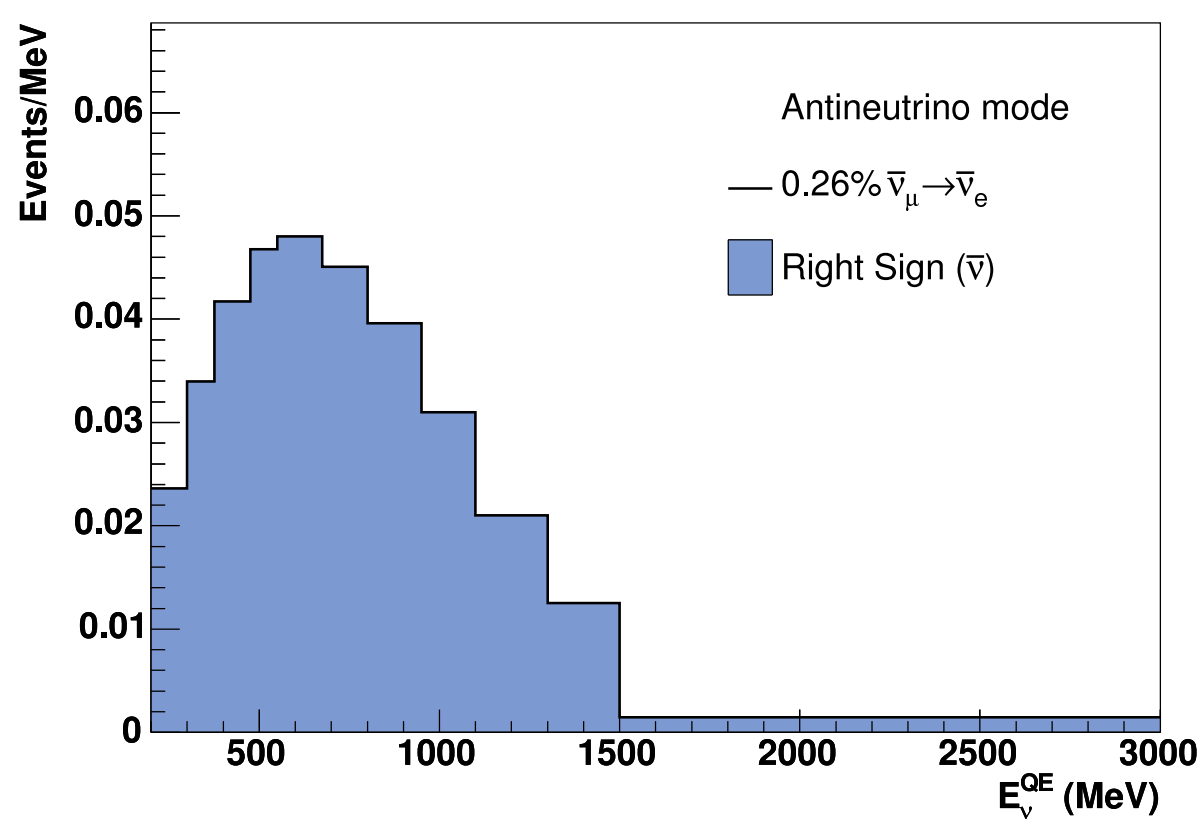

Figure 4-10: The $\nu_{e}$ CCQE signal prediction in the MiniBooNE $\bar{\nu}_{e}$ appearance fit, assuming all $\bar{\nu}_{\mu}$ in the beam oscillate with a flat $0.26 \%$ probability. The signal prediction has been corrected as will be discussed in Sec. 4.5.

\subsubsection{The $\nu_{\mu}$ CCQE Constraint in the $\nu_{e}$ and $\bar{\nu}_{e}$ Appearance Fits}

The $\nu_{\mu}$ CCQE sample is included in the fit to oscillations in order to constrain the overall $\nu_{e}$ CCQE prediction, by taking advantage of strong flux and cross-section correlations among the $\nu_{e} \mathrm{CCQE}$ and $\nu_{\mu} \mathrm{CCQE}$ event samples. To see this, recall that the observed event rates, $D^{\nu_{e}}$ and $D^{\nu_{\mu}}$, are products of flux, cross-section, and detector efficiencies, i.e.,

$$
\begin{aligned}
D^{\nu_{e}}(E) & =\Phi_{\nu_{e}}(E) \sigma_{\nu_{e}}(E) \epsilon_{\nu_{e}}(E)+\Phi_{\nu_{\mu}}(E) P\left(\nu_{\mu} \rightarrow \nu_{e}\right) \sigma_{\nu_{e}}(E) \epsilon_{\nu_{e}}(E), \text { and } \\
D^{\nu_{\mu}}(E) & =\Phi_{\nu_{\mu}}(E) \sigma_{\nu_{\mu}}(E) \epsilon_{\nu_{\mu}}(E),
\end{aligned}
$$

in which case the correlations and their respective effects on the fit are evident from the following considerations: 
1. The majority of any possible $\nu_{\mu} \rightarrow \nu_{e}$ signal, as well as some $\nu_{e}$ backgrounds, share the same cross-section (CCQE) as the majority of the $\nu_{\mu}$ CCQE reconstructed sample. Therefore, any data to MonteCarlo disagreement coming from poor understanding of the cross-section is expected to induce similar effects in both distributions, effectively canceling the associated uncertainties in a simultaneous fit to the two distributions.

2. The majority of events in both the $\nu_{e}$ signal prediction and the $\nu_{e}$ background prediction are related to $\nu_{\mu}$ CCQE events through the same $\pi^{+}$or $\pi^{-}$decay chain at production. Specifically, the same $\pi^{+} \rightarrow \mu^{+} \nu_{\mu}$ decay which produces a $\nu_{\mu}$ in neutrino mode could, alternatively, directly produce a $\nu_{e}$ through $\pi^{+} \rightarrow e+\nu_{e}$ decay, or indirectly produce a $\nu_{e}$ through the subsequent $\mu^{+} \rightarrow e^{+} \nu_{e}$ decay. Therefore, uncertainties in $\pi^{+}$production are expected to produce similar effects in the two samples. Of course, the ancestor overlap must be significant in order to maximize the effect of the correction. In neutrino mode, $>90 \%$ of $\nu_{\mu}$ CCQE and $\nu_{e}$ CCQE signal samples come from $\pi^{+}$decay, while $\nu_{e}$ from the $\pi^{+} \rightarrow \mu^{+}$decay chain make up $36.9 \%$ of the total $\nu_{e}$ CCQE background events between $475-3000 \mathrm{MeV}$; therefore, the correlation is significant. This overlap is demonstrated in Fig. 4-11. Analogous correlations also exist in events from $\pi^{-} \rightarrow \mu^{-}$decays, which are important in antineutrino mode.
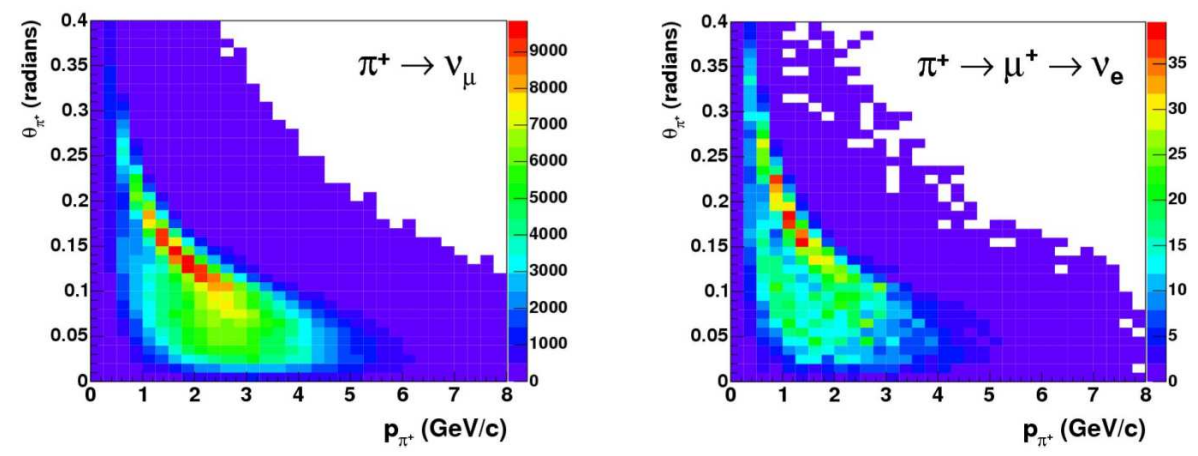

Figure 4-11: Ancestor kinematics phase-space overlap between $\nu_{e}$ and $\nu_{\mu}$ flux at the MiniBooNE detector. The figure is for neutrino mode [50]. 


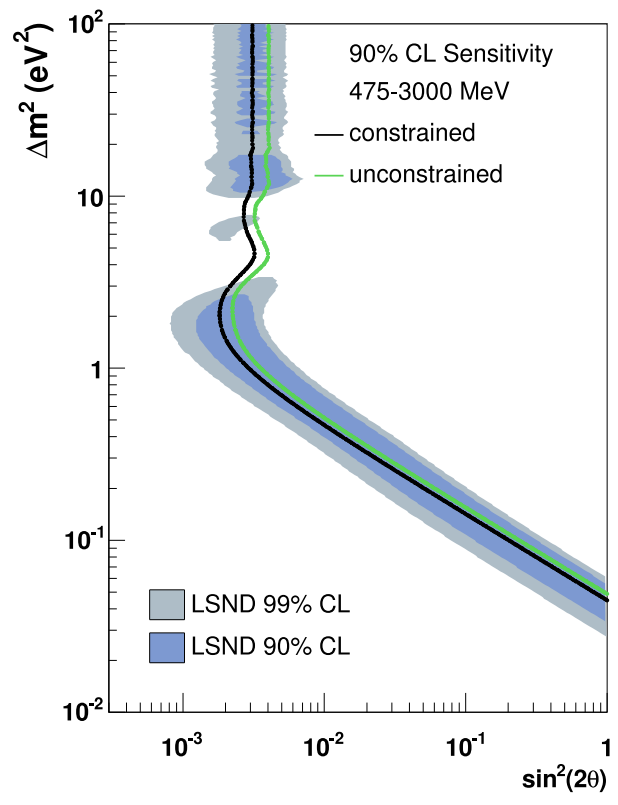

Figure 4-12: Effect of $\nu_{\mu}$ CCQE constraint on MiniBooNE's sensitivity to $\bar{\nu}_{\mu} \rightarrow \bar{\nu}_{e}$ oscillations. Antineutrino mode sensitivity, for $E_{\nu}^{Q E}>475 \mathrm{MeV}$. A similar but larger effect is seen in neutrino mode.

All information on the correlations enters the fit through the off-diagonal elements of a covariance matrix used in the $\chi^{2}$ calculation, relating the contents of the bins of the $\nu_{e} \mathrm{CCQE}$ and $\nu_{\mu} \mathrm{CCQE}$ distributions. This procedure maximizes the sensitivity to $\nu_{\mu} \rightarrow \nu_{e}$ oscillations when systematic uncertainties are included, as demonstrated in Fig. 4-12.

A more instructive description of how the constraint works is given in App. A.2.

\subsubsection{Scaling the Covariance Matrix}

When testing a particular oscillation hypothesis $\left(\Delta m^{2}, \sin ^{2} 2 \theta\right)$, one must properly account for the scaling of uncertainties and correlations between the $\nu_{e}$ CCQE signal prediction, $\nu_{e}$ CCQE background prediction, and $\nu_{\mu}$ CCQE prediction. In order to do so, a fractional systematics-only covariance matrix is input in the fit as a $3 \times 3$ block error matrix, which has the form ( $\nu_{e}$ fullosc, $\nu_{e}$ background, $\nu_{\mu}$ CCQE), as illustrated in Fig. 4-13 (left). This matrix is scaled, bin-by-bin, to three side-by-side 


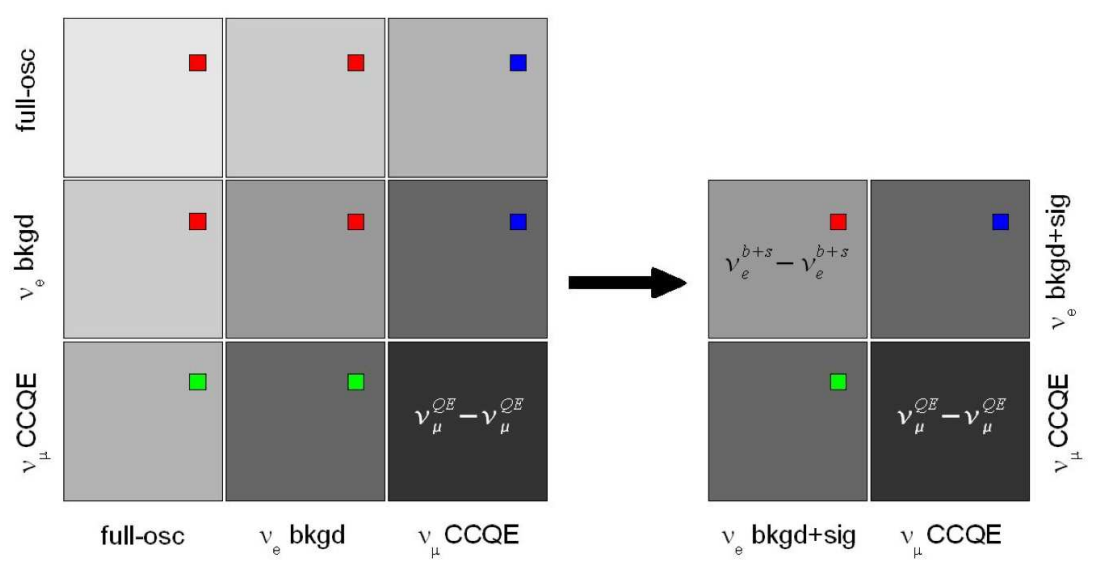

Figure 4-13: Schematic of the $3 \times 3$-block covariance matrix input to the MiniBooNE appearance fits (left), and compacted $2 \times 2$-block covariance matrix used in the $\chi^{2}$ definition. The matrix is collapsed by overlaying same-colored blocks in the figure. The fullosc parts of the matrix are scaled according to each set of oscillation parameters before the collapse, which properly propagates the signal systematic error onto the total $\nu_{e}$ CCQE prediction.

distributions, corresponding to the predicted $\nu_{e}$ signal (fullosc events weighted by the oscillation probability at that particular hypothesis), $\nu_{e}$ background, and $\nu_{\mu}$ CCQE central values,

$$
\mathcal{M}_{i j}^{3 \times 3, s y s}=\mathcal{M}_{i j}^{3 \times 3, f r a c} \cdot\left(P_{i} \cdot P_{j}\right)
$$

where $i, j=1, \ldots, N_{e}+N_{e}+N_{\mu}$.

A statistical error contribution corresponding to the signal prediction, $S_{k}(k=$ $\left.1, \ldots, N_{e}\right)$, and the predicted $\nu_{e}$ CCQE background and $\nu_{\mu}$ CCQE samples, $B_{k}^{\nu_{e}}(k=$ $\left.1, \ldots, N_{e}\right)$ and $B_{k}^{\nu_{\mu}}\left(k=1, \ldots, N_{\mu}\right)$, is added to the diagonal elements of $M_{j j}^{3 \times 3, s y s}$ for $j=1, \ldots, N_{e}+N_{e}+N_{\mu}$, in order to form the total $3 \times 3$ systematic plus statistical covariance matrix,

$$
\mathcal{M}_{i j}^{3 \times 3}=\mathcal{M}_{i j}^{3 \times 3, s y s}+\delta_{i j} \cdot P_{i} ; \quad i, j=1, \ldots, N_{e}+N_{e}+N_{\mu}
$$

Then, $\mathcal{M}_{i j}^{3 \times 3}$ is compacted to a $2 \times 2$-block error matrix, $\mathcal{M}_{i j}$, of the form $\left(\nu_{e}\right.$ signal $+\nu_{e}$ background, $\nu_{\mu} \mathrm{CCQE}$ ). This is done by superimposing the $N_{e} \times N_{e}$-dimensional blocks of the covariance matrix, bin-by-bin, as illustrated in Fig. 4-13. The resulting compacted $2 \times 2$ error matrix is what is used in Eq. 4.13 . 


\subsubsection{Finding the Best-fit Oscillation Parameters}

The best-fit point is found by performing an iterative $\chi^{2}$ fit across a grid of $\left(\Delta m^{2}, \sin ^{2} 2 \theta\right)$ parameters. Over the first iteration, the error matrix is kept fixed at the no oscillations prediction (null point on the grid, defined as $\Delta m^{2}=0$ and $\sin ^{2} 2 \theta=0$ ). Over each successive iteration, the error matrix is updated and kept fixed to reflect uncertainties at the best-fit point found in the previous iteration. The fit iterates until the $\chi^{2}$ convergence criterion

$$
\left|\chi_{\min , i}^{2}-\chi_{\min , i-1}^{2}\right| \leq 0.2
$$

is met, or for up to five iterations, in extreme cases. This iterative fit procedure avoids artificial minimization of the $\chi^{2}$ which can result by uncontrollably increasing the signal prediction (which in turn would increase $\mathcal{M}_{i j}$ ), and at the same time allows one to account for additional systematic and statistical uncertainties in the fit due to a non-zero signal prediction.

\subsubsection{On the Drawing of Confidence Level Intervals}

Allowed regions about the best fit point, $b f=\left(\Delta m_{b f}^{2}, \sin ^{2} 2 \theta_{b f}\right)$, are obtained using the $\Delta \chi^{2}$ definition

$$
\Delta \chi^{2}=\chi_{P}^{2}-\chi_{b f}^{2}
$$

mapped out over the full $P=\left(\Delta m^{2}, \sin ^{2} 2 \theta\right)$ parameter space surface, where the full systematics plus covariance matrix involved in the calculation of both $\chi_{P}^{2}$ and $\chi_{b f}^{2}$ corresponds to that of the best fit point.

The resulting $\Delta \chi^{2}$ surface is used to determine allowed regions about the best fit by drawing constant slices of $\Delta \chi^{2}$ across the grid points, defining those which are allowed at $\alpha$ C.L. as those which satisfy $\Delta \chi^{2} \leq \Delta \chi_{\alpha}^{2}, \Delta \chi_{\alpha}^{2}$ being the textbook $\chi^{2}$ cut values assuming two (2) degrees of freedom (d.o.f.). In reality, assuming two degrees of freedom across the full $\left(\Delta m^{2}, \sin ^{2} 2 \theta\right)$ parameter space is merely an approximation, as systematic correlations may exist between the fit parameters in certain regions of the parameter space. A more realistic approach in determining 
confidence level intervals is instead based on frequentist studies [167], where, through a series of "fake experiments" one may empirically determine the effective number of degrees of freedom at each point on the grid. In that case, $\Delta \chi_{\alpha}^{2}$ is $\left(\Delta m^{2}, \sin ^{2} 2 \theta\right)$ dependent.

In those studies, a large number $\mathcal{N}$ of fake data distributions is drawn from the full systematic plus statistical covariance matrix at each point on the grid, each representing potentially observed distributions at MiniBooNE assuming the particular point on the grid, $P_{T}$, represents a true oscillation (or no oscillation) scenario. Each draw corresponds to a "fake experiment". The full oscillation fit is performed for each draw $i=1, \ldots, \mathcal{N}$, resulting in a $\Delta \chi_{i}^{2}=\left(\chi_{P_{T}}^{2}-\chi_{b f}^{2}\right)_{i}$ distribution at each point, $P_{T}$, which, in an ideal world, is expected to correspond to two degrees of freedom, but in practice corresponds to $n$ effective degrees of freedom. One, then, may use $n$ to evaluate $\Delta \chi_{\alpha}^{2}$ at each point on the grid, and use that to determine whether such point is allowed within a confidence level $\alpha$ when performing a fit to real data. This is done by comparing $\Delta \chi_{\text {real }}^{2}$ to $\Delta \chi_{\alpha}^{2}$. Equivalently, mapping the $\Delta \chi^{2}$ distributions at each point on the grid allows for determining the $\Delta \chi_{\alpha}^{2}$ cut such that $\alpha$ of the experiments at each point have $\Delta \chi^{2} \leq \Delta \chi_{\alpha}^{2}$. This is illustrated in Fig. 4-14. We follow the latter definition.

Frequentist studies were performed in neutrino mode where it was confirmed that the $\Delta \chi_{i}^{2}$ distributions over the parameter regions where the MiniBooNE exclusion limit was drawn could be sufficiently approximated as distributions corresponding to two degrees of freedom [168]. Therefore, in the neutrino appearance search, the various confidence level regions are mapped using the standard, 2 d.o.f. $\Delta \chi_{\alpha}^{2}$ cuts summarized in Tab. 4.8. Similarly, when drawing exclusion limits, a 1-sided raster scan is used, where a constant $\Delta \chi^{2}$ cut is placed for each slice in $\Delta m^{2}$ with respect to the best fit $\sin ^{2} 2 \theta$ in each slice, assuming 1 degree of freedom. The resulting limit of confidence level $\alpha$ indicates the range of $\sin ^{2} 2 \theta$ values allowed at that confidence level for a given assumption of true value of $\Delta m^{2}$.

Results from fake data studies in antineutrino mode, however, warranted revision of the method by which the allowed regions and/or exclusion limits are drawn. 

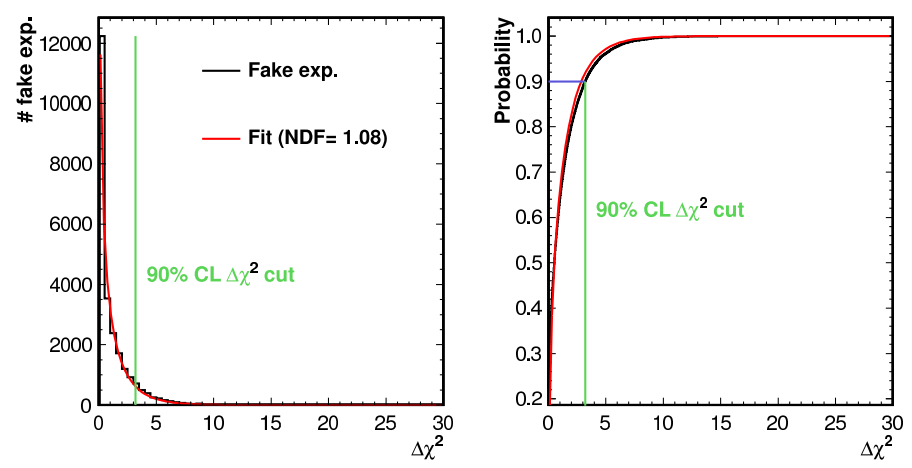

Figure 4-14: Fake data $\Delta \chi_{i}^{2}$ distribution at the null point (left), corresponding to $\left(\chi_{\text {null }}^{2}-\chi_{b f}^{2}\right)_{i}$ for $i=1, \ldots, \mathcal{N}$ fake experiments in antineutrino mode fits to $E_{\nu}^{Q E}>$ $475 \mathrm{MeV}$. The error matrix used in the $\chi_{\text {null }}^{2}$ and $\chi_{b f}^{2}$ calculations is that of the best fit for each $i$. Naively, one would expect the distribution to correspond to $n=2$. Following a real fit to data, the null point will be included in a $90 \%$ C.L. contour if $\Delta \chi_{\text {real }}^{2} \leq 2.86 .2 .86$ which is the $\Delta \chi_{90}^{2}$ cut value which encloses $90 \%$ of the fake experiments.

Specifically, the two degree of freedom approximation was found valid only in regions of the parameter space close to the LSND allowed region. At low $\sin ^{2} 2 \theta$ (as, e.g., in the case of the null point shown in Fig. 4-14), the effective degrees of freedom differ significantly from two, so that, given the inconclusive nature of the first antineutrino appearance results in [102], a more careful treatment of confidence level evaluation near the null point was deemed necessary. Therefore, in antineutrino mode, the modified frequentist method, described above, was followed, with $\Delta \chi_{\alpha}^{2}$ defined through

\begin{tabular}{l|cc}
\hline \hline Confidence level $\alpha$ & Standard, 2 d.o.f. & Fake data counting \\
\hline \hline $90 \%$ & 4.61 & 3.20 \\
$95 \%$ & 5.99 & 4.45 \\
$99 \%$ & 9.21 & 7.73 \\
$3 \sigma$ & 11.83 & 10.47 \\
$5 \sigma$ & 28.67 & - \\
\hline \hline
\end{tabular}

Table 4.8: $\Delta \chi_{\alpha}^{2}$ cuts used to map out different confidence level intervals. The $90 \%, 3$ $\sigma$, and $5 \sigma$ C.L. cuts are standard cuts used in the MiniBooNE neutrino mode analysis, assuming 2 degrees of freedom. For the antineutrino mode analysis, the $\Delta \chi_{\alpha}^{2}$ cuts for $90 \%, 95 \%$, and $99 \%$ C.L. have been determined as a function of $\left(\Delta m^{2}, \sin ^{2} 2 \theta\right)$. Shown here under "Fake data counting" are $\Delta \chi_{\alpha}^{2}$ cut values for the null point. 
fake data counting.

Similarly, to evaluate the sensitivity to $\bar{\nu}_{\mu} \rightarrow \bar{\nu}_{e}$ oscillations, fake experiments are generated at the null point, and the resulting $\Delta \chi_{i}^{2}=\left(\chi_{\text {null }}^{2}-\chi_{b f}^{2}\right)_{i}$ distribution is used to determine the cuts that have the same coverage as a single-sided cut, as is the case for the neutrino mode sensitivity; e.g., the $90 \%$ confidence level cut is defined by a cut in the $\chi^{2}$ distribution corresponding to $80 \%$. The resulting $\Delta \chi_{90}^{2}$ value used to determine the antineutrino sensitivity curve is 1.99 for a fit to $E_{\nu}^{Q E}>475$ $\mathrm{MeV}$, and 2.05 for a fit to $E>200 \mathrm{MeV}$, in contrast to 1.64, used for the neutrino sensitivity curve, the latter being the standard 1-sided cut assuming 2 degreees of freedom. Then, using a separate run of fake experiments, a surface of mean $\Delta \chi_{m}^{2}$ values extracted from the distributions of $\Delta \chi_{i}^{2}=\left(\chi_{\text {null }}^{2}-\chi_{b f}^{2}\right)_{i}$ at each point on the grid, $P_{T}$ is constructed. To obtain the $90 \%$ C.L. sensitivity, one finds the intercept of $\Delta \chi_{90}^{2}$ with the resulting surface of $\Delta \chi_{m}^{2}$ values.

Note that fake data studies do not significantly affect the sensitivity to oscillations, which is near the LSND region, but, as we will see later on, they have a dramatic effect at lower $\sin ^{2} 2 \theta$ values.

Finally, we note that what we have described is an alternative definition of $\chi^{2}$ to what is used in [165], which is based on a maximum likelihood fit. To be precise, in the case of the updated antineutrino appearance search results only, the $\chi^{2}$ definition we minimize and use to draw confidence levels throughout this thesis differs from that in [165]. The differences between the two methods have been studied extensively by the MiniBooNE collaboration in [169]; the maximum likelihood $\chi^{2}$ fit method has been found more powerful and less biased in drawing allowed regions under an oscillation hypothesis, in agreement with past studies in the literature [170].

\subsubsection{Quantifying Goodness-of-fit}

In order to quantify the agreement with a particular oscillation (or no oscillation) hypothesis $\left(\Delta m^{2}, \sin ^{2} 2 \theta\right)$, we use the standard $\chi^{2}$-probability definition, given the $\chi^{2}$ of Eq. 4.13 and the effective number of degrees of freedom at that point, $n d f$, 
approximated as

$$
n d f=N_{e}+N_{\mu}-n_{\text {fit params }}-1 .
$$

$n_{\text {fit params }}=2$ for any point other than the null point $\left(n_{\text {fit params }}=0\right)$, and 1 comes from the effective $N_{\pi}$ normalization correction we have introduced in the fit, obtained using a comparison of the $\nu_{\mu}$ CCQE prediction to the $\nu_{\mu}$ CCQE data (see Sec. 4.3.1).

In antineutrino mode, the number of degrees of freedom is cross-checked with fake data studies at the particular oscillation (or no oscillation) hypothesis. Fake data are generated at each hypothesis, $P_{T}$, and a distribution of $\left(\chi_{P_{T}}^{2}\right)_{i}$ is constructed. In that case, given the $\chi_{\text {real }}^{2}$ obtained from a fit to real data, one may read off the corresponding $\chi^{2}$-probability off of the fake data $\left(\chi_{P_{T}}^{2}\right)_{i}$ distribution at the null or best fit point, defining the $\chi^{2}$-probability as the fractional area of the distribution which satisfies $\left(\chi_{P_{T}}^{2}\right)_{i}>\chi_{\text {real }}^{2}$.

\subsection{Constraining the $\nu_{e}$ CCQE Background Pre- dictions}

The majority of predicted NC backgrounds to the $\nu_{e}$ CCQE predicted samples in Secs. 4.3.3 and 4.3.4 are constrained by actual measurements at MiniBooNE. These measurements use event samples from regions in reconstructed kinematic variables where any possible signal from $\nu_{\mu} \rightarrow \nu_{e}$ oscillations is negligible, in order to preserve blindness. The constrained backgrounds include NC $\pi^{0}, \Delta \rightarrow N \gamma$, and dirt events, and are discussed in Secs. 4.5.2, 4.5.3 and 4.5.4. All remaining backgrounds are constrained in various ways through the $\nu_{\mu} \mathrm{CCQE}$ data to MonteCarlo comparisons, both a priori to and during the $\nu_{e}-\nu_{\mu}$ combined fit to oscillations, as discussed in Sec. 4.5.1.

\subsubsection{Intrinsic $\nu_{e}$ and $\nu_{\mu}$ CCQE Mis-identified Backgrounds}

The comparison of $\nu_{\mu}$ CCQE data to MonteCarlo is used to check the accuracy of the $\nu_{\mu}$ CCQE MonteCarlo prediction, and adjust underlying flux and cross-section 
parameters, as necessary, to reach data to MonteCarlo agreement. Such parameters include, as discussed in Sec. 3.4.3, the CCQE cross-section parameters $m_{A}$ and $\kappa$. Not only are those same parameters used to predict the $\nu_{e}$ CCQE background and signal predictions, but also underlying variations on those parameters considered in assessing cross-section systematic uncertainties produce correlated effects in the predicted $\nu_{e}$ and $\nu_{\mu}$ CCQE event spectra. As we will see in Sec. 4.6, those correlations are built into the covariance matrix which is used in the appearance fit, and convey important energy-dependent information during the appearance fit, resulting in effectively reduced uncertainties. The effect of this energy-dependent constraint is discussed in more detail in App. A.2, but here we must point out that it is for that reason that a simultaneous fit to $\nu_{e}$ and $\nu_{\mu}$ CCQE reconstructed spectra is performed.

As discussed in Secs. 4.3.1 and 4.3.2, in both running modes, even after $m_{A}$ and $\kappa$ adjustments, an overall normalization difference is seen between reconstructed $\nu_{\mu}$ CCQE data and MonteCarlo prediction. This effect was attributed, in neutrino mode, to flux systematic effects, and was accounted for through a normalization correction, $N_{\pi}$, applied to all events from $\pi^{+}$parentage in the MonteCarlo predicted sample, which form the overwhelming majority of $\nu_{\mu}$ CCQE reconstructed events. In antineutrino mode, because a significant contribution of wrong-sign events (from $\pi^{+}$ rather than $\pi^{-}$) contributes to the sample, one must allow for separate normalization corrections for events from $\pi^{+}$and events from $\pi^{-}$. This allows for this difference being due to some systematic effect of the horn magnetic field. Right- versus wrong-sign differentiation is possible with reconstructed high-statistics $\nu_{\mu}$ CCQE data samples, by exploiting the fact that neutrino and antineutrino CCQE cross-sections have different $Q^{2}$ and $\cos \theta_{z}$ kinematic distributions (due to the $\pm \operatorname{sign}$ in Eq. 3.1). Therefore, comparisons of data and MonteCarlo predictions as a function of reconstructed $Q^{2}$ and/or $\cos \theta_{Z}$ can be used to extract two separate normalization factors: $N_{\pi^{+}}$, applied to predominantly neutrino events, and $N_{\pi^{-}}$, applied to predominantly antineutrino events [166].

The resulting $N_{\pi^{ \pm}}$correction factors extracted in each running mode are applied to all $\nu_{e}$ CCQE background events from $\pi^{ \pm} \rightarrow \mu^{ \pm}$, except for $\mathrm{NC} \pi^{0}, \Delta$, and dirt 
events, for reasons which become clear in the subsequent sections. However, all other backgrounds from mis-identified $\nu_{\mu}$ or $\bar{\nu}_{\mu}$ interactions receive the $\nu_{\mu}$ CCQE normalization correction according to their parentage at production $\left(\pi^{+}\right.$or $\left.\pi^{-}\right)$.

The effect of the $N_{\pi^{ \pm}}$normalization correction (which results in an increase in the signal prediction by $13 \%$ in neutrino mode and $20 \%$ in antineutrino mode for any value of $\sin ^{2} 2 \theta$ relative to $\left.N_{\pi^{ \pm}}=1.0\right)$ is an improvement on the sensitivity to oscillations, particularly at higher $\Delta m^{2}$ values (corresponding to higher-energy events, which are, on average, more correlated by cross-section).

\subsubsection{Mis-identified NC $\pi^{0}$ Backgrounds}

The NC $\pi^{0}$ predicted background events are directly constrained according to an in situ $\mathrm{NC} \pi^{0}$ rate measurement at MiniBooNE, described in detail in [155]. This measurement is particularly critical for the appearance search, as this process provides no background to the $\nu_{\mu}$ CCQE sample, and therefore the lack of correlation between $\mathrm{NC} \pi^{0}$ mis-identified events in the $\nu_{e}$ and $\nu_{\mu}$ CCQE samples makes it impossible to constrain otherwise.

The NC $\pi^{0}$ rate measurement at MiniBooNE uses events reconstructed near the $\pi^{0}$ mass peak to obtain a sample with $>90 \%$ purity in true $\mathrm{NC} \pi^{0}$ interactions. A direct comparison of data to MonteCarlo as a function of reconstructed $\pi^{0}$ momentum defines a correction function which can be applied as a function of true $\pi^{0}$ momentum bins, $p_{\pi^{0}}$, using MonteCarlo truth information to correct for efficiency and momentum unsmearing, in order to bring the simulated distribution in agreement with data. The correction function's ability to reproduce the observed $\mathrm{NC} \pi^{0}$ data in other important reconstructed kinematic distributions has been verified in $[155,171]$.

The same correction function is applied to $\mathrm{NC} \pi^{0}$ events predicted as backgrounds to the $\nu_{e}$ appearance analysis. The neutrino (antineutrino) mode extracted correction factors, $w^{\pi^{0}}$, applied to mis-identified effective ${ }^{10} \mathrm{NC} \pi^{0}$ events in the $\nu_{e}$ CCQE

\footnotetext{
${ }^{10}$ Referring to any event where a $\pi^{0}$ escapes the nucleus and decays in the detector, regardless of how it was generated, as we know that pion production is subject to charge exchange and pion absorption in the nucleus. In treating only effective $\pi^{0}$ events, we maintain consistency with the
} 
MonteCarlo as a function of 11 (8) bins of $p_{\pi^{0}}$, are shown along with their respective uncertainties and correlations expressed as a $11 \times 11(8 \times 8)$ covariance matrix in Tab. 4.9.

correction function, which was obtained using effective $\pi^{0}$ events. 


\begin{tabular}{|c|c|c|c|c|c|c|c|c|c|}
\hline $\mathbf{C} \mathbf{C V} p_{\pi^{0}}$ & $0.00-0.10$ & $0.10-0.15$ & $0.15-0.20$ & $0.20-0.25$ & $0.25-0.30$ & $0.30-0.40$ & $0.40-0.50$ & $0.50-0.60$ & 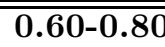 \\
\hline \multirow[t]{3}{*}{$\mathbf{C V} w^{\pi^{0}}$} & 1.3707 & 1.6794 & 1.2380 & 1.1379 & 1.0584 & 0.9702 & 0.8894 & 0.8385 & 0.7962 \\
\hline & & & & & & & & $0.80-1.00$ & $1.00-1.50$ \\
\hline & & & & & & & & 0.7692 & 0.7556 \\
\hline$w^{\pi^{\sigma}}$ & 1.4477 & 1.4794 & 1.1301 & 1.0414 & 0.9515 & 1.0241 & 0.7071 & 0.9638 & 0.9684 \\
\hline$\sigma_{i j}^{\pi^{0}}$ & $0.0-0.1$ & $0.1-0.2$ & $0.2-0.3$ & $0.3-0.4$ & $0.4-0.5$ & $0.5-0.6$ & $0.6-0.8$ & $0.8-1.0$ & $1.0-1.5$ \\
\hline $0.0-0.1$ & 0.1203 & 0.0401 & 0.0015 & -0.0079 & -0.0182 & -0.0455 & -0.0345 & -0.0672 & -0.0898 \\
\hline $0.1-0.2$ & 0.0401 & 0.0195 & 0.0008 & -0.0027 & -0.0074 & -0.0165 & -0.0161 & -0.0240 & -0.0392 \\
\hline $0.2-0.3$ & 0.0015 & 0.0008 & 0.0016 & 0.0001 & 0.0001 & -0.0003 & -0.0010 & -0.0024 & -0.0024 \\
\hline $0.3-0.4$ & -0.0079 & -0.0027 & 0.0001 & 0.0019 & 0.0009 & 0.0037 & 0.0024 & 0.0035 & 0.0063 \\
\hline 0.4-0.5 & -0.0182 & -0.0074 & 0.0001 & 0.0009 & 0.0054 & 0.0043 & 0.0076 & 0.0081 & 0.0170 \\
\hline 0.5-0.6 & -0.0455 & -0.0165 & -0.0003 & 0.0037 & 0.0043 & 0.0314 & 0.0104 & 0.0317 & 0.0412 \\
\hline 0.6-0.8 & -0.0345 & -0.0161 & -0.0010 & 0.0024 & 0.0076 & 0.0104 & 0.0198 & 0.0203 & 0.0400 \\
\hline 0.8-1.0 & -0.0672 & -0.0240 & -0.0024 & 0.0035 & 0.0081 & 0.0317 & 0.0203 & 0.0777 & 0.0540 \\
\hline 1.0-1.5 & -0.0898 & -0.0392 & -0.0024 & 0.0063 & 0.0170 & 0.0412 & 0.0400 & 0.0540 & 0.1274 \\
\hline
\end{tabular}

\begin{tabular}{r|ccccccccccc}
\hline \hline $\mathbf{C V ~} p_{\pi^{0}}$ & $\mathbf{0 . 0 - 0 . 1}$ & $\mathbf{0 . 1 - 0 . 2}$ & $\mathbf{0 . 2 - 0 . 3}$ & $\mathbf{0 . 3 - 0 . 4}$ & $\mathbf{0 . 4 - 0 . 5}$ & $\mathbf{0 . 5 - 0 . 6}$ & $\mathbf{0 . 6 - 1 . 0}$ & $\mathbf{1 . 0 - 1 . 5}$ & - & - & - \\
\hline $\mathbf{C V ~} w^{\pi^{0}}$ & 1.54504 & 1.16673 & 0.966318 & 0.860145 & 0.803898 & 0.774101 & 0.749241 & 0.741101 & - & - & - \\
\hline$w^{\pi^{0}}$ & 1.54504 & 1.16673 & 0.966318 & 0.860145 & 0.803898 & 0.774101 & 0.754078 & 0.744855 & 0.741101 & - & - \\
\hline \hline$\sigma_{i j}^{\pi^{0}}$ & $\mathbf{0 . 0 - 0 . 1}$ & $\mathbf{0 . 1 - 0 . 2}$ & $\mathbf{0 . 2 - 0 . 3}$ & $\mathbf{0 . 3 - 0 . 4}$ & $\mathbf{0 . 4 - 0 . 5}$ & $\mathbf{0 . 5 - 0 . 6}$ & $\mathbf{0 . 6 - 0 . 8}$ & $\mathbf{0 . 8 - 1 . 0}$ & $\mathbf{1 . 0 - 1 . 5}$ & - & - \\
\hline $\mathbf{0 . 0 - 0 . 1}$ & 0.1445 & 0.0337 & 0.0013 & -0.0070 & -0.0164 & -0.0367 & -0.0393 & -0.0554 & -0.0733 & - & - \\
$\mathbf{0 . 1 - 0 . 2}$ & 0.0337 & 0.0128 & 0.0005 & -0.0018 & -0.0049 & -0.0098 & -0.0136 & -0.0147 & -0.0237 & - & - \\
$\mathbf{0 . 2 - 0 . 3}$ & 0.0013 & 0.0005 & 0.0018 & 0.0001 & 0.0001 & -0.0002 & -0.0009 & -0.0016 & -0.0016 & - & - \\
$\mathbf{0 . 3 - 0 . 4}$ & -0.0070 & -0.0018 & 0.0001 & 0.0020 & 0.0006 & 0.0023 & 0.0021 & 0.0023 & 0.0040 & - & - \\
$\mathbf{0 . 4 - 0 . 5}$ & -0.0164 & -0.0049 & 0.0001 & 0.0006 & 0.0056 & 0.0027 & 0.0068 & 0.0053 & 0.0110 & - & - \\
$\mathbf{0 . 5 - 0 . 6}$ & -0.0367 & -0.0098 & -0.0002 & 0.0023 & 0.0027 & 0.0202 & 0.0084 & 0.0185 & 0.0238 & - & - \\
$\mathbf{0 . 6 - 0 . 8}$ & -0.0393 & -0.0136 & -0.0009 & 0.0021 & 0.0068 & 0.0084 & 0.0282 & 0.0167 & 0.0326 & - & - \\
$\mathbf{0 . 8 - 1 . 0}$ & -0.0554 & -0.0147 & -0.0016 & 0.0023 & 0.0053 & 0.0185 & 0.0167 & 0.0605 & 0.0319 & - & - \\
$\mathbf{1 . 0 - 1 . 5}$ & -0.0733 & -0.0237 & -0.0016 & 0.0040 & 0.0110 & 0.0238 & 0.0326 & 0.0319 & 0.0993 & - & - \\
\hline \hline
\end{tabular}

Table 4.9: Correction factors and input systematic covariance matrix $\sigma_{i j}^{\pi^{0}}$ applied to predicted $\pi^{0}$ and $\Delta \rightarrow N \gamma$ event rates, as a function of bins of true $\pi^{0}$ or single- $\gamma$ momentum (in $\mathrm{GeV} / c$ ). The systematic uncertainties include correlations for the scaling corrections. 
The NC $\pi^{0}$ rate correction also accounts for the possibility of coherent $\pi^{0}$ production contributing to the total effective $\pi^{0}$ rate at more or less than the level predicted by the underlying MonteCarlo. Coherently produced $\pi^{0}$ have a larger impact on the mis-identified NC $\pi^{0}$ backgrounds to the $\nu_{e}$ CCQE prediction; therefore, the coherent $\mathrm{NC} \pi^{0}$ rate and uncertainties are also effectively constrained and reduced, respectively. Ref. [155] constrains the fraction of coherent $\pi^{0}$ production in the total reconstructed NC $\pi^{0}$ rate by a fit to the two-dimensional distribution of $m_{\gamma \gamma} v s$. $E_{\pi^{0}}\left(1-\cos \theta_{\pi^{0}}\right)$ [171]. This fit exploits precisely the fact that coherent $\pi^{0}$ production is more forward-angle peaked. The relative fraction of coherent NC $\pi^{0}$ production is determined to be $19.5 \% \pm 2.5 \%$ in neutrino mode, and the fraction has been assumed to be the same for the $\bar{\nu}_{e}$ appearance analysis. Note that the measured relative rate of coherent versus resonant production in [156] relied on external, past results, which were accompanied by large uncertainties.

The overall size of the applied correction to the total $\mathrm{NC} \pi^{0}$ rate is less than $10 \%$, and the corresponding size of the correction on the total $\nu_{e}$ CCQE background prediction in neutrino and antineutrino running mode is shown as a function of $E_{\nu}^{Q E}$ in Figs. 4-15 and 4-16. Note that the neutrino mode analysis presented here uses finer $\pi^{0}$ momentum binning compared to [156], as well as a higher-statistics sample, which leads to lower systematic uncertainties in the $\mathrm{NC} \pi^{0}$ rate measurement relative to those in [156], particularly at low energy (200-475 MeV).

\subsubsection{Mis-identified $\Delta \rightarrow N \gamma$ Backgrounds}

Through the NC $\pi^{0}$ measurement, the $\Delta \rightarrow N \gamma$ rate is also indirectly constrained, as it is related to the measured $\pi^{0}$ rate through the relative rate of resonant production times a branching fraction of $(0.56 \pm 0.04) \%$.

The correction function and fractional uncertainties on the NC $\pi^{0}$ rate measurement are also applicable to the $\Delta$ radiative decay rate, as a function of single- $\gamma$ momentum. However, in this case, an additional uncertainty due to final state interactions is also assigned. The latter have the effect of eliminating or creating a $\pi^{0}$. Resulting correlations between the $\mathrm{NC} \pi^{0}$ and $\Delta$ uncertainties are also treated in the 

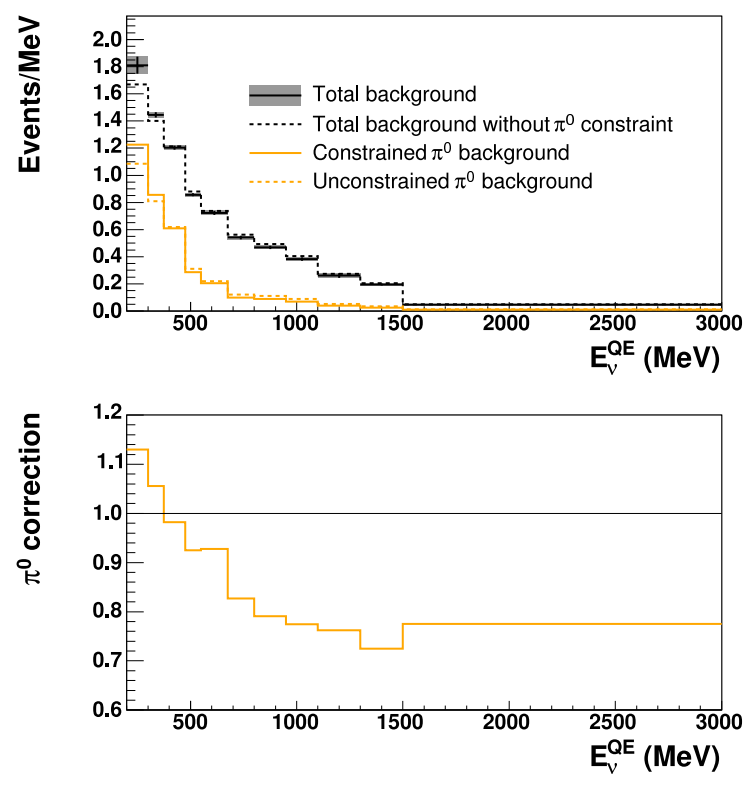

Figure 4-15: The effect of NC $\pi^{0}$ rate correction on the MiniBooNE $\nu_{e}$ CCQE background prediction. Neutrino mode.

oscillation analysis through the reweighting procedure discussed in Sec. 4.6.

\subsubsection{Dirt Backgrounds}

The rate of backgrounds from external interactions is constrained through a direct measurement at MiniBooNE, using a separate event sample where the rate of external interaction events is enhanced $[172,173]$. Such sample is obtained by isolating events reconstructed close to the detector boundaries, with tracks headed toward the center of the tank. In neutrino mode, comparisons suggested that the MonteCarlo over-predicted the absolute rate for such events. In neutrino mode, the observed to predicted dirt rate normalization difference was estimated from the study to be $0.7 \pm 0.1$. The extracted normalization difference was applied as a correction factor to the predicted dirt events in the $\nu_{e}$ CCQE sample, and a flat uncertainty of $15 \%$ was assigned on the dirt background as a function of reconstructed neutrino energy. In antineutrino mode, similar comparisons yielded a correction factor of $0.96 \pm 0.24$, also applied to the dirt background prediction as described for neutrino mode.

The dirt cut in Sec. 4.2.3 was specifically developed in [174] to reduce unnecessary 

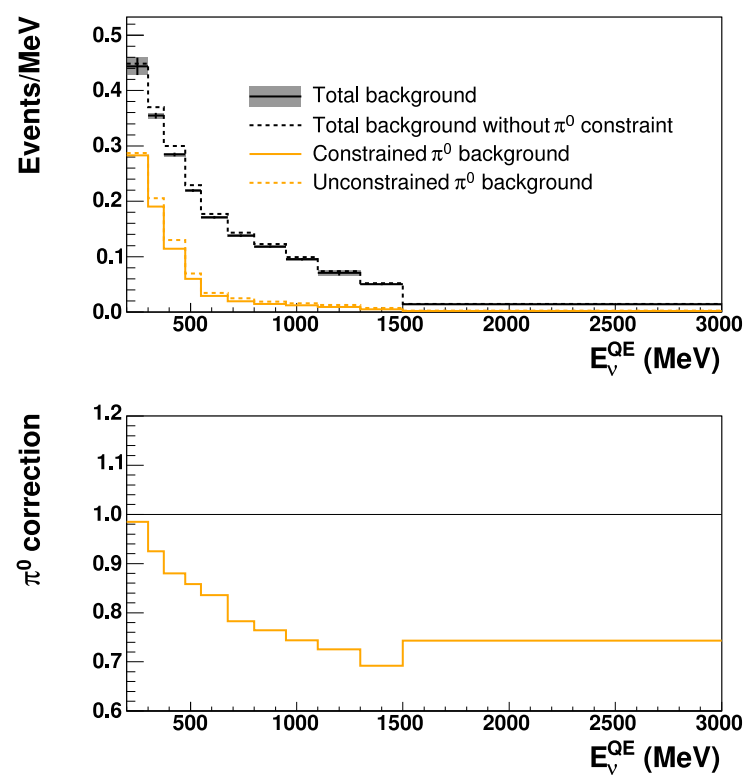

Figure 4-16: The effect of NC $\pi^{0}$ rate correction on the MiniBooNE $\nu_{e}$ CCQE background predictions. Antineutrino mode.

uncertainties due to those events at low energy (200-475 MeV). The cut significantly reduced (by $\sim 80 \%$ ) the contribution from dirt backgrounds in that range relative to [156], with minimal reduction $(\sim 20 \%)$ to expected $\nu_{e}$ events [174].

\subsection{Accounting for Systematic and Statistical Un- certainties}

Systematic uncertainties are determined by considering the effects on the $\nu_{\mu}$ and $\nu_{e}$ CCQE rate predictions which can be a result of variations of fundamental parameters used as input to the MiniBooNE MonteCarlo, within their associated uncertainty. A total of thirteen (assumed uncorrelated) general sources of systematic uncertainties are considered in the analysis, summarized in Tab. 4.10, and are discussed in more detail in the following subsections.

Each source of systematic uncertainty, $\sigma$, is mapped to a set of $\mathcal{N}$ variations of the predicted $\nu_{e}$ CCQE (background and fullosc) and $\nu_{\mu}$ CCQE central values, which are then compared to deduce systematic uncertainties on each sample as well as possible 
correlations between the samples, in the form of a systematics covariance matrix, $\mathcal{M}_{i j}^{\sigma}$. The thirteen resulting covariance matrices, assumed independent, are added to form

$$
\begin{aligned}
\mathcal{M}_{i j}= & \mathcal{M}_{i j}^{\pi^{+}}+\mathcal{M}_{i j}^{\pi^{-}}+\mathcal{M}_{i j}^{K^{+}}+\mathcal{M}_{i j}^{K^{-}}+\mathcal{M}_{i j}^{K^{0}}+\mathcal{M}_{i j}^{\text {beam }} \\
& +\mathcal{M}_{i j}^{\text {xsec }}+\mathcal{M}_{i j}^{\text {hadronic }}+\mathcal{M}_{i j}^{C C \pi^{+}}+\mathcal{M}_{i j}^{\pi^{0}}+\mathcal{M}_{i j}^{\text {dirt }} \\
& +\mathcal{M}_{i j}^{O \mathcal{M}}+\mathcal{M}_{i j}^{\text {det }},
\end{aligned}
$$

which corresponds to the total systematics covariance matrix to be used in the oscillation fit.

Each covariance matrix $\mathcal{M}_{i j}^{\sigma}$ corresponding to an underlying source of systematic uncertainty, $\sigma$, is constructed by considering $\mathcal{N}=1000$ separate varied distributions of the $\nu_{e}$ CCQE signal, background, and $\nu_{\mu}$ CCQE predictions as a function of $i$ bins of $E_{\nu}^{Q E}$, and then mapping their corresponding deviations from the central value prediction, $P_{i}$, onto the covariance matrix. The varied distributions are calculated each time by varying the underlying source of uncertainty (corresponding to $\sigma$ ) within its associated error band. Defining each varied distribution as $V_{i, n}^{\sigma}$, where $n=1, \ldots, \mathcal{N}$, the resulting covariance matrix is constructed as

$$
\mathcal{M}_{i j}^{\sigma}=\frac{1}{\mathcal{N}} \sum_{n=1}^{\mathcal{N}}\left(P_{i}-V_{i, n}^{\sigma}\right) \times\left(P_{j}-V_{j, n}^{\sigma}\right)
$$




\begin{tabular}{|c|c|}
\hline Category & Description of uncertainty \\
\hline $\begin{array}{r}\text { Neutrino Flux Uncertainties } \\
\pi^{+} \text {production } \\
\pi^{-} \text {production } \\
K^{0} \text { production } \\
K^{+} \text {production } \\
K^{-} \text {production } \\
\text { beam uncertainties }\end{array}$ & $\begin{array}{l}\text { uncertainty on the multiplicity and momentum distribution of } \pi^{+} \text {from the target } \\
\text { uncertainty on the multiplicity and momentum distribution of } \pi^{-} \text {from the target } \\
\text { uncertainty on the multiplicity and momentum distribution of } K^{0} \text { from the target } \\
\text { uncertainty on the multiplicity and momentum distribution of } K^{+} \text {from the target } \\
\text { uncertainty on the multiplicity and momentum distribution of } K^{-} \text {from the target } \\
\text { uncertainty on horn current, skin effect, nucleon interactions (cross-sections) in the target/horn, } \\
\text { pion cross-sections in the beamline }\end{array}$ \\
\hline $\begin{array}{r}\text { Neutrino Cross-Section Uncertainties } \\
\pi^{0} \text { rate } \\
\text { dirt event rate } \\
\mathrm{CC} \pi^{ \pm} \text {rate } \\
\text { all other cross-sections } \\
\text { Hadronic interactions }\end{array}$ & $\begin{array}{l}\text { uncertainty on momentum-dependent correction to the } \pi^{0} \text { rate } \\
\text { uncertainty on normalization correction to the dirt event rate } \\
\text { uncertainty on CC } \pi^{ \pm} \text {normalization correction to the } \mathrm{CC} \pi^{ \pm} \text {event rate } \\
\text { uncertainty on binding energy and Fermi momentum in }{ }^{12} C \text { model, } \\
\text { CCQE and other non-CCQE neutrino cross-section parameters } \\
\text { uncertainty on final state interactions in the oil }\end{array}$ \\
\hline $\begin{array}{r}\text { Detector Modeling } \\
\text { optical model } \\
\text { detector electronics }\end{array}$ & $\begin{array}{l}\text { uncertainties in modeling of light production, propagation, and detection by PMT's } \\
\text { variations in the electronics } q \text { and } t \text { response }\end{array}$ \\
\hline
\end{tabular}

Table 4.10: A summary of sources of systematic uncertainties contributing to the $\nu_{e}$ and $\bar{\nu}_{e}$ appearance analysis uncertainties. 


\begin{tabular}{lccc}
\hline \hline Low energy fractional (\%) uncertainties & & & \\
\hline Source of systematic & $\nu_{e}$ background & $\nu_{e}$ signal & $\nu_{\mu}$ CCQE \\
\hline \hline$\pi^{+}$production & $0.4 / 1.8$ & $0.1 / 6.8$ & $1.8 / 4.2$ \\
$\pi^{-}$production & $3.3 / 0.1$ & $15.0 / 0.2$ & $6.1 / 0.2$ \\
$K^{+}$production & $2.3 / 1.4$ & $0.0 / 0.2$ & $0.5 / 0.3$ \\
$K^{-}$production & $0.5 /-$ & $0.0 /-$ & $0.1 /-$ \\
$K^{0}$ production & $1.5 / 0.5$ & $0.0 /-$ & $0.1 / 0.0$ \\
Beam & $1.9 / 1.3$ & $2.2 / 3.0$ & $2.9 / 2.9$ \\
Cross-Sections & $6.4 / 5.9$ & $17.1 / 18.3$ & $15.3 / 14.9$ \\
$\pi^{0}$ rate & $1.7 / 1.4$ & $0.0 / 0.0$ & $-/-$ \\
Hadronic interactions & $0.5 / 0.8$ & $0.3 / 0.2$ & $0.0 / 0.0$ \\
CC $\pi$ reweighting & $-/-$ & $-/-$ & $4.9 /-$ \\
Dirt rate & $2.4 / 0.8$ & $-/-$ & $-/-$ \\
Detector electronics & $9.7 / 5.0$ & $4.6 / 6.7$ & $2.0 / 1.9$ \\
Optical model & $10.0 / 8.9$ & $13.1 / 20.4$ & $2.8 / 4.9$ \\
\hline Total & $16.4 / 12.3$ & $26.8 / 29.2$ & $17.9 / 16.7$ \\
\hline \hline
\end{tabular}

Table 4.11: Systematic fractional (\%) uncertainties on the $\nu_{e}$ CCQE background, $\nu_{e}$ CCQE signal, and $\nu_{\mu}$ CCQE samples in the antineutrino/neutrino appearance analysis. The values correspond to the low energy range: $200-475 \mathrm{MeV}$ for $\nu_{e} \mathrm{CCQE}$ background and $\nu_{e}$ CCQE signal, and $0-700 \mathrm{MeV}$ for $\nu_{\mu}$ CCQE.

The first eleven systematic uncertainties are determined via the use of reweighting of the MonteCarlo central value, $P_{i}$, in order to obtain each varied distribution $V_{i, n}^{\sigma}$ (see App. A.1 for more details on the reweighting procedure). The last two are determined via directly comparing the central values corresponding to different underlying MonteCarlo's to the central values for the default MonteCarlo, used in the oscillation fit.

The total fractional uncertainties on the $\nu_{e}$ CCQE background, $\nu_{e}$ CCQE expected signal, and $\nu_{\mu}$ CCQE predicted events are summarized in Tabs. 4.11 and 4.12, for low and high energy, respectively. The largest uncertainties are contributed from uncertainties in $\pi^{+}$production and cross-sections; however, those are effectively canceled in the simultaneous fit to $\nu_{e}$ and $\nu_{\mu}$ CCQE events, as discussed in Sec. 4.4.

In a simultaneous fit to $\nu_{e}$ and $\nu_{\mu}$ events, the largest contributing uncertainty to the neutrino mode search is that of optical modeling uncertainties. In antineutrino mode, the largest uncertainty after exploiting the $\nu_{\mu}$ CCQE constraint is that contributed by statistical uncertainties, followed by $\nu_{e}$ background systematic uncertainties due to $K^{0}$ production, at the level shown in Fig. 4-17. 


\begin{tabular}{lccc}
\hline \hline High energy fractional (\%) uncertainties & & & \\
\hline Source of systematic & $\nu_{e}$ background & $\nu_{e}$ fullosc & $\nu_{\mu}$ CCQE \\
\hline \hline$\pi^{+}$production & $0.7 / 2.2$ & $0.1 / 3.9$ & $3.3 / 4.1$ \\
$\pi^{+}$production & $2.2 / 0.2$ & $4.4 / 0.2$ & $3.6 / 0.2$ \\
$K^{+}$production & $4.9 / 5.7$ & $0.0 / 0.2$ & $0.6 / 0.4$ \\
$K^{-}$production & $1.1 /-$ & $0.0 /-$ & $0.1 /-$ \\
$K^{0}$ production & $5.7 / 1.5$ & $0.0 / 0.0$ & $0.1 / 0.0$ \\
Beam & $3.0 / 2.5$ & $2.8 / 3.6$ & $3.4 / 4.4$ \\
Cross-Sections & $12.9 / 11.9$ & $17.8 / 16.2$ & $16.3 / 16.1$ \\
$\pi^{0}$ Rate & $1.6 / 1.9$ & $0.0 / 0.0$ & $-/-$ \\
Hadronic interactions & $0.6 / 0.3$ & $0.3 / 0.1$ & $0.0 / 0.1$ \\
CC $\pi$ reweighting & $-/-$ & $-/-$ & $0.7 /-$ \\
Dirt rate & $1.2 / 0.4$ & $-/-$ & $-/-$ \\
Detector electronics & $3.0 / 1.7$ & $5.6 / 3.7$ & $2.9 / 2.4$ \\
Optical model & $3.2 / 2.3$ & $8.5 / 4.6$ & $2.1 / 2.7$ \\
\hline Total & $16.3 / 14.2$ & $21.2 / 18.1$ & $17.8 / 17.5$ \\
\hline \hline
\end{tabular}

Table 4.12: Systematic fractional (\%) uncertainties on the $\nu_{e}$ CCQE background, $\nu_{e}$ CCQE signal, and $\nu_{\mu}$ CCQE samples in the antineutrino/neutrino appearance analysis. The values correspond to the high energy range: $475-1100 \mathrm{MeV}$ for $\nu_{e}$ CCQE background and $\nu_{e}$ CCQE signal, and 700-1400MeV for $\nu_{\mu}$ CCQE.

\subsubsection{Flux Systematic Uncertainties}

The oscillation analysis accounts for flux uncertainties associated with both production cross-sections and beam modeling effects. A list of all underlying systematic uncertainties taken into account in predicting the MiniBooNE flux are shown in Tab. 4.13.

As discussed in Sec. 3.4.1, meson production at the target, in particular $\pi^{ \pm}, K^{+}$, and $K^{0}$ production, contribute the largest flux-related uncertainties on the $\nu_{e}$ and $\nu_{\mu}$ CCQE prediction. Most dominant are the uncertainties on $\pi^{+}$and $\pi^{-}$production, as those are the ones that contribute the most neutrino flux seen at the MiniBooNE detector. 


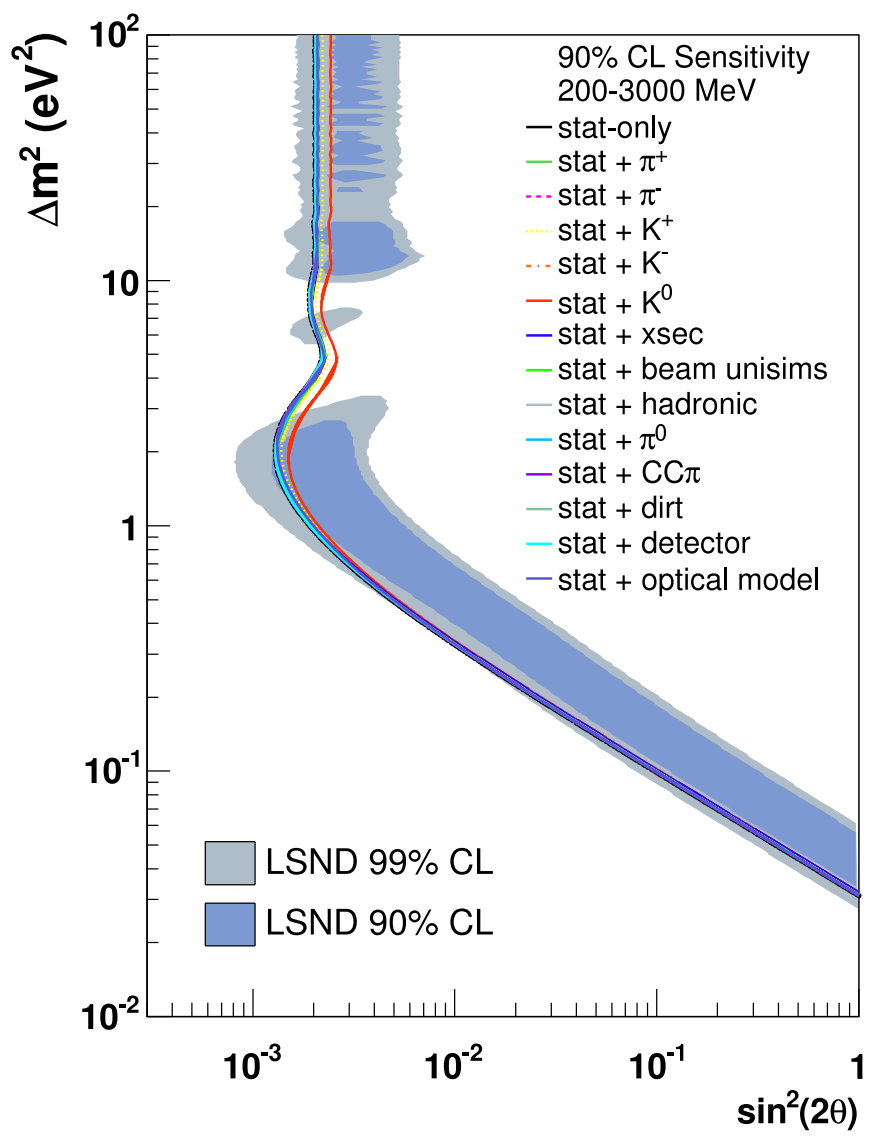

Figure 4-17: The effect of systematic uncertainties on MiniBooNE's sensitivity to $\bar{\nu}_{\mu} \rightarrow \bar{\nu}_{e}$ oscillations in antineutrino mode $\left(5.66 \times 10^{20} \mathrm{POT}\right) . K^{0}$ production uncertainties induce the most significant degradation in sensitivity. The sensitivity is most limited by statistical uncertainties. Shown here is the sensitivity for a fit to $E_{\nu}^{Q E}>200 \mathrm{MeV}$. 


\begin{tabular}{|c|c|c|}
\hline $\begin{array}{l}\text { Source of systematic } \\
\text { uncertainty }\end{array}$ & $\begin{array}{l}\text { Number of underlying } \\
\text { input parameters }\end{array}$ & $\begin{array}{l}\text { Nominal value of underlying input parameter(s) } \\
\text { and associated systematic uncertainties }\end{array}$ \\
\hline $\begin{array}{l}\text { Proton delivery: } \\
\text { POT rate }\end{array}$ & 1 & Total POT measured by toroids $\pm 2 \%$ (normalization) \\
\hline $\begin{array}{l}\text { Secondary production: } \\
\pi^{+} \text {production }\end{array}$ & 9 & $\begin{array}{l}\text { CV from SW fit, Tab. V in [125], uncertainties propagated } \\
\text { via spline fit interpolation [125]. }\end{array}$ \\
\hline$\pi^{-}$production & 9 & $\begin{array}{l}\text { CV from SW fit, Tab. VI in [125], uncertainties propagated } \\
\text { via spline fit interpolation [125]. }\end{array}$ \\
\hline$K^{+}$production & 7 & CV and uncertainties from FS fit, Tab. VII in [125]. \\
\hline$K^{-}$production & 1 & CV from MARS15 [144] MonteCarlo, $100 \%$ uncertainty. \\
\hline$K^{0}$ production & 9 & CV and uncertainties from SW fit, Tab. IX in [125]. \\
\hline $\begin{array}{l}\text { Hadronic interactions: } \\
p / n \sigma_{I N E}, \sigma_{Q E}, \sigma_{T O T} \\
\pi^{ \pm} \sigma_{I N E}, \sigma_{Q E}, \sigma_{T O T}\end{array}$ & $\begin{array}{l}3 \\
3\end{array}$ & $\begin{array}{l}\text { Defined within Secs. IV and VII.C in [125]. } \\
\text { Defined within Secs. IV and VII.C in [125]. }\end{array}$ \\
\hline Horn magnetic focusing: & & \\
\hline $\begin{array}{l}\text { Horn current } \\
\text { "Skin effect" }\end{array}$ & 1 & $\begin{array}{l}( \pm) 174 \pm 1 \mathrm{kA} \text {, with systematic excursions treated } \\
\text { as } 1 \sigma \text { deviations and propagated through } \\
\text { the neutrino flux in special beam MonteCarlo runs, Sec. VII.D. in [125] } \\
\text { Uncertainty on the } B \text { field decay length }\left(1.4_{-1.4}^{+0} \mathrm{~mm}\right) \\
\text { is treated as a } 1 \sigma \text { deviation and propagated through the } \\
\text { neutrino flux in a special MonteCarlo run, Sec. VII.D. in [125] }\end{array}$ \\
\hline $\begin{array}{l}\text { Beamline geometry: } \\
\text { None }\end{array}$ & & Negligible. \\
\hline
\end{tabular}

Table 4.13: Underlying systematic uncertainties taken into account in predicting the MiniBooNE flux. 
Uncertainties due to $\pi^{ \pm}$production are estimated by propagating the effect of variations on production differential cross-sections, through the MiniBooNE MonteCarlo, via reweighting. The underlying uncertainties are assessed as the production crosssection is varied within final uncertainties in the data provided by the HARP experiment. Rather than relying on the Sanford Wang (i.e., model-dependent) parametrization to draw variations on the production differential cross-section, a spline interpolation of the HARP data itself is used to extrapolate to meson kinematics regions where HARP data is unavailable [175] and propagate production uncertainties to the final $\nu_{e}$ and $\nu_{\mu}$ CCQE predictions. The above method provides an improvement with respect to the way $\pi^{ \pm}$production uncertainties were handled in the first MiniBooNE neutrino mode search for oscillations [156].

Uncertainties due to $K^{0}$ production are estimated by propagating, through the MiniBooNE MonteCarlo prediction, variations drawn within the uncertainties of the Sanford Wang fit parameters which are obtained from fits to the world's and $K^{0}$ production data. Uncertainties due to $K^{+}$production also come from propagating the error matrix from a Feynman Scaling fit to the world's $K^{+}$production data. A $100 \% K^{-}$production uncertainty is propagated through the MonteCarlo, since there are no available $K^{-}$production data to tune the predictions to.

The remaining beam related systematics, listed in Tab. 4.13, include: uncertainty on the horn current, defined within the simulation as $174 \pm 1 \mathrm{kA}$; uncertainty in the modeling of the "skin depth" effect, which allows for the magnetic field to penetrate into the inner conductor of the horn, effectively increasing the horn focusing power; and uncertainties in nucleon- $B e / A l$ and pion- $B e / A l$ hadronic cross-sections. The uncertainties on the $\nu_{e}$ and $\nu_{\mu}$ CCQE predictions due to the above beam systematics are estimated through special runs of the flux MonteCarlo, treating each uncertainty as a $1 \sigma$ excursion, and assuming the resulting flux variations correspond to $1 \sigma$ flux excursions. 


\subsubsection{Cross-Section Systematic Uncertainties}

The cross-section uncertainties are evaluated by propagating the uncertainties on a number of neutrino cross-section parameters used within the NUANCE neutrino event generator through the predicted CCQE event rates (and their backgrounds), via the use of reweighting.

The effects of all cross-section model parameters listed in Tab. 3.5 are considered, as well as that of additional uncertainties due to the various corrections adopted from MiniBooNE in situ measurements, as discussed in Sec. 4.5, which are summarized in Tab. 4.14. Those include:

- $\mathrm{NC} \pi^{0}$ rate uncertainty, as discussed in Sec. 4.5.2, corresponding to a $\sim 5 \%$ fluxintergrated uncertainty on the $\mathrm{NC} \pi^{0}$ prediction, once correlations are included.

- Dirt rate uncertainty, as discussed in Sec. 4.5.4..

- Uncertainties due to the correction to the relative fraction and overall normalization of coherent to resonant NC $\pi^{0}$ production, extracted from fits in [155], as discussed in Sec. 4.5.2.

- Uncertainty due to the $\Delta \rightarrow N \gamma$ radiative decay fraction (7\%) and correction of final state interaction effects $\left(10 \%\right.$, due to $\pi$ escape probability on $\left.{ }^{12} C\right)[176$, 177], added in quadrature and propagated along with the uncertainty from the $\mathrm{NC} \pi^{0}$ rate constraint, discussed in Sec. 4.5.3. This results in an overall $\sim 12.2 \%$ uncertainty on the $\Delta \rightarrow N \gamma$ background.

- Uncertainty on deep-inelastic scattering cross-sections (25\%).

- Uncertainty on the $\nu_{e}$ event rate and CCQE cross-section shape resulting from our choice of using the $\nu_{\mu}$ CCQE cross-section to predict the $\nu_{e}$ CCQE rates. This accounts for the fact that other choices in the RFG model parameters (instead of $m_{A}$ and $\kappa$ ) could have been made to improve the kinematic agreement between $\nu_{\mu}$ CCQE data and MonteCarlo prediction [178].

- Uncertainty in the $\nu_{e}$ and $\nu_{\mu}$ CCQE normalization correction. 


\begin{tabular}{l|cc}
\hline \hline $\begin{array}{l}\text { Cross-section } \\
\text { parameter }\end{array}$ & $\begin{array}{c}\text { Nominal value for } \nu_{e} \text { and } \bar{\nu}_{e} \\
\text { appearance analysis }\end{array}$ & Correlations \\
\hline \hline resonant $\pi^{0}$ fraction & $19.5 \% \pm 2.5 \%$ & $\rho($ res, coh $)=-1$ \\
coherent $\pi^{0}$ fraction & $80.5 \% \pm 2.5 \%$ & $\rho($ coh, res $)=-1$ \\
$\mathrm{BR}(\Delta \rightarrow N \gamma)$ & $(0.56 \pm 0.04) \%$ & - \\
$\Delta \rightarrow N \gamma$ normalization & $1.022 \pm 0.1245$ & - \\
$\pi$ absorption probability $\left({ }^{12} C\right)$ & (energy-dependent) $\pm 35 \%$ & - \\
$\pi$ charge exchange $\left({ }^{12} C\right)$ & (energy-dependent) $\pm 50 \%$ & - \\
$\pi$ escape probability $\left({ }^{12} C\right)$ & $62.5 \% \pm 7.5 \%$ & \\
deep-inelastic scattering & NUANCE default $\pm 25 \%$ & \\
$\mathrm{NC} \pi^{0}$ rate & $\left(p^{\pi^{0}}\right.$-dependent) & \\
$\mathrm{CCQE}$ cross-section normalization & \multicolumn{1}{l}{$1.0 \pm 0.10$} & \\
\hline \hline
\end{tabular}

Table 4.14: Additional cross-section uncertainties considered in neutrino and antineutrino MiniBooNE event rate predictions for the $\nu_{e}$ and $\bar{\nu}_{e}$ appearance analyses (see also Tab. 3.5). The quoted uncertainties for parameters extracted using MiniBooNE data do not include contributions from flux and detector uncertainties, to avoid double counting in the appearance analyses.

- Uncertainties on hadronic interaction processes such as photonuclear interactions, pion absorption, or pion charge exchange.

\subsubsection{Detector Systematic Uncertainties}

Detector systematic uncertainties are separated into optical modeling uncertainties, and detector electronics uncertainties. Optical modeling (OM) uncertainties result from uncertainties in light creation, propagation, and detection in the tank, which are controlled by a total of 35 parameters in the simulation. Unlike flux and cross-section effects, optical modeling effects cannot be propagated to $E_{\nu}^{Q E}$-dependent distributions using the standard reweighting technique in App. A.1, as they can affect the reconstruction of various types (and not just overall rate) of events non-trivially. Therefore, in the neutrino (antineutrino) appearance analysis, the entire detector MonteCarlo simulation, reconstruction, and event selection are re-generated $\mathcal{N}=66$ (130) times, where each time all 35 OM parameters are varied according to their covariance matrix, and propagated to the $\nu_{\mu}$ and $\nu_{e}$ CCQE predicted distributions. The parameter values have been generated as described in [163], and are constrained using Michel electron calibration data. 
Due the significantly larger computational requirements required in regenerating full MonteCarlo, the generation of both higher-statistics event samples in each MonteCarlo variation and a larger number of variations, $\mathcal{N}$, is impractical. As a consequence, each $i=1, \ldots, \mathcal{N}$ MonteCarlo variation, referred to as a "multisim", is generated with an intrinsic data-sized statistical uncertainty (due to finite statistics) which must be accounted for in the estimation of data statistical uncertainties, in order to avoid double-counting.

The multisim statistical uncertainty correction has the effect of subtracting off the statistical uncertainty contribution that is intrinsically built into the optical model uncertainties, accounting for the effects of possible MonteCarlo scaling corrections involved in obtaining $\mathcal{M}_{i j}^{O M}$. As discussed at the beginning of Sec. 4.6, uncertainties due to optical modeling are the dominant source of uncertainty in the neutrino mode analysis, which is systematics-limited.

Uncertainties due to detector electronics correspond to PMT charge and timing uncertainties, contributed by 1) variations in the PMT discriminator threshold, which, as described in Sec. 3.3, determines whether a hit was recorded or not, and 2) PMT charge amplitude and timing correlations which arise as different raw charge amplitudes take different times to reach threshold. The corresponding uncertainty on the central value predictions due to deviations in discriminator threshold level and charge-time correlations is determined by regenerating MonteCarlo for extreme excursions in each deviating quantity. These MonteCarlo variants, referred to as "unisims", are also subject to intrinsic statistical jitter due to finite MonteCarlo statistics, and in this case a smoothing procedure is implemented to remove that effect, as follows: For each unisim, $P_{i}^{n}$, a ratio of $P_{i}^{n} / P_{i}$ is constructed as function of $i$ bins of $E_{\nu}^{Q E}$, with respect to the central value prediction, $P_{i}$, and fitted to a fifth-order polynomial separately for each sample ( $\nu_{e}$ signal, $\nu_{e}$ background, and $\nu_{\mu}$ CCQE). The order of the polynomial is chosen to be large enough to account for systematic variations, but small enough compared to the number of bins, to allow for sufficient smoothing of bin-to-bin statistical variations. The resulting polynomial, $p_{i}$, with an expected value of 1.0 assuming no statistical and systematic fluctuations in $P_{i}^{n}$, is then used to 


\begin{tabular}{l|cc}
\hline \hline MonteCarlo sample & $\begin{array}{c}\text { Neutrino mode } \\
\text { generated POT }\end{array}$ & $\begin{array}{c}\text { Antineutrino mode } \\
\text { generated POT }\end{array}$ \\
\hline \hline Dirt events & $2.294 \times 10^{21}$ & $1.044 \times 10^{22}$ \\
Fullosc events & $1.328 \times 10^{20}$ & $3.249 \times 10^{21}$ \\
Background events & $4.108 \times 10^{21}$ & $2.790 \times 10^{22}$ \\
\hline \hline
\end{tabular}

Table 4.15: Generated MonteCarlo event statistics.

reweight the central value prediction and construct the covariance matrix according to

$$
\mathcal{M}_{i j}^{\text {det }}=\frac{1}{\mathcal{N}} \sum_{n=1}^{\mathcal{N}}\left(P_{i}-p_{i} P_{i}\right)\left(P_{j}-p_{j} P_{j}\right) .
$$

\subsubsection{Statistical Uncertainties}

A data-sized statistical uncertainty evaluated according to the $\nu_{e}$ CCQE background and $\nu_{\mu} \mathrm{CCQE}$ central value predictions, $P_{i}$, is added to the diagonal of the total covariance matrix used in the combined $\nu_{e}-\nu_{\mu}$ fit. An additional statistical uncertainty evaluated for the best-fit signal prediction is also included, as discussed in Sec. 4.4.3. This statistical uncertainty can vary during the fit, and is calculated using the MonteCarlo $\nu_{e}$ CCQE signal prediction for any particular set of underlying oscillation parameters in consideration $\left(\sin ^{2} 2 \theta, \Delta m^{2}\right)$.

In addition, a MonteCarlo statistical uncertainty is also included, to account for statistical uncertainties due to the finite MonteCarlo statistics. This is calculated according to the total POT that each MonteCarlo generated sample corresponds to, summarized in Tab. 4.15.

\subsection{Sensitivity to $\nu_{e}$ and $\bar{\nu}_{e}$ Appearance}

Given that a significant $(3.8 \sigma)$ excess of $\nu_{e}$ CCQE events had been observed in the original neutrino mode appearance analysis at low reconstructed neutrino energies, between $200-475 \mathrm{MeV}$ [156], the final oscillation fit results presented in this work use events with reconstructed neutrino energy above $475 \mathrm{MeV}$. This choice is justified in part by the fact that, in the simple, two-neutrino oscillation framework assumed, the 
LSND-like oscillations induce a most significant excess in the 475-1250 MeV energy range. This can be justified by simple $L / E$ considerations, given the LSND excess in Fig. 2-5.

We also note that while the first MiniBooNE antineutrino appearance results [165], corresponding to $3.39 \times 10^{20}$ POT, showed no evidence for a significant excess at low energy (within large statistical uncertainties), given the possibility of an excess becoming significant (with increased statistics) at low energy, the antineutrino oscillation fit energy range is also restricted to $475<E_{\nu}^{Q E}<3000 \mathrm{MeV}$. The source of an excess at only low energy is assumed to be from sources other than simple $\bar{\nu}_{\mu} \rightarrow \bar{\nu}_{e}$ two-neutrino oscillations.

The relative degradation in sensitivity that results from this energy range restriction from $200 \mathrm{MeV}$ to $475 \mathrm{MeV}$ is illustrated in Fig. 4-18. Due to the $\propto \Delta m^{2} / E$ dependence of the oscillation probability, excluding low energy information from the fit worsens the sensitivity to oscillations at lower $\Delta m^{2}$ values, but the effect on the overall sensitivity is almost negligible, in particular in neutrino mode.

With that said, fits to $200<E_{\nu}^{Q E}<3000 \mathrm{MeV}$ are also considered in this work, given that results presented here correspond to several updates to the analysis and fit method relative to $[156,102]$.

Figure 4-19 presents MiniBooNE's final sensitivity to $\nu_{\mu} \rightarrow \nu_{e}$ oscillations described by the oscillation probability in Eq. 4.1, from a simultaneous fit to the $\nu_{e}$ and $\nu_{\mu}$ CCQE distributions in neutrino mode. The sensitivity curves in neutrino mode are obtained from a fit to a fake no oscillations prediction, and using the one-sided raster scan method discussed in Sec. 4.4.5: at each $\Delta m^{2}$ on the grid, the rightmost value of $\sin ^{2} 2 \theta$ is found which satisfies $\Delta \chi^{2}=\chi^{2}\left(\sin ^{2} 2 \theta\right)-\chi_{b f}^{2}\left(\sin ^{2} 2 \theta_{b f}\right)=\Delta \chi_{\alpha}^{2}$, with respect to the best-fit $\sin ^{2} 2 \theta_{b f}$ at that particular $\Delta m^{2}$. Assuming one (1) degree of freedom, the $\Delta \chi_{\alpha}^{2}$ cut corresponding to $\alpha=90 \%$ C.L. is 1.64 . The $3 \sigma$ and 5 $\sigma$ C.L. sensitivity curves are obtained using the corresponding values for a two-sided gaussian distribution $\left(\Delta \chi_{3 \sigma}^{2}=9.0\right.$ and $\left.\Delta \chi_{5 \sigma}^{2}=25.0\right)$.

Figure 4-20 presents MiniBooNE's sensitivity to simple, two-neutrino $\bar{\nu}_{\mu} \rightarrow \bar{\nu}_{e}$ oscillations, governed by the same oscillation probability as in Eq. 4.1, using an- 

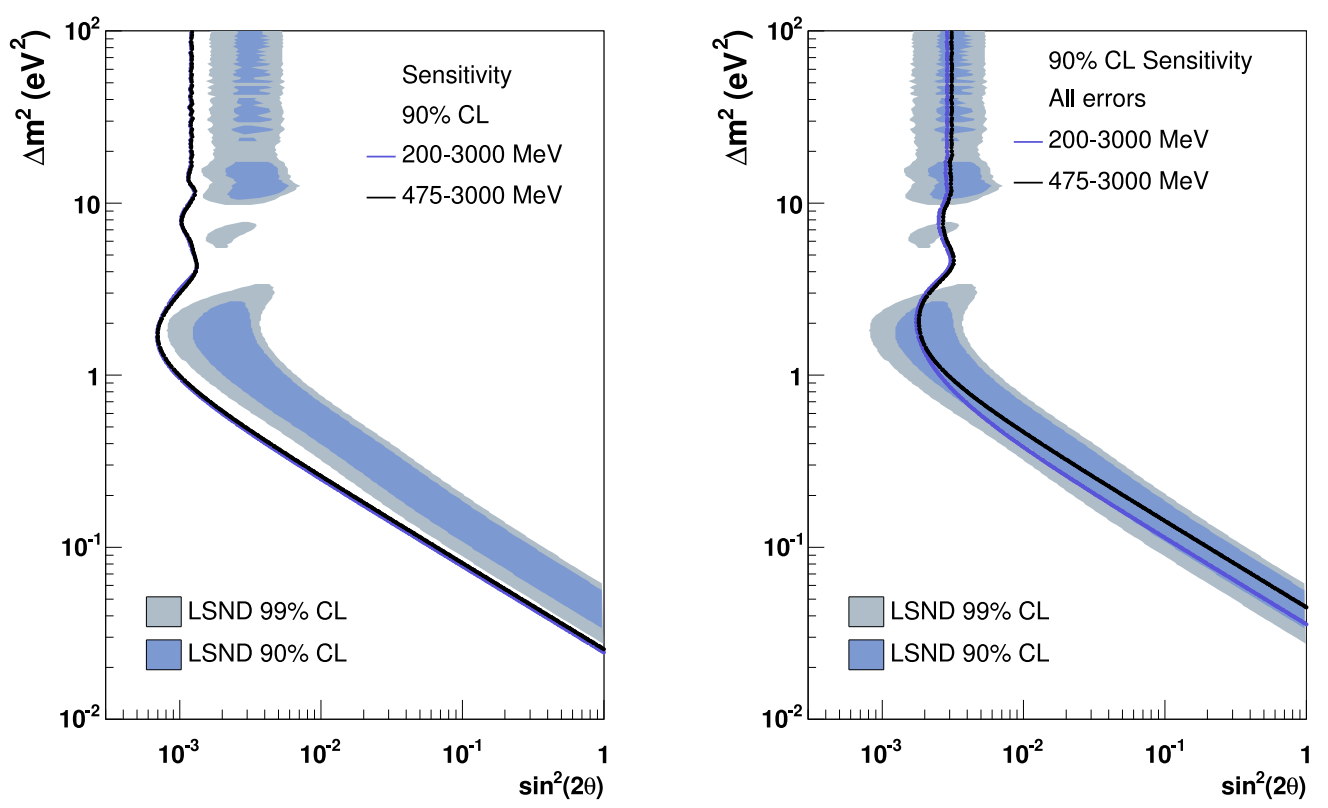

Figure 4-18: The effect of the $E_{\nu}^{Q E}>475 \mathrm{MeV}$ cut on MiniBooNE's sensitivity to $\nu_{\mu} \rightarrow \nu_{e}$ oscillations in neutrino mode $\left(6.46 \times 10^{20} \mathrm{POT}\right.$, left $)$ and $\bar{\nu}_{\mu} \rightarrow \bar{\nu}_{e}$ oscillations in antineutrino mode $\left(5.66 \times 10^{20} \mathrm{POT}\right.$, right). All sensitivity curves correspond to 90\% C.L.

tineutrino data corresponding to $5.66 \times 10^{20}$ POT. A different method is used to draw antineutrino sensitivities, as discussed in Sec. 4.4.5, which is approximately equivalent to the one used in neutrino mode. The figures illustrate that, while MiniBooNE does not presently have sufficient sensitivity to potentially exclude the full 90\% C.L. LSND-allowed region (left) in antineutrino mode, it has sufficient sensitivity to probe most of the $90 \%$ C.L. allowed region from a joint KARMEN and LSND $\bar{\nu}_{\mu} \rightarrow \bar{\nu}_{e}$ analysis (right).

In summary, the neutrino mode search provides a high-statistics, powerful test of the simplest LSND oscillation interpretation, which is based on a two-neutrino approximation and assumes no $C P($ or $C P T)$ violation $\left(P\left(\nu_{\mu} \rightarrow \nu_{e}\right)=P\left(\bar{\nu}_{\mu} \rightarrow\right.\right.$ $\left.\bar{\nu}_{e}\right)$ ). The antineutrino mode search provides a direct test of the LSND oscillation interpretation in a simple, two-neutrino approximation, independent of any $C P$ (or $C P T)$ assumptions. If two distinct oscillation patterns are observed in neutrino versus antineutrino running mode at MiniBooNE (i.e., $\left.P\left(\nu_{\mu} \rightarrow \nu_{e}\right) \neq P\left(\bar{\nu}_{\mu} \rightarrow \bar{\nu}_{e}\right)\right)$, possible 


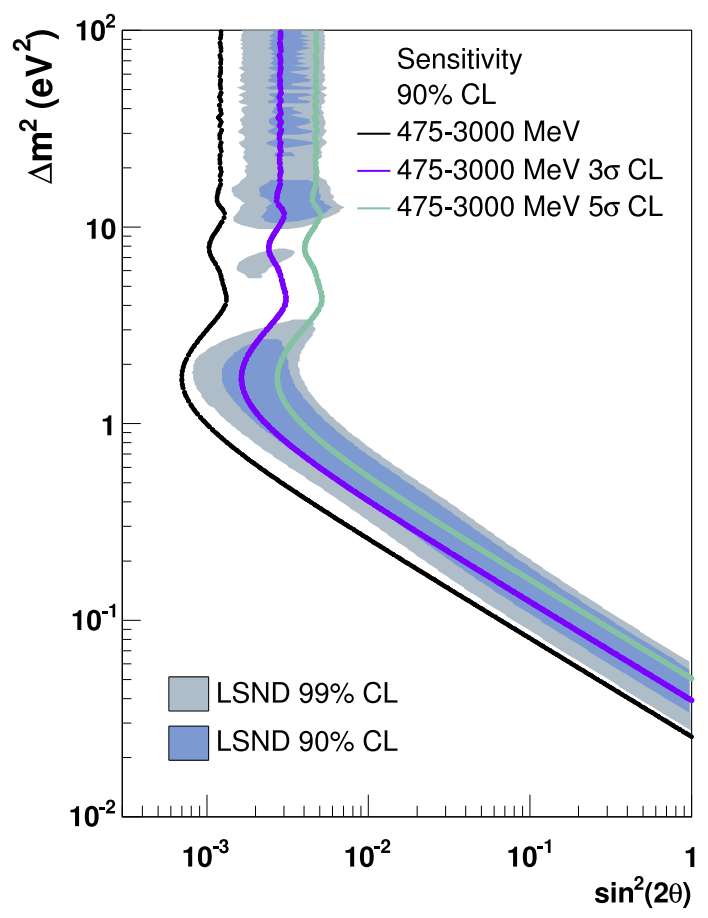

Figure 4-19: MiniBooNE's final sensitivity to $\nu_{\mu} \rightarrow \nu_{e}$ oscillations in neutrino mode, for $6.46 \times 10^{20} \mathrm{POT}$. If no oscillations exist due to underlying parameters to the right of each curve, MiniBooNE is able to exclude regions to the right of each curve at the level of confidence identified by each curve.

interpretations could involve sterile neutrino oscillations with $C P$ violation, or some other non-standard sterile neutrino oscillation scenario. We consider the former in Chapter 6 . 

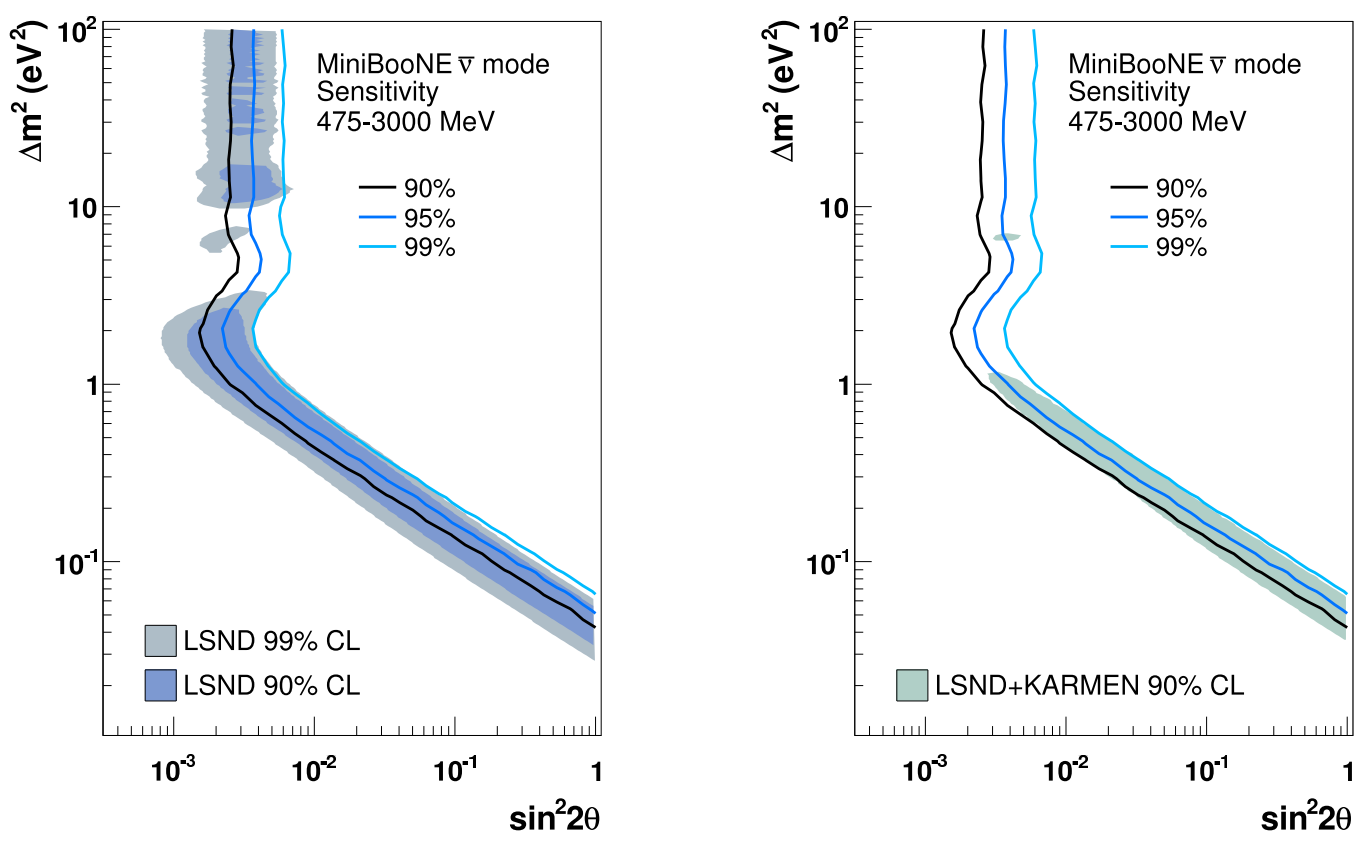

Figure 4-20: MiniBooNE's sensitivity to $\bar{\nu}_{\mu} \rightarrow \bar{\nu}_{e}$ oscillations in antineutrino mode, for $5.66 \times 10^{20} \mathrm{POT}$. If no oscillations exist due to underlying parameters to the right of each curve, MiniBooNE is able to exclude regions to the right of each curve at the level of confidence identified by each curve. The left figure compares MiniBooNE's sensitivity reach to LSND-allowed $\bar{\nu}_{\mu} \rightarrow \bar{\nu}_{e}$ oscillations. The right figure compares MiniBooNE's sensitivity reach to joint LSND+KARMEN-allowed $\bar{\nu}_{\mu} \rightarrow \bar{\nu}_{e}$ oscillations. 


\section{Chapter 5}

\section{MiniBooNE Appearance Results}

Following the analysis method and assumptions described in Chapter 4, this chapter presents results from a $\nu_{e}$ and a $\bar{\nu}_{e}$ appearance search at MiniBooNE. The $\nu_{e}$ appearance search was performed in neutrino running mode, and corresponds to $6.46 \times 10^{20} \mathrm{POT}$, while the $\bar{\nu}_{e}$ appearance search was performed in antineutrino running mode, and corresponds to $5.66 \times 10^{20}$ POT. $^{1}$

\section{$5.1 \quad \nu_{e}$ Appearance Results}

\subsubsection{Oscillation Search}

Figure 5-1 shows a comparison of the observed $\nu_{e}$ CCQE event distribution to the total background prediction, assuming no oscillations, as a function of the full reconstructed neutrino energy range. Assuming no oscillations, the resulting $\chi^{2} /$ d.o.f. from a fit to the full $200-3000 \mathrm{MeV}$ range is $22.2 / 18$, corresponding to a $\chi^{2}$-probability of $22 \%$.

In the 475-1250 MeV energy region, which is where a significant excess from twoneutrino oscillations would appear, MiniBooNE observes a total of $408 \nu_{e}$ CCQE candidate events, in agreement with the background prediction of $385.9 \pm 35.7$ events within statistical and constrained systematic uncertainties. However, while no evi-

\footnotetext{
${ }^{1}$ Final antineutrino appearance results, corresponding to a total of $\sim 10.0 \times 10^{20} \mathrm{POT}$, are expected in approximately two years from this writing.
} 


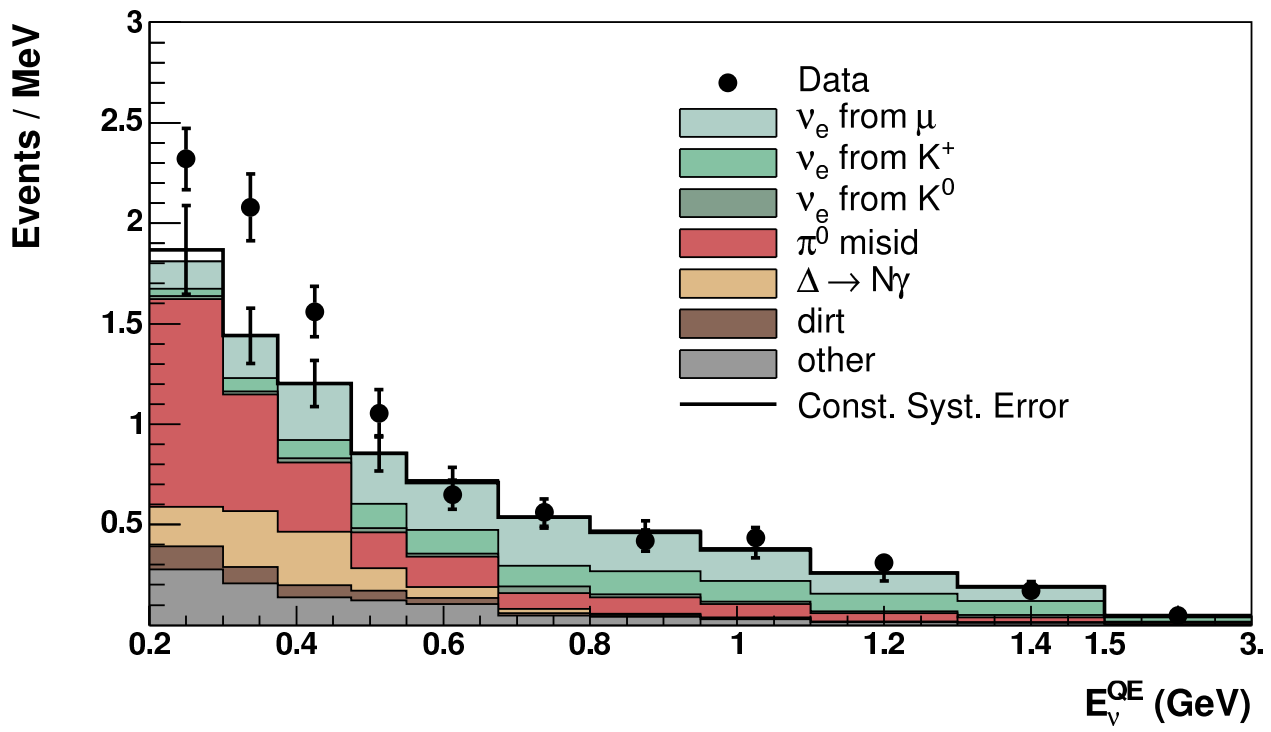

Figure 5-1: The distribution of $\nu_{e}$ CCQE observed data (points with statistical errors) and background prediction (histogram with constrained systematic errors) in neutrino mode, as a function of the full reconstructed neutrino energy range, $200<E_{\nu}^{Q E}<$ $3000 \mathrm{MeV}$.

dence of any signal from simple, two-neutrino $\nu_{\mu} \rightarrow \nu_{e}$ oscillations is seen, MiniBooNE still observes an excess of $\nu_{e}$-like events in the reconstructed neutrino energy range from 200 to $475 \mathrm{MeV} .^{2}$ Compared to an expectation of $415.2 \pm 43.4$ events, a total of $544 \nu_{e}$ CCQE candidate events are observed in this energy range, corresponding to an excess of $128.8 \pm 20.4 \pm 38.3$ events, or $3.0 \sigma$.

Figure 5-4 shows the observed excess of events (background-subtracted observed data) as a function of reconstructed neutrino energy and as a function of reconstructed visible energy of the outgoing electron, $E_{v i s}$. In both distributions, the excess is overlaid with various signal predictions for LSND-allowed $\nu_{\mu} \rightarrow \nu_{e}$ oscillations, including the one corresponding to the MiniBooNE best-fit parameters from a fit to $E_{\nu}^{Q E}>200$ $\mathrm{MeV}$. None of the sets of oscillation parameters within the LSND allowed region, including the best-fit parameters, can provide an adequate explanation of the sharplypeaked observed excess in terms of $\nu_{\mu} \rightarrow \nu_{e}$ oscillations. As shown by the overlaid signal predictions in solid lines, sets of parameters which can accommodate a large

\footnotetext{
${ }^{2} \mathrm{~A}$ similar excess was evident in the original $\nu_{e}$ appearance analysis at the $3.6 \sigma$ level [102].
} 
excess at low energy also contribute significantly to intermediate energies, where the background prediction is well constrained by the $\nu_{\mu}$ CCQE sample and no excess is observed. As a reference for the reader, the $\chi^{2} /$ d.o.f. between data and background plus signal prediction corresponding to $\sin ^{2} 2 \theta=0.2$ and $\Delta m^{2}=0.1 \mathrm{eV}^{2}$, shown by the magenta line in Fig. 5-4, is 21.6/16; therefore, even though those particular parameters could account for the excess at low energy, the fit excludes that possibility by making use of the strong energy-dependent correlations which enter through the off-diagonals of the covariance matrix.

Assuming no oscillations, a combined fit of $\nu_{e}$ and $\nu_{\mu}$ CCQE distributions with the $\nu_{e}$ CCQE reconstructed neutrino energy range restricted to $475-3000 \mathrm{MeV}\left(E_{\nu}^{Q E}>\right.$ $475 \mathrm{MeV}$ fit) yields a null $\chi^{2} /$ d.o.f. of $9.1 / 15$. The corresponding $\chi^{2}$-probability is $87 \%$. Once a fit to two-neutrino oscillations is performed, the resulting $\chi^{2} /$ d.o.f. at the best fit is $7.2 / 13$, corresponding to a $\chi^{2}$ probability of $89 \%$ for the best-fit parameters:

$$
\left(\Delta m^{2}, \sin ^{2} 2 \theta\right)=\left(3.46 \mathrm{eV}^{2}, 0.0012\right)
$$

In the two-neutrino oscillation scenario, the reduction in $\chi^{2}$ relative to the null hypothesis of $\Delta \chi^{2}=\chi^{2}-\chi_{\text {best-fit }}^{2}=8.8-7.2=1.6$, for 2 d.o.f., is not significant at $90 \%$ C.L. ${ }^{3}$

Consequently, in neutrino mode, MiniBooNE places an exclusion limit to twoneutrino oscillations at the $\Delta m^{2}$ range suggested by the LSND excess, as shown in Fig. 5-2. The limit presented here, corresponding to $6.46 \times 10^{20} \mathrm{POT}$, is comparable to that of the original neutrino oscillation analysis [156], indicated on the same figure by the black dashed line.

Note that a fit to oscillations over the full 200-3000 MeV range yields a best-fit $\chi^{2} /$ d.o.f. of $18.2 / 16$, corresponding to a $\chi^{2}$ probability of $31 \%$, and $\Delta \chi^{2}=22.6-$ $18.2=4.4$ relative to the null hypothesis, for 2 degrees of freedom, which is not significant to allow for oscillations at 90\% C.L. The fit to oscillations over the 200-

\footnotetext{
${ }^{3}$ Here we have used the $\Delta \chi^{2}$ definition of a $2 \mathrm{D}$ search for oscillations, where the error matrix used for both the null and best-fit $\chi^{2}$ calculation corresponds to the best-fit prediction, as discussed in Sec. 4.4.5.
} 


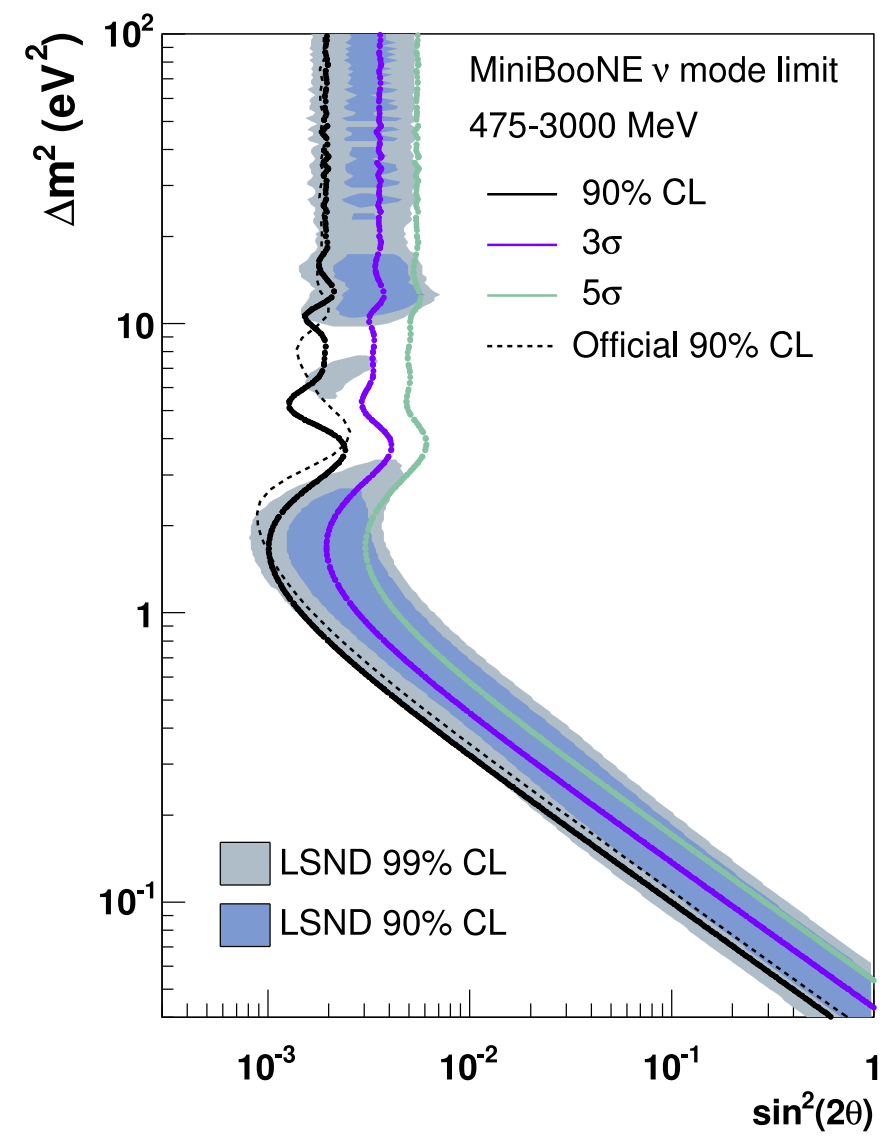

Figure 5-2: MiniBooNE's final limit to two-neutrino $\nu_{\mu} \rightarrow \nu_{e}$ oscillations in neutrino mode, for $6.46 \times 10^{20}$ POT. The dashed line corresponds to MiniBooNE's official result, from [156].

$3000 \mathrm{MeV}$ range yields the exclusion limits in Fig. 5-3. The fit to $E_{\nu}^{Q E}>200 \mathrm{MeV}$ also excludes the majority of the $90 \%$ C.L. allowed region of LSND.

\subsubsection{The Low Energy Excess}

As the low energy excess seems incompatible with two-neutrino oscillations at the LSND scale, it poses the question of whether it could be new physics, or whether it could be due to mis-estimated background. There are several physics interpretations that have been proposed as possible sources of this excess, such as non-standard sterile neutrino oscillations, or new interactions (both SM and non-SM). A nice review is 


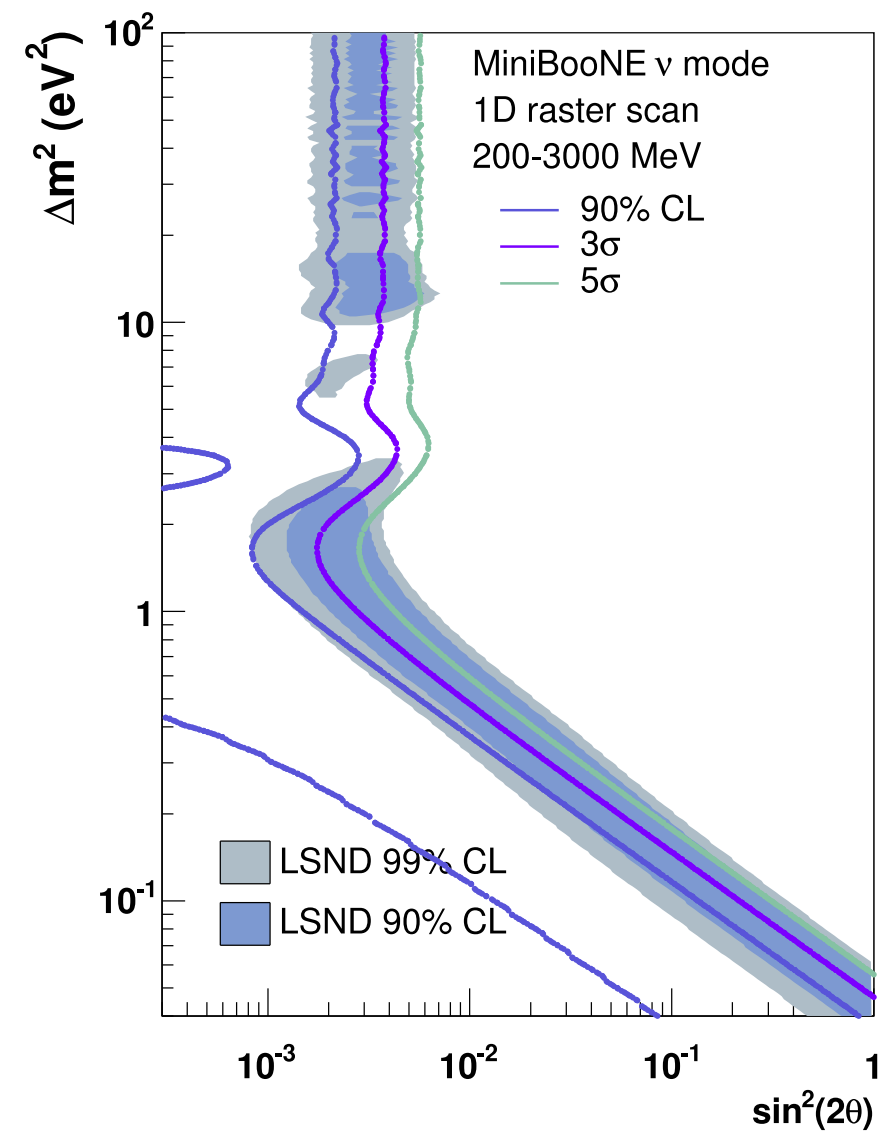

Figure 5-3: Limit to two-neutrino $\nu_{\mu} \rightarrow \nu_{e}$ oscillations in neutrino mode from a fit to $E_{\nu}^{Q E}>200 \mathrm{MeV}$, for $6.46 \times 10^{20} \mathrm{POT}$. 

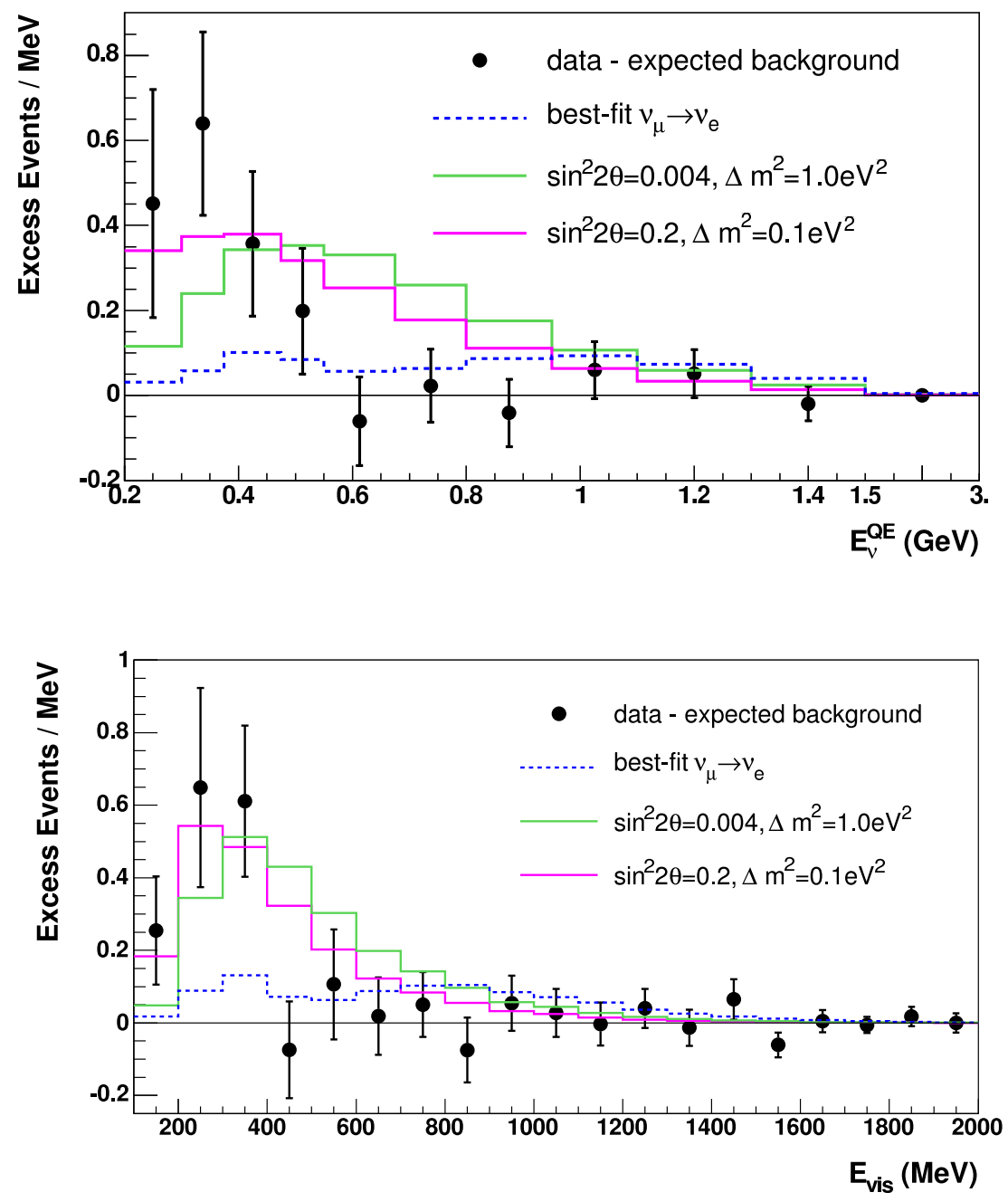

Figure 5-4: The $E_{\nu}^{Q E}$ (top) and $E_{v i s}$ (bottom) excess distributions (data-background prediction) with data statistical and background constrained systematic uncertainties in neutrino mode. Overlaid are the absolute predictions for various $\nu_{\mu} \rightarrow \nu_{e}$ oscillation signals, including that predicted by the $E_{\nu}^{Q E}$ best fit parameters, and two other predicted by two sets of oscillation parameters from the LSND allowed region. The distributions correspond to events with $200<E_{\nu}^{Q E}<3000 \mathrm{MeV}$. 
given in [179]. We will specifically consider the possibility of $(3+2)$ sterile neutrino oscillations with $C P$ violation in the following chapter. In the remainder of this section, we consider the possibility of the low energy excess being due to a possible background mis-estimation.

Of course, a comprehensive set of low-level cross-checks have been performed showing that the observed $\nu_{e}$ CCQE events in the $200-475 \mathrm{MeV}$ region do not look unusual as compared to the rest of the $\nu_{e}$ CCQE data set. Specifically, the observed low energy events are distributed evenly in time over the full MiniBooNE neutrino run, their reconstructed vertices are distributed evenly within the fiducial volume of the detector, and their reconstructed $R_{\text {back-to-wall }}$ and likelihood distributions show evenly distributed excesses with respect to MonteCarlo predicted $\nu_{e}$-like events [180].

Nevertheless, of particular interest are the $\mathrm{NC} \pi^{0}, \Delta \rightarrow N \gamma$ radiative decay and dirt backgrounds. Due to their NC nature, those backgrounds are reconstructed at low energy, and contribute the majority of background events in the 200-475 MeV range. To obtain additional handles on various background hypotheses, we restrict the studies to events in the low energy region where the excess is most significant (300$475 \mathrm{MeV})$, and compare the observed events to various background expectations as a function of three other reconstructed kinematic variables: $E_{v i s}, Q^{2}$, and $\cos \theta_{z} . E_{v i s}$ corresponds to the visible (kinetic) energy of the outgoing electron, as above. $\theta_{z}$ is the reconstructed scattering angle of the outgoing electron with respect to the incident neutrino direction, assuming CCQE scattering. $Q^{2}$, the four-momentum transfer in the neutrino interaction, is determined from $E_{v i s}$ and $\cos \theta_{z}$, also assuming CCQE scattering.

Figure 5-5 shows a comparison of the observed data and total background for events with $300<E_{\nu}^{Q E}<475 \mathrm{MeV}$, as a function of the above three kinematic variables. The total background in each distribution is also broken up in two classes: backgrounds from intrinsic $\nu_{e}$ produced in $K^{+}, K^{0}$, and $\pi^{+} \rightarrow \mu^{+}$beam decays, and backgrounds from $\nu_{\mu}$ mis-identified events, including mostly $\mathrm{NC} \pi^{0}, \Delta \rightarrow N \gamma$, and dirt events. As in the case of the $E_{\nu}^{Q E}$ distribution, in all three kinematic distributions, the backgrounds which most significantly contribute to the kinematic ranges where 

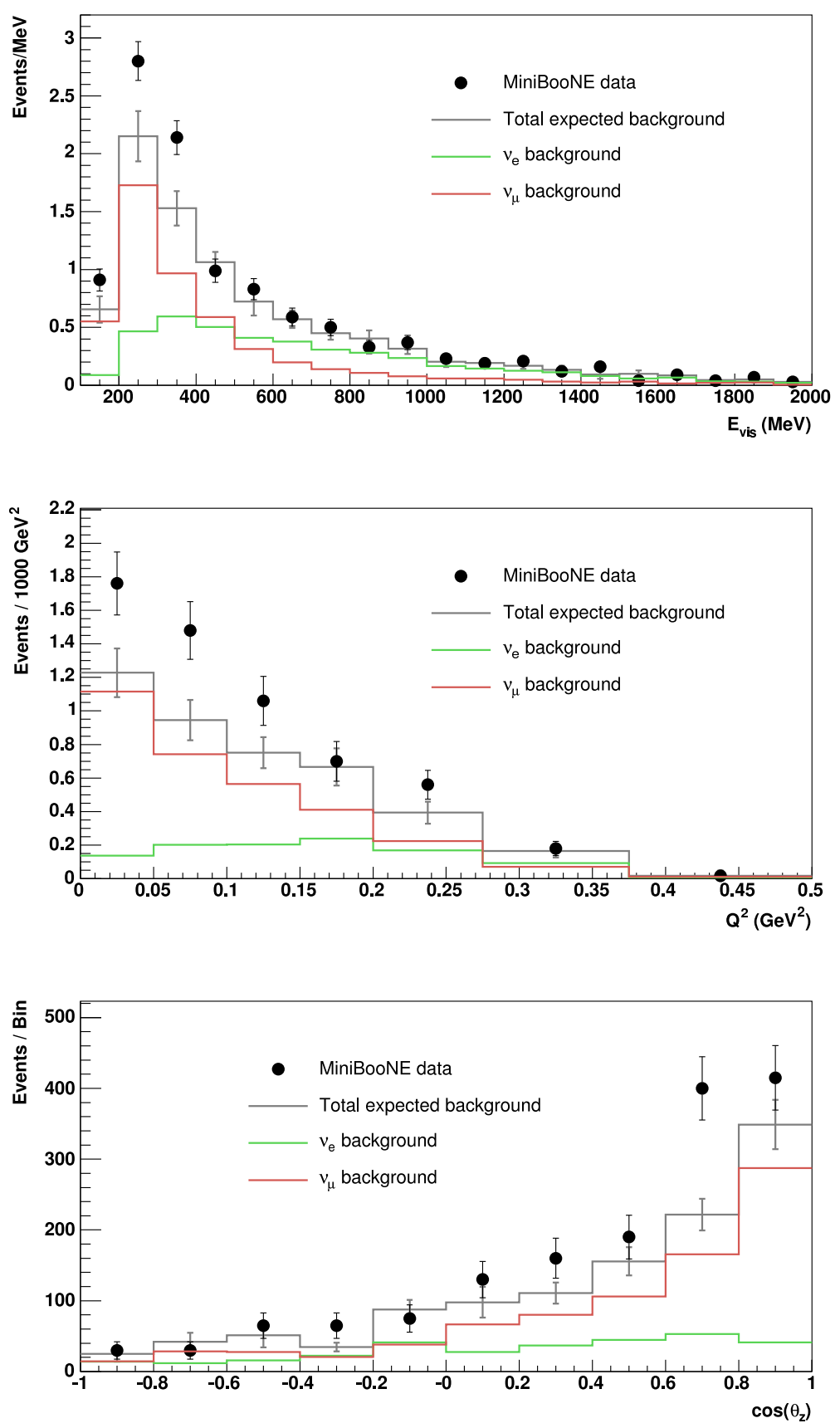

Figure 5-5: The $E_{\text {vis }}$ (top), $Q^{2}$ (middle) and $\cos \theta_{z}$ (bottom) distributions for data (points with statistical error bars) and $\nu_{e}$ CCQE background (histogram with constrained systematic error bars), for events with $300<E_{\nu}^{Q E}<475 \mathrm{MeV}$. Also shown are the expected distributions from intrinsic $\nu_{e}$ backgrounds from $K^{+}, K^{0}$, and $\pi^{+} \rightarrow \mu^{+}$ decays, as well as $\nu_{\mu}$ mis-identified backgrounds (NC $\pi^{0}, \Delta \rightarrow N \gamma$, and dirt). 

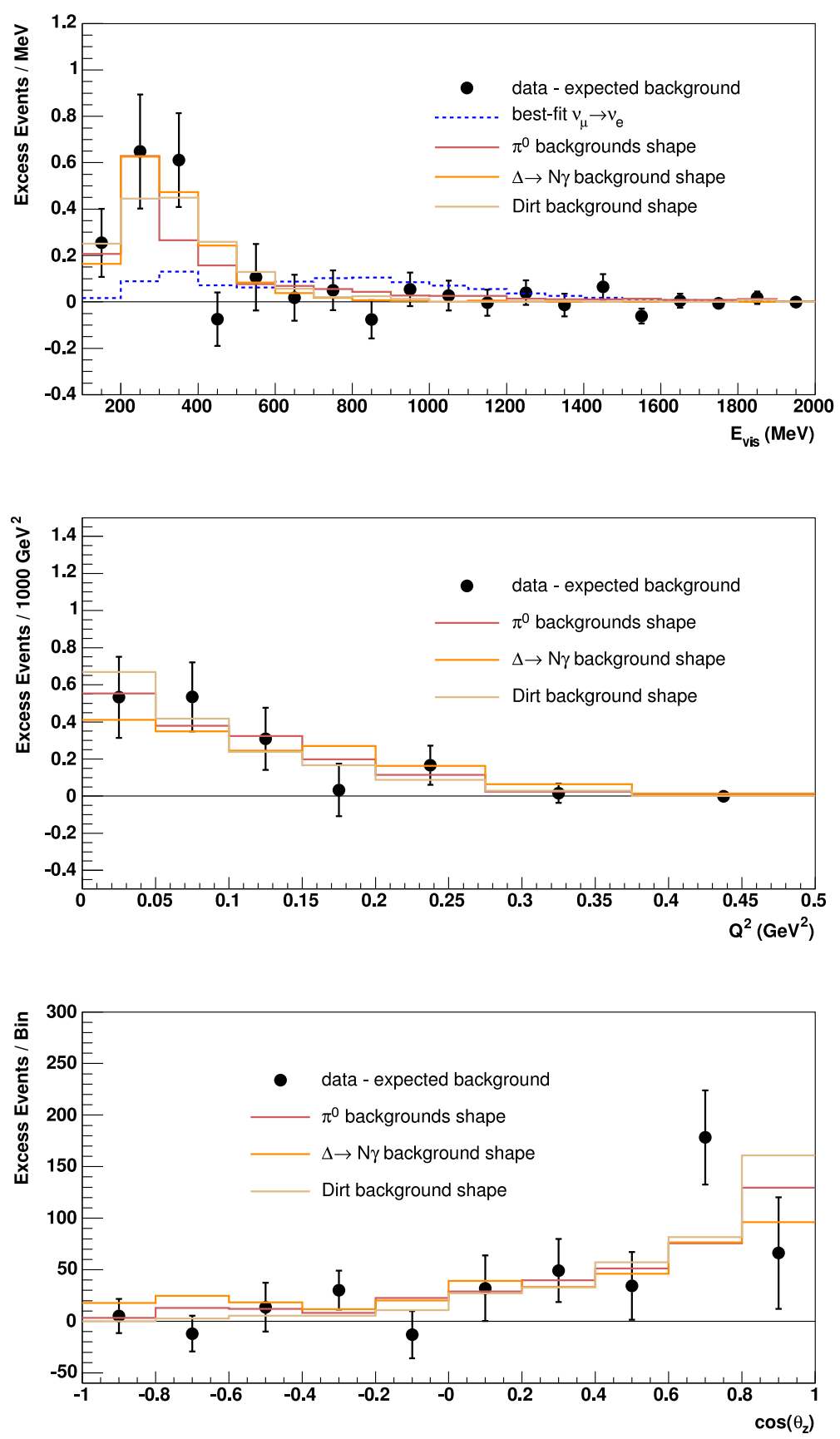

Figure 5-6: The $E_{v i s}$ (top), $Q^{2}$ (middle) and $\cos \theta_{z}$ (bottom) excess distributions (data-background prediction) with data statistical and background constrained shapeonly systematic uncertainties in neutrino mode. Overlaid are the shapes of $\nu_{\mu}$ and $\bar{\nu}_{\mu}$ mis-identified backgrounds in the $\nu_{e}$ CCQE sample (NC $\pi^{0}, \Delta \rightarrow N \gamma$, and dirt). The distributions correspond to events with $300<E_{\nu}^{Q E}<475 \mathrm{MeV}$. 

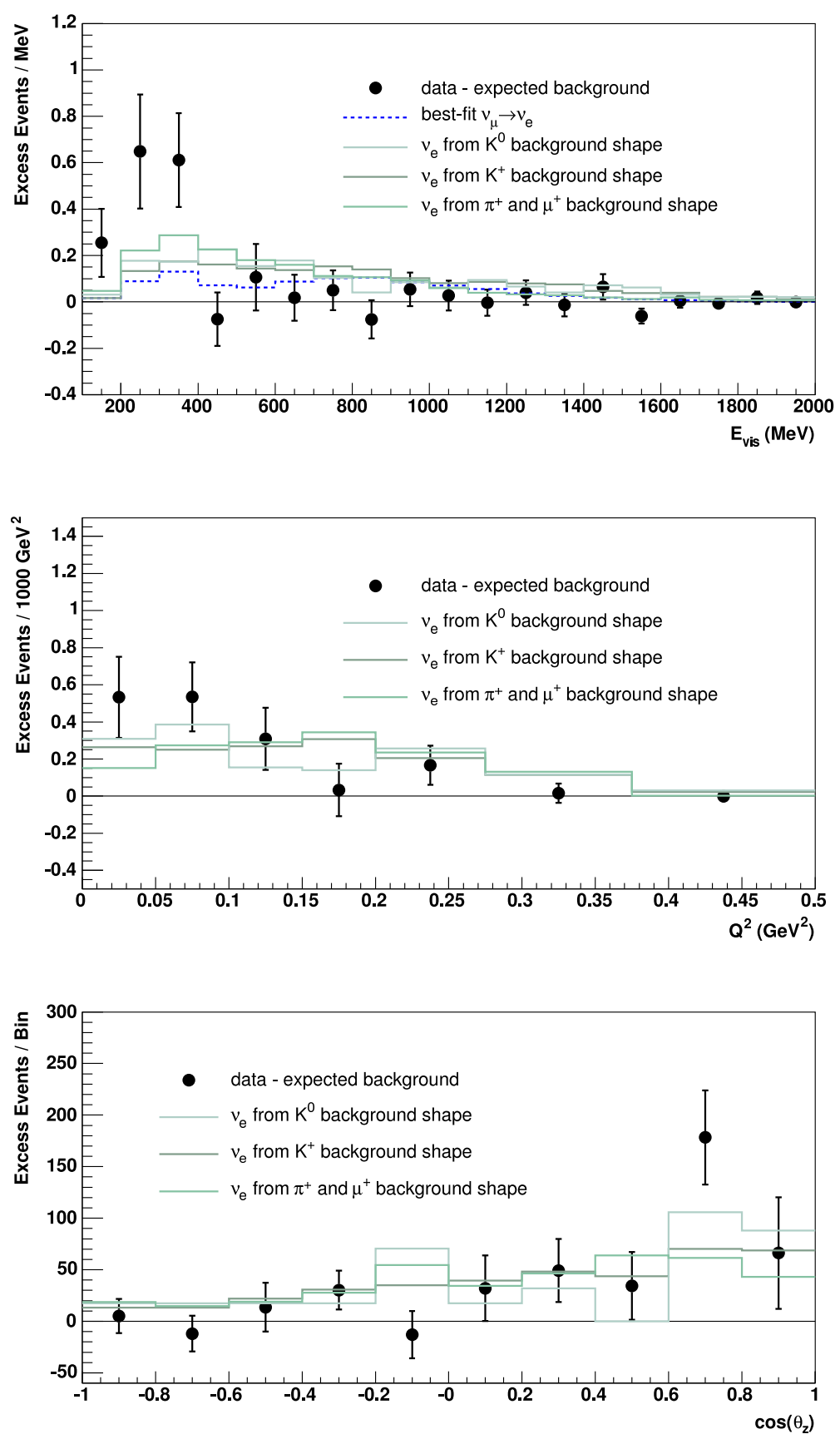

Figure 5-7: The $E_{v i s}$ (top), $Q^{2}$ (middle) and $\cos \theta_{z}$ (bottom) excess distributions (data-background prediction) with data statistical and background constrained shapeonly systematic uncertainties in neutrino mode. Overlaid are the shapes of intrinsic $\nu_{e}$ and $\bar{\nu}_{e}$ backgrounds from $K^{ \pm}, K^{0}$, and $\pi \rightarrow \mu$ decays. The distributions correspond to events with $300<E_{\nu}^{Q E}<475 \mathrm{MeV}$. 


\begin{tabular}{cccc}
\hline \hline Process & $\chi_{\cos \theta_{z}}^{2} / 9$ d.o.f. & $\chi_{Q^{2}}^{2} / 6$ d.o.f. & Factor increase \\
\hline \hline $\mathrm{NC} \pi^{0}$ & 13.46 & 2.18 & 2.0 \\
$\Delta \rightarrow N \gamma$ & 16.85 & 4.46 & 2.7 \\
$\nu_{e} C \rightarrow e^{-} X$ & 14.58 & 8.72 & 2.4 \\
$\bar{\nu}_{e} C \rightarrow e^{+} X$ & 10.11 & 2.44 & 65.4 \\
\hline \hline
\end{tabular}

Table 5.1: The $\chi^{2}$ values from comparisons of the event excess $Q^{2}$ and $\cos \theta_{z}$ distributions for $300<E_{\nu}^{Q E}<475 \mathrm{MeV}$ to the expected shapes from various NC and $\mathrm{CC}$ reactions. Also shown is the factor increase necessary for the estimated background for each process to explain the low-energy excess.

the excess is seen are $\nu_{\mu}$-induced.

Figures 5-6 and 5-7 show comparisons of the observed excess corresponding to each kinematic distribution of Fig. 5-7 to the predicted (relatively-normalized) shapes of different types of background: Fig. 5-6 considers the possibility that the excess in the $300-475 \mathrm{MeV}$ is due to mis-estimation of $\nu_{\mu} \mathrm{NC}$ induced backgrounds, which contribute a single photon in the detector, and Fig. 5-7 considers the possibility that the excess is due to mis-estimation of intrinsic $\nu_{e}$ backgrounds. The error bars correspond to constrained shape-only (see App. A.3) systematic uncertainties (on the total constrained background) plus data statistical uncertainty, propagated in quadrature. Indeed, the background hypotheses that seem to best match the shape of the excess, particularly as a function of $E_{v i s}$, are those of $\nu_{\mu}$-induced NC singlephoton backgrounds.

To properly quantify excess shape agreement to each background hypothesis, we calculate the $\chi^{2}$ between the observed excess and each relatively-normalized background prediction for the $\cos \theta_{z}$ and $Q^{2}$ distributions. The $\chi^{2}$ calculations are performed over the $\nu_{e}$-only CCQE distributions, assuming no oscillations, using shapeonly information. The calculation makes use of the $\nu_{e}$ CCQE part of the $\nu_{\mu}$ CCQEconstrained systematics covariance matrix $M_{i j}^{\text {constr.sys }}$, extracted separately for each kinematic distribution, where the overall normalization uncertainty has been removed as discussed in Appendix A.3.

Table 5.1 shows the resulting $\chi^{2}$ values for the two most preferred NC hypotheses: $\mathrm{NC} \pi^{0}$ and $\Delta \rightarrow N \gamma$, with the NC $\pi^{0}$ hypothesis yielding the lowest $\chi^{2}$ of the two. The 
required factor increase for each background hypothesis which would be necessary to fully account for the excess is shown on the right column. In the case of $\pi^{0}$, a factor of 2.0 would be necessary to explain the excess as a result of mis-estimated NC $\pi^{0}$ background. In the case of $\Delta \rightarrow N \gamma$, an even larger factor increase of 2.7 would be necessary. Recall that the assigned uncertainties on the overall rate of $\mathrm{NC} \pi^{0}$ and $\Delta \rightarrow N \gamma$ backgrounds are $7 \%$ and $12 \%$, respectively (see Sec. 4.6.2). Therefore, each hypothesis would require a factor increase that corresponds to a deviation from the (constrained) central value prediction of more than $5 \sigma$.

Also shown in Tab. 5.1 are the $\chi^{2}$ 's from a shape fit to two oscillation hypotheses, two-neutrino $\nu_{\mu} \rightarrow \nu_{e}$ oscillations with a flat $0.26 \%$ oscillation probability, and two-neutrino $\bar{\nu}_{\mu} \rightarrow \bar{\nu}_{e}$ oscillations due to the wrong-sign component of the neutrino beam, also assuming a flat $0.26 \%$ oscillation probability, and both assuming CCQE scattering. Largest shape agreement is found in the case of $\bar{\nu}_{e} C \rightarrow e^{+} X$ scattering; this is because the $\bar{\nu}_{\mu}$ component of the flux in neutrino mode peaks at lower energy (see Fig. 4-8 in Sec. 4.3.3). However, if interpreted as two-neutrino $\bar{\nu}_{\mu} \rightarrow \bar{\nu}_{e}$ oscillations, the oscillation probability amplitude that would best describe the size of the excess is maximal, and is incompatible with both the LSND allowed regions and the KARMEN limit.

In summary, while the observed low energy excess is kinematically consistent with the dominant backgrounds to the $\nu_{e}$ CCQE appearance search at low energy, the NC $\pi^{0}$ in situ measurement at MiniBooNE strongly disfavors the possibility of the low energy excess being due to mis-estimation of $\mathrm{NC} \pi^{0}$ and $\Delta \rightarrow N \gamma$ events.

\section{$5.2 \bar{\nu}_{e}$ Appearance Results}

\subsubsection{Oscillation Search}

Figure 5-8 shows the $E_{\nu}^{Q E}$ distribution for the $\nu_{e}$ CCQE observed data and background prediction in antineutrino mode. A total of 277 events pass the $\nu_{e}$ CCQE event selection requirements with $200<E_{\nu}^{Q E}<3000 \mathrm{MeV}$. The data are in excess of the 


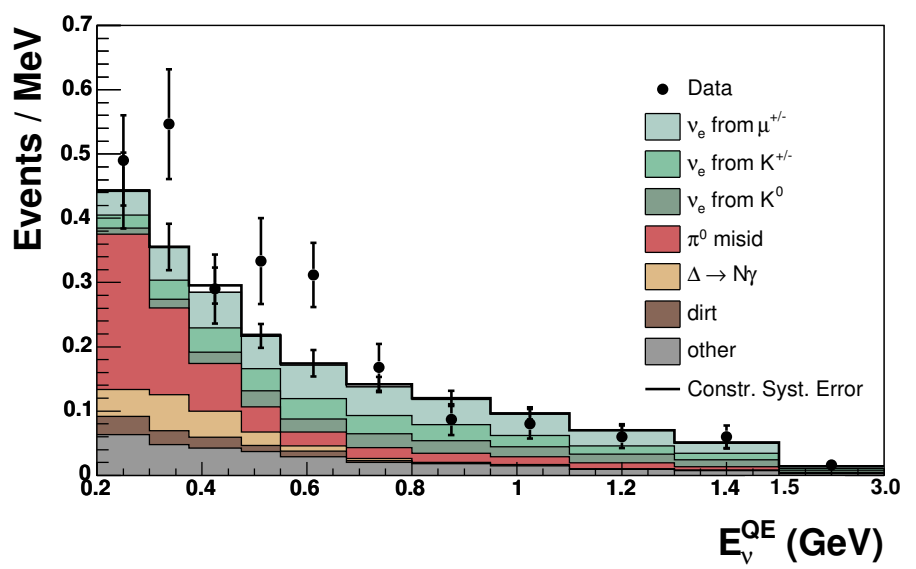

Figure 5-8: The distribution of $\nu_{e}$ CCQE observed data (points with statistical errors) and background prediction (histogram with constrained systematic errors), as a function of the full reconstructed neutrino energy range, $200<E_{\nu}^{Q E}<3000 \mathrm{MeV}$. Antineutrino mode.

background prediction of $233.8 \pm 22.5$ events in the corresponding energy range. The $\chi^{2} /$ d.o.f. from a fit over the full reconstructed neutrino energy range, 200-3000 MeV, assuming no oscillations is $33.2 / 18$, corresponding to a $\chi^{2}$-probability of $1.6 \%$.

The event excess as a function of $E_{\nu}^{Q E}$ is shown in Fig. 5-10, for both $200<$ $E_{\nu}^{Q E}<3000 \mathrm{MeV}$ (top figure), and $475<E_{\nu}^{Q E}<3000 \mathrm{MeV}$ (bottom figure). The small excess $(2.8 \sigma)$ originally seen in the $475-675 \mathrm{MeV}$ region in [102] has now become more evident, and the overall deficit which was seen in [102] at higher energies has now decreased. The present excess significance in the $475-675 \mathrm{MeV}$ region is $3.6 \sigma$. The excess significance over the full reconstructed energy range, 200-3000 MeV, corresponds to $1.9 \sigma$. A $1.3 \sigma$ excess is observed at low energy (200-475 MeV), while a $1.4 \sigma$ excess is observed at high energy $(475-3000 \mathrm{MeV})$.

The $\chi^{2}$ from a fit to $475-3000 \mathrm{MeV}$ (default oscillation fit energy range) assuming no oscillations corresponds to $\chi^{2} /$ d.o.f $=26.7 / 15$, and a $\chi^{2}$-probability of $3.1 \%$. The fit to oscillations above $475 \mathrm{MeV}$ yields a $\chi^{2} /$ d.o.f. at best fit of 16.4/13, corresponding to a $\chi^{2}$-probability of $23.0 \%$, and a change in $\chi^{2}$ relative to the null parameters of $\Delta \chi^{2}=\chi_{\text {null }}^{2}-\chi_{\text {best }-f i t}^{2}=21.1-16.3=4.8$, which is significant at $96 \%$ C.L., given the effective change in degrees of freedom in the fit $($ d.o.f. $=1.2)$. The best fit parameters 


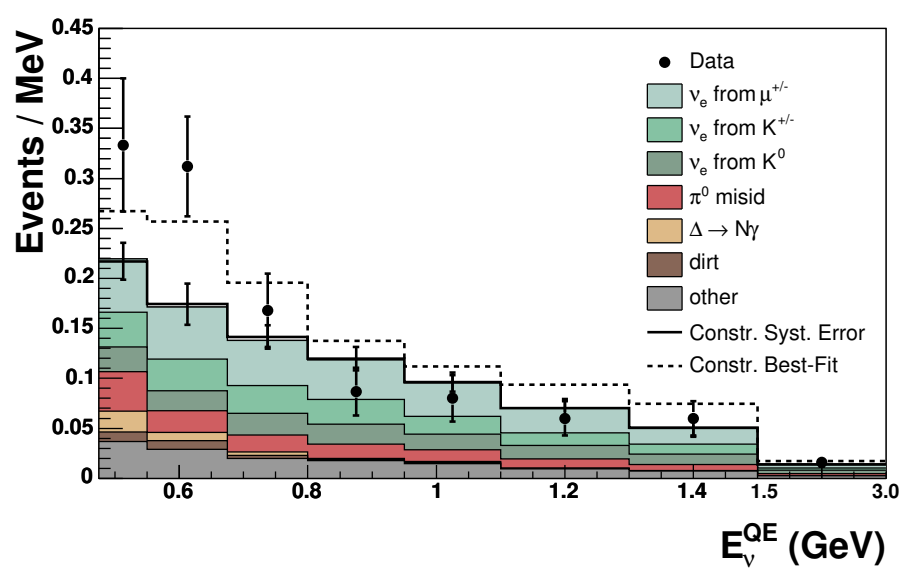

Figure 5-9: The distribution of $\nu_{e}$ CCQE observed data (points with statistical errors) and background prediction (stacked colored histogram with constrained systematic errors), as a function of reconstructed neutrino energy in the range $475<E_{\nu}^{Q E}<3000$ $\mathrm{MeV}$, in antineutrino mode. The dashed distribution corresponds to the total background and $\bar{\nu}_{\mu} \rightarrow \bar{\nu}_{e}$ signal prediction using the best-fit parameters from an oscillation fit to $475<E_{\nu}^{Q E}<3000 \mathrm{MeV}\left(\Delta m^{2}=4.64 \mathrm{eV}^{2}, \sin ^{2} 2 \theta=0.0063\right)$.

correspond to

$$
\left(\Delta m^{2}, \sin ^{2} 2 \theta\right)=\left(4.64 \mathrm{eV}^{2}, 0.0063\right)
$$

Figure 5-9 shows the $E_{\nu}^{Q E}$ distribution for $\nu_{e}$ CCQE observed data and background prediction for $475<E_{\nu}^{Q E}<3000 \mathrm{MeV}$, and compares the observed data distribution to that of the background plus best-fit oscillation hypothesis.

Figure 5-10 compares the observed excess to the expected signal from the bestfit $\bar{\nu}_{\mu} \rightarrow \bar{\nu}_{e}$ oscillation parameters returned by each fit, 200-3000 MeV and 475$3000 \mathrm{MeV}$, as well as to those from two other sets of neutrino oscillation parameters from the LSND allowed region. The best-fit oscillation parameters from both fits, 475-3000 MeV and 200-3000 MeV are driven by the observed excess between $475<$ $E_{\nu}^{Q E}<675 \mathrm{MeV}$. For comparison purposes, the oscillation fit to $200<E_{\nu}^{Q E}<3000$ $\mathrm{MeV}$ yields similar best fit parameters, corresponding to $\left(\Delta m^{2}, \sin ^{2} 2 \theta\right)=\left(4.42 \mathrm{eV}^{2}\right.$, 0.0063), with a best-fit $\chi^{2} /$ d.o.f. of $20.6 / 16$, corresponding to a $\chi^{2}$-probability of $19.4 \%$.

The best-fit oscillation parameters returned by the fit are summarized in Tab. 5.2. Both the 200-3000 MeV, and the 475-3000 MeV fit return very similar oscillation 


\begin{tabular}{l|cc}
\hline \hline$E_{\nu}^{Q E}$ range (MeV) & Best-fit $\Delta m^{2}$ & Best-fit $\sin ^{2} 2 \theta$ \\
\hline \hline $200-3000$ & $4.42 \mathrm{eV}^{2}$ & 0.0063 \\
$475-3000$ & $4.64 \mathrm{eV}^{2}$ & 0.0063 \\
\hline \hline
\end{tabular}

Table 5.2: Best-fit oscillation parameters returned from antineutrino appearance fits using $5.66 \times 10^{20}$ POT. For corresponding $\chi^{2}$ values see Tab. 5.4. For comparison, the best-fit parameters from the previous $\bar{\nu}_{e}$ search at MiniBooNE $\left(3.39 \times 10^{20} \mathrm{POT}\right)$ are $\left(\Delta m^{2}, \sin ^{2} 2 \theta\right)=\left(4.42 \mathrm{eV}^{2}, 0.0045\right)$ for $E_{\nu}^{Q E}>200 \mathrm{MeV}$, and $\left(\Delta m^{2}, \sin ^{2} 2 \theta\right)=$ $\left(4.42 \mathrm{eV}^{2}, 0.0047\right)$ for $E_{\nu}^{Q E}>475 \mathrm{MeV}$.

parameters, which are at the level of the LSND best-fit of $\Delta m^{2}=1.2 \mathrm{eV}^{2}$, and $\sin ^{2} 2 \theta=0.003$, but in fact fall outside the LSND 99\% C.L. allowed region from [107]. Note that the allowed regions in [107] are obtained from a combined fit to DAR and DIF data sets, the latter being a neutrino rather than antineutrino dominated sample, with a less significant excess than that seen in the LSND DAR data set. ${ }^{4}$ Nevertheless, there is significant overlap with the LSND allowed region at lower $\Delta m^{2}{ }^{5}$

The number of data, background, and excess events for different $E_{\nu}^{Q E}$ ranges are summarized in Tab. 5.3, and compared to the corresponding results from the neutrino mode appearance search [101].

Figure 5-11 shows the MiniBooNE 90\%, 95\%, and 99\% C.L. allowed regions obtained from a fit to 475-3000 MeV. The contours are drawn using the method described in Sec. 4.4.5, which uses a series of fake experiments to precisely quantify effective degrees of freedom in the fit across the full $\left(\Delta m^{2}, \sin ^{2} 2 \theta\right)$ parameter space. The 475-3000 MeV (default) fit to oscillations excludes the no oscillations hypothesis at $96 \%$ C.L. A fit to $200-3000 \mathrm{MeV}$ also excludes the null point at more than $95 \%$ C.L., as shown in Fig. 5-12, and is in agreement with the fit to 475-3000 MeV. As expected, the effect of the low energy region is negligible on the fit. The null and best fit $\chi^{2}$ 's returned by both fits are summarized in Table 5.4 for various oscillation hypotheses.

\footnotetext{
${ }^{4} \mathrm{~A}$ direct comparison of MiniBooNE antineutrino results from [102] with the LSND DAR (antineutrino-only) results will be seen in Chapter 6 .

${ }^{5}$ The maximum likelihood fit to the MiniBooNE data yields similar allowed regions, with best fit parameters of $\left(\Delta m^{2}, \sin ^{2} 2 \theta\right)=\left(0.064 \mathrm{eV}^{2}, 0.96\right)$ from a fit to $E_{\nu}^{Q E}>475 \mathrm{MeV}$, and $\left(\Delta m^{2}, \sin ^{2} 2 \theta\right)=$ $\left(4.42 \mathrm{eV}^{2}, 0.0066\right)$ from a fit to $E_{\nu}^{Q E}>200 \mathrm{MeV}$.
} 

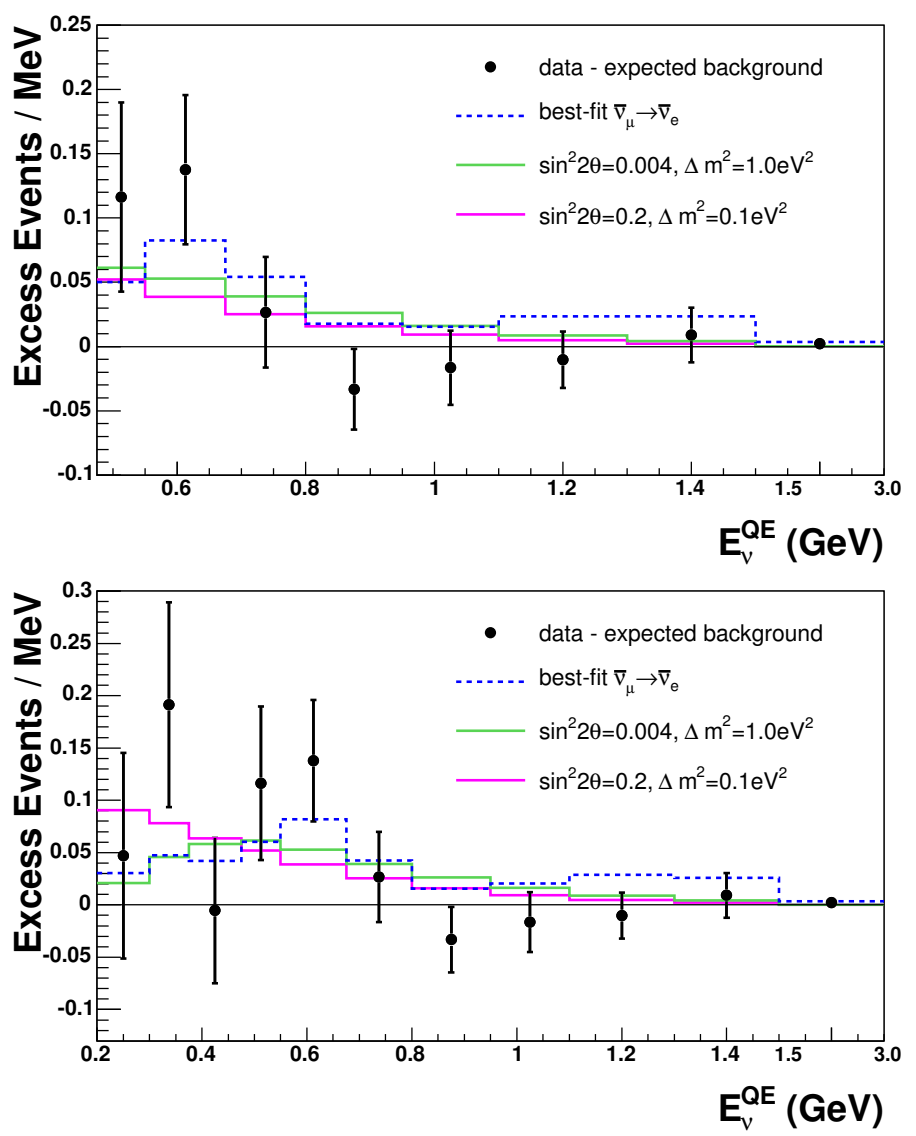

Figure 5-10: The $\nu_{e}$ CCQE event excess as a function of $E_{\nu}^{Q E}$ in antineutrino mode, compared to expectations from the best oscillation fit parameters from a fit to $E_{\nu}^{Q E}>475 \mathrm{MeV}$ (top figure, $\Delta m^{2}=4.64 \mathrm{eV}^{2}$, $\sin ^{2} 2 \theta=0.0063$ ), and a fit to $E_{\nu}^{Q E}>200 \mathrm{MeV}$ (bottom figure, $\Delta m^{2}=4.42 \mathrm{eV}^{2}, \sin ^{2} 2 \theta=0.0063$ ). Also shown are the signal predictions from two other sets of oscillation parameters in the LSND allowed region. The error bars include both data statistical and unconstrained background systematic uncertainties. 


\begin{tabular}{ccc}
\hline \hline Event sample & $\bar{\nu}_{e}$ Analysis & $\nu_{e}$ Analysis \\
& $\left(\mathbf{5 . 6 6} \times \mathbf{1 0}^{20} \mathbf{P O T}\right)$ & $\left(\mathbf{6 . 4 6} \times \mathbf{1 0}^{20} \mathbf{P O T}\right)$ \\
\hline \hline $200-475 \mathrm{MeV}$ & & \\
Data & 119 & 544 \\
Background & $100.5 \pm 14.3$ & $415.2 \pm 43.4$ \\
Excess & $18.5 \pm 14.3(1.3 \sigma)$ & $128.8 \pm 43.4(3.0 \sigma)$ \\
\hline $475-1250 \mathrm{MeV}$ & & \\
Data & 120 & 408 \\
Background & $99.1 \pm 14.0$ & $385.9 \pm 35.7$ \\
Excess & $20.9 \pm 14.0(1.50 \sigma)$ & $22.1 \pm 35.7(0.6 \sigma)$ \\
\hline \hline
\end{tabular}

Table 5.3: Summary of data, background, excess events, and excess significance in the MiniBooNE $\nu_{e}$ and $\bar{\nu}_{e}$ appearance analyses for different $E_{\nu}^{Q E}$ ranges. The uncertainties include both statistical and constrained systematic errors.

\begin{tabular}{l|cccc}
\hline \hline$E_{\nu}^{Q E}$ range $(\mathrm{MeV})$ & $\chi_{\text {null }}^{2} /$ d.o.f. & $\chi_{\text {null }}^{2} /$ d.o.f. & $\chi_{\text {bf }}^{2} /$ d.o.f. & $\chi_{\text {LSND bf }}^{2} /$ d.o.f. \\
\hline \hline$>200$ & $33.21 / 18$ & $26.28 / 16$ & $20.63 / 16$ & $25.19 / 18$ \\
$>475$ & $26.75 / 15$ & $21.10 / 13$ & $16.26 / 13$ & $19.46 / 15$ \\
\hline \hline
\end{tabular}

Table 5.4: $\chi^{2}$ obtained from fits to $E_{\nu}^{Q E}>200 \mathrm{MeV}$, and $E_{\nu}^{Q E}>475 \mathrm{MeV}$. From left to right: $\chi_{\text {null }}^{2}$, calculated between the data and background prediction with the null (no signal) error matrix; $\chi_{\text {null }}^{2}$, calculated between the data and background prediction with the best-fit error matrix; $\chi_{b f}^{2}$, calculated between the data and background+best-fit signal prediction with the best-fit error matrix; $\chi_{\text {LSNDbest-fit }}^{2}$, calculated between the data and background+signal prediction with LSND best fit parameters with the error matrix corresponding to the LSND best-fit parameters, $\left(\Delta m^{2}, \sin ^{2} 2 \theta\right)=(1.2,0.003)$. The best-fit parameters returned by the fit are summarized in Tab. 5.2.

Compared to the first antineutrino appearance results from MiniBooNE [102], the excess significance has increased at both low (200-475 MeV) and high (above $475 \mathrm{MeV}$ ) energy. Aside from a higher $\sin ^{2} 2 \theta$ preferred, driven by the higher excess significance now evident in the data, the best-fit parameters are consistent with those from [102]. Compatibility between the two results is discussed further in App. A.4.

In summary, with the increased ( $\sim 70 \%$ additional data relative to [102]) antineutrino statistics, MiniBooNE observes an excess of events consistent with LSND-like two-neutrino oscillations, and in a fit to two-neutrino oscillations it excludes the null hypothesis over an oscillation hypothesis at 96\% C.L. 

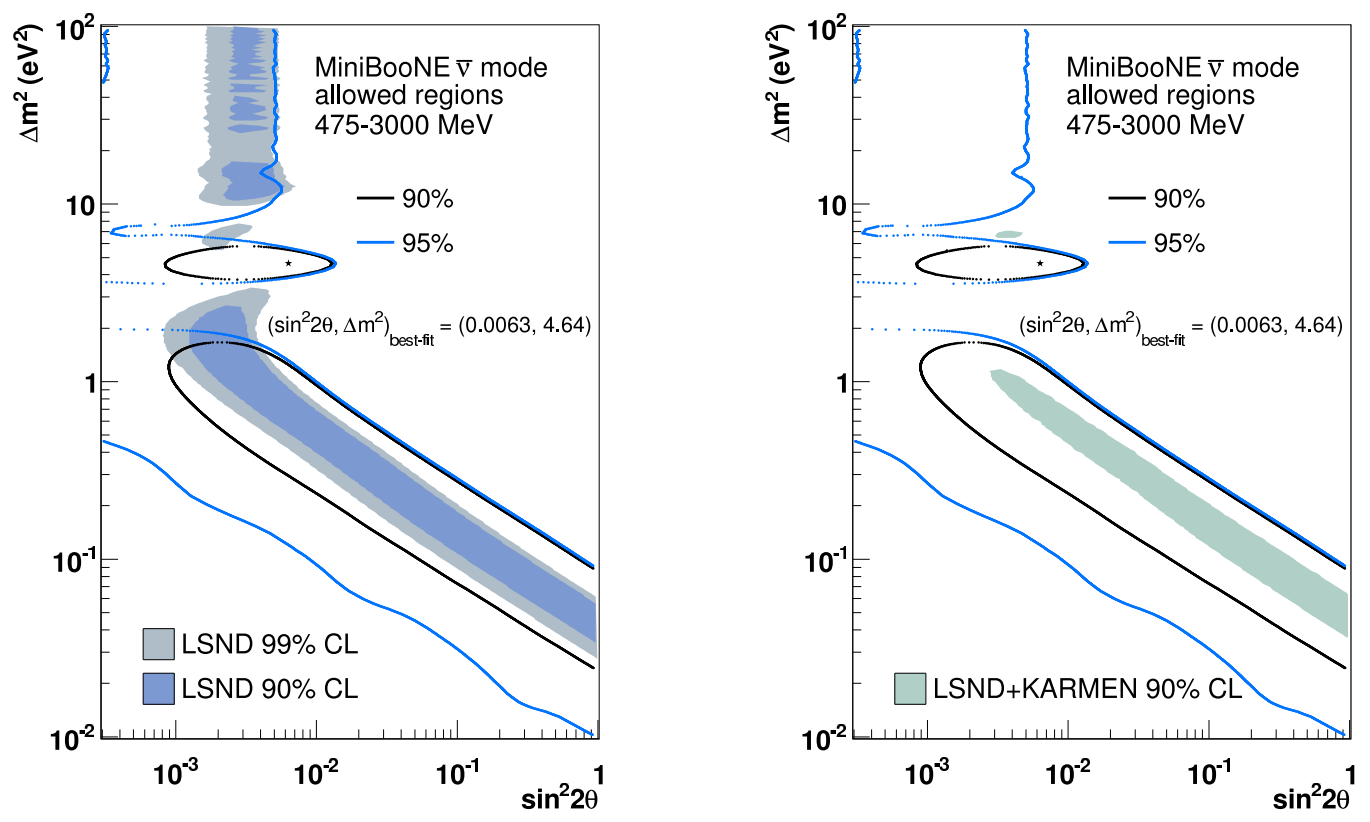

Figure 5-11: MiniBooNE $\bar{\nu}_{\mu} \rightarrow \bar{\nu}_{e} 90 \%$ and $95 \%$ C.L. allowed regions from a fit to $E_{\nu}^{Q E}>475 \mathrm{MeV}$ in antineutrino mode.
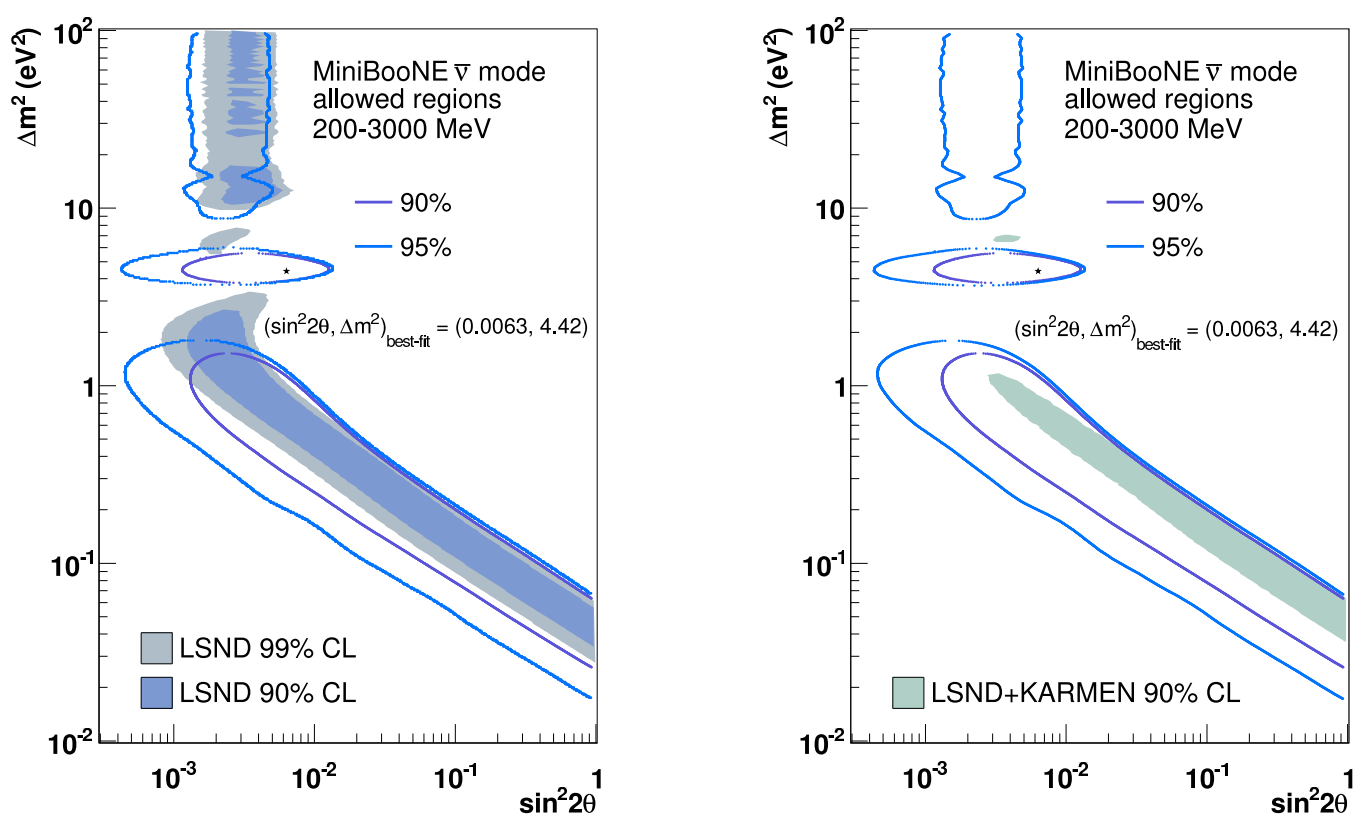

Figure 5-12: MiniBooNE $\bar{\nu}_{\mu} \rightarrow \bar{\nu}_{e} 90 \%$ and $95 \%$ C.L. allowed regions from a fit to $E_{\nu}^{Q E}>200 \mathrm{MeV}$ in antineutrino mode. 


\subsubsection{A Closer Look at the Antineutrino Mode Excess}

Besides $E_{\nu}^{Q E}$, the $\nu_{e}$ CCQE data also show a similar excess over predicted background in other reconstructed kinematic variables. Figure 5-13 shows the observed and predicted event distributions as functions of reconstructed $E_{v i s}, Q^{2}$ and $\cos \theta_{z}$ for events with $200<E_{\nu}^{Q E}<3000 \mathrm{MeV}$. Also shown in the figures are the predicted distributions from intrinsic $\nu_{e}$ and $\bar{\nu}_{e}$ backgrounds, and mis-identified $\nu_{\mu}$ and $\bar{\nu}_{\mu}$ events (mainly NC $\pi^{0}, \Delta \rightarrow N \gamma$ and dirt backgrounds, which are mostly events with a single-photon in the final state). The null $\chi^{2}$ values from these comparisons are high for the case of $E_{v i s}$, at $\chi_{\text {Evis }}^{2} /$ d.o.f. $=23.8 / 13$, but acceptable for $Q^{2}$ and $\cos \theta_{z}$, at $\chi_{Q^{2}}^{2} /$ d.o.f. $=14.5 / 11$, and $\chi_{\cos \theta_{z}}^{2} /$ d.o.f. $=13.6 / 11$. The $\chi^{2}$ values corresponding to the best-fit prediction calculated using the $E_{\nu}^{Q E}>475 \mathrm{MeV}$ best fit parameters $\left(\Delta m^{2}=4.64 \mathrm{eV}^{2}, \sin ^{2} 2 \theta=0.0063\right)$ are $\chi_{E v i s}^{2} /$ d.o.f. $=17.5 / 13, \chi_{Q^{2}}^{2} /$ d.o.f. $=10.7 / 11$, and $\chi_{\cos \theta_{z}}^{2}=12.6 / 11$, supporting the $E_{\nu}^{Q E}$ fit results, in that a signal hypothesis provides a better description of the observed distributions than the no oscillation hypothesis.

A comparison of $Q^{2}, \cos \theta_{z}$, and $E_{v i s}$ excess distributions to the $\bar{\nu}_{\mu} \rightarrow \bar{\nu}_{e}$ signal predicted from the above best-fit parameters is shown in Fig. 5-14. The best-fit signal prediction is in agreement with the excess distribution in all three reconstructed variables.

In order to quantify the level at which various background hypotheses agree with the observed $\nu_{e}$-like excess as a function of each kinematic variable, shape fits are performed to relatively-normalized excess and background predictions, following what was done for the low energy excess investigations in neutrino mode. The resulting background-subtracted observed events are shown as a function of each kinematic variable in Figs. 5-15 and 5-16, and compared with the relatively-normalized shape of each background hypothesis. The error bars correspond to constrained shape-only systematic uncertainties (on the total constrained background) plus data statistical uncertainty, propagated in quadrature. ${ }^{6}$ Unlike in neutrino mode, the shape of the

\footnotetext{
${ }^{6}$ The data statistical uncertainty is the dominant source of uncertainty in these fits.
} 

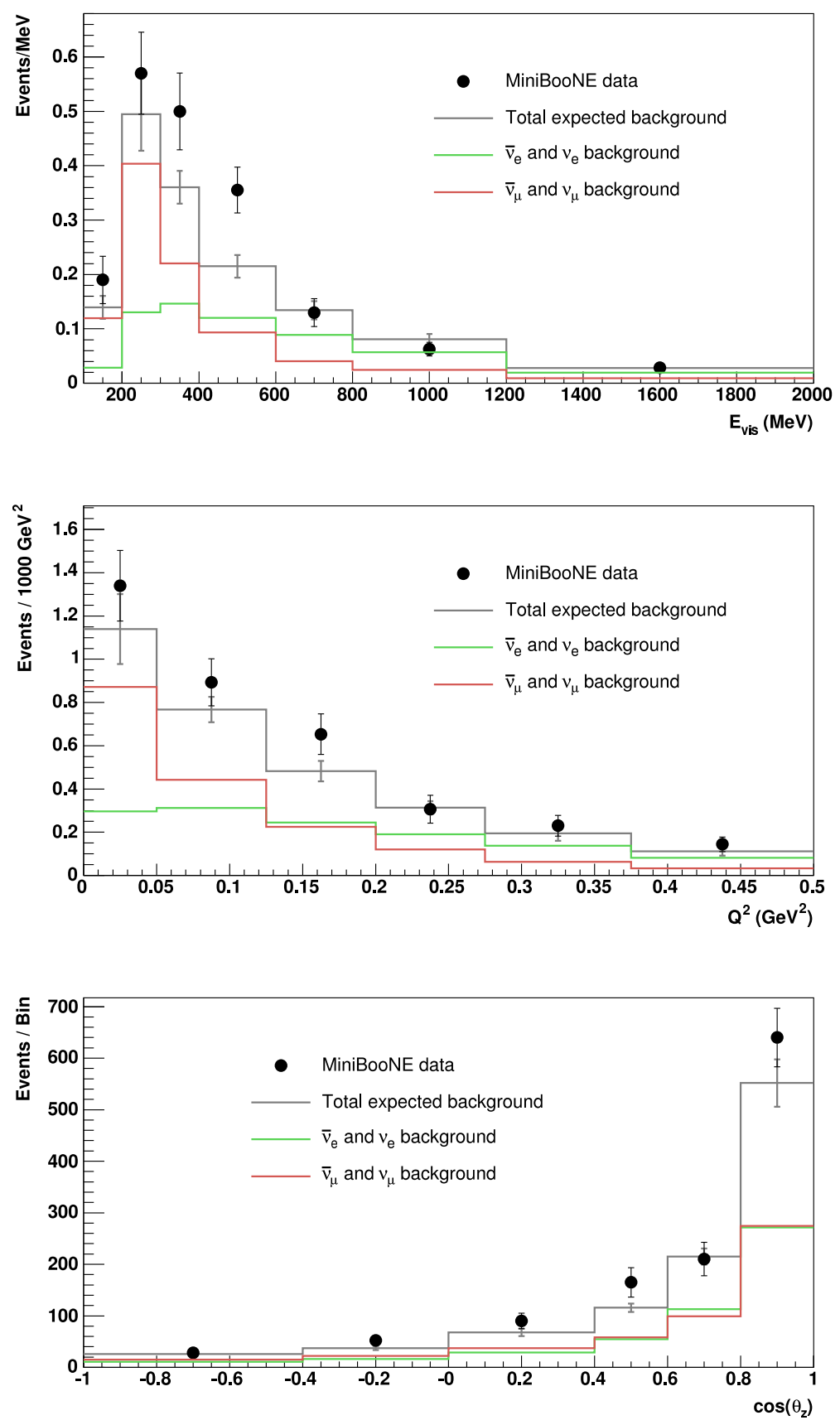

Figure 5-13: The $E_{v i s}$ (top), $Q^{2}$ (middle) and $\cos \theta_{z}$ (bottom) distributions for data (points with statistical error bars) and $\nu_{e}$ CCQE background (histogram with constrained systematic error bars) for events with $200<E_{\nu}^{Q E}<3000 \mathrm{MeV}$, in antineutrino mode. Also shown are the expected distributions from intrinsic $\bar{\nu}_{e}$ and $\nu_{e}$ backgrounds from $K^{ \pm}, K^{0}$, and $\pi \rightarrow \mu$ decays, as well as $\bar{\nu}_{\mu}$ and $\nu_{\mu}$ mis-identified backgrounds (NC $\pi^{0}, \Delta \rightarrow N \gamma$, and dirt). 

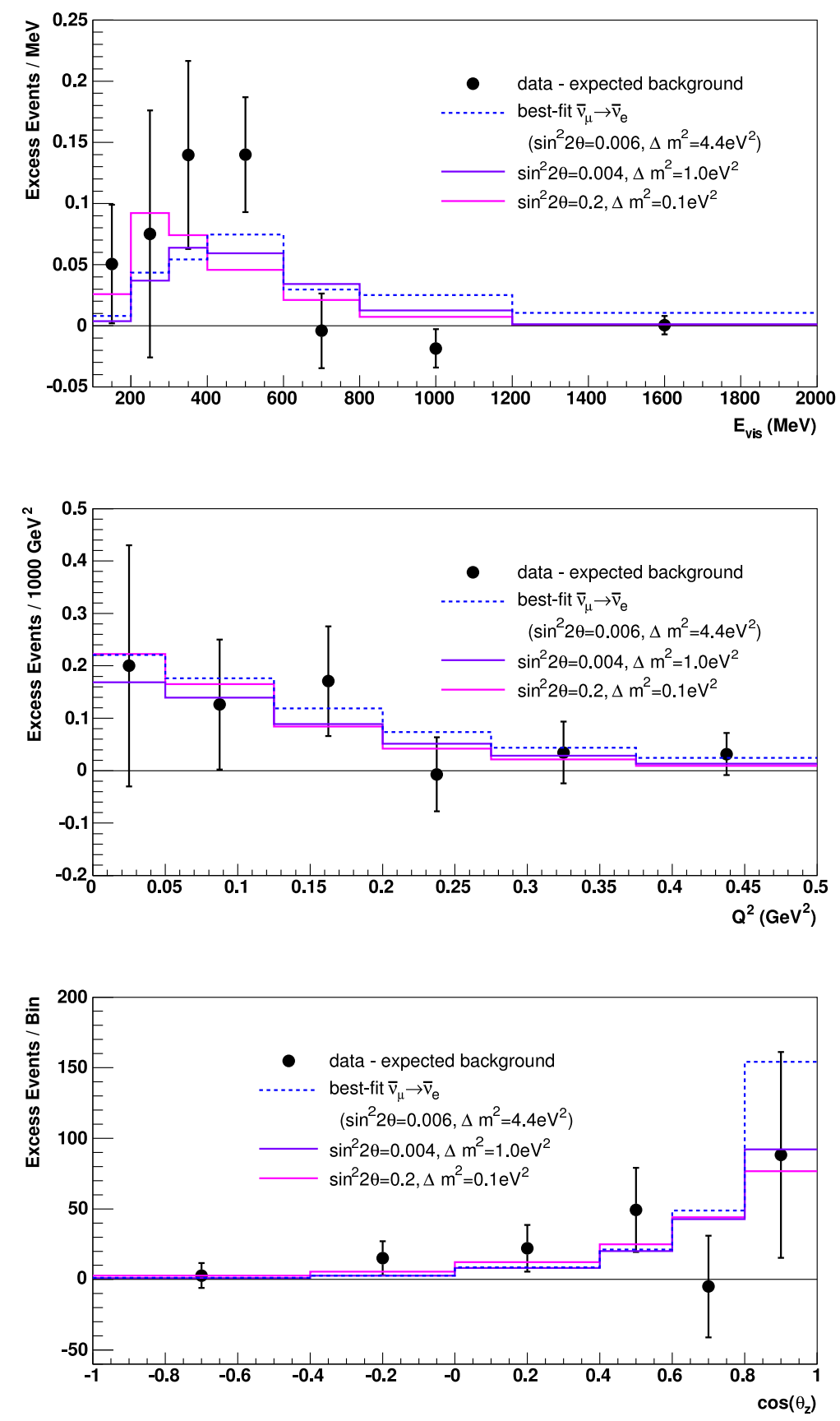

Figure 5-14: The $E_{\text {vis }}$ (top), $Q^{2}$ (middle) and $\cos \theta_{z}$ (bottom) excess distributions (data-background prediction) with data statistical and background constrained systematic uncertainties in antineutrino mode. Overlaid are the absolute predictions for various $\bar{\nu}_{\mu} \rightarrow \bar{\nu}_{e}$ oscillation signals, including that predicted by the $E_{\nu}^{Q E}$ best fit parameters, and two other predicted by two sets of oscillation parameters from the LSND allowed region. The distributions correspond to events with $200<E_{\nu}^{Q E}<3000 \mathrm{MeV}$. 

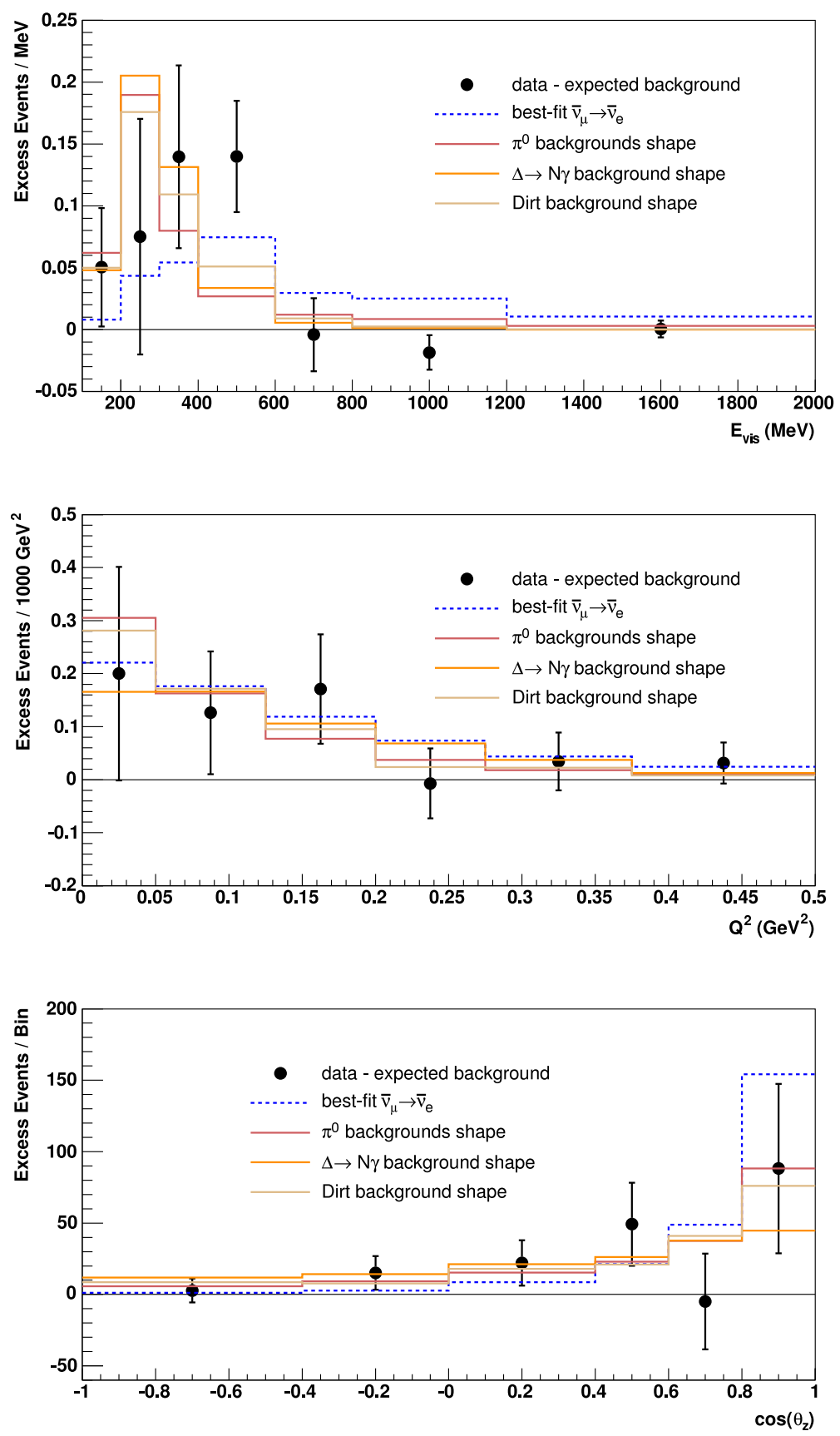

Figure 5-15: The $E_{\text {vis }}$ (top), $Q^{2}$ (middle) and $\cos \theta_{z}$ (bottom) excess distributions (data-background prediction) in antineutrino mode, with data statistical and background constrained shape-only systematic uncertainties. Overlaid are the shapes of $\bar{\nu}_{\mu}$ and $\nu_{\mu}$ mis-identified backgrounds in the $\nu_{e}$ CCQE sample (NC $\pi^{0}, \Delta \rightarrow N \gamma$, and dirt). The distributions correspond to events with $200<E_{\nu}^{Q E}<3000 \mathrm{MeV}$. 

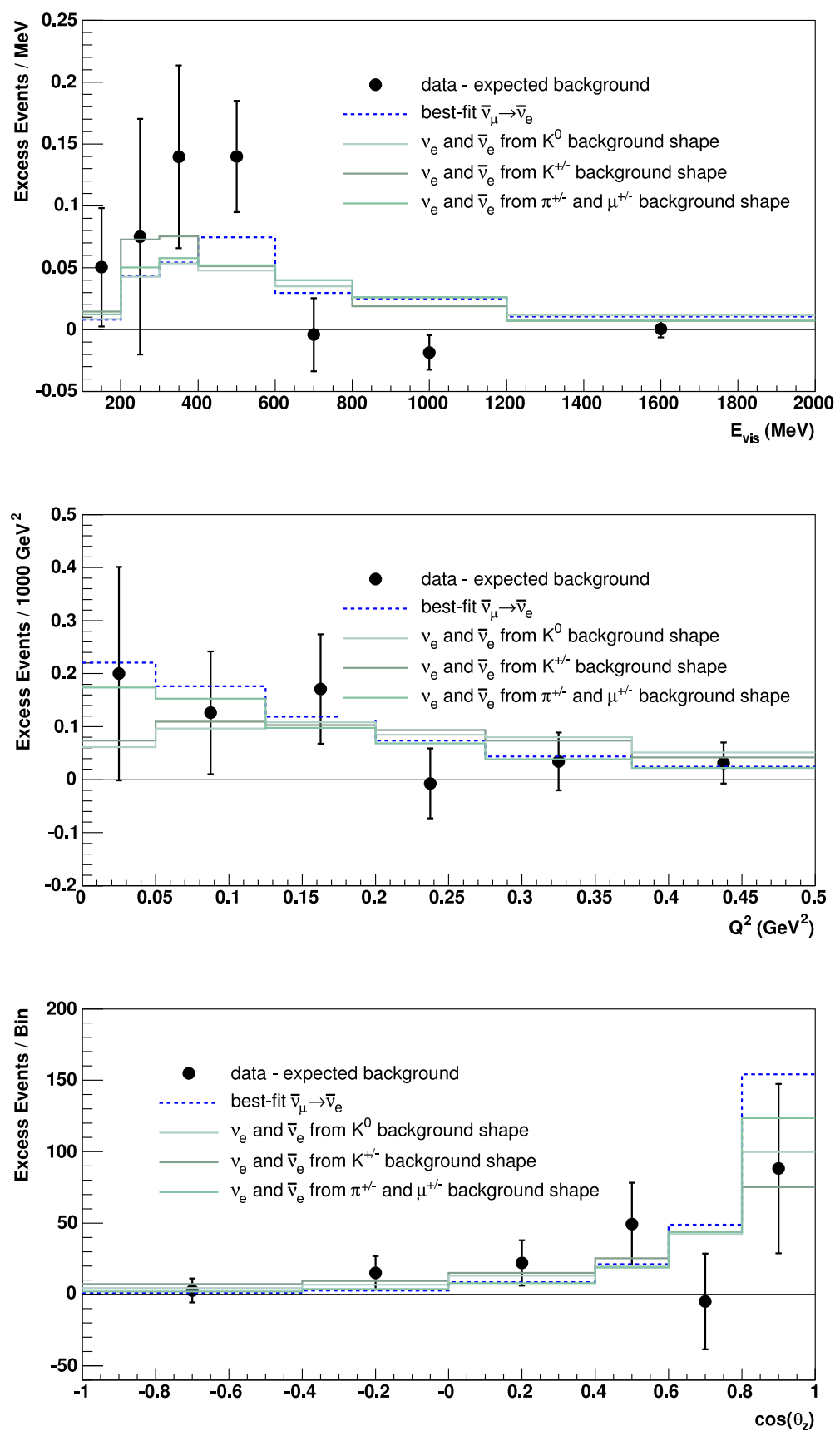

Figure 5-16: The $E_{\text {vis }}$ (top), $Q^{2}$ (middle) and $\cos \theta_{z}$ (bottom) excess distributions (data-background prediction) in antineutrino mode, with data statistical and background constrained shape-only systematic uncertainties. Overlaid are the shapes of intrinsic $\bar{\nu}_{e}$ and $\nu_{e}$ backgrounds from $K^{ \pm}, K^{0}$, and $\pi \rightarrow \mu$ decays. The distributions correspond to events with $200<E_{\nu}^{Q E}<3000 \mathrm{MeV}$. 
observed excess in antineutrino mode, as best illustrated in Fig. 5-15 (top), is not found to particularly resemble that of $\pi^{0}$ or $\Delta$ backgrounds, as those backgrounds are more sharply peaked at lower $E_{v i s}$. The intrinsic $\nu_{e}$ backgrounds do not provide a viable possibility either, as their energy spectra are relatively flat compared to the observed excess.

The resulting $\chi^{2}$ 's are summarized in Tabs. 5.5 through 5.7, for $E_{v i s}, \cos \theta_{z}$, and $Q^{2}$, respectively. Due to large uncertainties, the $Q^{2}$ and $\cos \theta_{z}$ shape-only fits yield $\chi^{2}$ 's which are relatively comparable for all background hypotheses, as well as the best-fit signal prediction. In the case of $E_{v i s}$, however, a larger differentiation among various hypotheses can be seen. In particular, the dirt background shape comparison provides the lowest $\chi^{2}$ and most probable shape hypothesis, by more than 10 units of $\chi^{2}$ relative to the $K^{0}$ shape hypothesis, for example.

For each background hypothesis, the normalization factor by which the total background prediction must be scaled to account for the excess is also given, and compared to the assumed (unconstrained) fractional uncertainty assigned to that particular background in the oscillation analysis. The uncertainty on each background accounts for all production and cross-section contributing uncertainties, and in the case of $\mathrm{NC} \pi^{0}, \Delta$ and dirt backgrounds it includes detector and optical modeling uncertainties.

As in Sec. 5.1.2, we quantify the level at which any particular hypothesis can account for the excess by comparing the factor increase necessary for each background hypothesis to account for the excess to the systematic uncertainty assigned to that particular background. Given the uncertainty on $\pi^{0}, \Delta$ and dirt backgrounds, the required factor increase which would be necessary to account for the excess in each case would imply a background modification at a level of more than $5 \sigma$, in all three reconstructed variables. In the case of intrinsic $\nu_{e}$ backgrounds from $K^{ \pm}, K^{0}$, and $\pi \rightarrow$ $\mu$ decays, the systematic uncertainties to which those backgrounds are susceptible are larger. Table 5.8 lists a more detailed breakup of systematic uncertainty contributions for $\nu_{e}\left(\right.$ and $\bar{\nu}_{e}$ ) from $K^{0}, \pi^{+} \rightarrow \mu^{+}$and $\pi^{-} \rightarrow \mu^{-}$, and $K^{+}$and $K^{-}$. 


\begin{tabular}{|c|c|c|c|c|c|}
\hline Background & Excess events & "Background events & Scale factor & "Background \% uncertainty & $\chi_{\text {constr shape-only }}^{2}$ \\
\hline$\overline{\bar{\pi} \pi^{0}}$ & 46.73 & 59.14 & 1.79 & $13.6(7.4)$ & 13.49 \\
\hline$\Delta$ & 46.73 & 15.86 & 3.95 & $15.5(14.2)$ & 10.48 \\
\hline dirt & 46.73 & 8.94 & 6.22 & $24.2(24.2)$ & 8.54 \\
\hline$K^{ \pm}$ & 46.73 & 32.16 & 2.45 & $32.9(38.6) / 84.2(84.4)$ & 15.28 \\
\hline$K^{0} / \bar{K}^{0}$ & 46.73 & 25.45 & 2.84 & $32.5(41.6) / 33.6(46.9)$ & 19.73 \\
\hline$\pi^{ \pm} \rightarrow \mu^{ \pm}$ & 46.73 & 53.13 & 1.88 & $24.6(23.7) / 20.1(27.2)$ & 18.84 \\
\hline $\bar{\nu}_{\mu} \rightarrow \bar{\nu}_{e}$ signal & 46.73 & 49.91 & - & - & 15.44 \\
\hline
\end{tabular}

Table 5.5: Investigation of different backgrounds as a possible source of the observed excess in antineutrino running mode (reconstructed energy range: $200-3000 \mathrm{MeV}$ ). The table lists the total number of excess events in the $100<E_{v i s}<2000 \mathrm{MeV}$ range, compared to the MonteCarlo prediction for each background hypothesis. The agreement between each background

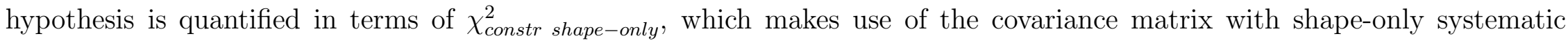
uncertainties and correlations corresponding to the total background and data statistical uncertainty, in order to calculate a $\chi^{2}$ between the excess distribution and the relatively-normalized background shape for each background hypothesis. The scale factor by which the background prediction must be multiplied to account for the excess (total excess/total background +1 ) is also given, along with the effective fractional uncertainty (from all sources, excluding detector and optical modeling uncertainties) on that particular background, prior to $\nu_{\mu}$ CCQE fit constraints. The uncertainty corresponds to high (low) energy. 


\begin{tabular}{|c|c|c|c|c|c|}
\hline Background & Excess events & Background events & Scale factor & Background \% uncertainty & $\chi_{\text {constr shape-only }}^{2}$ \\
\hline$\overline{\pi^{0}}$ & 42.99 & $\overline{599.94}$ & 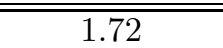 & $13.6(7.4)$ & 3.13 \\
\hline$\Delta$ & 42.99 & 15.86 & 3.71 & $15.5(14.2)$ & 4.24 \\
\hline dirt & 42.99 & 8.94 & 5.81 & $24.2(24.2)$ & 4.10 \\
\hline$K^{ \pm}$ & 42.99 & 32.66 & 2.32 & $32.9(38.6) / 84.2(84.4)$ & 3.77 \\
\hline$K^{0} / \bar{K}^{0}$ & 42.99 & 25.90 & 2.66 & $32.5(41.6) / 33.6(46.9)$ & 3.93 \\
\hline$\pi^{ \pm} \rightarrow \mu^{ \pm}$ & 42.99 & 53.74 & 1.80 & $24.6(23.7) / 20.1(27.2)$ & 5.02 \\
\hline $\bar{\nu}_{\mu} \rightarrow \bar{\nu}_{e}$ signal & 42.99 & 50.06 & & & 5.35 \\
\hline
\end{tabular}

$\overrightarrow{\&}$ Table 5.6: Investigation of different backgrounds as a possible source of the observed excess in antineutrino running mode (reconstructed energy range: $200-3000 \mathrm{MeV}$ ). The table lists the total number of excess events in the $-1<\cos \theta_{z}<+1$ range, compared to the MonteCarlo prediction for each background hypothesis. The agreement between each background hypothesis

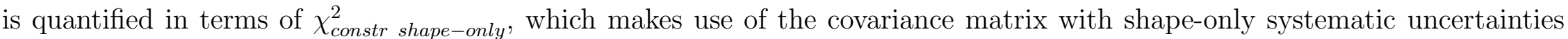
and correlations corresponding to the total background and data statistical uncertainty, in order to calculate a $\chi^{2}$ between the excess distribution and the relatively-normalized background shape for each background hypothesis. The scale factor by which the background prediction must be multiplied to account for the excess (total excess/total background +1 ) is also given, along with the effective fractional uncertainty (from all sources, excluding detector and optical modeling uncertainties) on that particular background, prior to $\nu_{\mu}$ CCQE fit constraints. The uncertainty corresponds to high (low) energy. 


\begin{tabular}{|c|c|c|c|c|c|}
\hline Background & Excess events & Background events & Scale factor & Background \% uncertainty & $\chi_{\text {constr shape-only }}^{2}$ \\
\hline$\overline{\pi^{0}}$ & 39.19 & 56.75 & $\overline{c 1.69}$ & $13.6(7.4)$ & 2.06 \\
\hline$\Delta$ & 39.19 & 15.41 & 3.54 & $15.5(14.2)$ & 2.21 \\
\hline dirt & 39.19 & 8.37 & 5.68 & $24.2(24.2)$ & 1.49 \\
\hline$K^{ \pm}$ & 39.19 & 25.94 & 2.51 & $32.9(38.6) / 84.2(84.4)$ & 3.51 \\
\hline$K^{0} / \bar{K}^{0}$ & 39.19 & 19.94 & 2.96 & $32.5(41.6) / 33.6(46.9)$ & 3.42 \\
\hline$\pi^{ \pm} \rightarrow \mu^{ \pm}$ & 39.19 & 48.84 & 1.80 & $24.6(23.7) / 20.1(27.2)$ & 2.05 \\
\hline $\bar{\nu}_{\mu} \rightarrow \bar{\nu}_{e}$ signal & 39.188844 & 46.16 & & & 1.79 \\
\hline
\end{tabular}

$\underset{v}{\overrightarrow{0}}$ Table 5.7: Investigation of different backgrounds as a possible source of the observed excess in antineutrino running mode (reconstructed energy range: $200-3000 \mathrm{MeV}$ ). The table lists the total number of excess events in the $0<Q^{2}<0.5 \mathrm{GeV}^{2}$ range, compared to the MonteCarlo prediction for each background hypothesis. The agreement between each background hypothesis

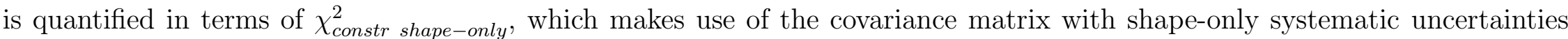
and correlations corresponding to the total background and data statistical uncertainty, in order to calculate a $\chi^{2}$ between the excess distribution and the relatively-normalized background shape for each background hypothesis. The scale factor by which the background prediction must be multiplied to account for the excess (total excess/total background +1 ) is also given, along with the effective fractional uncertainty (from all sources, excluding detector and optical modeling uncertainties) on that particular background, prior to $\nu_{\mu}$ CCQE fit constraints. The uncertainty corresponds to high (low) energy. 


\begin{tabular}{|c|c|c|c|c|c|c|c|c|}
\hline Background & "MC correction & 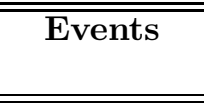 & $\begin{array}{c}\text { Scale factor } \\
E_{v i s} / \cos \theta_{z} / Q^{2} \\
\end{array}$ & $\begin{array}{l}\% \text { uncert. } \\
\text { cross-sect. }\end{array}$ & meson prod. & beam & hadronic int. & total (no det.) \\
\hline 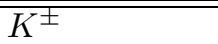 & & $25.15(8.15)$ & 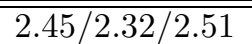 & & & & & \\
\hline$K^{+}$ & & $22.96(7.82)$ & & $16.1(19.8)$ & $28.0(29.1)$ & $6.3(15.8)$ & $0.3(0.5)$ & $32.9(38.6)$ \\
\hline$K^{-}$ & & $2.19(0.33)$ & & $15.8(15.2)$ & $81.2(81.2)$ & $15.7(16.6)$ & $1.6(4.8)$ & $84.2(84.4)$ \\
\hline $\bar{K}^{0} / \bar{K}^{0}$ & & $34.25(5.13)$ & $2.84 / 2.66 / 2.96$ & & & & & \\
\hline$\nu_{e}$ from $K^{0}$ & & $22.70(3.69)$ & & $16.6(22.1)$ & $27.1(32.6)$ & $6.7(13.5)$ & $0.7(0.4)$ & $32.5(41.6)$ \\
\hline $\bar{\nu}_{e}$ from $K^{0}$ & & $11.56(1.44)$ & & $18.1(15.6)$ & $27.6(43.6)$ & $5.9(7.5)$ & $1.7(1.2)$ & $33.6(46.9)$ \\
\hline$\pi^{ \pm} \rightarrow \mu^{ \pm}$ & $N \pi^{ \pm}$ & $41.15(13.44)$ & $1.88 / 1.80 / 1.80$ & & & & & \\
\hline$\pi^{+} \rightarrow \mu^{+}$ & $N \pi^{+}=0.99$ & $6.36(2.09)$ & & $16.8(19.9)$ & $12.5(5.5)$ & $12.7(11.6)$ & $1.4(1.4)$ & $24.6(23.7)$ \\
\hline$\pi^{-} \rightarrow \mu^{-}$ & $N \pi^{-}=1.20$ & $34.79(11.35)$ & & $18.0(16.6)$ & $8.1(21.4)$ & $3.8(3.4)$ & $0.8(0.9)$ & $20.1(27.2)$ \\
\hline
\end{tabular}

Table 5.8: Summary of intrinsic $\nu_{e}$ background contribution in the $475<E_{\nu}^{Q E}<3000 \mathrm{MeV}$ range $\left(200<E_{\nu}^{Q E}<475 \mathrm{MeV}\right.$ range), and assigned total (production $\times$ cross-section) systematic uncertainties in the $475<E_{\nu}^{Q E}<1100 \mathrm{MeV}\left(200<E_{\nu}^{Q E}<475\right.$ $\mathrm{MeV}$ ) range in the $\bar{\nu}_{e}$ appearance analysis. The scale factor necessary to account for the observed excess of $\nu_{e}$ CCQE events as a function of $E_{v i s}, \cos \theta_{z}$, and $Q^{2}$ is compared to the total flux $\times$ cross-section systematic uncertainty of each background. See text for more details. 
As seen in Tab. 5.8, systematic uncertainties on $\nu_{e}$ from $K^{0}$ are mostly dominated by $K^{0}$ production, at $\sim 30 \%$, followed by cross-section uncertainties, at $\sim 17 \%$, and beam systematic uncertainties, at $\sim 7 \%$. A scale factor of approximately 2.7 (minimum, from $\cos \theta_{z}$ distribution comparison) would therefore correspond to a $>4 \sigma$ effect in the flux $\times$ cross-section prediction (assuming a conservative $\sim 40 \%$ uncertainty).

Systematic uncertainties on $\nu_{e}$ from $K^{ \pm}$are mostly dominated by $K^{ \pm}$production, at $\sim 30 \%$ in the case of $K^{+}$, and $\sim 80 \%$ in the case of $K^{-}$, followed by cross-section at $\sim 15-20 \%$, and beam systematics at $\sim 6-17 \%$. A scale factor of approximately 2.3 (minimum, from $\cos \theta_{z}$ distribution comparison) would therefore correspond to a $>3 \sigma$ effect in the flux $\times$ cross-section prediction (assuming a conservative $\sim 40 \%$ uncertainty, average, weighted by $K^{ \pm}$background contributions).

In the case of $\nu_{e}$ from $\pi \rightarrow \mu$ decays, the dominant source of systematic error comes from cross-section uncertainties $(\sim 16-20 \%)$, followed by pion production uncertainties $\left(12.5 \%\right.$ for $\pi^{+} \rightarrow \mu^{+}$and $8 \%$ for $\left.\pi^{-} \rightarrow \mu^{-}\right)$and beam systematics $(\sim 3$ $13 \%$ ). Assuming a $\sim 25 \%$ uncertainty on the flux $\times$ cross-section prediction of intrinsic $\nu_{e}$ backgrounds from $\pi^{ \pm} \rightarrow \mu^{ \pm}$, a scale factor of 1.8 (minimum, from $\cos \theta_{z}$ and $Q^{2}$ distribution comparison) would imply a $3.2 \sigma$ modification to account for the size of the excess in the $E_{v i s}, \cos \theta_{z}$ and $Q^{2}$ distributions. This calculation assumes that both $\nu_{e}$ from $\pi^{+}$and $\nu_{e}$ from $\pi^{-}$decay chains have been misestimated. An even larger discrepancy would be obtained if one assumes only one of those sources (for example, only $\nu_{e}$ from $\pi^{-} \rightarrow \mu^{-}$) has been misestimated, since a larger background scale factor would be required to account for the excess. Note that this estimate is very conservative, as it ignores the constraint on flux and cross-section that comes from exploiting $\nu_{e}-\nu_{\mu}$ CCQE correlations. Also, the background prediction in this comparison already includes the $N \pi^{ \pm}$normalization correction, which effectively increases the background prediction normalization. A comparison without the $N \pi^{ \pm}$ correction would imply a larger effect to account for the excess as misestimated $\nu_{e}$ from $\pi^{ \pm} \rightarrow \mu^{ \pm}$ 


\subsubsection{On the Possibility of a Low Energy Excess in Antineu- trinos}

Unlike the first antineutrino appearance results, the updated appearance results presented in this section show a $1.3 \sigma$ excess of events above background prediction at low energy. Because of large uncertainties in the low energy region and relatively low $\bar{\nu}_{\mu}$ flux contribution to that range, the observed low energy excess has a relatively small impact to the oscillation fit results, including fit quality, best-fit parameters, and allowed regions. Nevertheless, the presence of a low energy excess at $1.3 \sigma$ amplifies the preference of a signal over the no oscillation hypothesis.

One may be interested to attribute the difference between observed excess and what is expected from the best-fit or no oscillations prediction to any one of the possible neutrino mode low energy excess interpretations. A detailed study is beyond the scope of this thesis; however, in this section we consider the possibility that the excess seen in neutrino mode, summarized in Tab. 5.3 is expected to scale with the neutrino content the beam from neutrino to antineutrino mode, and discuss its effect on the oscillation fits.

Under the hypothesis that the source of the neutrino mode excess is due to neutrinos in the beam (specifically, $\nu_{\mu}$ ), one would expect 11.6 excess events at low energy in antineutrino mode, estimated by scaling the neutrino mode observed low energy excess by the ratio of the wrong-sign $\left(\nu_{\mu}\right)$ content of the antineutrino flux to the total neutrino mode flux. The best fit oscillation hypothesis (from a fit to $E_{\nu}^{Q E}>475 \mathrm{MeV}$ ) corresponds to 6.9 signal events above background prediction between 200-475 MeV, to be compared to 18.5 observed excess events. The observed excess at low energy is in agreement with both the signal expected from a neutrino-only induced low energy excess hypothesis, and the sum of the two signal expectations (neutrino-only induced low energy excess plus $\bar{\nu}_{\mu} \rightarrow \bar{\nu}_{e}$ oscillations).

In general, as seen by the relatively small difference in fit results obtained from $E_{\nu}^{Q E}>475 \mathrm{MeV}$ and $E_{\nu}^{Q E}>200 \mathrm{MeV}$ fits, it is expected that ascribing the low energy excess to any hypothesis is expected to have a marginal effect on oscillations. 


\subsection{Sensitivity to $\bar{\nu}_{e}$ Appearance for $10.0 \times 10^{20} \mathrm{POT}$}

Figure 5-17 shows the MiniBooNE projected sensitivity to $\bar{\nu}_{\mu} \rightarrow \bar{\nu}_{e}$ oscillations for $10.0 \times 10^{20} \mathrm{POT}$, obtained assuming the current relative event compositions in the $\nu_{e}$ $\mathrm{CCQE}$ and $\nu_{\mu} \mathrm{CCQE}$ central values, and the same fractional systematic uncertainties.

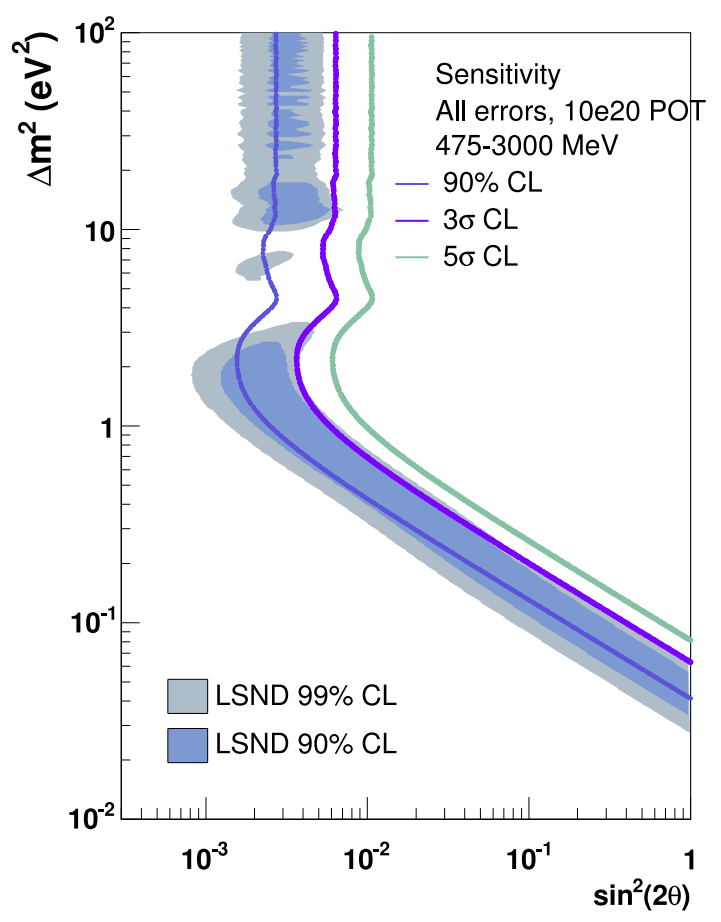

Figure 5-17: MiniBooNE projected 90\%, $3 \sigma$, and $5 \sigma$ C.L. $\bar{\nu}_{\mu} \rightarrow \bar{\nu}_{e}$ oscillation sensitivity for $10.0 \times 10^{20} \mathrm{POT}$. The sensitivity curves correspond to a one-sided raster scan, assuming 1 degree of freedom.

As shown in Fig. 5-18, assuming the best-fit parameters obtained from a fit to $E_{\nu}^{Q E}>475 \mathrm{MeV}$ with the present data set are true, MiniBooNE would be able to measure a non-zero oscillation signal at $>95 \%$ C.L., assuming 2 degrees of freedom. ${ }^{7}$ This confidence level is obtained from a fake fit to a signal due to $\left(\Delta m^{2}, \sin ^{2} 2 \theta\right)=$ $\left(4.64 \mathrm{eV}^{2}, 0.0063\right)$, assuming no statistical or systematic fluctuations in the input fake

\footnotetext{
${ }^{7}$ Note that a more precise determination of allowed regions requires fake data studies over the full parameter space with samples corresponding to $10.0 \times 10^{20}$ POT.
} 


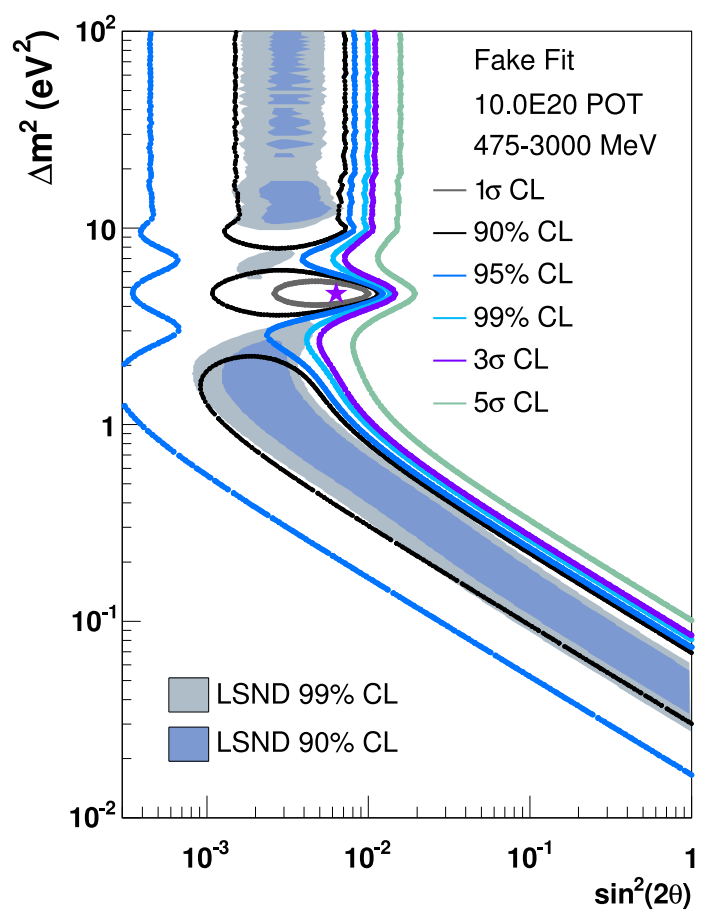

Figure 5-18: Allowed regions from a fake fit to $\Delta m^{2}=4.64 \mathrm{eV}^{2}$ and $\sin ^{2} 2 \theta=0.0063$ for $10.0 \times 10^{20}$ POT. $E_{\nu}^{Q E}>475 \mathrm{MeV}$. The contours shown are $2 \mathrm{D}$ contours, obtained using standard $\Delta \chi^{2}$ cuts, assuming 2 degrees of freedom.

signal.

Assuming the best-fit parameters obtained from a fit to $E_{\nu}^{Q E}>475 \mathrm{MeV}$ with the present data set are true, one can estimate the confidence level at which MiniBooNE is expected to exclude the no oscillation hypothesis under different POT assumptions, as shown in Fig. 5-19. Fake data studies at $\left(\Delta m^{2}, \sin ^{2} 2 \theta\right)=\left(4.64 \mathrm{eV}^{2}, 0.0063\right)$ as well as at the no oscillations point, corresponding to $5.66 \times 10^{2} 0 \mathrm{POT}$, are used to estimate the necessary $\Delta \chi^{2}$ cuts for various confidence levels. This approach assumes that the effective degrees of freedom in the fit are independent of POT, and correspond to those of $5.66 \times 10^{20}$ POT. The projected confidence level for $10.0 \times 10^{20}$ POT is $98.5 \% .^{8}$

Figure 5-20 shows the corresponding projected excess significance for $E_{\nu}^{Q E}>$

\footnotetext{
${ }^{8}$ Note that with a maximum likelihood fit method, the corresponding projected confidence level is estimated to be $>3 \sigma$.
} 


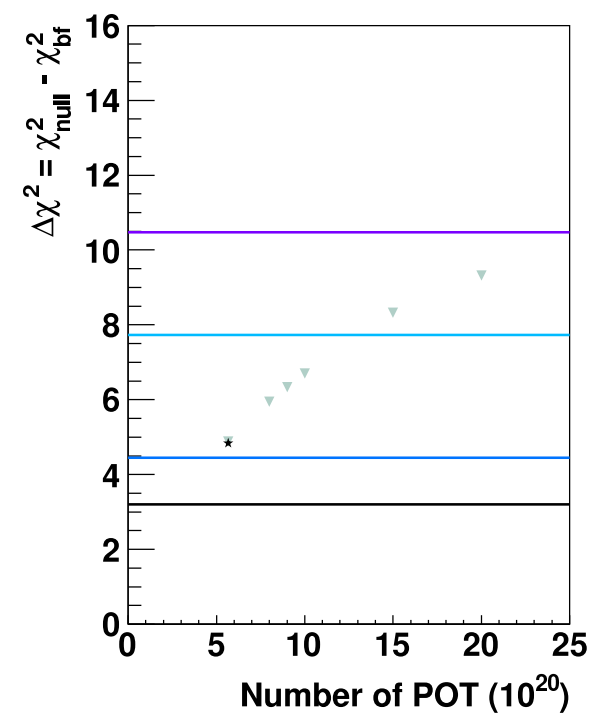

475-3000 MeV Fake Fit $\Delta \chi^{2}$

* True Data Fit $\Delta \chi^{2}$

$-3 \sigma \mathrm{CL}$ cut, $\Delta \chi^{2}=10.47$

- $99 \%$ CL cut, $\Delta \chi^{2}=7.73$

- 95\% CL cut, $\Delta \chi^{2}=4.45$

$-90 \%$ CL cut, $\Delta \chi^{2}=3.20$

Figure 5-19: The confidence level at which MiniBooNE is expected to exclude the no oscillation hypothesis, assuming current antineutrino best fit parameters, as a function of POT. The best fit parameters correspond to those obtained from an $E_{\nu}^{Q E}>475$ $\mathrm{MeV}$ fit to real data, for $5.66 \times 10^{20}$ POT.

$475 \mathrm{MeV}$ as a function of POT. The yellow points indicate statistical-only significance, defined as

$$
\left(D-B_{\text {constr }}\right) / \sqrt{B_{\text {constr }}}
$$

where $D$ and $B$ are the total data and background for $E_{\nu}^{Q E}>475 \mathrm{MeV}$. The green (blue) points indicate the significance with statistical and (constrained) systematic uncertainties included, using the statistical and (constrained) systematic uncertainty on the (constrained) background, i.e.,

$$
\left(D-B_{(\text {constr })}\right) / \sigma_{B_{(\text {constr })}}
$$

As expected, the statistical significance increases as the square root of the ratio of POT. The total (systematic and statistical) significance flattens out as the statistics 


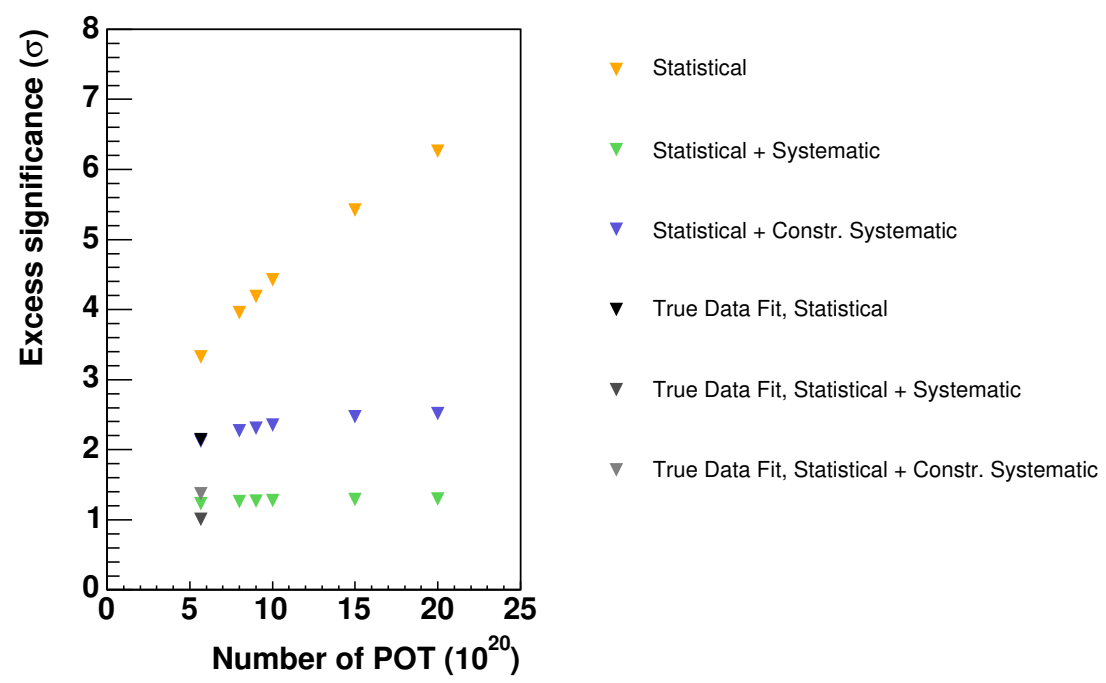

Figure 5-20: Significance for an excess due to current best fit parameters as a function of POT. The best fit parameters used to calculated the expected excess correspond to those obtained from an $E_{\nu}^{Q E}>475 \mathrm{MeV}$ fit to real data, for $5.66 \times 10^{20}$ POT.

increase, and the search becomes systematics limited. The gray points indicate the current significance, from a fit to true data. Since the fitted excess is larger than the true (observed) excess in the current data set, the observed excess significance for $5.66 \times 10^{20}$ POT is lower than the one implied by the fitted parameters, for the same POT. For $10.0 \times 10^{20}$ POT, the total excess significance for $E_{\nu}^{Q E}>475 \mathrm{MeV}$, assuming the current $\Delta m^{2}$ and $\sin ^{2} 2 \theta$ best-fit parameters, will be $2.35 \sigma$. 


\section{Chapter 6}

\section{Interpretation of MiniBooNE and LSND Appearance Results in Global Light Sterile Neutrino Oscillation Fits}

In this chapter, we address the level of compatibility among MiniBooNE and LSND results from [101, 102] and [107], respectively, and investigate the viability of light sterile neutrino oscillation models. These fits also consider constraints from shortbaseline oscillation experiments summarized in Sec. 2.2.3, which come from both neutrino and antineutrino experiments. Constraints from atmospheric oscillation experiments on muon neutrino disappearance to sterile states are also considered, as will be described later in this chapter.

We begin by considering MiniBooNE and LSND results in simultaneous fits to sterile neutrino oscillation parameters under a two-neutrino oscillation interpretation, or, equivalently, under the $(3+1)$ oscillation hypothesis. As will be seen, the three results (MiniBooNE neutrino results, MiniBooNE antineutrino results, and LSND results) are incompatible under this scenario. Next, we investigate the possibility of reconciling the MiniBooNE neutrino results with the MiniBooNE and LSND an- 
tineutrino results by invoking extra degrees of freedom in the fit and $C P$ violation, as allowed within a $(3+2)$ oscillation hypothesis. As in the case of the $(3+1)$ hypothesis, constraints from atmospheric and short-baseline experiments are also considered for the $(3+2)$ hypothesis.

The following paper (published in $[82]^{1}$ ) presents the assumptions, analysis fit method, and results from these fits, building upon previous work by the author and her collaborators $[181,113,81]$. The main results of this paper are summarized as follows:

1. While all antineutrino short-baseline oscillation data sets considered in the fits are compatible under a $(3+1)$ model, strong constraints arise from neutrino experiments, highly disfavoring a CPT-conserving $(3+1)$ hypothesis.

2. Fits with two sterile neutrinos, $(3+2)$, and $C P$ violation provide a small improvement to the compatibility of all short-baseline oscillation data relative to the $(3+1)$ hypothesis. Nevertheless, even those fits suffer substantially from strong constraints from CDHS and atmospheric data sets.

Note that the MiniBooNE antineutrino results included in these fits correspond to the first MiniBooNE antineutrino appearance results [102], corresponding to $3.39 \times 10^{20}$ POT, and not the updated antineutrino results presented in Chapter $5\left(5.66 \times 10^{20}\right.$ POT). Preliminary fits with the updated antineutrino data set from MiniBooNE, support and strengthen the conclusions presented here [182].

\footnotetext{
${ }^{1}$ The paper was followed by corrections in [82], erratum. The paper capture in this dissertation reflects those corrections.
} 


\title{
Viability of $\Delta m^{2} \sim 1 \mathrm{eV}^{2}$ sterile neutrino mixing models in light of MiniBooNE electron neutrino and antineutrino data from the Booster and NuMI beamlines
}

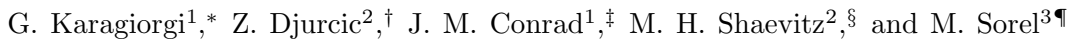 \\ ${ }^{1}$ Massachusetts Institute of Technology, Cambridge, MA 02139 \\ ${ }^{2}$ Department of Physics, Columbia University, New York, NY 10027 and \\ ${ }^{3}$ Instituto de Fisica Corpuscular, IFIC, CSIC and Universidad de Valencia, Spain
}

(Dated: May 7, 2010)

\begin{abstract}
This paper examines sterile neutrino oscillation models in light of recently published results from the MiniBooNE Experiment. The new MiniBooNE data include the updated neutrino appearance results, including the low energy region, and the first antineutrino appearance results, as well as first results from the off-axis NuMI beam observed in the MiniBooNE detector. These new global fits also include data from LSND, KARMEN, NOMAD, Bugey, CHOOZ, CCFR84, and CDHS. Constraints from atmospheric oscillation data have been imposed. We test the validity of the three-active plus one-sterile $(3+1)$ and two-sterile $(3+2)$ oscillation hypotheses, and we estimate the allowed range of fundamental neutrino oscillation parameters in each case. We assume CPTinvariance throughout. However, in the case of $(3+2)$ oscillations, CP violation is allowed. With the addition of the new MiniBooNE data sets, there are clear incompatibilities between neutrino and antineutrino experiments under a $(3+1)$ oscillation hypothesis. A better description of all shortbaseline data over a $(3+1)$ is provided by a $(3+2)$ oscillation hypothesis with $\mathrm{CP}$ violation. However, we still find large incompatibilities among appearance and disappearance experiments, consistent with previous analyses, as well as incompatibilities between neutrino and antineutrino experiments. Aside from LSND, the data sets responsible for this tension are the MiniBooNE neutrino data set, CDHS, and the atmospheric constraints. On the other hand, fits to antineutrino-only data sets, including appearance and disappearance experiments, are found significantly more compatible, even within a $(3+1)$ oscillation scenario.
\end{abstract}

PACS numbers: $14.60 . \mathrm{Pq}, 14.60 . \mathrm{St}, 12.15 . \mathrm{Ff}$

\section{INTRODUCTION}

Sterile neutrino oscillation models were proposed more than a decade ago as an explanation for the LSND anomaly [1-5], an excess of events consistent with $\bar{\nu}_{\mu} \rightarrow$ $\bar{\nu}_{e}$ oscillations at high $\Delta m^{2}$. These models relate $\nu_{e}$ appearance $\left(\nu_{\mu} \rightarrow \nu_{e}\right)$ with $\nu_{\mu}$ and $\nu_{e}$ disappearance $\left(\nu_{\mu} \rightarrow \nu_{\mu}\right.$ and $\left.\nu_{e} \rightarrow \nu_{\ell}\right)$, motivating combined fits in all three oscillation channels. Relatively early in the discussion of models, it was demonstrated $[5,6]$ that a threeactive plus one-sterile $(3+1)$ neutrino oscillation model could not reconcile the LSND result with existing null results from other short-baseline (SBL) experiments, including KARMEN [7], NOMAD [8], Bugey [9], CHOOZ [10], CCFR84 [11], and CDHS [12], which had similar high $\Delta m^{2}$ sensitivity. However, it was shown that a three-active plus two-sterile neutrino $(3+2)$ oscillation scenario provided a better description of these data sets $[5]$.

In 2001, the MiniBooNE experiment began running

*Electronic address: georgiak@mit.edu

$\dagger$ Electronic address: zdjurcic@nevis.columbia.edu

$\ddagger$ Electronic address: conrad@mit.edu

$\S$ Electronic address: shaevitz@nevis.columbia.edu

『Electronic address: sorel@ific.uv.es with the goal to test the LSND result using both neutrino and antineutrino beams. This is a short-baseline appearance and disappearance experiment located at Fermi National Accelerator Laboratory (Fermilab). MiniBooNE's first results, reported in 2007, described a search for $\nu_{\mu} \rightarrow \nu_{e}$ oscillations [13]. These results were incompatible with a simple two-neutrino oscillation interpretation of the LSND signal and, within this model, MiniBooNE excluded the LSND result at the $98 \%$ CL. However, this same analysis reported a $3.7 \sigma$ excess of electron neutrino candidate events at low energies, between 300-475 MeV, which remains unexplained. Reference [14] included the MiniBooNE first result in a global fit to all SBL experiments under the $(3+1)$ and $(3+2)$ oscillation scenarios. The analysis built on an earlier study, which introduced the possibility of CP violation $\left(P\left(\nu_{\mu} \rightarrow \nu_{e}\right) \neq\right.$ $\left.P\left(\bar{\nu}_{\mu} \rightarrow \bar{\nu}_{e}\right)\right)$ within $(3+2)$ fits [15]. Including the first MiniBooNE results into the global fit led to two observations in Ref. [14]: 1) MiniBooNE, LSND, and the null appearance experiments (KARMEN and NOMAD) are compatible under a $(3+2)$ sterile neutrino oscillation scenario with large $\mathrm{CP}$ violation. 2) There is severe tension between appearance and disappearance experiments, at a level of more than $3 \sigma$. In this paper we will consider both observations in light of new appearance data. Also, we will show that the incompatibility between appearance and disappearance experiments arises mainly from two $\nu_{\mu}$ disappearance data sets: CDHS and atmospheric 


\begin{tabular}{lc}
\hline \hline Data Set & Channel \\
\hline \hline Appearance experiments: & \\
LSND & $\bar{\nu}_{\mu} \rightarrow \bar{\nu}_{e}$ \\
BNB-MB $(\nu)$ & $\nu_{\mu} \rightarrow \nu_{e}$ \\
BNB-MB $(\bar{\nu})$ & $\bar{\nu}_{\mu} \rightarrow \bar{\nu}_{e}$ \\
NUMI-MB & $\nu_{\mu} \rightarrow \nu_{e}$ \\
KARMEN & $\bar{\nu}_{\mu} \rightarrow \bar{\nu}_{e}$ \\
NOMAD & $\nu_{\mu} \rightarrow \nu_{e}$ \\
\hline Disappearance experiments: & \\
Bugey & $\bar{\nu}_{e} \rightarrow \bar{\nu}_{\phi}$ \\
CHOOZ & $\bar{\nu}_{e} \rightarrow \bar{\nu}_{\phi}$ \\
CCFR 84 & $\nu_{\mu} \rightarrow \nu_{\mu}$ \\
CDHS & $\nu_{\mu} \rightarrow \nu_{\mu}$ \\
\hline \hline
\end{tabular}

TABLE I: Short-baseline oscillation data sets considered in this paper, and oscillation channel that each data set constrains.

constraints.

Motivated by three new results from MiniBooNE, this paper re-examines the $(3+1)$ and $(3+2)$ global fits to the SBL data. These new results are: 1) an updated $\nu_{\mu} \rightarrow \nu_{e}$ result [16]; 2) first results for a $\bar{\nu}_{\mu} \rightarrow \bar{\nu}_{e}$ search [17]; and 3) first $\nu_{\mu} \rightarrow \nu_{e}$ results from the NuMI off-axis beam at MiniBooNE [18]. We consider these new results in combination with seven SBL data sets. These provide constraints on: $\nu_{\mu}$ disappearance (from the CCFR84 and CDHS experiments), $\bar{\nu}_{e}$ disappearance (from the Bugey and CHOOZ experiments), $\nu_{\mu} \rightarrow \nu_{e}$ oscillations (from the NOMAD experiment), and $\bar{\nu}_{\mu} \rightarrow \bar{\nu}_{e}$ oscillations (from the LSND and KARMEN experiments). Furthermore, we have taken into account atmospheric constraints based on the analysis of Ref. [19]. These constraints have been incorporated in our analysis following the method described in Ref. [15], and are included in fits to all SBL experiments, null SBL experiments, or as explicitly stated. Table I summarizes all SBL data sets used in the fits presented in this paper.

In this work, we do not discuss experimental constraints on sterile neutrino models other than SBL and atmospheric neutrino ones. Constraints from the measurement of the electron spectrum near the endpoint in beta-decay experiments are relatively weak as long as the mostly-sterile mass states are heavier than the mostly-active ones, because of the small electron flavor of the former (see Refs. [5, 20]). We make this assumption throughout the paper, by requiring that the heavier sterile neutrino mass eigenstates, $m_{5}$ and $m_{4}$, obey $m_{5}>m_{4}>m_{1}$. Constraints on the energy density (and mass) in the Universe carried by sterile neutrinos from cosmic microwave background, matter power spectrum, and supernovae data have been studied in Ref. [21]. While relevant, these constraints are found to be weaker than SBL ones, since sterile neutrino states do not necessarily feature thermal abundances at decoupling. Constraints on the number of relativistic degrees of freedom from the observations of cosmological abundances of light elements produced at the epoch of Big Bang Nucleosynthesis may also be relevant, although model-dependent. For such a study involving one sterile neutrino species participating in the mixing, see for example Ref. [22].

The paper is organized as follows. In Section II, we provide a short description of the MiniBooNE experiment and the new data sets. In Section III, we specify the formalism used in this analysis to describe $(3+n)$ oscillations, where $n$ is the number of sterile neutrinos. In Section IV, we discuss the analysis method followed, and describe in detail the way in which the three MiniBooNE data sets have been incorporated. In Section V, we present the results obtained for the $(3+1)$ (CP-conserving only), and $(3+2) \mathrm{CP}$-conserving and $\mathrm{CP}$-violating hypotheses. For each hypothesis, we quote the compatibility between various sets of SBL experiments and report the best-fit neutrino mass and mixing parameters derived from the combined analysis of all experimental data sets. In the $(3+2) \mathrm{CP}$-violating case, we discuss the constraints on the $\mathrm{CP}$ violation phase, inferred from a combined analysis of all SBL oscillation results. Finally, in Section VI, we discuss constraints to the $(3+2)$ CP-violating models from each of the SBL experiments considered in this analysis. The goal of this particular study was to investigate whether the source of tension between appearance and disappearance experiments [14] is a result of a single experiment, other than LSND.

\section{THE NEW MINIBOONE DATA SETS}

The MiniBooNE experiment uses a muon neutrino beam produced by $8 \mathrm{GeV}$ protons from the Fermilab Booster Neutrino Beamline (BNB) impinging on a beryllium target. The target is located within a magnetic focusing horn [23]. The current of the horn can be reversed for running neutrinos or antineutrinos, allowing MiniBooNE to perform both neutrino and antineutrino oscillation searches. The detector [24] is located $L=541 \mathrm{~m}$ from the primary target, and the neutrino flux has an average energy of $\sim 0.75 \mathrm{GeV}$. This design maintains the LSND $L / E$ of $\sim 1 \mathrm{~m} / \mathrm{MeV}$. The detector consists of a spherical tank with a $610-\mathrm{cm}$ active radius, instrumented with 1520 8-inch photomultipliers. This is filled with 800 tons of pure mineral oil. An outer veto region rejects cosmic rays and neutrino events producing particles which cross the detector boundaries.

The MiniBooNE neutrino data set used in this analysis corresponds to the updated results recently reported by the MiniBooNE collaboration [16]. Compared to the first MiniBooNE result which was released in 2007 [13], the new result involves a re-analysis of the MiniBooNE low energy excess events and several updates to the Monte Carlo prediction. These updates include a new model of photonuclear effects, incorporation of new data on $\pi^{0}$ production and a better treatment of pion re-interaction in the detector following decay, an improved estimate and rejection method of the background from interactions 
outside the detector, and improvements to the determination of systematic errors. The updated low-energy analysis has resulted in a reduction to the significance of the excess from $3.7 \sigma$ in the original analysis to $3.4 \sigma$, along with some slight modification to the shape of the energy spectrum; specifically, the peak of the excess has shifted slightly to higher neutrino energies. In addition, the new analysis extends in energy down to $200 \mathrm{MeV}$, compared to $300 \mathrm{MeV}$ in the original analysis, which offers additional $L / E$ information. The new result also corresponds to modestly higher statistics, corresponding to the total data collected during the experiment's neutrino running of $6.46 \times 10^{20}$ protons on target (POT), compared to $5.58 \times 10^{20}$, previously.

More recently, the MiniBooNE Collaboration reported its first results from a search for $\bar{\nu}_{\mu} \rightarrow \bar{\nu}_{e}$ oscillations, using a muon antineutrino beam [17]. The antineutrino analysis performed by MiniBooNE mirrors the updated neutrino analysis [16], and includes the Monte Carlo prediction updates of the latter. The total antineutrino data set used in the analysis corresponds to $3.39 \times 10^{20} \mathrm{POT}$. However, due to meson production and cross-section effects, the antineutrino event sample, unlike the neutrino event sample, is statistically limited. Unlike the neutrino search, the MiniBooNE antineutrino search provides a direct test of the LSND result, similar to the search performed by KARMEN. The MiniBooNE sensitivity to $\bar{\nu}_{\mu} \rightarrow \bar{\nu}_{e}$ extends into the low- $\Delta m^{2}$ region allowed by a combined analysis of KARMEN and LSND data. Nevertheless, the MiniBooNE antineutrino search has observed no conclusive signal, and a limit has been set, which is considerably weaker than the sensitivity, and comparable to the KARMEN limit. The limit degradation with respect to the sensitivity is due to a $2.8 \sigma$ fluctuation of data above expected background observed in the $475-675 \mathrm{MeV}$ energy region. Thus, at present, the MiniBooNE antineutrino result is inconclusive with respect to oscillations allowed by LSND. However, MiniBooNE is in the process of collecting more antineutrino data. This is expected to improve the experiment's sensitivity to $\bar{\nu}_{\mu} \rightarrow \bar{\nu}_{e}$ oscillations. Updated results are expected after about three years of running.

The third new data set [18] arises from the fact that the MiniBooNE detector is illuminated by the off-axis $(110$ mrad) neutrino flux from the NuMI beamline at Fermilab. This analysis has reported a $1.2 \sigma$ excess of $\nu_{e}$-like events in the neutrino energy range below $900 \mathrm{MeV}$. The NuMI data set corresponds to a mean $L / E$ that is approximately the same as those of the MiniBooNE and LSND data sets, and therefore probes the same $\Delta m^{2}$ range, providing complementary information in oscillation fits with MiniBooNE and LSND.

\section{III. $(3+n)$ STERILE NEUTRINO OSCILLATION FORMALISM}

The formalism used in this paper follows that which was presented in Ref. [15]. We provide a brief summary here.

In sterile neutrino oscillation models, under the assumptions of CPT invariance and negligible matter effects, the probability for a neutrino produced with flavor $\alpha$ and energy $E$, to be detected as a neutrino of flavor $\beta$ after traveling a distance $L$, is given by $[25,26]$ :

$$
\begin{array}{r}
P\left(\nu_{\alpha} \rightarrow \nu_{\beta}\right)=\delta_{\alpha \beta}-4 \sum_{i>j} \mathcal{R}\left(U_{\alpha i}^{*} U_{\beta i} U_{\alpha j} U_{\beta j}^{*}\right) \sin ^{2} x_{i j}+ \\
2 \sum_{i>j} \mathcal{I}\left(U_{\alpha i}^{*} U_{\beta i} U_{\alpha j} U_{\beta j}^{*}\right) \sin 2 x_{i j}
\end{array}
$$

where $\mathcal{R}$ and $\mathcal{I}$ indicate the real and imaginary parts of the product of mixing matrix elements, respectively; $\alpha, \beta \equiv e, \mu, \tau$, or $s,(s$ being the sterile flavor $) ; i, j=$ $1, \ldots, 3+n$ ( $n$ being the number of sterile neutrino species); and $x_{i j} \equiv 1.27 \Delta m_{i j}^{2} L / E$. In defining $x_{i j}$, we take the neutrino mass splitting $\Delta m_{i j}^{2} \equiv m_{i}^{2}-m_{j}^{2}$ in $\mathrm{eV}^{2}$, the neutrino baseline $L$ in $\mathrm{km}$, and the neutrino energy $E$ in $\mathrm{GeV}$. For antineutrinos, the oscillation probability is obtained from Eq. 1 by replacing the mixing matrix $U$ with its complex-conjugate matrix. Therefore, if the mixing matrix is not real, neutrino and antineutrino oscillation probabilities can differ.

For $3+n$ neutrino species, there are, in general, $2+n$ independent mass splittings, $(3+n)(2+n) / 2$ independent moduli of parameters in the unitary mixing matrix, and $(2+n)(1+n) / 2$ Dirac CP-violating phases that may be observed in oscillations. In SBL neutrino experiments that are sensitive only to $\nu_{\mu} \rightarrow \nu_{\mu}, \nu_{e} \rightarrow \nu_{\phi}$, and $\nu_{\mu} \rightarrow \nu_{e}$ transitions, the set of observable parameters is reduced considerably. In this case, the number of observable parameters is restricted to $n$ independent mass splittings, $2 n$ moduli of mixing matrix parameters, and $n-1$ CPviolating phases. Therefore, for $(3+2)$ sterile neutrino models $(n=2$ case), for example, there are two independent mass splittings, $\Delta m_{41}^{2}$ and $\Delta m_{51}^{2}$, both defined to be greater than zero, four moduli of mixing matrix parameters $\left|U_{e 4}\right|,\left|U_{\mu 4}\right|,\left|U_{e 5}\right|,\left|U_{\mu 5}\right|$, and one CP-violating phase. The convention used for the CP-phase is:

$$
\phi_{45}=\arg \left(U_{\mu 5}^{*} U_{e 5} U_{\mu 4} U_{e 4}^{*}\right) .
$$

In that case, the general oscillation formula in Eq. 1 becomes:

$$
\begin{array}{r}
P\left(\nu_{\alpha} \rightarrow \nu_{\alpha}\right)=1-4\left[\left(1-\left|U_{\alpha 4}\right|^{2}-\left|U_{\alpha 5}\right|^{2}\right)\right. \\
\left(\left|U_{\alpha 4}\right|^{2} \sin ^{2} x_{41}+\left|U_{\alpha 5}\right|^{2} \sin ^{2} x_{51}\right)+ \\
\left.\left|U_{\alpha 4}\right|^{2}\left|U_{\alpha 5}\right|^{2} \sin ^{2} x_{54}\right]
\end{array}
$$

and

$$
\begin{array}{r}
P\left(\nu_{\alpha} \rightarrow \nu_{\beta \neq \alpha}\right)=4\left|U_{\alpha 4}\right|^{2}\left|U_{\beta 4}\right|^{2} \sin ^{2} x_{41}+ \\
4\left|U_{\alpha 5}\right|^{2}\left|U_{\beta 5}\right|^{2} \sin ^{2} x_{51}+ \\
8\left|U_{\alpha 5}\right|\left|U_{\beta 5}\right|\left|U_{\alpha 4}\right|\left|U_{\beta 4}\right| \sin x_{41} \sin x_{51} \cos \left(x_{54}-\phi_{45}\right)
\end{array}
$$


The formulas for antineutrino oscillations are obtained by substituting $\phi_{45} \rightarrow-\phi_{45}$.

For the case of $(3+1)$ sterile neutrino models $(n=$ 1 case), the corresponding oscillation probabilities are obtained from Eqs. 3 and 4 by setting $x_{51}=x_{54}=0$ and $\left|U_{\alpha 5}\right|=0$. Note that, under the above assumptions, no $\mathrm{CP}$ violation is allowed for $(3+1)$ models.

\section{ANALYSIS METHOD}

In this section, we first provide an overview of the fitting technique. We then focus on the method followed for including the MiniBooNE data sets. The physicsand statistical-assumptions for the other null SBL experiments and LSND, which are also included in the fit, are described in detail in Ref. [5]. The constraints from atmospheric experiments, according to Ref. [19], have been incorporated as described in Ref. [15].

\section{A. General Technique}

The Monte Carlo method used to apply the oscillation formalism described in Section III closely follows the one described in Ref. [15]. We start by randomly varying sets of oscillation parameters: $\Delta m_{41}^{2},\left|U_{e 4}\right|,\left|U_{\mu 4}\right|$ for the case of $(3+1) ; \Delta m_{41}^{2},\left|U_{e 4}\right|,\left|U_{\mu 4}\right|, \Delta m_{51}^{2},\left|U_{e 5}\right|,\left|U_{\mu 5}\right|, \phi_{45}$ for the case of $(3+2)$. Without loss of generality, we take $\Delta m_{51}^{2}>\Delta m_{41}^{2}$. In CP-conserving models, $\phi_{45}$ is set to 0 or $\pi$ by default, whereas in CP-violating models $\phi_{45}$ is allowed to vary within the full $(0,2 \pi)$ range. For each set of oscillation parameters, a signal prediction is obtained and compared to observed data for each SBL experiment, in the form of a $\chi^{2}$ for each experiment. For each set of oscillation parameters that is generated, the various $\chi^{2}$ 's are linearly summed together to form $\chi_{S B L}^{2}$, which is then used to extract the best-fit parameters and allowed regions.

A $\chi^{2}$ minimization is carried out using a Markov Chain [27]. This minimization procedure relies on calculating the $\chi^{2}$ difference between successive sets of parameters and using that as a measure of whether the new point in parameter space is a "good" point to step to, or whether a new set of parameters needs to be drawn again. This is realized in the form of a probability of accepting a new set of parameters, $P\left(x_{i} \rightarrow x_{i+1}\right)$, given by

$$
P\left(x_{i} \rightarrow x_{i+1}\right)=\min \left(1, e^{-\left(\chi_{i+1}^{2}-\chi_{i}^{2}\right) / T}\right),
$$

where $x_{i}$ and $x_{i+1}$ are two successive points in parameter space, and $\mathrm{T}$ is a Temperature parameter. Larger $\mathrm{T}$ values allow for larger $\Delta \chi^{2}$ jumps on the $\chi^{2}$ surface, and therefore by varying the $\mathrm{T}$ value, one can avoid local minima, as well as finely scan the parameter space. This minimization method is particularly preferred in fits with large parameter space dimensionality, as in the case of $(3+2)$ oscillation fits, due to its higher efficiency.
In extracting the various confidence level contours, we marginalize over the parameter space and report results obtained with $\Delta \chi^{2}$ levels corresponding to 1 degree of freedom for exclusion limits, and 2 degrees of freedom for allowed regions.

To quantify the statistical compatibility between various data sets, we use the Parameter Goodness-of-fit (PG) test introduced in [28]. In this test one quantifies how well various data sets are in agreement, by comparing the minimum $\chi^{2}$ obtained by a fit where all data sets have been included as constraints, $\chi_{m i n, a l l}^{2}$, to the sum of the $\chi^{2}$ minima obtained by independent fits for each experiment, i.e.,

$$
\chi_{P G}^{2}=\chi_{m i n, a l l}^{2}-\sum_{i} \chi_{m i n, i}^{2},
$$

where $i$ runs over experimental data sets in consideration. The PG is obtained from $\chi_{P G}^{2}$ based on the number of common underlying fit parameters, $n d f_{P G}$ :

$$
P G=\operatorname{prob}\left(\chi_{P G}^{2}, n d f_{P G}\right) .
$$

For example, for testing the compatibility between KARMEN and LSND for the $(3+2)$ CP-conserving oscillation hypothesis, we fit for both KARMEN and LSND simultaneously to extract $\chi_{\min , K+L}^{2}$, and for KARMEN and LSND separately to extract $\chi_{m i n, K}^{2}$, and $\chi_{m i n, L}^{2}$, respectively, and obtain:

$$
\chi_{P G}^{2}(K, L)=\chi_{\min , K+L}^{2}-\left(\chi_{\min , K}^{2}+\chi_{\min , L}^{2}\right) .
$$

As these are appearance experiments, there are 4 common fit parameters for a CP-conserving $(3+2)$ model $\left(\Delta m_{41}^{2}, \Delta m_{51}^{2},\left|U_{e 4}\right|\left|U_{\mu 4}\right|\right.$, and $\left.\left|U_{e 5}\right|\left|U_{\mu 5}\right|\right)$; therefore,

$$
P G(K, L)=\operatorname{prob}\left(\chi_{P G}^{2}(K, L), 4\right) .
$$

It should be noted that $\chi^{2}$-probabilities and PG tests can lead to drastically different results [28]. This is often a consequence of a large data set simultaneously fitted with small data sets, where the large data set dominates the $\chi^{2}$ of the simultaneous fit.

\section{B. Inclusion of the MiniBooNE Neutrino and Antineutrino Data Sets}

The MiniBooNE neutrino data set $(\operatorname{BNB}-\mathrm{MB}(\nu))$, described in Sec. II, is included in the fits in the form of two side-by-side distributions of $\nu_{e}$ and $\nu_{\mu}$ charged-current quasi-elastic (CCQE) events. Each distribution is a function of neutrino energy, reconstructed under the hypothesis of CCQE neutrino interaction kinematics, $E_{\nu}^{Q E}$. The full $200-3000 \mathrm{MeV}$ range of $\nu_{e} \mathrm{CCQE}$ data is used in the fit. The observed event distributions are compared to the corresponding Monte Carlo predicted distributions, and a $\chi^{2}$ is calculated using a covariance matrix which includes systematic and statistical uncertainties as well 
as systematic correlations between the predicted $\nu_{e}$ and $\nu_{\mu}$ distributions.

During the fit, we vary the $\nu_{e}$ distribution according to the sterile neutrino oscillation parameters, but keep the $\nu_{\mu}$ distribution unchanged. The $\nu_{\mu}$ distribution remains unchanged during the fit, despite the possibility of $\nu_{\mu}$ disappearance in the MiniBooNE data. In fact, MiniBooNE has released results from $\nu_{\mu}$ and $\bar{\nu}_{\mu}$ disappearance searches at $\Delta m^{2} \sim 1 \mathrm{eV}^{2}$ [29]. These results are relevant as constraints to sterile neutrino mixing parameters under consideration, but they have been purposefully omitted in this analysis, due to the fact that the $\nu_{\mu}$ and $\bar{\nu}_{\mu}$ CCQE samples used in the disappearance analysis [29] and the (different) $\nu_{\mu}$ and $\bar{\nu}_{\mu}$ CCQE samples used as constraints in the appearance analyses $[16,17]$ are highly correlated samples, and these correlations have not yet become available. We assume that including MiniBooNE $\nu_{\mu}$ disappearance would have a small effect on sterile neutrino fit results, given the large overlap of the $\nu_{\mu}$ disappearance limit from MiniBooNE with the corresponding limits from CDHS and CCFR84 [29]. However, the impact of the MiniBooNE disappearance results on the fits considered in this paper will be discussed. Nevertheless, we employ this side-by-side fitting method as it takes advantage of correlations in the $\nu_{\mu}$ and $\nu_{e}$ predictions and in order to effectively constrain the $\nu_{e}$ prediction and reduce systematic uncertainties in the $\nu_{\mu} \rightarrow \nu_{e}$ search.

The fit method follows the details described in [16], except that it uses a different definition for the covariance matrix used in the $\chi^{2}$ calculation. Ref. [16] involves an iterative fit method where the $\chi^{2}$ calculation for each point on the parameter space being probed uses the covariance matrix calculated according to the best-fit signal prediction. Instead, in the MiniBooNE fits presented here, the $\chi^{2}$ surface is estimated using the covariance matrix calculated according to the signal prediction at each point of the parameter space under consideration. The two fit methods yield similar results, although, by definition, the iterative method of [16] results in a relative shift of the allowed region to the left, i.e. towards smaller oscillation amplitudes.

The MiniBooNE antineutrino data set $(\mathrm{BNB}-\mathrm{MB}(\bar{\nu}))$, described in Sec. II, is included in the fits in the same way as the BNB-MB $(\nu)$ data set, in the form of two sideby-side $E_{\nu}^{Q E}$ distributions of $\bar{\nu}_{e}$ and $\bar{\nu}_{\mu}$ CCQE events. In this case, the disappearance limit obtained using the MiniBooNE $\bar{\nu}_{\mu}$ CCQE sample provides substantial coverage of so-far unexplored sterile neutrino mass and mixing parameter space [29]. Even though we do not explicitly fit the MiniBooNE $\bar{\nu}_{\mu}$ CCQE distribution for disappearance, we comment on the effect of the limit from [29] in Sec. V, and justify that excluding the MiniBooNE $\bar{\nu}_{\mu}$ disappearance information from the fits does not substantially affect the parameter space of interest. The full 200-3000 MeV range of $\bar{\nu}_{e}$ CCQE data is used in the fit. The BNB-MB $(\bar{\nu})$ data fit method also follows the details described in [17], except that it uses the definition for the covariance matrix described above.
In fits where both neutrino and antineutrino data are included, it has been assumed that the two data sets are fully uncorrelated. In reality, the two data sets have large systematic correlations. However, neglecting the effects of these correlations is a reasonable approximation, given that the antineutrino data set is statistics limited.

\section{Inclusion of the NuMI-beam Data Set}

The NUMI-MB data set [18], described in Sec. II, is included in the fits in the form of a distribution of $\nu_{e}$ CCQE events as a function of reconstructed neutrino energy, $E_{\nu}^{Q E}$. The predicted $\nu_{e}$ distribution is obtained by adding to the expected $\nu_{e}$ CCQE background any contribution from $\nu_{\mu}$ to $\nu_{e}$ oscillations. The contribution is estimated as follows: First, a fully (100\%) oscillated NUMI-MB $\nu_{\mu} \rightarrow \nu_{e}$ sample is obtained by reweighting the BNB-MB fully oscillated $\nu_{\mu} \rightarrow \nu_{e}$ Monte Carlo predicted sample according to the ratio of the NuMI-beam flux from [18] to the BNB-MB flux [23], as a function true neutrino energy. As the oscillation parameters vary during the fit, a signal prediction is calculated by rescaling the number of events in this fully oscillated sample by the corresponding oscillation probability, according to the true neutrino energy and distance travelled, from production to detection, of each event. We assume a constant $L$ of 700 meters. The prediction is compared to the observed $\nu_{e}$ CCQE events as a function of 10 bins of $E_{\nu}^{Q E}$. The background and signal prediction are assumed to have the same fractional systematic uncertainties, and a statistical uncertainty is calculated for each point in the parameter space according to the signal prediction of each point under consideration. The data and background central value and systematic uncertainty per $E_{\nu}^{Q E}$ bin have been estimated from [18]. Unlike the systematic uncertainties of the BNB-MB $\nu_{e}$ and $\bar{\nu}_{e}$ CCQE data sets, the NUMI-MB $\nu_{e}$ CCQE systematics have not been constrained using information from the $\nu_{\mu}$ CCQE spectrum from the NuMI beamline. Furthermore, we have not considered potential systematic correlations among the $\nu_{e}$ CCQE bins as a function of $E_{\nu}^{Q E}$.

\section{V. (3+1) AND (3+2) MODELS AFTER THE NEW MINIBOONE $\nu, \bar{\nu}$, AND NUMI RESULTS}

\section{A. (3+1) FIT RESULTS}

In this section, the new MiniBooNE results are examined under a $(3+1)$ oscillation hypothesis and compared to LSND and other null SBL experiments. The new data sets are studied first within the context of appearanceonly experiments, and subsequently in fits involving both appearance and disappearance data. Fits to only antineutrino and only neutrino SBL experiments are also explored. 


\begin{tabular}{|c|c|c|c|c|c|c|c|c|c|c|c|}
\hline \multirow[t]{2}{*}{ Fit } & \multicolumn{11}{|c|}{ Data Sets } \\
\hline & $\operatorname{BNB}-\mathrm{MB}(\nu)$ & BNB-MB $(\bar{\nu})$ & LSND & NUMI-MB & KARMEN & NOMAD & $\mathrm{CHOOZ}$ & Bugey & CCFR & $\mathrm{CDHS}$ & atm \\
\hline APP & $\sqrt{ }$ & $\checkmark$ & $\sqrt{ }$ & $\sqrt{ }$ & $\sqrt{ }$ & $\sqrt{ }$ & & & & & \\
\hline DIS & & & & & & & $\sqrt{ }$ & $\sqrt{ }$ & $\sqrt{ }$ & $\sqrt{ }$ & $\sqrt{ }$ \\
\hline$\nu$ & $\sqrt{ }$ & & & $\checkmark$ & & $\checkmark$ & & & $\checkmark$ & $\checkmark$ & $\checkmark$ \\
\hline$\nu \mathrm{APP}$ & $\checkmark$ & & & $\checkmark$ & & $\checkmark$ & & & & & \\
\hline $\bar{\nu}$ & & $\checkmark$ & $\checkmark$ & & $\checkmark$ & $\sqrt{ }$ & $\checkmark$ & & & & \\
\hline $\bar{\nu} \mathrm{APP}$ & & $\checkmark$ & $\checkmark$ & & $\checkmark$ & & & & & & \\
\hline signal & $\checkmark$ & $\checkmark$ & $\sqrt{ }$ & & & & & & & & \\
\hline signal APP & $\sqrt{ }$ & $\checkmark$ & $\sqrt{ }$ & & & & & & & & \\
\hline & & & & $\checkmark$ & $\checkmark$ & $\checkmark$ & $\checkmark$ & $\checkmark$ & $\checkmark$ & $\checkmark$ & $\checkmark$ \\
\hline null APP & & & & $\sqrt{ }$ & $\sqrt{ }$ & $\sqrt{ }$ & & & & & \\
\hline
\end{tabular}

TABLE II: Short-baseline oscillation fits considered in this paper.

$\operatorname{BNB}-\mathrm{MB}(v)$

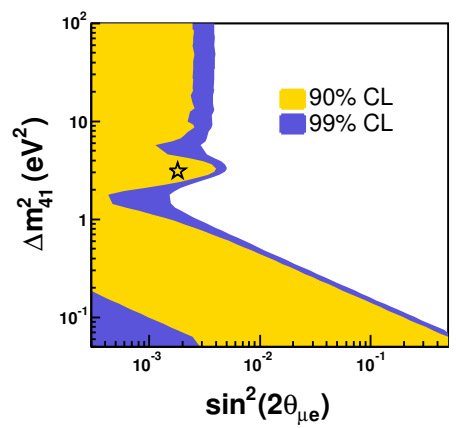

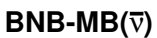

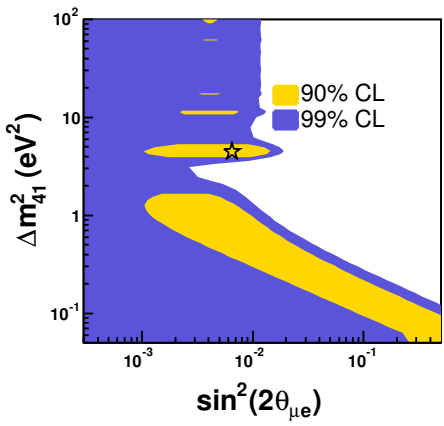

LSND

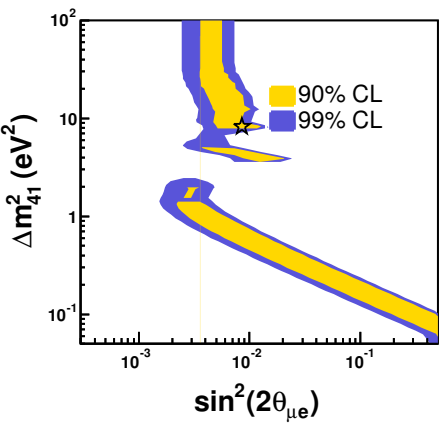

FIG. 1: Allowed regions (filled areas) at $90 \%$ and $99 \%$ CL from $\operatorname{BNB}-\mathrm{MB}(\nu)$-only, BNB-MB $(\bar{\nu})$-only, and LSND-only $(3+1)$ fits. These fits are, by construction, CP-conserving. The stars indicate the three respective best-fit points. All three data sets show closed contours at $90 \%$ CL. See text for more details.

Table II provides a reference for all the different data set combinations explored in fits in this paper.

\section{Studies with appearance-only experiments}

Figure 1 shows the allowed regions obtained by independent fits to each of the following three data sets: $\operatorname{BNB}-\mathrm{MB}(\nu), \mathrm{BNB}-\mathrm{MB}(\bar{\nu})$, and LSND. The regions are estimated using a 2-dimensional global scan of the $(3+1)$ parameter space $\left(\sin ^{2} 2 \theta_{\mu e}, \Delta m_{41}^{2}\right)$. Each contour is drawn by applying a flat $\Delta \chi^{2}=\chi^{2}\left(\sin ^{2} 2 \theta_{\mu e}, \Delta m_{41}^{2}\right)-$ $\chi_{\min }^{2}$ cut over the $\chi^{2}$ surface, with respect to the global $\chi^{2}$ minimum returned by the fit. The figure shows that, similarly to LSND, both BNB-MB data sets yield contours which exclude the no-oscillations (null) hypothesis at $90 \% \mathrm{CL}$. The null $\chi^{2}$ 's correspond to 22.2 and 24.5 for $\operatorname{BNB}-\mathrm{MB}(\nu)$ and $\mathrm{BNB}-\mathrm{MB}(\bar{\nu})$, respectively. The closed contours reflect a contradiction to the oscillation results published by the MiniBooNE collaboration; this is a consequence of the different $\chi^{2}$ definition involved in the fit method used here, as pointed out in Sec. IV B. All three data sets, $\operatorname{BNB}-\mathrm{MB}(\nu), \operatorname{BNB}-\mathrm{MB}(\bar{\nu})$, and LSND, yield similar best-fit parameters, indicated by the stars on the three graphs, of $\Delta m_{41}^{2}$ of order a few $\mathrm{eV}^{2}$ and $\sin ^{2} 2 \theta_{\mu e}$ of order $10^{-2}-10^{-3}$. The minimum $\chi^{2}$ and best-fit parameters returned by each experiment are summarized in Table III.

In light of the above BNB-MB results and the already established LSND anomaly, we find it interesting to study sterile neutrino oscillations with the LSND, BNB-MB $(\nu)$, and $\mathrm{BNB}-\mathrm{MB}(\bar{\nu})$ data sets assumed to be (positive) "signal" experiments under both the $(3+1)$ and the $(3+2)$ models. This classification is based on the fact that all three data sets exclude the null result at $90 \%$ confidence level under a $(3+1)$ oscillation hypothesis. Figure 2 shows the $\operatorname{BNB}-\mathrm{MB}(\nu)$ and BNB-MB $(\bar{\nu})$ event distributions for both the null and the best-fit $(3+1)$ oscillation hypothesis for each data set. In the case of the $\operatorname{BNB}-\operatorname{MB}(\nu)$ data set, even though the best-fit hypothesis provides a better description of the event spectrum at $90 \% \mathrm{CL}$ $\left(\Delta \chi^{2}=\chi_{\text {null }}^{2}-\chi_{\text {best }- \text { fit }}^{2}=4.7\right.$, for 2 fit parameters $)$, it fails to fully explain the low energy excess. Therefore, the $(3+1)$ oscillation hypothesis alone seems inadequate 


\begin{tabular}{|c|c|c|c|c|c|c|}
\hline Data Set & $\chi^{2}($ dof $)$ & $\chi^{2}$-probability & $\Delta m_{41}^{2}$ & $\sin ^{2} 2 \theta_{\mu e}$ & $\sin ^{2} 2 \theta_{\mu \mu}$ & $\sin ^{2} 2 \theta_{e e}$ \\
\hline \multicolumn{7}{|l|}{ Appearance-only fits: } \\
\hline LSND & $3.4(3)$ & $34 \%$ & 8.19 & 0.0085 & - & - \\
\hline $\operatorname{BNB}-\mathrm{MB}(\nu)$ & $17.5(16)$ & $35 \%$ & 3.12 & 0.0018 & - & - \\
\hline $\mathrm{BNB}-\mathrm{MB}(\bar{\nu})$ & $17.6(16)$ & $35 \%$ & 4.46 & 0.0065 & - & - \\
\hline NUMI-MB & $2.0(8)$ & $98 \%$ & 6.97 & 0.020 & - & - \\
\hline KARMEN & $6.0(7)$ & $54 \%$ & 6.81 & 0.00096 & - & - \\
\hline NOMAD & $33.3(28)$ & $31 \%$ & 53.3 & 0.00012 & - & - \\
\hline signal APP & $50.3(39)$ & $11 \%$ & 0.045 & 0.98 & - & - \\
\hline signal APP* & $50.4(39)$ & $10 \%$ & 0.15 & 0.090 & - & - \\
\hline null APP & $46.6(47)$ & $49 \%$ & 0.040 & 1.00 & - & - \\
\hline APP & $97.1(88)$ & $24 \%$ & 0.045 & 1.00 & - & - \\
\hline $\mathrm{APP}^{*}$ & $97.2(88)$ & $24 \%$ & 0.15 & 0.090 & - & - \\
\hline $\mathrm{LSND}+\mathrm{MB}-\mathrm{BNB}(\bar{\nu})$ & $22.3(21)$ & $38 \%$ & 4.55 & 0.0074 & - & - \\
\hline $\operatorname{LSND}+\operatorname{MB}-\mathrm{BNB}(\bar{\nu})^{*}$ & $22.3(21)$ & $38 \%$ & 4.55 & 0.0074 & - & - \\
\hline $\mathrm{LSND}+\mathrm{MB}-\mathrm{BNB}(\bar{\nu})+\mathrm{KARMEN}$ & $33.6(30)$ & $29 \%$ & 0.57 & 0.0097 & - & - \\
\hline $\operatorname{BNB}-\mathrm{MB}(\nu)+$ NUMI-MB + NOMAD & $57.8(56)$ & $40 \%$ & 0.033 & 1.00 & - & - \\
\hline \multicolumn{7}{|l|}{ Appearance and disappearance fits: } \\
\hline all SBL* & $197.4(196)$ & $46 \%$ & 0.92 & 0.0025 & 0.13 & 0.073 \\
\hline$\nu$ & $90.5(90)$ & $47 \%$ & 0.19 & 0.031 & 0.031 & 0.034 \\
\hline $\bar{\nu}$ & $87.9(103)$ & $86 \%$ & 0.91 & 0.0043 & 0.35 & 0.043 \\
\hline
\end{tabular}

TABLE III: Comparison of best-fit values for mass splittings and mixing angles obtained from (3+1) fits to appearance data sets and appearance+disappearance data sets. Mass splittings are shown in $\mathrm{eV}^{2}$. The minimum $\chi^{2}$ from each fit, as well as the $\chi^{2}$-probability are also given. The signal appearance (APP) data sets include BNB-MB $(\nu), \mathrm{BNB}-\mathrm{MB}(\bar{\nu})$ and LSND. The null APP data sets include KARMEN, NOMAD and NUMI-MB; the maximal best-fit $\sin ^{2} 2 \theta_{\mu e}$ in this case is inconsequential, as it corresponds to a best-fit $\Delta m^{2}$ region of very poor sensitivity. See text for more details.

* In these fits, the electron and muon content of the sterile neutrino mass eigenstate have been explicitly constrained to $<0.3$.
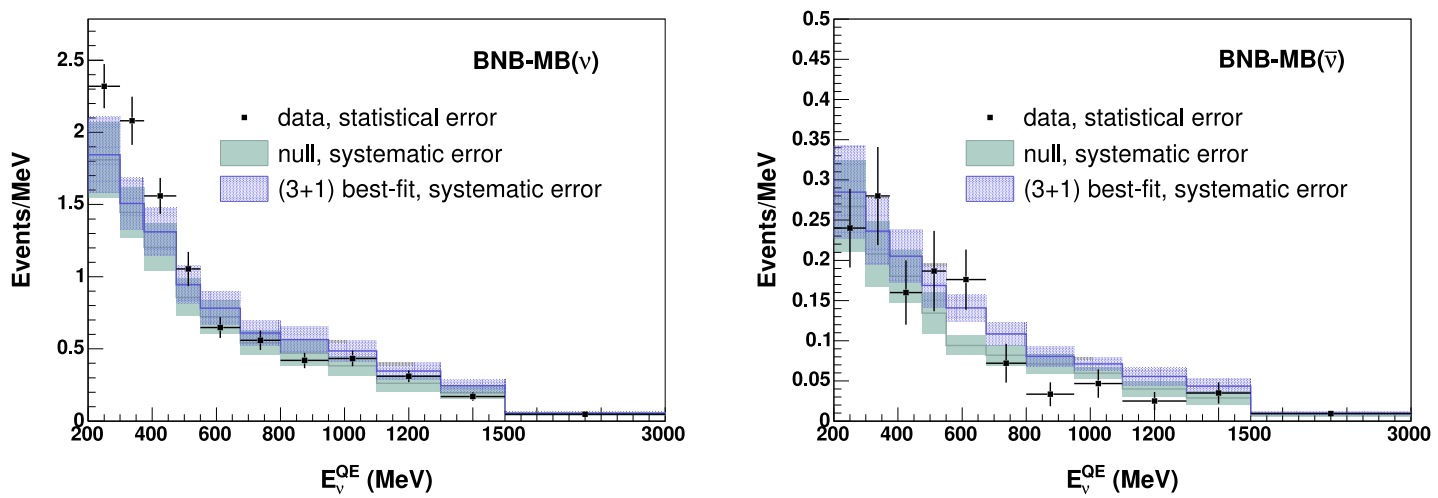

FIG. 2: Left: Null and (3+1) best-fit predicted event distributions $\left(\Delta m_{41}^{2}, \sin ^{2}\left(2 \theta_{\mu e}\right)\right)=(3.12,0.0018)$ for $\operatorname{BNB}-\mathrm{MB}(\nu)$. Right: Null and $(3+1)$ best-fit predicted event distributions $\left(\Delta m_{41}^{2}, \sin ^{2}\left(2 \theta_{\mu e}\right)\right)=(4.46,0.0065)$ for BNB-MB $(\bar{\nu})$. The event distributions are shown as functions of reconstructed neutrino energy, $E_{\nu}^{Q E}$. The data are shown in black points with statistical uncertainty. The null (no-oscillation) prediction is shown by the light gray histogram with (solid) systematic error band. The best-fit prediction (signal and background) is shown by the blue (dark gray) histogram with (shaded) systematic error band.

as an explanation for the low energy excess, as also reported by the MiniBooNE collaboration $[13,16]$. In the case of the $\operatorname{BNB}-\mathrm{MB}(\bar{\nu})$ data set, the best-fit hypothesis provides a better description of the data in the 500-700 $\mathrm{MeV}$ range. However, the statistical uncertainties are too large to allow for a strong conclusion.
The allowed regions obtained by a joint analysis of $\operatorname{BNB}-\mathrm{MB}(\nu)+\operatorname{BNB}-\mathrm{MB}(\bar{\nu})+\mathrm{LSND}$, as well as a joint analysis of BNB-MB $(\bar{\nu})+$ LSND are shown on the left panels of Figs. 3 and 4, respectively. In the case of the combined fit of all three data sets (Fig. 3), due to the difference in preferred mixing amplitudes mentioned in 


\begin{tabular}{|c|c|c|c|c|}
\hline Data Set & $\chi^{2}$-probability (\%) & $\mathrm{PG}(\%)$ & & \\
\hline APP & 24 & PG( BNB-MB $(\nu)$, BNB-MB $(\bar{\nu})$, LSND,NUMI-MB,KARMEN,NOMAD ) & $=\operatorname{Prob}(17.3,10)=$ & 6.8 \\
\hline signal APP & 11 & $\operatorname{PG}(\operatorname{BNB}-\mathrm{MB}(\nu), \mathrm{BNB}-\mathrm{MB}(\bar{\nu}), \mathrm{LSND})$ & $=\operatorname{Prob}(11.9,4)=$ & 1.8 \\
\hline $\mathrm{LSND}+\mathrm{MB}-\mathrm{BNB}(\bar{\nu})$ & 38 & $\mathrm{PG}(\mathrm{BNB}-\mathrm{MB}(\bar{\nu}), \mathrm{LSND})$ & $=\operatorname{Prob}(1.4,2)=$ & 49.0 \\
\hline $\bar{\nu} \mathrm{APP}$ & 29 & PG( BNB-MB $(\bar{\nu})$, LSND,KARMEN ) & $=\operatorname{Prob}(6.7,4)=$ & 15.3 \\
\hline$\nu \mathrm{APP}$ & 40 & PG( BNB-MB $(\nu)$, NUMI-MB,NOMAD ) & $=\operatorname{Prob}(4.9,4)=$ & 29.8 \\
\hline all SBL* & 46 & $\begin{array}{l}\mathrm{PG}(\mathrm{BNB}-\mathrm{MB}(\nu), \mathrm{BNB}-\mathrm{MB}(\bar{\nu}), \mathrm{NUMI}-\mathrm{MB}, \mathrm{LSND}, \mathrm{KARMEN}, \\
\quad \text { NOMAD,Bugey,CHOOZ,CCFR84,CDHS,ATM}) \\
\mathrm{PG}(\mathrm{APP}, \mathrm{DIS}) \\
\mathrm{PG}(\nu, \bar{\nu})\end{array}$ & $\begin{array}{l}=\operatorname{Prob}(42.0,18)= \\
=\operatorname{Prob}(14.8,2)= \\
=\operatorname{Prob}(18.8,3)=\end{array}$ & $\begin{array}{l}0.11 \\
0.06 \\
0.03 \\
\end{array}$ \\
\hline$\nu$ & 47 & PG( BNB-MB $(\nu)$, NUMI-MB,NOMAD,CCFR84,CDHS,ATM ) & $=\operatorname{Prob}(14.7,8)=$ & 6.5 \\
\hline $\bar{\nu}$ & 86 & PG( BNB-MB $(\bar{\nu})$, LSND,KARMEN,Bugey,CHOOZ ) & $=\operatorname{Prob}(8.43,7)=$ & 29.9 \\
\hline
\end{tabular}

TABLE IV: Summary of $\chi^{2}$-probabilities for $(3+1)$ fits with different combinations of SBL data sets, and PG results testing compatibility among different data sets. See text for more details.

${ }^{*}$ In these fits, the electron and muon content of the sterile neutrino mass eigenstate have been explicitly constrained to <0.3.

the previous paragraph, the best-fit point ends up shifting from an intermediate $\Delta m^{2}$ and small mixing amplitude to a smaller $\Delta m^{2}$ and maximal mixing amplitude of 0.98. Obviously a maximal mixing amplitude is unphysical in the case of sterile neutrino oscillations. If the fits are repeated with the electron and muon content of the sterile mass eigenstate limited to values less than 0.3 [38], the returned $\chi^{2}$-probabilities are $10 \%$ and $38 \%$, for $\mathrm{BNB}-\mathrm{MB}(\nu)+\mathrm{BNB}-\mathrm{MB}(\bar{\nu})+\mathrm{LSND}$ and BNB-MB $(\bar{\nu})$ + LSND, respectively; the reduction in $\sin ^{2} 2 \theta_{\mu e}$ space has essentially no effect on these results. The best-fit parameters from these fits are also given in Table III.

Perhaps a more interesting observation regarding Fig. 1 is the striking similarity of $\operatorname{BNB}-\mathrm{MB}(\bar{\nu})$ and LSND 90\% CL allowed regions and best-fit oscillation parameters, keeping in mind that both data sets describe antineutrino oscillations. It should be noted that in a $(3+1)$ oscillation scenario, under the assumption of CPT invariance, there can be no difference between neutrino and antineutrino oscillation probabilities. However, a PG test, as described in Sec. IV A, suggests a significantly higher compatibility $(49 \%)$ between $\operatorname{BNB}-\mathrm{MB}(\bar{\nu})$ and LSND, rather than for all three signal experiments $(\mathrm{BNB}-\mathrm{MB}(\nu)$, BNB-MB $(\bar{\nu})$ and LSND) combined (1.8\%). This is also supported by the $\chi^{2}$-probabilities returned by the fits: $11 \%$ in the case of the BNB-MB $(\nu)+\operatorname{BNB}-\mathrm{MB}(\bar{\nu})+$ LSND fit, and $38 \%$ in the case of the $\operatorname{BNB}-\mathrm{MB}(\bar{\nu})+$ LSND fit. This incompatibility is due to the fact that the $\operatorname{BNB}-\mathrm{MB}(\nu)$ data set prefers a mixing amplitude $\sim 3$ times smaller than the amplitude preferred by LSND or $\operatorname{BNB}-\mathrm{MB}(\bar{\nu})$, and excludes the LSND and BNB-MB $(\bar{\nu})$ best-fits at $99 \% \mathrm{CL}$. Table IV provides a summary of the above $\chi^{2}$-probabilities and PG test results.

Figures 3 and 4 also illustrate the limits from various combinations of the remaining three (null) SBL appearance experiments, KARMEN, NOMAD, and NUMI-MB, under a $(3+1)$ oscillation scenario, overlaid on the allowed regions described above.

The $90 \%$ and $99 \%$ CL limits obtained by each of the null appearance experiments are shown on the left panel of Fig. 3. These limits correspond to the upper $\sin ^{2} 2 \theta_{\mu e}$ values allowed at each $\Delta m_{41}^{2}$, estimated using a onesided raster scan of the parameter space. It is interesting to point out that, despite the indication of a slight excess $(1.2 \sigma)$ of observed $\nu_{e}$-like events for neutrino energies below $900 \mathrm{MeV}$ found in the NuMI analysis [18], the currently assumed NUMI-MB systematic and statistical uncertainties are quite large, resulting in a limit that is much weaker relative to the limits of KARMEN and NOMAD. In fact, due to this excess and the large systematic uncertainties, the NUMI-MB data set provides a very good fit to $(3+1)$ models, with a $\chi^{2}$-probability of $98 \%$. The event distributions for the null and bestfit $(3+1)$ oscillation hypothesis for the NUMI-MB data set are shown in Fig. 5. The observed distribution fits nicely to an oscillation signal at $\left(\Delta m_{41}^{2}, \sin ^{2}\left(2 \theta_{\mu e}\right)\right)=$ $(7.36,0.019)$. Such large signal, however, would be in disagreement with the $\mathrm{BNB}-\mathrm{MB}(\nu)$ results. Additional data and reduced systematic uncertainties in the NUMI$\mathrm{MB}$ analysis are necessary for higher sensitivity and more conclusive results. This is currently an ongoing effort and new results are expected soon. The limits from a combined NUMI-MB + KARMEN + NOMAD analysis are shown on the middle panel of Fig. 3. Both panels illustrate that the null appearance experiments provide essentially no constraints to the parameter space allowed by the BNB-MB and LSND data sets, except at higher $\Delta m^{2}$.

The best-fit parameters obtained independently from the NUMI-MB and KARMEN data sets, shown in Table III, are similar to those of LSND, BNB-MB $(\nu)$, and $\operatorname{BNB}-\mathrm{MB}(\bar{\nu})$. The NOMAD data set, on the other hand, prefers a much larger $\Delta m_{41}^{2} \sim 50 \mathrm{eV}^{2}$, and a much smaller $\sin ^{2} 2 \theta_{\mu e} \sim 10^{-4}$.

A combined analysis of all appearance data yields a $\chi^{2}$-probability of $24 \%$ for the best-fit hypothesis, both in the case where maximal mixing is allowed in the fit, and in the case where the electron and muon content of the sterile mass eigenstate has been limited to small values $(<0.3)$. The allowed region obtained by a joint analysis 

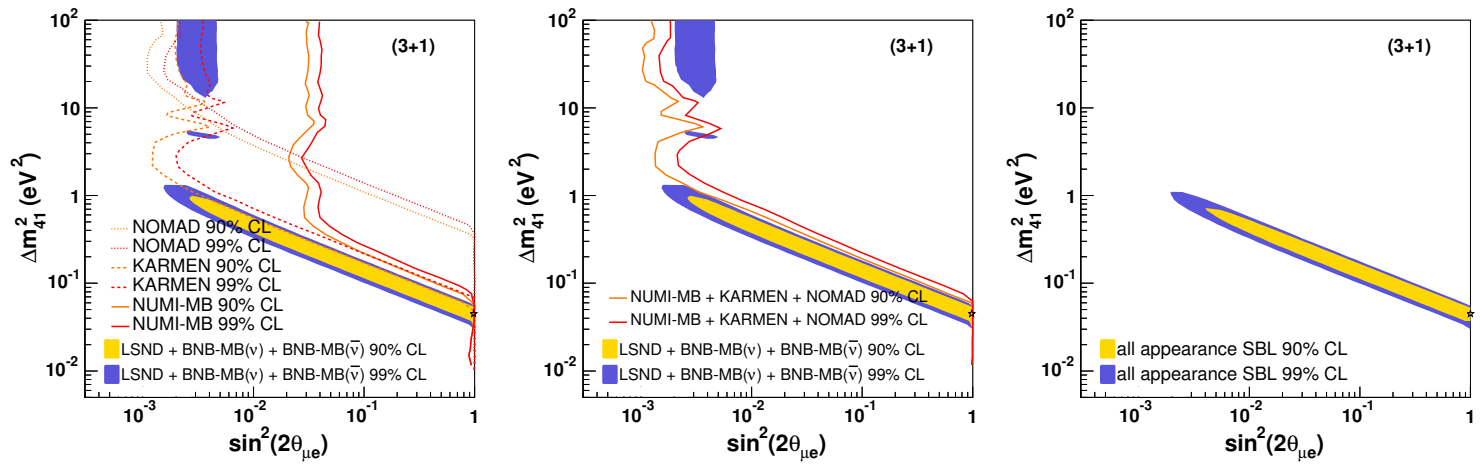

FIG. 3: Left: Allowed 90\% and 99\% CL regions (light and dark filled areas, respectively) from a combined analysis of BNB$\operatorname{MB}(\nu), \operatorname{BNB}-\mathrm{MB}(\bar{\nu})$ and LSND data sets, and $90 \%$ and $99 \%$ exclusion limits (light and dark curves, respectively) from each of the null appearance experiments, NUMI-MB (solid curves), KARMEN (dashed curves) and NOMAD (dotted curves). Middle: The same allowed region with overlayed $90 \%$ and $99 \%$ exclusion limits from a combined analysis of all null appearance experiments. Right: Allowed region obtained by a combined analysis of all appearance data sets, signal and null. See text for more details.
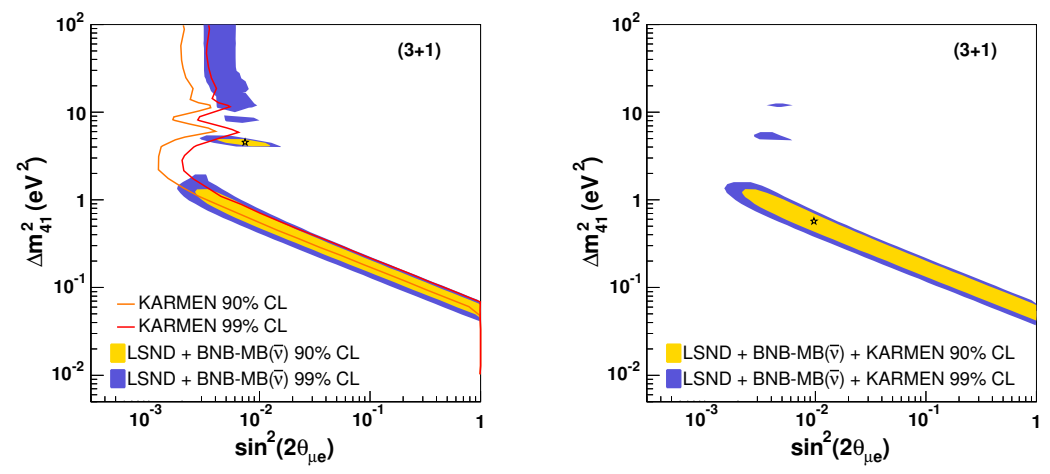

FIG. 4: Left: The allowed $90 \%$ and 99\% CL regions (light and dark filled areas, respectively) from a combined analysis of BNB-MB $(\bar{\nu})$ and LSND data sets, and $90 \%$ and $99 \%$ exclusion limits (light and dark curves, respectively) from KARMEN. A comparison of only these three experiments is interesting, as these three experiments have searched for antineutrino oscillations at short baselines. Right: The allowed regions obtained from a combined analysis of all three experiments (BNB-MB $(\bar{\nu}), \mathrm{LSND}$, and KARMEN). See text for more details.

of all appearance experiments under a $(3+1)$ oscillation scenario is shown in the right panel of Fig. 3.

Similarly, Fig. 4 (left) corresponds to the allowed region obtained by a joint analysis of $\operatorname{BNB}-\mathrm{MB}(\bar{\nu})+$ LSND. The limit shown is that of the KARMEN experiment, which is the only other SBL experiment to perform an appearance search with antineutrinos. The KARMEN limit provides substantial coverage of the joint LSND and
$\mathrm{BNB}-\mathrm{MB}(\bar{\nu})$ allowed region, excluding the best-fit point of the LSND + BNB-MB $(\bar{\nu})$ fit at $>99 \%$ CL. However, KARMEN imposes little constraint to the lower- $\Delta m^{2}$ allowed solutions. A joint analysis of all three data sets yields a $\chi^{2}$-probability of $29 \%$ for the best-fit hypothesis, and an allowed region shown on the right panel of Fig. 4. The $\chi^{2}$-probability remains the same for fits where the electron and muon content of the sterile mass 


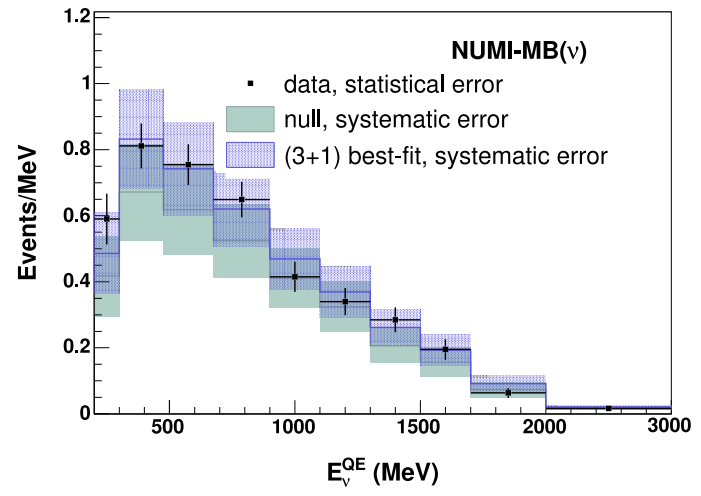

FIG. 5: Null and $(3+1)$ best-fit predicted event distributions $\left(\Delta m_{41}^{2}, \sin ^{2}\left(2 \theta_{\mu e}\right)\right)=(7.36,0.019)$ for NUMI-MB. The data are shown in black points with statistical uncertainty. The null (no-oscillation) prediction is shown by the light gray histogram with (solid) systematic error band. The best-fit prediction (signal and background) is shown by the blue (dark gray) histogram with (shaded) systematic error band.

eigenstates have been limited to values less than 0.3. As shown in Table IV, the three data sets are compatible at $15.3 \%$. New results from MiniBooNE with increased antineutrino statistics should be able to provide more information to these fits [17].

\section{Studies with appearance and disappearance experiments}

Much stronger constraints than those of the null appearance experiments are provided by the null disappearance experiments (CCFR84, CDHS, CHOOZ, and Bugey) and atmospheric constraints, under the assumptions of CPT conservation and unitarity of the neutrino mixing matrix. The $90 \%$ and $99 \%$ CL exlusion limits from a combined analysis of all null data sets (NUMI-MB, KARMEN, NOMAD, Bugey, CHOOZ, CCFR84, CDHS, and atmospheric constraints) are shown in Fig. 6. The figure shows that the parameter space jointly-allowed by $\operatorname{BNB}-\mathrm{MB}(\nu)+\mathrm{BNB}-\mathrm{MB}(\bar{\nu})+$ and LSND at $99 \%$ CL is excluded by a combined analysis of all null SBL experiments, appearance and disappearance, at 99\% CL. The severe tension between LSND and the null SBL experiments [14] continues to exist and in fact increases further with the addition of $\operatorname{BNB}-\mathrm{MB}(\nu)$ and $\operatorname{BNB}-\mathrm{MB}(\bar{\nu})$ data. The signal results show low $(0.15 \%)$ compatibility with null results. The LSND result remains to be mostly responsible for the low compatibility, as the $\operatorname{BNB}-\mathrm{MB}(\nu)$ and BNB-MB $(\bar{\nu})$ experiments show $14 \%$ and $3.7 \%$ compatibility with the null experiments, respectively.

Figure 7 shows the allowed region obtained by the joint BNB-MB $(\bar{\nu})+$ LSND + KARMEN + Bugey + CHOOZ analysis. Here, the $\bar{\nu}_{e}$ disappearance constraints

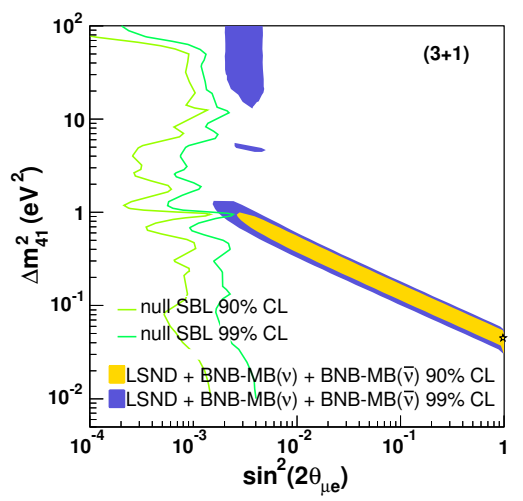

FIG. 6: The allowed $90 \%$ and $99 \%$ CL regions (light and dark filled areas, respectively) from a combined analysis of BNB$\operatorname{MB}(\nu)+\operatorname{BNB}-\mathrm{MB}(\bar{\nu})+\mathrm{LSND}$ data sets, and $90 \%$ and $99 \%$ exclusion limits (light and dark curves, respectively) from a combined analysis of all remaining (null, appearance and disappearance) SBL experiments. The null fit includes atmospheric constraints. The null SBL experiments exclude the joint $99 \%$ CL allowed region at 99\% CL.

from Bugey and $\mathrm{CHOOZ}$ are interesting to consider from the perspective of a joint analysis of only antineutrino SBL experiments. In a joint fit, all of the above (antineutrino) experiments yield a high $\chi^{2}$-probability of $86 \%$, and $29.9 \%$ compatibility. In these fits, Bugey and CHOOZ constrain $\left|U_{e 4}\right|$, but provide no direct constraints on $\left|U_{\mu 4}\right|$. However, a joint analysis with the LSND, BNB-MB $(\bar{\nu})$, and KARMEN appearance experiments, which are sensitive to the product of $\left|U_{e 4}\right|\left|U_{\mu 4}\right|$, provides indirect constraints to the $\left|U_{\mu 4}\right|$ mixing element. Figure 7 (left) also shows that a fit to all antineutrino experiments without LSND yields similar closed contours at $90 \% \mathrm{CL}$, which include the best-fit point. Current constraints from MiniBooNE on $\bar{\nu}_{\mu}$ disappearance [29] provide relatively small constraints to the $\sin ^{2} 2 \theta_{\mu \mu}$ allowed space, as illustrated in the right panel of Fig. 7. However, new results from a joint MiniBooNE and SciBooNE [30] $\bar{\nu}_{\mu}$ disappearance search, which are expected soon [31], may be able to probe this region with higher sensitivity, and will be interesting within the context of CPT-violating models. According to the best-fit oscillation parameters from a fit to only antineutrino SBL data, MiniBooNE should observe muon antineutrino disappearance with an amplitude of $\sin ^{2} 2 \theta_{\mu \mu} \sim 0.35$, at $\Delta m^{2} \sim 0.91 \mathrm{eV}^{2}$. The MINOS experiment [32] should also have sensitivity to these oscillation parameters in antineutrino running mode; muon antineutrino disappearance search results from MINOS are expected soon [33]. Incorporation of the upcoming MiniBooNE and MINOS disappearance results in these fits is currently being investigated.

Neutrino-only fits also yield a reasonably high $\chi^{2}$ - 

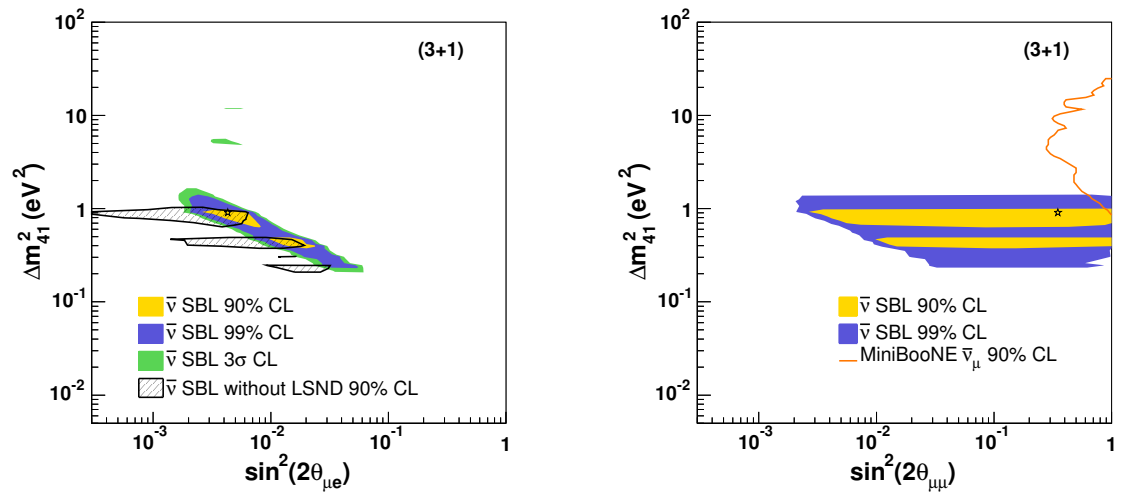

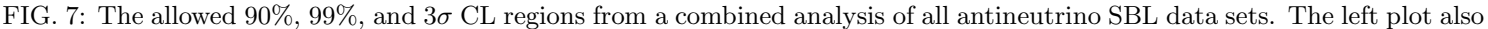
shows the 90\% CL allowed region obtained from a combined analysis of all antineutrino experiments except LSND (KARMEN, BNB-MB $(\bar{\nu})$, Bugey, and CHOOZ). The right plot also shows the 90\% CL exclusion limit from [29]. The MiniBooNE $\bar{\nu}_{\mu}$ disappearance search excludes the parameter space to the right of the line at $90 \%$ CL. See text for more details.
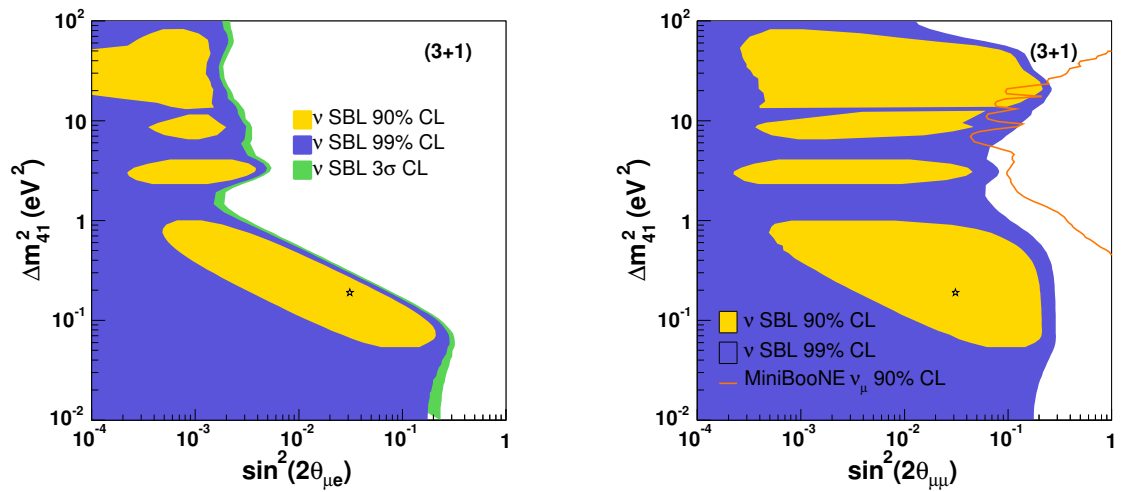

FIG. 8: The allowed $90 \%, 99 \%$, and $3 \sigma$ CL regions from a combined analysis of all neutrino SBL data sets. The right plot also shows the 90\% CL exclusion limit from [29]. The MiniBooNE $\nu_{\mu}$ disappearance search excludes the parameter space to the right of the line at $90 \%$ CL. See text for more details.

probability of $47 \%$; the corresponding allowed regions are shown in Fig. 8. Current constraints from MiniBooNE $\nu_{\mu}$ disappearance are shown on the right panel of Fig. 8. Interestingly, fits to only neutrino SBL data also yield a closed contour at 90\% CL. The parameter space, however, points to smaller mixing amplitudes relative to those preferred by the antineutrino-only fit. Neutrino-only fits and antineutrino-only fits are incompatible, with a PG of $0.03 \%$, as shown in Table IV. The large incompatibility between antineutrino and neutrino
SBL results suggests that the neutrino and antineutrino data sets cannot be accommodated within a $(3+1)$ CPTconserving sterile neutrino oscillation scenario. However, the constraining power of antineutrino SBL experiments alone on $\Delta m_{41}^{2}$ and $\sin ^{2} 2 \theta_{\mu e}$ is remarkable, and invites exploration of models that provide the possibility of different oscillation patterns for neutrinos versus antineutrinos.

Figure 9 (left) shows a comparison of the BNB-MB $(\nu)$, $\operatorname{BNB}-\mathrm{MB}(\bar{\nu})$, and NUMI-MB event distributions for the 

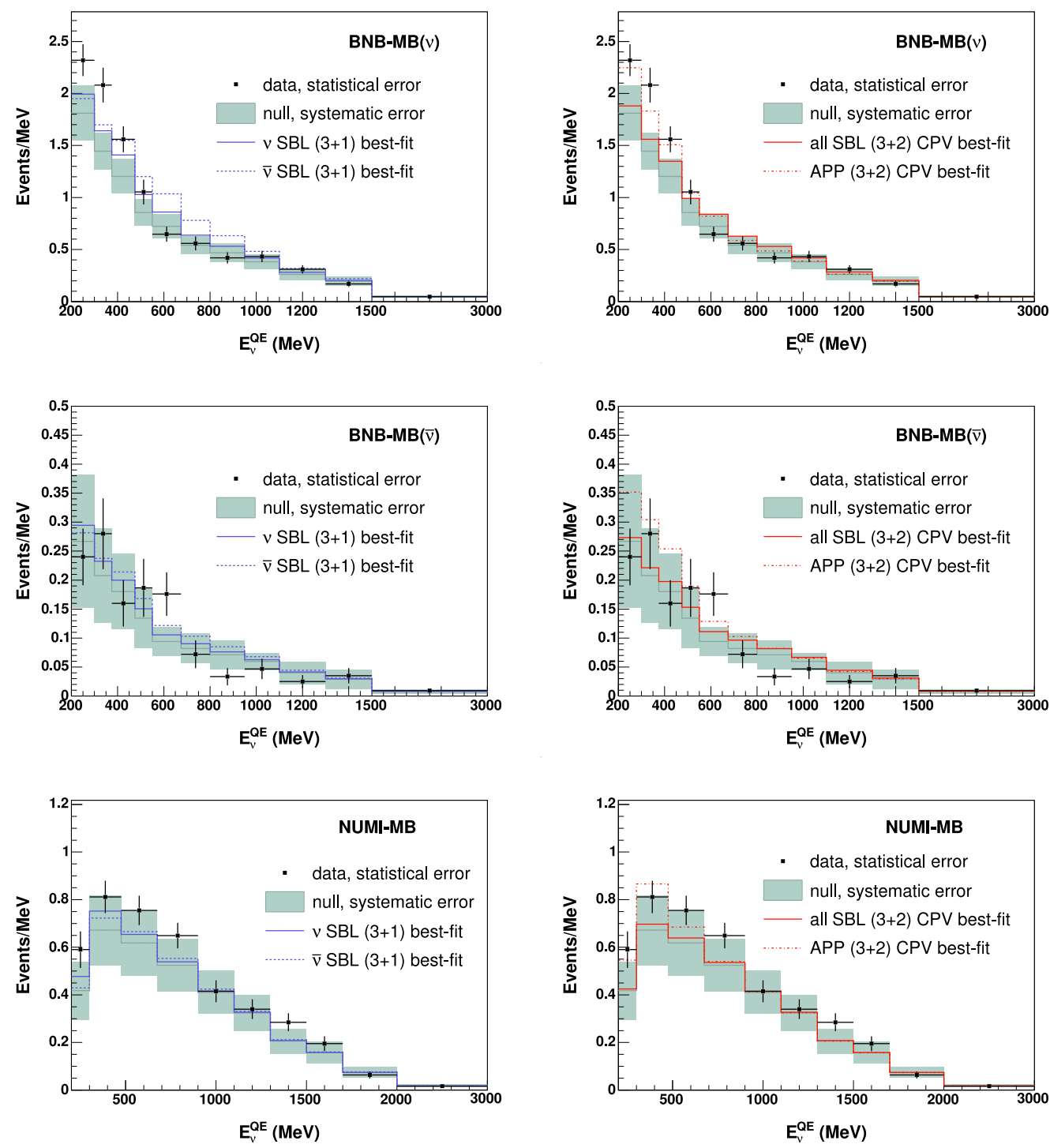

FIG. 9: Left: MiniBooNE predicted event distributions using the neutrino-only $(3+1)$ best-fit parameters $\left(\Delta m^{2}, \sin ^{2}(2 \theta)\right)=(0.19, \quad 0.031)$ in blue (dark gray) solid line and antineutrino-only $(3+1)$ best-fit parameters $\left(\Delta m^{2}, \sin ^{2}(2 \theta)\right)=(0.91,0.0043)$ in blue (dark gray) dashed line. The null predictions are shown in light gray with systematic error bands. The observed data are shown in black points with statistical error bars. Right: MiniBooNE predicted event distributions using the best-fit parameters obtained from a (3+2) CP-violating fit to all SBL experiments and appearance-only SBL experiments, in red (dark gray) solid line and red (dark gray) dashed line, respectively.

neutrino-only best-fit parameters and antineutrino-only best-fit parameters. The neutrino best-fit parameters provide a better description to BNB-MB $(\nu)$ and NUMIMB distributions than the antineutrino best-fit parameters, with $\chi_{B N B-M B(\nu)}^{2}=18.4$ and $\chi_{N U M I-M B}^{2}=4.4$, compared to $\chi_{B N B-M B(\nu)}^{2}=32.4$ and $\chi_{N U M I-M B}^{2}=$ 4.8. On the other hand, the antineutrino best-fit parameters provide a better description to $\mathrm{BNB}-\mathrm{MB}(\bar{\nu})$ than the neutrino best-fit parameters $\left(\chi_{B N B-M B(\bar{\nu})}^{2}=19.7\right.$, compared to $\left.\chi_{B N B-M B(\bar{\nu})}^{2}=21.7\right)$. 

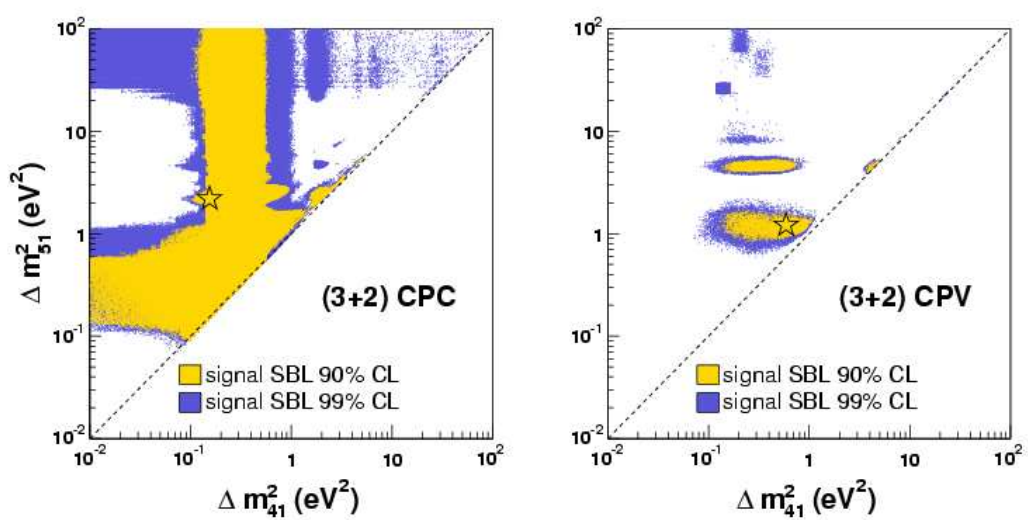

FIG. 10: Allowed regions in $\left(\Delta m_{41}^{2}, \Delta m_{51}^{2}\right)$ space for fits to CP-conserving (CPC, left) and CP-violating (CPV, right) $(3+2)$ oscillation models. Only the BNB-MB $(\nu), \operatorname{BNB}-\mathrm{MB}(\bar{\nu})$ and LSND data sets have been included in the fit.

The best-fit results from the $(3+1)$ oscillation fit involving all SBL data sets are summarized in Table III. The best-fit parameters from neutrino-only and antineutrino-only fits are also shown.

\section{B. (3+2) FIT RESULTS}

Neutrino oscillation models with more than one sterile neutrino have been of particular interest because they open up the possibility of observable $\mathrm{CP}$ violation effects in short-baseline neutrino oscillations. If $(3+n)$ sterile neutrino oscillations are realized in nature, with $n>1$, $\mathrm{CP}$ violation becomes a natural possibility, which is very appealing from the perspective of theories attempting to explain the matter-antimatter asymmetry in our universe [34].

In this section, the new MiniBooNE results are examined under both a CP-conserving (CPC) and a CPviolating $(\mathrm{CPV})(3+2)$ oscillation hypothesis. The new results are studied first within the context of appearanceonly experiments, and subsequently in fits involving both appearance and disappearance data.

From the point of view of the data at hand from LSND, $\operatorname{BNB}-\mathrm{MB}(\nu)$, and $\mathrm{BNB}-\mathrm{MB}(\bar{\nu})$ (see Fig. 1 ), CP violation offers the potential of reconciling two experimental signatures - an excess in LSND data at $3.8 \sigma$ significance and one suggested at 90\% CL in BNB-MB antineutrino data, both pointing to relatively large mixing, reconciled with a possible excess found in BNB-MB neutrino data suggesting relatively small mixing, both at a similar $L / E$ - as manifestations of the same underlying oscillation hypothesis.

It should be noted that in the studies presented in this section, due to the larger dimensionality of the fits, the electron and muon content of the sterile mass eigenstates have been limited to values less than 0.3. This is a realistic assumption for sterile neutrino oscillation models.

\section{Studies with appearance-only experiments}

Allowing for $\mathrm{CP}$ violation in $(3+2)$ fits to LSND and $\operatorname{BNB}-\mathrm{MB}(\nu$ and $\bar{\nu})$ data leads to a significant reduction in absolute $\chi^{2}$ of 12.2 , for 1 degree of freedom (dof), corresponding to a best-fit CPV phase $\phi_{45}=1.1 \pi$. The $\chi^{2}$-probability of the fit increases from $13 \%$ in the CPC case to $53 \%$ in the CPV case. The same test can be performed using all appearance data. In this case, allowing for $\mathrm{CP}$ violation leads to a reduction in $\chi^{2}$ of 13.3 for 1 dof, with a best-fit CPV phase $\phi_{45}=1.1 \pi$. The $\chi^{2}$ probability from the CPV fit is comparable to that of a signal-only fit, at $56 \%$.

The $90 \%$ and $99 \%$ CL allowed $\left(\Delta m_{41}^{2}, \Delta m_{51}^{2}\right)$ parameter space obtained by a combined fit to $\operatorname{BNB}-\mathrm{MB}(\nu)$ $+\operatorname{BNB}-\mathrm{MB}(\bar{\nu})+$ LSND is shown in Fig. 10. The figure illustrates that a CPV scenario (right panel) is much more restrictive in $\Delta m^{2}$ parameters compared to a CPC scenario (left panel). That is true both at $90 \%$ and $99 \%$ $\mathrm{CL}$, shown by the significant reduction in allowed regions around $\Delta m_{41}^{2}=0.5 \mathrm{eV}^{2}$ and $\Delta m_{51}^{2}=1 \mathrm{eV}^{2}$.

A similar effect is seen in the case of fits to all appearance experiments, as shown in Fig. 11. Allowing for CP violation in fits to neutrino and antineutrino appearance data sets lead to a considerable improvement in the fit quality, and provides strong constraints to the $\Delta m_{41}^{2}$ and $\Delta m_{51}^{2}$ parameters of the model.

The best-fit parameters for the signal-only and appearance-only fits are summarized in Table V. 

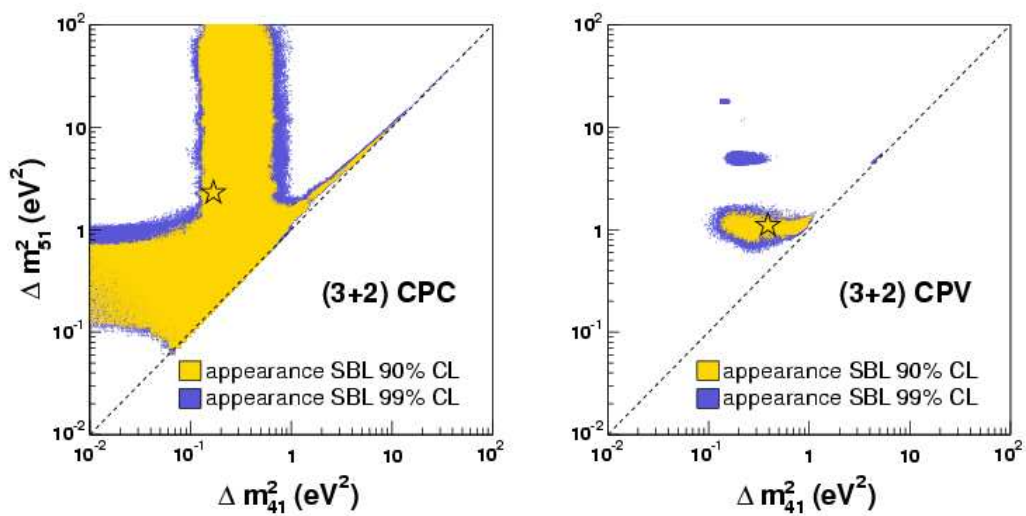

FIG. 11: Allowed regions in $\left(\Delta m_{41}^{2}, \Delta m_{51}^{2}\right)$ space for fits to CP-conserving (CPC, left) and CP-violating (CPV, right) $(3+2)$ oscillation models. Only appearance data sets have been included in the fit.
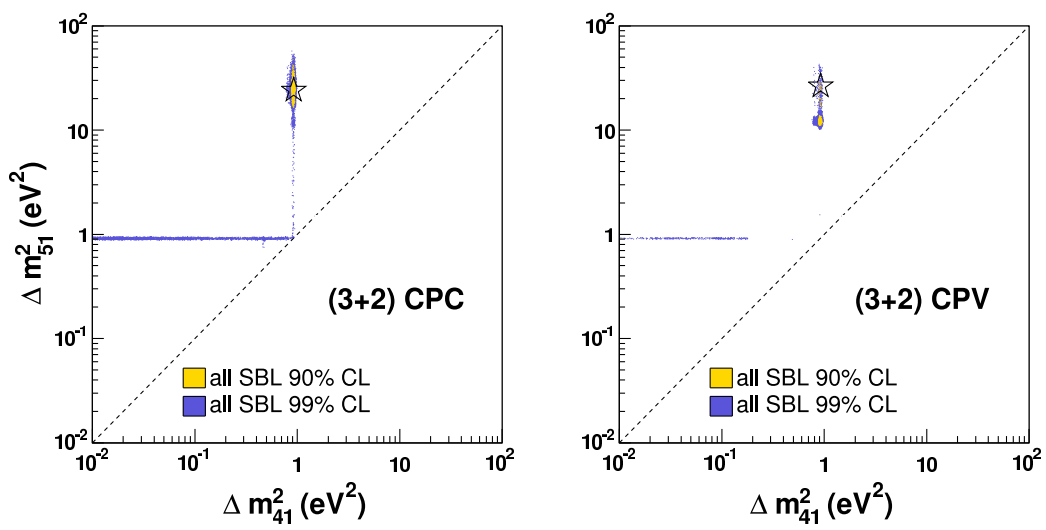

FIG. 12: Allowed regions in $\left(\Delta m_{41}^{2}, \Delta m_{51}^{2}\right)$ space for fits to CP-conserving (CPC, left) and CP-violating (CPV, right) $(3+2)$ oscillation models. All SBL data sets (appearance and disappearance) and atmospheric constraints have been included in the fit.

\section{Studies with appearance and disappearance experiments}

A dramatic reduction in the allowed $\left(\Delta m_{41}^{2}, \Delta m_{51}^{2}\right)$ parameter space occurs once all SBL data sets are considered in the fit, as shown in Fig. 12. Compared to the CPC hypothesis, with the addition of disappearance constraints, the CPV hypothesis fails to provide a substantially better description of the data, reflected by the reduction in $\chi^{2}$ of $\chi_{C P C}^{2}-\chi_{C P V}^{2}=2.2$ for 1 dof. Furthermore, mainly due to CDHS [5], the allowed $\Delta m^{2}$ regions shift to higher $\Delta m_{51}^{2}$ values near $\Delta m_{51}^{2}=25 \mathrm{eV}^{2}$.
The returned $\chi^{2}$-probabilities from fits to all SBL data are $52 \%$ for the CPC fit, and $54 \%$ for the CPV fit. A PG test among all experimental data sets for the CPV case yields a compatibility of $7.0 \%$. While the $\chi^{2}$-probability and compatibility for the $(3+2)$ CPV scenario are perfectly acceptable, as will be discussed in Sec. VI, an underlying source of tension exists due LSND and three other data sets: $\mathrm{BNB}-\mathrm{MB}(\nu)$, CDHS, and atmospheric constraints. The best-fit parameters extracted from a fit to all SBL data are also summarized in Table V.

A comparison of Tables III and V suggests that, with the addition of the new data sets from MiniBooNE, the 


\begin{tabular}{|c|c|c|c|c|c|c|c|c|c|c|}
\hline Data Set & Fit & $\chi^{2}(\operatorname{dof})$ & $\chi^{2}$-probability & $\Delta m_{41}^{2}$ & $\Delta m_{51}^{2}$ & $\left|U_{e 4}\right|$ & $\left|U_{\mu 4}\right|$ & $\left|U_{e 5}\right|$ & $\left|U_{\mu 5}\right|$ & $\phi_{45}$ \\
\hline signal APP & $\mathrm{CPV}$ & $34.7(36)$ & $53 \%$ & 0.59 & 1.21 & 0.19 & 0.33 & 0.20 & 0.16 & $1.1 \pi$ \\
\hline signal APP & $\mathrm{CPC}$ & $46.9(37)$ & $13 \%$ & 2.01 & 2.22 & 0.42 & 0.24 & 0.33 & 0.33 & 0 \\
\hline APP & CPV & $82.5(85)$ & $56 \%$ & 0.39 & 1.10 & 0.40 & 0.20 & 0.21 & 0.14 & $1.1 \pi$ \\
\hline APP & $\mathrm{CPC}$ & $95.8(86)$ & $22 \%$ & 0.18 & 2.31 & 0.32 & 0.38 & 0.086 & 0.071 & 0 \\
\hline all SBL & $\mathrm{CPV}$ & $189.3(192)$ & $54 \%$ & 0.92 & 26.5 & 0.13 & 0.13 & 0.078 & 0.15 & $1.7 \pi$ \\
\hline all SBL & $\mathrm{CPC}$ & $191.5(193)$ & $52 \%$ & 0.92 & 24.0 & 0.12 & 0.14 & 0.070 & 0.14 & 0 \\
\hline
\end{tabular}

TABLE V: Comparison of best-fit values for mass splittings and mixing parameters for $(3+2) \mathrm{CP}$-conserving $(\mathrm{CPC})$ and $\mathrm{CP}-$ violating $(\mathrm{CPV})$ models. Mass splittings are shown in $\mathrm{eV}^{2}$. The appearance experiments include $\mathrm{BNB}-\mathrm{MB}(\nu$ and $\bar{\nu}), \mathrm{LSND}$, NUMI-MB, KARMEN, and NOMAD. The signal experiments include LSND, BNB-MB $(\nu)$, and BNB-MB $(\bar{\nu})$. See text for more details.

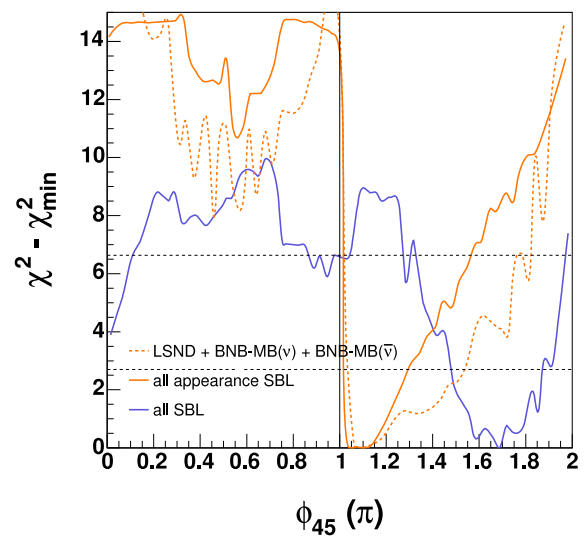

FIG. 13: Projection of $\Delta \chi^{2}=\chi^{2}-\chi_{\min }^{2}$ as a function of the $\mathrm{CP}$-violating phase $\phi_{45}$. The dashed horizontal lines indicate the $90 \%$ and $99 \%$ CL $\Delta \chi^{2}$.

$(3+2)$ CPV oscillation hypothesis provides a better description of all SBL data, compared to the $(3+1)$ hypothesis. Compared to $(3+1)$ models, $(3+2)$ CP-conserving models give a reduction of $5.9 \chi^{2}$ units for 3 additional fit parameters, while $(3+2) \mathrm{CP}$-violating models give a reduction of $8.1 \chi^{2}$ units with 4 additional parameters. This represents a relative improvement that is significantly smaller than that found in Ref. [5] from fits using data sets prior to atmospheric constraints and the new MiniBooNE results.

The MiniBooNE event distributions for the $(3+2)$ CPV best-fit parameters are shown on the right panels of Fig. 9. The resulting $\chi^{2}$-probabilities are $8.6 \%, 6.7 \%$, and $33.6 \%$, for $\operatorname{BNB}-\mathrm{MB}(\nu)$, BNB-MB $(\bar{\nu})$, and NUMI$\mathrm{MB}$, respectively, obtained using the best-fit parameters from a $(3+2)$ CPV fit to all SBL data. Notice, however, that the best-fit parameters from a $(3+2) \mathrm{CPV}$ fit to appearance-only SBL data provide a better description of all three MiniBooNE data sets than the best-fit parameters from a $(3+2) \mathrm{CPV}$ fit to all SBL data, particularly for the $\mathrm{BNB}-\mathrm{MB}(\nu)$ data set. Furthermore, in the case of NUMI-MB, the $(3+2)$ CPV appearance-only best-fit distribution, shown in dashed red (dark gray) on the right $\left(\chi^{2}\right.$-probability $\left.=61 \%\right)$, has comparable agreement with data as the $(3+1)$ neutrino-only best-fit distribution, shown in solid blue (dark gray) on the left $\left(\chi^{2}\right.$-probability $\left.=82 \%\right)$. In the case of $\operatorname{BNB}-\mathrm{MB}(\nu)$, the $(3+2)$ CPV appearance-only best-fit parameters are actually more preferred than the $(3+1)$ neutrino-only bestfit parameters, with a $\chi^{2}$-probability of $56.9 \%$, rather than $30.1 \%$. However, in the case of BNB-MB $(\bar{\nu})$ the $\chi^{2}-$ probability is highest $(23.4 \%)$ for the $(3+1)$ antineutrinoonly best fit parameters.

Figure 13 shows the projection of $\Delta \chi^{2}=\chi^{2}-\chi_{\min }^{2}$ as a function of the CP-violating phase $\phi_{45}$ for the three fits discussed in this section: the appearance-only fit projection is shown in the solid orange (light gray) line, the BNB-MB $(\nu)+\mathrm{BNB}-\mathrm{MB}(\bar{\nu})+\mathrm{LSND}$ fit projection in dashed orange (light gray), and the projection from a fit to all SBL experiments is shown in blue (dark gray). Both the fit to the three signal experiments (BNB$\operatorname{MB}(\nu)+\mathrm{BNB}-\mathrm{MB}(\bar{\nu})+\mathrm{LSND})$ and the fit to appearanceonly experiments seem to strongly prefer a CPV phase at $\phi_{45}=1.1 \pi$, as illustrated by the three overlapping dips in the $\Delta \chi^{2}$ distribution. However, when fits to all SBL data are considered, the strong dependence disappears and a CPV phase at $\phi_{45}=1.7 \pi$ is preferred.

\section{CONSTRAINTS TO (3+2) CP-VIOLATING FITS FROM EACH SBL EXPERIMENT}

In this section we study the constraints to experimentally allowed $(3+2)$ CP-violating oscillations by each of the SBL experiments considered in our fits. This is accomplished through a study where fits are performed using all-but-one experiment at a time. Within this study, we are also interested in examining the source of incompatibility between appearance and disappearance data, as well as testing compatibility between neutrino and antineutrino appearance search results within a CPviolating scenario. The latter is motivated by the large incompatibility found in neutrino versus antineutrino fits, as well as appearance versus disappearance fits.

Table VI summarizes the $\chi^{2}$-probability and PG results from $(3+2)$ CP-violating fits. The upper rows 


\begin{tabular}{|c|c|c|c|c|c|}
\hline Data Set & $\chi^{2}($ dof $)$ & $\chi^{2}$-probability $(\%)$ & $\mathrm{PG}(\%)$ & & \\
\hline all SBL & $189.3(192)$ & 54.2 & $\begin{array}{l}\text { PG( BNB-MB }(\nu), \text { BNB-MB }(\bar{\nu}), \text { NUMI-MB,LSND,KARMEN, } \\
\quad \text { NOMAD,Bugey,CHOOZ,CCFR84,CDHS,ATM }) \\
\text { PG( APP,DIS ) } \\
\text { PG }(\nu, \bar{\nu})\end{array}$ & $\begin{array}{l}=\operatorname{Prob}(53.9,40)= \\
=\operatorname{Prob}(25.5,4)= \\
=\operatorname{Prob}(25.4,7)=\end{array}$ & $\begin{array}{c}7.0 \\
0.004 \\
0.06 \\
\end{array}$ \\
\hline$\overline{\mathrm{APP}}$ & $82.5(85)$ & 55.7 & $\begin{array}{l}\text { PG( BNB-MB }(\nu), \text { BNB-MB }(\bar{\nu}), \text { NUMI-MB,LSND,KARMEN, } \\
\quad \text { NOMAD ) }\end{array}$ & $=\operatorname{Prob}(20.2,25)=$ & 73.6 \\
\hline DIS & $81.3(103)$ & 94.4 & PG( Bugey,CHOOZ,CCFR84,CDHS,ATM ) & $=\operatorname{Prob}(8.14,11)=$ & 70.1 \\
\hline$\nu$ & $81.3(86)$ & 62.4 & PG( BNB-MB $(\nu)$,NUMI-MB,NOMAD,CCFR84,CDHS,ATM ) & $=\operatorname{Prob}(17.3,17)=$ & 43.4 \\
\hline $\bar{\nu}$ & $82.6(99)$ & 88.3 & PG( BNB-MB $(\bar{\nu})$, KARMEN,LSND,Bugey,CHOOZ ) & $=\operatorname{Prob}(11.2,16)=$ & 79.7 \\
\hline$\nu \mathrm{APP}$ & $45.1(53)$ & 77.0 & PG( BNB-MB $(\nu)$, NUMI-MB,NOMAD ) & $=\operatorname{Prob}(3.07,10)=$ & 98.0 \\
\hline $\bar{\nu} \mathrm{APP}$ & $27.1(27)$ & 46.0 & $\begin{array}{l}\mathrm{PG}(\mathrm{BNB}-\mathrm{MB}(\bar{\nu}), \mathrm{KARMEN}, \mathrm{LSND}) \\
\mathrm{PG}(\nu \mathrm{APP}, \bar{\nu} \mathrm{APP})\end{array}$ & $\begin{array}{l}=\operatorname{Prob}(6.88,10)= \\
=\operatorname{Prob}(10.3,5)=\end{array}$ & $\begin{array}{r}73.7 \\
6.8 \\
\end{array}$ \\
\hline all - BNB-MB $(\nu)$ & $167.3(174)$ & 62.8 & $\mathrm{PG}($ all SBL - BNB-MB $(\nu), \operatorname{BNB}-\mathrm{MB}(\nu))$ & $=\operatorname{Prob}(15.7,5)=$ & 0.78 \\
\hline all - BNB-MB $(\bar{\nu})$ & $167.4(174)$ & 62.6 & PG( all SBL - BNB-MB $(\bar{\nu})$, BNB-MB $(\bar{\nu}))$ & $=\operatorname{Prob}(8.62,5)=$ & 13 \\
\hline all - NUMI-MB & $183.7(182)$ & 45.1 & PG( all SBL - NUMI-MB , NUMI-MB ) & $=\operatorname{Prob}(3.90,5)=$ & 56 \\
\hline all - LSND & $175.2(187)$ & 72.2 & PG( all SBL - LSND, LSND ) & $=\operatorname{Prob}(12.5,5)=$ & 2.9 \\
\hline all - KARMEN & $179.4(183)$ & 56.1 & PG( all SBL - KARMEN, KARMEN ) & $=\operatorname{Prob}(4.53,5)=$ & 48 \\
\hline all - NOMAD & $153.2(162)$ & 67.8 & PG( all SBL - NOMAD , NOMAD ) & $=\operatorname{Prob}(1.96,5)=$ & 86 \\
\hline all - Bugey & $140.4(132)$ & 29.2 & PG( all SBL - Bugey, Bugey ) & $=\operatorname{Prob}(3.90,4)=$ & 42 \\
\hline all - $\mathrm{CHOOZ}$ & $179.9(178)$ & 44.6 & PG( all SBL - CHOOZ, CHOOZ ) & $=\operatorname{Prob}(3.09,4)=$ & 54 \\
\hline all - CCFR84 & $174.3(174)$ & 47.9 & PG( all SBL - CCFR84, CCFR84) & $=\operatorname{Prob}(0.35,4)=$ & 99 \\
\hline all - CDHS & $172.8(177)$ & 57.5 & PG( all SBL- CDHS, CDHS ) & $=\operatorname{Prob}(9.21,4)=$ & 5.6 \\
\hline all - ATM & $184.0(190)$ & 60.9 & PG( all SBL - ATM , ATM ) & $=\operatorname{Prob}(5.31,1)=$ & 2.1 \\
\hline
\end{tabular}

TABLE VI: Comparison of $\chi^{2}$-probabilities for $(3+2)$ CP-violating fits with different combinations of SBL data sets. Also shown are $\mathrm{PG}$ results testing compatibility among different data sets. The last eleven rows of the table provide the compatibility (PG) between the experiment being removed from each fit and all remaining experiments. See text for more details.

\begin{tabular}{l|c}
\hline \hline Data Sets & PG (\%) \\
\hline \hline APP vs. DIS & 0.004 \\
APP (no BNB-MB $(\nu))$ vs. DIS (no CDHS + ATM) & 23.7 \\
APP (no BNB-MB $(\nu))$ vs. DIS (no CDHS) & 0.36 \\
APP (no BNB-MB $(\nu))$ vs. DIS (no ATM) & 0.52 \\
APP (no BNB-MB $(\nu))$ vs. DIS & 0.067 \\
APP vs. DIS (no CDHS + ATM) & 2.9 \\
APP vs. DIS (no CDHS) & 0.027 \\
APP vs. DIS (no ATM) & 0.019 \\
\hline \hline
\end{tabular}

TABLE VII: Comparison of compatibility between appearance (APP) and disappearance (DIS) experiments, within a $(3+2) \mathrm{CP}$-violating scenario. The $\mathrm{BNB}-\mathrm{MB}(\nu)$ data set, CDHS data set, and atmospheric constraints (ATM) are removed from the fits as specified in order to establish the source of tension between appearance and disappearance experiments. Compatibilities are obtained using $n d f_{P G}=4$. See text for more details.

summarize $\chi^{2}$-probabilities and PG's from fits to all SBL experiments, as well as fits to appearance-only, disappearance-only, neutrino-only, antineutrino-only, neutrino appearance-only, and antineutrino appearanceonly data sets. Appearance and disappearance data sets, as well as neutrino and antineutrino data sets, are incompatible with a PG of less than $0.1 \%$. Grouping SBL appearance-only data sets according to whether they are neutrino or antineutrino experiments yields significantly higher compatibilities - $98 \%$ among $\nu$ appearance exper-

\begin{tabular}{l|c}
\hline \hline Data Sets & PG $(\%)$ \\
\hline \hline$\nu$ vs. $\bar{\nu}$ & 0.06 \\
$\nu($ no BNB-MB $(\nu)+$ CDHS + ATM) vs. $\bar{\nu}$ & 56.5 \\
$\nu($ no BNB-MB $(\nu)+$ CDHS) vs. $\bar{\nu}$ & 3.7 \\
$\nu$ (no BNB-MB $(\nu)+$ ATM) vs. $\bar{\nu}$ & 4.4 \\
$\nu$ (no BNB-MB $(\nu))$ vs. $\bar{\nu}$ & 1.1 \\
$\nu$ (no CDHS + ATM) vs. $\bar{\nu}$ & 2.3 \\
$\nu$ (no CDHS $)$ vs. $\bar{\nu}$ & 0.07 \\
$\nu($ no ATM $)$ vs. $\bar{\nu}$ & 0.21 \\
\hline \hline
\end{tabular}

TABLE VIII: Comparison of compatibility between neutrino $(\nu)$ and antineutrino $(\bar{\nu})$ experiments, within a $(3+2) \mathrm{CP}-$ violating scenario. The BNB-MB $(\nu)$ data set, CDHS data set, and atmospheric constraints (ATM) are removed from the fits as specified in order to establish the source of tension between neutrino and antineutrino experiments. Compatibilities are obtained using $n d f_{P G}=7$. See text for more details.

iments, and $74 \%$ among $\bar{\nu}$ appearance experiments. The compatibility between $\nu$ and $\bar{\nu}$ appearance-only results is lower, at $6.8 \%$ but still acceptable. In the case where disappearance experiments are included in the comparison between neutrino and antineutrino fits, the compatibility among all $\bar{\nu}$ SBL data sets remains considerably high, at $80 \%$, as does the compatibility among all $\nu$ SBL data sets, at $43 \%$. However the compatibility between $\bar{\nu}$ and $\nu$ results is only $0.06 \%$.

The remaining rows of Table VI provide the $\chi^{2}$ - 
probabilities of global fits under the same oscillation scenario where one experiment is excluded from the fit at a time (as indicated by the "-" sign in the table). The $\chi^{2}$ probabilities of all fits are acceptable, ranging between $29.2 \%$ for a fit excluding the Bugey data set, and $72.2 \%$ for a fit excluding the LSND data set. Aside from LSND, three experiments stand out as having the poorest compatibility when compared to a global fit with all other SBL data sets: 1) BNB-MB $(\nu), 2)$ CDHS and 3) atmospheric constraints (ATM). These three experiments have been identified as the possible source of tension between appearance and disappearance experiments, or neutrino and antineutrino experiments. The remaining combinations yield reasonably high compatibilities of at least $42 \%$, with the exception of LSND and BNB-MB $(\bar{\nu})$ which are compatible with the remaining data sets at $2.9 \%$ and $13 \%$, respectively.

To further test the hypothesis that the tension between appearance and disappearance experiments is a result of the BNB-MB $(\nu)$ and CDHS data sets and atmospheric constraints, the compatibility between appearance and disappearance experiments is re-evaluated several times. Each time, a different combination of these three experiments is excluded from the fits. The results are summarized in Table VII. The compatibility among appearance and disappearance experiments with BNB-MB $(\nu)$, CDHS, and atmospheric constraints excluded from the fits is high, at $23.7 \%$. The $\mathrm{BNB}-\mathrm{MB}(\nu)$ data set alone is not responsible for the disagreement between appearance and disappearance experiments, as suggested by the sixth row of the Table VII. Even with BNB-MB $(\nu)$ included in the fit, a compatibility of $2.9 \%$ can be obtained if CDHS and atmospheric constraints are excluded from the fit.

The same test can be performed between neutrino and antineutrino experiments. The results are summarized in Table VIII. Again, the compatibility between neutrino and antineutrino experiments is re-evaluated several times; each time, a different combination of the $\operatorname{BNB}-\mathrm{MB}(\nu)$, CDHS, and atmospheric constraint data sets is excluded from the fits. Here, the compatibility among neutrino and antineutrino experiments with $\mathrm{BNB}-\mathrm{MB}(\nu)$, CDHS, and atmospheric constraints excluded from the fits is even higher, at $56 \%$. The BNB$\operatorname{MB}(\nu)$ data set is just as responsible for the disagreement between neutrino and antineutrino experiments as the CDHS data set and atmospheric constraints alone. The tension seems to be caused by all three experiments, as none of them independently excluded from the fit can accound for the increase in compatibility from $\sim 1 \%$ (or less) to $56 \%$.

It is possible that higher compatibility between BNB$\operatorname{MB}(\nu)$ and all remaining SBL data sets may be achieved if the fits are to be repeated with the low energy region $\left(200<E_{\nu}^{Q E}<475 \mathrm{MeV}\right)$ excluded from the BNB-MB $(\nu)$ data set.

A global analysis with $\mathrm{BNB}-\mathrm{MB}(\nu)$, CDHS, and atmospheric constraints excluded from the fit yields a $\chi^{2}$ - probability of $82 \%$ and $>90 \%$ compatibility among all experiments.

\section{CONCLUSIONS}

We have re-examined global fits to sterile neutrino oscillation models, using new data from MiniBooNE. Those include the final MiniBooNE neutrino mode results and the first, low statistics MiniBooNE antineutrino results, as well as first results from the off-axis NuMI beam observed in the MiniBooNE detector.

Within a $(3+1) \mathrm{CP}$ - and CPT-conserving scenario, we have found that the data set collected by MiniBooNE using the NuMI off-axis beam (NUMI-MB) currently provides very weak constraints to sterile neutrino fits, due to large systematic uncertainties. Updated NuMI results, expected soon, should have a greater impact on these fits.

Within the same oscillation framework, the MiniBooNE antineutrino data set (BNB-MB $(\bar{\nu})$ ) is found in agreement with LSND, yielding, in a combined analysis with LSND and KARMEN under a $(3+1)$ oscillation hypothesis, a $\chi^{2}$-probability of $29 \%$, and best-fit parameters similar to those of LSND. Updated MiniBooNE antineutrino appearance results, with almost twice the current statistics, are expected in the near future.

The MiniBooNE neutrino data set (BNB-MB $(\nu)$ ), although suggestive of an excess that could be described by a $(3+1)$ oscillation hypothesis with a $\chi^{2}$ probability of $35 \%$, is found incompatible with the signals from the MiniBooNE antineutrino and LSND results.

The remaining null appearance and disappearance experiments (NUMI-MB $(\nu)$, KARMEN, NOMAD, Bugey, CHOOZ, CDHS, CCFR84) and atmospheric oscillation data impose strong constraints to the parameter space allowed by a combined $(3+1)$ analysis of MiniBooNE neutrino and antineutrino and LSND data, excluding the 99\% CL allowed region at 99\% CL. However, the constraints from antineutrino disappearance experiments on the parameter space allowed by antineutrino appearance experiments (BNB-MB $(\bar{\nu})$, LSND, and KARMEN) are weaker. In a $(3+1)$ oscillation framework, all antineutrino experiments yield a best-fit $\chi^{2}$-probability of $86 \%$, and exclude the no-oscillations hypothesis at $>5.0 \sigma$. The best-fit parameters are similar to those of LSND, and correspond to a muon antineutrino disappearance amplitude of 0.35 , which may be addressed by upcoming results from MiniBooNE and MINOS on muon antineutrino disappearance. Additionally, fits to all neutrino experiments yield a best-fit $\chi^{2}$-probability of $47 \%$ and exclude the null hypothesis at $>90 \%$ CL.

Furthermore, we find that with the addition of the new MiniBooNE data sets, the $(3+2)$ oscillation models provide a much better description of all SBL data sets compared to $(3+1)$ models. In the case of $(3+2)$ fits, $\mathrm{CP}$ violation allows for a significant improvement in $\chi^{2}$ probability for fits to only $\mathrm{BNB}-\mathrm{MB}(\nu)+\mathrm{BNB}-\mathrm{MB}(\bar{\nu})+$ LSND, and fits to only appearance experiments. In the 
case of global fits, however, the effect of $\mathrm{CP}$ violation is muted, as allowing for $\mathrm{CP}$ violation results in a relatively small improvement in the fit. The $\chi^{2}$-probability for the best-fit (3+2) CPV hypothesis is $54 \%$, compared to $52 \%$ for the CPC case. The best-fit corresponds to large but not maximal $\mathrm{CP}$ violation $\left(\phi_{45}=1.7 \pi\right)$.

The high incompatibility among appearance and disappearance data seen in the past [14] in the case of $(3+2)$ $\mathrm{CP}$-violating fits still remains with the addition of the new MiniBooNE results. We have shown that the incompatibility is a result of the $\operatorname{BNB}-\operatorname{MB}(\nu)$ and CDHS data sets and atmospheric constraints. The compatibility between appearance and disappearance data with these three experiments excluded from the fits is significantly higher, at $24 \%$.

Neutrino and antineutrino results are also incompatible within a $(3+2) \mathrm{CP}$-violating scenario, with a PG of less than $0.1 \%$. The compatibility improves to $6.8 \%$ in the case of comparing appearance-only neutrino versus antineutrino results.

Overall, allowing for mixing with multiple sterile neutrino states and $\mathrm{CP}$ violation does not seem sufficient to allow incorporating all SBL experiments within a CPTconserving, sterile neutrino oscillation framework. It may be that there is an issue with one or more of the following data sets: LSND, BNB-MB $(\nu)$, including the low-energy excess, CDHS, or atmospheric constraints; alternatively, theories with CPT-violating oscillations or effective CPT violation [35-37] may succeed in reconciling all shortbaseline oscillation signatures, and should be explored.

\section{Acknowledgments}

We thank Bill Louis for valuable discussions. We also thank the National Science Foundation for their support.
[1] C. Athanassopoulos et al. [LSND Collaboration], Phys. Rev. Lett. 77, 3082 (1996) [arXiv:nucl-ex/9605003]; C. Athanassopoulos et al. [LSND Collaboration], Phys. Rev. C 58, 2489 (1998) [arXiv:nucl-ex/9706006]; A. Aguilar et al. [LSND Collaboration], Phys. Rev. D 64, 112007 (2001) [arXiv:hep-ex/0104049].

[2] O. L. G. Peres and A. Y. Smirnov, Nucl. Phys. B 599, 3 (2001) [arXiv:hep-ph/0011054].

[3] A. Strumia, Phys. Lett. B 539, 91 (2002) [arXiv:hep$\mathrm{ph} / 0201134]$.

[4] W. Grimus and T. Schwetz, Eur. Phys. J. C 20, 1 (2001) [arXiv:hep-ph/0102252].

[5] M. Sorel, J. M. Conrad and M. H. Shaevitz, Phys. Rev. D 70, 073004 (2004) [arXiv:hep-ph/0305255].

[6] M. Maltoni, T. Schwetz, M. A. Tortola and J. W. F. Valle, Nucl. Phys. B 643, 321 (2002) [arXiv:hep-ph/0207157].

[7] B. Armbruster et al. [KARMEN Collaboration], Phys. Rev. D 65, 112001 (2002) [arXiv:hep-ex/0203021].

[8] P. Astier et al. [NOMAD Collaboration], Phys. Lett. B 570, 19 (2003) [arXiv:hep-ex/0306037]; D. Gibin, Nucl. Phys. Proc. Suppl. 66, 366 (1998); V. Valuev [NOMAD Collaboration], SPIRESentry Prepared for International Europhysics Conference on High-Energy Physics (HEP 2001), Budapest, Hungary, 12-18 Jul 2001

[9] Y. Declais et al., Nucl. Phys. B 434, 503 (1995).

[10] M. Apollonio et al., [arXiv:hep-ex/0301017].

[11] I. E. Stockdale et al., Phys. Rev. Lett. 52, 1384 (1984).

[12] F. Dydak et al., Phys. Lett. B 134, 281 (1984).

[13] A. A. Aguilar-Arevalo et al. [The MiniBooNE Collaboration], Phys. Rev. Lett. 98, 231801 (2007) [arXiv:hepex/0704.1500].

[14] M. Maltoni and T. Schwetz, Phys. Rev. D 76, 093005 (2007) [arXiv:hep-ph/0705.0107]

[15] G. Karagiorgi et al., Phys. Rev. D 75, 013011 (2007) [arXiv:hep-ph/0609177].

[16] A. A. Aguilar-Arevalo et al. [MiniBooNE Collaboration], Phys. Rev. Lett. 102, 101802 (2009) [arXiv:hep$\mathrm{ex} / 0812.2243]$.
[17] A. A. Aguilar-Arevalo et al. [MiniBooNE Collaboration], [arXiv:hep-ex/0904.1958].

[18] P. Adamson et al., Phys. Rev. Lett. 102, 211801 (2009) [arXiv:hep-ex/0809.2447].

[19] M. Maltoni, T. Schwetz, M. Tortola and J. W. F. Valle, New J. Phys. 6, 122 (2004) [arXiv:hep-ph/0405172].

[20] S. Goswami and W. Rodejohann, JHEP 0710, 073 (2007) [arXiv:0706.1462 [hep-ph]].

[21] A. Melchiorri, O. Mena, S. Palomares-Ruiz, S. Pascoli, A. Slosar and M. Sorel, JCAP 0901, 036 (2009) [arXiv:0810.5133 [hep-ph]].

[22] A. D. Dolgov and F. L. Villante, Nucl. Phys. B 679, 261 (2004) [arXiv:hep-ph/0308083].

[23] A. A. Aguilar-Arevalo et al. [MiniBooNE Collaboration], Phys. Rev. D 79, 072002 (2009) [arXiv:hep$\mathrm{ex} / 0806.1449]$.

[24] A. A. Aguilar-Arevalo et al. [MiniBooNE Collaboration], Nucl. Instrum. Meth. A 599, 28 (2009) [arXiv:hepex/0806.4201].

[25] V. Barger, Y. B. Dai, K. Whisnant and B. L. Young, Phys. Rev. D 59, 113010 (1999) [arxiv:hep-ph/9901388].

[26] B. Kayser, [arXiv:hep-ph/0211134].

[27] P. Brâemaud, Markov chains: Gibbs fields, Monte Carlo simulation, and queues, Springer, New York, 1999.

[28] M. Maltoni and T. Schwetz, Phys. Rev. D 68, 033020 (2003) [arXiv:hep-ph/0304176].

[29] A. A. Aguilar-Arevalo et al. [MiniBooNE Collaboration], Phys. Rev. Lett. 103, 061802 (2009) [arXiv:0903.2465 [hep-ex]].

[30] A. A. Aguilar-Arevalo et al. [SciBooNE Collaboration], arXiv:hep-ex/0601022.

[31] K. B. M. Mahn, private communication.

[32] I. Ambats et al. [MINOS Collaboration], FERMILABDESIGN-1998-02.

[33] MINOS, J. Hartnell, (2009), FNAL Seminar, 15 May 2009. URL:http://theory.fnal.gov/jetp/talks/Hartnell.pdf.

[34] A. D. Sakharov, Pisma Zh. Eksp. Teor. Fiz. 5, 32 (1967) [JETP Lett. 5, 24 (1967 SOPUA,34,392-393.1991 
UFNAA,161,61-64.1991)].

[35] G. Barenboim and J. D. Lykken, Phys. Lett. B 554, 73 (2003) [arXiv:hep-ph/0210411].

[36] G. Barenboim and J. D. Lykken, arXiv:0908.2993 [hep$\mathrm{ph}$.

[37] S. Hollenberg and H. Pas, arXiv:0904.2167 [hep-ph].
[38] This constraint is imposed under the assumption that mixing with sterile neutrinos should be small. 


\section{Chapter 7}

\section{A Search for Mixing Freedom at MiniBooNE}

Chapter 4 presented the method by which MiniBooNE searches for $L / E$-dependent $\nu_{e}$ appearance in a $\nu_{\mu}$-dominated beam as well as $\bar{\nu}_{e}$ appearance in a $\bar{\nu}_{\mu}$-dominated beam, both at an $L / E$ of $\sim 1 \mathrm{eV}^{2}$. The fit method and results presented in Chapters 4 and 5 investigate one specific scenario of mixing freedom, namely light sterile neutrinos (scenario 1 in Sec. 2.1.1).

In this chapter, a similar approach is followed to search for a more general effect of mixing freedom, that of instantaneous flavor transitions. Due to MiniBooNE's short baseline $(L \sim 0)$ and low intrinsic $\nu_{e}$ and $\bar{\nu}_{e}$ contamination in either running mode, MiniBooNE is an ideal experiment to constrain $\left|\left(U U^{\dagger}\right)_{e \mu}\right|$ and $\left|\left(U U^{\dagger}\right)_{\mu e}\right|$, given a $3 \times 3$ neutrino mixing matrix, $U$. Assuming no new physics which can lead to mixing freedom, those two parameters should be exactly zero. A significant deviation from zero could be an indication of mixing freedom, and it would manifest in MiniBooNE as an excess of $\nu_{e}$ and/or $\bar{\nu}_{e}$ CCQE events over background prediction in each running mode.

The search described in this chapter is applicable to scenario 2 in Chapter 2, as well as other underlying physics scenarios. In actuality, it applies to scenario 2 only within the approximation that the very light sterile neutrinos have negligibly small mixings and therefore do not significantly participate in oscillations, so that 
the $\Delta m^{2}$-dependent term of Eq. 2.16 can be safely ignored. An alternative scenario is one where only the heavier sterile neutrinos exist.

In the following two sections, we give a brief overview of the method by which the MiniBooNE neutrino and antineutrino appearance data sets from [101, 102] are fit (independently) to search for these mixing freedom effects. The results are presented in Sec. 7.4. We warn that the results are not competitive with constraints from electroweak measurements; however, the fit method followed in this analysis sets up the technique and acts as an example of how one may directly probe mixing freedom in the neutrino sector.

The remainder of this chapter will not distinguish between $\nu$ and $\bar{\nu}$ rates, except where explicitly stated otherwise. Specifically, by $\nu$ we refer to both $\nu$ and $\bar{\nu}$.

\subsection{General Assumptions for a Search for Instan- taneous Transitions}

This search examines the MiniBooNE neutrino and antineutrino results first under the hypothesis of only $\nu_{\mu} \rightarrow \nu_{e}$ instantaneous transitions, which is the channel in which the MiniBooNE experiment has the highest sensitivity, and then under the hypothesis of both $\nu_{\mu} \rightarrow \nu_{e}$ instantaneous transitions and $\nu_{\mu} \rightarrow \nu_{\mu}$ and $\nu_{e} \rightarrow \nu_{\phi}$ instantaneous transitions.

In general, it is artificial to assume that only $\nu_{\mu} \rightarrow \nu_{e}$ transitions due to mixing freedom will take place in a given experiment. As in the case of standard threeneutrino oscillations, in the presence of mixing freedom, both $\nu_{\mu} \rightarrow \nu_{e}$ and $\nu_{\mu} \rightarrow \nu_{x}$ and/or $\nu_{e} \rightarrow \nu_{x}$ transitions can occur, ${ }^{1}$ the latter corresponding to $\left|\left(U U^{\dagger}\right)_{\mu \mu}\right| \neq 1$ and $\left|\left(U U^{\dagger}\right)_{e e}\right| \neq 1$, respectively. The simultaneous occurrence of these effects can lead to a more complicated experimental signature, and is therefore more appropriate as well as interesting to explore.

In practice, in a more general scenario where those transitions are also allowed to

\footnotetext{
${ }^{1}$ Limiting this statement to experiments with sensitivity only to muon and electron type neutrinos, as in the case of MiniBooNE.
} 
occur, both the MiniBooNE signal prediction and the $\nu_{e}$ and $\nu_{\mu}$ background predictions must be modified accordingly.

The first scenario we consider (only $\nu_{\mu} \rightarrow \nu_{e}$ transitions) may be thought of as an appearance search with an oscillation probability which is independent of $L$ and $E$. In this scenario, which we consider under "Fit I", muon neutrinos in the beam which we assume are primarily produced in $\mathrm{CC}$ weak decays can undergo an instantaneous transition into electron neutrinos, and are then detected (as electron neutrinos) via the CCQE interaction in the MiniBooNE detector.

The second scenario (both $\nu_{\mu} \rightarrow \nu_{e}$ as well as $\nu_{\mu} \rightarrow \nu_{\mu}$ and $\nu_{e} \rightarrow \nu_{q}$ transitions) may be thought of as a $\nu_{\mu} \rightarrow \nu_{e}$ instantaneous appearance search where the $\nu_{e}$ background and $\nu_{\mu} \mathrm{CCQE}$ predictions are also subject to disappearance. The effect of the instantaneous disappearance is to decrease normalizations for $\nu_{\mu}$ CCQE and various $\nu_{e}$ background rates expected in the detector. Again, all three oscillation probabilities are independent of $L$ and $E$, and each is allowed to vary independently.

We perform each of the above searches with both the neutrino and antineutrino appearance data sets from $[101,102]{ }^{2}$ In the case of a neutrino mode search, it is conservative to consider separately a fit to $E>200 \mathrm{MeV}$, and a fit to $E>475 \mathrm{MeV}$. The $E>200 \mathrm{MeV}$ is motivated by the question of whether the low energy excess can be explained through instantaneous transitions due to mixing freedom. However, from the perspective on placing constraints to mixing freedom under the null hypothesis, the $E>475 \mathrm{MeV}$ fit is the default choice. In the case of the antineutrino mode search, since the data set in consideration shows no significant low energy excess, the fit is performed over the full $E_{\nu}^{Q E}$ range.

Note that no constraints from near detectors or other oscillation experiments on the $\left|\left(U U^{\dagger}\right)_{\alpha \beta}\right|$ elements have been considered in these fits. Such combined analyses have been performed using experimental data sets other than MiniBooNE (see, e.g. Ref. [89]), and would be interesting to revisit given constraints from MiniBooNE extracted in this work.

\footnotetext{
${ }^{2}$ Note that the antineutrino data used in these fits are from the first MiniBooNE antineutrino search, corresponding $3.39 \times 10^{20}$ POT, and not the data presented in Chapter 5 of this thesis.
} 


\subsection{Predicting MiniBooNE Event Rates When Mix- ing Is Free}

In either running mode, neutrino or antineutrino, in order to obtain a total $\nu_{e}$ prediction for both signal and background, one must account for mixing freedom effects both at neutrino production and at neutrino detection. These effects can vary depending on the type of interaction by which neutrinos are produced and/or detected [88].

For simplicity, the fit assumes that all neutrino event rates subject to instantaneous transitions are due to neutrinos that were both produced and detected through $\mathrm{CC}$ interactions. We will demonstrate later on that, in the case of MiniBooNE, this is a reasonable approximation. In that case, assuming a free $3 \times 3$ neutrino mixing matrix, the number of expected events from transitions is given by [89]

$$
n=\int d E \frac{d \Phi_{\alpha}(E)}{d E} P_{\alpha \beta}(L / E) \sigma_{\beta}(E) \epsilon(E)
$$

In the above equation, $\Phi_{\alpha}(E)$ is the flux of neutrinos of flavor $\alpha$ (which is affected by mixing freedom); $P_{\alpha \beta}(L / E)$ is the oscillation probability given in Eq. 2.16, with appropriate normalization factors for a scenario with mixing freedom; and $\sigma_{\beta}(E)$ is the detection cross-section for a neutrino of flavor $\beta$ (which also depends on mixing freedom). The factor $\epsilon(E)$ is the detector and event selection efficiency as a function of energy.

Since we have assumed that all neutrinos are produced and detected only through $\mathrm{CC}$ interactions, the expressions for the flux and cross-section can be written as

$$
\frac{d \Phi_{\alpha}(E)}{d E}=\frac{d \Phi_{\alpha}^{C C}(E)}{d E}=\frac{d \Phi_{\alpha}^{C C(S M)}(E)}{d E}\left|\left(U U^{\dagger}\right)_{\alpha \alpha}\right|
$$

and

$$
\sigma_{\beta}(E)=\sigma_{\beta}^{C C}(E)=\sigma_{\beta}^{C C(S M)}(E)\left|\left(U U^{\dagger}\right)_{\beta \beta}\right|
$$

where $\Phi_{\alpha}^{C C(S M)}(E)$ and $\sigma_{\beta}^{C C(S M)}(E)$ are the flux and cross-section for neutrino $\alpha$ and 
$\beta$, respectively, extracted based on Standard-Model (no mixing freedom) assumptions. The factor $\left|\left(U U^{\dagger}\right)_{\alpha \alpha}\right|$ simply implies that, if there are instantaneous transitions $\left(\left|\left(U U^{\dagger}\right)_{\alpha \alpha}\right| \neq 1\right)$, not all decays producing neutrinos will contribute to the $\nu_{\alpha}$ flux. Likewise, the factor $\left|\left(U U^{\dagger}\right)_{\beta \beta}\right|$ implies that not all $\nu_{\beta}$ neutrinos interacting in the detector will produce a $\beta$ lepton, as expected, because mixing is free.

Note that, in many neutrino experiments, what is used as $\Phi_{\alpha}^{(S M)}(E)$ may already include mixing freedom effects, for example in the case where the flux prediction uses experimentally determined parameters as input. That is certainly true in the case of the MiniBooNE flux predictions, which use as input production cross-section parameters which come from external experimental measurements, as discussed in Sec. 3.4.1. The same applies in the case of neutrino cross-section parameters. This makes a search for transitions of the form $\alpha=\beta$ particularly challenging, if not impossible.

For instantaneous transitions due to mixing freedom, the oscillation probability of Eq. 7.1 is given by

$$
P_{\alpha \beta}(L / E=0)=\frac{\left|\left(U U^{\dagger}\right)_{\beta \alpha}\right|^{2}}{\left|\left(U U^{\dagger}\right)_{\beta \beta}\right|\left|\left(U U^{\dagger}\right)_{\alpha \alpha}\right|} .
$$

Therefore, the expression for the expected number of events becomes

$$
n=\int d E \frac{d \Phi_{\alpha}^{C C(S M)}(E)}{d E}\left|\left(U U^{\dagger}\right)_{\beta \alpha}\right|^{2} \sigma_{\beta}^{C C(S M)}(E) \epsilon(E)
$$

The above equation applies to a neutrino beam. In the case of an antineutrino beam, the probability changes to

$$
\bar{P}_{\alpha \beta}(L / E=0)=\frac{\left|\left(U U^{\dagger}\right)_{\alpha \beta}\right|^{2}}{\left|\left(U U^{\dagger}\right)_{\beta \beta}\right|\left|\left(U U^{\dagger}\right)_{\alpha \alpha}\right|} .
$$

That is, a $\nu_{\alpha} \rightarrow \nu_{\beta}$ instantaneous transition search constrains $\left|\left(U U^{\dagger}\right)_{\beta \alpha}\right|$, whereas a $\bar{\nu}_{\alpha} \rightarrow \bar{\nu}_{\beta}$ search constrains $\left|\left(U U^{\dagger}\right)_{\alpha \beta}\right|$. 


\subsection{Fit Method}

\subsubsection{Fit I: $\nu_{\mu} \rightarrow \nu_{e}$ Transitions}

The expected number of $\nu_{e}$ events from $\nu_{\mu} \rightarrow \nu_{e}$ instantaneous transitions is given by Eq. 7.5 for $\alpha=\mu$ and $\beta=e$,

$$
\begin{aligned}
n & =\int d E \frac{d \Phi_{\mu}^{C C(S M)}(E)}{d E}\left|\left(U U^{\dagger}\right)_{e \mu}\right|^{2} \sigma_{e}^{C C(S M)}(E) \epsilon(E) \\
& =\left|\left(U U^{\dagger}\right)_{e \mu}\right|^{2} \int d E \frac{d \Phi_{\mu}^{C C(S M)}(E)}{d E} \sigma_{e}^{C C(S M)}(E) \epsilon(E) \\
& =\left|\left(U U^{\dagger}\right)_{e \mu}\right|^{2} \cdot N^{\nu_{\mu} \rightarrow \nu_{e}}(E)
\end{aligned}
$$

In the above expression, $N^{\nu_{\mu} \rightarrow \nu_{e}}(E)$ is the number of electron neutrino candidate events MiniBooNE expects to see assuming all muon neutrinos produced in the MiniBooNE beam interact in the detector and as $\nu_{e}$ 's. This sample can be identified as the fully-oscillated (fullosc) sample used in the MiniBooNE appearance analyses $[101,102] .^{3}$ These events are indeed both produced and detected primarily through CC interactions, which retroactively justifies the assumption made in Sec. 7.1.

In neutrino mode, the total $\nu_{e}$ CCQE prediction, $n_{i}^{\text {expected }}$, is given by

$$
\begin{aligned}
n_{i}^{\text {expected }} & =\left|\left(U U^{\dagger}\right)_{e \mu}\right|^{2} \cdot N_{i}^{\nu_{\mu} \rightarrow \nu_{e}}+n_{i}^{\nu_{e} \text { bkgd }} \\
& =n_{i}^{\nu_{\mu} \rightarrow \nu_{e}}+n_{i}^{\nu_{e} b k g d}
\end{aligned}
$$

as a function of reconstructed neutrino energy bins $i$, where $n_{i}^{\nu_{e} b k g d}$ are the predicted background electron neutrino candidate events given in [101]. Similarly, in antineutrino mode we obtain

$$
\begin{aligned}
n_{i}^{\text {expected }} & =\left|\left(U U^{\dagger}\right)_{\mu e}\right|^{2} \cdot N_{i}^{\nu_{\mu} \rightarrow \nu_{e}}+n_{i}^{\nu_{e} \text { bkgd }} \\
& =n_{i}^{\nu_{\mu} \rightarrow \nu_{e}}+n_{i}^{\nu_{e} b k g d}
\end{aligned}
$$

\footnotetext{
${ }^{3}$ Strictly speaking, it would be precisely the fullosc sample from MiniBooNE, had MiniBooNE not relied on experimental production or cross-section measurements to constrain the normalization of this sample.
} 
A Markov chain fit [183] is performed for each running mode, where $\left|\left(U U^{\dagger}\right)_{e \mu}\right|$ or $\left|\left(U U^{\dagger}\right)_{\mu e}\right|$ are varied randomly within $[0,1]$, and a new expected distribution, $n_{i}^{\text {expected }}$, is obtained for each new value of the varied parameter and compared to the data, $n_{i}^{\text {observed }}$, using the following $\chi^{2}$ definition,

$$
\chi^{2}=\sum_{i, j}\left(n_{i}^{\text {observed }}-n_{i}^{\text {expected }}\right) M_{i j}^{-1}\left(n_{j}^{\text {observed }}-n_{j}^{\text {expected }}\right) .
$$

The $\chi^{2}$ is calculated by summing over both the $\nu_{e}$ and the $\nu_{\mu}$ CCQE predictions from $[101,102]$ side-by-side. $M_{i j}^{-1}$ is the inverse of the full systematic + statistical covariance matrix for the $\nu_{e}$ CCQE (including background and signal from $\nu_{\mu} \rightarrow \nu_{e}$ transitions) and $\nu_{\mu} \mathrm{CCQE}$ predictions, calculated following the same procedure as described in Sec. 4.4.3.

\subsubsection{Fit II: $\nu_{\mu} \rightarrow \nu_{e}, \nu_{\mu} \rightarrow \nu_{\mu}$ and $\nu_{e} \rightarrow \nu_{\phi}$ Transitions}

In this fit, one follows the same procedure as in Sec. 7.3.1, except now both the $\nu_{e}$ CCQE background and $\nu_{\mu}$ CCQE predictions must also be modified according to mixing freedom parameters.

Because the $\nu_{e} \mathrm{CCQE}$ and $\nu_{\mu} \mathrm{CCQE}$ predicted samples have been subject to experimental constraints, including constraints obtained from an actual measurement of the $\nu_{\mu}$ CCQE data-to-MonteCarlo normalization which was also propagated to the $\nu_{e}$ CCQE signal and background predictions (this was done assuming no significant $\nu_{\mu}$ transitions of any kind), one must carefully undo these effects before attempting to fit for instantaneous transitions using the $\nu_{\mu}$ CCQE and $\nu_{e}$ CCQE background predictions. We begin by describing in more detail how these constraints enter in the predictions, and the assumptions we must make in the fit to undo those effects while allowing for sufficient sensitivity to $\nu_{e}$ and $\nu_{\mu}$ instantaneous transitions.

As discussed in Sec. 4.5.1, MonteCarlo predicted events from $\pi^{ \pm}$have received an approximately flat normalization correction of $N_{\pi}$ according to their parentage, which is a constraint that comes from forcing the $\nu_{\mu}$ CCQE Monte-Carlo prediction to agree with the measured $\nu_{\mu} \mathrm{CCQE}$ rate at MiniBooNE. In neutrino mode, this factor 
corresponds to $N_{\pi} \sim 1.28$. In antineutrino mode, the corresponding normalization correction comes from a weighted average of $N_{\pi^{+}}$and $N_{\pi^{-}}$, and corresponds to $N_{\pi} \sim$ 1.15, accounting for the relative contribution of $\nu_{\mu}$ from $\pi^{+}$and $\pi^{-}$in the $\nu_{\mu}$ CCQE prediction.

The backgrounds contributing to the MiniBooNE $\nu_{e}$ appearance search are of two general types:

1. Intrinsic $\nu_{e}$ backgrounds. These are highly correlated with the $\nu_{\mu}$ CCQE sample through flux and cross-section. A large fraction of these is produced in $\pi \rightarrow$ $\mu \rightarrow e$ decays, and those therefore receive the same normalization correction as that of the $\nu_{\mu} \mathrm{CCQE}$ and $\nu_{e} \mathrm{CCQE}$ signal samples.

2. Backgrounds from mis-identified $\mathrm{NC} \pi^{0}$ or radiative $\Delta$ decay events and $\nu_{\mu^{-}}$ induced dirt events. Each of these backgrounds is constrained through an in situ measurement.

Of course the simplest approach in this fit would be to assume that the backgrounds are mostly intrinsic $\nu_{e}$ 's produced and detected mostly through CC interactions, but this would be a very crude approximation, and certainly false at low energies, where backgrounds from $\nu_{\mu}$-induced, mis-identified events dominate. Instead, we break up the predicted $\nu_{e}$ background events into three categories, and treat each of them separately:

1. intrinsic $\nu_{e}$ 's from muon decay, $n^{\nu_{e}}$ from $\mu$,

2. other intrinsic $\nu_{e}{ }^{\prime} \mathrm{s}, n^{\nu_{e}}$ other , and

3. mis-identified $\nu_{\mu}$ events, $n^{\nu_{\mu}}$ mis-ID, which include $\pi^{0}, \Delta$, and dirt events.

With the above distinctions in mind, assuming no transitions, the number of expected $\nu_{e}$ background events is given by

$$
\begin{aligned}
n^{\nu_{e} \text { bkgd }}(E) & =n^{\nu_{e} \text { from } \mu}(E)+n^{\nu_{e} \text { other }}(E)+n^{\nu_{\mu} \text { mis-ID }} \\
& =N^{\nu_{e} \text { from } \mu}(E)+N^{\nu_{e} \text { other }}(E)+N^{\nu_{\mu} \text { mis-ID }}(E),
\end{aligned}
$$


where uppercase $N$ correspond to the MiniBooNE predictions in $[101,102]$.

$N^{\nu_{e}}$ from $\mu(E)$ events have received the $N_{\pi}$ correction, and $N^{\nu_{\mu}}$ mis-ID $(E)$ have either received the $N_{\pi}$ correction, or been measured directly. Neglecting the correction for a moment, if the unitarity constraint is relaxed, the different background components would become subject to instantaneous transitions, and so the number of expected events would become

$$
\begin{aligned}
n^{\nu_{e} b k g d}(E)= & \left|\left(U U^{\dagger}\right)_{e e}\right|^{2} \cdot N^{\nu_{e} \text { from } \mu}(E) \\
+ & \left|\left(U U^{\dagger}\right)_{e e}\right|^{2} \cdot N^{\nu_{e} \text { other }}(E) \\
+ & \left|\left(U U^{\dagger}\right)_{\mu \mu}\right|^{2} \cdot N^{\nu_{\mu}} \operatorname{mis-ID}(E) .
\end{aligned}
$$

In the above expression, the $N^{\nu_{e}}$ from $\mu$ and $N^{\nu_{e}}$ other samples include mostly events that are both produced and detected through CC interactions; However, the $N^{\nu_{\mu}}$ mis-ID sample includes events that are detected primarily through NC interactions. The latter sample fails our assumption that all events detected through CC interactions, but that turns out not to be an issue because of reasons we discuss next.

At this point, what remains to be done is the reversal of the effect of the $N_{\pi}$ correction. As mentioned earlier, the predicted $N^{\nu_{e}}$ from $\mu(E)$ events and a fraction of the predicted $N^{\nu_{\mu}}$ mis-ID events have been roughly scaled by a factor of $\sim 1.28$ in neutrino mode, and 1.15 in antineutrino mode. An important realization is that the underlying models considered in Chapter 2 cannot induce $\left|\left(U U^{\dagger}\right)_{\alpha \alpha}\right|>1$. Therefore, the MiniBooNE extracted normalization corrections taken at face value cannot be attributed to instantaneous transitions. In that case, we can account for transitions of the form $\left|\left(U U^{\dagger}\right)_{\alpha \alpha}\right|<1$, assuming that the measured $N_{\pi}$ normalizations are be due to possible $\nu_{\mu}$ instantaneous transitions plus some systematic normalization difference, $\kappa$, which is varied in the fit within uncertainty. Following that reasoning, the $\nu_{\mu} \rightarrow \nu_{e}$ signal prediction and $\nu_{e}$ background from $\mu$ decay prediction should also be modified. Starting with their corresponding first principles predictions, those should receive a 
correction of $\kappa$ and not $N_{\pi}$. That is,

$$
\begin{aligned}
& n^{\nu_{\mu} \rightarrow \nu_{e}}(E)=\left|\left(U U^{\dagger}\right)_{e \mu}\right|^{2} \cdot N^{\nu_{\mu} \rightarrow \nu_{e}}(E) \cdot \frac{\kappa}{N_{\pi}}, \quad \text { and } \\
& n^{\nu_{e} \text { from } \mu}=\left|\left(U U^{\dagger}\right)_{e e}\right|^{2} \cdot N^{\nu_{e} \text { from } \mu}(E) \cdot \frac{\kappa}{N_{\pi}} \text {. }
\end{aligned}
$$

The same would apply to all $\nu_{\mu}$ mis-identified events except those which are identified as dirt, NC $\pi^{0}$ and $\Delta$ events, which make up the majority of $N^{\nu_{\mu}}$ mis-ID events in the $\nu_{e}$ background prediction. The latter are constrained to exactly match the observed MiniBooNE data using respective high-purity and high-statistics reconstructed data samples. As a result, any potential mixing freedom effect which may be affecting the mis-identified samples effectively cancels out through the direct measurement. Therefore, the mis-identified distributions are kept fixed during the fit, and no information on mixing freedom can be extracted from that sample. The same reasoning also allows to ignore the fact that the instantaneous transitions event rate in Eq. 7.5 assumes that all events are produced and detected only through CC events, a clearly incorrect assumption for the majority of $N^{\nu_{\mu}}$ mis-ID events.

The overall $\nu_{e}$ prediction in the presence of mixing freedom, including $\nu_{\mu} \rightarrow \nu_{e}$ transitions, is given by

$$
\begin{aligned}
n_{i}^{\text {expected }}= & n_{i}^{\nu_{\mu} \rightarrow \nu_{e}}+n_{i}^{\nu_{e} \text { bkgd }} \\
= & \left|\left(U U^{\dagger}\right)_{e \mu}\right|^{2} \cdot N_{i}^{\nu_{\mu} \rightarrow \nu_{e}}(E) \cdot \frac{\kappa}{N_{\pi}} \\
& +\left|\left(U U^{\dagger}\right)_{e e}\right|^{2} \cdot N_{i}^{\nu_{e}} \text { from } \mu(E) \cdot \frac{\kappa}{N_{\pi}} \\
& +\left|\left(U U^{\dagger}\right)_{e e}\right|^{2} \cdot N_{i}^{\nu_{e} \text { other }}(E) \\
& +N_{i}^{\nu_{\mu}} \text { mis-ID }(E)
\end{aligned}
$$

in neutrino mode, for $N_{\pi}=1.28$, and

$$
\begin{aligned}
n_{i}^{\text {expected }} & =n_{i}^{\nu_{\mu} \rightarrow \nu_{e}}+n_{i}^{\nu_{e} \text { bkgd }} \\
& =\left|\left(U U^{\dagger}\right)_{\mu e}\right|^{2} \cdot N_{i}^{\nu_{\mu} \rightarrow \nu_{e}}(E) \cdot \frac{\kappa}{N_{\pi}}
\end{aligned}
$$




$$
\begin{aligned}
& +\left|\left(U U^{\dagger}\right)_{e e}\right|^{2} \cdot N_{i}^{\nu_{e} \text { from } \mu}(E) \cdot \frac{\kappa}{N_{\pi}} \\
& +\left|\left(U U^{\dagger}\right)_{e e}\right|^{2} \cdot N_{i}^{\nu_{e} \text { other }}(E) \\
& +N_{i}^{\nu_{\mu}} \operatorname{mis}^{-I D}(E)
\end{aligned}
$$

in antineutrino mode, for $N_{\pi}=1.15$.

Similarly, the $\nu_{\mu}$ CCQE prediction used in the side-by-side $\nu_{e}$ and $\nu_{\mu}$ CCQE fit to mixing freedom is also subject to $\nu_{\mu} \rightarrow \nu_{\mu}$ transitions. The $\nu_{\mu}$ CCQE prediction is given by

$$
n_{i}^{\text {expected }}=\left|\left(U U^{\dagger}\right)_{\mu \mu}\right|^{2} \cdot N_{i}^{\nu_{\mu} C C Q E}(E) \cdot \frac{\kappa}{N_{\pi}}
$$

in both neutrino and antineutrino mode.

The $\chi^{2}$ is calculated as in Sec. 7.3.1, except now a pull term, $\chi_{\text {constraint }}^{2}$, is added to the total $\chi^{2}$, so that

$$
\chi^{2}=\sum_{i, j}\left(n_{i}^{\text {observed }}-n_{i}^{\text {expected }}\right) M_{i j}^{-1}\left(n_{j}^{\text {observed }}-n_{j}^{\text {expected }}\right)+\chi_{\text {constraint }}^{2}
$$

where

$$
\chi_{\text {constraint }}^{2}=\frac{\left(N_{\pi}-\kappa\right)^{2}}{\sigma_{\text {stat }}^{2}} .
$$

$\sigma_{\text {stat }}$ corresponds to the fractional statistical uncertainty of the $\nu_{\mu}$ CCQE prediction in neutrino mode $(0.3 \%)$ or antineutrino mode $(1 \%)$. Note that $M_{i j}$ is recalculated for each set of $\left|\left(U U^{\dagger}\right)_{\alpha \beta}\right|$ and $\kappa$ parameters to include new statistical uncertainties according to the $\nu_{e}$ background and $\nu_{\mu}$ CCQE prediction after mixing freedom effects. 


\begin{tabular}{l|ll}
\hline \hline Neutrino mode fit & $\left|\left(U U^{\dagger}\right)_{e \mu}\right|$ & $\chi_{\min }^{2} /$ d.o.f. \\
\hline \hline MB200 & \multicolumn{3}{|}{} \\
best fit & 0.024 & $20.8 / 17$ \\
90\% C.L. limits & $(0,0.038)$ & \\
99\% C.L. limits & $(0,0.044)$ & \\
\hline MB475 & & \\
best fit & 0.021 & $7.8 / 14$ \\
90\% C.L. limits & $(0,0.036)$ & \\
99\% C.L. limits & $(0,0.042)$ & \\
\hline \hline
\end{tabular}

Table 7.1: MiniBooNE 90\% and 99\% C.L. limits from a search to $\nu_{\mu} \rightarrow \nu_{e}$ transitions in neutrino mode. The limits on $\left|\left(U U^{\dagger}\right)_{e \mu}\right|$ are obtained using a $\delta \chi^{2} \equiv \chi^{2}-\chi_{\min }^{2}$ cut of 2.90 (90\% C.L.) and 6.63 (99\% C.L.). The null $\chi^{2}$ 's, corresponding to $\left|\left(U U^{\dagger}\right)_{e \mu}\right|=0$, are 22.2 (18 d.o.f.) for MB200 and 8.8 (15 d.o.f.) for MB475, respectively.

\begin{tabular}{l|ll}
\hline \hline Antineutrino mode fit & $\left|\left(U U^{\dagger}\right)_{\mu e}\right|$ & $\chi_{\min }^{2} /$ d.o.f. \\
\hline \hline MB200 & & \\
best fit & 0.044 & $22.2 / 17$ \\
90\% C.L. limits & $(0,0.069)$ & \\
99\% C.L. limits & $(0,0.083)$ & \\
\hline \hline
\end{tabular}

Table 7.2: MiniBooNE $90 \%$ and $99 \%$ C.L. limits from a search to $\bar{\nu}_{\mu} \rightarrow \bar{\nu}_{e}$ in antineutrino mode. The limits on $\left|\left(U U^{\dagger}\right)_{\mu e}\right|$ are obtained using a $\delta \chi^{2} \equiv \chi^{2}-\chi_{\min }^{2}$ cut of 2.90 (90\% C.L.) and 6.63 (99\% C.L.). The null $\chi^{2}$, corresponding to $\left|\left(U U^{\dagger}\right)_{\mu e}\right|=0$, is 24.4 (18 d.o.f.).

\subsection{Fit Results}

\subsubsection{Fit I: $\nu_{\mu} \rightarrow \nu_{e}$ Transitions}

Table 7.1 summarizes the results from a fit to MiniBooNE neutrino-mode data allowing for possible instantaneous $\nu_{\mu} \rightarrow \nu_{e}$ transitions due to mixing freedom. Fits to both energy ranges $\left(200<E_{\nu}^{Q E}<3000 \mathrm{MeV}(\mathrm{MB} 200)\right.$ and $475<E_{\nu}^{Q E}<3000 \mathrm{MeV}$ (MB475)) have been considered.

In the neutrino mode search for mixing freedom, the best-fit values in Tab. 7.1 correspond to a change in $\chi^{2}$ relative to a null hypothesis of 1.4 and 1.0 for MB475 and MB200, for one fit parameter. The relatively small reduction in the fit $\chi^{2}$ implies that $\nu_{\mu} \rightarrow \nu_{e}$ transitions are not significantly preferred over the null hypothesis. Thus, 


\begin{tabular}{l|llll}
\hline \hline Neutrino mode fit & $\left|\left(U U^{\dagger}\right)_{e \mu}\right|$ & $\left|\left(U U^{\dagger}\right)_{e e}\right|$ & $\left|\left(U U^{\dagger}\right)_{\mu \mu}\right|$ & $\chi_{\min }^{2} /$ d.o.f. \\
\hline \hline MB200 & & & & \\
best fit & 0.013 & 1.00 & 0.954 & $20.3 / 15$ \\
90\% C.L. limits & $(0,0.036)$ & $(0.94,1)$ & $(0.89,1)$ & \\
99\% C.L. limits & $(0,0.043)$ & $(0.87,1)$ & $(0.86,1)$ & \\
\hline MB475 & & & & \\
best fit & 0.021 & 1.00 & 1.00 & $7.9 / 12$ \\
90\% C.L. limits & $(0,0.044)$ & $(0.76,1)$ & $(0.93,1)$ & \\
99\% C.L. limits & $(0,0.057)$ & $(0.51,1)$ & $(0.88,1)$ & \\
\hline \hline
\end{tabular}

Table 7.3: MiniBooNE results from a search for $\nu_{\mu} \rightarrow \nu_{e}, \nu_{\mu} \rightarrow \nu_{\mu}$ and $\nu_{e} \rightarrow \nu_{\phi}$ instantaneous transitions in neutrino mode. The limits are obtained using a $\Delta \chi^{2} \equiv$ $\chi^{2}-\chi_{\min }^{2}$ cut of 2.90 (90\% C.L.) and 6.63 (99\% C.L.). The null $\chi^{2}$ 's, obtained using $\kappa=N_{\pi}$, are 22.2 (18 d.o.f.) for MB200 and 8.8 (15 d.o.f.) for MB475, respectively.

MiniBooNE places limits on $\left|\left(U U^{\dagger}\right)_{e \mu}\right|$ and as shown in the same table. At 90\%C.L., MiniBooNE constraints $\left|\left(U U^{\dagger}\right)_{e \mu}\right|$ to be less than 0.04. This result is independent of the $\nu_{e}$ CCQE energy range considered in the fit $(200-3000 \mathrm{MeV}$, or $475-3000 \mathrm{MeV})$.

Analogous results obtained in from the search for mixing freedom in antineutrino mode are summarized in Tab. 7.2 . The best-fit values in Tab. 7.2 correspond to a change in $\chi^{2}$ relative to a null hypothesis of 2.2 , for one fit parameter. In this case the improvement on the fit quality that mixing freedom introduces is larger than in neutrino mode, but still not significant at 90\% C.L. Thus, MiniBooNE places limits on $\left|\left(U U^{\dagger}\right)_{\mu e}\right|$ and as shown in Table 7.2. At 90\%C.L., MiniBooNE constraints $\left|\left(U U^{\dagger}\right)_{\mu e}\right|$ to be less than 0.07 . This result is obtained from a fit over the full $\nu_{e}$ CCQE energy range $(200-3000 \mathrm{MeV})$.

\subsubsection{Fit II: $\nu_{\mu} \rightarrow \nu_{e}, \nu_{\mu} \rightarrow \nu_{\mu}$ and $\nu_{e} \rightarrow \nu_{\phi}$ Transitions}

Tables 7.3 and 7.4 summarize results from fits to MiniBooNE data with simultaneous $\nu_{\mu} \rightarrow \nu_{e}, \nu_{\mu} \rightarrow \nu_{\mu}$ and $\nu_{e} \rightarrow \nu_{q}$ instantaneous transitions due to mixing freedom. Again, fits to both energy ranges $\left(200<E_{\nu}^{Q E}<3000 \mathrm{MeV}\right.$ and $475<E_{\nu}^{Q E}<3000$ $\mathrm{MeV}$ ) have been considered in neutrino mode, and fits to $200<E_{\nu}^{Q E}<3000 \mathrm{MeV}$ have been considered in antineutrino mode.

In neutrino mode, both fits, MB200 and MB475, yield a change in $\chi^{2}$ relative 


\begin{tabular}{l|llll}
\hline \hline Antineutrino mode fit & $\left|\left(U U^{\dagger}\right)_{\mu e}\right|$ & $\left|\left(U U^{\dagger}\right)_{e e}\right|$ & $\left|\left(U U^{\dagger}\right)_{\mu \mu}\right|$ & $\chi_{\min }^{2} /$ d.o.f. \\
\hline \hline MB200 & & & & \\
best fit & 0.044 & 1.00 & 1.00 & $22.2 / 15$ \\
$90 \%$ C.L. & $(0.008,0.089)$ & $(0.056,1)$ & $(0.96,1)$ & \\
$99 \%$ C.L. & $(0,0.11)$ & $(0,1)$ & $(0.91,1)$ & \\
\hline \hline
\end{tabular}

Table 7.4: MiniBooNE results from a search for $\bar{\nu}_{\mu} \rightarrow \bar{\nu}_{e}, \bar{\nu}_{\mu} \rightarrow \bar{\nu}_{\mu}$ and $\bar{\nu}_{e} \rightarrow \bar{\nu}_{\phi}$ instantaneous transitions in antineutrino mode. The limits are obtained using a $\delta \chi^{2} \equiv \chi^{2}-\chi_{\min }^{2}$ cut of 2.90 (90\% C.L.) and 6.63 (99\% C.L.). The null $\chi^{2}$, obtained using $\kappa=N_{\pi}$, is 24.4 (18 d.o.f.).

the the null hypothesis of 1.9 and 1.0 for three effective fit parameters, respectively. Once again the small change in the fit quality implies that transitions due to mixing freedom are only marginally preferred over the null hypothesis. MiniBooNE therefore places limits on $\left|\left(U U^{\dagger}\right)_{e \mu}\right|,\left|\left(U U^{\dagger}\right)_{e e}\right|$, and $\left|\left(U U^{\dagger}\right)_{\mu \mu}\right|$, as shown on Tab. 7.3. The limits on each $\left|\left(U U^{\dagger}\right)_{\alpha \beta}\right|$ parameter are obtained by marginalizing all other fit parameters; a one-dimensional $\delta \chi^{2}$ cut, assuming 1 degree of freedom, is placed with respect to the $\left|\left(U U^{\dagger}\right)_{\alpha \beta}\right|$ value which minimizes the $\chi^{2}$ in the fit, and thus the lower and upper bounds on $\left|\left(U U^{\dagger}\right)_{\alpha \beta}\right|$ are extracted. At 90\%C.L., MiniBooNE constraints $\left|\left(U U^{\dagger}\right)_{e \mu}\right|$ to be less than 0.044, and $\left|\left(U U^{\dagger}\right)_{e e}\right|$ and $\left|\left(U U^{\dagger}\right)_{\mu \mu}\right|$ to be 1.0 within $24 \%$ and $7 \%$, respectively. Note that, as expected, due to large normalization uncertainties from flux and cross-section, MiniBooNE's constraints on $\left|\left(U U^{\dagger}\right)_{e e}\right|$ and $\left|\left(U U^{\dagger}\right)_{\mu \mu}\right|$ are much looser.

In antineutrino mode, the fit yields a change in $\chi^{2}$ relative the the null hypothesis of 2.2 for three effective fit parameters, respectively. Again the relative improvement on the fit quality is small. Notice, however, that $\left|\left(U U^{\dagger}\right)_{\mu e}\right|=0$ is now just barely excluded at $90 \%$ C.L. The best fit $\left|\left(U U^{\dagger}\right)_{\mu e}\right|$ parameter corresponds to 0.044 . The upper limit on $\left|\left(U U^{\dagger}\right)_{\mu e}\right|$ corresponds to 0.089 , at $90 \%$ C.L. As in the case of the neutrino mode data sets, the limits on $\left|\left(U U^{\dagger}\right)_{e e}\right|$ and $\left|\left(U U^{\dagger}\right)_{\mu \mu}\right|$ are considerably weaker, as shown on Table 7.3. At 90\%C.L., MiniBooNE constraints $\left|\left(U U^{\dagger}\right)_{e e}\right|$ and $\left|\left(U U^{\dagger}\right)_{\mu \mu}\right|$ to be 1.0 within $44 \%$ and $4 \%$, respectively.

The projected $90 \%$ C.L. and $99 \%$ C.L. allowed regions in $\left(\left|\left(U U^{\dagger}\right)_{e e}\right|,\left|\left(U U^{\dagger}\right)_{e \mu}\right|\right)-$, $\left(\left|\left(U U^{\dagger}\right)_{\mu \mu}\right|,\left|\left(U U^{\dagger}\right)_{e \mu}\right|\right)-$, and $\left(\left|\left(U U^{\dagger}\right)_{\mu \mu}\right|,\left|\left(U U^{\dagger}\right)_{e e}\right|\right)$-space are shown in Figs. 7-1 and 

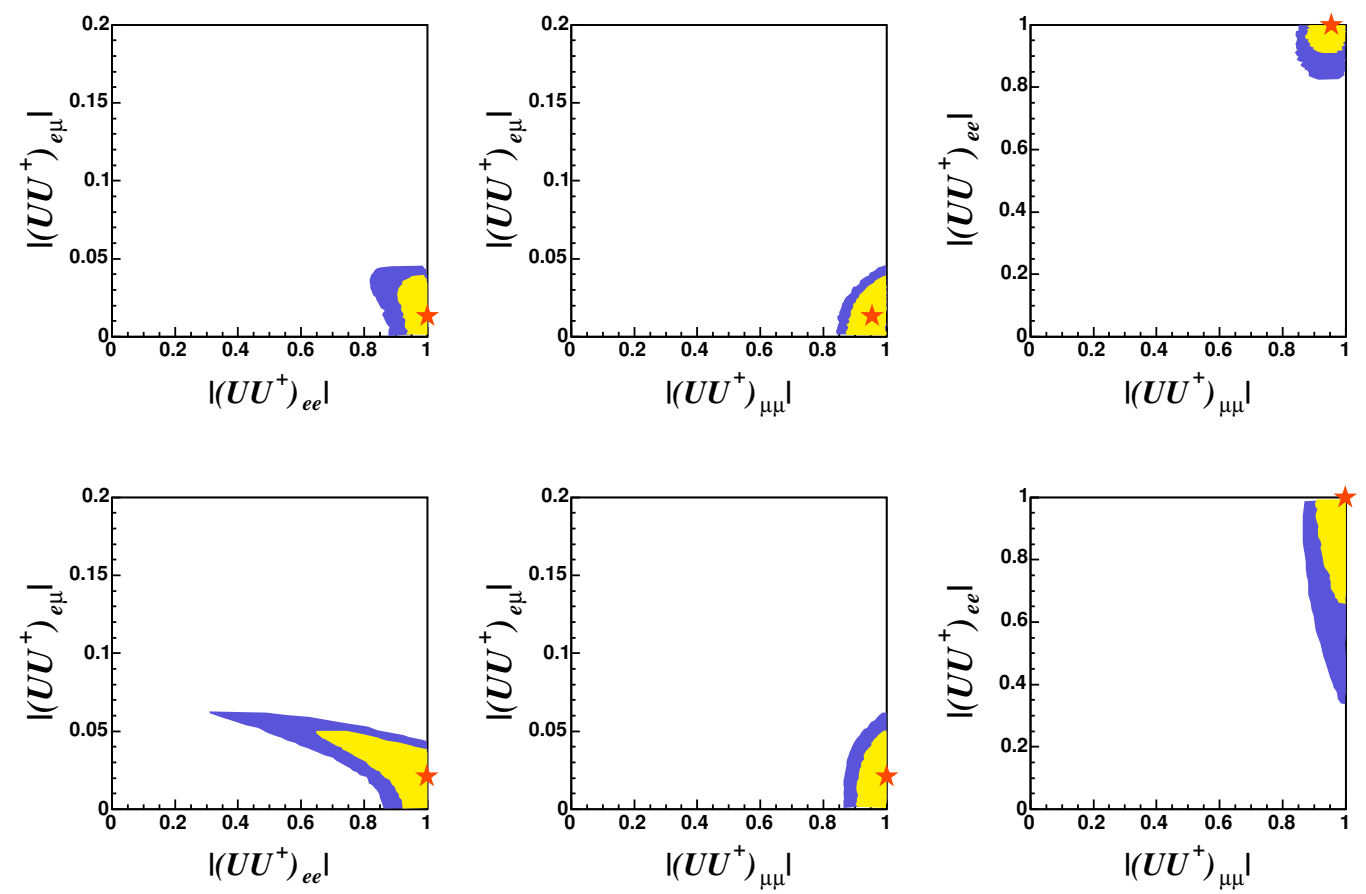

Figure 7-1: $\left|\left(U U^{\dagger}\right)_{e \mu}\right|,\left|\left(U U^{\dagger}\right)_{e e}\right|$, and $\left|\left(U U^{\dagger}\right)_{\mu \mu}\right|$ allowed regions at $90 \%$ (yellow) and $99 \%$ (blue) C.L. from fits to MiniBooNE neutrino data, obtained using a $\delta \chi^{2} \equiv$ $\chi^{2}-\chi_{\min }^{2}$ cut of 4.61 (90\% C.L.) and 9.21 (99\% C.L.). The stars indicate the best fit point. Top row: MB200 fit; bottom row: MB475 fit.
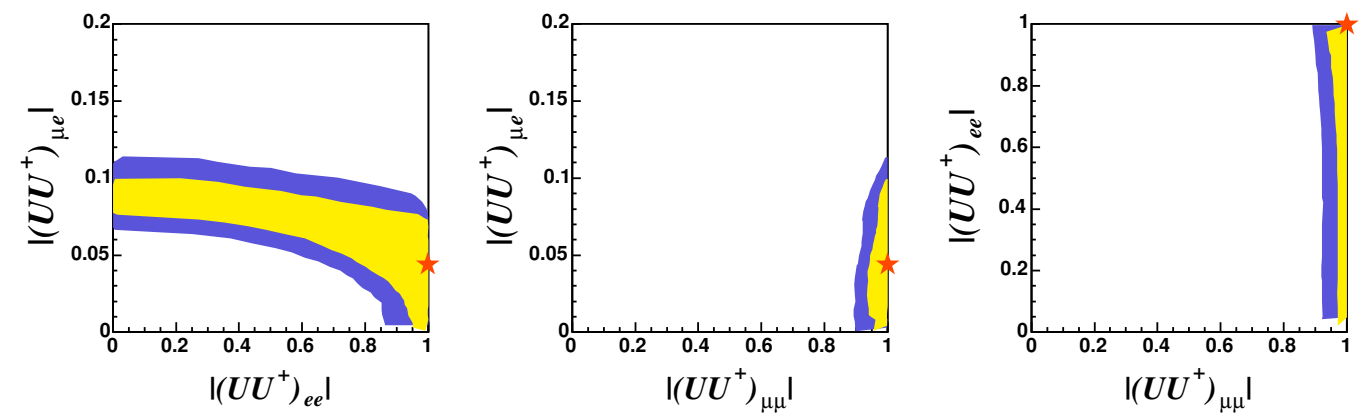

Figure 7-2: $\left|\left(U U^{\dagger}\right)_{\mu e}\right|,\left|\left(U U^{\dagger}\right)_{e e}\right|$, and $\left|\left(U U^{\dagger}\right)_{\mu \mu}\right|$ allowed regions at $90 \%$ (yellow) and 99\% (blue) C.L. from fits to MiniBooNE antneutrino data, obtained using a $\delta \chi^{2} \equiv$ $\chi^{2}-\chi_{\min }^{2}$ cut of 4.61 (90\% C.L.) and 9.21 (99\% C.L.). The stars indicate the best fit point. 
7-2. Those correspond to two-dimensional projections of the full parameter space, obtained using a $\delta \chi^{2} \equiv \chi^{2}-\chi_{\min }^{2}$ cut of 4.61 (90\% C.L.) and 9.21 (99\% C.L.), assuming 2 d.o.f.

\subsection{Final Remarks}

Figures 7-3 and 7-4 compare the MiniBooNE observed $\nu_{e}$ and $\bar{\nu}_{e}$ CCQE energy spectra to the predicted $\nu_{e}$ and $\bar{\nu}_{e}$ CCQE energy spectra for both the null case (MiniBooNE background prediction assuming no instantaneous transitions), and the case where transitions due to mixing freedom are allowed, corresponding to the best-fit parameters of Tables 7.1 through 7.4.

In neutrino mode, both fits (MB200 and MB475) yield consistent results, and prefer no $\nu_{\mu} \rightarrow \nu_{e}$ transitions. That can be understood once one considers the energy distribution of events due to $\nu_{\mu} \rightarrow \nu_{e}$ transitions. These events contribute to the $\nu_{e}$ CCQE prediction mostly at intermediate energy $(\sim 500 \mathrm{MeV})$, where the MiniBooNE observed $\nu_{e}$ distribution shows good agreement with the predicted $\nu_{e}$ background distribution.

Note that, as the top panel of Fig. 7-3 shows, mixing freedom cannot account for the observed neutrino mode low energy excess, even in the most general mixing freedom scenario which allows for both $\nu_{\mu} \rightarrow \nu_{e}$ and $\nu_{\mu} \rightarrow \nu_{\mu}$ and $\nu_{e} \rightarrow \nu_{q}$ instantaneous transitions.

On the other hand, the antineutrino mode fit likes a non-negligible contribution from $\bar{\nu}_{\mu} \rightarrow \bar{\nu}_{e}$ instantaneous transitions, driven by the small (2.8 $\sigma$ significant) excess seen in the $475-675 \mathrm{MeV}$ range. This is true for both fits to only $\bar{\nu}_{\mu} \rightarrow \bar{\nu}_{e}$ instantaneous transitions and fits to $\bar{\nu}_{\mu} \rightarrow \bar{\nu}_{e}, \bar{\nu}_{e} \rightarrow \bar{\nu}_{e}$, and $\bar{\nu}_{\mu} \rightarrow \bar{\nu}_{\mu}$ instantaneous transitions. However, it becomes more significant in the latter case, because of the extra freedom to reduce intrinsic $\nu_{e}$ backgrounds (for $\left|\left(U U^{\dagger}\right)_{e e}\right|<1$ ) and accommodate the deficit seen at higher energies.

MiniBooNE's constraints on mixing freedom are summarized in Tab. 7.5. The

limits obtained are evidently not competitive with limits on $\left|\left(U U^{\dagger}\right)_{e \mu}\right|$ which can be 

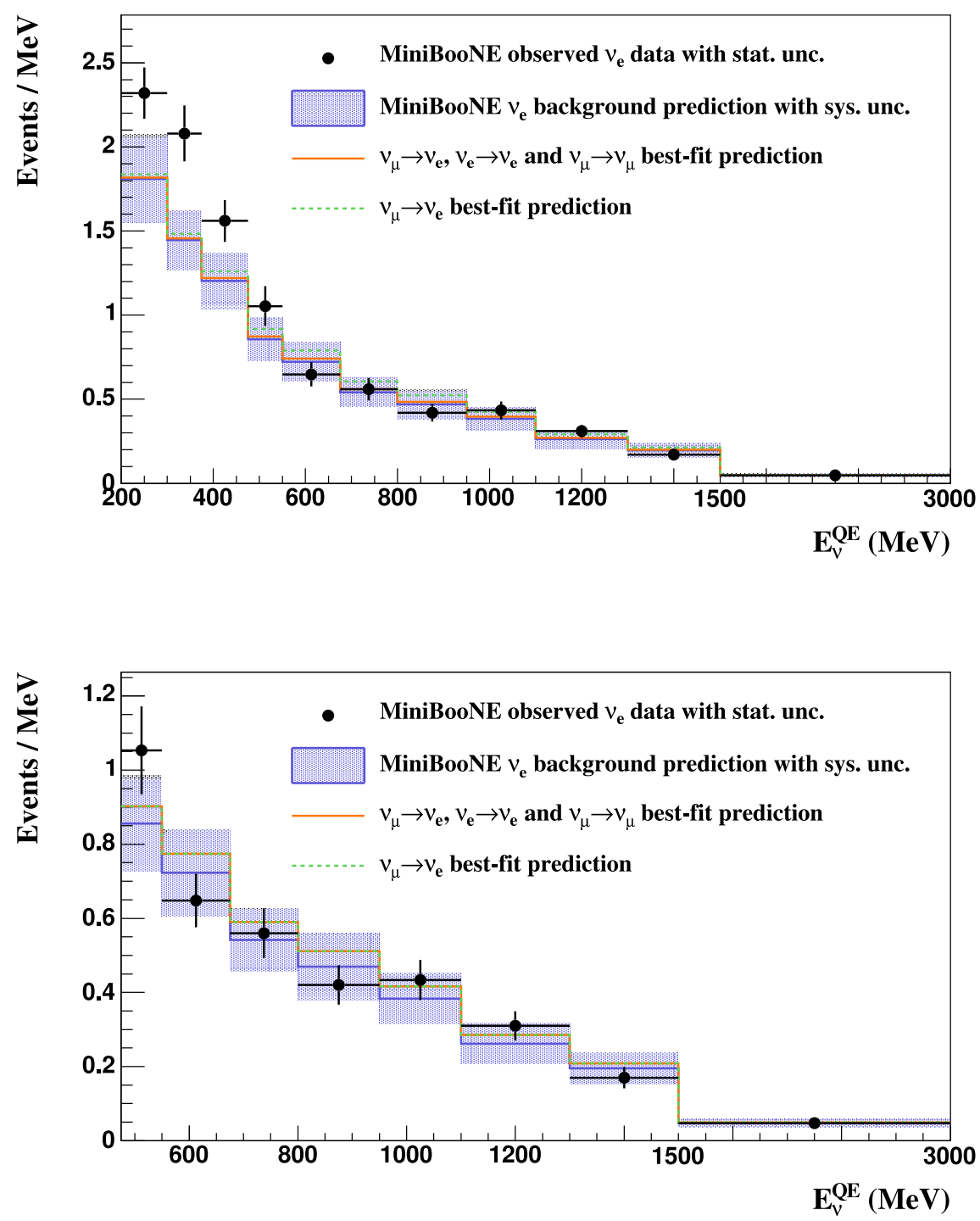

Figure 7-3: Comparison of MiniBooNE observed neutrino mode $\nu_{e}$ CCQE data from [101] (black points) to predicted background (blue histogram) and best-fit prediction in a mixing freedom scenario (red and green histograms). The error bars indicate data statistical uncertainty, whereas the hashed bands indicate systematic uncertainty on the background prediction (null hypothesis). Top: MB200 fit; Bottom: MB475 fit. 


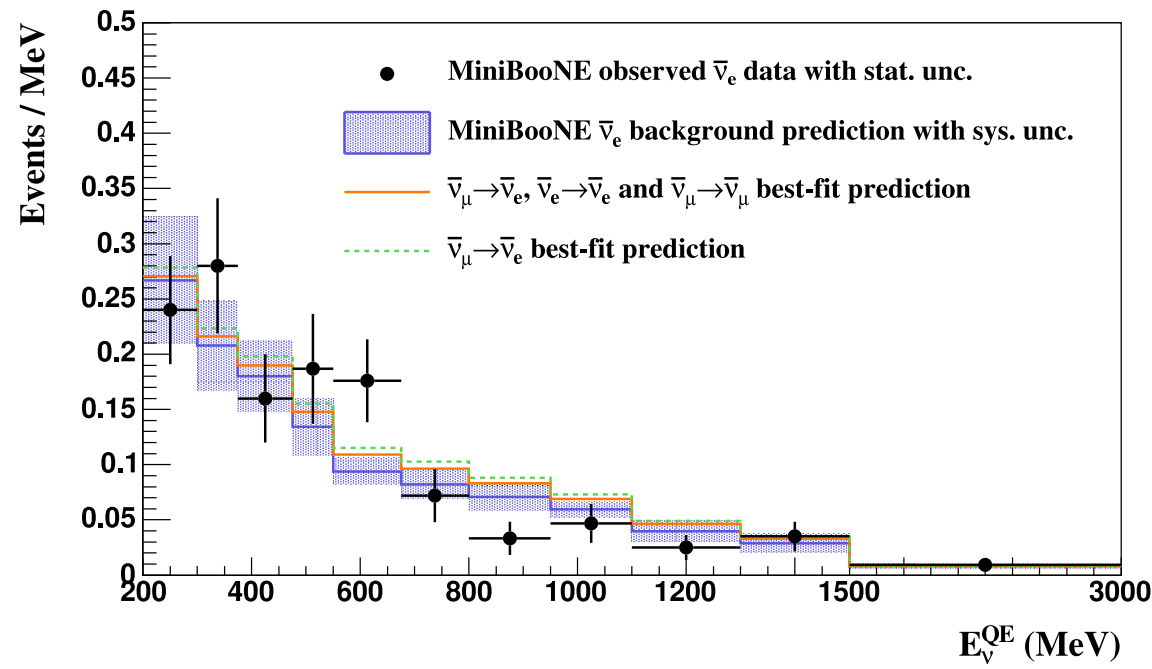

Figure 7-4: Comparison of MiniBooNE observed antineutrino mode $\nu_{e}$ CCQE data from [102] (black points) to predicted background (blue histogram) and best-fit prediction in a mixing freedom scenario (red and green histograms). The error bars indicate data statistical uncertainty, whereas the hashed bands indicate systematic uncertainty on the background prediction (null hypothesis). MB200 fit only. Note that this data set corresponds to lower statistics than the data set in Chapter 5 .

\begin{tabular}{c|cccc}
\hline \hline & $\left|\left(U U^{\dagger}\right)_{e \mu}\right|$ & $\left|\left(U U^{\dagger}\right)_{\mu e}\right|$ & $\left|\left(U U^{\dagger}\right)_{e e}\right|$ & $\left|\left(U U^{\dagger}\right)_{\mu \mu}\right|$ \\
\hline 90\% C.L. allowed & $(0,0.044)$ & $(0.008,0.089)$ & $(0.76,1)$ & $(0.93,1)$ \\
\hline \hline
\end{tabular}

Table 7.5: Summary of constraints to mixing freedom from MiniBooNE, at 90\% C.L. All limits are obtained from an $E_{\nu}^{Q E}>475 \mathrm{MeV}$ fit to the neutrino mode data set from [101], except for that of $\left|\left(U U^{\dagger}\right)_{\mu e}\right|$, which is obtained from an $E_{\nu}^{Q E}>200 \mathrm{MeV}$ fit to the antineutrino mode data set from [102]. 
placed through $\mu \rightarrow e \gamma$ searches [89], and which are of the order of $10^{-4}$. Nevertheless, a MiniBooNE search is interesting as a direct probe of mixing freedom, and the resulting limits compare competitively with those of other $\nu_{\mu} \rightarrow \nu_{e}$ oscillation experiments, such as the ones considered in [89].

We point out that the uncertainties in the underlying neutrino flux and crosssections predicted from first principles - in the case of MiniBooNE reflected in the observed $N_{\pi}$ normalization differences - are what currently impede sensitivity to instantaneous transitions due to mixing freedom. Further reduction of those uncertainties through experimental constraints, such as constraints from external neutrino cross-section measurements, comes the caveats mentioned in Sec. 7.2. Future improvements on the theoretical understanding of neutrino interactions will help push this idea further. 


\section{Chapter 8}

\section{Conclusions}

"Has MiniBooNE found new physics?"

MiniBooNE has performed searches for small-amplitude $\nu_{\mu} \rightarrow \nu_{e}$ and $\bar{\nu}_{\mu} \rightarrow \bar{\nu}_{e}$ oscillations at $L / E \sim 0.5-1.0 \mathrm{~m} / \mathrm{MeV}$. When looking for such oscillations in neutrino mode, MiniBooNE sees a $3.0 \sigma$ evidence of an excess at $L / E>1 \mathrm{~m} / \mathrm{MeV}$. The observed excess, however, is too sharply peaked at low energy in order to be consistent with an excess expected from simple, $C P T$ - and $C P$-conserving two-neutrino oscillations. As such, it is incompatible with the single sterile neutrino oscillation interpretation of LSND. By excluding the low energy region from the oscillation fit, MiniBooNE places a limit which rules out the LSND two-neutrino oscillation interpretation at $98 \%$ C.L. This limit assumes $C P T$ and $C P$ conservation.

The source of the MiniBooNE low energy excess, as of this writing, remains unknown. It is kinematically consistent with single-photon backgrounds to the appearance search; however, such backgrounds at MiniBooNE are well-constrained through several in situ measurements. The MicroBooNE experiment, which employs a liquidargon time-projection-chamber detector, is currently under construction in the Fermilab Booster neutrino beamline, and it is planning to investigate the viability of various proposed interpretations.

On the other hand, when looking for $\bar{\nu}_{\mu} \rightarrow \bar{\nu}_{e}$ two-neutrino oscillations in antineutrino mode, MiniBooNE sees an excess at $L / E \sim 0.5-1.0 \mathrm{~m} / \mathrm{MeV}$ which is consistent 


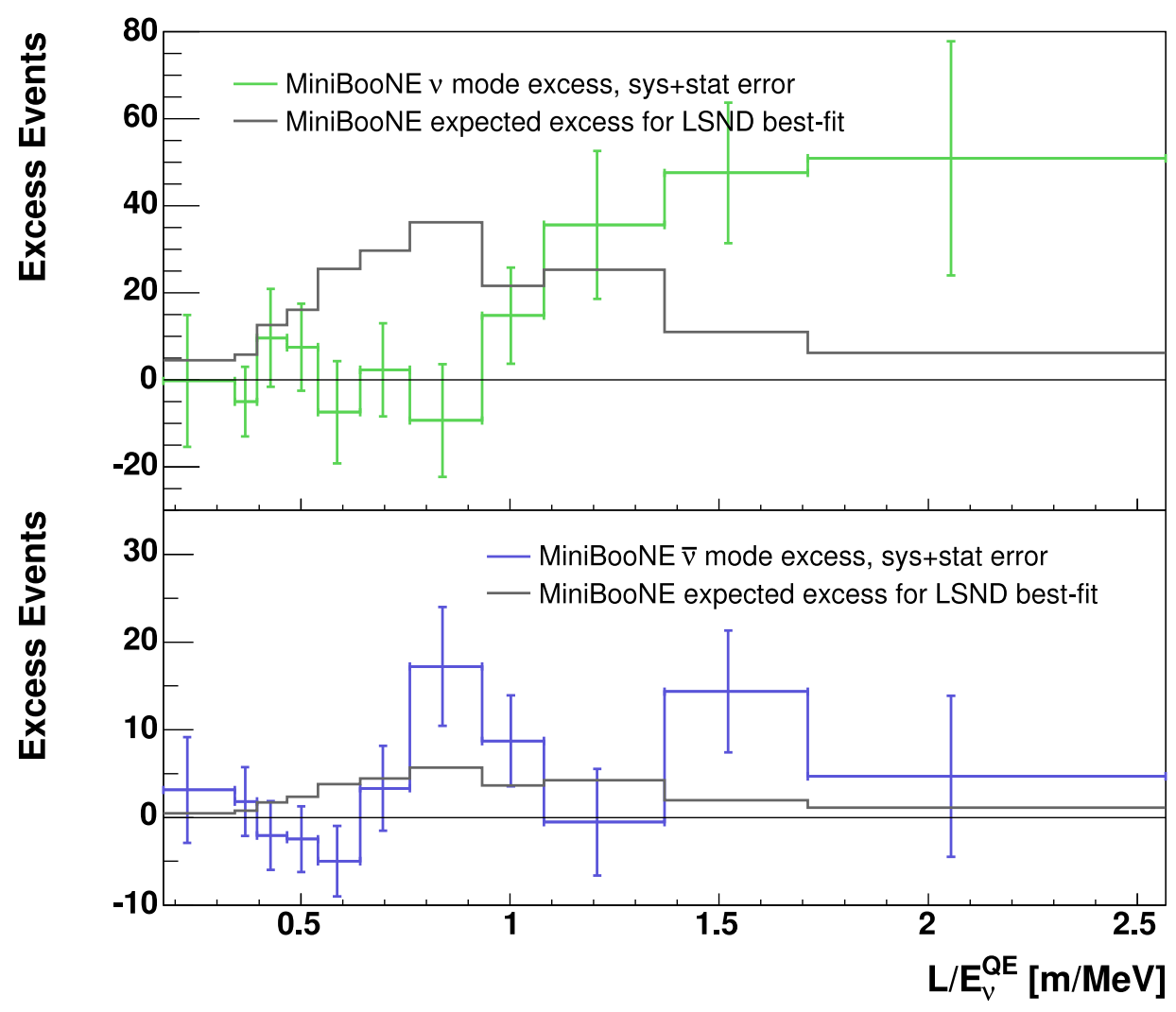

Figure 8-1: The MiniBooNE neutrino (top) and antineutrino (bottom) mode observed excess distributions as a function of $L / E$. $L$ is the true neutrino baseline, approximated as a constant $(513.6 \mathrm{~m})$, while $E$ corresponds to reconstructed neutrino energy, $E_{\nu}^{Q E}$. The error bars include systematic and statistical uncertainties. Overlaid are MiniBooNE signal predictions corresponding to the LSND best fit parameters in a $C P$ - and $C P T$-conserving two-neutrino oscillation approximation. Note that oscillation signals are always calculated as a function of true energy.

with LSND-like oscillations at greater than $96 \%$ C.L. At the same time, a hint of an excess is seen at larger $L / E$, though not as significant as the corresponding one seen in neutrino mode. Further antineutrino running at MiniBooNE will provide increased statistics and improved sensitivity to $\bar{\nu}_{\mu} \rightarrow \bar{\nu}_{e}$ oscillations.

The two excess signatures from MiniBooNE, summarized in Fig. 8-1, are found consistent with the LSND signature as a function of $L / E$ only in a scenario with two sterile neutrinos (two independent $\Delta m^{2}$ ) and $C P$ violation. However, this interpretation becomes problematic once confronted with constraints from atmospheric and short-baseline muon neutrino disappearance experiments.

The excess seen in antineutrino mode at MiniBooNE is also consistent with an 
effectively non-unitary $3 \times 3$ neutrino mixing matrix, at the $90 \%$ C.L. This holds under the assumption of heavy, mostly sterile neutrino mass eigenstates beyond the three currently assumed $\nu_{1}, \nu_{2}$ and $\nu_{3}$ mass eigenstates, and under the approximation that $m_{h}^{2} \ll E / L$ at MiniBooNE.

Has MiniBooNE found new physics? Maybe. 


\section{Appendix A}

\section{MiniBooNE Analysis Tools and Cross Checks}

\section{A.1 Reweighting}

In certain cases, instead of running fresh MonteCarlo each time an underlying parameter is varied, one can take advantage of truth information stored in the MonteCarlo prediction, and adjust the prediction appropriately by simply reweighting events within histograms that would be affected by the variation. For example, adjustments in neutrino cross-section are only energy and neutrino type dependent. In that case, one can apply a reweighting function for different types of neutrinos, changing the population of neutrinos as a function of true neutrino energy. This technique allows for easy comparisons between MonteCarlo central values obtained with different flux and cross-section parameters, without the need of regenerating new high-statistics MonteCarlo, which simplifies error analysis.

In the MiniBooNE appearance analysis, this technique is used to produce variations in the central value histograms used in the oscillation fits, as a function of recon-

structed neutrino energy, $E_{\nu}^{Q E}$, according to fluctuations in fundamental parameters input to the MonteCarlo simulation, as allowed by their associated uncertainty. This allows for easy error propagation from fundamental input parameter uncertainties onto the final central value predictions. The reweighed histograms are studied as a 
function of the following bin boundaries (in $\mathrm{GeV}$ ):

- $\nu_{e}$ CCQE fullosc (18 bins):

$(0.200,0.250,0.300,0.375,0.475,0.550,0.600,0.675,0.750,0.800,0.950,1.100$, $1.150,1.250,1.300,1.500,1.700,1.900,3.000)$

- $\nu_{e}$ CCQE background (18 bins):

$(0.200,0.250,0.300,0.375,0.475,0.550,0.600,0.675,0.750,0.800,0.950,1.100$, $1.150,1.250,1.300,1.500,1.700,1.900,3.000)$

- $\nu_{\mu} \operatorname{CCQE}(17$ bins):

$(0.0,0.4,0.5,0.6,0.7,0.8,0.9,1.0,1.1,1.2,1.3,1.4,1.5,1.6,1.7,1.8,1.9,3.0)$

\section{A.2 The $\nu_{\mu}-\nu_{e}$ Combined Fit Constraint}

The MiniBooNE fit for oscillations involves a simultaneous fit to $\nu_{e}$ CCQE and $\nu_{\mu}$ CCQE predictions, where information gained by comparing the observed highstatistics $\nu_{\mu}$ CCQE event sample to the MonteCarlo prediction can be related to the $\nu_{e}$ CCQE prediction (both signal and background) through systematic correlations accounted for within the fit, and therefore used to constrain the uncertainties and absolute rate of the $\nu_{e}$ CCQE prediction.

In this appendix, we provide a more instructive description of how this constraining procedure works within the fit, using a two-bin example. The two bins correspond to one bin for the full $\nu_{e}$ CCQE distribution, and one bin for the full $\nu_{\mu}$ CCQE distribution. In that case, the $\chi^{2}$ calculation in the fit reads as

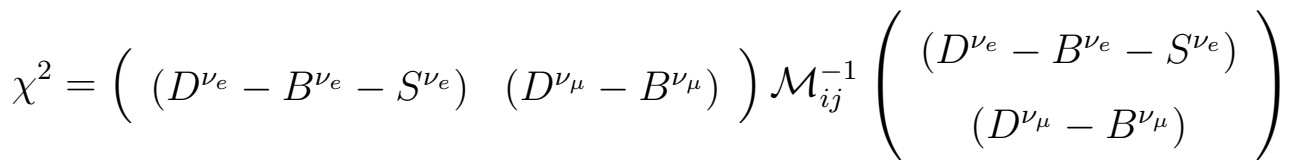

where $\mathcal{M}_{i j}^{-1}$ is the inverse of the $2 \times 2$ covariance matrix including the systematic $(\sigma)$ 
plus statistical $(s)$ uncertainty on both $\nu_{e}$ and $\nu_{\mu}$ predicted events,

$$
\mathcal{M}_{i j}=\left(\begin{array}{cc}
s_{\nu_{e}}^{2}+\sigma_{\nu_{e}}^{2} & \rho \sigma_{\nu_{e}} \sigma_{\nu_{\mu}} \\
\rho \sigma_{\nu_{e}} \sigma_{\nu_{\mu}} & s_{\nu_{\mu}}^{2}+\sigma_{\nu_{\mu}}^{2}
\end{array}\right)
$$

as well as the systematics correlation $\rho \sigma_{\nu_{e}} \sigma_{\nu_{\mu}}$ between the $\nu_{e}$ and $\nu_{\mu}$ events. The inverse is given by

$$
\mathcal{M}_{i j}^{-1}=\frac{1}{|\mathcal{M}|}\left(\begin{array}{cc}
s_{\nu_{\mu}}^{2}+\sigma_{\nu_{\mu}}^{2} & -\rho \sigma_{\nu_{e}} \sigma_{\nu_{\mu}} \\
-\rho \sigma_{\nu_{e}} \sigma_{\nu_{\mu}} & s_{\nu_{e}}^{2}+\sigma_{\nu_{e}}^{2}
\end{array}\right)
$$

where $|\mathcal{M}|=\left(s_{\nu_{e}}^{2}+\sigma_{\nu_{e}}^{2}\right)\left(s_{\nu_{\mu}}^{2}+\sigma_{\nu_{\mu}}^{2}\right)-\left(\rho \sigma_{\nu_{e}} \sigma_{\nu_{\mu}}\right)^{2}$ is the determinant of $\mathcal{M}_{i j}$. Then, expanding the $\chi^{2}$ expression yields

$$
\begin{aligned}
\chi^{2}= & \frac{1}{|\mathcal{M}|}\left(\left(s_{\nu_{\mu}}^{2}+\sigma_{\nu_{\mu}}^{2}\right)\left(D^{\nu_{e}}-B^{\nu_{e}}-S^{\nu_{e}}\right)^{2}\right. \\
& -2 \rho \sigma_{\nu_{e}} \sigma_{\nu_{\mu}}\left(D^{\nu_{e}}-B^{\nu_{e}}-S^{\nu_{e}}\right)\left(D^{\nu_{\mu}}-B^{\nu_{\mu}}\right) \\
& \left.+\left(s_{\nu_{e}}^{2}+\sigma_{\nu_{e}}^{2}\right)\left(D^{\nu_{\mu}}-B^{\nu_{\mu}}\right)^{2}\right) .
\end{aligned}
$$

Following a fit to oscillations, minimizing the above $\chi^{2}$ expression, or equivalently setting its first derivative with respect to $S^{\nu_{e}}$ to zero, yields

$$
S^{\nu_{e}}=\left(D^{\nu_{e}}-B^{\nu_{e}}\right)-\left(\frac{\sigma_{\nu_{e}} \sigma_{\nu_{\mu}}}{s_{\nu_{\mu}}^{2}+\sigma_{\nu_{\mu}}^{2}}\right) \rho\left(D^{\nu_{\mu}}-B^{\nu_{\mu}}\right)
$$

which corresponds to the best-fit signal prediction.

Of course, if we were to ignore systematic correlations between the $\nu_{e}$ and $\nu_{\mu}$ events in the fit $(\rho \equiv 0)$, the best-fit signal prediction would correspond precisely to the difference between observed and predicted $\nu_{e}$ events. However, once systematic correlations are taken into account, the number of best-fit signal events will be corrected accordingly if there are any differences between the observed and predicted $\nu_{\mu}$ CCQE events (i.e., if $\left.\left(D^{\nu_{\mu}}-B^{\nu_{\mu}}\right) \neq 0\right)$. The correction in fact is propagated to both the signal and the $\nu_{e}$ background prediction. For example, if an excess is 
seen in the observed $\nu_{\mu}$ events relative to the MonteCarlo prediction, and assuming a positive correlation $(\rho>1)$ between $\nu_{\mu}$ and $\nu_{e}$ background events, the fit will induce an increase in $\nu_{e}$ background prediction, and relative reduction in signal prediction.

Similarly, by calculating the inverse of the second derivative of the $\chi^{2}$ in Eq. A.4 with respect to $S^{\nu e}$, and multiplying by two, we obtain

$$
\sigma_{S}^{2}=s_{\nu_{e}}^{2}+\sigma_{\nu_{e}}^{2}-\left(\frac{\rho^{2}}{s_{\nu_{\mu}}^{2} / \sigma_{\nu_{\mu}}^{2}+1}\right) \sigma_{\nu_{e}}^{2}
$$

which corresponds to the uncertainty on the number of best-fit signal events.

Again, assuming no systematic correlations between the $\nu_{e}$ and $\nu_{\mu}$ events in the fit $(\rho \equiv 0)$, the total systematic plus statistical uncertainty is simply that corresponding to the $\nu_{e}$ background predicted uncertainty. Once correlations are included, then the uncertainty is always reduced according to the level of correlation between the $\nu_{e}$ and $\nu_{\mu}$ events, and reduced further with the level of statistical precision of the $\nu_{\mu}$ sample (as $s_{\nu_{\mu}}^{2} \rightarrow 0$ ). Assuming negligibly small statistical errors on the $\nu_{\mu}$ sample, and in the extreme case that the $\nu_{e}$ and $\nu_{\mu}$ samples are fully correlated $(\rho=1)$, the resulting uncertainty on the signal prediction is simply the statistical uncertainty on the $\nu_{e}$ background. In practice, this results in a partial reduction to the systematic uncertainty on the signal prediction.

Note that the expectation that the fit will correct for any possible normalization discrepancies is also what allows us to fix the overall normalization of events from $\pi^{+}$and $\pi^{-}$decays according to the normalization extracted from a fit to $\nu_{\mu}$ CCQE only events prior to the oscillation fit. The reason why the normalization is corrected in practice a priori to the fit, is to account for proper calculation of systematics and correlations between the samples, using the correct relative contribution from each background component, after $N_{\pi}$ scaling, which can otherwise lead to different effective corrections during the fit [184].

This procedure is used to constrain the signal predictions and systematic uncertainties both at the null and at the best fit point, and when it is expanded to multiplebin distributions, as in the actual fit, it leads to small, energy-dependent corrections. 
The resulting uncertainties are quoted within this thesis as "constrained."

\section{A.3 Obtaining a Shape-only Error Matrix}

In Chapter 5, we summarize results from shape-only fits of observed excess distributions to relatively normalized shapes of various backgrounds or absolute oscillation hypotheses. Here, we present how those fits are performed in more detail.

Each $\chi^{2}$ calculation is performed over the $\nu_{e}$ CCQE distribution predicted as a function of a particular kinematic variable $\left(K=E_{v i s}, \cos \theta_{z}\right.$, or $\left.Q^{2}\right)$, assuming no oscillations, and using the $\nu_{e}$ CCQE part of the $\nu_{\mu}$ CCQE-constrained systematics covariance matrix $M_{i j}^{\text {constr.sys }}(K)$, where the overall normalization uncertainty has been removed as will be discussed in the following paragraphs.

The constrained systematics-only error matrix, obtained as discussed in App. A.2 (expanded from a two-bin to an $\left(N_{\nu_{e}}+N_{\nu_{\mu}}\right)$-bin fit), is used to evaluate the shapeonly constrained systematic error matrix, $M_{i j}^{\text {constr.sys,shape }}$, given a total $\nu_{e}$ CCQE background prediction, $B_{i}^{T}$, as follows:

$$
M_{i j}^{\text {constr.sys,shape }}=M_{i j}^{\text {constr.sys }}-B_{i}^{T} P_{j} /\left(\sum_{k} B_{k}^{T}\right)-B_{j}^{T} P_{i} /\left(\sum_{k} B_{k}^{T}\right)+B_{i}^{T} B_{j}^{T} N^{2}
$$

where $P_{i}=\sum_{j} M_{i j}^{\text {constr.sys }}$ (sum over rows), $P_{j}=\sum_{i} M_{i j}^{\text {constr.sys }}$ (sum over columns), and $N$ is the fractional normalization uncertainty of $M_{i j}^{\text {constr.sys }}$ on the total background, given by

$$
N=\sqrt{\left(\sum_{i, j} M_{i j}^{\text {constr.sys }}\right) /\left(\sum_{k} B_{k}^{T}\right)^{2}} .
$$

In that case, the shape-fit $\chi^{2}$ for each hypothesis is given by

$$
\chi_{\text {shape }}^{2}=\sum_{i, j}\left(\Delta_{i}-B_{i}\right)\left(M_{i j}^{\text {constr.sys,shape }}+\delta_{i j} D_{i}\right)\left(\Delta_{j}-B_{j}\right),
$$

where $\Delta_{i}=D_{i}-T_{i}^{\text {constr }}$ is the observed excess (data minus constrained total background prediction), and $B_{i}$ is the particular background hypothesis under consid- 
eration, $B_{i}^{\text {true }}$, relatively-normalized to the total excess over all bins $i$, i.e., $B_{i}=$ $B_{i}^{\text {true }} \cdot\left(\sum_{i} \Delta_{i}\right) /\left(\sum_{i} B_{i}^{\text {true }}\right)$. In the case of a comparison to a signal prediction, $B_{i}$ is replaced by $S_{i}^{\text {true }}$. Note that in that case the signal prediction is not relativelynormalized to the total excess. The number of degrees of freedom in these fits corresponds to the number of $\nu_{e}$ bins involved in the $\chi^{2}$ calculation, minus one (1) due to fixing the normalization, i.e., $N_{\nu_{e}}-1=6,5$, and 5 , for the $E_{v i s}, Q^{2}$, and $U_{z}$ fits, respectively.

\section{A.4 Absorber Studies in Antineutrino Mode}

During antineutrino running, one of the absorber plates installed above the MiniBooNE decay pipe accidentally fell in the MiniBooNE beamline, and remained in place for a period of time, during which data corresponding to $0.57 \times 10^{20}$ POT was collected. A second plate also fell following the one-absorber running period, and remained in place along with the first plate for an additional $0.61 \times 10^{20}$ POT. All available data from all three absorber running periods (0-, 1-, and 2-absorbers in the beam decay region) is used in the antineutrino analysis presented in this thesis (corresponding to a total of $5.66 \times 10^{20} \mathrm{POT}$ ). The total one- and two-absorber POT correspond to $21 \%$ of the total antineutrino POT.

The purpose of installing absorber plates above the beamline is to allow for optional cross-checks of flux systematics [116]. Specifically, lowering absorber plates in the decay tunnel suppresses the relative amount of neutrinos from decays of long-lived particles, such as muons (decay length $\sim 500 \mathrm{~m}$ ) and neutral kaons (decay length $\sim 100$ $\mathrm{m}$ ), relative to charged kaons and pions (decay length $\sim 20 \mathrm{~m}$ and $\sim 10 \mathrm{~m}$ ). Therefore, careful modeling of the absorber plates and their effect on the neutrino flux is essential for an accurate prediction of $\nu_{\mu}$ CCQE events and even more so for $\nu_{e}$ CCQE events, since $\nu_{e}$ from $K^{0}$ decay contribute significantly to the $\nu_{e}$ CCQE background prediction. To some extent, the plates also absorb pions before they can decay but this will affect both the $\nu_{e}$ and the $\nu_{\mu}$ CCQE samples, and so by involving the constraint described in App. A.2, the contribution from pions can be constrained regardless. On 
the other hand, $K^{0}$ decays affect only the $\nu_{e}$ CCQE background, and therefore cannot be constrained by the $\nu_{\mu}$ CCQE sample.

The effect of the absorber on the MiniBooNE flux has been accounted for in all $\nu_{\mu} \mathrm{CCQE}$ and $\nu_{e} \mathrm{CCQE}$ MonteCarlo predictions presented in this thesis, by means of flux reweighting as a function of true neutrino energy, as discussed in App. A.1, for each neutrino type $\left(\nu_{e}, \bar{\nu}_{e}, \nu_{\mu}\right.$, and $\left.\bar{\nu}_{\mu}\right)$, and according to neutrino parent $\left(\pi^{+}, \pi^{-}, K^{+}\right.$, etc.). The accuracy of the absorber reweighting technique in reproducing available two-subevent (mostly $\nu_{\mu}$ CCQE events), three-subevent (mostly CC $\pi$ events), and one-subevent (signal-blind) samples has been studied [185], and was verified using MiniBooNE data prior to unblinding. Specifically, it was found to reproduce similar (within uncertainties) data to MonteCarlo agreement for each absorber configuration, and for each of the above three samples.

Absorber data quality checks have been performed separately for the $\nu_{\mu} \mathrm{CCQE}$ sample, and are discussed in [118]. Here, we limit the discussion to $\nu_{e}$ CCQE checks.

Figure A-1 shows the absolute number of observed $\nu_{e}$ CCQE events per POT for the three absorber periods, 0,1 , and 2. The jul07 and jul07_update correspond to all data used in the $3.39 \times 10^{20}$ POT analysis [102], which include the 1 and 2 absorber running periods, whereas sep09 and mar10 correspond to the additional, 0 -absorber-only data collected since then, which are included only in the $5.66 \times 10^{20}$ POT analysis presented in this thesis. Without accounting for flux differences, the 0 -absorber POT-normalized observed data in the $200<E_{\nu}^{Q E}<3000 \mathrm{MeV}$ range are systematically higher than those corresponding to 1- and 2-absorber, as expected.

Comparing only 0-absorber observed event rates per corresponding POT, and accounting for statistical uncertainties and a $2 \%$ normalization uncertainty due to POT systematics (see Sec. 4.6.1), yields a compatibility of $22.3 \%\left(\chi^{2} /\right.$ d.o.f. $\left.=1.5 / 2\right)$ between the 0-absorber data used in the analysis of [102] (118 observed events for $2.20 \times 10^{20} \mathrm{POT}$ ) and the additional 0-absorber data used in the $5.66 \times 10^{20}$ analysis presented in this thesis (142 observed events for $2.28 \times 10^{20} \mathrm{POT}$ ). The observed $\nu_{e}$ CCQE candidate events per POT are shown as a function of time in Fig. A-2. Besides the absorber running periods, there are no anomalous event rate periods. 
MonteCarlo studies determine that the expected reduction in overall event rate due to the presence of a single absorber plate in the decay pipe is $23 \%$, and $31 \%$ for two absorber plates, relative to no absorber. The absorber reweighting applied to MonteCarlo predicted events is speficically designed to account for any difference in event rates due to the different absorber configurations. Therefore, a comparison of observed data to MonteCarlo prediction in each running period is more instructive. Figure A-3 shows a comparison of observed data to MonteCarlo predictions for different absorber running configurations, as a function of reconstructed neutrino energy. In the top panel of Fig. A-3, the distributions are shown separately for the total (jul07+jul07_update+sep09+mar10) 0-absorber data set, 1-absorber data set, and 2-absorber data set. In the bottom panel of Fig. A-3, the 1- and 2-absorber data sets have been combined to enhance statistics. From the figures, one can clearly see a statistically significant excess in the 0-absorber data set, but not in the 1- and 2-absorber data sets; the statistical uncertainties, however, are larger for the 1- and 2-absorber running periods. The ratio of observed data to MonteCarlo prediction is shown in Fig. A-4 for each running configuration. All three data to MonteCarlo ratios agree within statistical uncertainties.

The compatibility between the two running periods, the one corresponding to the analysis presented in [102] (period 1), and the one corresponding to the additional data set considered in the analysis presented in this thesis, i.e. $(5.66-3.39) \times 10^{20}$ POT (period 2), can be calculated by comparing the ratio of observed data to MonteCarlo background prediction (which has been absorber-reweighted as appropriate for each period) using events from all absorber configurations. For the second running period, the MonteCarlo predicts 101.4 events compared to 133 observed events in the $200<E_{\nu}^{Q E}<3000 \mathrm{MeV}$ range, corresponding to a ratio of $1.31 \pm 0.12$ (stat). For the first running period, the MonteCarlo predicts $^{1} 132.4$ events compared to 144 observed events, corresponding to a ratio of $1.09 \pm 0.09$ (stat). The resulting compatibility is $12.3 \%\left(\chi^{2} /\right.$ d.o.f. $=2.4 / 2$, calculated about the weighted average of the two mea-

\footnotetext{
${ }^{1}$ Using the MonteCarlo reweighting correction settings updated for the $5.66 \times 10^{20}$ POT analysis, except for the absorber reweighting, and therefore differs from the 139.2 events quoted in [102].
} 

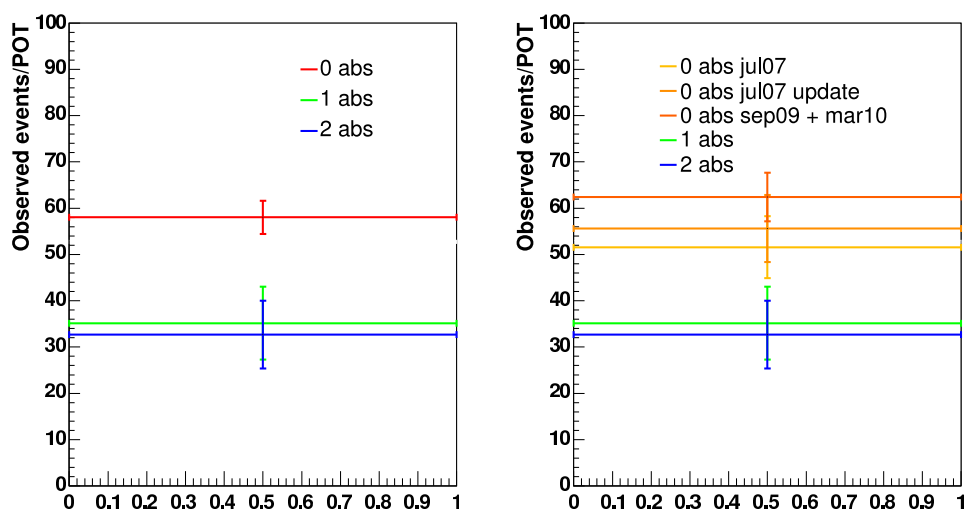

Figure A-1: Total number of observed $\nu_{e}$ CCQE candidate events (for $200<$ $E_{\nu}^{Q E}<3000 \mathrm{MeV}$ ) per POT, for different running periods in antineutrino mode. MiniBooNE sees a total of 239 candidate events in the 0-absorber data set, and 20 and 18 events in the 1- and 2-absorber data sets, respectively.

surements), and increases to $13.1 \%$ once POT normalization systematic uncertainties $( \pm 2 \%)$ are included.

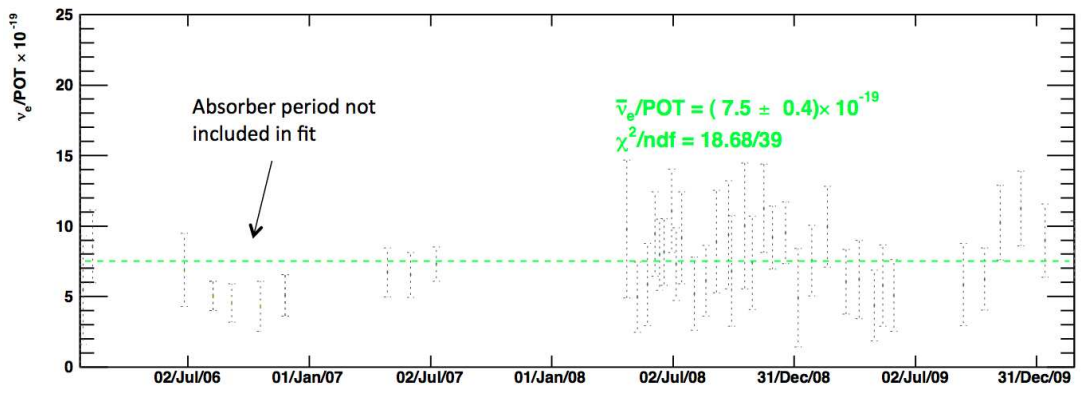

Figure A-2: The number of observed $\nu_{e}$ CCQE candidate events per POT over time. Antineutrino running mode. 

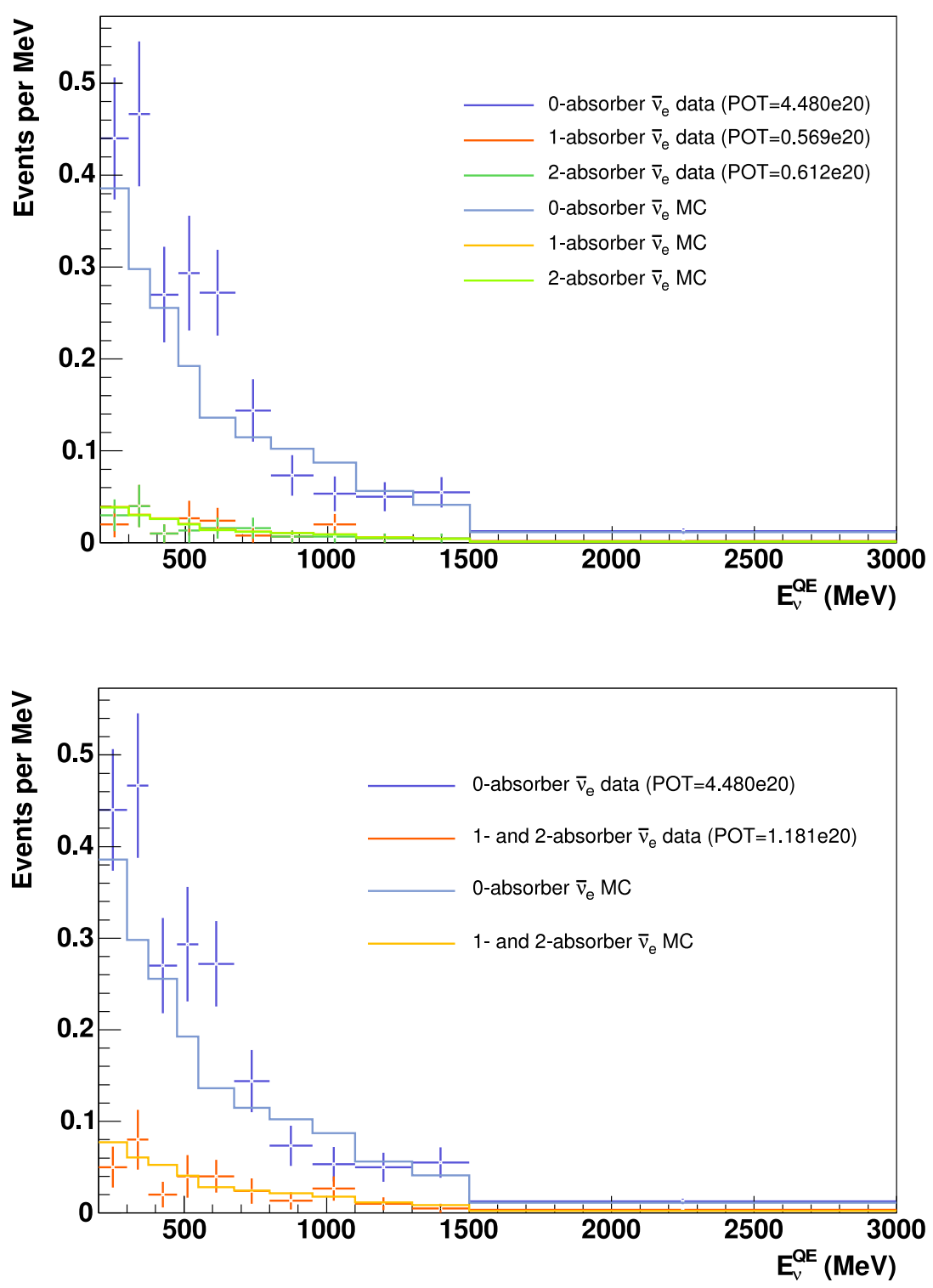

Figure A-3: $E_{\nu}^{Q E}$ distributions for observed and MonteCarlo-predicted $\nu_{e}$ CCQE candidate events for different absorber running periods in antineutrino mode. The MonteCarlo predicts a total of 197 candidate events in the 0-absorber data set, and 20 events in each of the 1- and 2-absorber data sets. The corresponding observed data events are 239, 20, and 18. In the bottom panel, the 1- and 2-absorber data are combined to enhance statistical significance. 


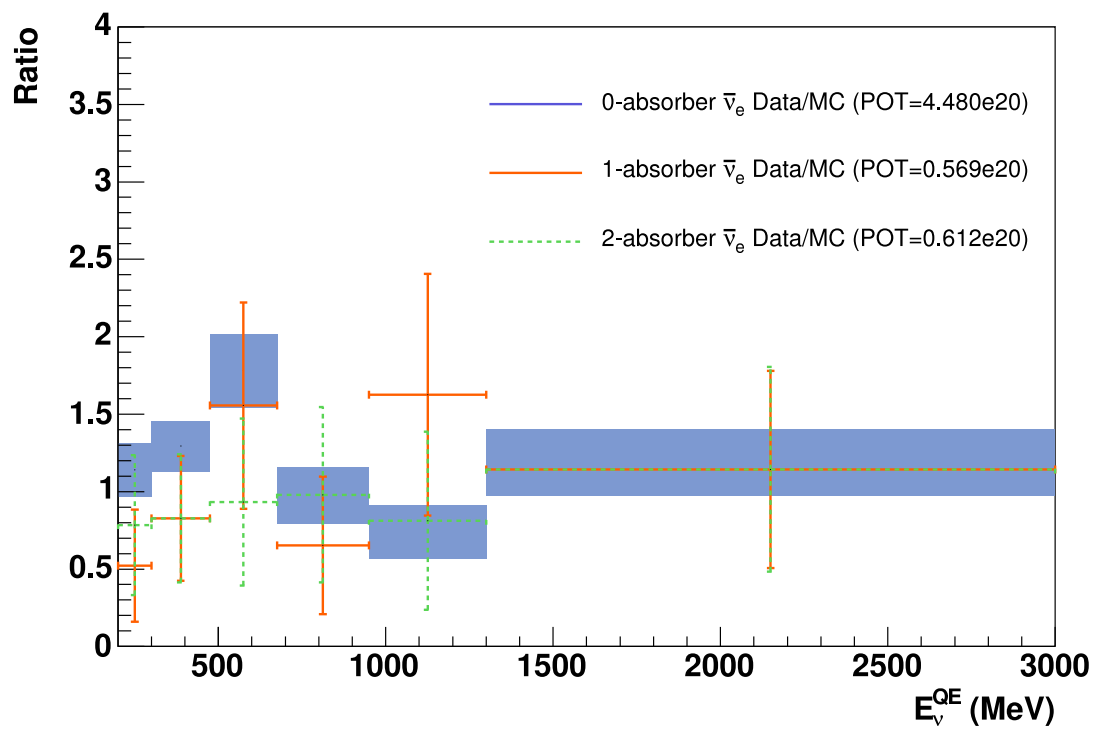

Figure A-4: $E_{\nu}^{Q E}$ distributions for the ratio of observed to MonteCarlo-predicted $\nu_{e}$ CCQE candidate events for different absorber running periods in antineutrino mode. 


\section{Appendix B}

\section{Future Searches: MicroBooNE}

\section{B.1 Introduction}

The MicroBooNE experiment is a new neutrino experiment proposed to run in the booster neutrino beamline at Fermi National Accelerator Laboratory [115]. MicroBooNE is a demonstration experiment for large-scale Liquid-Argon Time Projection Chamber (LArTPC) experiments. Those are of high interest for the future U.S. LongBaseline Neutrino Experiment (LBNE) program [186], ${ }^{1}$ due to their extraordinarily precise event reconstruction, as well as their potential for scalability ${ }^{2}$ to multi-kiloton (>100 kton) sizes [187].

In addition to useful $\mathrm{R} \& \mathrm{D}$ in laying the groundwork for the next generation of LArTPC's, MicroBooNE's goals include important physics measurements, such as neutrino cross-section measurements on argon in the $1 \mathrm{GeV}$ energy range, which is the energy range targeted by LBNE experiments [188]. The primary physics goals of MicroBooNE revolve around searches for new physics in the neutrino sector. Specifically, MicroBooNE's primary goal is to investigate possible interpretations of the low energy excess observed by MiniBooNE in neutrino running mode. This chapter

\footnotetext{
${ }^{1}$ Future long-baseline neutrino experiments aim to address the questions of neutrino mass hierarchy, size of $\theta_{13}$, and possibility of $C P$ violation in the neutrino sector.

${ }^{2}$ Competing technologies in terms of particle identification and resolution capability include bubble chambers and emulsion detectors. However, these require image scanning systems which lead to considerable delay in data processing.
} 
focuses on MicroBooNE's sensitivity in resolving the nature of the MiniBooNE low energy excess, but also investigates MicroBooNE's sensitivity to light sterile neutrino oscillations.

Sections B. 3 and B.4 briefly introduce some important concepts of the experiment, including the detector conceptual design, with emphasis on the light detection system, and expected neutrino event rates for MicroBooNE's current run plan. In Sec. B.5 we discuss MicroBooNE's sensitivity in resolving the MiniBooNE neutrino low energy excess as either electron-like, or photon-like in nature. Motivated by the MiniBooNE antineutrino appearance results presented in Chapter 5, in Sec. B.5.3 we explore MicroBooNE's sensitivity to $\bar{\nu}_{\mu} \rightarrow \bar{\nu}_{e}$ oscillations for a potential antineutrino run. Finally, in Sec. B.6, we present preliminary R\&D studies performed which are relevant for the MicroBooNE light collection detector system.

At the time of this writing, MicroBooNE has been successfully reviewed by the Fermilab Directorate and the Department of Energy for CD-1 phase approval [189], and commissioning is expected to being in 2012 [190].

\section{B.2 Background on LArTPC's}

LArTPC detectors are particularly appealing to the neutrino oscillation community from the perspective of $\nu_{\mu} \rightarrow \nu_{e}$ and $\bar{\nu}_{\mu} \rightarrow \bar{\nu}_{e}$ searches. The high resolution event topology of which these detectors are capable provides a significant signal to background rejection compared to Cherenkov detectors, because of the ability to differentiate between $\nu_{e}$ (signal) events and single-photon events which form an irreducible background in Cherenkov detectors. Unlike LArTPC's, Cherenkov detectors, e.g., as we have seen in the case of MiniBooNE, are limited by those backgrounds.

LArTPC's were first proposed in 1976 by H. Chen. Since then, considerable $R \& D$ progress has been made in this field, especially by the ICARUS collaboration in Europe [197], which has been able to demonstrate the successful operation of a 300 ton LArTPC detector. Table B.1 highlights some of the important milestones in the history of LArTPC technology. Today, a large fraction of the LArTPC R\&D 


\begin{tabular}{llc}
\hline \hline Time frame & Highlight & Ref. \\
\hline \hline 1976 & H. Chen proposes a tracking LAr detector (FNAL P496). & {$[191]$} \\
\hline late 70's & W. Willis \& V. Radeka begin successful electronics development & {$[192]$} \\
through today & which culminates in the MicroBooNE proposal. & {$[115]$} \\
\hline 1977 & Carlo Rubbia, et al., begin ICARUS program, & {$[193,194]$} \\
through today & which has produced 30 years of results. & $e . g .,[195]$ \\
\hline 2007 & ArgoNeuT program begins, & {$[196]$} \\
through today & presently taking data at Fermilab. & \\
\hline \hline
\end{tabular}

Table B.1: A few highlights in LArTPC development prior to MicroBooNE .

effort focuses on demonstrating their scalability to multi-kiloton sizes, e.g., [197, 198]. Along with ICARUS, MicroBooNE, with a total size of 150 tons, acts as an important step in a phased program [199].

\section{B.3 The MicroBooNE Detector}

The MicroBooNE detector is a $\sim 70$ ton fiducial volume (150 ton total volume) LArTPC detector. The detector design is illustrated in Fig. B-1.

The detector volume is filled with high purity liquid argon, and serves as the neutrino target and tracking medium for charged particles produced in neutrino interactions. Neutrino detection, event reconstruction and particle identification rely on measuring the ionization energy loss $(d E / d x)$ of particles produced in neutrino interactions with argon.

The liquid argon and active detector components are enclosed in a cylindrical cryostat, which is a foam-insulated vessel made of stainless steel.

Operation of the detector relies on several component systems: the cryogenics system, which is designed to keep the liquid-argon temperature stable at $87.3 \mathrm{~K}$, the purification system, which is designed to purify and keep the liquid-argon impurities (for example, oxygen levels) at less than $1 \mathrm{ppm}$, and the data acquisition and high-voltage electronics system, which provide live feeds to and from the active components of the detector. The latter consist of the TPC and a light collection system of photomultiplier tubes (PMT's). The wall along the top of the cryostat is equipped 


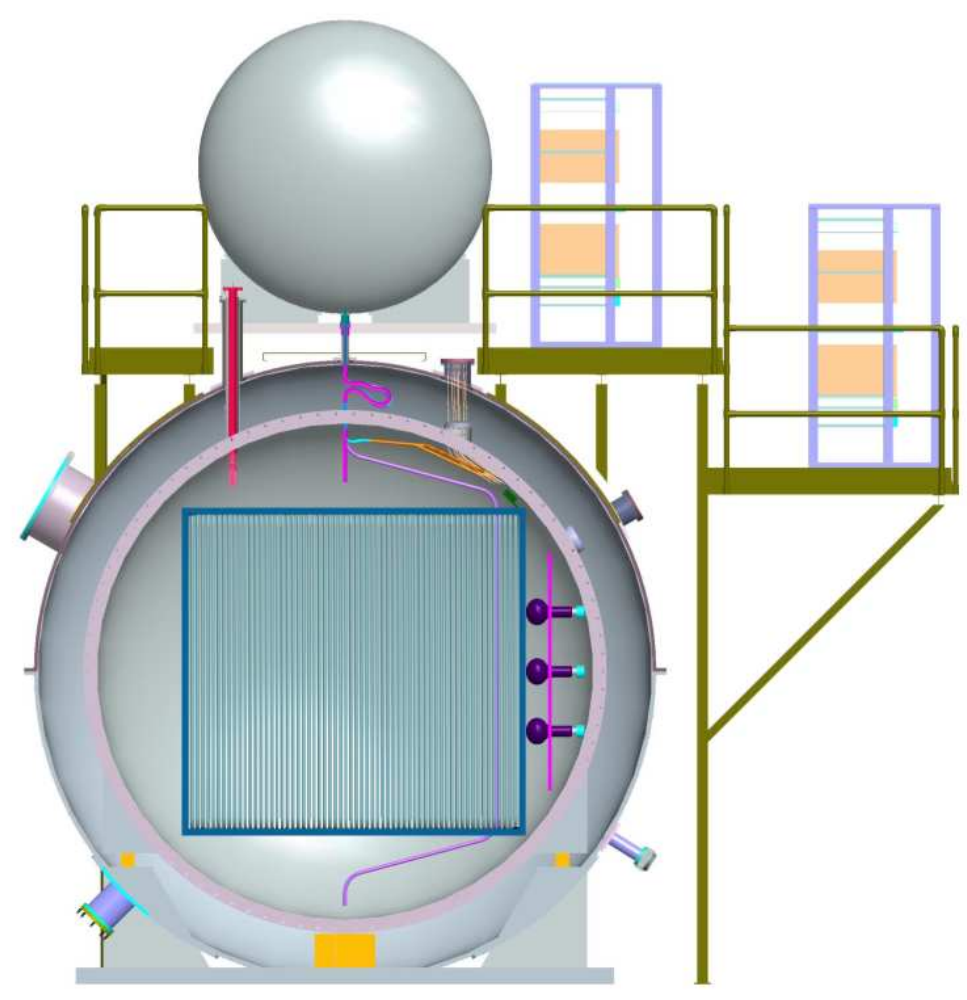

Figure B-1: Schematic of the MicroBooNE detector conceptual design. Neutrino beam axis view. The MicroBooNE active detector components (TPC surrounded by a field cage shown in blue in the center of the detector, and PMT's in the beam right side of the detector) are enclosed in a cryostat cylindrical vessel. On top of the MicroBooNE detector is a liquid-argon expansion vessel, which is part of the detector's cryogenics system.

with several feedthroughs, shared among the above systems, which allow for signals to come out of the detector, and HV and power to be supplied to the active detectors and electronics components.

Ionization signals produced by the passage of charge particles ${ }^{3}$ through the liquid argon are detected by the TPC. The TPC is defined by two parallel, conductive planes, positioned diametrically opposite each other about the neutrino beam axis. The planes are held at a specific electric potential configuration, which creates a uniform electric within the liquid argon volume suspended between them. The TPC active volume dimensions are $2.3 \mathrm{~m} \times 2.6 \mathrm{~m} \times 10.4 \mathrm{~m}$, and those determine the fiducial volume of the experiment.

\footnotetext{
${ }^{3} \mathrm{~A}$ minimum ionizing particle in liquid argon produces $\sim 6000$ electrons per mm.
} 

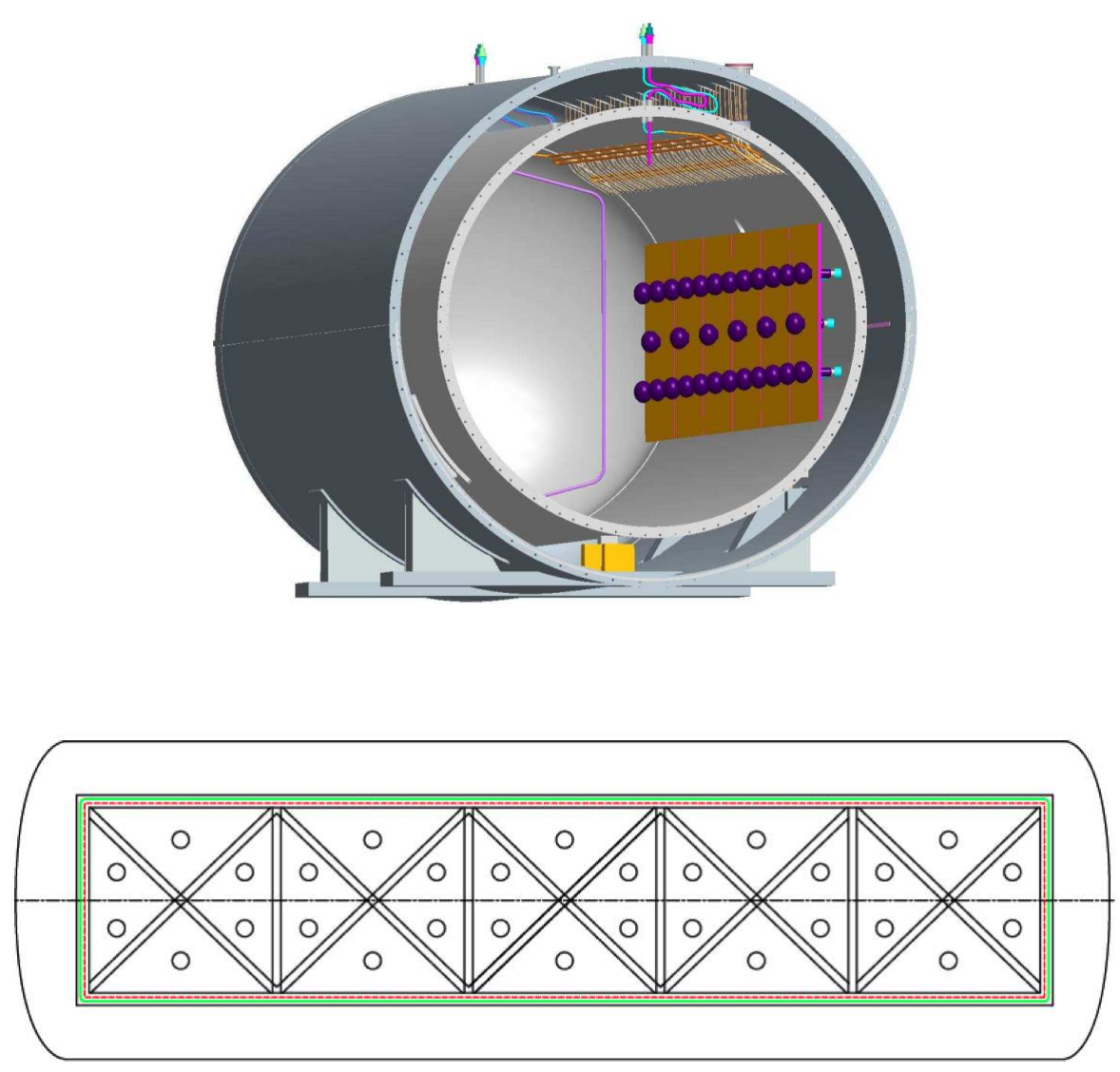

Figure B-2: A close-up 3D view (top) and schematic (bottom) of the PMT arrangement in the MicroBooNE detector. The PMT's (30 total) are arranged in the beam right side of the MicroBooNE detector, behind the TPC anode plane.

The ionization electrons produced across a particle track drift through the medium, driven by an electric field of $\sim 500 \mathrm{~V} / \mathrm{cm}$ applied between the TPC anode and cathode planes, and get collected at the anode plane. The anode plane is made up of three parallel wire planes, at $30^{\circ}, 150^{\circ}$, and $90^{\circ}$ with respect to the beam direction, located on the beam right side of the detector. The electric potential of each anode wire plane is chosen so that the electrons pass through the first two (induction) wire planes and are collected on third (collection) wire plane. The induced and direct electrical signals on each wire are sampled at $2 \mathrm{MHz}$ and recorded for further processing. Both shape and timing information for each wire signal is collected.

Because the wire positions are known, the time at which the signal arrives at each wire, with respect to the time the interaction took place, can be used to reconstruct the charged particle trajectory. Since each charged particle produces ionization across 


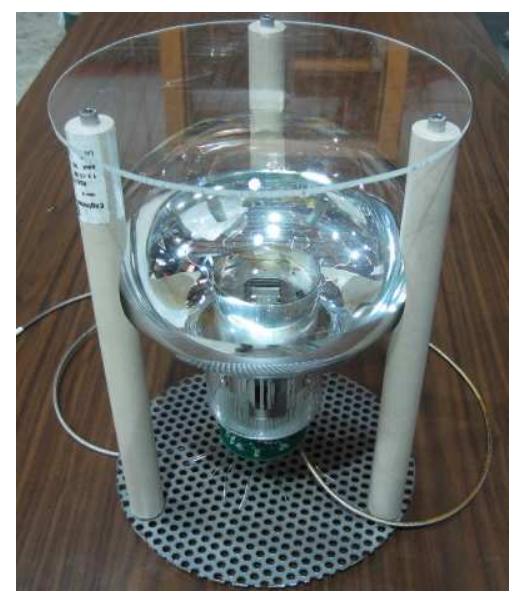

Figure B-3: MicroBooNE PMT supporting frame mock-up, made of three 1-inch diameter grooved PEEK posts surrounding the PMT in at three equidistant positions.
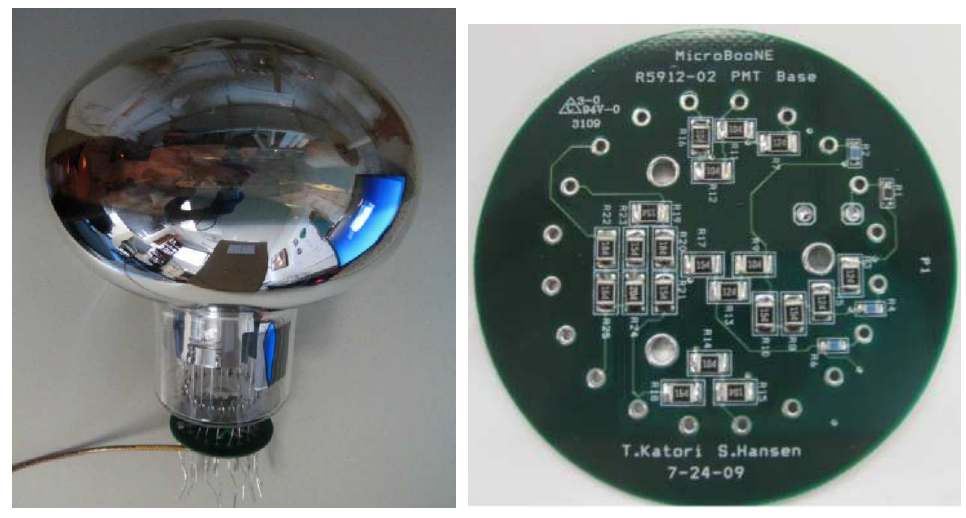

Figure B-4: R5912-02MOD PMT and custom-made PMT base used in the MicroBooNE experiment.

its extended track, the energy loss per unit distance $(d E / d x)$ can be determined through the ionization charge projection across the wire plane. The amplitude of the ionization electron signal provides a measure of the charge and therefore the energy loss of the particle. Because the ionization energy loss is characteristic of a particle's mass, charge, and momentum, a measurement of $d E / d x$ allows for an estimate of the particle's momentum and identification of the particle itself.

As expected, the wire spacing affects the charge sampling and therefore the position and energy resolution of the experiment. Desired position resolution for MicroBooNE is at the millimeter scale or better, which requires a wire spacing of $3 \mathrm{~mm}$ 
given the charge diffusion and attachment properties in liquid argon at MicroBooNE's operating electric field, temperature and purity conditions.

Because typical drift times for ionization charge in LAr are large (electron drift velocity $v_{D}\left(e^{-}\right)=1.60 \mathrm{~mm} / \mu \mathrm{s}$ for $E=500 \mathrm{~V} / \mathrm{cm}$ ) and further vary with temperature and electric field, they do not allow for a precise determination of the time the interaction takes place within the beam spill (1.6 $\mu \mathrm{s}$ long) and, consequently, the driftdirection coordinate of the interaction vertex. Therefore, precise interaction timing information is provided with the use of a light detection system which is installed in the MicroBooNE vessel as part of the active detectors system. Because liquid argon produces ample and fast ${ }^{4}$ scintillation light when excited electromagnetically, the light detection system exploits the scintillation light signal produced by charged particles produced in neutrino interactions, in order to determine the initial interaction time. This technique has been demonstrated as a precise method of obtaining the initial time of an event in $[197,201,202]$.

The use of scintillation light as a neutrino interaction trigger within each beam spill significantly reduces the amount of (relatively low information) beam spill data to be processed, and therefore significantly reduces the data acquisition system processing and storage requirements.

The light detection system consists of an array of 30 PMT's, located behind the anode plane on the beam right side lune of the detector, as illustrated in Fig. B-2. The PMT structure is supported by a frame mounted to the walls of the cryostat. Each PMT is held in place by a set of three PEEK posts attached to the supporting frame structure, as shown in Fig. B-3. The PMT's to be used in MicroBooNE are 8-inch R5912-02MOD Hamamatsu PMT's with a platinum-coated bialkali $\left(K_{2} C s S b+P t\right)$ photocathode, and $C s S b$ dynode, shown in Fig. B-4, which are optimized for efficient operation at low-temperature. ${ }^{5}$ The PMT's provide $0.8 \%$ photocathode coverage.

\footnotetext{
${ }^{4}$ Scintillation in liquid argon proceeds through two de-excitation modes, resulting in a prompt scintillation time component, $\tau_{\text {prompt }}=6 \mathrm{~ns}$, and a slow scintillation component, $\tau_{\text {slow }}=1590 \mathrm{~ns}$ [200].

${ }^{5}$ Because of the semiconductor nature of most photocathode materials, most widely used PMT's show a large increase in resistivity at low temperature, which renders them unusable in liquid-argon detectors.
} 
The minimum photocathode coverage requirement is driven by the physics goals of the experiment, specifically MicroBooNE's potential for supernova neutrino detection, which requires sensitivity down to a few $\mathrm{MeV}$ in neutrino energies. ${ }^{6}$

The MicroBooNE detector will be installed where the MiniBooNE detector is currently located, which will expose the detector to both the BNB and the NuMI beamline. MicroBooNE's current BNB run plan consists of a three year operating period in neutrino running, for a total of $6.0 \times 10^{20}$ POT delivered to the BNB target. The BNB will be operated in the same configuration as that used during MiniBooNE neutrino mode operation, in order to minimize beam systematic uncertainties in the comparison of MicroBooNE data with MiniBooNE data.

For more detailed descriptions of the MicroBooNE detector see [115, 203].

\begin{tabular}{ll|r|r}
\hline \hline Interaction & Expected events & Fraction of total \\
\hline \hline Muon neutrino rates: & & \\
\hline $\mathrm{CCQE}$ & $\nu_{\mu} n \rightarrow \mu^{-} p$ & 52,500 & $45.0 \%$ \\
$\mathrm{CC} \pi^{+}$(Res. and Coh.) & $\nu_{\mu} X \rightarrow \mu^{-} X \pi^{+}$ & 24,250 & $20.8 \%$ \\
$\mathrm{CC} \pi^{0}$ & $\nu_{\mu} n \rightarrow \mu^{-} p \pi^{0}$ & 6,100 & $5.2 \%$ \\
$\mathrm{NC} \pi^{0}$ (Res. and Coh.) & $\nu_{\mu} X \rightarrow \nu_{\mu} X \pi^{0}$ & 7,950 & $6.9 \%$ \\
$\mathrm{NC}$ elastic & $\nu_{\mu} N \rightarrow \nu_{\mu} N$ & 16,900 & $14.5 \%$ \\
Other & 5,700 & $5.0 \%$ \\
\hline \hline Electron neutrino rates: & & $37.2 \%$ \\
\hline $\mathrm{CCQE}$ & $\nu_{e} n \rightarrow e^{-} p$ & 285 & $23.5 \%$ \\
$\mathrm{CC} \pi^{+}$(Res. and Coh.) & $\nu_{e} X \rightarrow e^{-} X \pi^{+}$ & 180 & $6.3 \%$ \\
$\mathrm{CC} \pi^{0}$ & $\nu_{e} n \rightarrow e^{-} p \pi^{0}$ & 50 & $6.7 \%$ \\
$\mathrm{NC} \pi^{0}$ (Res. and Coh.) & $\nu_{e} X \rightarrow \nu_{e} X \pi^{0}$ & 50 & $11.7 \%$ \\
NC elastic & $\nu_{e} N \rightarrow \nu_{e} N$ & 90 & $8.4 \%$ \\
Other & & 60 & \\
\hline \hline
\end{tabular}

Table B.2: Expected MicroBooNE BNB $\nu_{\mu}$ and $\nu_{e}$ event rates for various interactions, assuming neutrino mode running for $6.0 \times 10^{20}$ POT. The rates are approximate, from [204], and correspond to 70 ton fiducial volume, without efficiency considerations or any selection cuts. The "other" category includes DIS and NC $\pi^{ \pm}$events.

\footnotetext{
${ }^{6}$ This was estimated assuming liquid argon scintillation yield of the order of $\sim 10^{4}$ photons per $\mathrm{MeV}$ [197].
} 


\section{B.4 Neutrino Interactions in MicroBooNE}

Because MicroBooNE will be located at the same position as MiniBooNE, the neutrino flux seen by the MicroBooNE detector will be very similar to that seen by the MiniBooNE detector. Therefore, for many planned physics analyses, the interactions of interest in MicroBooNE and MiniBooNE are the same. Table B.2 summarizes the absolute $\nu_{\mu}$ and $\nu_{e}$ interaction rates expected in MicroBooNE in neutrino running mode, corresponding to $6.0 \times 10^{20}$ POT delivered to the BNB target. The dominant interaction channel, like in the case of MiniBooNE, is CCQE, followed by CC $\pi^{+}$.

Of course, differences in fiducial volume, detector and reconstruction efficiencies, neutrino target, and others, summarized in Tab. B.3, will introduce substantial differences in the reconstructed event rates. In the MicroBooNE sensitivity studies presented in Sec. B.3, crude approximations of detector efficiency and fiducial volume differences are taken into consideration.

\begin{tabular}{|c|c|c|}
\hline 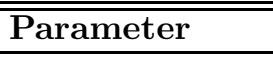 & MiniBooNE & "MicroBooNE \\
\hline Neutrino target & ${ }^{12} \mathrm{C}, \mathrm{H}$ & $\mathrm{Ar}$ \\
\hline Fiducial Volume & 450 ton & 70 ton \\
\hline PID efficiency & $<40 \%$ for $\nu_{e}$ CCQE events & $\sim 80 \%$ for $\nu_{e}$ CCQE events \\
\hline Acceptance & & $\begin{array}{l}\text { expected to be lower for } \nu_{\mu} \text { CCQE events } \\
\text { at higher energies }[205]\end{array}$ \\
\hline Energy threshold & $\begin{array}{l}140 \mathrm{MeV} \text { visible energy } \\
\text { for } \nu_{e} \mathrm{CCQE} \text { analysis }\end{array}$ & $\sim$ a few $\mathrm{MeV}$ visible energy \\
\hline
\end{tabular}

Table B.3: Comparison of MicroBooNE and MiniBooNE detector parameters driving reconstructed event rate differences.

Compared to MiniBooNE, MicroBooNE will have significantly better photon versus electron differentiation, which will lead to a significant signal to background improvement in the low energy excess interpretation studies.

Previous analyses have demonstrated that LArTPC's can provide excellent differentiation between electromagnetic showers induced by electrons and electromagnetic showers induced by photons [206]. This differentiation is achieved by placing a selection requirement on energy deposition in the first $2.4 \mathrm{~cm}$ of a reconstructed shower. A single photon will convert at approximately $18 \mathrm{~cm}\left(=\frac{9}{7} \times\right.$ radiation length $)$ from 
the interaction vertex into an $e^{+} e^{-}$pair, which will deposit energy corresponding to two minimum ionizing particles (mips) in the first stage of the shower. In contrast, a single electron from, say, a $\nu_{e}$ CCQE interaction, will deposit energy corresponding to a single mip in the first stage of the shower. Therefore, by measuring the energy deposition in the first $2.4 \mathrm{~cm}$ of an electromagnetic shower, MicroBooNE can identify the shower as created by either an electron or a photon. In Ref. [206], this method leads to an efficiency of $90 \%$ for detecting single electrons, with an inefficiency in rejecting single photons at $6 \%$, when one is specifically looking for electrons.

For MicroBooNE, the same differentiation technique is demonstrated in Fig. B5. Note that the same technique can be applied to search for single-photon events, in which case the efficiencies are reversed. For MicroBooNE's sensitivity studies, a conservative $80 \%$ signal efficiency is assumed for electrons with a $6 \%$ inefficiency in rejecting photons, and vice versa.

\section{B.5 MicroBooNE Physics Potential}

In this section, we investigate MicroBooNE's sensitivity to a possible low energy excess under two hypotheses:

1. The excess is due to single-photon events.

2. The excess is due to single-electron events.

In addition, we show preliminary estimates for MicroBooNE's sensitivity to either $\nu_{\mu} \rightarrow \nu_{e}$ or $\bar{\nu}_{\mu} \rightarrow \bar{\nu}_{e}$ oscillations, where for the latter we assume MicroBooNE running in antineutrino mode, which is beyond the scope of the current MicroBooNE run plan.

The event rates and sensitivity plots described in this section assume a fiducial volume of 70 tons, implying that events with a reconstructed vertex within that amount of volume are considered in the analysis. An upper limit for the fiducial volume of MicroBooNE is estimated using the TPC active volume dimensions, where 

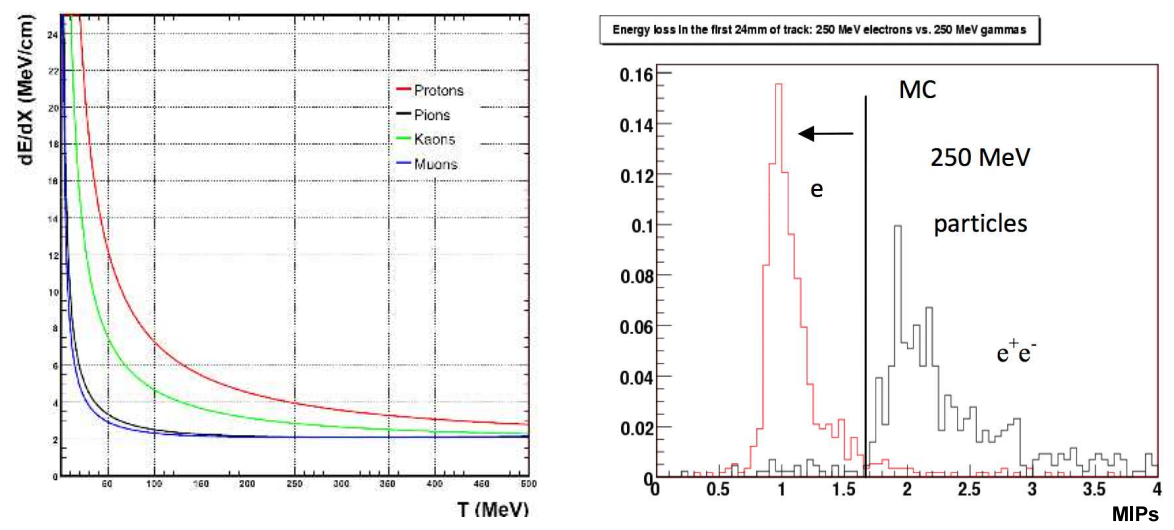

Figure B-5: MicroBooNE $d E / d x$ differentiation capability. The energy loss for different particles in LAr [207] is shown on the left panel, and MicroBooNE's electron versus photon separation capability [203] is shown on the right panel. MicroBooNE can reject $94 \%$ of events producing a single photon when looking for electron-like events, and vice versa, by placing a $d E / d x$ cut based on the number of minimum ionizing particles produced in the first $2.4 \mathrm{~cm}$ of the electromagnetic shower [208].

$230 \mathrm{~cm}, 260 \mathrm{~cm}$ and $1040 \mathrm{~cm}$ correspond to the transverse horizontal, transverse vertical and beam horizontal TPC dimensions, and accounting for a $5 \mathrm{~cm}$ electric field non-uniformity region at the volume edge. The resulting fiducial volume corresponds to approximately 80 tons for liquid argon $\left(\rho=1.4 \mathrm{~g} / \mathrm{cm}^{3}\right)$. However, a more conservative fiducial volume choice is 70 tons, made specifically for the oscillation and low energy excess sensitivity studies. This number is obtained by accounting for the same $5 \mathrm{~cm}$ non-uniformity region, as well as an additional $12 \mathrm{~cm}\left(=\frac{2}{3} \times\right.$ photon conversion length) cut on all sides, arbitrarily defined and expected to minimize backgrounds due to photon escape following a $\pi^{0}$ decay near the detector boundary. ${ }^{7}$

\footnotetext{
${ }^{7}$ Note that the value of 70 tons has been calculated assuming $\mathrm{Z}=1152 \mathrm{~cm}$ rather than $1040 \mathrm{~cm}$, for historical reasons.
} 


\section{B.5.1 Investigation of MiniBooNE Low Energy Excess}

While MiniBooNE has determined that its low energy excess is electromagnetic in nature, it cannot provide further insight on the source of the excess, as the detector cannot distinguish an electromagnetic shower caused by an electron to that caused by a single photon converting into $e^{+} e^{-}$in the detector. Present interpretations of the excess in the literature cover both signatures: those which predict a single electron visible in the detector, and those which predict a single photon visible in the detector. Therefore, the MicroBooNE analysis must proceed under two separate hypotheses: one which assumes the excess is due to an electron and one which assumes the excess is due to a single photon.

To obtain the event rate expectations under each hypothesis, we start with the MiniBooNE predicted $\nu_{e}$ CCQE background event rates and separate them into two classes, depending on whether they correspond to effectively single-electron or singlephoton events. All NC $\pi^{0}, \Delta \rightarrow N \gamma$ and dirt events mis-identified as $\nu_{e}$ CCQE events in MiniBooNE are considered photon-like, while all others are considered electron-like, including mostly events from intrinsic $\nu_{e}$ backgrounds. Note that the generalization of events other than $\mathrm{NC} \pi^{0}, \Delta \rightarrow N \gamma$, dirt, and intrinsic $\nu_{e}$ as electron-like overestimates the background prediction in the electron-like hypothesis, and underestimates the background prediction in the photon-like hypothesis. Nevertheless, it is a reasonable approximation. ${ }^{8}$

The resulting distributions are mapped into MicroBooNE predictions by assuming an energy-independent scaling of a factor of two, to account for MicroBooNE's higher particle reconstruction and identification efficiency ( $\sim 80 \%$ as opposed to $\sim 40 \%$ ), and an additional factor of 0.154 , to account for the difference in fiducial volume (see Tab. B.3).

The reconstructed energy spectrum for the electron interpretation is shown in Fig. B-6. For this hypothesis, MicroBooNE would expect to see 36.8 excess events

\footnotetext{
${ }^{8}$ Those events come from processes such as NC elastic scattering on nucleons, CC and NC resonant single-pion production, multi-pion resonant processes, and coherent pion production, most of which will likely be rejected from both samples in MicroBooNE using event topology information.
} 


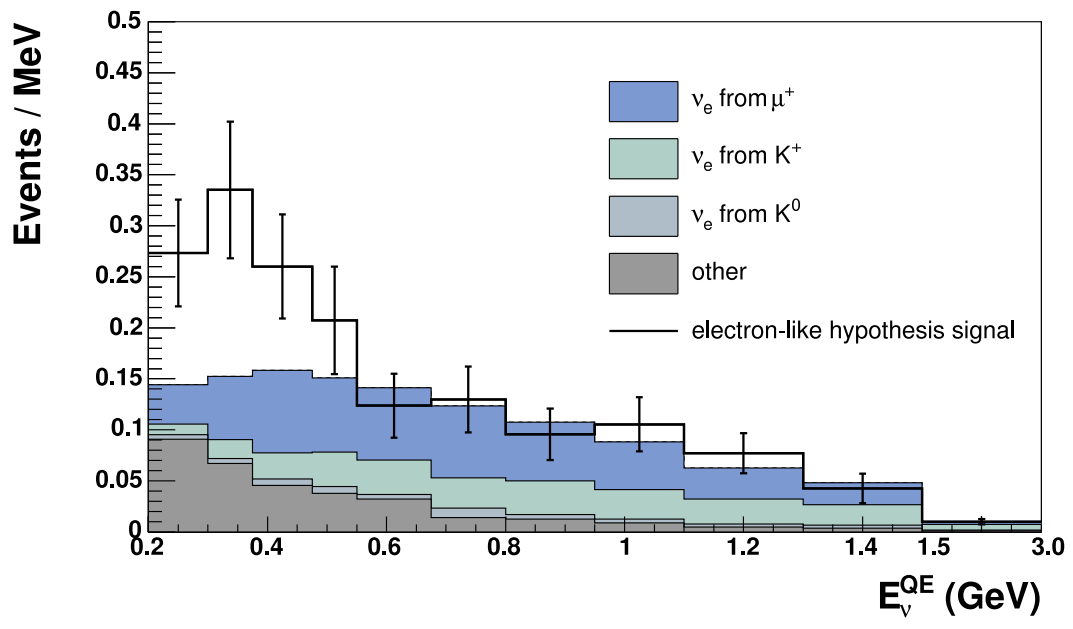

Figure B-6: MicroBooNE expected energy spectrum for electron-like events. The black line corresponds to the MicroBooNE reconstructed data distribution assuming the low energy excess observed by the MiniBooNE detector is due to an electron-like signal. The event predictions account for MicroBooNE detector efficiency, fiducial volume, and electron-photon separation efficiency. The prediction assumes data collected for $6.0 \times 10^{20} \mathrm{POT}$ in neutrino mode. The error bars on the dashed histogram indicate background+excess statistical uncertainty.

in the $200-475 \mathrm{MeV}$ reconstructed neutrino energy range, compared to a background prediction of $41.6 \pm 6.4$ events. This corresponds to an excess statistical significance of $5.7 \sigma$.

The reconstructed energy spectrum for the photon interpretation is shown in Fig. B-7. For this hypothesis, MicroBooNE would expect to see 36.8 excess events, compared to a background prediction of $78.9 \pm 8.9$ events. This corresponds to an excess statistical significance of $4.1 \sigma$.

In summary, MicroBooNE would be able to resolve the nature of the MiniBooNE low energy excess at 5.7(4.1) $\sigma$, assuming that the excess is due to events reconstructed as single-electrons (single-photons) in the MicroBooNE detector. 


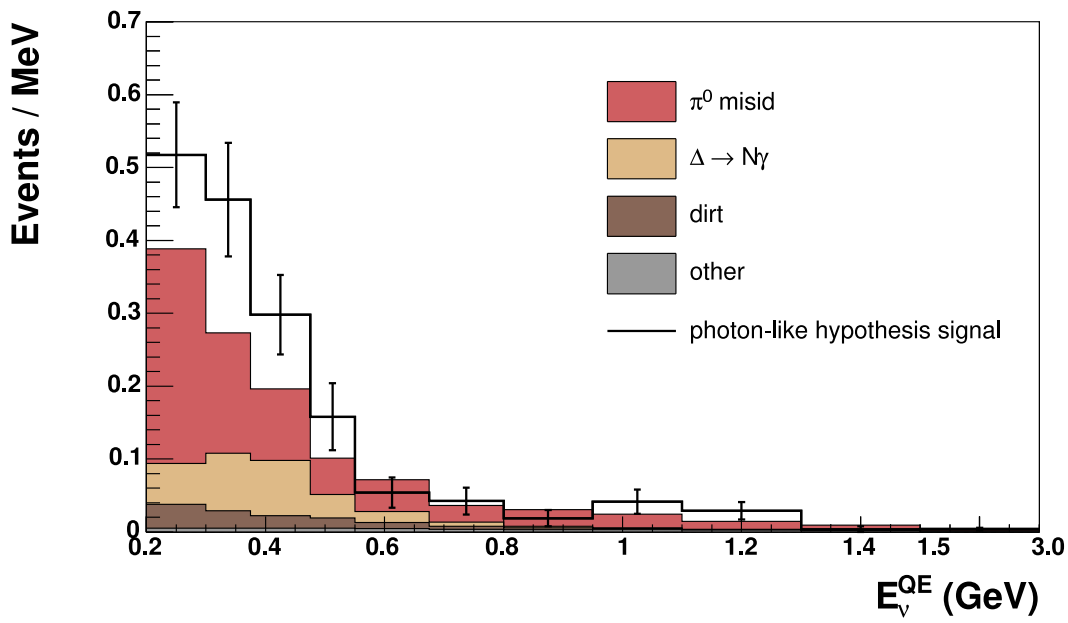

Figure B-7: MicroBooNE expected energy spectrum for photon-like events. The black line corresponds to the MicroBooNE reconstructed data distribution assuming the low energy excess observed by the MiniBooNE detector is due to a photon-like signal. The event predictions account for MicroBooNE detector efficiency, fiducial volume, and electron-photon separation efficiency. The prediction assumes data collected for $6.0 \times 10^{20} \mathrm{POT}$ in neutrino mode. The error bars on the dashed histogram indicate background+excess statistical uncertainty.

\section{B.5.2 Neutrino Oscillations}

Being at a similar $L / E$ as MiniBooNE, MicroBooNE can also perform a search for $\nu_{\mu} \rightarrow \nu_{e}$ oscillations at $\Delta m^{2} \sim 1 \mathrm{eV}^{2}$.

Figure B-8 shows the MicroBooNE sensitivity to $\nu_{\mu} \rightarrow \nu_{e}$ oscillations, for a fit to electron-like events with reconstructed energy above $475 \mathrm{MeV}$, and assuming statistical-only uncertainties (no systematic uncertainties). For comparison, Fig. B-8 also shows the MiniBooNE limit obtained from neutrino mode running, corresponding to $6.46 \times 10^{20} \mathrm{POT}$. The MicroBooNE background prediction assumed for the $\nu_{\mu} \rightarrow \nu_{e}$ oscillation search is identical to that of Fig. B-6, for $E_{\nu}^{Q E}>475 \mathrm{MeV}$.

Due to MicroBooNE's relatively small size, the sensitivity to oscillations is primarily limited by large statistical uncertainties. On the other hand, MicroBooNE's sensitivity benefits from a signal to background enhancement due to the ability to reject photon backgrounds which would otherwise be mis-identified as electrons in a 


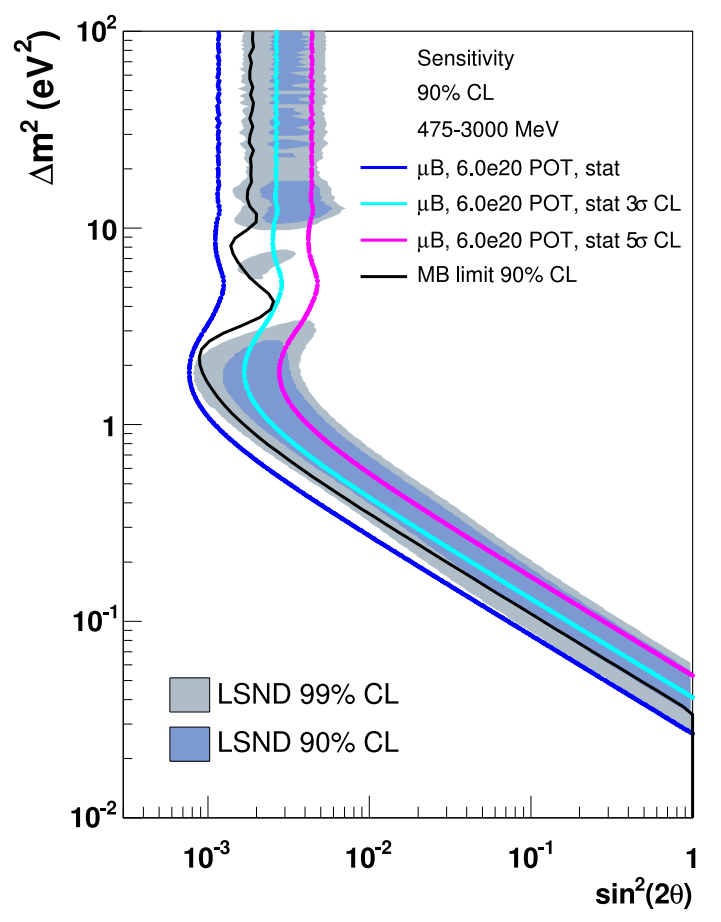

Figure B-8: Comparison of MiniBooNE oscillation result in neutrino mode (MB) to MicroBooNE sensitivity $(\mu \mathrm{B})$ for $\nu_{\mu} \rightarrow \nu_{e}$ oscillations. The MicroBooNE sensitivity is comparable to MiniBooNE's because of the improved detection technique, despite the fact that MicroBooNE is $\sim 5$ times smaller than MiniBooNE. The MicroBooNE sensitivity assumes statistical only uncertainties.

Cherenkov detector like MiniBooNE. As a result, MicroBooNE's sensitivity is comparable to that of MiniBooNE, for similar POT rates $\left(6.0 \times 10^{20}\right.$ and $6.46 \times 10^{20}$, respectively).

The reconstructed energy spectrum for electron neutrino events and the expected excess from the LSND best-fit for $\nu_{\mu} \rightarrow \nu_{e}$ oscillations are illustrated in Fig. B-9. Under the simple, two-neutrino hypothesis, MicroBooNE would expect to observe 43.3 excess events above a background prediction of 110.8 events $(475-3000 \mathrm{MeV})$.

Because for $6.0 \times 10^{20}$ POT the MicroBooNE search for oscillations is expected to be statistics- rather than systematics-limited, it may prove beneficial to consider additional signal channels as a way to enhance statistics. For example one could look 


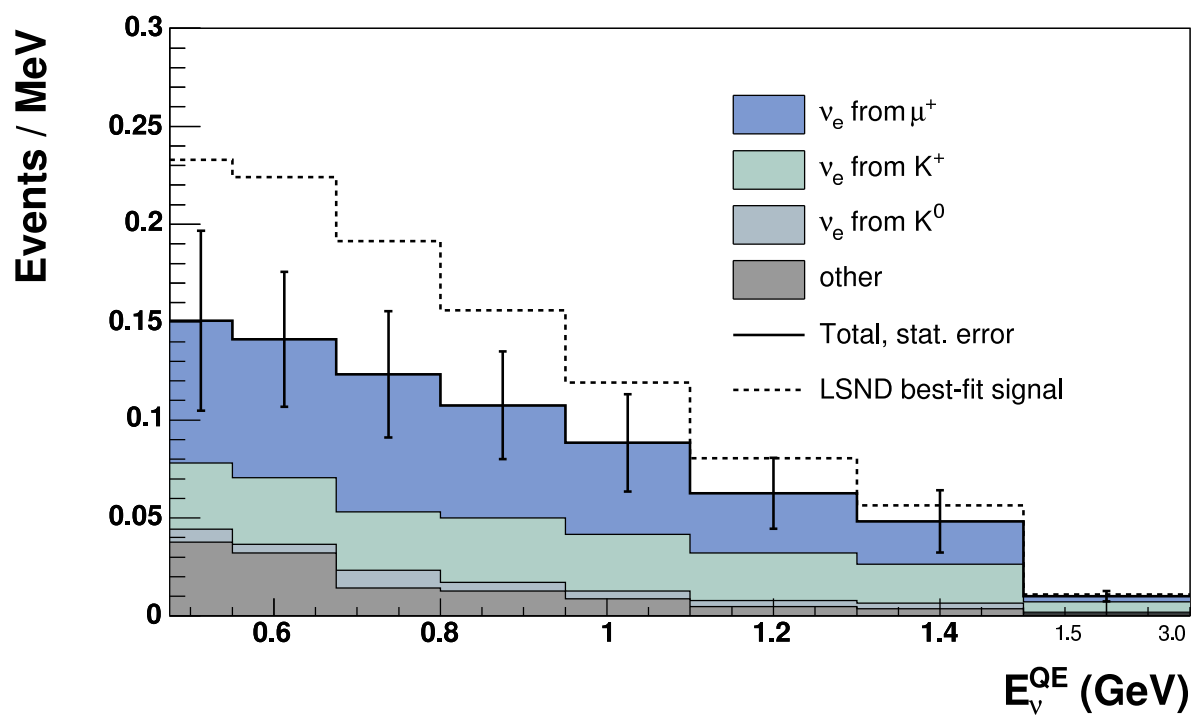

Figure B-9: MicroBooNE $\nu_{e}$ CCQE background spectrum and $\nu_{\mu} \rightarrow \nu_{e}$ signal expectation at the LSND best-fit parameters $\left(\Delta m^{2}=1.2 \mathrm{eV}^{2}, \sin ^{2} 2 \theta=0.003\right)$. Neutrino mode.

for signal from $\nu_{e} \mathrm{CC} \pi^{+}$interactions. CC $\pi^{+}$interactions account for $23.5 \%$ of the total $\nu_{e}$ interaction rate expected in MicroBooNE, and form the next most abundant interaction channel after CCQE (37.2\%) which can also provide a flavor tag for the incoming neutrino (due to the presence of the charged lepton in the final state).

\section{B.5.3 Antineutrino Oscillations}

While the current MicroBooNE run plan focuses on neutrino mode running (motivated primarily by the low energy excess in the MiniBooNE neutrino mode results [101]), given the latest MiniBooNE antineutrino mode results presented in Chapter 5, it is interesting to consider MicroBooNE's sensitivity to oscillations in antineutrino running mode.

To investigate MicroBooNE's sensitivity to $\bar{\nu}_{\mu} \rightarrow \bar{\nu}_{e}$ oscillations, a similar analysis approach is followed as in Sec. B.5.2, except here we start with the full MiniBooNE antineutrino running event rates, and then scale to MicroBooNE rates in a similar fashion. 


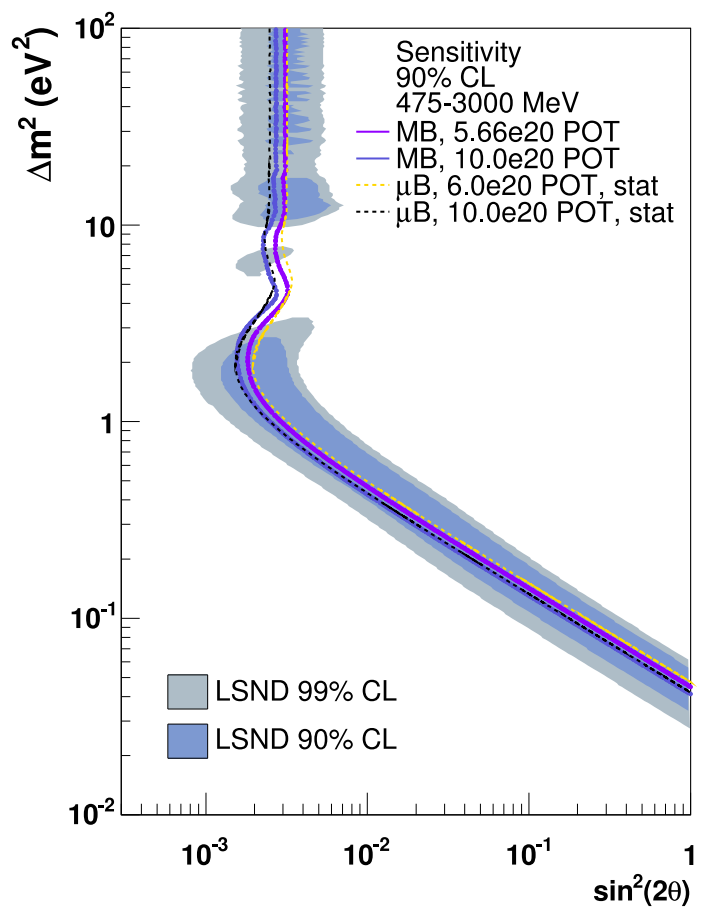

Figure B-10: Comparison of MiniBooNE sensitivity (MB) to MicroBooNE sensitivity $(\mu \mathrm{B})$ in antineutrino mode. The MicroBooNE sensitivity is comparable to MiniBooNE's because of the improved detection technique, despite the fact that MicroBooNE is $\sim 5$ times smaller than MiniBooNE. The MicroBooNE sensitivity is obtained assuming statistical only uncertainties.

Figure B-10 illustrates MicroBooNE's sensitivity to oscillations in antineutrino running mode, assuming statistical only uncertainties, and $6.0 \times 10^{20}$ POT. As in the case of the MiniBooNE $\bar{\nu}_{\mu} \rightarrow \bar{\nu}_{e}$ search, the oscillation signal prediction is assumed to be contributed by the right-sign-only component of the beam. As shown in the figure, MicroBooNE's sensitivity is comparable to that of MiniBooNE, for similar POT, for the same reasoning as in Sec. B.5.2.

The event distribution expected at MicroBooNE for the best-fit parameters suggested by MiniBooNE's latest antineutrino appearance results is shown in the left panel of Fig. B-11. The right panel shows the expected distribution for LSND's best fit oscillation parameters. The number of background and signal events expected in each case, along with the signal statistical significance, are summarized in Tab. B.4. 

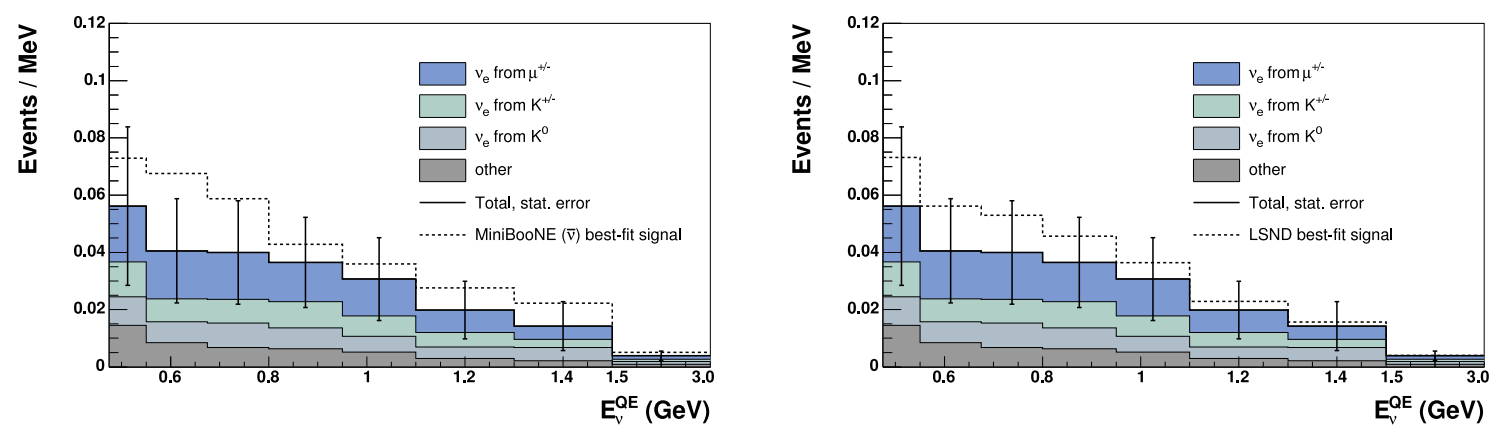

Figure B-11: MicroBooNE $\nu_{e}$ CCQE background spectrum and possible $\bar{\nu}_{\mu} \rightarrow \bar{\nu}_{e}$ signal expectations in antineutrino mode, corresponding to $6.0 \times 10^{20} \mathrm{POT}$. The left plot shows the signal expectation at the MiniBooNE antineutrino best-fit parameters $\left(\Delta m^{2}=4.6 \mathrm{eV}^{2}, \sin ^{2} 2 \theta=0.006\right)$. The right plot shows the signal expectation at the LSND best-fit parameters $\left(\Delta m^{2}=1.2 \mathrm{eV}^{2}, \sin ^{2} 2 \theta=0.003\right)$.

Note that, as in the case of the neutrino mode oscillation search, one may consider additional signal channels, such as $\bar{\nu}_{e} \mathrm{CC} \pi^{-}$events, as a way to enhance statistics. Furthermore, the sensitivity can be further enhanced by rejecting wrong-sign $\nu_{e}$ CCQE backgrounds. This can be done in MicroBooNE by looking for energy deposited by the presence of a proton rather than a neutron at the neutrino interaction vertex. Assuming the same right- versus wrong-sign background composition as in the MiniBooNE (true) CCQE background prediction in the antineutrino mode, a $\sim 40 \%$ background reduction, which corresponds to ignoring the wrong-sign CCQE component of that sample, is expected. The corresponding statistical-only sensitivity, with wrong-sign CCQE-only backgrounds removed from the background prediction, is shown in Fig. B-12.

\section{B.6 R\&D Studies for LAr Scintillation Light De- tection}

The development of a data acquisition system that will be capable of triggering and processing neutrino events in a LArTPC at a large enough rate and with high enough 


\begin{tabular}{lrcc}
\hline \hline & & $\mathbf{6 . 0} \times \mathbf{1 0}^{20} \mathbf{P O T}$ & $\mathbf{1 0 . 0} \times \mathbf{1 0}^{20} \mathbf{P O T}$ \\
\hline \hline Background prediction & $:$ & $37.0 \pm 6.1$ & $61.6 \pm 7.8$ \\
\hline Signal prediction & MiniBooNE best fit: & 13.6 & 22.7 \\
& LSND best fit: & 8.1 & 13.6 \\
\hline Stat. signif. & MiniBooNE best fit: & $2.2 \sigma$ & $2.9 \sigma$ \\
& LSND best fit: & $1.3 \sigma$ & $1.7 \sigma$ \\
\hline \hline
\end{tabular}

Table B.4: MicroBooNE excess statistical significance in a $\bar{\nu}_{\mu} \rightarrow \bar{\nu}_{e}$ oscillation hypothesis. Two specific cases are considered, $\bar{\nu}_{\mu} \rightarrow \bar{\nu}_{e}$ oscillations at the MiniBooNE antineutrino best-fit parameters (MB BF), and $\bar{\nu}_{\mu} \rightarrow \bar{\nu}_{e}$ oscillations at the LSND best-fit parameters (LSND BF). The uncertainties are background statistical only. The statistical significance exceeds $3 \sigma$ for $11.0 \times 10^{20}$ POT, and $5 \sigma$ for $30.0 \times 10^{20}$ POT (obtained using the MiniBooNE best-fit parameters).

efficiency is challenging. An attractive possibility is the use of a light collection system in simultaneous operation with the TPC, triggering on the presence of liquid argon scintillation light [200, 201, 202], which is typically emitted by products of neutrino interactions, in coincidence with the beam spill.

Scintillation light detection, in general, can provide precise timing information due to scintillators' fast time response and recovery time, compared to other detection techniques. In certain cases, scintillation light can provide a variety of other information as well, including energy deposited in the material, ${ }^{9}$ through pulse shape discrimination techniques. It can therefore potentially be used to aid in particle identification.

A challenge in employing this technique in LArTPC's is posed by the fact that liquid argon scintillates in the VUV range (128 nm scintillation wavelength). Because commercially available PMT's are optimized for visible light detection (they are typically made of glass, which is transparent to VUV light), one needs to introduce wavelength shifting materials in the detector, which will convert VUV scintillation light to visible light before PMT detection.

The remainder of this section discusses a set of $R \& D$ studies performed to address the question of how one can efficiently detect liquid argon scintillation light in Mi-

\footnotetext{
${ }^{9}$ Above a certain minimum energy, the light output of a scintillator is directly proportional to the deposited energy.
} 


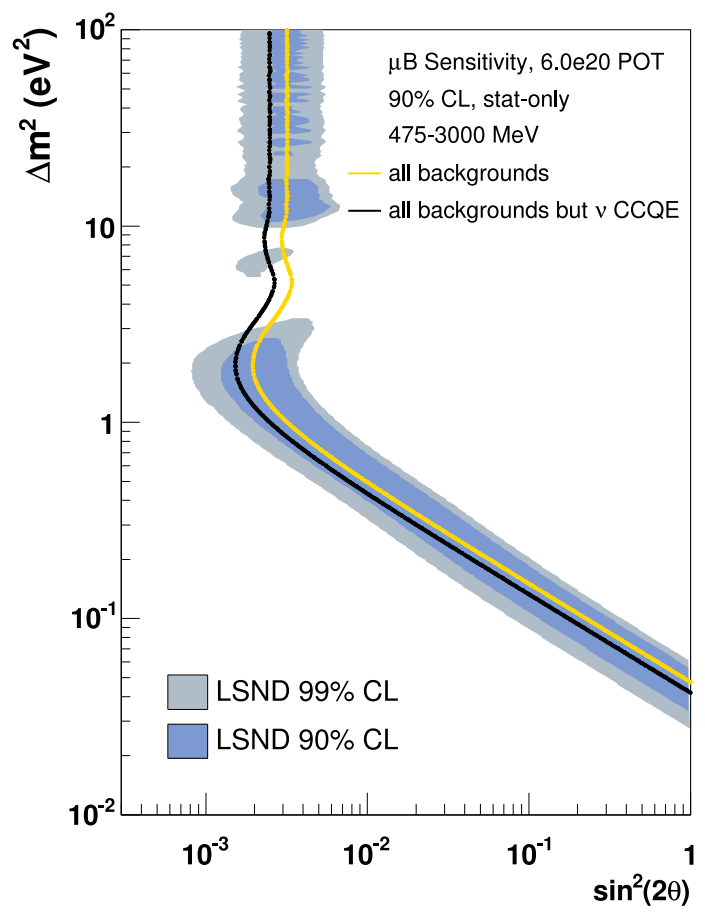

Figure B-12: MicroBooNE estimates for improved sensitivity to $\bar{\nu}_{\mu} \rightarrow \bar{\nu}_{e}$ oscillations in antineutrino running mode. The black curve corresponds to wrong-sign $\nu_{e}$ and (mis-identified) $\nu_{\mu}$ CCQE events removed from the background prediction, assuming $100 \%$ efficiency in identifying the recoil proton in the interaction.

croBooNE with the use of R5912-02MOD Hamamatsu PMT's. These R\&D studies are currently being continued by the MicroBooNE Active Detector Working Group (ADWG).

\section{B.6.1 TPB As a VUV to Visible Wavelength Shifting Mate- rial}

Tetra-Phenyl-Butadiene (TPB) is an attractive fluorescent wavelength shifter which has been successfully used in liquid argon detectors such as WARP, ICARUS, and XMASS [209, 197, 210].

Figure B-13 illustrates the light properties of TPB when illuminated by liquid 


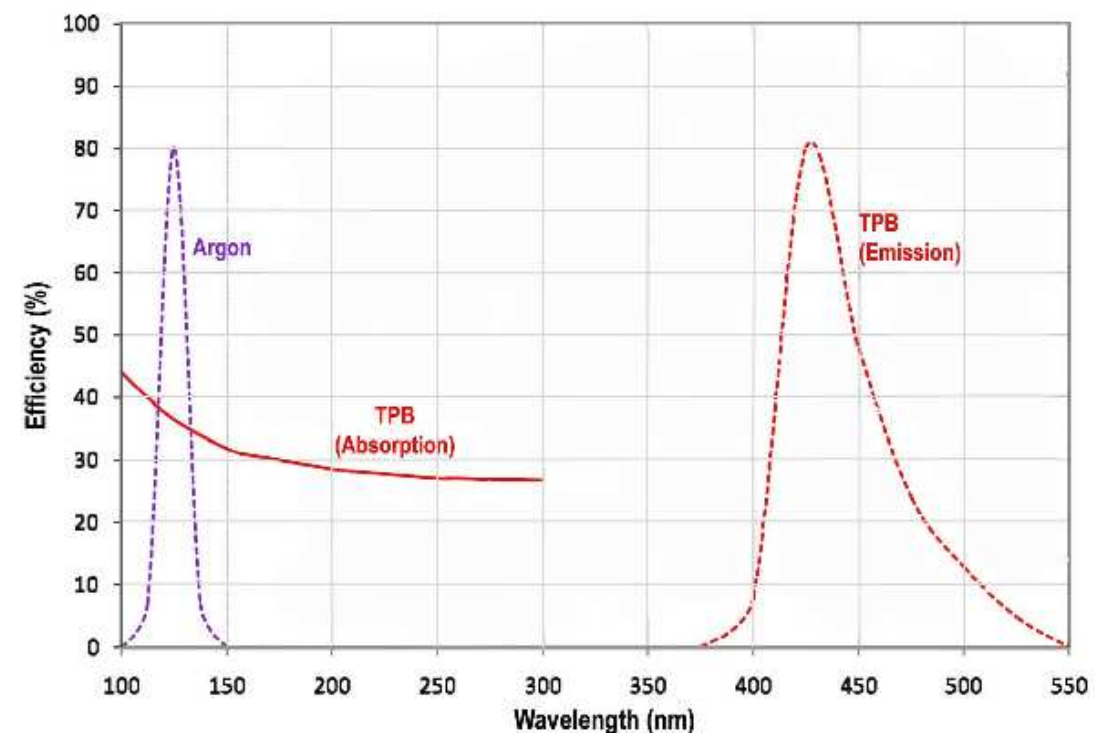

Figure B-13: TPB absorption and emission spectra for liquid argon scintillation light. Scintillation light emitted by liquid argon (128 nm, shown by the purple curve) is absorbed by TPB with roughly $35 \%$ efficiency (shown by the solid red curve) and re-emitted in the visible $(450 \mathrm{~nm})$ range, as shown by the dashed red curve [211].

argon scintillation light. The absorption curve of TPB, shown by the solid red curve, is a good match to the scintillation emission spectrum of liquid argon, shown by the dashed blue line, while the emission spectrum of TPB, shown by the dashed red curve, is a good match to the spectral sensitivity of the PMT's which will be used in the MicroBooNE detector [212], shown in Fig. B-14.

There are two viable options that have been considered as a way to efficiently introduce TPB in the MicroBooNE light collection system. One involves coating the PMT glass surface itself by a thin layer of TPB so that VUV light reaching the PMT surface is converted into visible before penetrating the PMT glass and reaching the photocathode, a method developed for the ICARUS experiment. The second one involves the use of wavelength-shifting TPB-coated plates, positioned in front of each PMT inside the MicroBooNE detector. The two configurations are illustrated in Fig. B-15. 


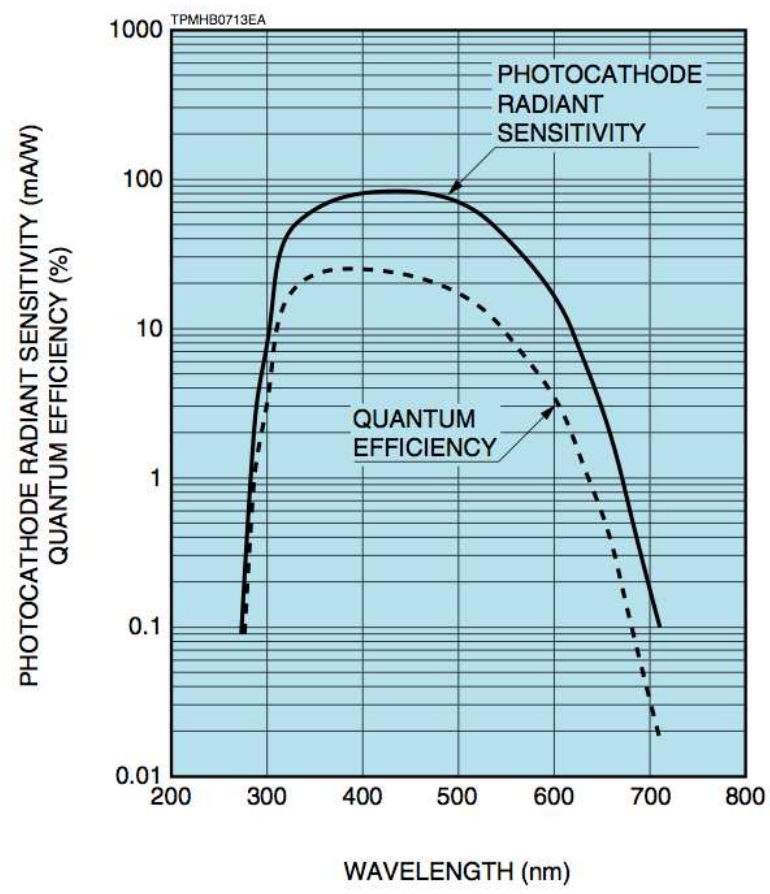

Figure B-14: Spectral efficiency of Hamamatsu R5912-02MOD PMT's [212].

Past experiments faced with this issue have resolved to coating the PMT surface with a thin layer of TPB, in the form of either pure TPB or films of TPB dissolved in polystyrene (PS). Instead, in order to avoid issues related to PMT storage and handling, the MicroBooNE ADWG is more interested in the TPB-coated plate configuration. This technique offers the advantages of easy production, storage, transportation, and installation of both plates and PMT's in the MicroBooNE detector, with minimal risk of damaging the TPB coating and/or PMT, during both production and installation.

\section{B.6.2 Light Collection Efficiency of Two Geometry Configu- rations}

A preliminary study has been performed to investigate the relative light collection efficiency of the two TPB coating configurations of Fig. B-15. The study considers purely geometrical factors to compare the two configurations, given the fact that the 

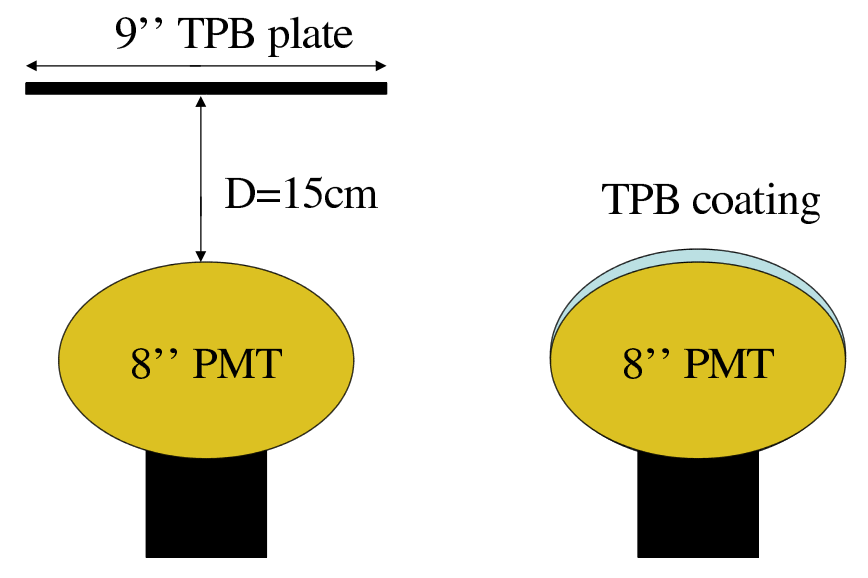

Figure B-15: Cartoon illustration of two light collection system configurations being explored for the MicroBooNE detector. The TPB-coated plate configuration is shown on the left; several plate diameters and positions (D) with respect to PMT surface have been explored. The TPB-coated PMT configuration is shown on the right. The figure is not to scale.

PMT light collection efficiency is highly dependent on the angle of incident light [124]. This dependence is illustrated in Fig. B-16.

Of course, TPB on either surface will emit blue light isotropically. As a result, a relatively larger fraction of converted visible light is redirected away from the PMT face in the case of the TPB-coated plate configuration, so that the TPB-coated PMT configuration has a higher angular acceptance for TPB-emitted blue light. However, the freedom to use a larger plate diameter in the TPB-coated plate configuration counteracts the lower angular acceptance in this configuration, by increasing the TPB cross-section for VUV light conversion, and therefore effectively increasing the photocathode coverage of the PMT.

A study was performed in order to quantify the relative amount of light reaching the PMT cathode for the two configurations. The study assumes parallel (with respect to PMT axis) light illuminating each configuration, a spherical PMT surface, infinitely thin TPB coating and plate, and no absorption, refraction, or reflection. The study accounts for the isotropic emission of TPB light, and the PMT angular efficiency from 


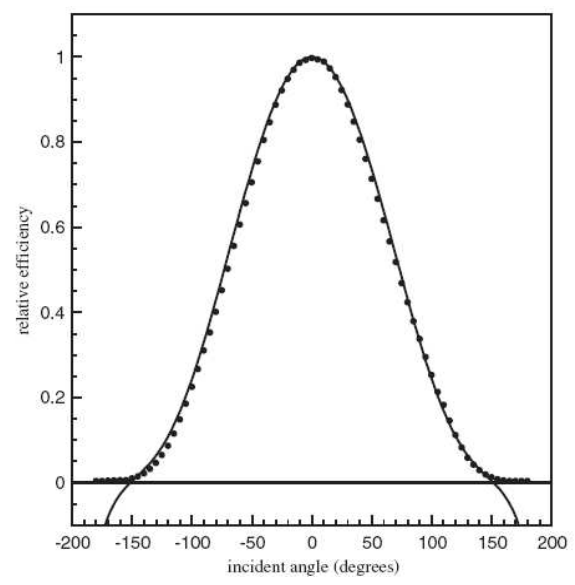

Figure B-16: Relative angular efficiency of an 8-inch Hamamatsu R5912 PMT for a wide beam of parallel light. The plot is from Fig. 12 in [124]. The angle $(\phi)$ is measured with respect to the PMT axis. A fit to data from several PMT's yields an average $\epsilon(\phi)=1-1.182 \times 10^{-4} \phi^{2}+4.959 \times 10^{-9} \phi^{4}-7.371 \times 10^{-11} \phi^{6}$, where $\phi$ is in degrees.

Fig. B-16 [124].

Figure B-17 summarizes the findings of the study. Based on geometrical factors alone, a TPB-coated plate configuration provides an equally viable option to directly coating the PMT surface. Specifically, given geometrical factors alone, the geometry of a 12-inch diameter TPB-coated plate at zero distance from the PMT apex results in comparable efficiency relative to direct PMT surface coating. The efficiency could be increased further with wider plates installed in front of the PMT's.

\section{B.6.3 Coating Options and Plate Materials}

The TPB-coated plate configuration is faced with multiple permutations of possible plate materials and TPB coating methods.

One critical factor in determining acceptable options is the mechanical quality and behavior of the TPB-coated plates at cryogenic temperatures. To determine which plate coating method behaves best in cryogenic conditions, several plates were coated using different coating methods and then tested in a liquid-nitrogen cryocycling test. 


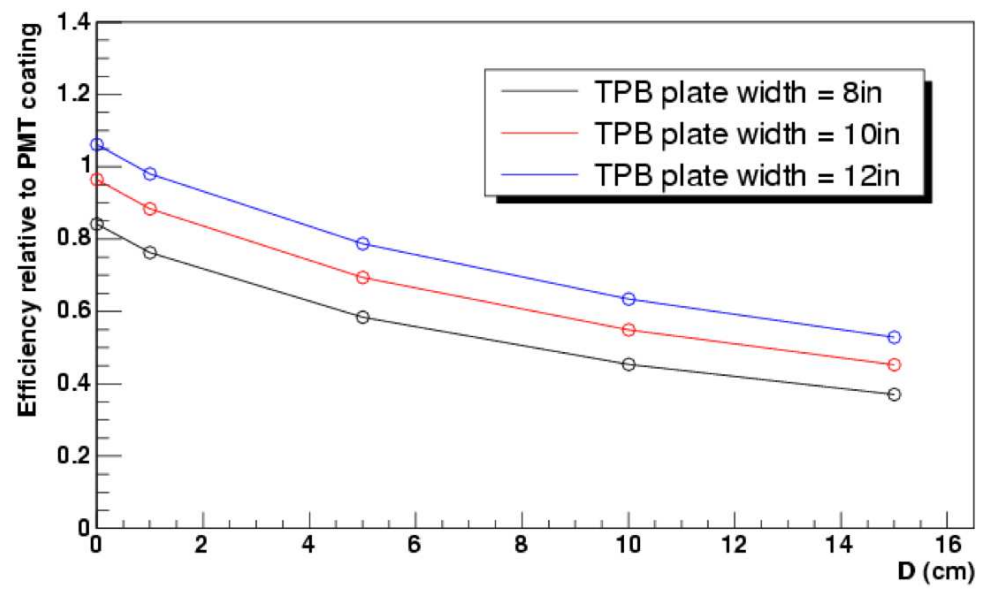

Figure B-17: Light collection efficiency for an 8-, 10-, and 12-inch diameter TPBcoated plate at distance $D$ from the PMT surface apex, relative to a TPB-coated 8-inch PMT surface. For a fixed distance $D$, a wider plate leads to higher light collection efficiency, by diverting more (incident parallel) light to the PMT. For a fixed plate diameter, a smaller distance $(D)$ from the PMT surface leads to higher light collection efficiency, as it allows for a larger solid angle coverage of re-emitted light by the PMT.

Several plate materials, including quartz, borosilicate glass, and acrylic (common and military grade), of similar thickness and surface area were tested, coated with various techniques:

- acrylic (common) plates coated with a TPB-PS mixture using the "drip coating method" (see below),

- glass (thin and thick quartz, and borosilicate) coated with with a TPB-PS mixture using the "drip coating method",

- acrylic (common and military grade) coated with pure TPB using the "paint coating method" (see below).

Because of the powder nature of TPB, the coating is best applied by dissolving certain amounts of TPB in a liquid solvent which is then left to evaporate after the coating is applied (in liquid form) on the surface of interest (acrylic, glass, etc.). The two coating methods considered in this study are described as follows:

- Drip coating: 
Plates were coated using a TPB-PS-toluene mixture which was prepared as follows: $0.33 \mathrm{~g}$ of TPB were dissolved in $50 \mathrm{~mL}$ of toluene, and $1.00 \mathrm{~g}$ of scintillator grade PS pellets were added to the mixture once the TPB was completely dissolved; the mixture was agitated until the PS was completely dissolved. To coat, $3 \mathrm{~mL}$ of the mixture were applied to a clean plate, gently rocking the plate to wet the entire surface. The plate was then left in a horizontal position in a fume hood until the toluene evaporated (typically over $24 \mathrm{hrs}$ ). This resulted in a coating of $25 \%$ TPB concentration, defined as the fraction, by weight, of TPB to TPB plus PS. To produce $15 \%$ and 33\% TPB-PS coatings, $0.18 \mathrm{~g}$ and $0.50 \mathrm{~g}$ of TPB were used, respectively.

- Paint coating:

Plates were coated using a $0.1 \mathrm{~g}$ to $30 \mathrm{~mL}$ TPB to toluene mixture. To coat, roughly $1 \mathrm{~mL}$ of the mixture was applied to the surface using a clean acid brush, in two coating layers. The plate was then left in a horizontal position in the fume hood until the toluene evaporated (typically over $24 \mathrm{hrs}$ ), leaving behind only a layer of pure TPB.

Note that plates prepared using the drip method have a clear coating, while plates prepared using the paint method are cloudy (this is due to the powder nature of TPB).

An additional coating method is that of evaporative coating of TPB. ${ }^{10}$ Preliminary tests suggest that this method yields coatings with optical and mechanical qualities comparable to those of the paint coating method, with the advantage that, with the former method, significantly more uniform TPB coatings across plates as large as 8-inch in diameter, as shown in Fig. B-18, can be obtained. However, because in this coating method TPB is merely deposited on a highly polished surface, the coating can be easily rubbed off, and requires special handling.

Once dried, the various samples were cryocycled in liquid nitrogen $\left(L N_{2}\right)$, one by one. Due to expansion coefficient mismatch between the coating and the plate ma-

\footnotetext{
${ }^{10}$ This coating technique has been developed at Fermilab by E. Hahn. Uniform TPB coatings onto glass or acrylic surfaces are obtained by sublimating TPB from an electrically heated filament onto the plate, in a vacuum environment.
} 


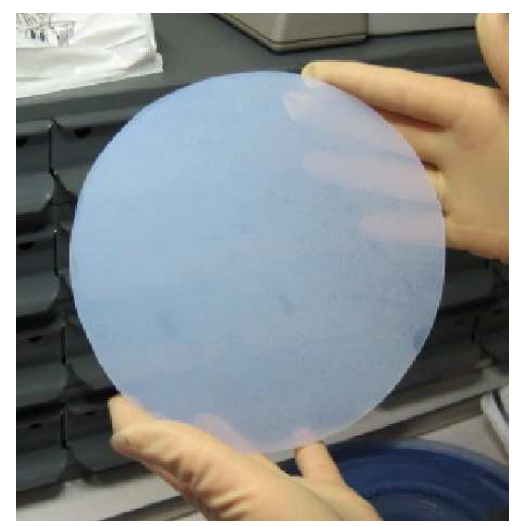

Figure B-18: Evaporatively coated TPB plate.

terial, the TPB-PS coating cracked and de-laminated over the full surface of siliconbased plates (quartz, borosilicate glass, and high-temperature silica) after two immersions in $L N_{2}$, as shown in Fig. B-19. Depending on the thickness of the TPB-PS coating, some acrylic plates also showed de-lamination at the edge of the plate surface. Some representative results are illustrated in Fig. B-19. Plates with thinner TPB-PS coatings $(<0.1 \mathrm{~mm}),{ }^{11}$ show essentially no visible coating degradation after multiple immersions in $L N_{2}$.

The paint coating method (no PS) also showed promising results for acrylic plates. In this case, acrylic degradation during application of the toluene-based mixture and, therefore, mild mixing of acrylic with TPB result in better adhesion of TPB to the plate. Several tests have been performed, which show no visible coating degradation after multiple cryocycling.

Finally, the issue of TPB-PS films bubbling off of acrylic plates during a potential MicroBooNE detector vessel evacuation has also been raised. Bubbling and deformation of the coating could occur if a significant amount of air is trapped between the acrylic and the TPB-PS layer during coating. To test this possibility, two $4 \times 4$ inch $^{2}$ drip-coated plates, one with $15 \%$ and one with $25 \%$ TPB-PS concentration,

\footnotetext{
${ }^{11}$ This is particularly true in the case of plates coated with a variation of the drip coating technique, which used an airbrushing technique to apply much thinner, and more uniform TPB-PS coatings onto acrylic plates.
} 

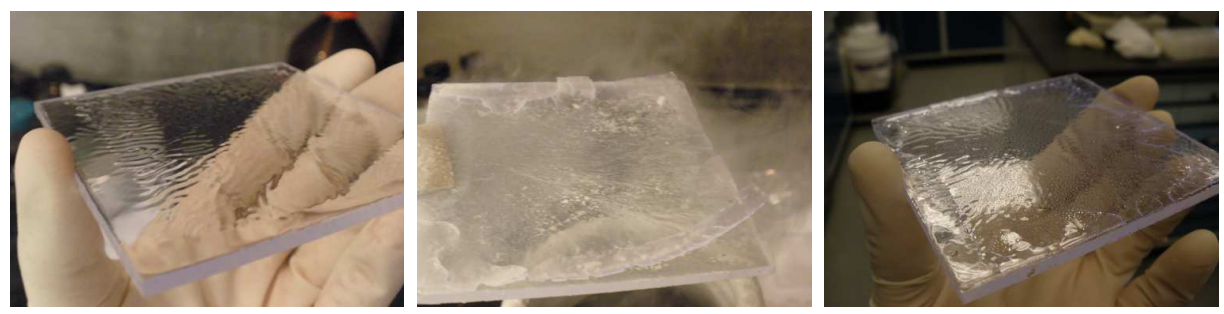

Figure B-19: Testing of TPB-PS drip-coated acrylic plates in liquid nitrogen. Acrylic sample coated with TPB-PS before immersion in liquid nitrogen (left); immediately after immersion (middle); and an hour after immersion (right). The TPB-PS coating detaches from the acrylic surface near the edges of the plate, and visible cracks form across the full surface of the coating. The plate itself shows no signs of cracking.

were tested under vacuum.

A bell jar was used, shown in Fig. B-20. An oil roughing pump was used to pump air out of the jar for roughly 5 hours, during which no evidence of bubbling was found, for an estimated ${ }^{12}$ vacuum level of the order of $10^{-2}$ to $10^{-3}$ Torr. After the test was completed, no visible changes on the coating were found on either one of the plates.

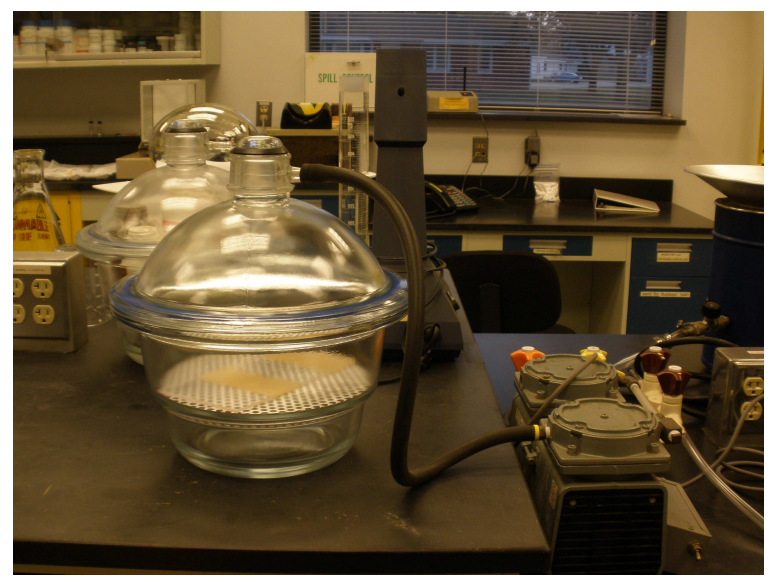

Figure B-20: Vacuum test setup for TPB-coated plates. Two drip-coated plates were placed in a bell jar, connected to a roughing pump (GAST diaphragm pump, model no. DAA-V111-EB, S/N 0888) through a plastic foam tube.

\footnotetext{
${ }^{12}$ The vacuum level was estimated from the vacuum pump specifications.
} 


\section{B.6.4 Light Transmittance and Fluorescence Studies}

Light transmittance and fluorescence measurements in the visible range were performed for various types of plates in consideration for use in the MicroBooNE detector. The plates were coated using the methods described in Sec. B.6.3.

\section{Transmittance Studies}

The transmittance measurement study was performed using a commercial Agilent 8453 UV/Visible Spectrometer. Plate samples, 1/8-inch thick, were compared in terms of their transmittance relative to air at STP for wavelengths ranging between 200-800 nm. The test setup schematic is illustrated in Fig. B-21. Both acrylic and glass samples were studied. Each plate sample measurement was repeated up to five times, to ensure reproducibility of the results.

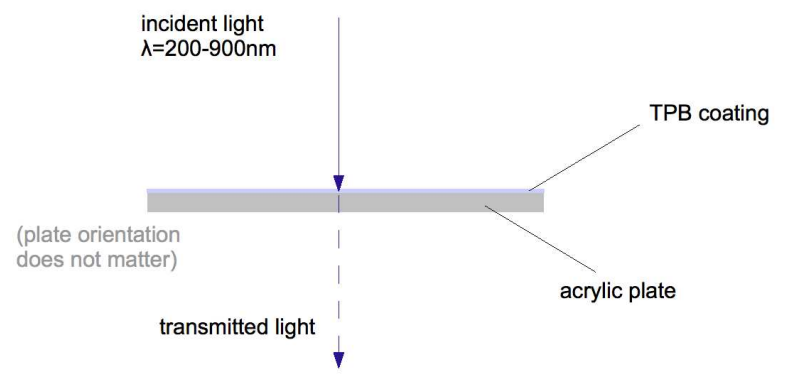

Figure B-21: TPB-coated plate transmittance measurement setup.

Figure B-22 shows representative transmittance curves from each of the plates tested. According to Fig. B-22, quartz and borosilicate glass show the highest transmittance overall, extending down to 200 and $250 \mathrm{~nm}$, respectively. Common acrylic has similar transmittance for wavelengths greater than $400 \mathrm{~nm}$, but once coated with TPB and PS the cutoff wavelength increases to $420 \mathrm{~nm}$, and the transmittance at 450 $\mathrm{nm}$ is reduced by a few \%. The higher wavelength cutoff is presumed to be a result of PS in the coating. Acrylic coated with only TPB, using the paint method, shows an overall reduction in transmittance relative to acrylic with no coating, especially 
around 400-450 nm, due to the opaque nature of TPB powder.

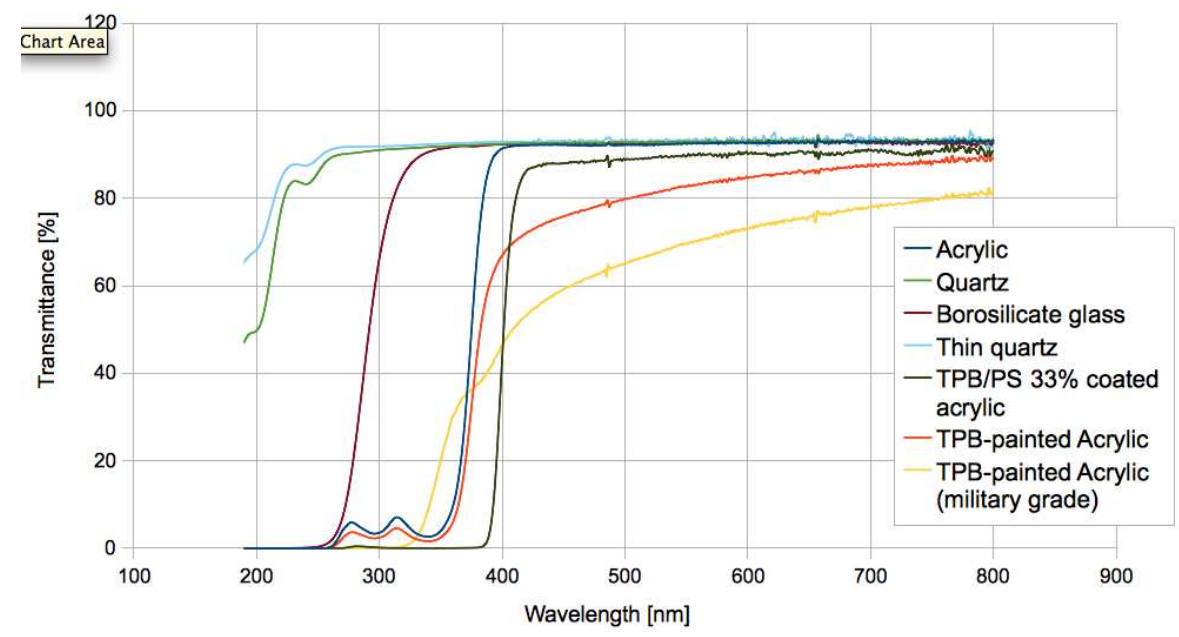

Figure B-22: Transmittance for different 1/8-inch thick plate materials relative to air, with and without TPB coating. Thin quartz corresponds to 1/16-inch thick quartz plate.

Figure B-23 shows a comparison of transmittance for three plates which were coated using the drip method, each with a different relative TPB to PS concentration. All three coatings show similar results, with higher TPB concentration corresponding to lower transmittance for wavelengths greater than $420 \mathrm{~nm}$. However, this effect is roughly within uncertainty, which is of the order of $2-3 \%$.

\section{Fluorescence Studies}

The fluorescence measurement study was performed using a commercial f4500 FL Spectrometer. The test setup is illustrated schematically in Fig. B-24. The spectrometer uses a Xenon lamp with a diffraction grating setup to isolate $200 \pm 10 \mathrm{~nm}$ light. ${ }^{13}$ The light is directed onto a (coated) plate sample, incident at -45 degrees with respect to the normal on the plate surface. Light absorbed by the TPB is reemitted isotropically in the blue range, and detected in the +45 degree direction over the $200-800 \mathrm{~nm}$ range.

\footnotetext{
${ }^{13}$ The study was performed in air, therefore the minimum wavelength available for study was 200 $\mathrm{nm}$.
} 


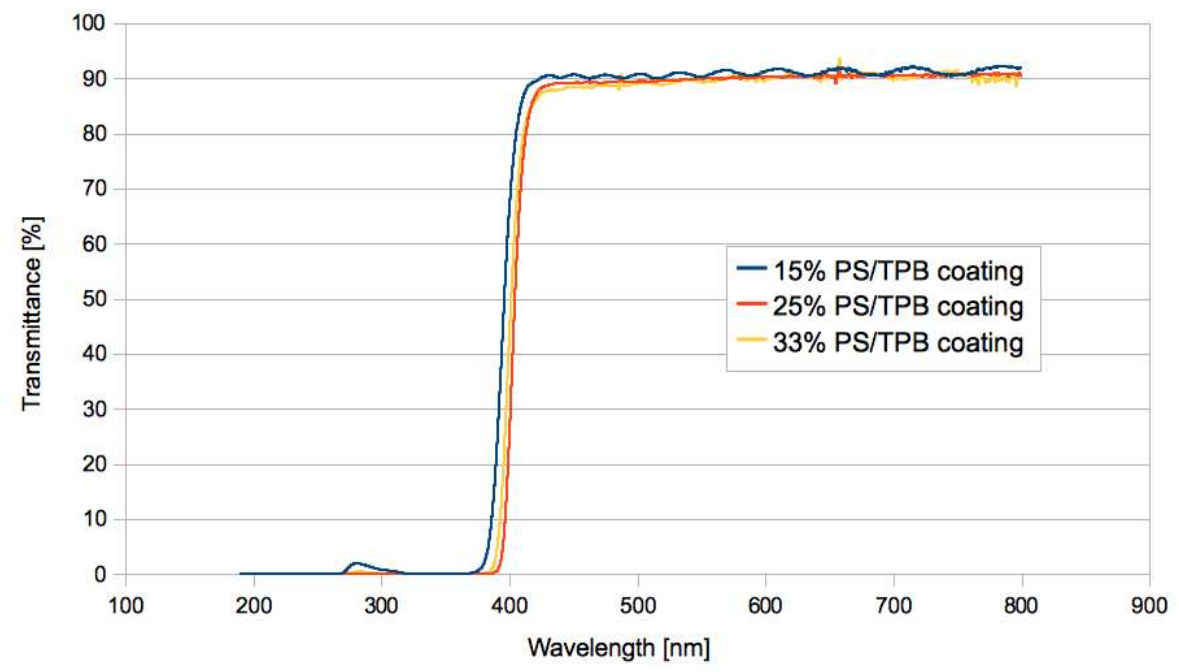

Figure B-23: Transmittance for 1/8-inch acrylic plates relative to air, drip-coated with TPB and PS at different concentrations.

Figure B-25 shows the fluorescence spectra obtained for various types of TPB coatings on acrylic plates. All plates were coated using the drip or paint method outlined in Sec. B.6.3. The scale on the y-axis is arbitrary. However, all panels have been properly normalized relative to a blank acrylic reference plate, so that relative comparisons can be made.

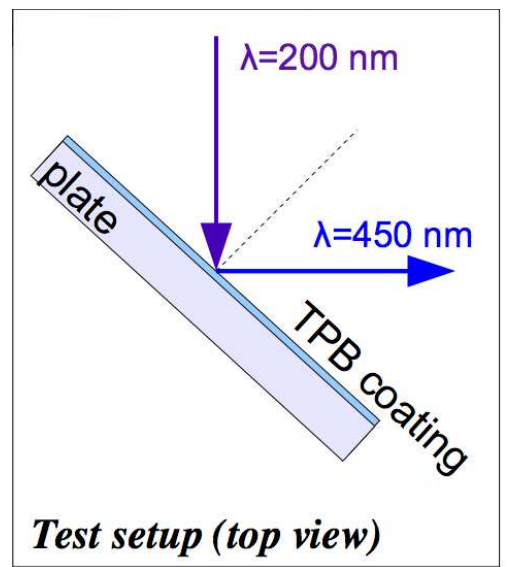

Figure B-24: Schematic of TPB fluorescence measurement test setup.

At least two different plates were measured for each of the $15 \%, 25 \%$, and $33 \%$ 


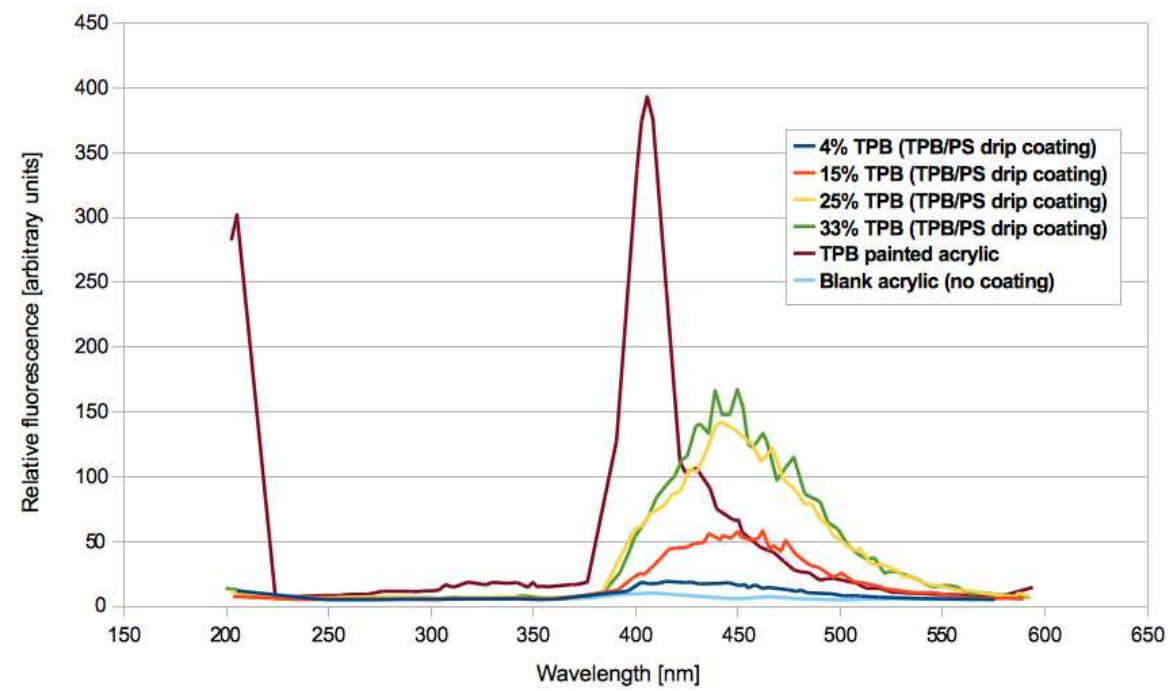

Figure B-25: Fluorescence spectra for various TPB coatings on acrylic plates, for 200 $\mathrm{nm}$ incident wavelength. The peak at $200 \mathrm{~nm}$ is due to partially reflected incident light.

TPB-PS concentration measurements, to ensure consistency. In each case, all plates gave similar results. Fluorescence amplitudes were found to increase with TPB concentration, so a higher concentration of TPB (up to approximately 25\%) would be desirable, if the drip method is chosen.

Plates which were painted with TPB-toluene mixtures showed significantly higher fluorescence amplitudes; however, significant amplitude variation was found across the plate surface (up to $10 \times$ variation in a $4 \times 4$ inch $^{2}$ surface area). An additional worry for this coating method is the lower transmittance yield. Plate comparison measurements in liquid argon currently in progress by the group are sensitive to the combined effect of fluorescence and transmittance, and will therefore conclusively determine which coating method yields the highest light conversion efficiency. Preliminary results in air using a picoquant light source suggest that plates coated with only TPB, using the paint method, show higher overall efficiency than TPB-PS coated plates [213].

An interesting observation was made with plates coated with $33 \%$ TPB-PS mix, which showed evidence of TPB crystallization over time. Those plates also showed 
fluorescence peaks that resembled those of the TPB-painted samples, when the measurement was performed over isolated regions with crystallization.

\section{B.6.5 Summary of R\&D Studies}

Acrylic or glass plates transparent to $400 \mathrm{~nm}$ light and coated with TPB may be installed in the MicroBooNE detector and act as VUV to visible wavelength shifting devices, allowing for efficient detection of $128 \mathrm{~nm}$ LAr scintillation light by PMT's optimized for $400 \mathrm{~nm}$ light detection.

Transmittance measurements for different types of plate materials in air indicate that quartz and borosilicate plates show high transmittance across the widest range of wavelengths (310-800 nm) compared to other plates considered in the study; acrylic plates show comparable transmittance over $380-800 \mathrm{~nm}$.

In terms of coating methods, acrylic plates coated with thin films of TPB-PS efficiently transmit light for wavelengths greater than $420 \mathrm{~nm}$, while acrylic plates coated with pure TPB can partially transmit light at lower wavelengths ( $\geq 360 \mathrm{~nm}$ ), but show significantly lower transmittance above 400-450 nm, compared to samples coated with TPB and PS. Coatings of different TPB-PS concentrations (15\%, 25\%, and 33\%) show no significant difference in transmittance above $420 \mathrm{~nm}$.

On the other hand, fluorescent studies have shown that light fluorescence yield increases with TPB-PS concentration in the coating. Coatings with $25 \%$ concentration are currently the most conservative choice, since there is no dramatic difference in fluorescence yield compared to $33 \%$ concentration, and this choice avoids possible non-uniformities seen in 33\% coatings due to crystallization of TPB, which could complicate light simulation.

Pure TPB coatings show significantly higher fluorescence yields; however, for this coating method, non-uniformity and low transmittance may prove to be an issue, as suggested Fig. B-26. Evaporative coating of TPB is expected to show similar behavior to that of the paint coating method; however, evaporative coating is expected to be advantageous over the paint method because higher TPB uniformity over the plate surface can be achieved with that method. 
If the TPB-PS drip-coating method is chosen as the default, acrylic plates are the conservative choice, due to mechanical considerations alone. The coefficient of expansion of acrylic better matches that of PS, which results in less crazing during cryocycling. 

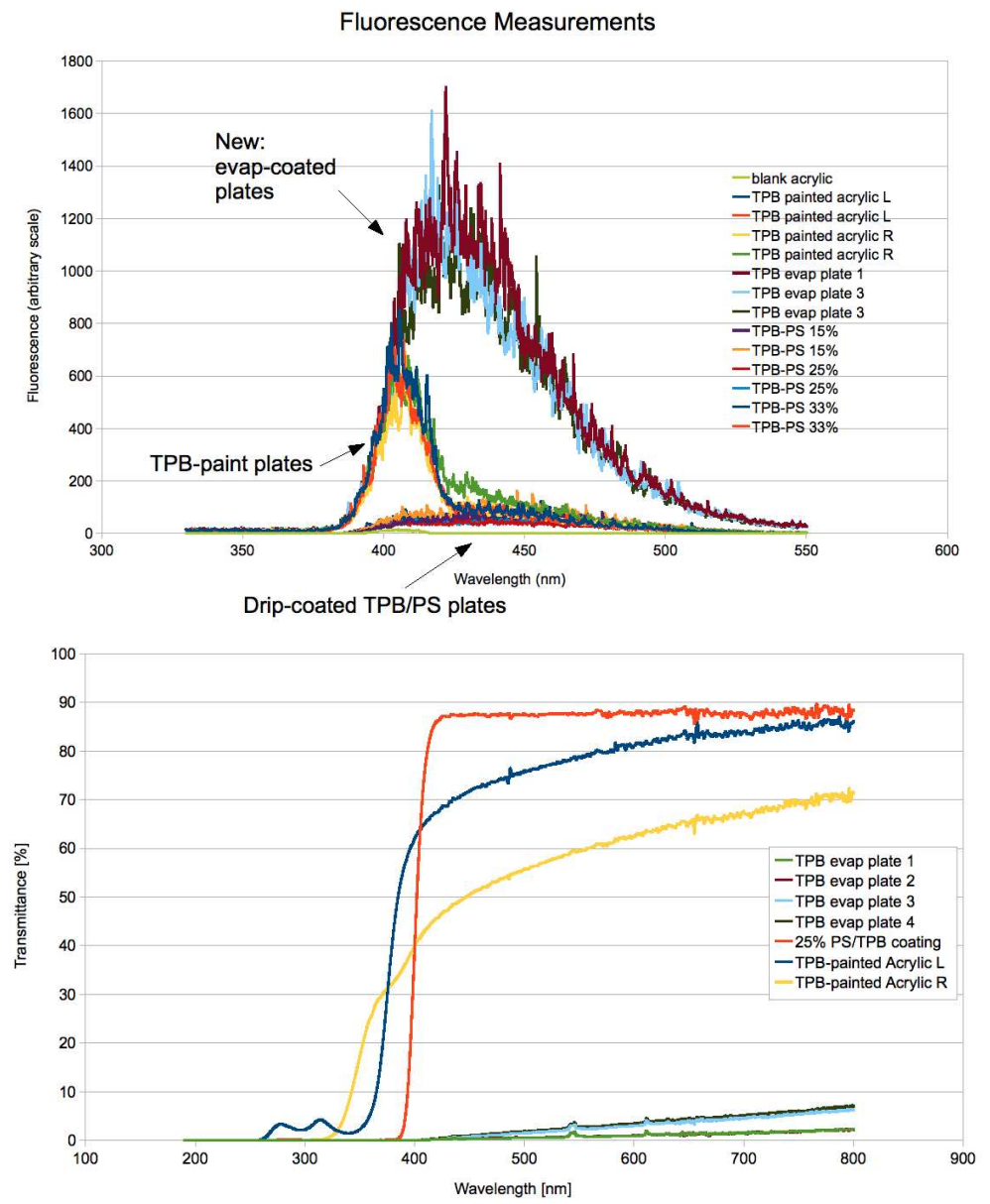

Figure B-26: Summary of fluorescence (top) and transmittance (bottom) measurements for various TPB coatings on acrylic plates. Evaporatively coated plates of various plate (1/8" for plates 1 and 3 , and 1/16" for plates 2 and 4 ) and coating (1.6 $\mu \mathrm{A}$ for plates 1 and 2 , and $2 \mu \mathrm{A}$ for plates 3 and 4 ) thicknesses show the highest fluorescence yield; however they show low transmittance to visible light, due to the powder nature of TPB. 


\section{Bibliography}

[1] F. Abe et al. [CDF Collaboration], Phys. Rev. Lett. 74, 2626 (1995).

[2] S. Abachi et al. [D0 Collaboration], Phys. Rev. Lett. 74, 2422 (1995).

[3] K. Kodama et al. [DONUT Collaboration], Phys. Lett. B 504, 218 (2001).

[4] Wikipedia contributors, "Standard Model," Wikipedia, http://en.wikipedia. org/wiki/File:Standard_Model_of_Elementary_Particles.svg (accessed May 12, 2010).

[5] S. L. Glashow, Nucl. Phys. 22, 579 (1961).

[6] S. Weinberg, Phys. Rev. Lett. 19, 1264 (1967).

[7] A. Salam, in the Proceedings of 8th Nobel Symposium, Lerum, Sweden, 19-25 May 1968, pp 367-377.

[8] Wikipedia contributors, "Standard Model," Wikipedia, http://en.wikipedia. org/wiki/File:Elementary_particle_interactions.svg (accessed May 12, 2010).

[9] K. Nakamura et al. [Particle Data Group], J. Phys. G 37, 075021 (2010).

[10] F. Englert and R. Brout, Phys. Rev. Lett. 13, 321 (1964).

[11] P. W. Higgs, Phys. Rev. Lett. 13, 508 (1964).

[12] G. S. Guralnik, C. R. Hagen and T. W. B. Kibble, Phys. Rev. Lett. 13, 585 (1964). 
[13] Y. Nambu and G. Jona-Lasinio, Phys. Rev. 122, 345 (1961).

[14] J. Goldstone, Nuovo Cim. 19, 154 (1961).

[15] G. Arnison et al. [UA1 Collaboration], Phys. Lett. B 122, 103 (1983).

[16] G. Arnison et al. [UA1 Collaboration], Phys. Lett. B 126, 398 (1983).

[17] C. L. Cowan, F. Reines, F. B. Harrison, H. W. Kruse and A. D. McGuire, Science 124, 103 (1956).

[18] G. Danby, J. M. Gaillard, K. A. Goulianos, L. M. Lederman, N. B. Mistry, M. Schwartz and J. Steinberger, Phys. Rev. Lett. 9, 36 (1962).

[19] M. L. Perl et al., Phys. Rev. Lett. 35, 1489 (1975).

[20] T. D. Lee and C. N. Yang, Phys. Rev. 104, 254 (1956).

[21] C. S. Wu, E. Ambler, R. W. Hayward, D. D. Hoppes and R. P. Hudson, Phys. Rev. 105, 1413 (1957).

[22] M. Goldhaber, L. Grodzins and A. W. Sunyar, Phys. Rev. 109, 1015 (1958).

[23] T. D. Lee and C. N. Yang, Phys. Rev. 105, 1671 (1957).

[24] E. C. G. Sudarshan and R. e. Marshak, Phys. Rev. 109, 1860 (1958).

[25] R. J. Davis, D. S. Harmer and K. C. Hoffman, Phys. Rev. Lett. 20, 1205 (1968).

[26] V. N. Gribov and B. Pontecorvo, Phys. Lett. B 28, 493 (1969).

[27] K. S. Hirata et al. [KAMIOKANDE-II Collaboration], Phys. Lett. B 205, 416 (1988).

[28] R. Becker-Szendy et al., Phys. Rev. D 46, 3720 (1992).

[29] C. Berger et al. [Frejus Collaboration], Phys. Lett. B 227, 489 (1989).

[30] C. Berger et al. [Frejus Collaboration], Phys. Lett. B 245, 305 (1990). 
[31] Q. R. Ahmad et al. [SNO Collaboration], Phys. Rev. Lett. 87, 071301 (2001).

[32] B. Aharmim et al. [SNO Collaboration], Phys. Rev. C 72, 055502 (2005).

[33] P. Minkowski, Phys. Lett. B 67, 421 (1977).

[34] M. Gell-Mann, P. Ramond and R. Slansky, PRINT-80-0576-CERN (1980).

[35] R. N. Mohapatra and G. Senjanovic, Phys. Rev. Lett. 44, 912 (1980).

[36] R. Foot, H. Lew, X. G. He and G. C. Joshi, Z. Phys. C 44, 441 (1989).

[37] G. Lazarides, Q. Shafi and C. Wetterich, Nucl. Phys. B 181, 287 (1981).

[38] J. Schechter and J. W. F. Valle, Phys. Rev. D 25, 774 (1982).

[39] J. W. F. Valle, arXiv:1001.5189 [hep-ph].

[40] S. Dawson and R. N. Mohapatra, Hackensack, USA: World Scientific (2008).

[41] R. N. Mohapatra, eConf C040802, L011 (2004) [New J. Phys. 6, 82 (2004)].

[42] T. Yanagida, Prog. Theor. Phys. 64, 1103 (1980).

[43] R. N. Mohapatra and G. Senjanovic, Phys. Rev. D 23, 165 (1981).

[44] J. H. Christenson, J. W. Cronin, V. L. Fitch and R. Turlay, Phys. Rev. Lett. 13, 138 (1964).

[45] C. Amsler et al. [Particle Data Group], Phys. Lett. B 667, 1 (2008).

[46] G. L. Fogli, E. Lisi and G. Scioscia, Phys. Rev. D 52, 5334 (1995).

[47] J. Schechter and J. W. F. Valle, Phys. Rev. D 23, 1666 (1981).

[48] J. F. Nieves and P. B. Pal, Phys. Rev. D 64, 076005 (2001).

[49] A. de Gouvea, B. Kayser and R. N. Mohapatra, Phys. Rev. D 67, 053004 (2003).

[50] D. W. Schmitz, FERMILAB-THESIS-2008-26. 
[51] D. S. Ayres et al. [NOvA Collaboration], arXiv:hep-ex/0503053.

[52] M. Maltoni, T. Schwetz, M. A. Tortola and J. W. F. Valle, New J. Phys. 6, 122 (2004).

[53] Y. Fukuda et al. [Super-Kamiokande Collaboration], Phys. Rev. Lett. 81, 1562 (1998).

[54] Y. Ashie et al. [Super-Kamiokande Collaboration], Phys. Rev. D 71, 112005 (2005).

[55] Y. Ashie et al. [Super-Kamiokande Collaboration], Phys. Rev. Lett. 93, 101801 (2004).

[56] D. G. Michael et al. [MINOS Collaboration], Phys. Rev. Lett. 97, 191801 (2006).

[57] M. H. Ahn et al. [K2K Collaboration], Phys. Rev. D 74, 072003 (2006).

[58] M. Sanchez, Fermilab PAC presentation 2008.

[59] L. Wolfenstein, in West Lafayette 1978, Proceedings, Neutrinos 'ry, West Lafayette 1978, C3-C6 and Washington 1978, Proceedings, Long-distance Neutrino Detection, 108-112.

[60] M. C. Gonzalez-Garcia and M. Maltoni, Phys. Rept. 460, 1 (2008).

[61] T. Araki et al. [KamLAND Collaboration], Phys. Rev. Lett. 94, 081801 (2005).

[62] S. Abe et al. [KamLAND Collaboration], Phys. Rev. Lett. 100, 221803 (2008).

[63] M. Apollonio et al. [CHOOZ Collaboration], Eur. Phys. J. C 27, 331 (2003).

[64] Y. Declais et al., Nucl. Phys. B 434, 503 (1995).

[65] F. Boehm et al., Phys. Rev. D 64, 112001 (2001).

[66] G. L. Fogli, E. Lisi, A. Marrone, A. Palazzo and A. M. Rotunno, Prog. Part. Nucl. Phys. 57, 71 (2006). 
[67] M. C. Gonzalez-Garcia, M. Maltoni and J. Salvado, JHEP 1004, 056 (2010).

[68] F. Ardellier et al. [Double Chooz Collaboration], arXiv:hep-ex/0606025.

[69] J. K. Ahn et al. [RENO Collaboration], arXiv:1003.1391 [hep-ex].

[70] H. Steiner [Daya Bay Collaboration], Prog. Part. Nucl. Phys. 64, 342 (2010).

[71] M. C. Sanchez [MINOS Collaboration], arXiv:0909.2362 [hep-ex].

[72] Y. Itow et al. [The T2K Collaboration], arXiv:hep-ex/0106019.

[73] L. Bornschein [KATRIN Collaboration], Nucl. Phys. A 752, 14 (2005).

[74] S. Kraft-Bermuth [MARE Collaboration], J. Phys. Conf. Ser. 136, 042055 (2008).

[75] C. Kraus et al., Eur. Phys. J. C 40, 447 (2005).

[76] R. Barate et al. [ALEPH Collaboration], Eur. Phys. J. C 2, 395 (1998).

[77] D. Denegri, B. Sadoulet and M. Spiro, Rev. Mod. Phys. 62, 1 (1990).

[78] U. Seljak, A. Slosar and P. McDonald, JCAP 0610, 014 (2006).

[79] J. Lesgourgues and S. Pastor, Phys. Rept. 429, 307 (2006).

[80] A. Melchiorri, O. Mena, S. Palomares-Ruiz, S. Pascoli, A. Slosar and M. Sorel, JCAP 0901, 036 (2009).

[81] G. Karagiorgi, A. Aguilar-Arevalo, J. M. Conrad, M. H. Shaevitz, K. Whisnant, M. Sorel and V. Barger, Phys. Rev. D 75, 013011 (2007) [Erratum-ibid. D 80, $099902(2009)]$.

[82] G. Karagiorgi, Z. Djurcic, J. M. Conrad, M. H. Shaevitz and M. Sorel, Phys. Rev. D 80, 073001 (2009) [Erratum-ibid. D 81, 039902 (2010)].

[83] B. Bekman, J. Gluza, J. Holeczek, J. Syska and M. Zralek, Phys. Rev. D 66, 093004 (2002). 
[84] S. M. Bilenky and C. Giunti, Phys. Lett. B 300, 137 (1993).

[85] V. Barger, S. Geer and K. Whisnant, New J. Phys. 6, 135 (2004).

[86] W. Loinaz, N. Okamura, T. Takeuchi and L. C. R. Wijewardhana, Phys. Rev. D 67, 073012 (2003).

[87] W. Loinaz, N. Okamura, S. Rayyan, T. Takeuchi and L. C. R. Wijewardhana, Phys. Rev. D 70, 113004 (2004).

[88] B. Kayser, G. Karagiorgi and J. M. Conrad, in preparation.

[89] S. Antusch, C. Biggio, E. Fernandez-Martinez, M. B. Gavela and J. Lopez-Pavon, JHEP 0610, 084 (2006).

[90] B. Kayser and L. Stodolsky, Phys. Lett. B 359, 343 (1995).

[91] C. Giunti, arXiv:hep-ph/0402217.

[92] E. Komatsu et al., arXiv:1001.4538 [astro-ph.CO].

[93] K. Abazajian, N. F. Bell, G. M. Fuller and Y. Y. Y. Wong, Phys. Rev. D 72, $063004(2005)$.

[94] G. Gelmini, S. Palomares-Ruiz and S. Pascoli, Phys. Rev. Lett. 93, 081302 (2004).

[95] Y. Farzan and A. Y. Smirnov, Phys. Lett. B 557, 224 (2003).

[96] Y. Farzan, O. L. G. Peres and A. Y. Smirnov, Nucl. Phys. B 612, 59 (2001).

[97] P. Gorla [CUORE Collaboration], AIP Conf. Proc. 1182, 116 (2009).

[98] A. de Gouvea, J. Jenkins and N. Vasudevan, Phys. Rev. D 75, 013003 (2007).

[99] [ALEPH Collaboration and DELPHI Collaboration and L3 Collaboration], Phys. Rept. 427, 257 (2006).

[100] H. B. Li et al. [TEXONO Collaboration], Phys. Rev. Lett. 90, 131802 (2003). 
[101] A. A. Aguilar-Arevalo et al. [MiniBooNE Collaboration], Phys. Rev. Lett. 102, $101802(2009)$.

[102] A. A. Aguilar-Arevalo et al. [MiniBooNE Collaboration], Phys. Rev. Lett. 103, 111801 (2009).

[103] K. Eitel [KARMEN Collaboration], Nucl. Phys. Proc. Suppl. 91, 191 (2001).

[104] P. Astier et al. [NOMAD Collaboration], Nucl. Phys. B 611, 3 (2001).

[105] C. Athanassopoulos et al. [LSND Collaboration], Nucl. Instrum. Meth. A 388, 149 (1997).

[106] C. Athanassopoulos et al. [LSND Collaboration], Phys. Rev. Lett. 77, 3082 (1996).

[107] A. Aguilar et al. [LSND Collaboration], Phys. Rev. D 64, 112007 (2001).

[108] B. Armbruster et al. [KARMEN Collaboration], Phys. Rev. D 65, 112001 (2002).

[109] E. D. Church, K. Eitel, G. B. Mills and M. Steidl, Phys. Rev. D 66, 013001 (2002).

[110] G. W. Sullivan [E-0776 Collaboration], UMI-90-21764 (1990).

[111] I. E. Stockdale et al., Phys. Rev. Lett. 52, 1384 (1984).

[112] F. Dydak et al., Phys. Lett. B 134, 281 (1984).

[113] M. Sorel, UMI-31-74896.

[114] A. O. Bazarko [BooNe Collaboration], arXiv:hep-ex/9906003.

[115] H. Chen et al. [MicroBooNE Collaboration], FERMILAB-PROPOSAL-0974.

[116] E. Church et al. [BooNe Collaboration], FERMILAB-PROPOSAL-0898. 
[117] A. A. Aguilar-Arevalo et al. [MiniBooNE Collaboration], Phys. Rev. Lett. 103, $061802(2009)$.

[118] K. B. M. Mahn, FERMILAB-THESIS-2009-22.

[119] I. Stancu et al. [MiniBooNE Collaboration], http://www-boone.fnal.gov/ about_boone/publicpages/8gevtdr_2.0.ps.gz

[120] T. Katori, FERMILAB-THESIS-2008-64.

[121] C. M. Bhat, MiniBooNE Technical Note 49.

[122] A. A. Aguilar-Arevalo et al. [MiniBooNE Collaboration], Nucl. Instrum. Meth. A 599, 28 (2009).

[123] S. J. Brice et al., Nucl. Instrum. Meth. A 562, 97 (2006).

[124] B. T. Fleming, L. Bugel, E. Hawker, V. Sandberg, S. Koutsoliotas, S. McKenney and D. Smith, IEEE Trans. Nucl. Sci. 49, 984 (2002).

[125] A. A. Aguilar-Arevalo et al. [MiniBooNE Collaboration], Phys. Rev. D 79, $072002(2009)$.

[126] GEANT4 Reference Manual, http://geant4.web.cern.ch/geant4/ UserDocumentation/UsersGuides/PhysicsReferenceManual/html/node110. html

[127] S. Agostinelli et al. [GEANT4 Collaboration], Nucl. Instrum. Meth. A 506, 250 (2003).

[128] J. Allison et al., IEEE Trans. Nucl. Sci. 53, 270 (2006).

[129] M. G. Catanesi et al., Eur. Phys. J. C 52, 29 (2007).

[130] I. Chemakin et al. [E910 Collaboration], Phys. Rev. C 77, 015209 (2008) [Erratum-ibid. C 77, 049903 (2008)].

[131] Yu. D. Aleshin, I. A. Drabkin and V. V. Kolesnikov, ITEP-80-1977. 
[132] T. Abbott et al. [E-802 Collaboration], Phys. Rev. D 45, 3906 (1992).

[133] J. V. Allaby et al. CERN Report No. CERN 70-12, unpublished.

[134] D. Dekkers et al., Phys. Rev. 137, B962 (1965).

[135] T. Eichten et al., Nucl. Phys. B 44, 333 (1972).

[136] R. A. Lundy, T. B. Novey, D. D. Yovanovitch and V. L. Telegdi, Phys. Rev. Lett. 14, 504 (1965).

[137] G. J. Marmer et al., Phys. Rev. 179, 1294 (1969).

[138] I. A. Vorontsov, G. A. Safronov, A. A. Sibirtsev, G. N. Smirnov and Yu. V. Trebukhovsky, ITEP-88-011.

[139] F. Abe et al., Phys. Rev. D 36, 1302 (1987).

[140] I. Chemakin et al. [E910 Collaboration], Nucl. Phys. A 639, 407 (1998).

[141] J. R. Sanford and C. L. Wang, BNL-11479.

[142] R. P. Feynman, Phys. Rev. Lett. 23, 1415 (1969).

[143] M. H. Shaevitz, MiniBooNE Technical Note 209.

[144] N. V. Mokhov, S. I. Striganov, A. Van Ginneken, S. G. Mashnik, A. J. Sierk and J. Ranft, arXiv:nucl-th/9812038.

[145] D. Casper, Nucl. Phys. Proc. Suppl. 112, 161 (2002).

[146] A. A. Aguilar-Arevalo et al. [MiniBooNE Collaboration], Phys. Rev. Lett. 100, 032301 (2008).

[147] C. H. Llewellyn Smith, Phys. Rept. 3, 261 (1972).

[148] R. Bradford, A. Bodek, H. S. Budd and J. Arrington, Nucl. Phys. Proc. Suppl. 159, 127 (2006). 
[149] V. Bernard, L. Elouadrhiri and U. G. Meissner, J. Phys. G 28, R1 (2002).

[150] R. Gran et al. [K2K Collaboration], Phys. Rev. D 74, 052002 (2006).

[151] J. L. Alcaraz-Aunion and J. Walding [SciBooNE Collaboration], AIP Conf. Proc. 1189, 145 (2009).

[152] R. A. Smith and E. J. Moniz, Nucl. Phys. B 43, 605 (1972) [Erratum-ibid. B 101, $547(1975)]$.

[153] J. Grange, MiniBooNE Technical Note 273.

[154] A. A. Aguilar-Arevalo et al. [MiniBooNE Collaboration], Phys. Rev. Lett. 103, 081801 (2009).

[155] A. A. Aguilar-Arevalo et al. [MiniBooNE Collaboration], Phys. Lett. B 664, 41 (2008).

[156] A. A. Aguilar-Arevalo et al. [The MiniBooNE Collaboration], Phys. Rev. Lett. 98, 231801 (2007).

[157] P. Meyers and H. Tanaka, MiniBooNE Technical Note 228.

[158] P. Meyers, MiniBooNE Technical Note 135.

[159] C. Zeitnitz and T. A. Gabriel, Nucl. Instrum. Meth. A 349, 106 (1994).

[160] G. Battistoni et al., AIP Conf. Proc. 896, 31 (2007).

[161] B. C. Brown et al., IEEE Nucl. Sci. Symp. Conf. Rec. 1, 652 (2004).

[162] J. L. Raaf et al., IEEE Trans. Nuc. Sci. NS-49 (2002) 957.

[163] R. B. Patterson, FERMILAB-THESIS-2007-19 (2007).

[164] R. B. Patterson, E. M. Laird, Y. Liu, P. D. Meyers, I. Stancu and H. A. Tanaka, Nucl. Instrum. Meth. A 608, 206 (2009). 
[165] A. A. Aguilar-Arevalo et al. [The MiniBooNE Collaboration], arXiv:1007.1150 [hep-ex].

[166] J. Grange, MiniBooNE Technical Note 283.

[167] G. J. Feldman and R. D. Cousins, Phys. Rev. D 57, 3873 (1998).

[168] A. A. Aguilar-Arevalo, MiniBooNE Technical Note 223, see also Addendum I and II.

[169] Z. Pavlovic, MiniBooNE Technical Note 291.

[170] F. James, "Statistical Methods in Experimental Physics."

[171] A. A. Aguilar-Arevalo et al. [MiniBooNE Collaboration], Phys. Rev. D 81, 013005 (2010).

[172] Z. Djurcic and W. C. Louis, MiniBooNE Technical Note 249.

[173] Z. Djurcic and W. C. Louis, MiniBooNE Technical Note 280.

[174] R. Van de Water, MiniBooNE Technical Note 251.

[175] S. Brice, MiniBooNE Technical Note 257.

[176] G. Garvey, MiniBooNE Technical Note 242, see also Addendum I.

[177] G. Zeller, G. Garvey and W. C. Louis, MiniBooNE Technical Note 245.

[178] T. Katori, R. Tayloe and G. Zeller, MiniBooNE Technical Note 219.

[179] T. Schwetz, arXiv:0805.2234 [hep-ph].

[180] M. Sterbenz, MiniBooNE Technical Note 229.

[181] M. Sorel, J. M. Conrad and M. Shaevitz, Phys. Rev. D 70, 073004 (2004).

[182] G. Karagiorgi, Neutrino 2010, June 14-19, 2010, Athens, Greece. 
[183] P. Brâemaud, Markov chains: Gibbs fields, Monte Carlo simulation, and queues, Springer, New York, 1999.

[184] M. Shaevitz, MiniBooNE Technical Note 255.

[185] Z. Pavlovic, MiniBooNE Collaboration Meeting, August 2008.

[186] Fermilab Steering Group Report, August 2007.

[187] L. Bartoszek et al., arXiv:hep-ex/0408121.

[188] D. Finley et al., FERMILAB-FN-0776-E (2005).

[189] Fermilab Directorate Report, "Director's Initial Review of the MicroBooNE Project", 2009, MicroBooNE doc-db 691.

[190] Fermilab Accelerator Experiments' Run Plan, 2010, http: //www.fnal.gov/directorate/program_planning/schedule/ Draft10-13LongRangeSchedule-v8_06_15_10.pdf

[191] H. H. . Chen, P. E. Condon, B. C. Barish and F. J. Sciulli, FERMILABPROPOSAL-0496 (1976).

[192] E. Gatti et al., IEEE Trans. Nucl. Sci. Vol. NS-26 (1978) 2910.

[193] C. Rubbia, CERN-EP-INT-77-08 (1977).

[194] ICARUS Collaboration, "ICARUS: a proposal for the Gran Sasso Laboratory", INFN/AE-85/7, Frascati, Italy, 1985.

[195] P. Cennini et al. [ICARUS Collaboration], ICARUS-PROPOSAL (1993).

[196] The ArgoNeut Project at Fermilab, http://t962.fnal.gov

[197] S. Amerio et al. [ICARUS Collaboration], Nucl. Instrum. Meth. A 527, 329 (2004).

[198] M. Soderberg [ArgoNeuT Collaboration], arXiv:0910.3433 [physics.ins-det]. 
[199] "Recommendations to the Department of Energy and the National Science Foundation on a Future U.S. Program in Neutrino Oscillations", submitted by the Neutrino Scientific Assesment Group, http://www.science.doe.gov/hep/ hepap_reports.shtm

[200] A. Hitachi, T. Takahashi, N. Funayama, K. Masuda, J. Kikuchi and T. Doke, Phys. Rev. B 27, 5279 (1983).

[201] P. Cennini et al., Nucl. Instrum. Meth. A 432, 240 (1999).

[202] T. Doke et al., Nucl. Instrum. Meth. A 269, 291 (1988).

[203] MicroBooNE Conceptual Design Report, February 2010, MicroBooNE doc-db 340.

[204] J. Spitz, MicroBooNE doc-db 277.

[205] J. Spitz, MicroBooNE doc-db 783.

[206] E. Kearns et al. [T2K Collaboration], "A Proposal for a Detector 2 km Away From the T2K Neutrino Source", 2007.

[207] A. Martinez de la Ossa Romero, arXiv:hep-ex/0703026.

[208] J. Spitz, MicroBooNE doc-db 115.

[209] R. Brunetti et al. [WARP Collaboration], arXiv:astro-ph/0411491.

[210] Y. Suzuki [XMASS Collaboration], PoS IDM2008, 001 (2008).

[211] K. Arisaka, ICEPP Seminar, University of Tokyo (2008).

[212] R5912 PMT spec, http://sales.hamamatsu.com/en/products/ electron-tube-division/detectors/photomultiplier-tubes/ part-r5912.php

[213] L. Bugel, MicroBooNE doc-db 910. 